\title{
Documentation, User Support, and Verification of Wind Turbine and Plant Models
}

Sponsoring Office:

U.S. Department of Energy (DOE)

Golden Field Office

1617 Cole Blvd.

Golden, CO 80401

Awarded To:

EnerNex LLC

Attn: Jeffrey Lamoree

620 Mabry Hood Road, Suite 300

Knoxville, TN 37932

Tel.: (865) 218-4600 ext. 6131

Fax.: (865) 218-8999

Email: jeff@enernex.com

www.enernex.com

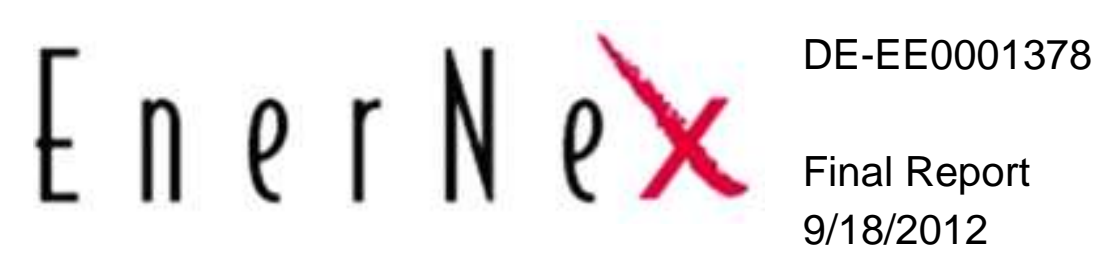


This report is based upon work supported by the U. S. Department of Energy under Award No. DE-EE0001378. 


\section{Disclaimer}

Any findings, opinions, and conclusions or recommendations expressed in this report are those of the authors and do not necessarily reflect the views of the Department of Energy. 


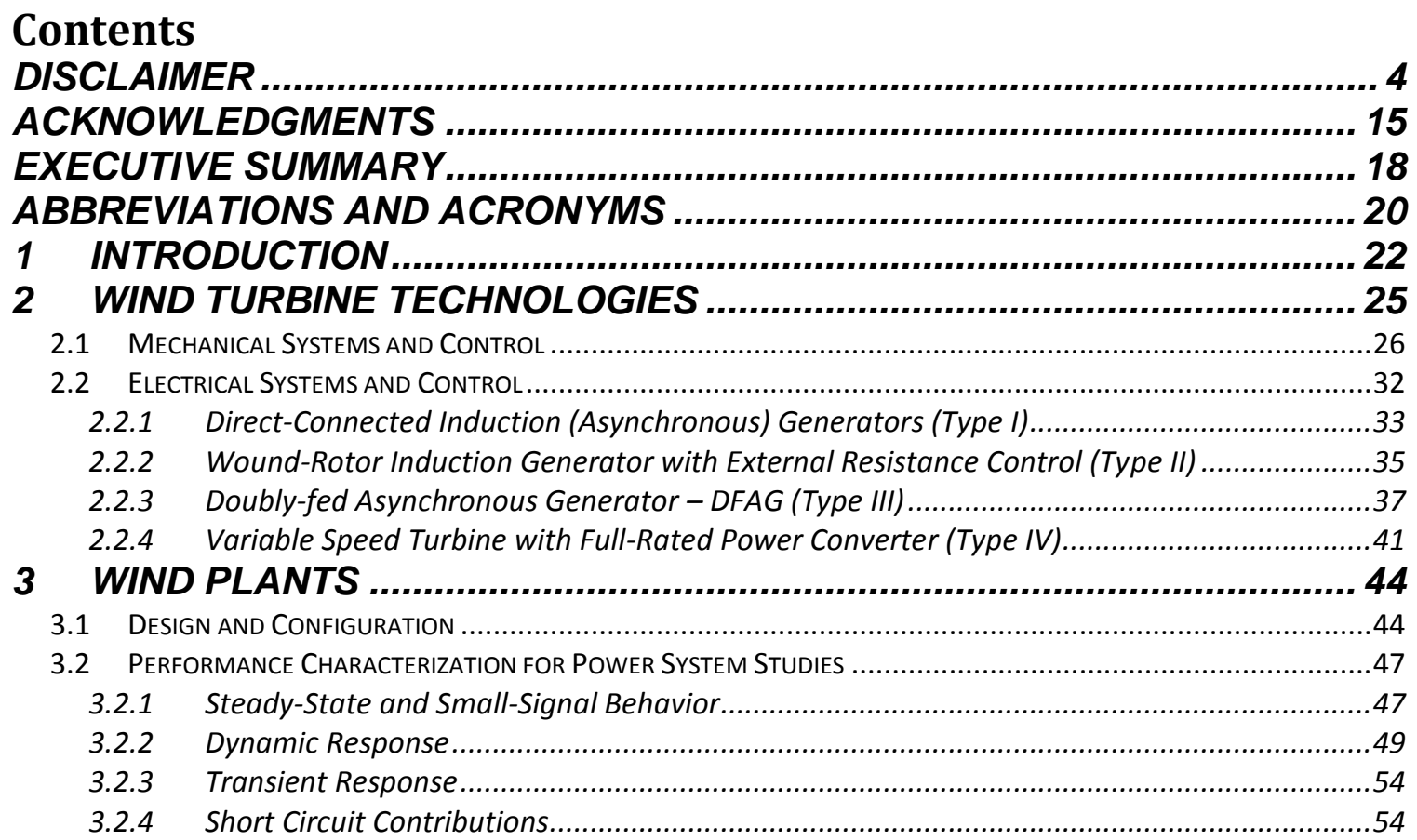

4 WIND GENERATION TECHNOLOGY AND APPLICATION TRENDS...... 59

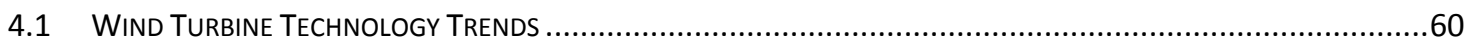

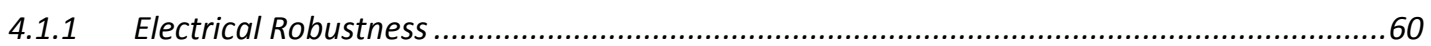

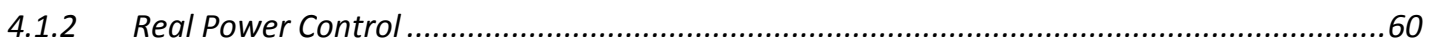

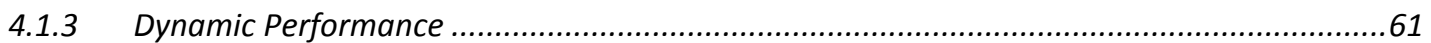

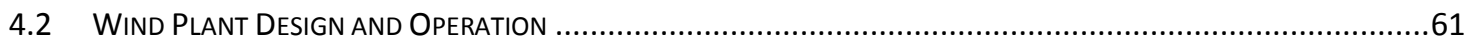

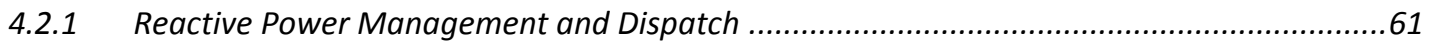

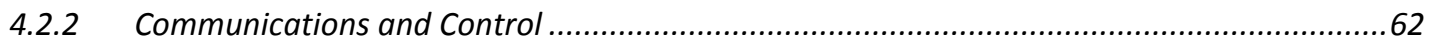

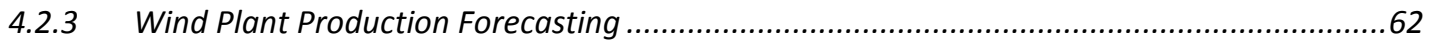

5 CHARACTERISTICS OF THE CURRENT FLEET ................................. 64

6 GENERIC MODELS............................................................................. 70

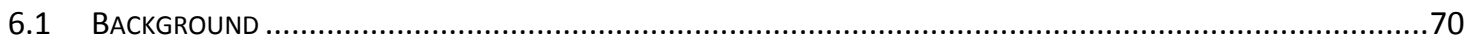

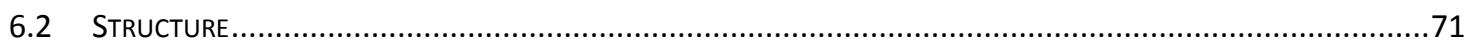

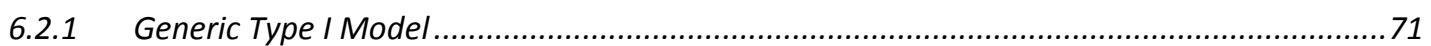

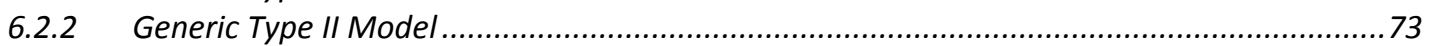

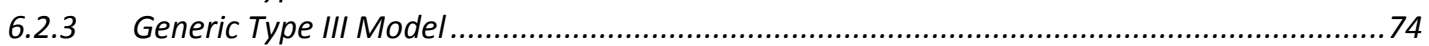

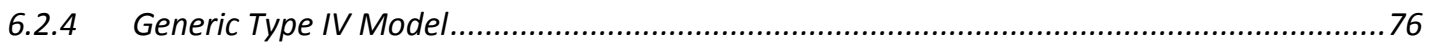

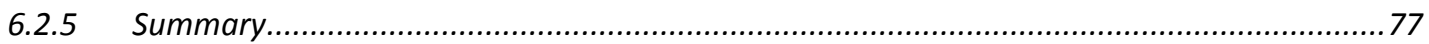

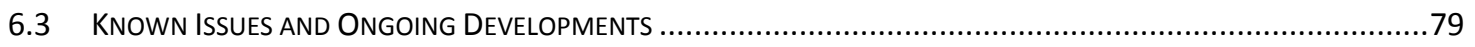

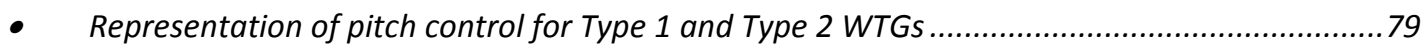

- Generalization of Type 3 and Type 4 WTG Control Structure ....................................................79

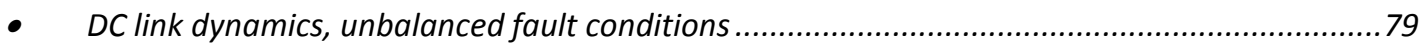

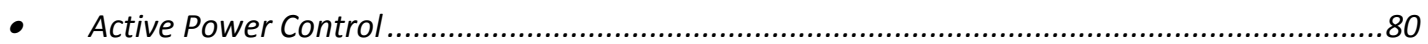

$7 \quad$ WIND TURBINE DATA BASE ........................................................... 81

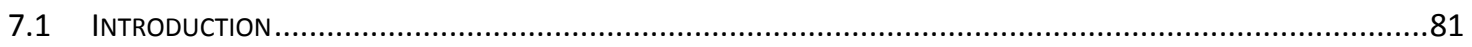

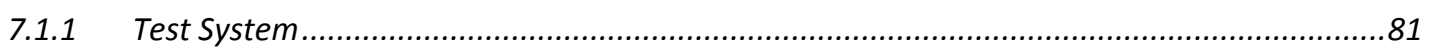

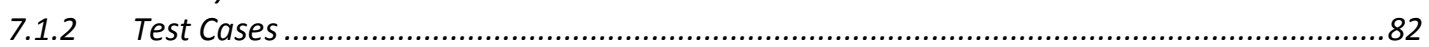

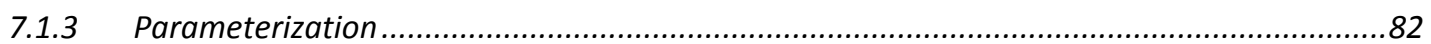

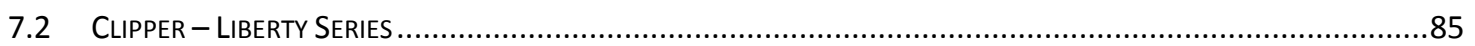

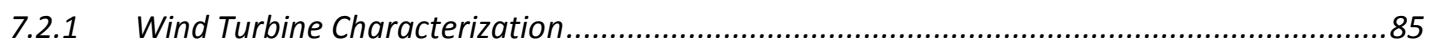

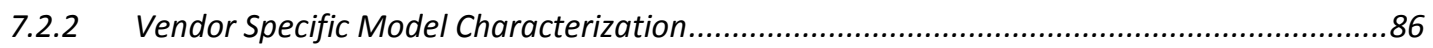


7.2.3 Generic Model Parameterization....................................................................................94

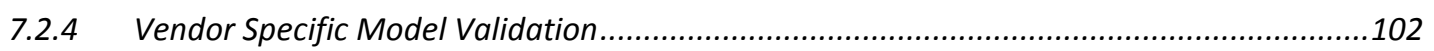

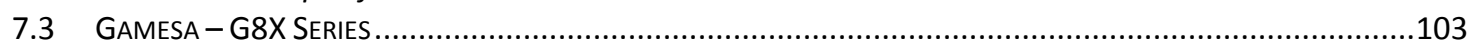

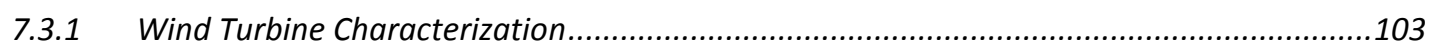

7.3.2 Vendor Specific Model Characterization........................................................................103

7.3.3 Generic Model Parameterization ..................................................................................103

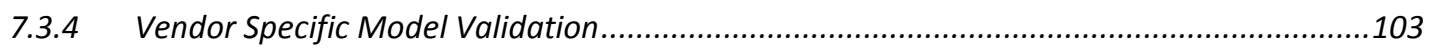

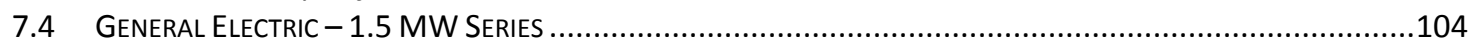

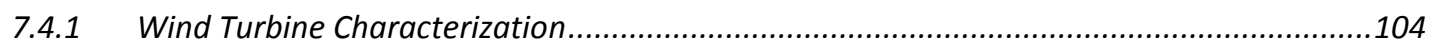

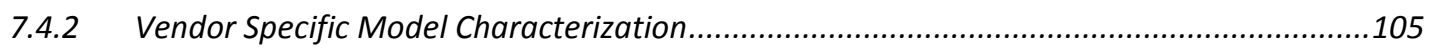

7.4.3 Generic Model Parameterization ......................................................................................112

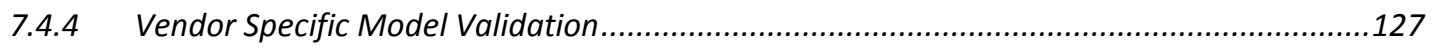

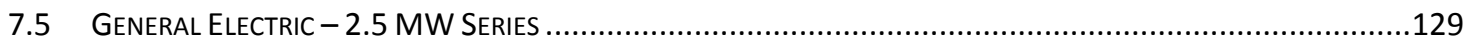

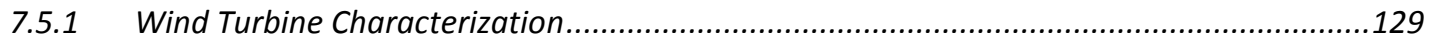

7.5.2 Vendor Specific Model Characterization........................................................................130

7.5.3 Generic Model Parameterization.................................................................................139

7.5.4 Vendor Specific Model Validation .................................................................................153

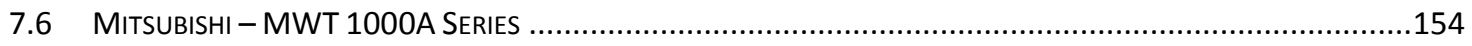

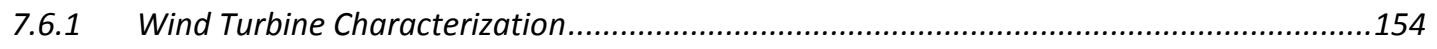

7.6.2 Vendor Specific Model Characterization.....................................................................155

7.6.3 Generic Model Parameterization............................................................................155

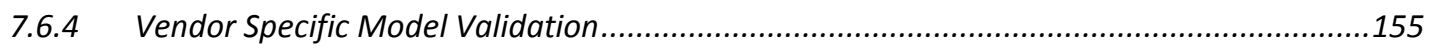

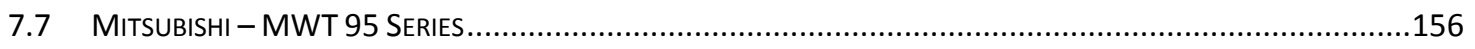

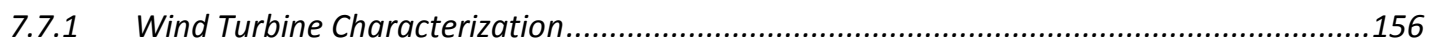

7.7.2 Vendor Specific Model Characterization .........................................................................156

7.7.3 Generic Model Parameterization..................................................................................157

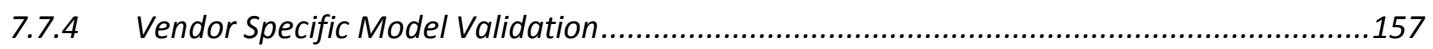

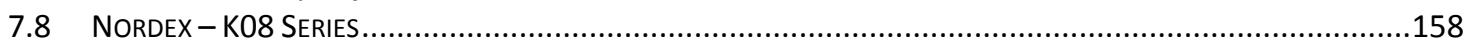

7.8.1 Wind Turbine Characterization ................................................................................ 158

7.8.2 Vendor Specific Model Characterization......................................................................159

7.8.3 Generic Model Parameterization......................................................................................159

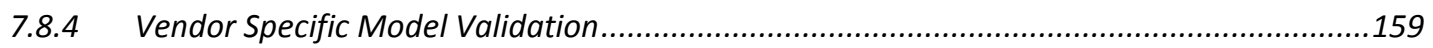

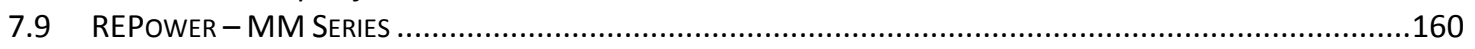

7.9.1 Wind Turbine Characterization ............................................................................ 160

7.9.2 Vendor Specific Model Characterization .........................................................................160

7.9.3 Generic Model Parameterization ..................................................................................161

7.9.4 Vendor Specific Model Validation .....................................................................161

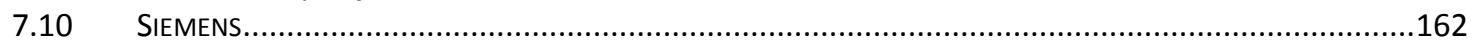

7.10.1 Wind Turbine Characterization ........................................................................... 162

7.10.2 Vendor Specific Model Characterization ............................................................162

7.10.3 Generic Model Parameterization ........................................................................162

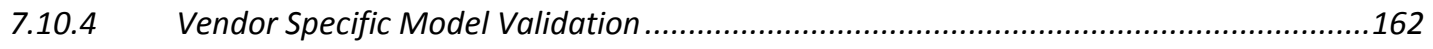

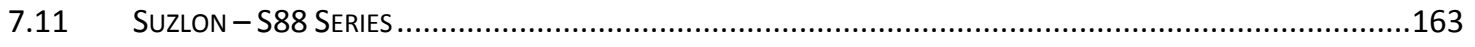

7.11.1 Wind Turbine Characterization ..............................................................................163

7.11.2 Vendor Specific Model Characterization .................................................................163

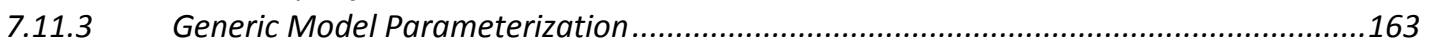

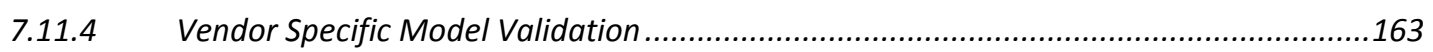

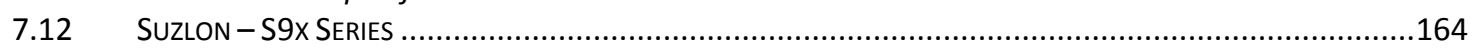

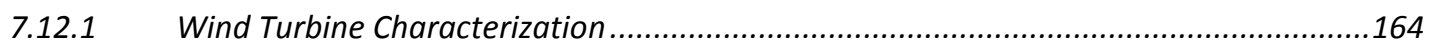

7.12.2 Vendor Specific Model Characterization ...................................................................164

7.12.3 Generic Model Parameterization ...................................................................................164

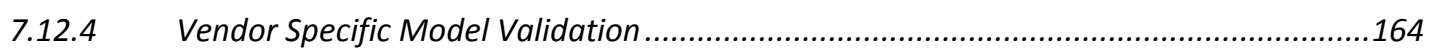

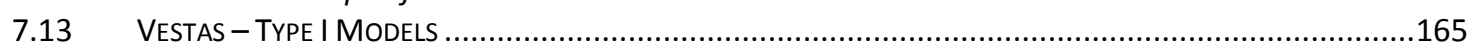


7.13.1 Example List of Vestas Type I Wind Turbines .......................................................165

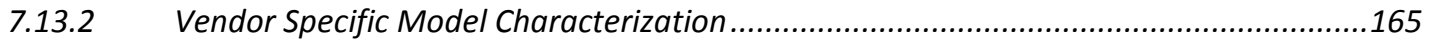

7.13.3 Generic Model Parameterization .............................................................................173

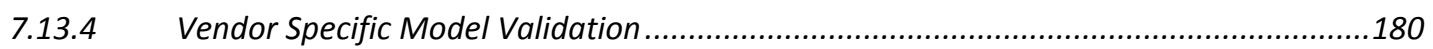

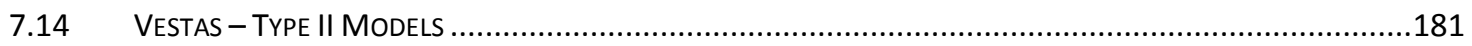

7.14.1 Example List of Vestas Type II Wind Turbines .............................................................181

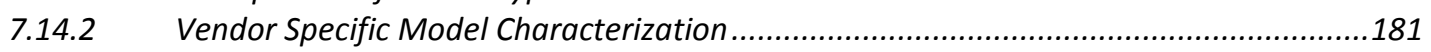

7.14.3 Generic Model Parameterization .....................................................................................190

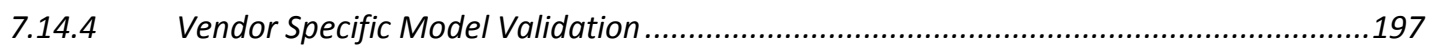

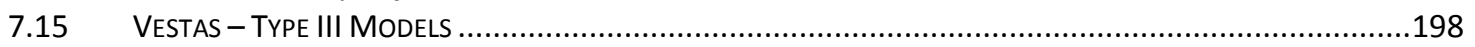

7.15.1 Example List of Vestas Type III Wind Turbines ............................................................198

7.15.2 Vendor Specific Model Characterization ..................................................................198

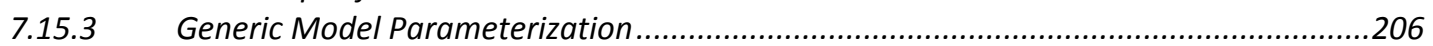

7.15.4 Vendor Specific Model Validation .......................................................................213

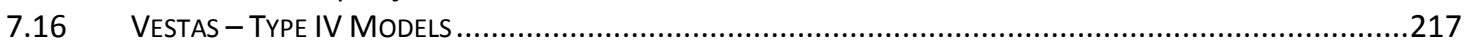

7.16.1 Example List of Vestas Type IV Wind Turbines.......................................................217

7.16.2 Vendor Specific Model Characterization ...................................................................217

7.16.3 Generic Model Parameterization ........................................................................226

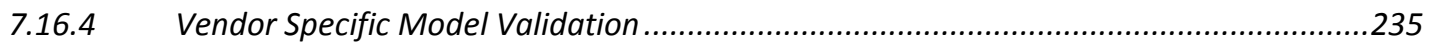

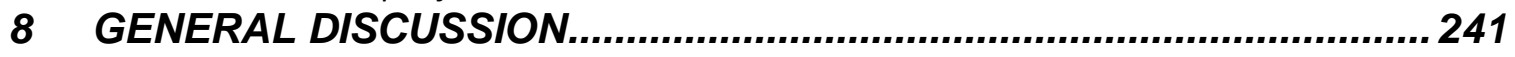

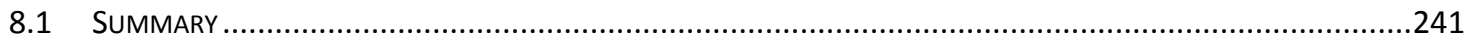

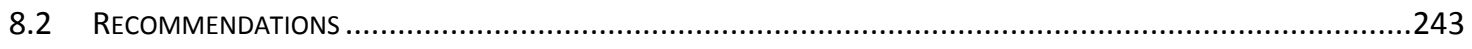

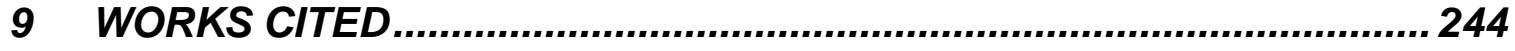




\section{Figures}

Figure 2-1: Generalized wind turbine model with control elements and hierarchy.

Figure 2-2: Coefficient of performance $\left(C_{p}\right)$ for a modern wind turbine blade assembly as a function of tipspeed ratio $(\lambda)$ and blade pitch $(\beta$, in degrees).

Figure 2-3: Power curve for a variable-speed, pitch-controlled wind turbine. Note "flatness" of output for

wind speeds at or above rated value.

Figure 2-4: Simplified model of wind turbine mechanical system. Two mass model with gearbox (top) and model with equivalent gearbox inertia and reference of all components to high-speed shaft (bottom)....31

Figure 2-5: Illustration of type I wind turbine generator. ..................................................................33

Figure 2-6: Torque vs. Speed characteristic for an induction machine used in a commercial wind turbine.34

Figure 2-7: Variation of real (solid) and reactive (dash) power with slip for a squirrel-cage induction

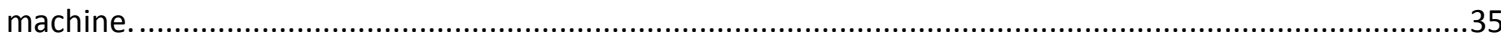

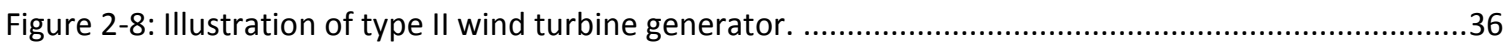

Figure 2-9: Torque vs Speed curve of a Type II turbine at various rotor resistances. .................................37

Figure 2-10: Illustration of type III wind turbine generator. ..............................................................3

Figure 2-11: Configuration of a wind turbine with four-quadrant power converter supplying rotor circuit of

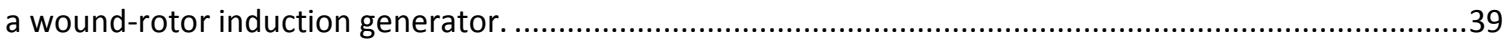

Figure 2-12: Configuration of a type IV wind turbine generator......................................................41 Figure 2-13: Simple output current control stage for a static power converter in a grid-tied DG application.

Figure 3-1: Illustration of the impact of collector line reactive losses on the net reactive power capability

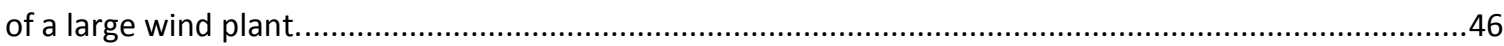
Figure 3-2: Wind turbine dynamic response to fault on the grid. ...................................................... Figure 3-3: Expanded view of Figure 3-2 .......................................................................................5. Figure 3-4: Contribution by a 2.0 MVA induction generator to a symmetrical three-phase fault. Shown are voltage at the machine terminals (top), stator currents (middle), and real and reactive power (generator convention) at the machine terminals (bottom) .................................................................................56 Figure 3-5: Transient model of a doubly-fed induction generator with vector control of rotor currents. ...57 Figure 3-6: Short-circuit contribution from the GE $1.5 \mathrm{MW}$ wind turbine for $150 \mathrm{~ms}$ grid fault. Shown are voltage at the machine terminals (top), stator currents (middle), and real and reactive power (generator

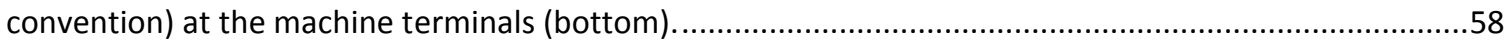

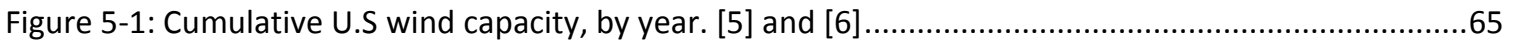

Figure 5-2: Wind power capacity market share by regional entity by the end of 2010. [5] and [6] ...........66

Figure 5-3: Wind power capacity market share by vendor by the end of 2010. [5] and [6] ......................67

Figure 5-4: Wind power capacity market share by turbine type by the end of 2010. [5] and [6] ...............69

Figure 5-5: Share of the different turbine types within the regional entities' wind capacity by the end of

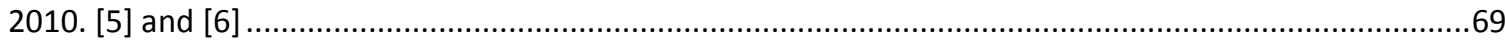

Figure 6-1: Type 1 wind turbine model connectivity diagram in PSLF. ..............................................72

Figure 6-2: Type 1 wind turbine model connectivity diagram in PSSE. ..............................................72

Figure 6-3: Type 2 wind turbine model connectivity diagram in PSLF. ..............................................73

Figure 6-4: Type 2 wind turbine model connectivity diagram in PSSE. ..............................................74

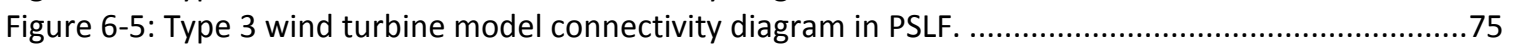

Figure 6-6: Type 3 wind turbine model connectivity diagram in PSSE. ...............................................76

Figure 6-7: Type 4 wind turbine model connectivity diagram in PSLF. ..................................................77

Figure 6-8: Type 4 wind turbine model connectivity diagram in PSSE. ..................................................77

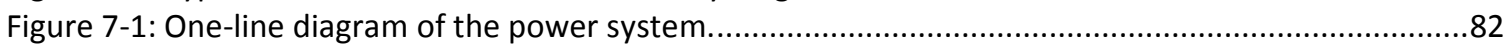

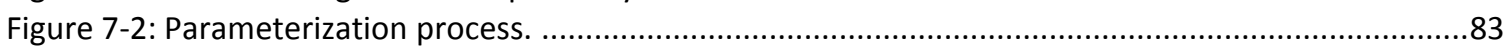

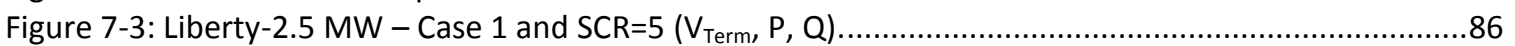

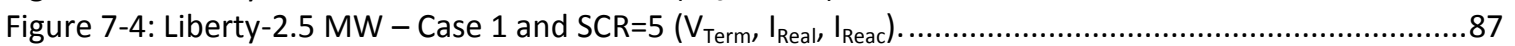

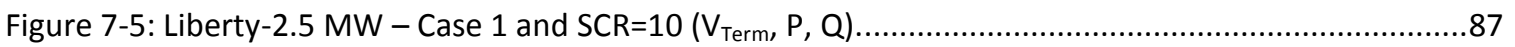

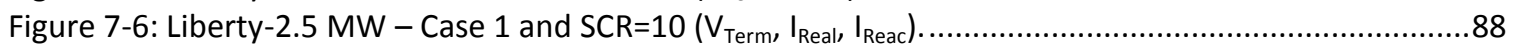

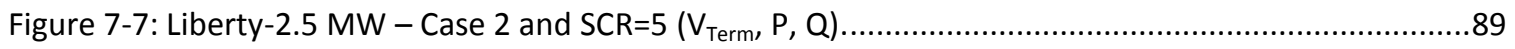




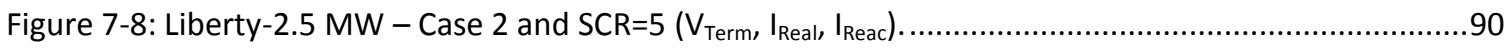

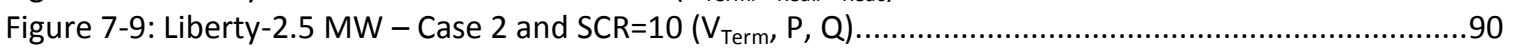

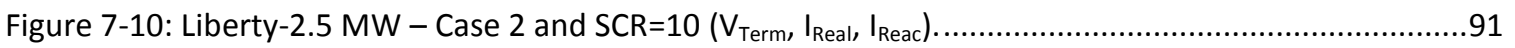

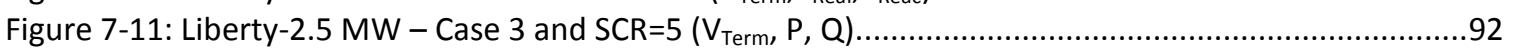

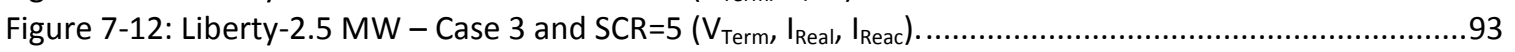

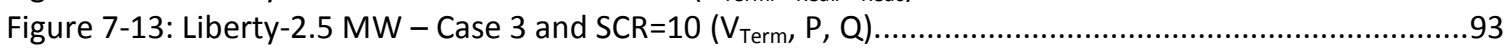

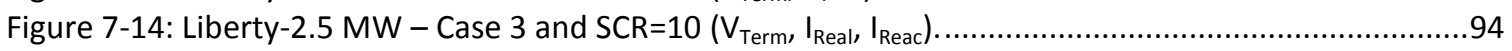

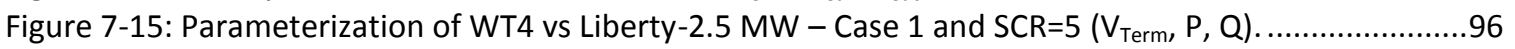

Figure 7-16: Parameterization of WT4 vs Liberty-2.5 MW - Case 1 and SCR=5 $\left(V_{\text {Term, }} I_{\text {Real }}, I_{\text {Reac }}\right) . \ldots \ldots \ldots . . . . .96$

Figure 7-17: Parameterization of WT4 vs Liberty-2.5 MW - Case 1 and SCR=10 ( $\left.\mathrm{V}_{\text {Term }}, \mathrm{P}, \mathrm{Q}\right)$...................97

Figure 7-18: Parameterization of WT4 vs Liberty-2.5 MW - Case 1 and SCR=10 $\left(V_{\text {Term }}, I_{\text {Real }}, I_{\text {Reac }}\right)$.............97

Figure 7-19: Parameterization of WT4 vs Liberty-2.5 MW - Case 2 and SCR=5 $\left(V_{\text {Term }}, P, Q\right)$....................98

Figure 7-20: Parameterization of WT4 vs Liberty-2.5 MW - Case 2 and SCR=5 ( $\left.\mathrm{V}_{\text {Term, }} \mathrm{I}_{\text {Real }}, \mathrm{I}_{\text {Reac }}\right) . \ldots \ldots \ldots . . . . .98$

Figure 7-21: Parameterization of WT4 vs Liberty-2.5 MW - Case 2 and SCR=10 ( $\left.\mathrm{V}_{\text {Term }} \mathrm{P}, \mathrm{Q}\right)$...................99

Figure 7-22: Parameterization of WT4 vs Liberty-2.5 MW - Case 2 and $S C R=10\left(V_{\text {Term }}, I_{\text {Real }}, I_{\text {Reac }}\right)$.............99

Figure 7-23: Parameterization of WT4 vs Liberty-2.5 MW - Case 3 and SCR=5 ( $\left.\mathrm{V}_{\text {Term }}, \mathrm{P}, \mathrm{Q}\right)$...................100

Figure 7-24: Parameterization of WT4 vs Liberty-2.5 MW - Case 3 and SCR=5 ( $\left.V_{\text {Term, }} I_{\text {Real }}, I_{\text {Reac }}\right) . \ldots \ldots \ldots . . . .100$

Figure 7-25: Parameterization of WT4 vs Liberty-2.5 MW - Case 3 and SCR=10 ( $\left.\mathrm{V}_{\text {Term }}, \mathrm{P}, \mathrm{Q}\right)$................101

Figure 7-26: Parameterization of WT4 vs Liberty-2.5 MW - Case 3 and $S C R=10\left(V_{\text {Term }}, I_{\text {Real }}, I_{\text {Reac }}\right)$...........101

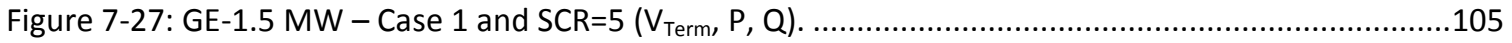

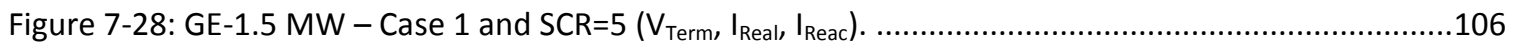

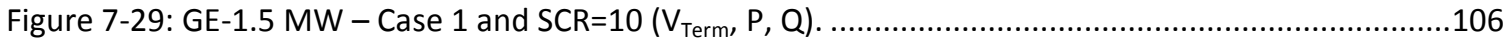

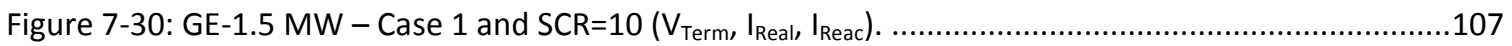

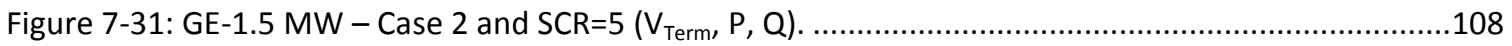

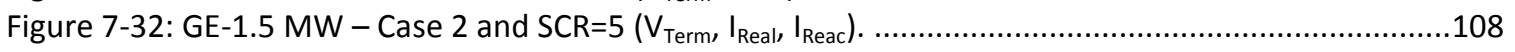

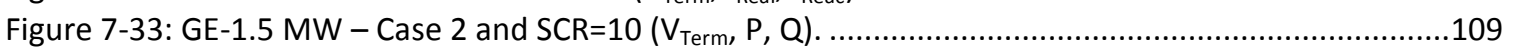

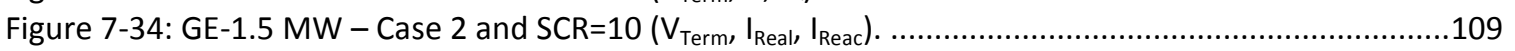

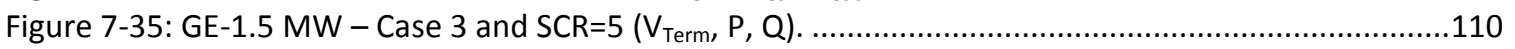

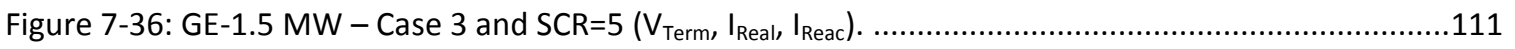

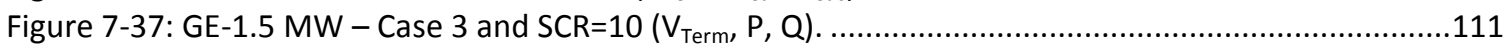

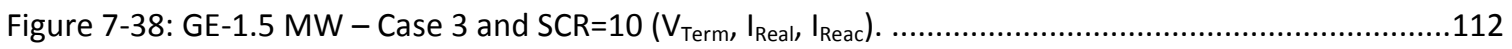

Figure 7-39: Parameterization of WT3 vs GE-1.5 MW - Case 1 and SCR=5 ( $\left.\mathrm{V}_{\text {Term }}, \mathrm{P}, \mathrm{Q}\right) \ldots \ldots \ldots \ldots \ldots \ldots \ldots . . . . . . . . . .114$

Figure 7-40: Parameterization of WT3 vs GE-1.5 MW - Case 1 and SCR=5 $\left(V_{\text {Term }}, I_{\text {Real }}, I_{\text {Reac }}\right) \ldots \ldots \ldots \ldots \ldots . . . . .114$

Figure 7-41: Parameterization of WT3 vs GE-1.5 MW - Case 1 and SCR=10 $\left(V_{\text {Term }}, P, Q\right) \ldots \ldots \ldots \ldots \ldots \ldots \ldots . . . . . . . . . .115$

Figure 7-42: Parameterization of WT3 vs GE-1.5 MW - Case 1 and SCR=10 ( $\left.V_{\text {Term, }} I_{\text {Real }}, I_{\text {Reac }}\right) \ldots \ldots \ldots \ldots . . . . . .115$

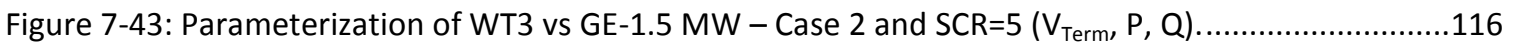

Figure 7-44: Parameterization of WT3 vs GE-1.5 MW - Case 2 and SCR=5 $\left(\mathrm{V}_{\text {Term }}, \mathrm{I}_{\text {Real, }}, \mathrm{I}_{\text {Reac }}\right) \ldots \ldots \ldots \ldots \ldots \ldots . . . . . .116$

Figure 7-45: Parameterization of WT3 vs GE-1.5 MW - Case 2 and SCR=10 ( $\left.\mathrm{V}_{\text {Term }}, \mathrm{P}, \mathrm{Q}\right)$.......................117

Figure 7-46: Parameterization of WT3 vs GE-1.5 MW - Case 2 and SCR=10 ( $\left.\mathrm{V}_{\text {Term, }} \mathrm{I}_{\text {Real }}, \mathrm{I}_{\text {Reac }}\right) \ldots \ldots \ldots \ldots \ldots . . . . . .117$

Figure 7-47: Parameterization of WT3 vs GE-1.5 MW - Case 3 and SCR=5 ( $\left.\mathrm{V}_{\text {Term }}, \mathrm{P}, \mathrm{Q}\right)$........................118

Figure 7-48: Parameterization of WT3 vs GE-1.5 MW - Case 3 and SCR=5 $\left(\mathrm{V}_{\text {Term, }} \mathrm{I}_{\text {Real }}, \mathrm{I}_{\text {Reac }}\right) \ldots \ldots \ldots \ldots \ldots . . . . . .118$

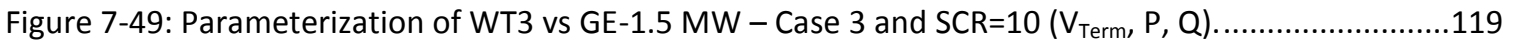

Figure 7-50: Parameterization of WT3 vs GE-1.5 MW - Case 3 and SCR=10 $\left(\mathrm{V}_{\text {Term, }} \mathrm{I}_{\text {Real }}, \mathrm{I}_{\text {Reac }}\right) \ldots \ldots \ldots \ldots \ldots . . . .119$

Figure 7-51: Parameterization of WT3 vs GE-1.5 MW - Case 1 and SCR=5 ( $\left.\mathrm{V}_{\text {Term }}, \mathrm{P}, \mathrm{Q}\right)$.......................121

Figure 7-52: Parameterization of WT3 vs GE-1.5 MW - Case 1 and SCR=5 $\left(\mathrm{V}_{\text {Term, }}, \mathrm{I}_{\text {Real, }} \mathrm{I}_{\text {Reac }}\right) \ldots \ldots \ldots \ldots \ldots . . . . . .121$

Figure 7-53: Parameterization of WT3 vs GE-1.5 MW - Case 1 and SCR=10 $\left(V_{\text {Term }}, P, Q\right) \ldots \ldots \ldots \ldots \ldots \ldots . . . . .122$

Figure 7-54: Parameterization of WT3 vs GE-1.5 MW - Case 1 and SCR=10 $\left(V_{\text {Term }}, I_{\text {Real }}, I_{\text {Reac }}\right) \ldots \ldots \ldots \ldots \ldots . . . .122$

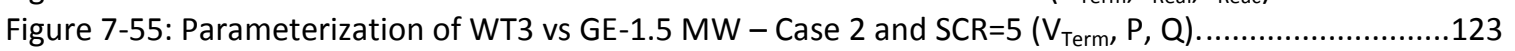

Figure 7-56: Parameterization of WT3 vs GE-1.5 MW - Case 2 and SCR=5 $\left(\mathrm{V}_{\text {Term }}, \mathrm{I}_{\text {Real }}, \mathrm{I}_{\text {Reac }}\right) \ldots \ldots \ldots \ldots \ldots \ldots . . . . . . .123$

Figure 7-57: Parameterization of WT3 vs GE-1.5 MW - Case 2 and SCR=10 $\left(V_{\text {Term }}, P, Q\right) \ldots \ldots \ldots \ldots \ldots \ldots . . . . . . . . . . . .124$

Figure 7-58: Parameterization of WT3 vs GE-1.5 MW - Case 2 and SCR=10 ( $\left.V_{\text {Term, }} I_{\text {Real }}, I_{\text {Reac }}\right) \ldots \ldots \ldots \ldots \ldots . . . .124$

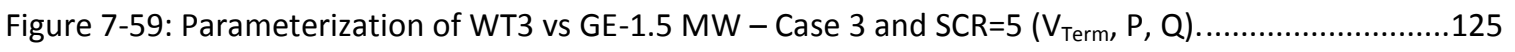

Figure 7-60: Parameterization of WT3 vs GE-1.5 MW - Case 3 and SCR=5 $\left(V_{\text {Term }}, I_{\text {Real, }} I_{\text {Reac }}\right) \ldots \ldots \ldots \ldots \ldots \ldots . . . . . . . .125$ 
Figure 7-61: Parameterization of WT3 vs GE-1.5 MW - Case 3 and SCR=10 ( $\left.\mathrm{V}_{\text {Term }}, \mathrm{P}, \mathrm{Q}\right) \ldots \ldots \ldots \ldots \ldots \ldots \ldots . . . . . . . . . . .126$

Figure 7-62: Parameterization of WT3 vs GE-1.5 MW - Case 3 and SCR=10 ( $\left.V_{\text {Term, }} I_{\text {Real }}, I_{\text {Reac }}\right) \ldots \ldots \ldots \ldots . . . . . .126$

Figure 7-63: POI Voltage - test measurements vs. GE-VSM and Generic Type III simulations. .................127

Figure 7-64: POI Reactive Power - test measurements (blue) vs. GE-VSM (red) and Generic Type III (green)

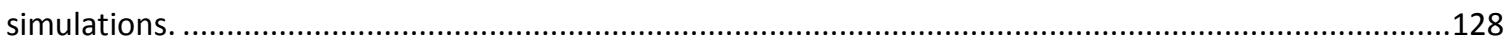

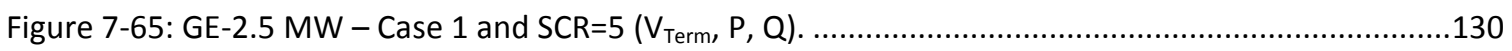

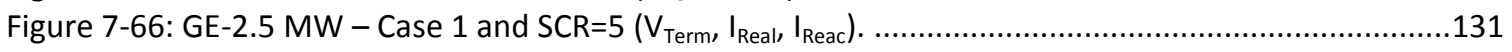

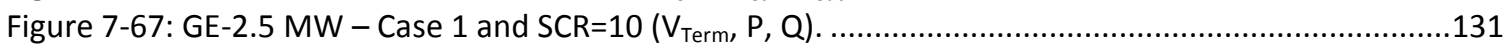

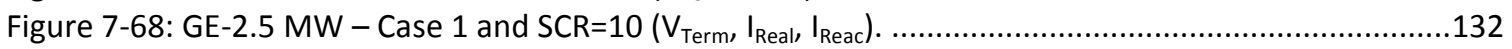

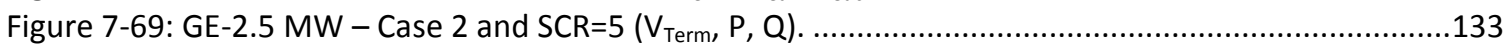

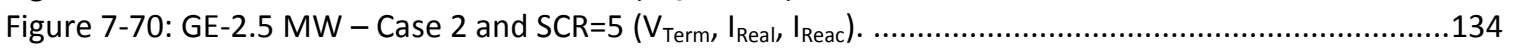

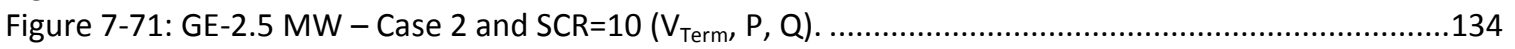

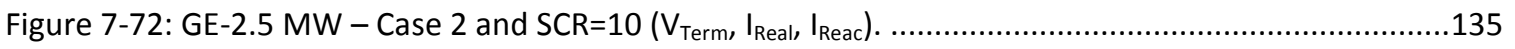

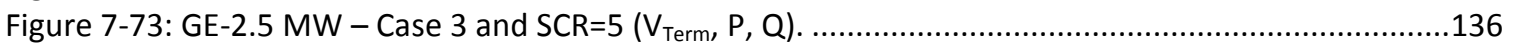

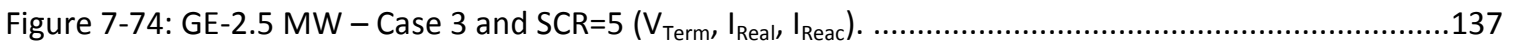

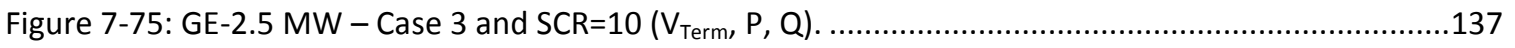

Figure 7-76: GE-2.5 MW - Case 3 and SCR=10 ( $\left.V_{\text {Term, }} I_{\text {Real }} I_{\text {Reac }}\right)$. ....................................................138

Figure 7-77: Parameterization of WT4 vs GE-2.5 MW - Case 1 and SCR=5 ( $\left.\mathrm{V}_{\text {Term, }} \mathrm{P}, \mathrm{Q}\right) \ldots \ldots \ldots \ldots \ldots \ldots \ldots . . . . . . . . . . .140$

Figure 7-78: Parameterization of WT4 vs GE-2.5 MW - Case 1 and SCR=5 $\left(V_{\text {Term }}, I_{\text {Real }}, I_{\text {Reac }}\right) \ldots \ldots \ldots . . . . . . . . .140$

Figure 7-79: Parameterization of WT4 vs GE-2.5 MW - Case 1 and SCR=10 $\left(\mathrm{V}_{\text {Term }}, \mathrm{P}, \mathrm{Q}\right)$........................141

Figure 7-80: Parameterization of WT4 vs GE-2.5 MW - Case 1 and SCR=10 $\left(\mathrm{V}_{\text {Term, }} \mathrm{I}_{\text {Real }}, \mathrm{I}_{\text {Reac }}\right) \ldots \ldots \ldots \ldots \ldots . . . . .141$

Figure 7-81: Parameterization of WT4 vs GE-2.5 MW - Case 2 and SCR $=5\left(\mathrm{~V}_{\text {Term, }} \mathrm{P}, \mathrm{Q}\right)$.........................142

Figure 7-82: Parameterization of WT4 vs GE-2.5 MW - Case 2 and SCR=5 $\left(\mathrm{V}_{\text {Term }}, \mathrm{I}_{\text {Real }}, \mathrm{I}_{\text {Reac }}\right) \ldots \ldots \ldots \ldots \ldots \ldots . . . . . . . .142$

Figure 7-83: Parameterization of WT4 vs GE-2.5 MW - Case 2 and SCR=10 $\left(V_{\text {Term }}, P, Q\right) \ldots \ldots \ldots \ldots \ldots \ldots . . . . . . . . . . .143$

Figure 7-84: Parameterization of WT4 vs GE-2.5 MW - Case 2 and SCR=10 $\left(V_{\text {Term }}, I_{\text {Real }}, I_{\text {Reac }}\right) \ldots \ldots \ldots \ldots \ldots . . . .143$

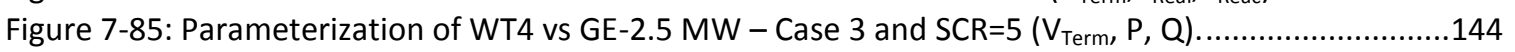

Figure 7-86: Parameterization of WT4 vs GE-2.5 MW - Case 3 and SCR=5 $\left(V_{\text {Term }}, I_{\text {Real }}, I_{\text {Reac }}\right) \ldots \ldots \ldots \ldots \ldots . . . . . .144$

Figure 7-87: Parameterization of WT4 vs GE-2.5 MW - Case 3 and SCR=10 $\left(V_{\text {Term }}, P, Q\right) \ldots \ldots \ldots \ldots \ldots \ldots . . . . . . . . . . . .145$

Figure 7-88: Parameterization of WT4 vs GE-2.5 MW - Case 3 and SCR=10 ( $\left.\mathrm{V}_{\text {Term, }} \mathrm{I}_{\text {Real }}, \mathrm{I}_{\text {Reac }}\right) \ldots \ldots \ldots \ldots \ldots . . . . . .145$

Figure 7-89: Parameterization of WT4 vs GE-2.5 MW - Case 1 and SCR=5 ( $\left.\mathrm{V}_{\text {Term, }} \mathrm{P}, \mathrm{Q}\right)$........................147

Figure 7-90: Parameterization of WT4 vs GE-2.5 MW - Case 1 and SCR=5 $\left(V_{\text {Term }}, I_{\text {Real }}, I_{\text {Reac }}\right) \ldots \ldots \ldots \ldots \ldots \ldots . . . . . . . .147$

Figure 7-91: Parameterization of WT4 vs GE-2.5 MW - Case 1 and SCR=10 $\left(V_{\text {Term }}, P, Q\right) \ldots \ldots \ldots \ldots \ldots \ldots . . . . . . . . . . .148$

Figure 7-92: Parameterization of WT4 vs GE-2.5 MW - Case 1 and SCR=10 ( $\left.V_{\text {Term, }} I_{\text {Real }}, I_{\text {Reac }}\right) \ldots \ldots \ldots \ldots \ldots . . . . . .148$

Figure 7-93: Parameterization of WT4 vs GE-2.5 MW - Case 2 and SCR=5 ( $\left.\mathrm{V}_{\text {Term }}, \mathrm{P}, \mathrm{Q}\right)$........................149

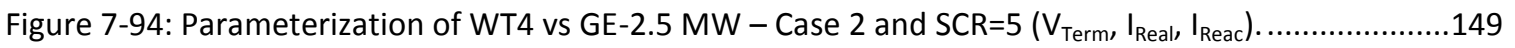

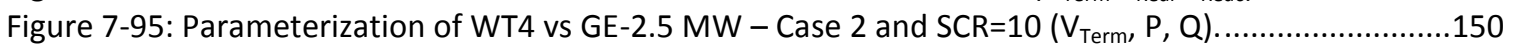

Figure 7-96: Parameterization of WT4 vs GE-2.5 MW - Case 2 and SCR=10 $\left(\mathrm{V}_{\text {Term }}, \mathrm{I}_{\text {Real }}, \mathrm{I}_{\text {Reac }}\right) \ldots . . . . . . . . . . . .150$

Figure 7-97: Parameterization of WT4 vs GE-2.5 MW - Case 3 and SCR=5 $\left(\mathrm{V}_{\text {Term }}, \mathrm{P}, \mathrm{Q}\right) \ldots \ldots \ldots \ldots \ldots \ldots \ldots . . . . . . . . . . .151$

Figure 7-98: Parameterization of WT4 vs GE-2.5 MW - Case 3 and SCR=5 $\left(V_{\text {Term }}, I_{\text {Real }}, I_{\text {Reac }}\right) \ldots \ldots \ldots \ldots \ldots \ldots . . . . . . . .151$

Figure 7-99: Parameterization of WT4 vs GE-2.5 MW - Case 3 and SCR=10 $\left(V_{\text {Term }}, P, Q\right) \ldots \ldots \ldots \ldots \ldots \ldots \ldots . . . . . . . . . . .152$

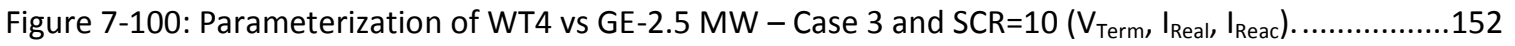

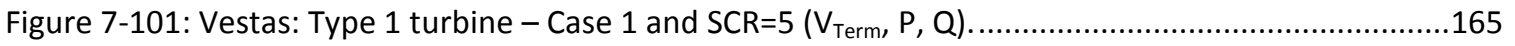

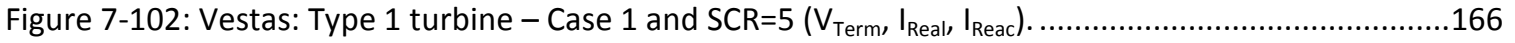

Figure 7-103: Vestas: Type 1 turbine - Case 1 and $S C R=10\left(V_{\text {Term }}, \mathrm{P}, \mathrm{Q}\right)$.........................................166

Figure 7-104: Vestas: Type 1 turbine - Case 1 and $S C R=10\left(V_{\text {Term }}, I_{\text {Real }}, I_{\text {Reac }}\right)$.......................................167

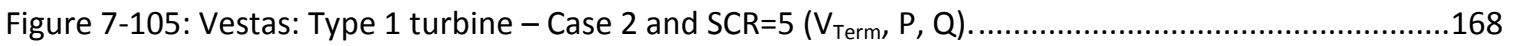

Figure 7-106: Vestas: Type 1 turbine - Case 2 and $S C R=5\left(V_{\text {Term }}, I_{\text {Real }}, I_{\text {Reac }}\right)$......................................169

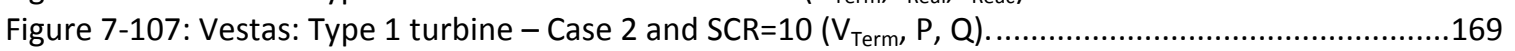

Figure 7-108: Vestas: Type 1 turbine - Case 2 and $S C R=10\left(V_{\text {Term }}, I_{\text {Real }}, I_{\text {Reac }}\right)$......................................170

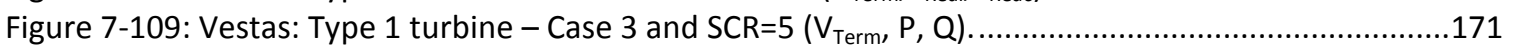

Figure 7-110: Vestas: Type 1 turbine - Case 3 and $S C R=5\left(V_{\text {Term }}, I_{\text {Real }}, I_{\text {Reac }}\right)$.......................................172

Figure 7-111: Vestas: Type 1 turbine - Case 3 and $S C R=10\left(V_{\text {Term }}, P, Q\right)$............................................172

Figure 7-112: Vestas: Type 1 turbine - Case 3 and $S C R=10\left(V_{\text {Term }}, I_{\text {Real }}, I_{\text {Reac }}\right)$......................................173 
Figure 7-113: Parameterization of WT1 vs Vestas: Type 1 turbine - Case 1 and $S C R=5\left(V_{\text {Term }}, P, Q\right)$.......174 Figure 7-114: Parameterization of WT1 vs Vestas: Type 1 turbine - Case 1 and $S C R=5\left(V_{\text {Term }}, I_{\text {Real, }} I_{\text {Reac }}\right) . .174$ Figure 7-115: Parameterization of WT1 vs Vestas: Type 1 turbine - Case 1 and $S C R=10\left(V_{T e r m}, P, Q\right)$......175 Figure 7-116: Parameterization of WT1 vs Vestas: Type 1 turbine - Case 1 and $S C R=10\left(V_{\text {Term }}, I_{\text {Real }}, I_{\text {Reac }}\right) .175$ Figure 7-117: Parameterization of WT1 vs Vestas: Type 1 turbine - Case 2 and $S C R=5\left(V_{\text {Term }}, P, Q\right)$........176 Figure 7-118: Parameterization of WT1 vs Vestas: Type 1 turbine - Case 2 and $S C R=5\left(V_{\text {Term }}, I_{\text {Real, }} I_{\text {Reac }}\right)$. .176 Figure 7-119: Parameterization of WT1 vs Vestas: Type 1 turbine - Case 2 and SCR=10 $\left(V_{\text {Term }}, P, Q\right) . \ldots . .177$ Figure 7-120: Parameterization of WT1 vs Vestas: Type 1 turbine - Case 2 and SCR=10 $\left(V_{\text {Term }}, I_{\text {Real }}, I_{\text {Reac }}\right) .177$ Figure 7-121: Parameterization of WT1 vs Vestas: Type 1 turbine - Case 3 and $S C R=5\left(V_{\text {Term }}, P, Q\right) \ldots \ldots . .178$ Figure 7-122: Parameterization of WT1 vs Vestas: Type 1 turbine - Case 3 and SCR $=5\left(V_{\text {Term }}, I_{\text {Real, }}, I_{\text {Reac }}\right) . .178$ Figure 7-123: Parameterization of WT1 vs Vestas: Type 1 turbine - Case 3 and $S C R=10\left(V_{T e r m}, P, Q\right) . . . . .179$ Figure 7-124: Parameterization of WT1 vs Vestas: Type 1 turbine - Case 3 and $S C R=10\left(V_{\text {Term }}, I_{\text {Real }}, I_{\text {Reac }}\right) .179$

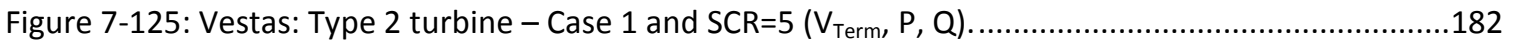

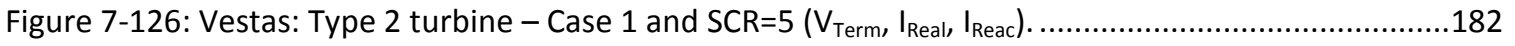

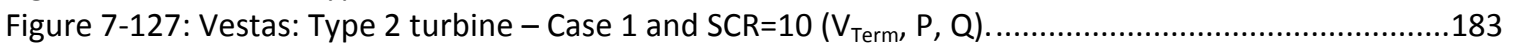

Figure 7-128: Vestas: Type 2 turbine - Case 1 and $S C R=10\left(V_{\text {Term }}, I_{\text {Real }}, I_{\text {Reac }}\right)$......................................183

Figure 7-129: Vestas: Type 2 turbine - Case 2 and $S C R=5\left(V_{\text {Term }}, \mathrm{P}, \mathrm{Q}\right)$..............................................184

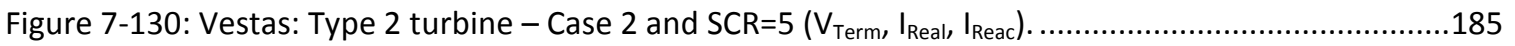

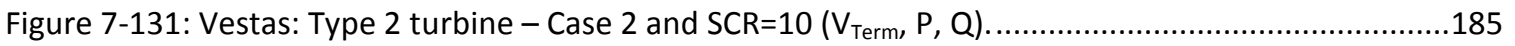

Figure 7-132: Vestas: Type 2 turbine - Case 2 and $S C R=10\left(V_{\text {Term }}, I_{\text {Real }}, I_{\text {Reac }}\right)$.......................................186

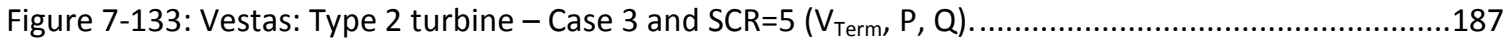

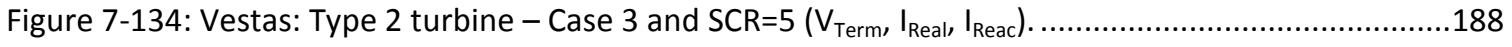

Figure 7-135: Vestas: Type 2 turbine - Case 3 and $S C R=10\left(V_{\text {Term }}, P, Q\right)$............................................188

Figure 7-136: Vestas: Type 2 turbine - Case 3 and $S C R=10\left(V_{\text {Term }}, I_{\text {Real }}, I_{\text {Reac }}\right)$.......................................189

Figure 7-137: Parameterization of WT2 vs Vestas: Type 2 turbine - Case 1 and SCR $=5\left(V_{\text {Term }}, P, Q\right)$........191

Figure 7-138: Parameterization of WT2 vs Vestas: Type 2 turbine - Case 1 and $S C R=5\left(V_{\text {Term }}, I_{\text {Real, }} I_{\text {Reac }}\right)$. .191

Figure 7-139: Parameterization of WT2 vs Vestas: Type 2 turbine - Case 1 and $S C R=10\left(V_{T e r m}, P, Q\right) . \ldots . .192$

Figure 7-140: Parameterization of WT2 vs Vestas: Type 2 turbine - Case 1 and SCR=10 $\left(V_{\text {Term }}, I_{\text {Real }}, I_{\text {Reac }}\right) \cdot 192$ Figure 7-141: Parameterization of WT2 vs Vestas: Type 2 turbine - Case 2 and $S C R=5\left(V_{\text {Term }}, P, Q\right) \ldots . . . .193$ Figure 7-142: Parameterization of WT2 vs Vestas: Type 2 turbine - Case 2 and SCR=5 $\left(V_{\text {Term }}, I_{\text {Real, }}, I_{\text {Reac }}\right) . .193$ Figure 7-143: Parameterization of WT2 vs Vestas: Type 2 turbine - Case 2 and $S C R=10\left(V_{T e r m}, P, Q\right) . . . . .194$ Figure 7-144: Parameterization of WT2 vs Vestas: Type 2 turbine - Case 2 and SCR=10 $\left(V_{\text {Term }}, I_{\text {Real }}, I_{\text {Reac }}\right) .194$ Figure 7-145: Parameterization of WT2 vs Vestas: Type 2 turbine - Case 3 and $S C R=5\left(V_{\text {Term }}, P, Q\right)$........195 Figure 7-146: Parameterization of WT2 vs Vestas: Type 2 turbine - Case 3 and $S C R=5\left(V_{\text {Term }}, I_{\text {Real, }} I_{\text {Reac }}\right) .195$ Figure 7-147: Parameterization of WT2 vs Vestas: Type 2 turbine - Case 3 and $S C R=10\left(V_{T e r m}, P, Q\right)$......196 Figure 7-148: Parameterization of WT2 vs Vestas: Type 2 turbine - Case 3 and SCR=10 $\left(V_{\text {Term }}, I_{\text {Real }}, I_{\text {Reac }}\right) .196$

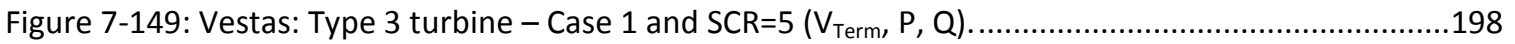

Figure 7-150: Vestas: Type 3 turbine - Case 1 and $S C R=5\left(V_{\text {Term }}, I_{\text {Real, }} I_{\text {Reac }}\right)$.......................................199

Figure 7-151: Vestas: Type 3 turbine - Case 1 and $S C R=10\left(V_{\text {Term }}, \mathrm{P}, \mathrm{Q}\right)$.............................................199

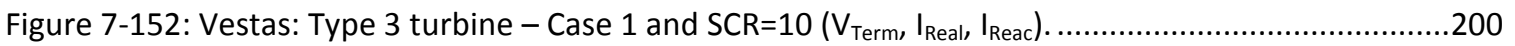

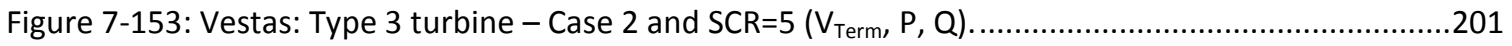

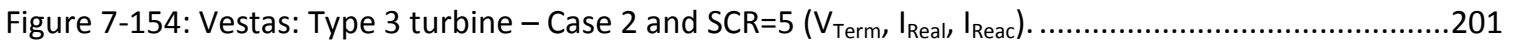

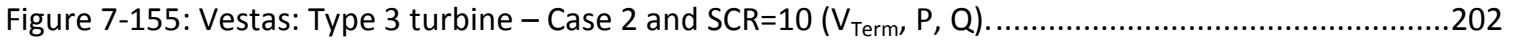

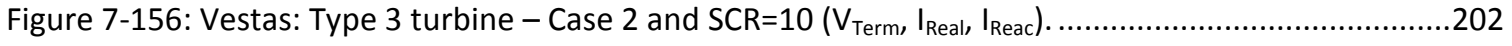

Figure 7-157: Vestas: Type 3 turbine - Case 3 and SCR=5 $\left(V_{\text {Term }}, \mathrm{P}, \mathrm{Q}\right)$.................................................203

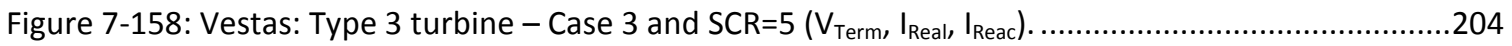

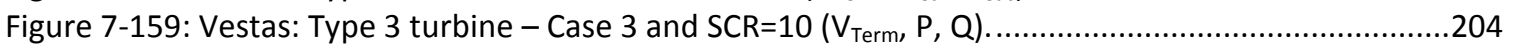

Figure 7-160: Vestas: Type 3 turbine - Case 3 and $S C R=10\left(V_{\text {Term }}, I_{\text {Real }}, I_{\text {Reac }}\right)$......................................205

Figure 7-161: Parameterization of WT3 vs Vestas: Type 3 turbine - Case 1 and $S C R=5\left(V_{\text {Term }}, P, Q\right)$.......207

Figure 7-162: Parameterization of WT3 vs Vestas: Type 3 turbine - Case 1 and SCR $=5\left(V_{\text {Term }}, I_{\text {Real, }} I_{\text {Reac }}\right) \ldots .207$ Figure 7-163: Parameterization of WT3 vs Vestas: Type 3 turbine - Case 1 and $S C R=10\left(V_{T e r m}, P, Q\right) . \ldots . .208$ Figure 7-164: Parameterization of WT3 vs Vestas: Type 3 turbine - Case 1 and $S C R=10\left(V_{\text {Term }}, I_{\text {Real }}, I_{\text {Reac }}\right) .208$ Figure 7-165: Parameterization of WT3 vs Vestas: Type 3 turbine - Case 2 and SCR $=5\left(V_{\text {Term }}, P, Q\right)$........209 
Figure 7-166: Parameterization of WT3 vs Vestas: Type 3 turbine - Case 2 and $S C R=5\left(V_{\text {Term }}, I_{\text {Real, }} I_{\text {Reac }}\right)$. .209 Figure 7-167: Parameterization of WT3 vs Vestas: Type 3 turbine - Case 2 and SCR $=10\left(V_{\text {Term }}, P, Q\right)$......210 Figure 7-168: Parameterization of WT3 vs Vestas: Type 3 turbine - Case 2 and $S C R=10\left(V_{\text {Term }}, I_{\text {Real }}, I_{\text {Reac }}\right) .210$ Figure 7-169: Parameterization of WT3 vs Vestas: Type 3 turbine - Case 3 and $S C R=5\left(V_{\text {Term }}, P, Q\right)$.......211 Figure 7-170: Parameterization of WT3 vs Vestas: Type 3 turbine - Case 3 and $S C R=5\left(V_{\text {Term }}, I_{\text {Real, }}, I_{\text {Reac }}\right) . .211$ Figure 7-171: Parameterization of WT3 vs Vestas: Type 3 turbine - Case 3 and SCR $=10\left(V_{\text {Term }}, P, Q\right)$......212 Figure 7-172: Parameterization of WT3 vs Vestas: Type 3 turbine - Case 3 and $S C R=10\left(V_{\text {Term }}, I_{\text {Real }}, I_{\text {Reac }}\right) .212$ Figure 7-173: Active power and reactive power validation during a 0.3 pu residual voltage and a dip duration of $2 \mathrm{sec}$...... Figure 7-174: Stator voltage and PCC voltage validation during a 0.3 pu residual voltage and a dip duration of $2 \mathrm{sec}$. 214

Figure 7-175: Active power and reactive power validation during a 0.3 pu residual voltage and a dip duration of $0.6 \mathrm{sec}$. .215

Figure 7-176: PCC voltage and stator voltage validation during a 0.3 pu residual voltage and a dip duration of $0.6 \mathrm{sec}$.

Figure 7-177: Active power and reactive power validation during a 0 pu residual voltage and a dip duration of $0.3 \mathrm{sec}$. 216

Figure 7-178: PCC voltage and stator voltage validation during a 0 pu residual voltage and a dip duration

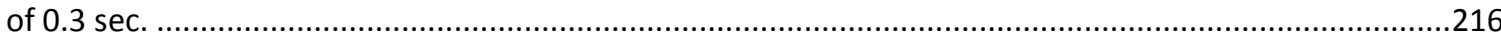

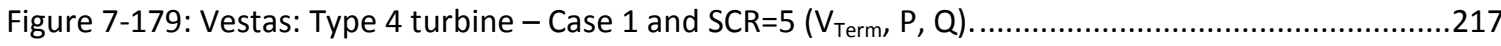

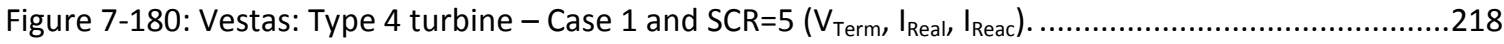

Figure 7-181: Vestas: Type 4 turbine - Case 1 and $S C R=10\left(V_{\text {Term }}, \mathrm{P}, \mathrm{Q}\right)$..........................................218

Figure 7-182: Vestas: Type 4 turbine - Case 1 and $S C R=10\left(V_{\text {Term, }} I_{\text {Real }}, I_{\text {Reac }}\right)$.......................................219

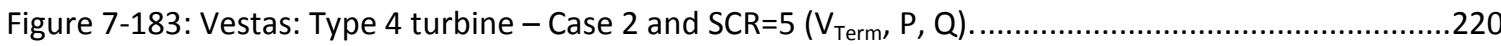

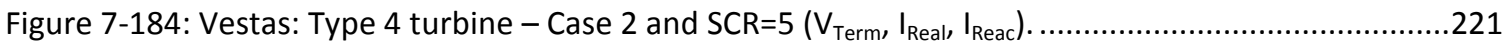

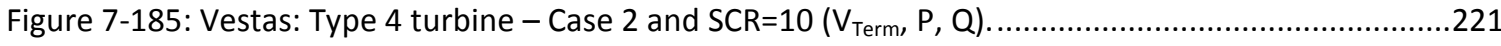

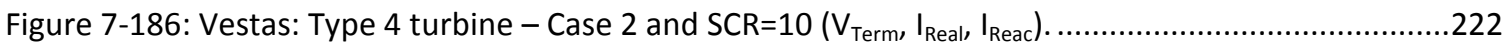

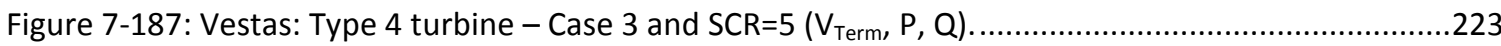

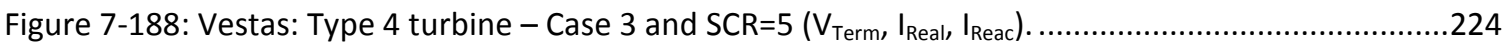

Figure 7-189: Vestas: Type 4 turbine - Case 3 and SCR=10 $\left(V_{\text {Term }}, \mathrm{P}, \mathrm{Q}\right)$..............................................224

Figure 7-190: Vestas: Type 4 turbine - Case 3 and SCR=10 (VTerm, IReal, IReac)..................................225

Figure 7-191: Parameterization of WT4 vs Vestas: Type 4 turbine - Case 1 and SCR=5 ( $\left.\mathrm{V}_{\text {Term }}, \mathrm{P}, \mathrm{Q}\right)$........227 Figure 7-192: Parameterization of WT4 vs Vestas: Type 4 turbine - Case 1 and SCR=5 $\left(V_{\text {Term }}, I_{\text {Real }}, I_{\text {Reac }}\right) \ldots .228$ Figure 7-193: Parameterization of WT4 vs Vestas: Type 4 turbine - Case 1 and $S C R=10\left(V_{T e r m}, P, Q\right) . \ldots . .228$ Figure 7-194: Parameterization of WT4 vs Vestas: Type 4 turbine - Case 1 and $S C R=10\left(V_{\text {Term }}, I_{\text {Real }}, I_{\text {Reac }}\right) .229$ Figure 7-195: Parameterization of WT4 vs Vestas: Type 4 turbine - Case 2 and $S C R=5\left(V_{\text {Term }}, P, Q\right)$........230 Figure 7-196: Parameterization of WT4 vs Vestas: Type 4 turbine - Case 2 and SCR=5 $\left(V_{\text {Term }}, I_{\text {Real }}, I_{\text {Reac }}\right)$. .230 Figure 7-197: Parameterization of WT4 vs Vestas: Type 4 turbine - Case 2 and $S C R=10\left(V_{T e r m}, P, Q\right)$......231 Figure 7-198: Parameterization of WT4 vs Vestas: Type 4 turbine - Case 2 and SCR $=10\left(V_{\text {Term }}, I_{\text {Real }}, I_{\text {Reac }}\right) .231$ Figure 7-199: Parameterization of WT4 vs Vestas: Type 4 turbine - Case 3 and SCR $=5\left(V_{\text {Term }}, P, Q\right)$........232 Figure 7-200: Parameterization of WT4 vs Vestas: Type 4 turbine - Case 3 and SCR=5 $\left(V_{\text {Term }}, I_{\text {Real, }}, I_{\text {Reac }}\right) . .232$ Figure 7-201: Parameterization of WT4 vs Vestas: Type 4 turbine - Case 3 and SCR $=10\left(V_{\text {Term }}, P, Q\right)$......233 Figure 7-202: Parameterization of WT4 vs Vestas: Type 4 turbine - Case 3 and SCR=10 $\left(V_{\text {Term }}, I_{\text {Real }}, I_{\text {Reac }}\right) .233$ Figure 7-203: Active power and reactive power validation during a 0.9 pu residual voltage and a dip duration of $0.5 \mathrm{sec}$. 235 Figure 7-204: PCC voltage and stator voltage validation during a 0.9 pu residual voltage and a dip duration of $0.5 \mathrm{sec}$. 236

Figure 7-205: Active power and reactive power validation during a 0.5 pu residual voltage and a dip duration of $0.5 \mathrm{sec}$. 237 Figure 7-206: PCC voltage and stator voltage validation during a 0.5 pu residual voltage and a dip duration of $0.5 \mathrm{sec}$. 238 Figure 7-207: Active power and reactive power validation during a 0.2 pu residual voltage and a dip duration of $0.3 \mathrm{sec}$. 
Figure 7-208: PCC voltage and stator voltage validation during a 0.2 pu residual voltage and a dip duration

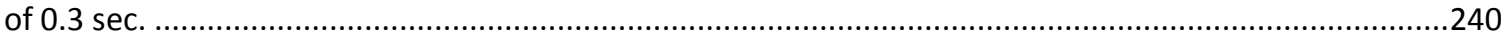




\section{Tables}

Table 5-1: US wind power cumulative capacity by state by the end of 2010. [4], [5] and [6] ....................64

Table 5-2: NERC wind power cumulative capacity by interconnection by the end of 2010. [5] and [6] .......66

Table 5-3: Current wind turbine models by type (not a complete list) . ................................................68

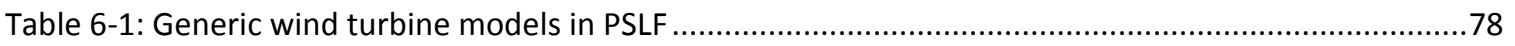

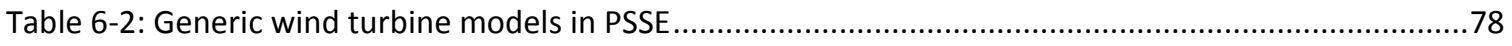

Table 7-1: PSLF generic model parameter values. ......................................................................95

Table 7-2: PSLF generic model parameter values .........................................................................113

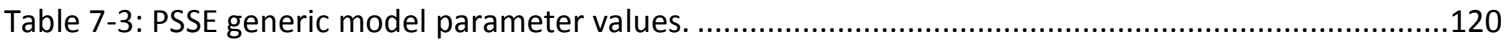

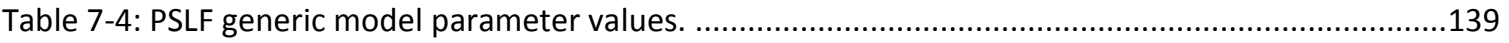

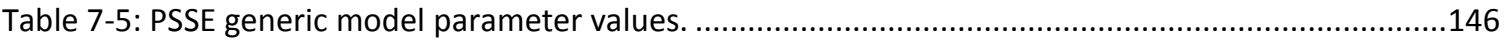

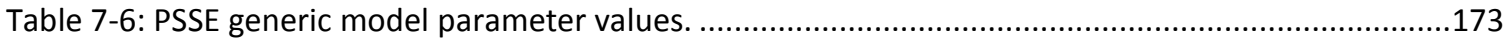

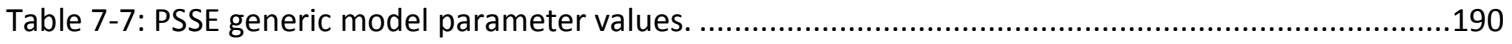

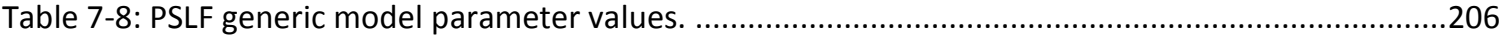

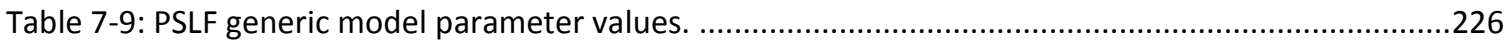




\section{Acknowledgments}

\section{Principal Investigator:}

Robert Zavadil - EnerNex LLC

\section{With contributions from:}

$\begin{array}{llll}\text { Vadim Zheglov } & \text { Yuriy Kazachkov } & \text { Bo Gong } & \text { Juan Sanchez-Gasca } \\ \text { EnerNex LLC } & \text { Siemens Energy } & \text { Siemens Energy } & \text { GE Energy }\end{array}$

Workshops and Outreach:

Four dissemination workshops took place during the course of this work. The authors would like to acknowledge the host organizations, the primary organizers, and the presenters and contributors for their efforts:

\begin{tabular}{|l|l|l|l|}
\hline \multicolumn{1}{|c|}{ Host } & \multicolumn{1}{c|}{ Organizer } & \multicolumn{1}{c|}{ Venue } & \multicolumn{1}{c|}{ Dates } \\
\hline WECC & $\begin{array}{l}\text { Donald Davies } \\
\text { Brittany Power }\end{array}$ & Salt Lake City, UT & June 23, 2011 \\
\hline NYISO/ISO-NE & $\begin{array}{l}\text { John Adams } \\
\text { Kathleen Hockford }\end{array}$ & Rensselaer, NY & July 5-6, 2011 \\
\hline SPP/MISO & $\begin{array}{l}\text { David Duebner } \\
\text { Toni Schewe }\end{array}$ & St. Paul, MN & August 16-17, 2011 \\
\hline PJM & $\begin{array}{l}\text { Chuck Liebold } \\
\text { Evan Wilcox }\end{array}$ & Pataskala, OH & September 26-27, 2011 \\
\hline
\end{tabular}

Presenters and contributors:

Abraham Ellis - Sandia National Laboratories

Bernardo Fernandes - Siemens Energy

Bo Gong - Siemens Energy

Douglas Brown - Siemens Energy

Eduard Muljadi - NREL

Jason MacDowell - GE Energy

Jay Senthil - Siemens Energy
Jim Feltes - Siemens Energy

Juan Sanchez-Gasca - GE Energy

Mark Lauby - NERC

Pouyan Pourbeik - EPRI

Robert Zavadil - EnerNex

Vadim Zheglov - EnerNex

Yuriy Kazachkov - Siemens Energy 
Close to 200 individuals from numerous organizations attended the stakeholder workshops:

\begin{tabular}{|c|c|c|c|}
\hline $\begin{array}{l}\text { Alberta Electric } \\
\text { System Operator }\end{array}$ & $\begin{array}{l}\text { Electric Power } \\
\text { Research Institute }\end{array}$ & $\begin{array}{l}\text { Montana-Dakota } \\
\text { Utilities Co }\end{array}$ & $\begin{array}{l}\text { Richard C. Gross P.E., } \\
\text { Inc. }\end{array}$ \\
\hline Ameren Corporation & $\begin{array}{l}\text { Electric Reliability } \\
\text { Council of Texas }\end{array}$ & National Grid & $\begin{array}{l}\text { Sandia National } \\
\text { Laboratories }\end{array}$ \\
\hline $\begin{array}{l}\text { American Electric } \\
\text { Power }\end{array}$ & $\begin{array}{l}\text { Empire District Electric } \\
\text { Company }\end{array}$ & $\begin{array}{l}\text { National Renewable } \\
\text { Energy Laboratory }\end{array}$ & Siemens Energy Inc. \\
\hline $\begin{array}{l}\text { American } \\
\text { Transmission } \\
\text { Company }\end{array}$ & GE Energy & $\begin{array}{l}\text { Nebraska Public Power } \\
\text { District }\end{array}$ & Siemens-PTI \\
\hline AWS Truepower & Hydro One & $\begin{array}{l}\text { New Brunswick System } \\
\text { Operator }\end{array}$ & Slater Consulting \\
\hline $\begin{array}{l}\text { Basin Electric Power } \\
\text { Cooperative }\end{array}$ & $\begin{array}{l}\text { Independence Power } \\
\text { \& Light }\end{array}$ & New York ISO & Southwest Power Pool \\
\hline $\begin{array}{l}\text { Bonneville Power } \\
\text { Administration }\end{array}$ & $\begin{array}{l}\text { Independent } \\
\text { Electricity System } \\
\text { Operator }\end{array}$ & $\begin{array}{l}\text { New York Power } \\
\text { Authority }\end{array}$ & $\begin{array}{l}\text { Suzlon Wind Energy } \\
\text { Corporation }\end{array}$ \\
\hline California ISO & ISO New England & Nordex & Texas Tech University \\
\hline $\begin{array}{l}\text { City Utilities of } \\
\text { Springfield MO }\end{array}$ & ITC Midwest & $\begin{array}{l}\text { North American Electric } \\
\text { Reliability Corporation }\end{array}$ & $\begin{array}{l}\text { Utility System } \\
\text { Efficiencies. Inc. }\end{array}$ \\
\hline Clipper Windpower & Lincoln Electric System & $\begin{array}{l}\text { Northeast Power } \\
\text { Coordinating Council }\end{array}$ & Vestas Americas \\
\hline ComEd & Manjure & Northern Power System & Weber \\
\hline Con Edison & Midwest Energy & $\begin{array}{l}\text { Otter Tail Power } \\
\text { Company }\end{array}$ & Westar Energy Inc \\
\hline Constellation Energy & Midwest ISO & Pacificorp & $\begin{array}{l}\text { Western Area Power } \\
\text { Administration }\end{array}$ \\
\hline $\begin{array}{l}\text { Customized Energy } \\
\text { Solutions }\end{array}$ & $\begin{array}{l}\text { Midwest Reliability } \\
\text { Organization }\end{array}$ & PJM & $\begin{array}{l}\text { Western Electricity } \\
\text { Coordinating Council }\end{array}$ \\
\hline $\begin{array}{l}\text { Federal Energy } \\
\text { Regulatory } \\
\text { Commission }\end{array}$ & Minnesota Power & PSM Consulting, Inc. & $\begin{array}{l}\text { Wisconsin Public } \\
\text { Service Corp }\end{array}$ \\
\hline $\begin{array}{l}\text { Department of Public } \\
\text { Safety }\end{array}$ & $\begin{array}{l}\text { Minnesota Renewable } \\
\text { Energy Society }\end{array}$ & $\begin{array}{l}\text { Public Service } \\
\text { Enterprise Group }\end{array}$ & $\begin{array}{l}\text { Wolverine Power } \\
\text { Supply Cooperative, Inc }\end{array}$ \\
\hline Duke Energy & $\begin{array}{l}\text { Mitsubishi Power } \\
\text { Systems }\end{array}$ & Puget Sound Energy & Xcel Energy \\
\hline
\end{tabular}


At the 'UWIG Fall Technical Workshop', held October 11-14, 2011, the preliminary results and findings were shared with groups of invited individuals from key stakeholder sectors. The authors wish to acknowledge following individuals for their insightful inputs:

Brendan Kirby - Consult Kirby John Daniel - ABB

Brian Parsons - NREL

Charles Smith - UWIG

Lisa Dangelmaier - Hawaii Electric Light Company

Charleton Clark - DOE

Marko Ibsch - GL Garrad Hassan

Dale Osborn - DISGEN Corp.

Reigh Walling - GE Energy

Diane Broad - Ecofys US

Rob Cleveland - GL Garrad Hassan

James Cote - Electric Power Systems

Steve Saylors - Vestas Wind Systems

Finally, the authors express their sincere gratitude to following individuals and their organizations for sharing their simulation models, validation data, professional expertise, and time which significantly helped in gathering the required information for the wind turbine data base:

Atsushi Uchiyama - Mitsubishi Power Systems

Babak Badrzadeh - Vestas Technology R\&D

Chester Zapotoczny - Suzlon Wind Energy

Frank Bergh - Nordex

Hanif Mashal - Clipper WindPower
Juan Sanchez-Gasca - GE Energy

Jun Li - EnerNex

Paul Gonzalez - REPower USA Corp.

Robert Nelson - Siemens Energy

Yuriy Kazachkov - Siemens Energy 


\section{Executive Summary}

As part of the Utility Wind Energy Integration Group (UWIG) and EnerNex's Wind Turbine Modeling Project, EnerNex has received ARRA (federal stimulus) funding through the Department of Energy (DOE) to further the progress of wind turbine and wind plant models. Despite the large existing and planned wind generation deployment, industry-standard models for wind generation have not been formally adopted. Models commonly provided for interconnection studies are not adequate for use in general transmission planning studies, where public, non-proprietary, documented and validated models are needed. NERC MOD (North American Electric Reliability Corporation) reliability standards require that power flow and dynamics models be provided, in accordance with regional requirements and procedures.

The goal of this project is to accelerate the appropriate use of generic wind turbine models for transmission network analysis. Through the developments and activities proposed for this effort, transmission planning engineers will be able to confidently apply the generic models for network studies involving commercial wind turbines sold into the U.S. market, in full compliance with NERC standards and guidelines.

The project objectives are accomplished through execution of the following initiatives:

- Defining proposed enhancements to the generic wind turbine model structures that would allow representation of more advanced features such as power control, automatic curtailment, inertial and governor response;

- Comparative testing of the generic models against more detailed (and sometimes proprietary) versions developed by turbine vendors;

- Developing recommended parameters for the generic models to best mimic the performance of specific commercial wind turbines;

- Documenting results of the comparative simulations in an application guide for users;

- Acquiring test data from all available sources for the purpose of validating the performance of the appropriately specified generic models in actual case studies; 
- Conducting technology transfer activities in regional workshops for dissemination of knowledge and information gained, and to engage electric power and wind industry personnel in the project while underway;

- Designing of a "living" homepage to establish an online resource for transmission planners by including: (1) turbine technology overview, (2) model characteristics and parameters, and (3) generic representations (http://www.uwig.org:8080/index.php?title=Main_Page). 


\section{Abbreviations and Acronyms}

AGC: Automatic Gain Control

AGC: Automatic Generation Control

ASCC: Alaska System Coordinating Council

AWEA: American Wind Energy Association

DFAG: Doubly-Fed Asynchronous Generator

DOE: Department of Energy

ERCOT: Electric Reliability Council of Texas

FACTS: Flexible Alternating Current Transmission Systems

FERC: Federal Energy Regulatory Commission

FRCC: Florida Reliability Coordinating Council

GE: General Electric Corporation

HQ: Hydro Quebec

IEC: International Electromechanical Council

IGBT: Insulated Gate Bipolar Transistor

IEEE: Institute of Electrical and Electronics Engineers

IPP: Independent Power Producer

ISO: Independent System Operator

MRO: Midwest Reliability Organization

NERC: North American Electric Reliability Corporation

NPCC: Northeast Power Coordinating Council

PSLF: Positive Sequence Load Flow

PSSE: Power System Simulation for Engineering

RAS: Remedial Action Scheme

REMTF: Renewable Energy Task Force 
RFC: Reliability First Corporation

SCADA: Supervisory Control and Data Acquisition

SERC: SERC Reliability Corporation

SPP: Southwest Power Pool

SPS: Special Protective System

TRE: Texas Reliability Entity

UWIG: Utility Wind Integration Group

VSM: Vendor Specific Model

VSM: Vendor Specific Model

WECC: Western Electricity Coordinating Council

WPP: Wind Power Plant

WTG: Wind Turbine Generator 


\section{Introduction}

Significant progress in the development of models for the study of wind plant interaction with the electric power system has been made over the past decade. However, with the pace of wind plant development surging over the past few years (and expected to continue), it is apparent that significant work remains in the wind turbine and wind plant model development process.

Transmission planning engineers, charged with ensuring that the electric power system meets the needs of its users now, next week, and ten years from now, rely on sophisticated analysis tools in all aspects of their work.

The electric power system is a very complicated machine. Maintaining high reliability requires much vigilance, whether the time frame of interest is the next hour or ten years from now. Any prospective changes, such as the connection of a new generator, must be studied thoroughly to make certain that the performance of the transmission network will not be degraded. For large systems, the technical analyses that form the basis for these studies rely on complex computer calculations and simulations. All elements of the system are represented - transmission lines, transformers, generators, auxiliary equipment such as excitation systems and governors, etc.

The suite of studies performed are based on two fundamental types of analyses:

- power flow, which for a specified dispatch of generation will compute the voltages at each bus and flows through each branch of the model,

- dynamic analysis, where the behavior of the network following a disturbance is simulated over a short period of time, usually 5 to 20 seconds.

Models for conventional electrical equipment to facilitate the various types of analysis that must be conducted have been developed over a number of years, and in most cases have undergone much refinement. Standard models have been evolved for many devices, so that, for example, a new generating unit can be studied by selected standard representations for the synchronous machine, exciter, and governor, and plugging in the appropriate parameters.

When it emerged on the transmission planning scene, wind generation was initially problematic because the underlying technology was unique, unlike conventional 
generation equipment, and did not fit into the templates provided by standard models. As the electric power industry became more certain that wind energy would be a significant part of the generation mix going forward, attention turned to the characterization of the technology for system studies.

The scale of the challenge has been often noted in industry meetings and workshops. Only partially in jest, it has been noted that the industry must do in about five years time for wind generation what it has taken over 50 years to accomplish with more familiar electrical equipment.

In response to this DOE solicitation, EnerNex and UWIG have assembled a project team to carry out the next important phase of wind plant model development, and a Technical Review Committee (TRC) to guide the effort. With support from DOE, the necessary continued development of these models can be accelerated by several years. Achieving this goal, however, is critically dependent on broad participation from the electric power and wind industries. By leveraging the resources and membership of the UWIG Modeling and Interconnection User Group, such a project team was assembled.

This project builds on efforts begun in 2005 by the WECC to develop a suite of generic wind turbine models appropriate for bulk transmission system studies. This earlier effort has progressed to where a prototype generic model for each of the major wind turbine generator topologies has been conceptualized and implemented in the major platforms for power system dynamic simulation in North America - PSS/E and PSLF. This work continues to be guided and managed by Abraham Ellis, previously of Public Service Company of New Mexico, currently with Sandia National Labs, and a member of the TRC.

Building on work begun earlier in Europe, the WECC team determined that because of the unique and varying technologies being employed for wind generation, a single "model" was neither appropriate nor achievable. Instead, four separate models, each corresponding to one of the topologies in the commercial marketplace were identified as a starting point for the model discussion. These are:

- Type I- a turbine with a squirrel-cage induction generator to perform the mechanical to electrical energy conversion.

- Type II - mechanical to electrical energy conversion performed with wound rotor induction generation, where external rotor circuit resistance is controlled to provide a limited variable speed range of operation. 
- Type III - a wound rotor induction generator with the rotor circuit connected back to the grid through a four-quadrant ac/dc/ac power convertor; presently the workhorse machine in the industry.

- Type IV - all electrical power from the rotating machine (which can be of almost any type - synchronous, induction, permanent magnet, dc, etc.) is processed through an static power converter on its way to the grid.

Recently, a new turbine topology has entered the U.S. market that is based on a variable-speed mechanical coupling and a conventional synchronous generator. Nominally, this is being referred to as the "Type V" turbine, but because of it's relatively conventionally topology, most of the modeling is expected to be straightforward using traditional and standard elements.

The effort lead by the WECC progressed through technical discussions and proof of concept, ultimately leading to the first revision of proposed generic models for the major topologies in the commercial market. These proposed models have or are being implemented in the PSS/E and PLSF model libraries. The next steps in this initiative are uncertain, as the flag has been passed to the IEEE Power and Energy Society Wind Generation Modeling Task Force, which part of the Power System Dynamics Committee.

With member funding, the UWIG is preparing much-needed documentation of these models. This however, is only the first small step towards the general application of the models for power system studies. This project seeks to develop enhanced documentation, user support, and application materials that will assist users of these major computer analysis platforms in the evaluation of bulk power system impacts of an ever-increasing fleet of wind generation.

Following development of these basic materials, the very difficult but absolutely vital task of validating commercial turbine-specific implementations of the generic models was undertaken. Validation of any model against actual performance data from the field is necessary, and will become mandatory as more stringent interconnection standards are established. Work from the previous phases of the effort was extended to cover this important aspect. The key objective of this phase was developing model-bymodel comparisons to detailed vendor-specific models, transient models, and field measurements. As always, acquisition of measurement data was a major challenge.

This later phase is also expected to be "work in progress" over an extended period of time due to the anticipated difficulties in acquiring the necessary validation data and the sheer scope due to the number of commercial turbines in the existing fleet. 


\section{Wind Turbine Technologies}

Models for conventional power system elements such as generators and their various control systems, switched and static compensation devices, load, and transmission network elements are well understood by power system analysts. Wind plants, however, pose several new challenges. The fundamental nature of a commercial bulk wind plant, with large numbers of relatively small wind turbine generators (WTGs) interconnected by a substantial medium-voltage network, requires equivalencing and simplification without loss of important detail. For some phenomena, the wind plant can be treated as a single entity - transmission power flow is an example, where definition of real and reactive power injection at the point of interconnection with the transmission system at an instant in time provides adequate representation. In dynamic studies, it would be desirable to treat the wind plant as a single large plant, but such treatment may not represent the full range of dynamic behavior or be appropriate for all types of system disturbances. Because each individual wind turbine is a relatively sophisticated machine, determining the dynamic behavior of the aggregate plant model can be difficult.

The technology employed in commercial wind turbines deviates from the much better understood conventional generation equipment. Induction machines, rather than synchronous generators, are used in nearly all U.S. commercial wind turbines. Further, some of the turbine designs employ sophisticated power electronic controllers that alter the fundamental behavior of the induction machines in both steady state and dynamic operation. The characteristics of other wind turbine elements and wind plants that may have an influence on the design, operation, or security of the bulk power system, such as the rotational inertias and torsional constants of the mechanical systems or the variation of real and reactive power output as functions of time, are unknown to power system analysts.

For analytical studies of large power systems, the time frames of interest range from tens of milliseconds to steady state. Device and component models, therefore, must accurately reflect behavior over the entire bandwidth, and properly account for any phenomena outside of the simulation bandwidth that may have an "aliasing" effect on the time frame of interest. Because the purpose of the models is to facilitate investigation of electrical power system issues, certain details of the mechanical system or energy conversion process may not be represented if they have no impact on 
electrical performance. In conventional models for large power plants, for example, details of the mechanical system, e.g. combustion process, steam cycle control, governor, etc., are included only to the extent that they influence the electrical behavior of the plant during the time frames of interest in a particular study.

A generalized wind turbine model is shown in Figure 2-1, and illustrates the major subsystems and control hierarchy that may influence the behavior of a single wind turbine in the time horizon of interest for large power system studies.

A wind turbine converts kinetic energy in a moving air stream to electric energy. Mechanical torque created by aerodynamic lift from the turbine blades is applied to a rotating shaft. An electrical generator on the same rotating shaft produces an opposing electromagnetic torque. In steady operation, the magnitude of the mechanical torque is equal to that of the electromagnetic torque, so the rotational speed remains constant, real power (the product of rotational speed and torque) is delivered to the grid. Since the wind speed is not constant, a variety of control mechanisms are employed to manage the conversion process and protect the mechanical and electrical equipment from conditions that would result in failure or destruction.

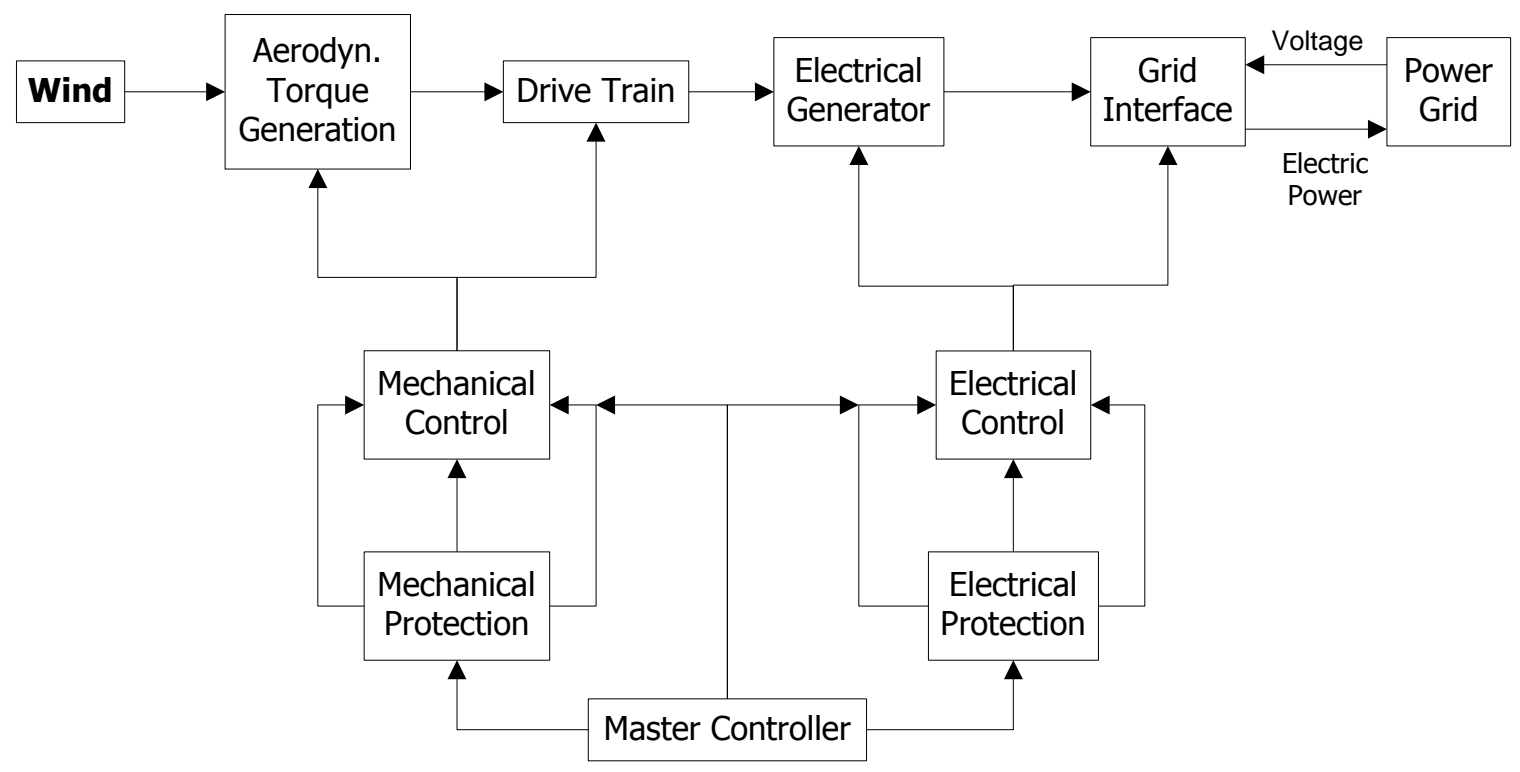

Figure 2-1: Generalized wind turbine model with control elements and hierarchy.

\subsection{Mechanical Systems and Control}

Mechanically, the turbine must be protected from rotational speeds above some value that could lead to catastrophic failure. Mechanical brakes are provided for stopping the turbine in emergency conditions, but are not used in normal operations. 
Controlling the power (and hence, torque) extracted from the moving air stream is the primary means for protecting the turbine from over-speed under all but emergency shutdown conditions.

In fairly steady conditions, the power extracted from the air stream by the turbine blades can be characterized by:

$$
P=\frac{1}{2} \cdot \rho \cdot \pi \cdot R^{2} \cdot v^{3} \cdot C_{p}
$$

where

$\rho=$ air density (nominally $1.22 \mathrm{~kg} / \mathrm{m} 3$ )

$\mathrm{R}=$ radius of area swept by the turbine blades

$v$ = speed of moving air stream

$C_{p}=$ "coefficient of performance" for the composite airfoil (rotating blades)

$C_{p}$ itself is not a constant for a given airfoil, but rather is dependent on a parameter $\lambda$, called the tip-speed ratio, which is the ratio of the speed of the tip of the blade to the speed of the moving air stream.

Since wind speed and air density cannot be controlled, and the radius of the blades is fixed, the performance coefficient is the only means for torque control. In some wind turbines, blades are designed so that $C_{p}$ falls dramatically at high wind speeds. This method of aerodynamic torque control is known as stall regulation, and is limited to preventing turbine over-speed during extreme gust conditions and limiting maximum shaft power in winds at or above the rated value.

Large wind turbines employ a more sophisticated method of aerodynamic torque regulation that has benefits in addition to preventing mechanical over-speed. The performance coefficient can also be changed by adjusting the "angle of attack" of the blades, as is done on some modern propeller-driven aircraft. Figure 2-2 shows $C_{p}$ as a function of $\lambda$ for a modern wind turbine. Blade pitch adjustment allows the energy capture to be optimized over a wide range of wind speeds (even if the rotational speed of the shaft is relatively constant), while still providing for over-speed protection through large adjustments in pitch angle. 


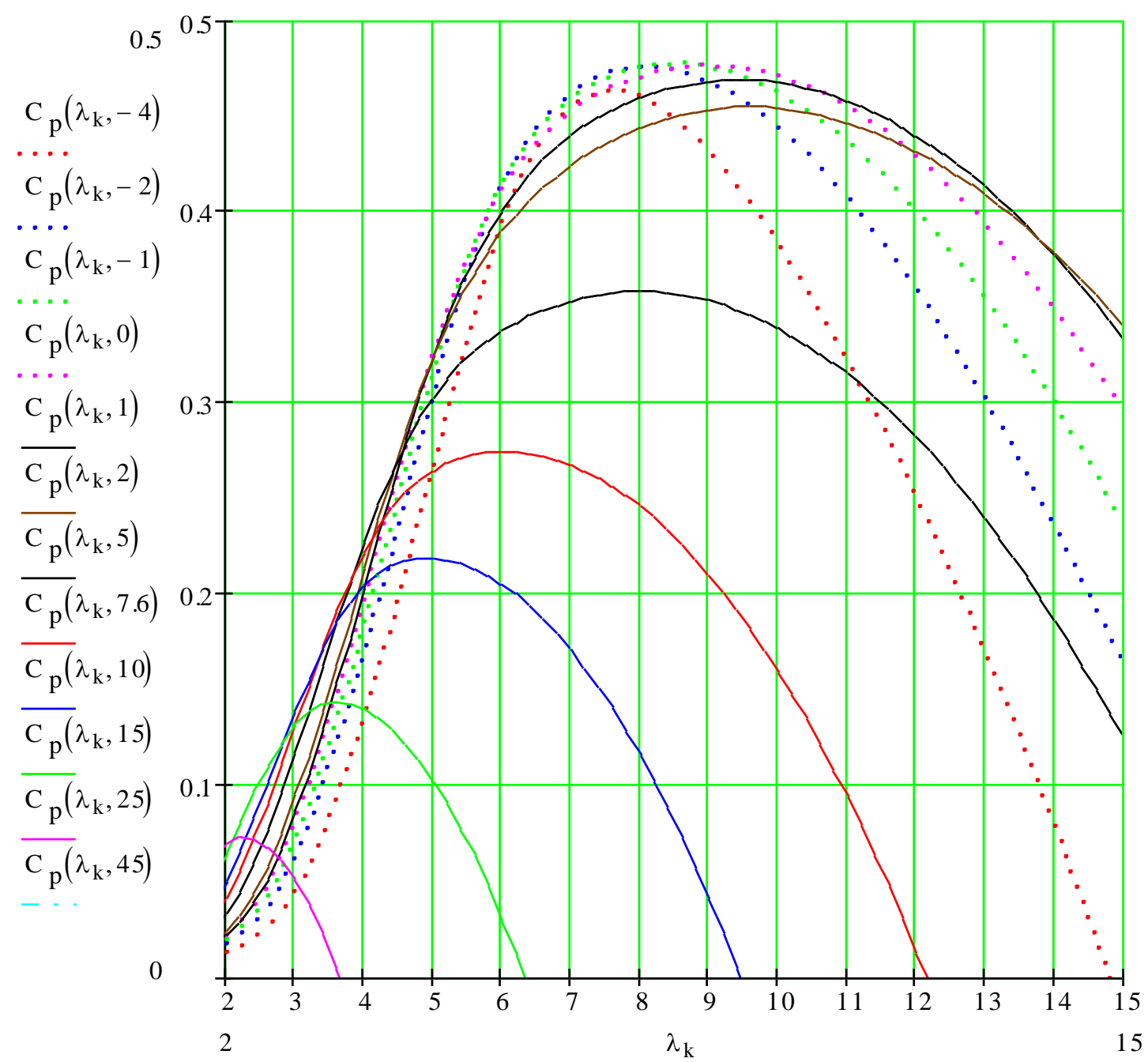

Figure 2-2: Coefficient of performance $\left(C_{p}\right)$ for a modern wind turbine blade assembly as a function of tip-speed ratio $(\lambda)$ and blade pitch ( $\beta$, in degrees).

The pitch of the turbine blades is controlled by an actuator in the hub that rotates each blade about a longitudinal axis. The inertia of the blade about this axis and the forces opposing such a rotation of the blades are not negligible. Pitching of the blades, therefore, does not happen instantaneously, with the dynamics governed by the longitudinal inertia of the blades, forces acting on the blade (which can be wind speed and pitch dependent), and the torque capability of the pitch actuator mechanism.

The characteristic shown in Figure 2-2 is a "quasi-static" depiction of the blade performance, in that is does not account for turbulence effects, blade vibration with respect to the average speed of rotation, or other asymmetries such as tower shadowing. It does, however, provide a much simpler means of incorporating the otherwise very complex details of the aerodynamic conversion process into models for electrical-side studies of the turbine. 
The overall conversion of wind energy to electric power is normally described by a turbine "power curve", which shows turbine electrical output as a function of steady wind speed (Figure 2-3). Such a representation is accurate only for steady-state operation, since the inherent dynamics of the mechanical and electrical systems along with all possible control functionality is neglected.

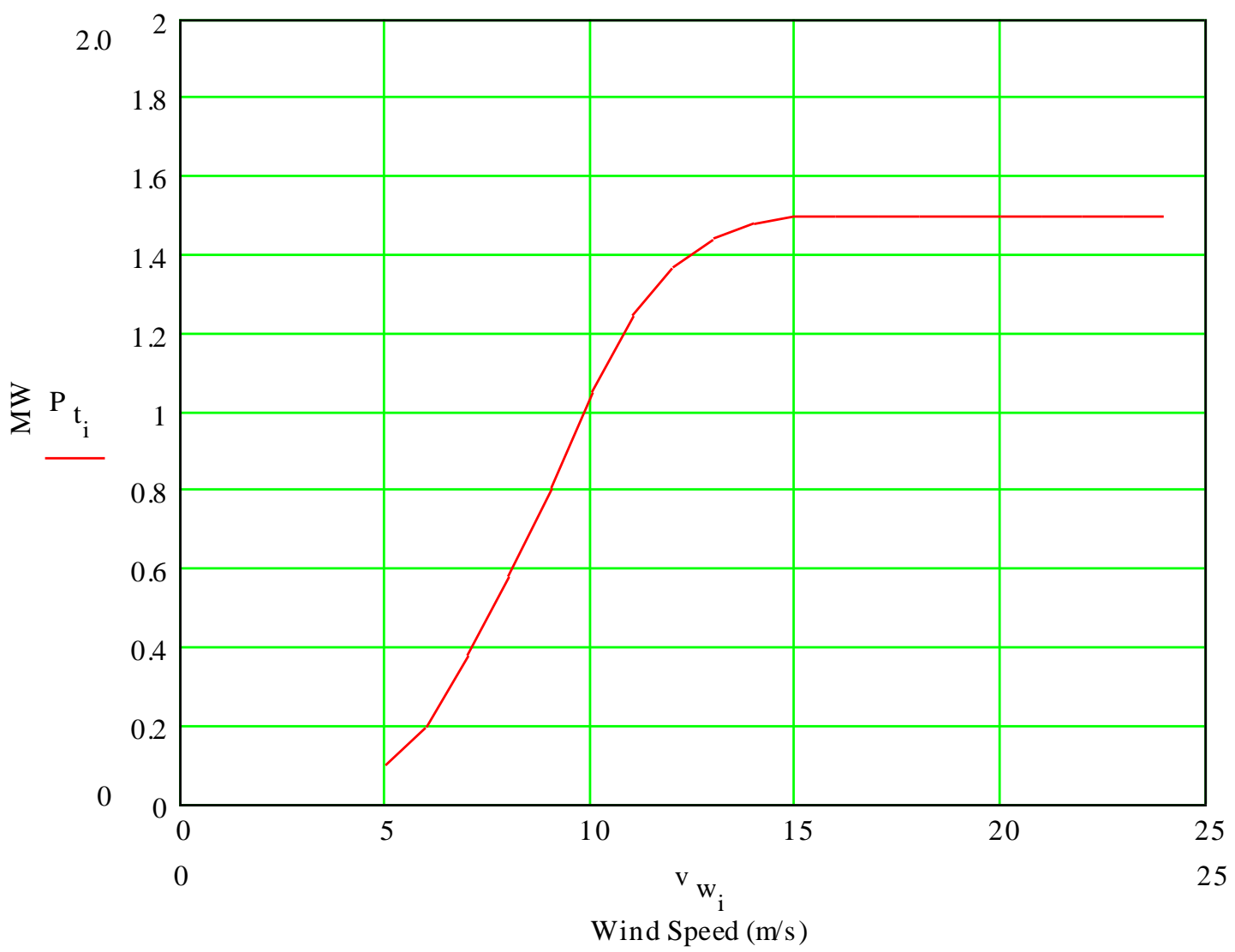

Figure 2-3: Power curve for a variable-speed, pitch-controlled wind turbine. Note "flatness" of output for wind speeds at or above rated value.

Rotational speeds of large wind turbines are partly limited by maximum blade tip speed, and so for megawatt-class turbines with long blades are relatively low, in the 15 to $30 \mathrm{rpm}$ range. With conventional electrical generators, a gearbox is necessary to match the generator speed to the blade speed. The resulting mechanical system, then, has low-speed and high-speed sections, with a gearbox in between, as shown in Figure 2-4 (top). An even simpler representation is shown at the bottom of Figure 2-4, where the gearbox inertia is added to the inertia of the generator, and all components are referred to the high-speed shaft by the square of the gear ratio.

For megawatt-scale turbines, the mechanical inertia is relatively large, with typical inertia constants $(\mathrm{H})$ of 3.0 seconds or larger (the inertia constant for the generator only 
will typically be about $0.5 \mathrm{~s}$ ). The mechanical inertia is an important factor in the dynamic behavior of the turbine, because the large inertia implies relatively slow changes in mechanical speed for both normal variations in wind speed and disturbances on the grid. In addition, the various control systems in the turbine may utilize turbine speed as an input or disturbance signal, so that large inertia will then govern the response time.

With a two-mass mechanical model, there will be one oscillatory mode. With relatively flexible drive shafts in large wind turbines, the natural frequency of this primary mode of oscillation will be in the range of 1 to $2 \mathrm{~Hz}$. 


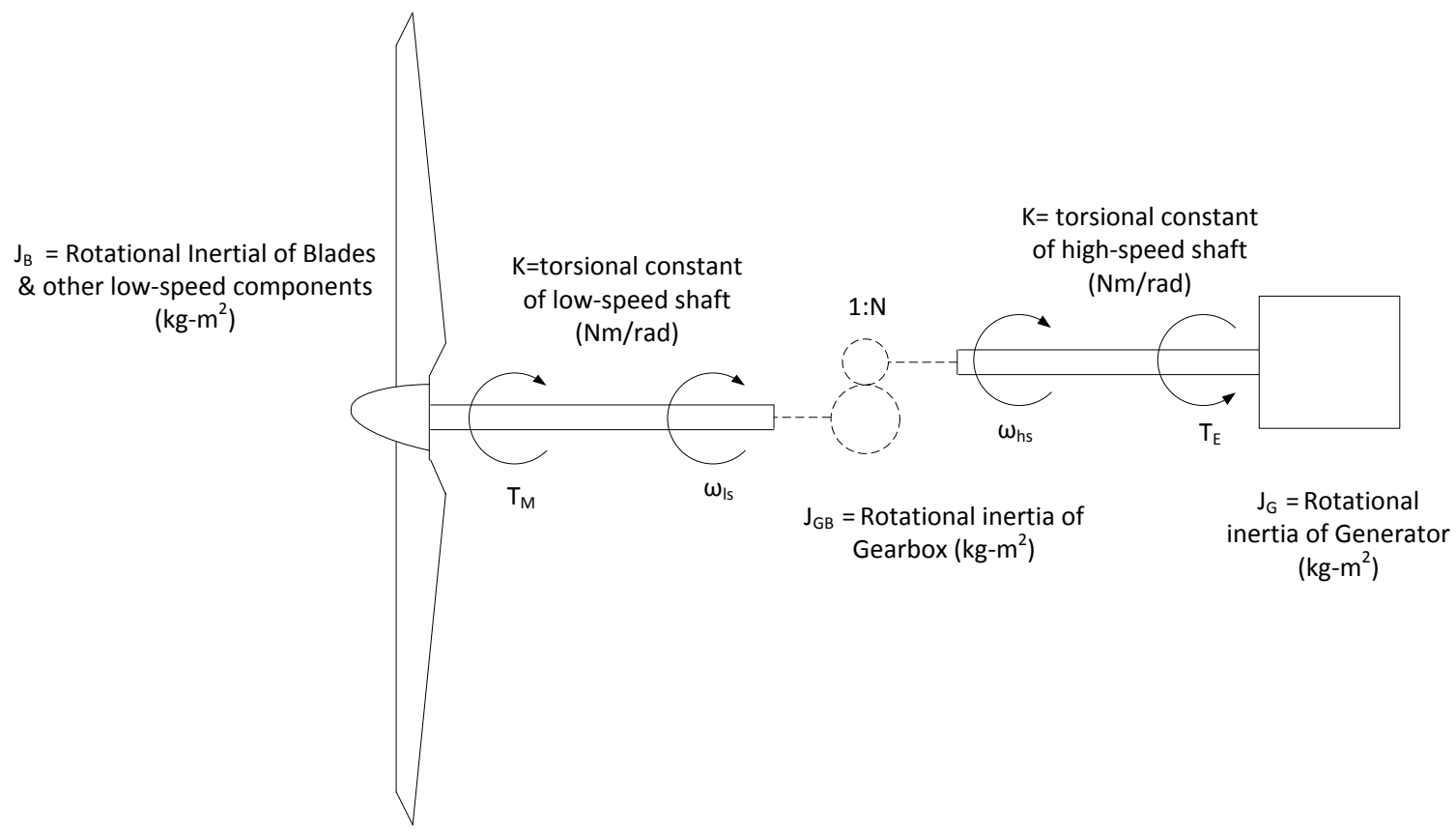

Blades

$\mathrm{J}_{\mathrm{B}}=$ Rotational Inertial of Blades

\& other low-speed components referred to high-speed base $\left(\mathrm{kg}-\mathrm{m}^{2}\right)$

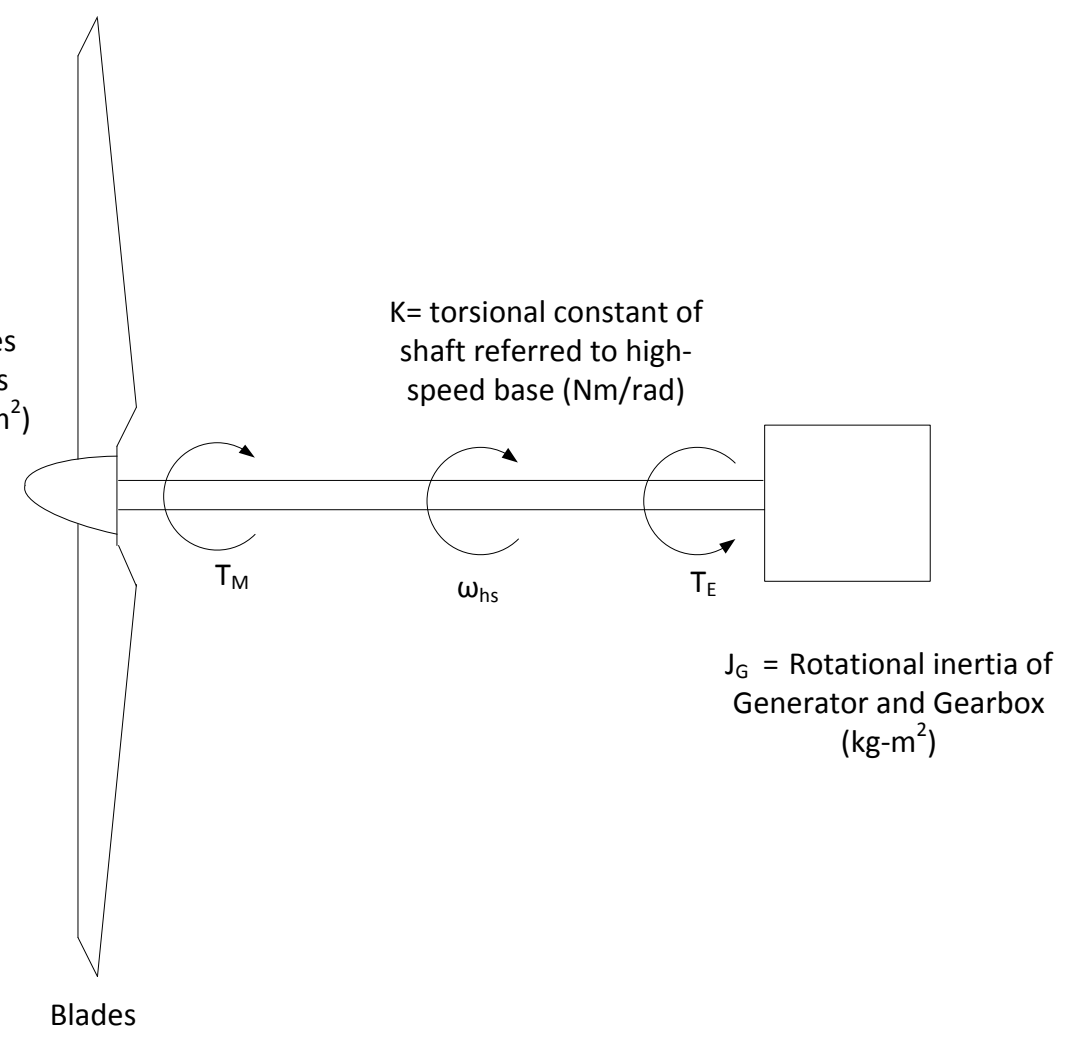

Figure 2-4: Simplified model of wind turbine mechanical system. Two mass model with gearbox (top) and model with equivalent gearbox inertia and reference of all components to high-speed shaft (bottom). 


\subsection{Electrical Systems and Control}

Induction machines are the energy conversion devices of choice in commercial wind turbine design. In addition to their robustness and reliability, they provide a "softer" coupling between the grid and the mechanical system of the turbine. Wind turbine manufacturers have also moved beyond the basic induction generator systems with technologies for improving control and overall efficiencies. These technologies have a definite impact on the electrical and dynamic performance of wind turbines, even to the extent of masking or overriding the dynamic characteristics that would normally be associated with rotating machinery. Almost all of the wind turbines deployed in large wind generation facilities in the U.S. over the past decades can be generally described by one of the following configurations ( [1], [2], and [3]):

- Type I WTG: Stall-regulated (fixed-pitch) blades connected to a hub, which is coupled via a gearbox to a conventional squirrel-cage induction generator. The generator is directly connected to the line, and may have automatically switched shunt capacitors for reactive power compensation and possibly a soft-start mechanism which is bypassed after the machine has been energized. The speed range of the turbine is fixed by the torque vs. speed characteristics of the induction generator.

- Type II WTG: A wound rotor induction generator with a mechanism for controlling the magnitude of the rotor current through adjustable external rotor circuit resistors, and pitch regulation of the turbine blades to assist in controlling speed. The speed range of the turbine is widened because of the external resistors.

- Type III WTG: A wound rotor induction generator where the rotor circuit is coupled to the line terminals through a four-quadrant power converter. The converter provides for vector (magnitude and phase angle) control of the rotor circuit current, even under dynamic conditions, and substantially widens the operating speed range of the turbine. Turbine speed is primarily controlled by actively adjusting the pitch of the turbine blades.

- Type IV WTG: A variable-speed wind turbine with a full-rated power converter between the electrical generator and the grid. The power converter provides substantial decoupling of the electrical generator dynamics from the grid, such that the portion of the converter connected directly to the electrical system defines most of the characteristics and behavior important for power system studies. 
The four major types of generator technologies used in today's commercial wind turbines are discussed in the following sections.

\subsubsection{Direct-Connected Induction (Asynchronous) Generators (Type I)}

Most wind generators installed at the end of the $20^{\text {th }}$ century were ordinary asynchronous (induction) generators, usually with fixed capacitance to correct for the reactive power demands of that type of generator (see Figure 2-5). An induction generator is essentially an induction motor where the slip is negative, i.e. the rotor speed is slightly ahead of the rotating flux in the stator winding. The induction generator has a squirrel cage rotor which draws magnetizing current from the stator giving rise to a high reactive power demand when fluxing, as when the generator circuit breaker is first closed.

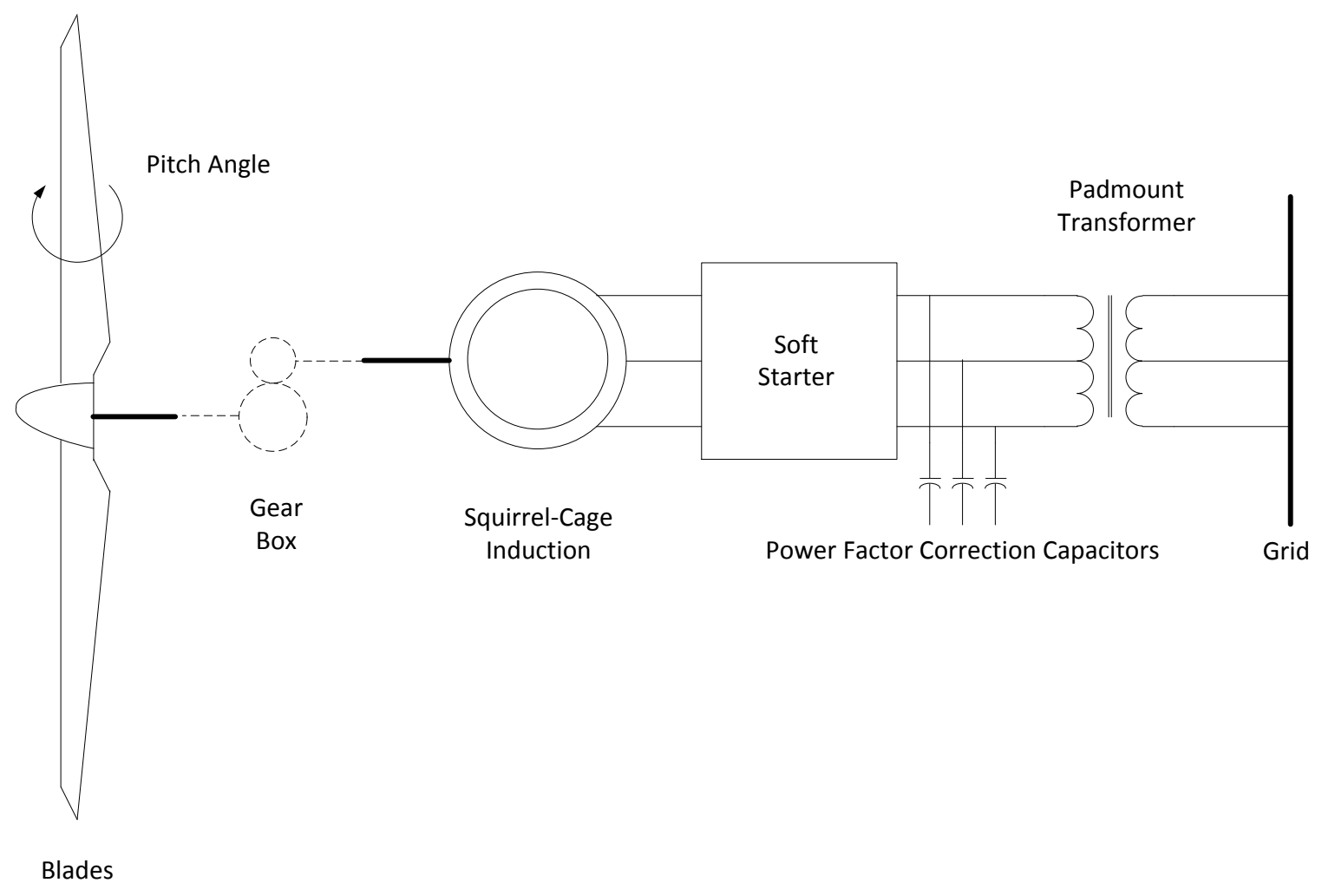

Figure 2-5: Illustration of type I wind turbine generator.

Wind turbines with squirrel-cage induction generators connected directly to the line are the simplest electrically. While for purposes of aerodynamic efficiency they operate at nearly constant speed, the slight variation of speed with torque (and power) can significantly reduce mechanical torque transients associated with gusts of wind and gridside disturbances. 
The speed range of the turbine is dictated by the torque vs. speed characteristic of the induction generator (Figure 2-6). For large generators in today's commercial turbines, slip at rated torque is less than $1 \%$, which results in very little speed variation over the operating range of the turbine. For a given wind speed, the operating speed of the turbine under steady conditions is a nearly linear function of torque, as illustrated by the torque vs. speed characteristic of Figure 2-6. For sudden changes in wind speed, the mechanical inertia of the drive train will limit the rate of change in electrical output.

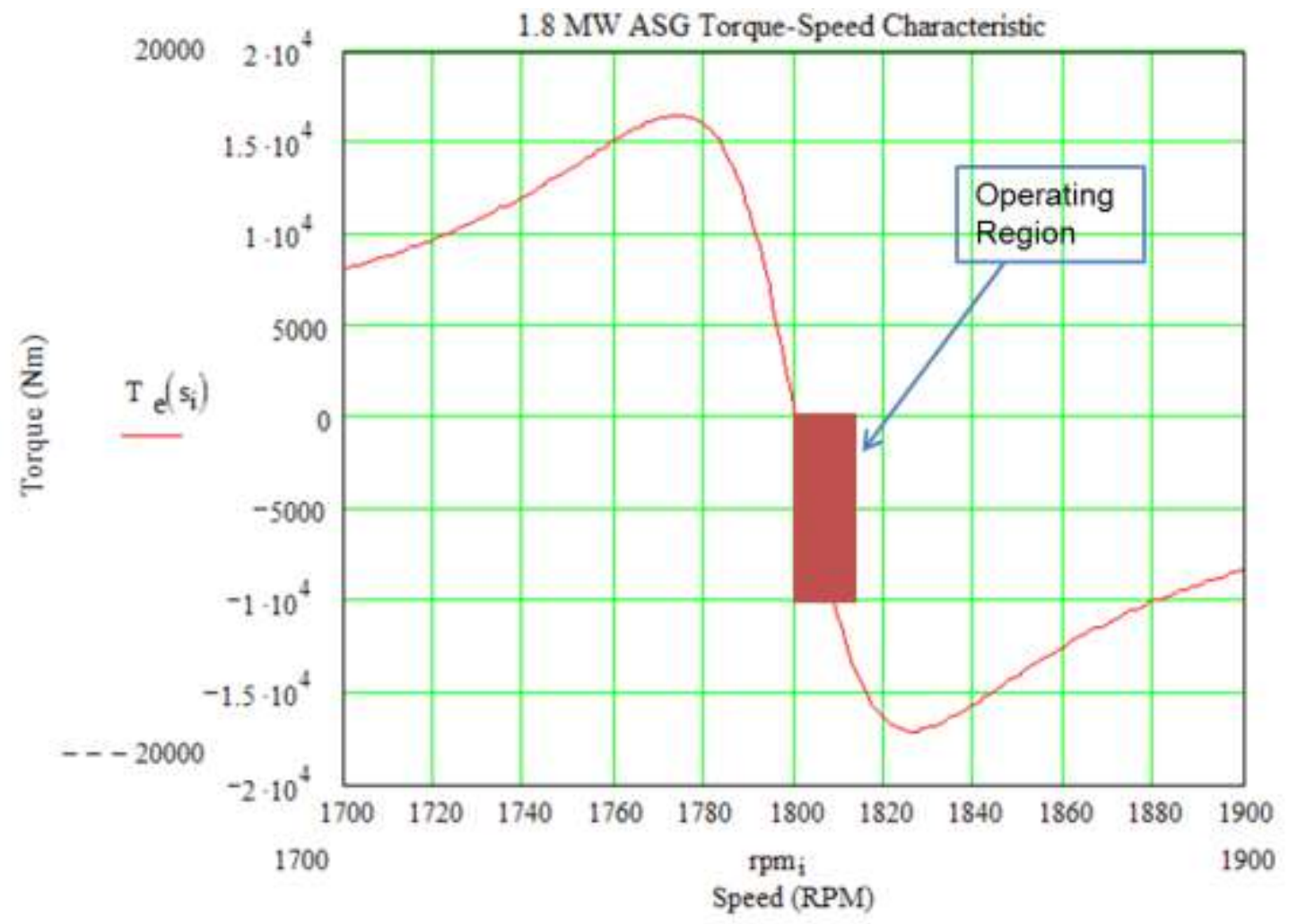

Figure 2-6: Torque vs. Speed characteristic for an induction machine used in a commercial wind turbine.

Because the induction generator derives its magnetic excitation from the grid, the response of the turbine during a grid disturbance will be influenced by the extent to which the excitation is disrupted.

Figure 2-7 shows how dramatically the reactive power demand from the network increases as the generator departs from a tight slip regime. For the machine shown, rated slip is about $0.8 \%$, at which point the machine would draw 340 kVAR from a line with rated voltage. If the slip were increased to just $1.0 \%$, the reactive power requirement increases to almost $480 \mathrm{kVAR}$. At $2.0 \%$ slip, reactive power consumption grows to $900 \mathrm{kVAR}$. 


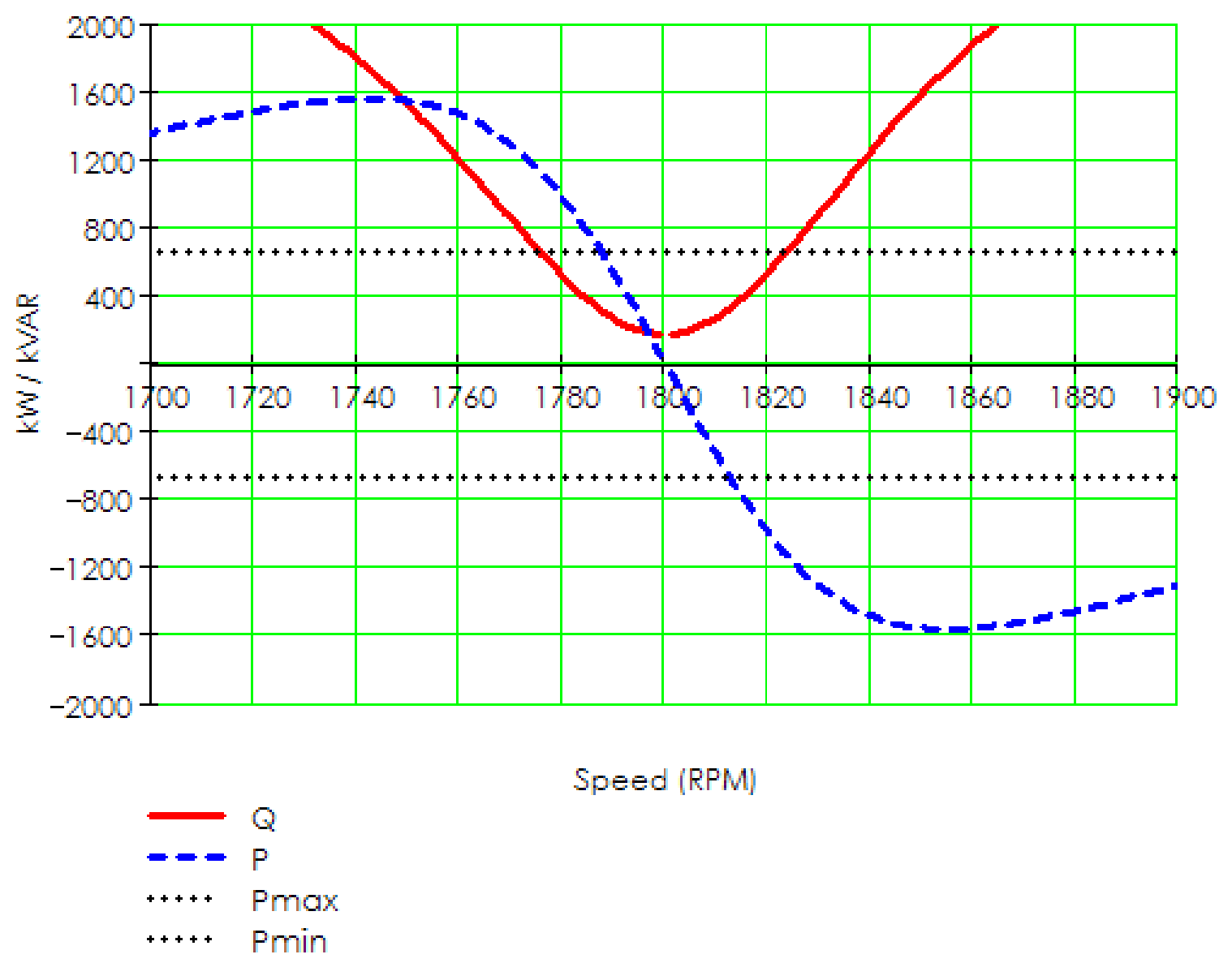

Figure 2-7: Variation of real (solid) and reactive (dash) power with slip for a squirrel-cage induction machine.

\subsubsection{Wound-Rotor Induction Generator with External Resistance Control (Type II)}

In a squirrel-cage induction generator, the rotor "circuits" are fictitious and not accessible external to the machine, and the induced currents responsible for torque generation are strictly a function of the slip speed. The turbine shown in Figure 2-8 utilizes a wound-rotor induction machine, where each of the three discrete rotor winding assemblies is electrically accessible via slip rings on the machine shaft. This provides for modification of the rotor circuit quantities and manipulation of the rotor currents, and therefore the electromagnetic torque production. The Type II turbines utilize a system for controlling the magnitude of the rotor currents in the induction generator over the operating speed range of the turbine. The current controller system usually consists of an external resistor network and a power electronics module that modulates the voltage across the resistors to maintain a commanded rotor current magnitude. The operation of the controller is quite fast, such that it is capable of holding 
the turbine output power constant for even gusting winds above rated wind speed, and significantly influences the dynamic response of the turbine to disturbances on the grid.

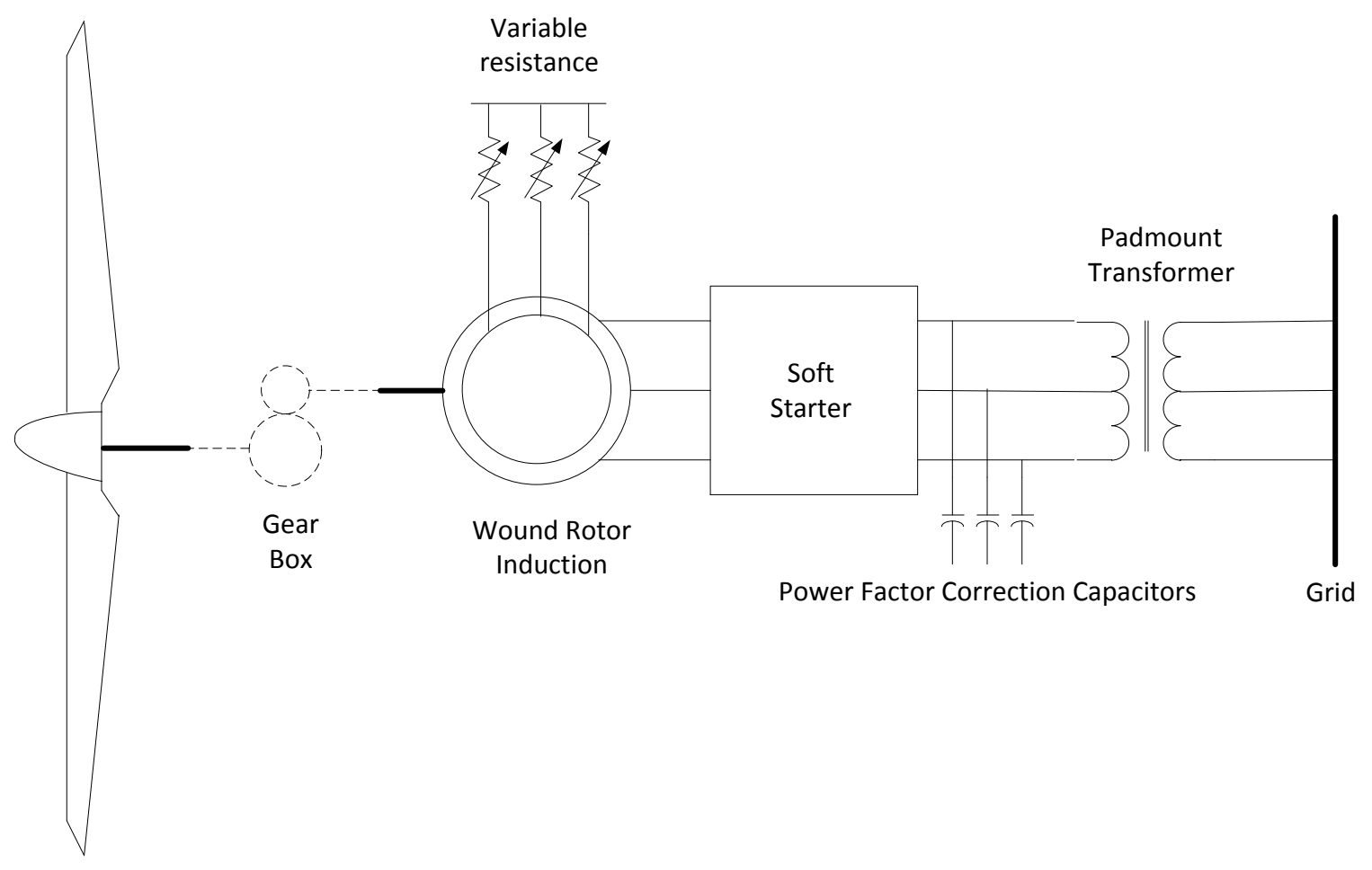

Blades

Figure 2-8: Illustration of type II wind turbine generator.

The operation of the scalar rotor current control under steady conditions is illustrated in Figure 2-9. The continuously variable rotor resistance effectively allows the generator to operate on an infinite number of torque vs. speed curves. In the figure, curves are shown for $10 \%$ increments of the total external rotor resistance. The maximum torque line shown on the graph corresponds to constant power at the nominal value. The curve is sloped as torque must decrease as speed increases to maintain constant power. 


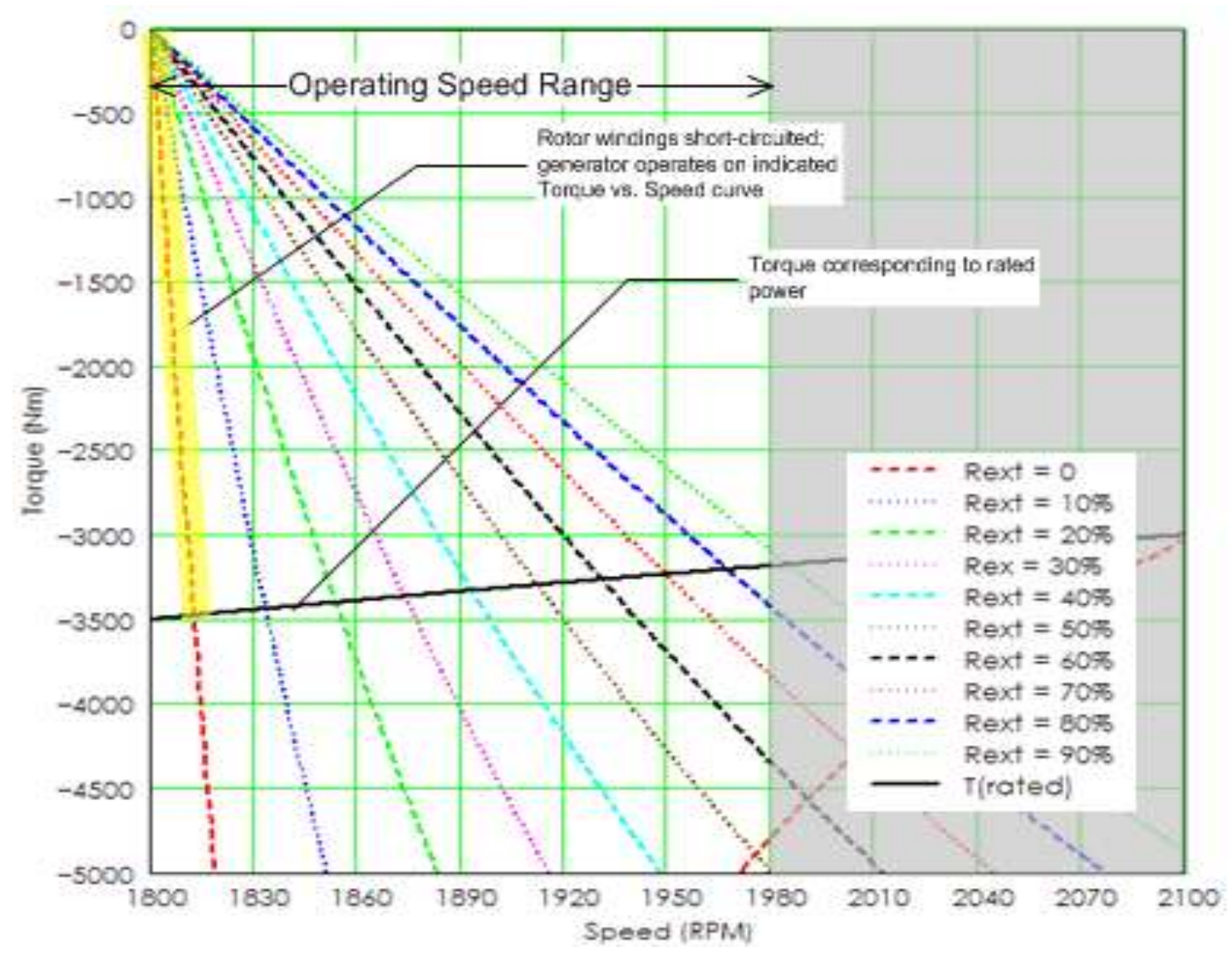

Figure 2-9: Torque vs Speed curve of a Type II turbine at various rotor resistances.

\subsubsection{Doubly-fed Asynchronous Generator - DFAG (Type III)}

An even more sophisticated rotor current control scheme can be employed in a doubly-fed asynchronous generator as shown in Figure 2-10. Here the rotor circuit is supplied with current from a four-quadrant voltage-source, current-regulated power converter. With respect to the grid frequency, such a converter can provide nearly instantaneous regulation of its output currents. Under steady operating conditions, the machine-side converter controls the magnitude and phase of currents in the rotor circuit to achieve desired values of electromagnetic torque. Reactive power flow into the line-connected stator terminals of the generator can also be controlled. 


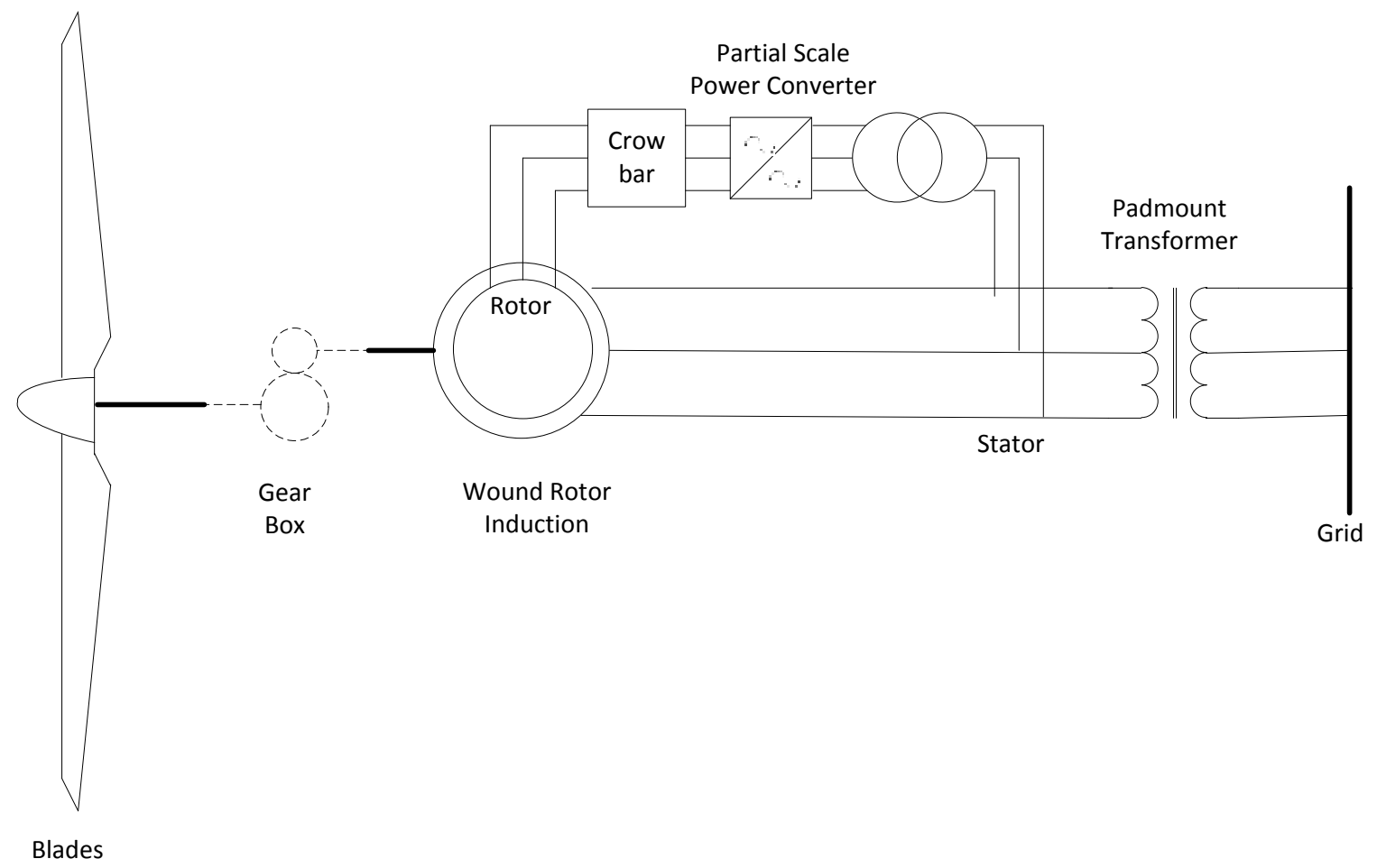

\section{Figure 2-10: Illustration of type III wind turbine generator.}

Field-oriented or vector control of induction machines is a well-known technique used in high-performance industrial drive systems, and its application to wind turbines brings similar advantages. In an earlier version of this turbine, the torque command (and therefore the magnitude of the rotor current component responsible for torque production) was linked to the speed of the machine via a "look-up" table (Figure 2-11). The field-orientation algorithm effectively creates an algebraic relationship between rotor current and torque, and removes the dynamics normally associated with an induction machine. The response of the power converter and control is fast enough to maintain proper alignment of the torque-producing component of the rotor current with the rotor flux so that the machine remains under relative control even during significant grid disturbances. 


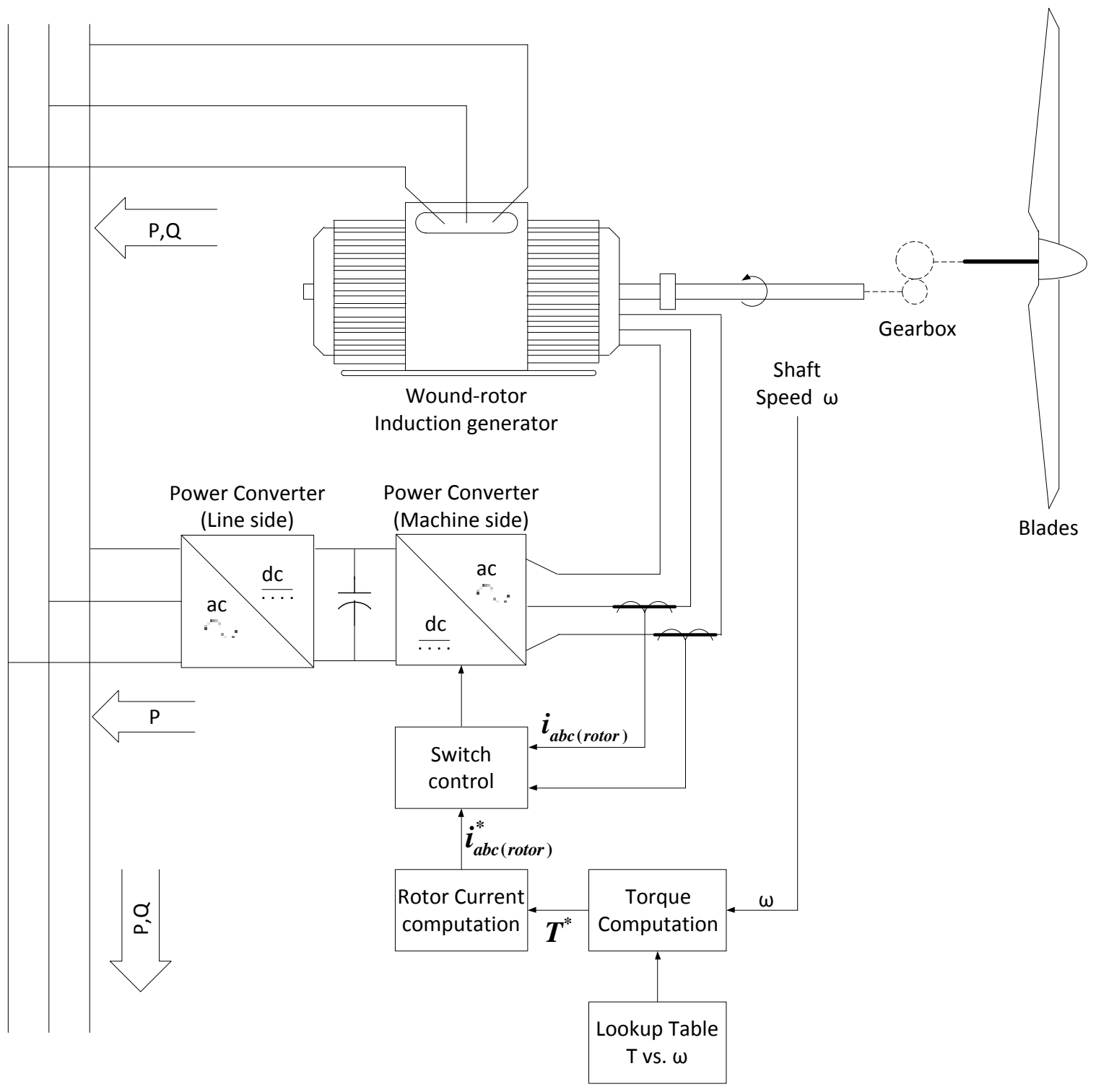

Figure 2-11: Configuration of a wind turbine with four-quadrant power converter supplying rotor circuit of a wound-rotor induction generator.

The line-side converter either absorbs or provides real power to the grid, depending on the operating speed of the generator. If the generator is operating below the synchronous speed for the grid frequency and pole number, some amount of real power will flow through the line-side converter to the dc link, then from the machine-side power converter into the rotor circuit. If the turbine is operating above synchronous speed, real power will flow in the opposite direction.

The stator is connected to the low voltage side of the wind turbine transformer, but in the case or the DFAG the rotor is a 3-phase coil winding connected to a variable frequency power electronic drive via slip rings rather than an internally short circuited winding. Active power is drawn from the grid to supply the rotor via an AC/DC and 
$\mathrm{DC} / \mathrm{AC}$ voltage source converter link and the rotor currents can be completely controlled by the IGBT control circuit. The frequency, which the rotor side converter targets, is that which when superimposed on the rotor speed gives rise to a synchronously rotating field in the air gap.

The active power exchange with the grid is the sum of the power supplied to the grid from the generator stator and the power exchanged with the rotor (minus the converter losses). The stator always exports active power to the grid.

The DFAG machine has a number of advantages over the induction generator. Because the rotor frequency is essentially decoupled from the grid it can operate over a wider slip range - $10 \%$ to $-16 \%$ compared to $0 \%$ to $-2 \%$ for an induction generator. The connected DFAG wind turbine is not restricted to a single unique operating speed. This allows the blade tip speed to be varied over a range to better match the wind speed and maintain an efficient operating position for a range of wind speeds.

Reactive power is controllable. The initial machine magnetization flux is established from the rotor and the grid side converter still draws only active power. The reactive power is created by the rotor side converter by the firing angle and thus the field angle relative to the rotating field in the stator. The stator similarly appears as a unity power factor device even at starting. Where the turbine control system is required to supply or absorb reactive power, the lead or lag of the rotor field can be controlled by the rotor side converter. Since the synchronous rotor field vectors can be shifted angularly as in the lead and lag of a synchronous machine, the machine can be modeled with direct (d) and quadrature (q) axis components.

The phase difference and magnitude of the rotor voltage determine the active and reactive power which is delivered to the terminals of a DFAG. In an ideal device, maximum active power exchange takes place at $\alpha=90^{\circ}$ or $270^{\circ}$ at which time there is no reactive power exchange. Reactive power exchange is maximized at $\alpha=0^{\circ}$ and $180^{\circ}$.

The DFAG can therefore manage active and reactive power independently by controls on the grid and rotor side converters. It requires a converter -inverter arrangement, but the maximum expected power through the converter to or from the rotor is about $25 \%$ of the total output power of the generator. Therefore the converters are cost effective. There is however a downside to the technology. Under serious system fault conditions the grid voltage may be close to collapse and attempts to meet a target voltage or power factor will drive very high currents into the rotor and hence through the power converters. The converters would be thermally damaged by these currents, so the equipment is generally closely protected by an electronic rotor short circuiting 
mechanism, often called the "crowbar". This can be active within $20 \mathrm{~ms}$ and is followed immediately by a machine trip. Conventional synchronous generators supply reactive energy to the fault and then on fault clearance they participate in the system recovery.

\subsubsection{Variable Speed Turbine with Full-Rated Power Converter (Type IV)}

For Type 4 Wind Turbine Generators (WTGs), the generator is connected to a full power converter. In this configuration, a wide range of electrical generator topologies could be employed - Permanent Magnet Synchronous Generator, Wound Rotor Synchronous Generator, or Wound Rotor Induction Generator. Because all of the power from the turbine is processed by the static power converter, the specific characteristics and dynamics of the electrical generator are effectively isolated from the power grid.

Full power conversion means that a back-to-back voltage-source converter is connected to the generator as illustrated in Figure 2-12. The generator frequency and grid frequency are decoupled by using a voltage-source converter.

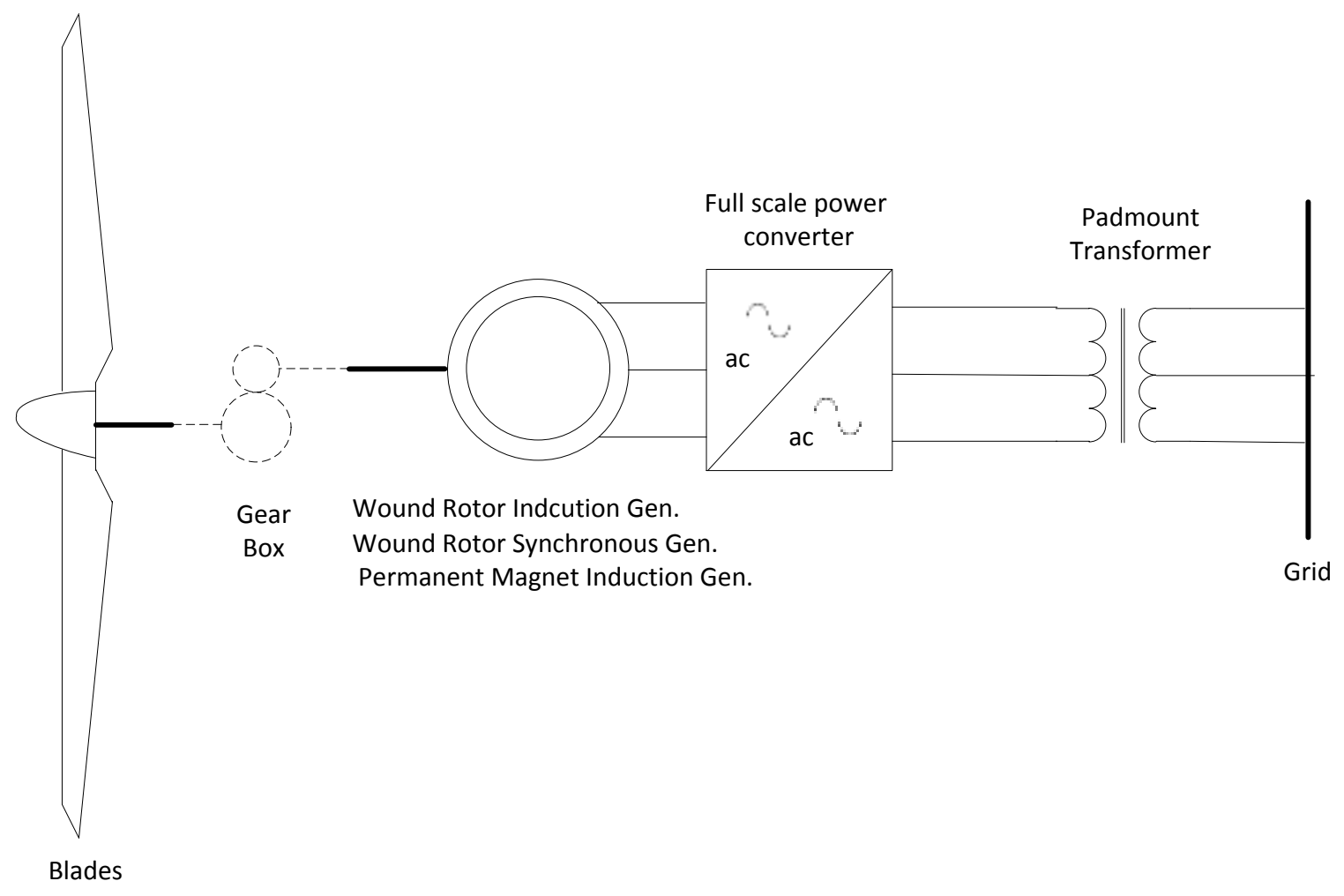

Figure 2-12: Configuration of a type IV wind turbine generator.

A modern static power converter utilizes power semiconductor devices (i.e. switches) that are capable of both controlled turn-on as well as turn-off. Further, the device characteristics enable switch transitions to occur very rapidly relative to a single 
cycle of $60 \mathrm{~Hz}$ voltage - nominal switching frequencies of a couple to several $\mathrm{kHz}$ are typical. This rapid switching speed, in combination with very powerful and inexpensive digital control, provides several advantages for distributed generation interface applications:

- Low waveform distortion with little passive filtering

- High-performance regulating capability

- High conversion efficiency

- Fast response to abnormal conditions, including disturbances, such as shortcircuits on the power system

- Capability for reactive power control

Figure 2-13 depicts a simplified control schematic for a static power converter in grid-parallel operation. Since an individual wind turbine is likely small in rating relative to the short-circuit capability of the system to which it is connected, the voltage magnitude at this point will only be slightly influenced by the operation of the turbine. The control scheme, therefore, is designed to directly regulate the currents to be injected into this "stiff" voltage source.

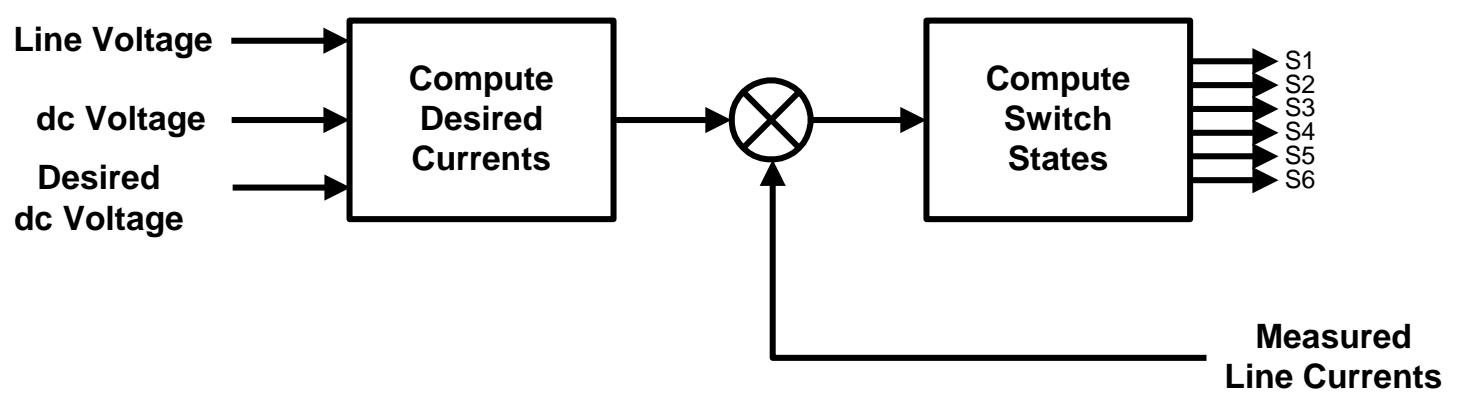

Figure 2-13: Simple output current control stage for a static power converter in a grid-tied DG application.

The ac line voltages, dc link voltage, and two of the three ac line currents - for a three-wire connection - are measured and provided to the main controller. The ac voltage and line currents are measured at a high resolution relative to $60 \mathrm{~Hz}$, so that the controller is working with instantaneous values. By comparing the measured dc voltage to the desired value, the controller determines if the real power delivered to the ac system should be increased, decreased, or held at the present value. Such a simple regulation scheme works because there is no electric energy storage in the converter (except for that in the dc filter capacitor), so the energy flowing into the dc side of the converter must be matched at all times to that injected into the ac line. If these quantities do not match, the dc link voltage will either rise or fall, depending on the 
algebraic sign of the mismatch. The error in the dc voltage is fed into a PI (proportionalintegral) regulator to generate a value representing the desired rms magnitude of the ac line currents. Another section of the control is processing the instantaneous value of the ac line voltage to serve as a reference or "template" for the currents to be produced by the converter. The desired instantaneous value of the line current is computed by multiplying the desired rms current magnitude by the present value from the template waveform. In the next stage of the control, often times called the "modulator" section, the desired instantaneous value of line current is compared to the measure value (in each phase). The modulator then determines the desired state of the six switches in the matrix based on the instantaneous current error in each phase of the line currents. The states are transmitted to the IGBT gate drivers, which then implement the state of each IGBT in the matrix as commanded by the controller. The process is then repeated at the next digital sampling interval of the overall control. The process is repeated thousands of times per single cycle of $60 \mathrm{~Hz}$ voltage. By using the line voltage as a template for the shape of the currents to be synthesized, synchronism is assured. Additionally, if there is no intentional phase shift introduced in the control calculations, the currents will be almost precisely - save for small delays introduced by the control itself - in phase with the line voltages, for unity power factor operation. 


\section{Wind Plants}

\subsection{Design and Configuration}

Wind turbines are just one (albeit an important) component of bulk wind plants. With individual turbine sizes now exceeding $1 \mathrm{MW}$, nameplate ratings for single wind plants of many tens to hundreds of MW are common. The geographic extent of the wind plant must be large enough to not only accommodate the dozens to a hundred or more turbines, but also allow optimal spacing and utilization of local terrain features that will maximize energy production. The infrastructure for connecting a large number of widely distributed turbines to a single point of interconnection with the transmission system has important influence over the electrical characteristics of the wind plant.

The installed and proposed utility-scale wind plants in the U.S. have some common design characteristics that offer potential simplifications for constructing aggregated models for transmission system studies. These commonalities stem from practicalities and optimizations regarding the local wind regime, micro-siting of individual turbines, electric system design, and operations and maintenance economies. The result is that, from the power system modeling perspective, large wind plants have the following features in common:

- A single turbine type - Since wind turbines are complex machines that require preventative, predictive, and on-demand maintenance to achieve the highest availability, it is better from a maintenance and operations perspective to utilize the same turbine throughout the wind plant and have a maintenance and operations staff that specializes in all aspects of this single turbine design.

- Medium voltage collector systems and interconnect equipment - The electrical infrastructure which "collects" power generated by each turbine in the plant and delivers it to the transmission system utilizes standard overhead and underground medium voltage (15 to $35 \mathrm{kV}$ ) equipment and design practices. Some variations from standard utility practice for medium voltage design are necessary, however, as the operation of wind turbines varies significantly from the distributed end-use loads for which the utility practice is optimized. For example, voltage regulation and protection schemes must be modified to account for generation, rather than load, 
distributed along the collector lines. The collector lines are an integral part of the wind plant; i.e. they are not utilized to serve non-wind plant load or other electric utility customers.

- Reactive compensation - Maintaining voltages within tolerances at individual turbines within a wind plant while at the same time meeting power factor or voltage regulation requirements at the point of interconnection with the transmission system requires careful management of reactive power. Typical locations for reactive power compensation within a wind plant are 1) at each individual turbine, dependent on the reactive power requirements and characteristics of the rotating machinery in the turbine; 2) at the interconnect substation in the form of switched shunt capacitor banks; and 3) at locations along the medium voltage collector lines depending on the layout of the plant. Some plants have the ability to dynamically control reactive power from each turbine, which offers the possibility of reactive power management for transmission system considerations to be accomplished by the turbines themselves. Terminal voltages at individual turbines, however, may be a constraint on the amount of reactive power that can be delivered to the interconnect substation during periods of high wind generation. In addition, when reactive power is required at the point of interconnection to the transmission network to support voltage, substantial reactive power may be "lost" in the medium voltage collector system between individual wind turbines and the interconnect substation.

- SCADA and Plant Control - Large wind plants typically have fairly extensive means for remote operation of individual turbines and collection of highresolution operating data. Interfaces to power system operations centers are also being implemented, allowing automated implementation of control area operator commands during certain system conditions - e.g. automatic curtailment.

The most important influence of the wind plant infrastructure on the interconnection bus bar characteristics of the wind plant is on the net reactive power capability of the wind plant. Voltage profiles along the collector lines are an internal issue. For purposes of characterizing the plant for transmission studies, the static, dynamic, and load-dependent effects of the collector system on the net reactive power at the interconnection substation must be characterized. Figure 3-1 illustrates this 
influence with an example from an operating wind plant. Wind plant generation and net reactive power requirements are shown as functions of wind speed. In the figure, the net reactive power is entirely a function of reactive losses in the lengthy overhead collector lines, since the turbines are assumed to be operating at unity power factor. The stepped line shows how staged shunt capacitor banks on the collector lines might be deployed to account for this load-dependent reactive loss. Not shown on the diagram is how such a scheme would contribute to the dynamic nature of the plant. As wind speeds - and power output - vary, so will the net reactive requirements. Details of the capacitor switching scheme are critical here, since there will be time delays and hysteresis associated with the capacitor bank controls. These parameters must be selected with some knowledge of the time variation of wind generation on the collector line to prevent unnecessary capacitor switching operations and potentially associated voltage flicker.

\section{Generation, Reactive Consumption, and Capacitor Switching Buffalo Ridge Feeder BRI 321 (Alpha/Zulu)}

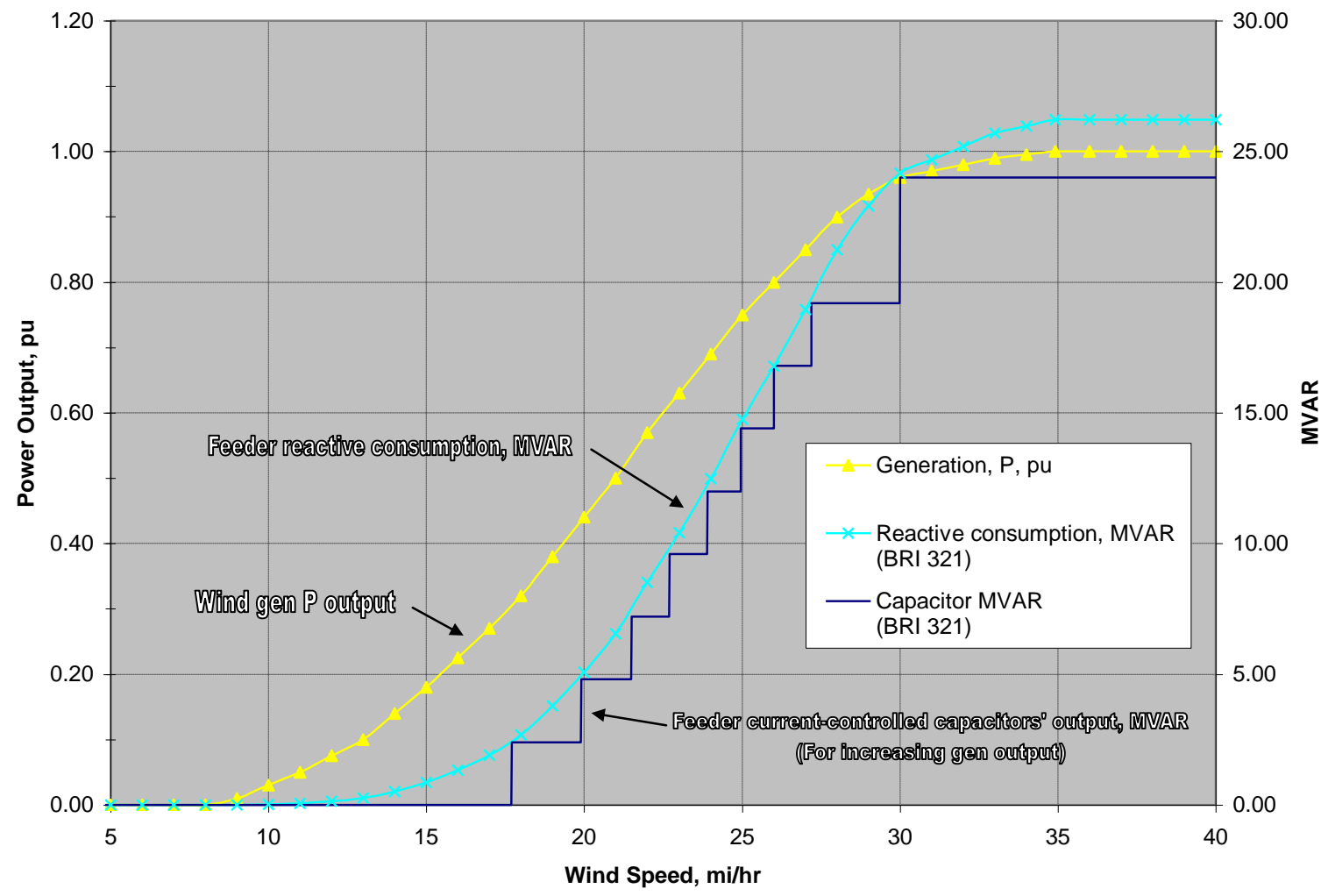

Figure 3-1: Illustration of the impact of collector line reactive losses on the net reactive power capability of a large wind plant. 


\subsection{Performance Characterization for Power System Studies}

Models that capture the aggregated behavior of all components in a wind plant as seen from the interconnection point to the transmission network are the most useful and sometimes practically required for large power system studies. This section discusses how these components contributed to behavior of the wind plant as a single entity connected to the transmission network for steady-state and dynamic conditions.

\subsubsection{Steady-State and Small-Signal Behavior}

For power flow calculations, a wind plant can obviously be represented as a single generating unit at the interconnection substation. Determining the equivalent "reactive capability" of the plant, however, can be complicated since it will be a function of a large number of elements within the plant - turbine reactive compensation, reactive losses in collector lines, auxiliary compensation equipment such as collector line capacitor banks, etc. While fairly standard and well-known for conventional generating units, this characteristic has not been considered explicitly for many of the plants developed over the past decade.

Net reactive power is also a function of voltage if shunt capacitors are present as part of the plant reactive compensation scheme.

The dynamic nature of the wind resource can introduce a new dimension to power system studies, especially where the transmission interconnection is weak. Reactive power support for maintaining target voltages at the transmission interconnection will vary with the real power injected. Temporal variation of wind plant aggregate power is a very complicated function of a number of plant parameters and variables, but it also can be a defining factor for the dynamic characteristics of the reactive compensation system.

Additionally, the reactive compensation devices within the plant - turbines (shunt capacitors or advanced control), collector line capacitor bank, and possibly interconnect substation-based equipment - are dynamic devices themselves, with set points and delay for toggling on or off of switched devices and continuous control for static var capabilities.

Some of the factors that influence the variability of the aggregate production of a wind plant include

- Variations in wind speed at each turbine location in the plant; 
- Topographical features that introduce turbulence and shear into the moving air stream across the geographical expanse of the wind plant;

- The mechanical inertia of individual turbines, which influences how the wind speed variations, turbulence, and wind shear affect the output of individual turbines

- The wind turbine control scheme, including the generator control and pitch regulation systems that determine how the electric power at the terminals of the turbine is influenced by fluctuating prime mover input;

- The number of turbines within the plant, since a larger number of turbines implies a larger geographical area for plant, and more statistical diversity in the local characteristics that contribute to output fluctuations;

- The grouping of turbines within the plant - if turbines are grouped into "strings", rather than more uniformly distributed over the area of the plant, local fluctuations in wind speed will affect more than a single turbine at an instant of time.

Wind generation is often characterized as "intermittent", but, to better understand how it can impact power system operations, it is useful to consider the output variability in more detail.

On the shortest time scales, say tens of seconds to minutes, the output of a wind plant can fluctuate because of varying wind speeds at the individual turbines comprising the plant due to effects of terrain and turbulence in the moving air stream. This is more likely the case in light to moderate winds, as modern wind turbines are capable of holding the output power "flat" for wind speed at or above the rated value. Measurement data shows that the fluctuations on this time scale as a fraction of the plant rating decrease in magnitude as the number of turbines in the plant increases.

Over longer time periods - tens of minutes to hours - wind plant generation will again exhibit fluctuation, and may also trend down or up as the larger scale meteorology responsible for the wind changes. Passage of a weather front is an example. Experience is showing that these trends can be predicted, but the accuracy of the prediction degrades quickly with time. Forecasts for the next hour, for instance will be much better than those for several hours ahead.

Longer-term forecasting for the next day or week is even less accurate, especially when timing is important. Predictions of a weather front passing an area tomorrow can 
be relatively accurate, but the accuracy for predicting which hour it will pass will be much lower.

"Intermittent", as the term is applied to wind generation, encompasses both the fluctuating characteristics along with the degree of uncertainty about when the resource will actually produce. Both of these attributes are important for power system engineers and operators who have come to understand well the fluctuations and uncertainties inherent in conventional generating resources and system loads. Because wind generation is new, these characteristics are only beginning to be quantified, and procedures for rigorously considering them in system studies have yet to be developed.

As of this writing, there are no practical analytical methods for characterizing the output fluctuations from a large wind plant. Direct measurements from operating wind plants, however, are providing some important insights into the complicated interaction of the factors listed above. The National Renewable Energy Laboratory (NREL) launched a program in CY2000 to collect high-resolution electrical measurement data from operating wind plants across the U.S. The database being compiled by NREL consists of continuous one-second samples of voltage, current, real power, and reactive power from wind plants in the Pacific Northwest, the upper Great Plains, and West Texas.

Preliminary analysis of this data has revealed much about the behavior of bulk-scale wind plants consisting of large numbers of individual turbines spread out over a significant geographical area. As the number of individual turbines increases, the perunit variations in the aggregate output decline substantially. This characteristic is a critical factor for grid studies, as it provides a basis for bounding the changes in real and reactive power over time that can influence system voltage and related indices such as flicker, as well as the impact on the generation/load balance in the control area.

\subsubsection{Dynamic Response}

The electrical and mechanical technologies which comprise commercial wind turbines differ dramatically from the familiar synchronous generator and auxiliary systems that are used to represent almost all conventional generating equipment. And, instead of a small number of very large generating units, bulk wind plants can be made up of a very large number of relatively small machines. Until quite recently, these attributes have presented a difficult challenge to power system engineers engaged in evaluating transmission system impacts of large wind generation facilities.

Evaluating the dynamic response of the electric power system during and immediately following major disturbances such as faults is a critical engineering function 
for ensuring system security and reliability. Now that wind plants make up a nonnegligible fraction of the generation assets in some control areas, their contribution to the system dynamic performance must be considered.

When subjected to a sudden and substantial change in terminal voltage or frequency, both the mechanical and electrical elements of the turbine along with the associated control systems influence its behavior. Consider the doubly-fed induction generator with flux vector control of torque via the power converter on the rotor circuit (Figure 2-11). When a fault on the transmission network causes the voltage at its terminals to sag to some fraction of normal,

- The magnitude of the main flux in the machine begins to decay in response to the reduced terminal voltage, and the position of the flux vector may suddenly change if there is a phase shift associated with the fault voltage.

- The rotor power converter control almost instantaneously adjusts the quadrature axis rotor currents to "line up" with the new rotor flux vector.

- Since the rotor flux is no longer at the pre-fault value, the stator power of the machine is reduced accordingly. In response, the power converter control may try to increase the torque-producing component of rotor current.

- Because the electrical power output of the machine is now lower than the pre-fault value, there is net accelerating torque on the mechanical system which will increase the rotational speed of the machine.

- The increased rotational speed will cause the turbine blades to begin pitching to reduce the mechanical torque input to the machine and reduce speed.

- When the fault is cleared and the terminal voltage returns to near normal, the rotor power converter control will readjust the position of the rotor current vector to again line up with the rotor flux vector.

- Electric power output will jump back to (or slightly above, if the rotor current had been increased by the controller during the fault) the pre-fault value. Since mechanical power had been reduced by the pitch system, net decelerating torque on the mechanical system will cause rotational speed to decrease. 
- The sudden changes in electromagnetic torque applied by the generator to the rotating shaft (at fault inception and clearing) excite the main mechanical resonance between the turbine blades and the generator inertia, such that these masses are now oscillating out of phase around the average speed of the rotating system.

- The oscillations in generator speed may be fed through the control system to produce oscillations in electric power at the stator terminals of the machine.

The response is depicted graphically in Figure 3-2, and Figure 3-3 shows an expanded view around the initiation and clearing of the system fault. Note how the vector control algorithm maintains control of the rotor (and stator) currents except for a few milliseconds at the beginning and end of the fault.

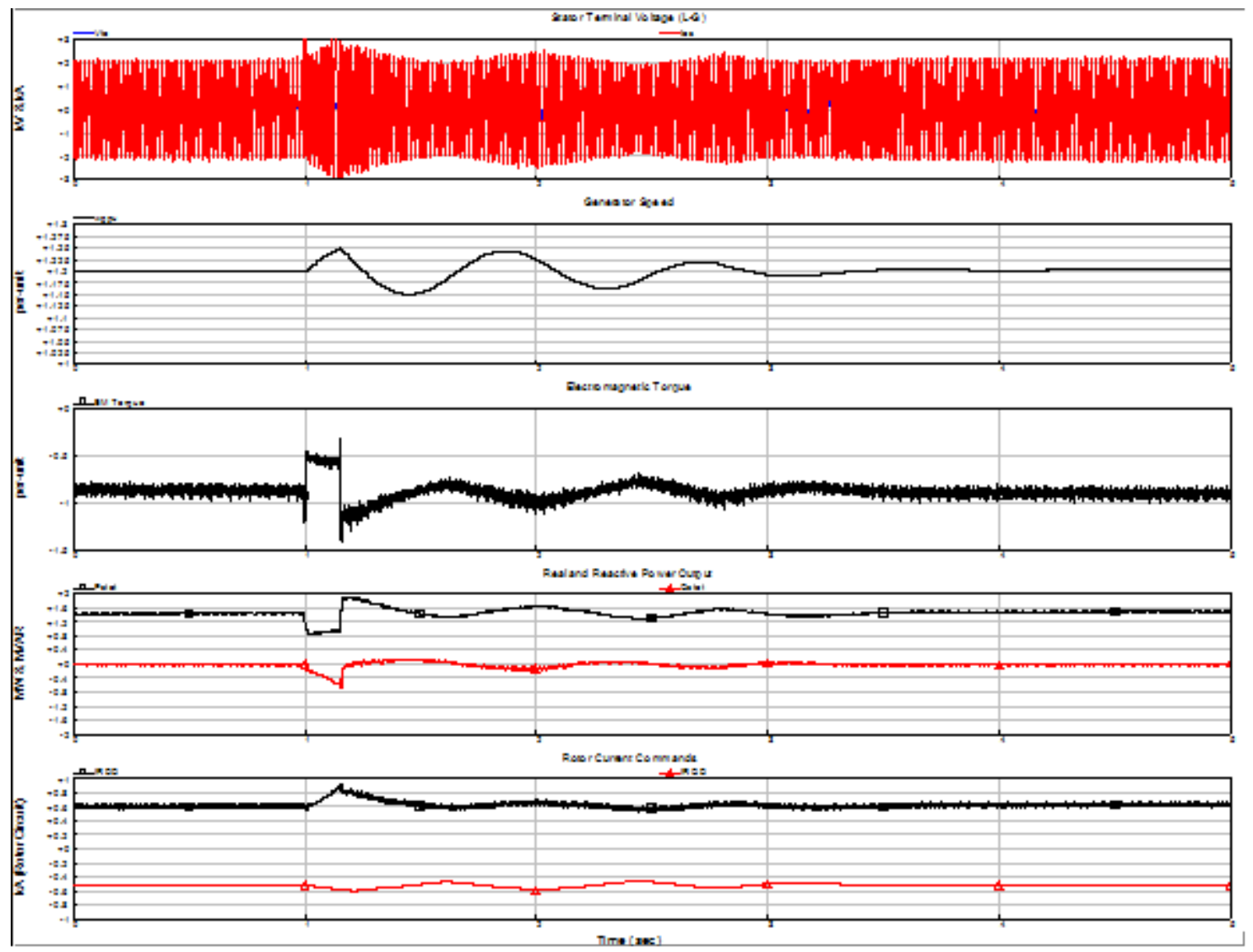

Figure 3-2: Wind turbine dynamic response to fault on the grid. 


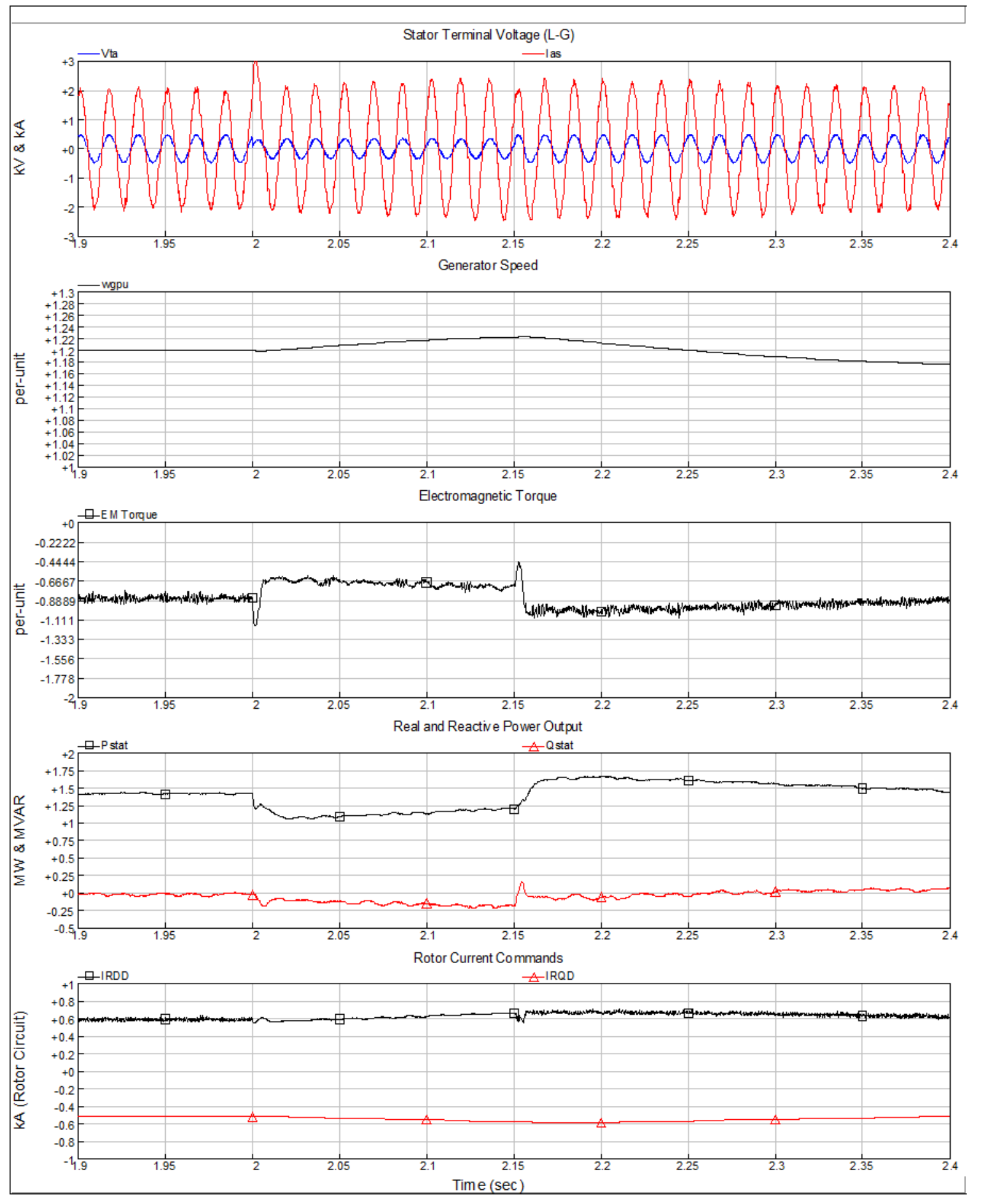

Figure 3-3: Expanded view of Figure 3-2.

While there are some similarities to the response of a synchronous generator to the same disturbance, the markedly different equipment and control comprising the wind turbine lead to a difference dynamic response. While the sequence above is only an 
example for one type of wind turbine, it is indicative of the behavior that needs to be represented in dynamic simulations of the entire power system.

In addition, the response described is for a single turbine. What is important from the perspective of the power system is the aggregate response of all the turbines in the wind plant, along with the influence of any other dynamic elements such as static compensation or switched elements.

Research is only beginning into electro-dynamic equivalents for wind plants. There is agreement on a few general guidelines and principles for developing these dynamic equivalents. For remote disturbances - those originating on the transmission network, not within the wind plant itself - individual turbines can be considered coherent, i.e. they response as if they were a large single machine of equivalent aggregate rating. This assumption is based on all turbines being of identical type and parameters, and that they "see" the disturbance at precisely the same instant and in roughly the same degree.

With some turbine technologies, there are nonlinearities in certain of the control blocks such that the response may be dependent on the pre-fault conditions at the turbine, namely the assumed generation level as a fraction of the rated value. If maximum generation conditions are being studied, then all turbines at the same prefault generation level is a good one. If for some reason partial generation conditions are of interest, aggregate dynamic performance of the plant could depend on how the total generation is allocated to individual turbines.

Because of the extensive medium voltage collector system that is part of many large wind plants, there is potentially an issue with differing pre-fault terminal voltages at turbines dependent on generation level and electrical location within the plant. And, as with the steady-state and small signal characterizations, the response of the plant in terms of reactive power may also be difficult to capture, unless the behavior at the interconnection bus bar is dominated by a single device such as a static var compensator located at the substation.

Fortunately, most of these detailed questions are likely of secondary importance, especially where the focus is on the power system as a whole and not some particular aspect of the wind plant response. Until new research findings indicate otherwise, relatively simple dynamic equivalents consisting of a single or small number of equivalent machines at the interconnection substations is the recommended approach. 


\subsubsection{Transient Response}

Dynamic simulations and studies of the interconnected power system are based on a number of assumptions to facilitate some simplifications in the representation of the dynamic components of the system. For some investigations, such simplifications are not valid or can obscure the aspects of the system model critical for the study.

Studies of sub-synchronous torsional interaction, control interactions, inadvertent islanding, etc. may require models with more detail than those used for system dynamic studies. Full transient models of all but the simple wind turbine technologies require information and engineering detail that can only be obtained from the wind turbine manufacturer. Studies of these types should be conducted collaboratively with technical personnel from the turbine designer.

\subsubsection{Short Circuit Contributions}

Little guidance exists for calculating short-circuit contributions from large wind generation facilities. Analytical approaches are complicated for the following reasons:

- Commercial wind turbines employ induction machines for electromechanical energy conversion, which do not strictly conform to the standard procedures and assumptions used in calculation of short-circuit contributions on the transmission network.

- Generator control technologies employed in wind turbines- e.g. scalar or vector control of rotor current in a wound-rotor induction machine - can substantially modify the behavior of the induction machine in response to a sudden drop in terminal voltage, further complicating calculation of terminal currents during such conditions

- Wind plants are composed of large numbers of relatively small generators, interconnected by an extensive medium-voltage network that itself influence fault contributions

The short-circuit behavior of a squirrel-cage induction generator is fairly well known, and procedures are spelled out in the technical literature (such as the IEEE Brown Book) for considering these machines in short-circuit studies. These recommendations, however, apply most directly to fault studies within large industrial facilities, and may require adaptation for transmission system fault studies.

In the remaining cases of the wound-rotor induction machines, the external components and accompanying control have very significant influence on the machine 
under network fault conditions, assuming that the control systems themselves are not bypassed or rendered inoperative as a consequence of reduced terminal voltage at the turbine.

The following paragraphs are intended as a qualitative description of the characteristics of the various wind turbine generator technologies under network fault conditions.

\subsubsection{Direct-Connect Squirrel Cage Induction Generator}

Induction generators are essentially induction motors that are driven at speed above their nameplate synchronous speed by some prime mover. Magnetic excitation necessary for torque production and power flow is drawn from the power supply system. The electric current necessary for magnetizing the iron core is responsible for much of the reactive power required by an induction machine.

When the source of excitation is removed from an induction machine, the main flux field collapses and torque production or power flow is no longer possible. It does take a finite amount of time for this field to collapse, however, during which time an induction machine will contribute current to a short-circuit on the power system. Also, if voltage is just reduced rather than removed completely as the result of a downstream fault, the main flux will decay to some new value, but provide necessary excitation for the machine to contribute to the fault. Contributions from induction motors are rarely considered in utility fault studies, but can be an important consideration for protective device coordination and rating within some industrial facilities. The IEEE Brown Book (Standard 399-1997) “IEEE Recommended Practice for Industrial and Commercial Power Systems Analysis" details procedures for calculating induction motor and generator contributions to short-circuits within facilities.

Figure 3-4 illustrates the behavior of a wind turbine employing a line-connected induction generator during a fault on the supply network. In the first cycle following fault inception, stator currents quickly build up to a value several times the rated current of the machine. The contribution during the first cycle can be estimated as the sum of: 1) a sinsusoidal component approximately equal to the pre-fault terminal voltage divided by the sum of the subtransient reactance of the generator and the reactance of the equivalent network to the point of fault, and 2) a uni-directional (dc) component that depends on the reactance to resistance $(X / R)$ ratio of the equivalent system impedance and the precise point on the terminal voltage wave where the fault is initiated. Both components decay in magnitude as the fault persists. The dc component 
decays at a rate governed by the $X / R$ ratio. The decrease in the magnitude of the sinusoidal component is due to the decay in the main flux of the machine.

After a few cycles, the dc component has vanished, and the sinusoidal component has decreased in magnitude. It should be noted here that precise calculation of the short-circuit contribution requires a time-domain computer simulation with a relatively detailed differential equation representation of the induction machine. The aforementioned IEEE Brown Book acknowledges as much, and prescribes an approximate method for defining two equivalent reactances for the induction machine one to be used for calculating the first cycle contribution, the other for a later time during the fault that would be associated with breaker clearing or interrupting requirements.

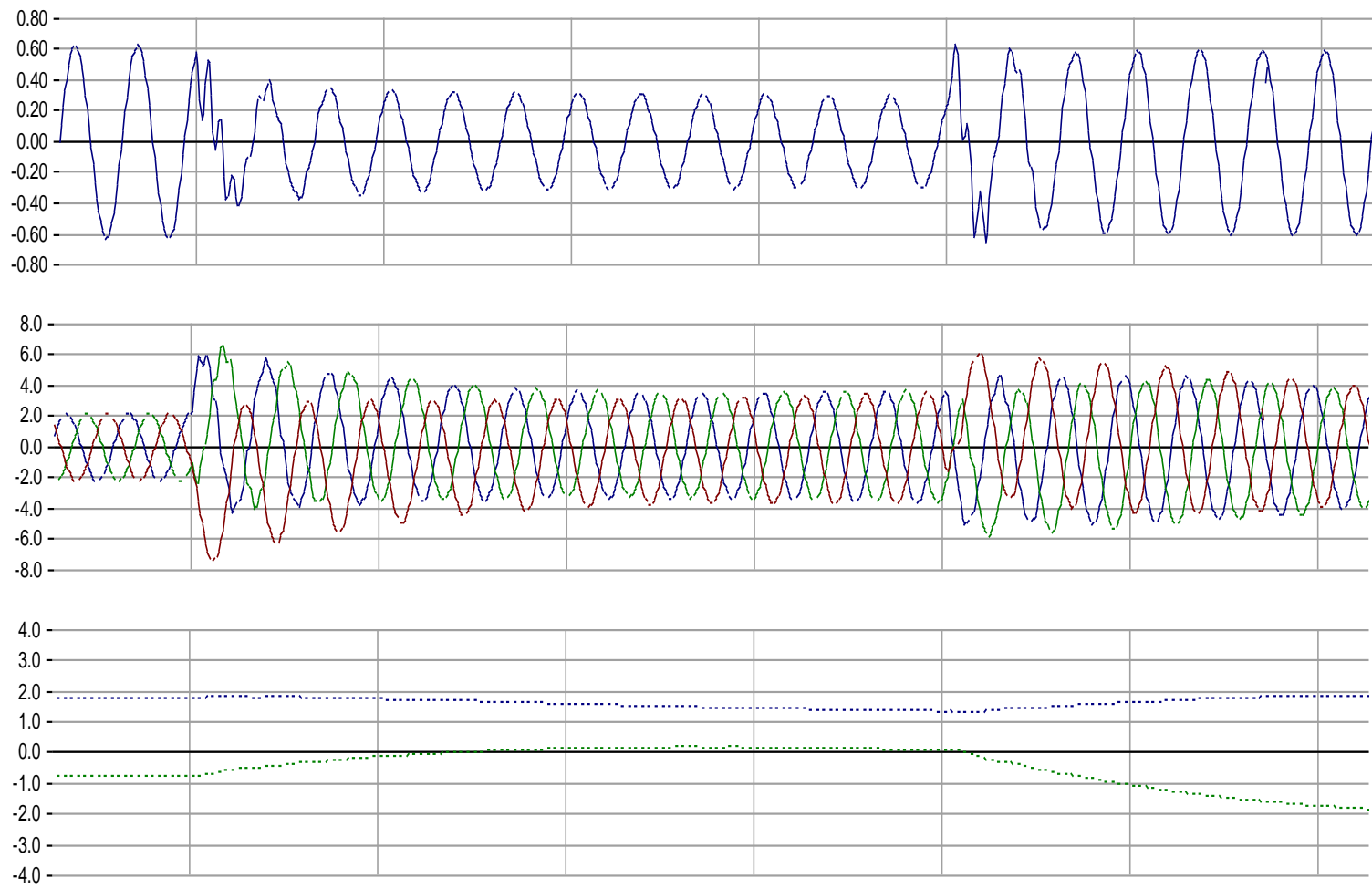

Figure 3-4: Contribution by a 2.0 MVA induction generator to a symmetrical three-phase fault. Shown are voltage at the machine terminals (top), stator currents (middle), and real and reactive power (generator convention) at the machine terminals (bottom).

\subsubsection{Doubly-Fed Induction Generator with Vector Control of Rotor Currents}

The 1.5 MW wind turbine from GE and its predecessor, the $750 \mathrm{~kW}$ turbines from Enron, are also based on a wound rotor induction generator. In these turbines, however, the rotor circuit is powered by a bi-directional static power converter (Figure 3-5). The fast response of the power converter coupled with sophistical algorithms in 
the turbine and converter controller sections allows for precise and continuous adjustment of the instantaneous currents in the rotor circuits of the induction machine. Nearly instantaneous control of electromagnetic torque and turbine power factor is possible with this scheme.

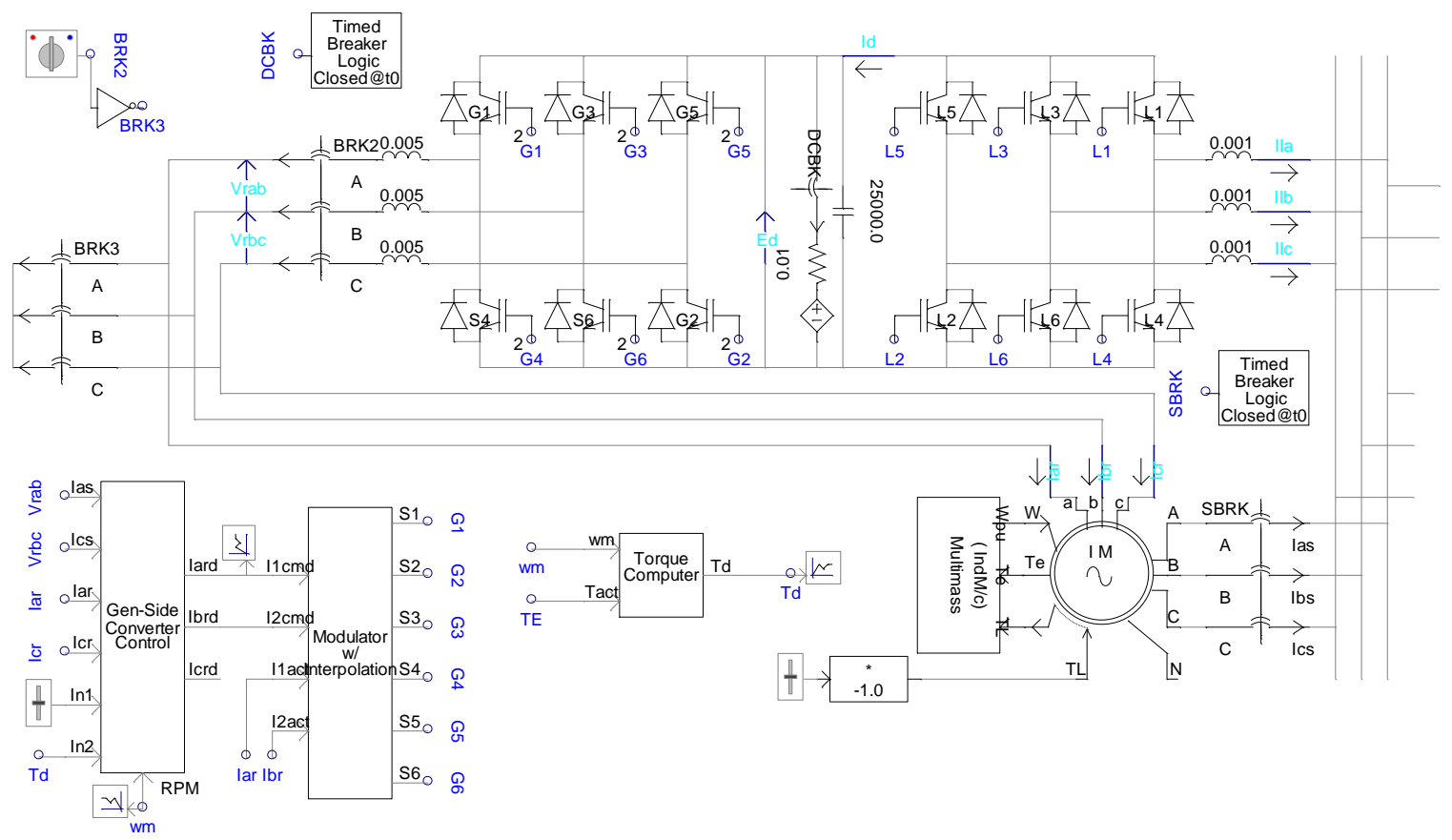

Figure 3-5: Transient model of a doubly-fed induction generator with vector control of rotor currents.

The fast action of the turbine and converter controls can limit the stator currents during a fault on the grid. Figure 3-6 details the turbine operation during a $150 \mathrm{~ms}$ grid fault. When the fault is initiated, the sudden change in terminal voltage magnitude and phase angle causes the power converter to momentarily "lose control" of the rotor currents, which is manifested as a one-quarter cycle "surge" in the stator current. Control is regained quickly, and the stator currents settle down to near their pre-fault value for the duration of the fault event (The slight rise in stator current magnitude during the fault is due to control actions attempting to restore the electromagnetic torque to the pre-fault value). When the fault is cleared, the phase and magnitude of terminal voltage again change suddenly, inducing another short-duration transient in the stator current. Again, however, control is regained, and stator currents return to the level desired by the turbine control. 

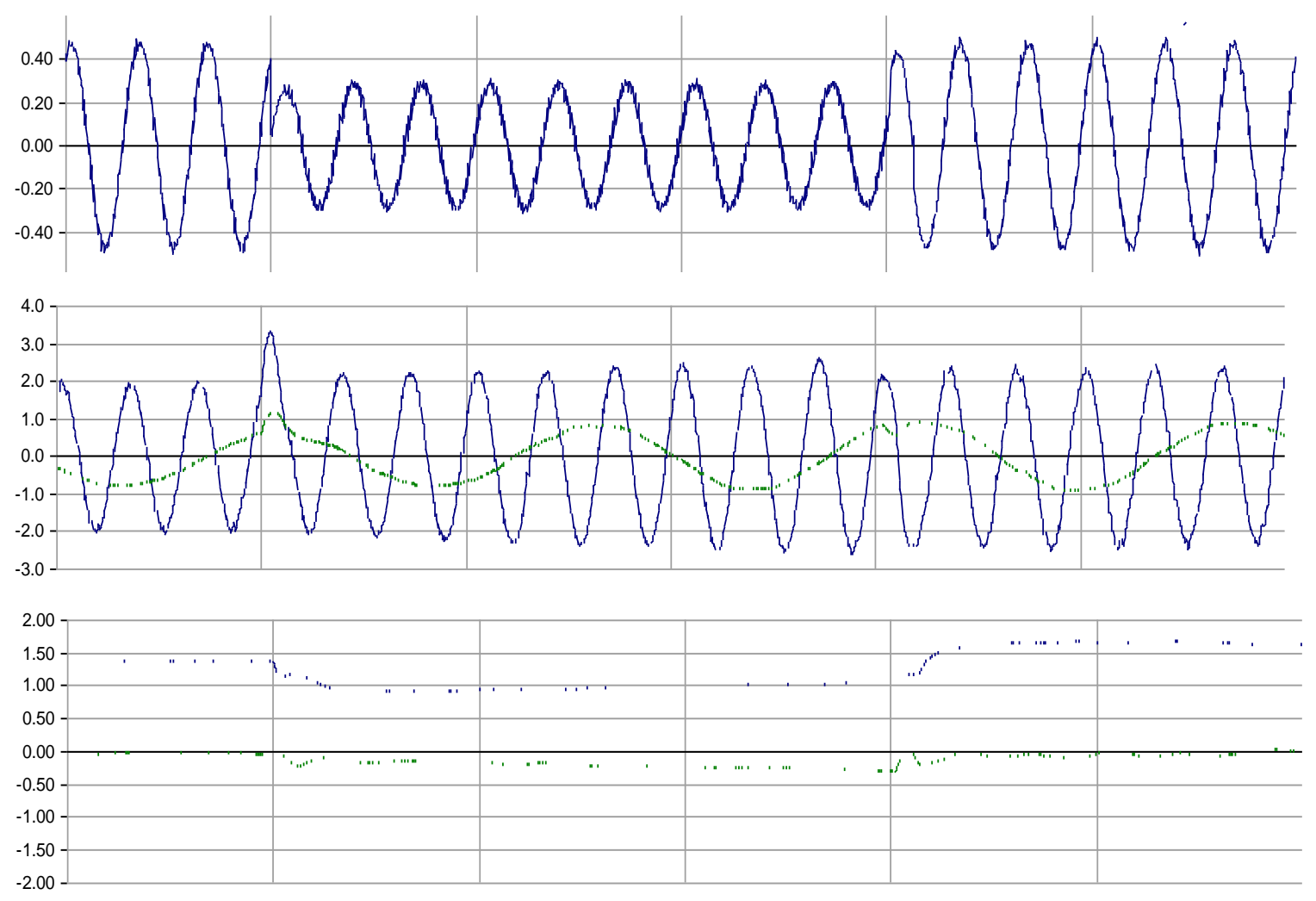

Figure 3-6: Short-circuit contribution from the GE 1.5 MW wind turbine for $150 \mathrm{~ms}$ grid fault. Shown are voltage at the machine terminals (top), stator currents (middle), and real and reactive power (generator convention) at the machine terminals (bottom).

It should be noted, however, that if the rotor power converter is bypassed, such as might be done to protect it from high rotor circuit voltage, the behavior of the turbine during the fault would be better characterized as a conventional induction machine. GE Power System Energy Consulting has developed an internal white paper specifying how the GE Wind Turbine would perform under conditions of rotor converter bypass. 


\section{Wind Generation Technology and Application Trends}

The turbine types described in Chapter 2 have served the U.S. wind industry from the beginning of the explosive growth in the mid 1990's. Newer, bigger turbine models have been introduced along the way, but the technological improvements and modifications in the new commercial introductions have not changed the basic electrical behavior of the turbines.

Reducing the cost of energy is still today the primary driver for ongoing developments in wind turbine technology. Experiences from large wind projects are, however, beginning to influence wind turbine developments, and are expected to have even more impact going forward. Wind turbine vendors now recognize that some features and enhancements to the electrical performance of their products are or will be demanded by customers and are critical for further expanding the overall market potential for wind generation in the U.S.

Wind plant design is always undergoing some evolution. Plant operators and project developers are gaining important experience from the first generations of large wind plants developed since the mid-1990's. Awareness is growing of the importance of the portion of the wind plant between the turbines and the interconnection point to the transmission network to plant availability, turbine performance, and successful operation with the grid.

Finally, with wind generation becoming a visible fraction of the generating assets in some control areas, transmission service providers are beginning a push for more stringent wind plant performance requirements and interconnection standards.

These influences will have a positive impact on the characteristics of wind generation facilities as viewed from the transmission network over the coming years. This section describes technological changes that will lead to new wind plant features and capabilities over the coming years that will affect electrical performance and integration with the grid. 


\subsection{Wind Turbine Technology Trends}

The value of variable speed technology for large wind turbines has been proven in the marketplace over the past decade, and will be the predominate technology going forward. Variable speed operation has benefits in terms of managing mechanical loads on the turbine blades, drive train, and structure. The grid-side benefits are also significant, and include dynamic reactive power control, increased dynamic control over electric power generation, and opportunities for further enhancement of gridintegration features of the turbine.

\subsubsection{Electrical Robustness}

Wind turbine vendors are now well aware of the need for improving turbine electric robustness, especially in terms of the ability to ride-through faults on the transmission system. Enhanced low-voltage ride through is already an option for several commercial turbines, and will likely be a standard feature in the coming few years. Farther down the road, it is expected that wind turbines will be no more sensitive in terms of tripping for transmission system faults than conventional generators, and will provide flexibility with respect to "programming" their shutdown modes for grid events.

\subsubsection{Real Power Control}

At present, commercial wind turbines generally operate to maximize energy production. When winds are at or above the rated speed, electrical output is "capped" at the nameplate rating. In light to moderate winds, however, the turbine is operated to capture as much energy as possible, such that the output will fluctuate when wind speed fluctuates.

These fluctuations are not optimal from the perspective of the grid, as they can lead to voltage variations and potentially increase the regulation burden at the control area level. In future generations of wind turbines, it will be possible to "smooth" these fluctuations to a greater degree than is achieved now with mechanical inertia alone. More sophisticated pitch regulation schemes, improved blade aerodynamic designs, and wider operating speed ranges will provide means for limiting the short-term changes in turbine output while at the same time minimizing the loss of production. Such a feature could be enabled only where and when it has economic value in excess of the lost production.

Extending this type of control would allow wind turbines to participate in Automatic Generation Control (AGC). In this mode, the turbine would have to operate at a level somewhat below the maximum available from the wind to provide room for "ramping 
up" in response to EMS commands. Again, the value of providing this service would have to be evaluated against the cost in terms of lower production as well as the cost of procuring this service from a different source. Technically, though, such operation is possible even with some of the present commercial wind turbine and wind plant technology.

\subsubsection{Dynamic Performance}

The dynamic characteristics of the more advanced commercial turbine technologies are complicated functions of the overall turbine design and control schemes. Little consideration has been given thus far to what would constitute desirable dynamic behavior from the perspective of the power system. Much of the attention to date in this area has been focused on the ride-through question. Once that matter is resolved, there may be opportunities to fine-tune the dynamic response of the turbine to transmission network faults so that it provides maximum support for system recovery and enhances overall stability.

Given the sophistication inherent in the topology and control schemes of future wind turbines, it should be possible to program the response to a degree to achieve such stability benefits. Such a feature would allow a wind turbine / wind plant to participate in a wide-area Remedial Action Scheme (RAS) or Special Protective System (SPS) as is sometimes done now with HVDC converter terminals and emerging FACTS devices.

\subsection{Wind Plant Design and Operation}

Realizing the benefits of enhanced capabilities of wind turbines will depend in large part on the overall wind plant design, since the actions of a large number of relatively small wind turbines must be coordinated to have positive impacts on the overall power system.

\subsubsection{Reactive Power Management and Dispatch}

Because of the fast pace at which the wind industry has emerged and grown over the last decade, the reactive power characteristics of a wind plant are more often than not an "outcome" rather than a design requirement. With more stringent interconnection requirements, more attention and analysis will be given to this topic for plants built over even the next few years. The required reactive power capability of a wind plant will be determined from the results of the interconnection study, and will drive the overall wind plant design, possibly impacting even turbine selection. 
Where the transmission system interconnection is weak or vulnerable, there will be more use of auxiliary equipment such as static var compensators. As design experience accumulates, the ability of the wind plant to provide for the needs of the transmission system at the point of interconnection will be much improved.

\subsubsection{Communications and Control}

The communications and control infrastructure of even present-day wind plants is quite sophisticated, with high-speed SCADA to each turbine and other critical devices or points within the collector system. This sophisticated infrastructure has yet to be exploited for purposes of improving the interconnection performance and integration of the wind plant with the power system; mostly it has been used for maximizing plant production and availability.

In the future, this infrastructure will be the foundation upon which many of the advanced features and capabilities will be based. The interface between the wind plant control center and power system control area operations will also be developed to a much higher degree. Advanced wind plant performance such as AGC participation will likely be accomplished by the control area EMS interacting with the wind plant control center, rather than from EMS to individual wind turbines. Such an interface would also facilitate other plant capabilities that could benefit power system security and reliability, such as automatic full - or partial-curtailment of wind generation under severe system contingencies.

\subsubsection{Wind Plant Production Forecasting}

While the fast fluctuations in wind plant output can create problems with respect to voltage flicker and reactive power management, somewhat longer term fluctuations in wind plant production appear to be of the most consequence for control area operators. More specifically, the uncertainty over what wind plant production will be during the next hour, or by hour for the next day is the major question. Planning conservatively to cover a possible reduction in wind plant generation results in higher reserve margins and higher cost if not needed. Backing down economic generation to accommodate a sudden increase in wind generation can also increase costs.

All of the analytical studies of the impacts of wind generation on power system operations have one theme in common: Better predictions of what wind generation will do at some time in the future allow the control area operators to better plan for the most economic set of resources to meet the remaining load. Much research work is ongoing in the area of wind production forecasting, and at least two commercial 
services have been launched to assist wind plant operators with sophisticated meteorological and statistical methodologies for improving the accuracy of production forecasts.

Wind generation forecasting has already been incorporated into the market rules for the California ISO, in exchange for preferable treatment with respect to settlement of imbalance energy and unscheduled deliveries. Continued focus on wind generation forecasting as a way to mitigate the uncertainty in future wind energy deliveries are expected to improve the science and methods that underlie these systems.

Assumptions about the ability to forecast wind generation at some time in the future can make a critical difference in the analysis of wind generation of power system operations and control. On shorter time scales, say for the next hour, the uncertainty about average wind speed will be smaller, so the variations in wind plant production compared with an average or scheduled value have more to do with the variability of the resource over the expanse of the wind plant and the factors discussed previously. As the forecast moves out into the future, the meteorological details take precedence. 


\section{Characteristics of the Current Fleet}

This Chapter provides an overview of the current wind turbine fleet operating within North American Electric Reliability Corporation's (NERC's) regional reliability entities.

In 2010 the US wind industry grew by 15\% totaling in roughly 5,400 MW of newly installed wind power [4]. This wind power has been installed across 29 states, with Texas (686 MW), Illinois (497 MW), and California (455 MW) being the states with the highest contribution. Table 5-1 summarizes the cumulative installed capacity by sate by the end of 2010.

Table 5-1: US wind power cumulative capacity by state by the end of 2010. [4], [5] and [6]

\begin{tabular}{|c|c|c|}
\hline State & Cumulative Capacity (MW) & $\mathbf{2 0 1 0}$ Installed Capacity (MW) \\
\hline Texas & 10,200 & 686 \\
\hline Iowa & 3,670 & 71 \\
\hline California & 3,603 & 455 \\
\hline Illinois & 2,417 & 497 \\
\hline Minnesota & 2,328 & 395 \\
\hline Washington & 2,220 & 256 \\
\hline Oregon & 2,104 & 346 \\
\hline Wyoming & 1,412 & 313 \\
\hline New York & 1,387 & 0 \\
\hline Oklahoma & 1,383 & 451 \\
\hline Indiana & 1,337 & 303 \\
\hline Colorado & 1,299 & 54 \\
\hline North Dakota & 1,298 & 221 \\
\hline Kansas & 1,026 & 53 \\
\hline Pennsylvania & 748 & 0 \\
\hline New Mexico & 597 & 102 \\
\hline Idaho & 499 & 206 \\
\hline Missouri & 457 & 149 \\
\hline Wisconsin & 450 & 20 \\
\hline West Virginia & 431 & 101 \\
\hline Rest of U.S. & 2,145 & 726 \\
\hline Total & 41,010 & 5,404 \\
\hline
\end{tabular}


With an annual growth of 35\% during the 2005-2010 period the U.S. wind industry finished 2010 with $41 \mathrm{GW}$ of wind power capacity. Overall, the installed wind capacity in U.S. grew by $2451 \%$ since 1997 (Figure 5-1).

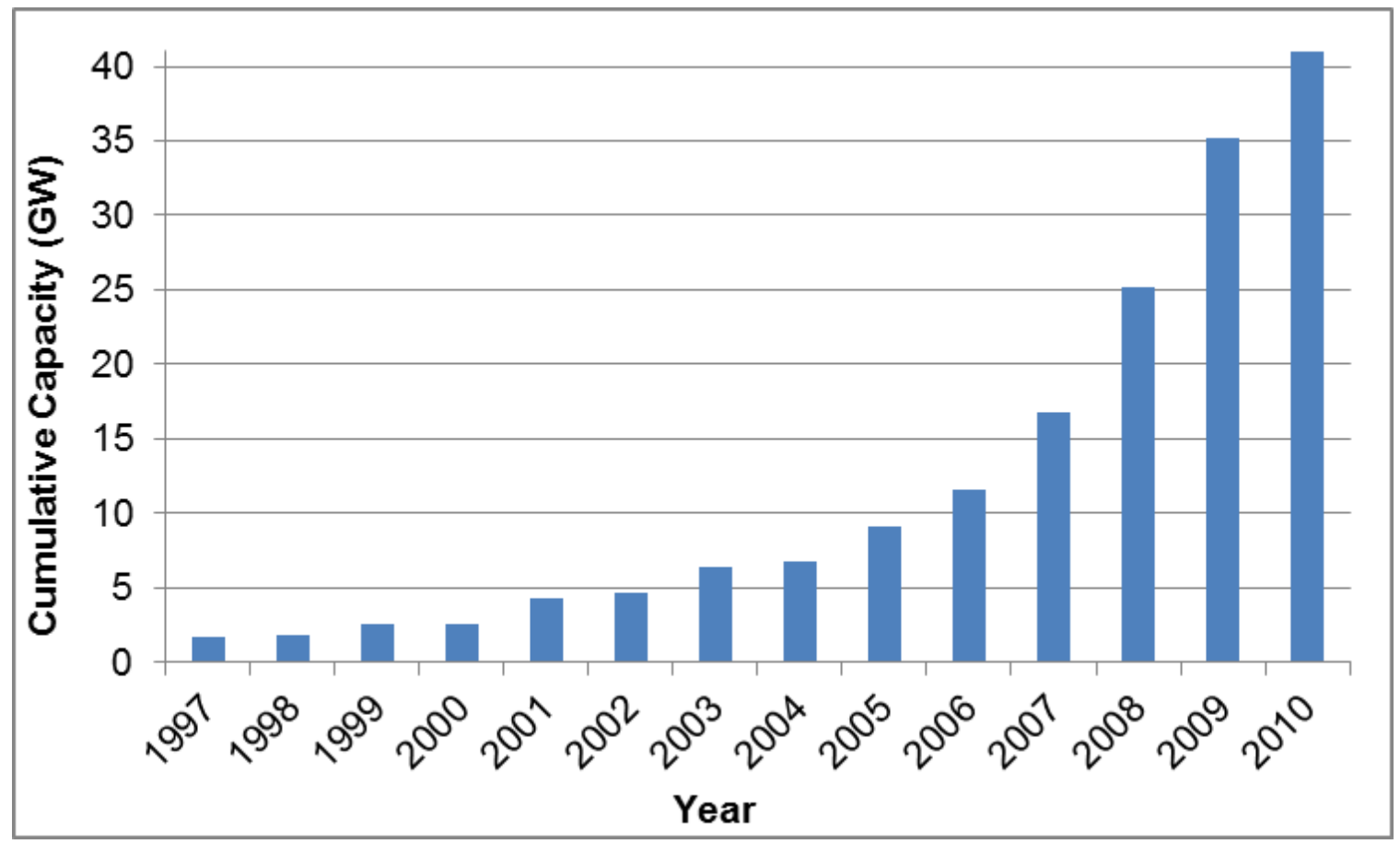

Figure 5-1: Cumulative U.S wind capacity, by year. [5] and [6]

The eight regional entities under NERC together with Hydro Quebec, Alaska Systems Coordinating Council (ASCC), and Hawaii came to 45 GW of installed wind capacity by the end of $2010^{1}$. The Western Electricity Coordinating Council (WECC), Texas Reliability Entity (TRE) and Midwest Reliability Organization (MRO) have a combined wind capacity of over $32 \mathrm{GW}$ or roughly $70 \%$ of all NERC wind power. Table 5-2 summarizes the cumulative installed capacity by regional entity by the end of 2010.

\footnotetext{
${ }^{1}$ Note that FRCC has no utility-scale $(>100 \mathrm{~kW})$ wind capacity.
} 
Table 5-2: NERC wind power cumulative capacity by interconnection by the end of 2010 . [5] and [6]

\begin{tabular}{|c|c|}
\hline Interconnection & Cumulative Capacity (MW) \\
\hline WECC & 13,629 \\
\hline TRE & 9,622 \\
\hline MRO & 8,951 \\
\hline RFC & 4,510 \\
\hline NPCC & 4,058 \\
\hline SPP & 2,257 \\
\hline SERC & 1,304 \\
\hline Hydro Quebec & 808 \\
\hline Hawaii & 63 \\
\hline ASCC & 0.1 \\
\hline Total & 45,202 \\
\hline
\end{tabular}

The wind capacity market share by regional entity by the end of 2010 is shown in Figure 5-2.

\section{Market Share by Regional Entity}

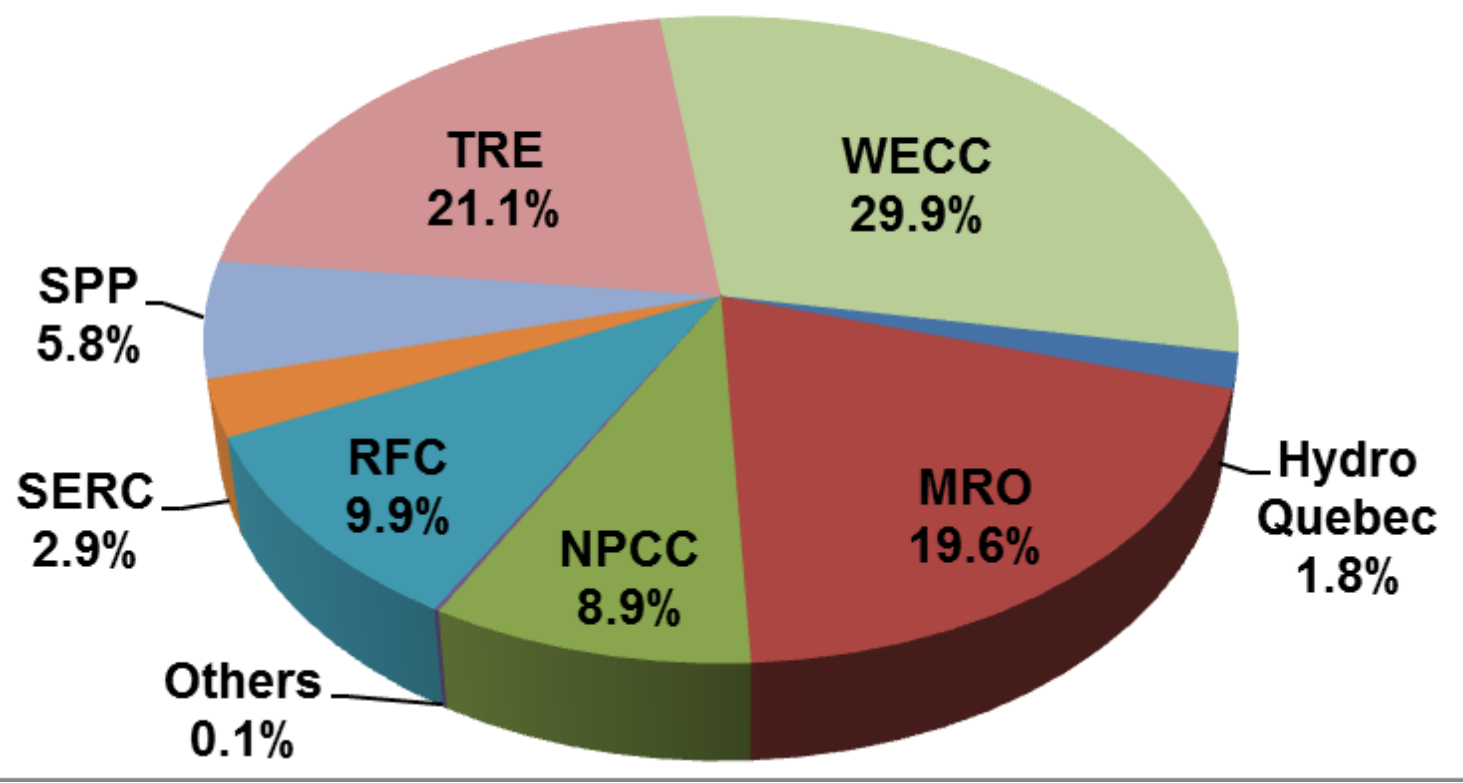

Figure 5-2: Wind power capacity market share by regional entity by the end of 2010. [5] and [6]

According to a DOE released report [7], manufacturers contributing to the North American wind market hail from United States, Europe, Japan, India, China, and South Korea. Altogether, eighteen different WTG manufacturers supplied the market in $2010^{1}$.

${ }^{1}$ Considered are utility-scale $(>100 \mathrm{~kW})$ installations. 
The vendors with the largest share of the considered market, by the end of 2010, were GE Energy (39\%), Vestas (18\%), and Siemens (11\%). Figure 5-3 shows the shows the wind capacity market share by vendor by the end of 2010 .

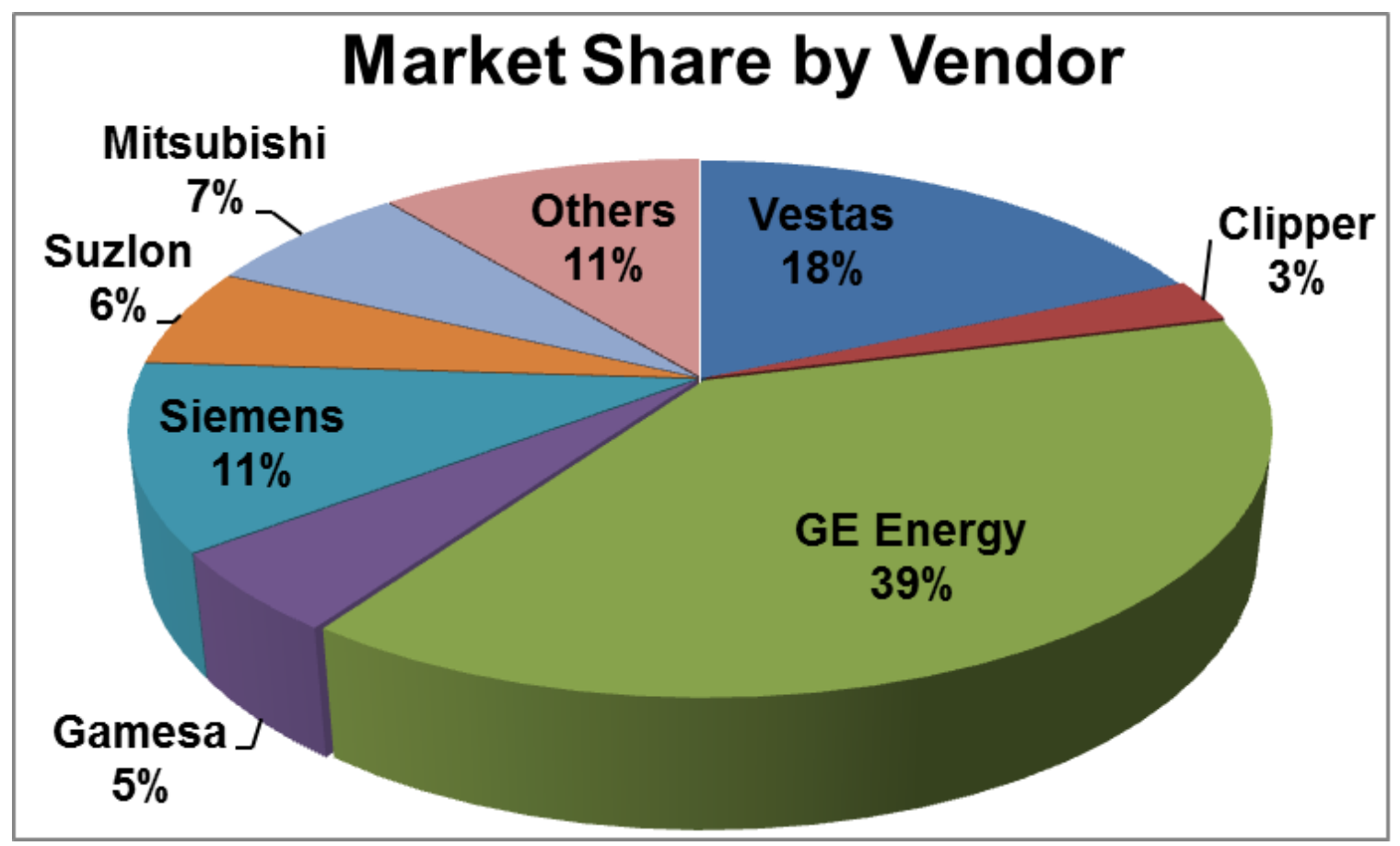

Figure 5-3: Wind power capacity market share by vendor by the end of 2010. [5] and [6]

In today's market the most commonly installed wind turbine generator technologies are the type 3 and type 4 units. Currently, all major vendors supply either type 3, type 4 or both of these technologies (Table 5-3). However, even with the installation numbers of type 1 and type 2 turbines in decline, they still represented roughly $30 \%$ of the operating wind capacity by the end of 2010 (Figure 5-4). 
Table 5-3: Current wind turbine models by type (not a complete list).

\begin{tabular}{|c|c|c|c|c|}
\hline WTG Models & Type I & Type II & Type III & Type IV \\
\hline Acciona & & & AW-Series & \\
\hline Bonus & B-Series & & & \\
\hline Clipper & & & & Liberty \\
\hline DeWind & & & D-Series & \\
\hline $\begin{array}{l}\text { Elecon } \\
\text { Engineering }\end{array}$ & T600-48DS & & & \\
\hline $\begin{array}{l}\text { Emergya Wind } \\
\text { Technologies }\end{array}$ & & $\begin{array}{l}\text { DW } \\
52 / 54\end{array}$ & & DW 90/96 \\
\hline Enercon & & & & E66, E70 \\
\hline Envision & & & E-Series & \\
\hline Fuhrlaender & & & FL-Series & \\
\hline Gamesa & & G80 & G80, G83, G87, G90 & \\
\hline GE & & & $1.5 \mathrm{MW}$ & $2.5 \mathrm{MW}$ \\
\hline Goldwind & & & & GW-Series \\
\hline Mitsubishi & MWT1000A & & MWT - 92; 95; 100 & \\
\hline Nordex & & & $\mathrm{N}$-Series & \\
\hline $\begin{array}{l}\text { Nordic } \\
\text { Windpower }\end{array}$ & N1000 & & & \\
\hline $\begin{array}{l}\text { Northern Power } \\
\text { Systems }\end{array}$ & & & & $\begin{array}{l}\text { Northern Power } \\
2.3\end{array}$ \\
\hline PowerWind & & & & $\begin{array}{l}\text { PowerWind } \\
56 / 60 / 90 / 100\end{array}$ \\
\hline REPower & & & MM - Series & \\
\hline Samsung & & & & $25 s, 25 x$ \\
\hline Siemens & & & & SWT93 \\
\hline Suzlon & S66 & S88 & & \\
\hline Turbo Winds & $\mathrm{T}$ - Series & & & \\
\hline Vestas & V82 & $\begin{array}{l}\text { V80, V47, } \\
\text { V66 }\end{array}$ & $\begin{array}{l}\text { V54 VCS; V80 VCS/VCUS; V90 } \\
\text { VCS/VCUS/VCRS; V100 } \\
\text { VCS/VCUS }\end{array}$ & $\begin{array}{l}\text { V80 GS; V90 GS; } \\
\text { V112 GS }\end{array}$ \\
\hline
\end{tabular}




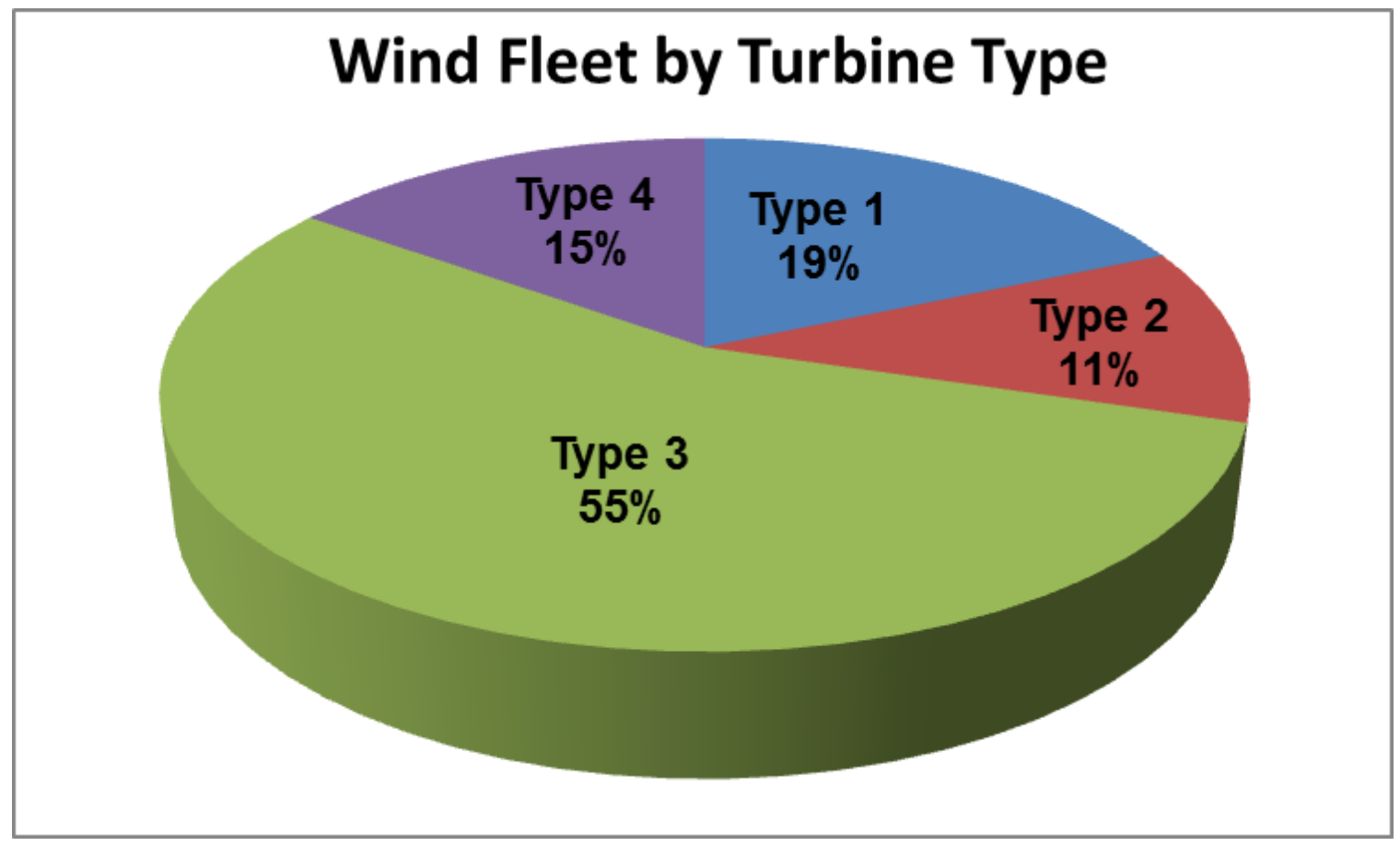

Figure 5-4: Wind power capacity market share by turbine type by the end of 2010. [5] and [6]

Figure 5-5 summarizes the share of different turbine types within the regional entities' wind capacity by the end of 2010 .

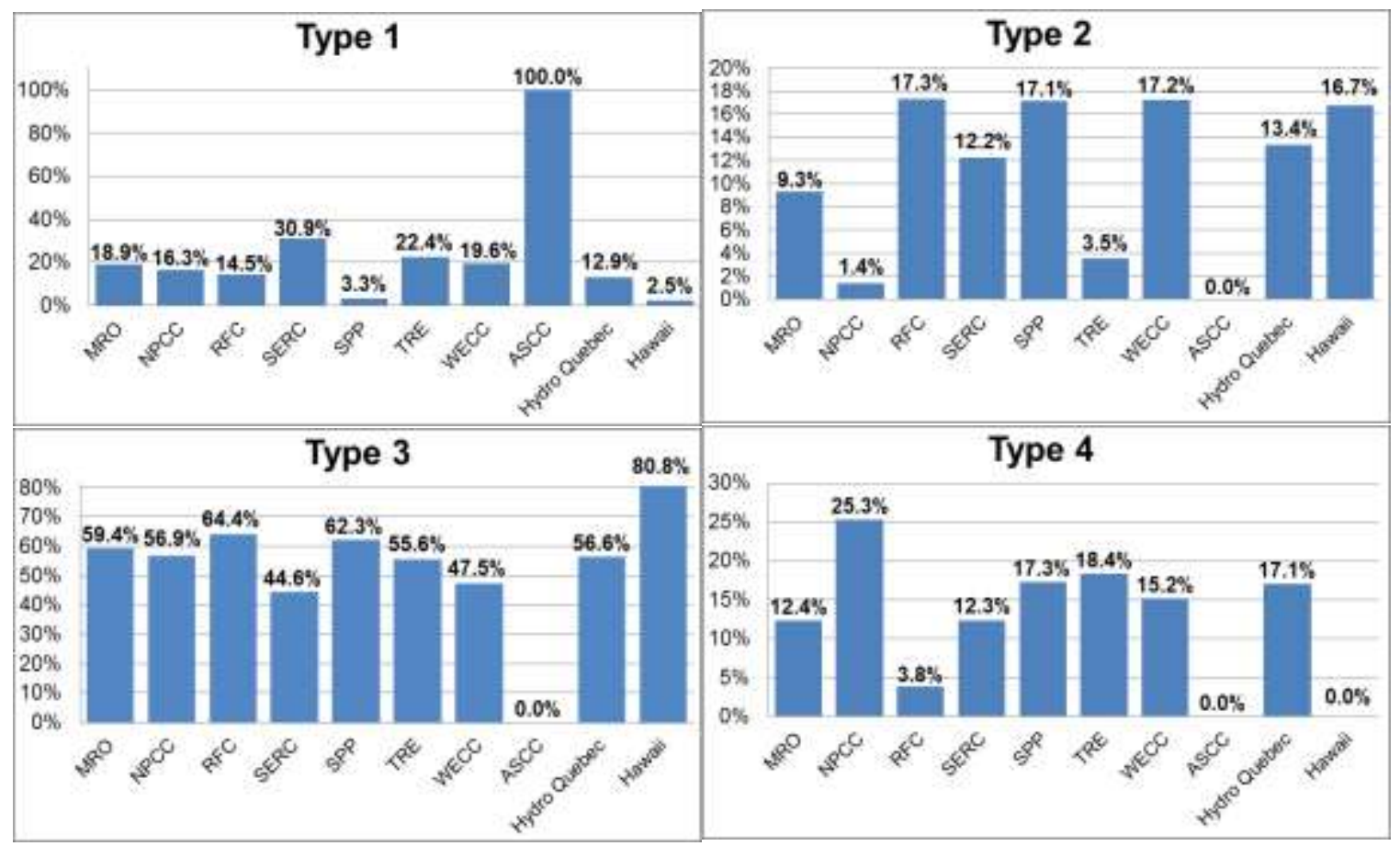

Figure 5-5: Share of the different turbine types within the regional entities' wind capacity by the end of 2010. [5] and [6] 


\section{Generic Models}

\subsection{Background}

The term generic model refers to non-proprietary dynamic models that can be used to represent wind turbine generators (WTGs) with similar physical and control topology, regardless of the manufacturer. In principle, generic WTG models should exhibit the following characteristics: a) allow for an easy exchange of model data between interested parties, b) facilitate comparisons of system dynamic performance between different simulation programs, c) allow for the implementation of WTG models in different simulation programs, and d) provide a mechanism by which manufacturers can tune the model parameters to best represent their equipment, without having to reveal proprietary information [8].

The design guidelines and modeling assumptions established for the generic models include [8]:

- The models are intended for the simulation of events in a time associated with typical transient stability simulations, i.e., ten to twenty seconds.

- It is assumed that in the simulation time frame the wind speed remains constant.

- The models are not designed for use in simulations that involve severe frequency excursions.

- The models allow for the use of a single mass (equivalent to the generator and turbine inertias) or two separate masses.

- The models are suitable for representing individual WTGs or the equivalent of a wind power plant [8].

- The main model components do not include protective functions. These functions are to be modeled externally.

Over the years, the notion of generic models has been advanced significantly, driven by efforts to standardize models for WTGs and wind power plants (WPPs). In North America, recent activities on generic model development are led by the Renewable Energy Task Force (REMTF) of the Western Electricity Coordinating Council (WECC), in coordination with manufacturers and software developers. The International 
Electromechanical Council (IEC) is currently working on standardized WTG and WPP model specifications, which will further guide model implementation activities. Despite the progress that has been made, it is recognized that additional effort is required. The existing generic models must be refined over time, and eventually should become standard models, similar to standard models for other power system components. [8]

\subsection{Structure}

First generation generic simulation models for all four turbine types are currently available as part of the main model libraries for the two most widely used commercial power system simulation tools in North America. These tools are Siemens PTI - Power System Simulation for Engineering (PSSE) and GE - Positive Sequence Load Flow (PSFL). This Section summarizes the model description, nomenclature, and function for all four turbine types as described in user manuals of PSLF [9] and PSSE [10] ${ }^{1}$. The work behind the development of the generic models has been documented in different publications and workshops see [8], [11], [12], [13].

\subsubsection{Generic Type I Model}

The type 1 wind turbine (wt1) is represented by three generic models in PSLF. These models are wt1g, wt1t, and wt1p. The wt1g model includes the generator dynamics. The wt1t model includes the single or double mass shaft compliance model. Finally, the wt1p model contains the pseudo governor model. In this arrangement, the turbine model computes the shaft speed from the electrical and mechanical torques provided by the generator and pseudo-governor models. The generator is a standard induction generator; however, unlike a conventional generator model, it contains no mechanical state variables for the machine rotor - these are included in the wind turbine model. Type 1 WTGs typically include requires reactive compensation, i.e., a capacitor bank, at the machine terminals. The user must represent shunt compensation separately in power flow. Figure 6-1 shows the type 1 wind turbine model connectivity diagram in PSLF.

\footnotetext{
${ }^{1}$ Note that an ongoing effort is focused on development of second generation generic models addressing shortfalls of the currently available models. [14]
} 


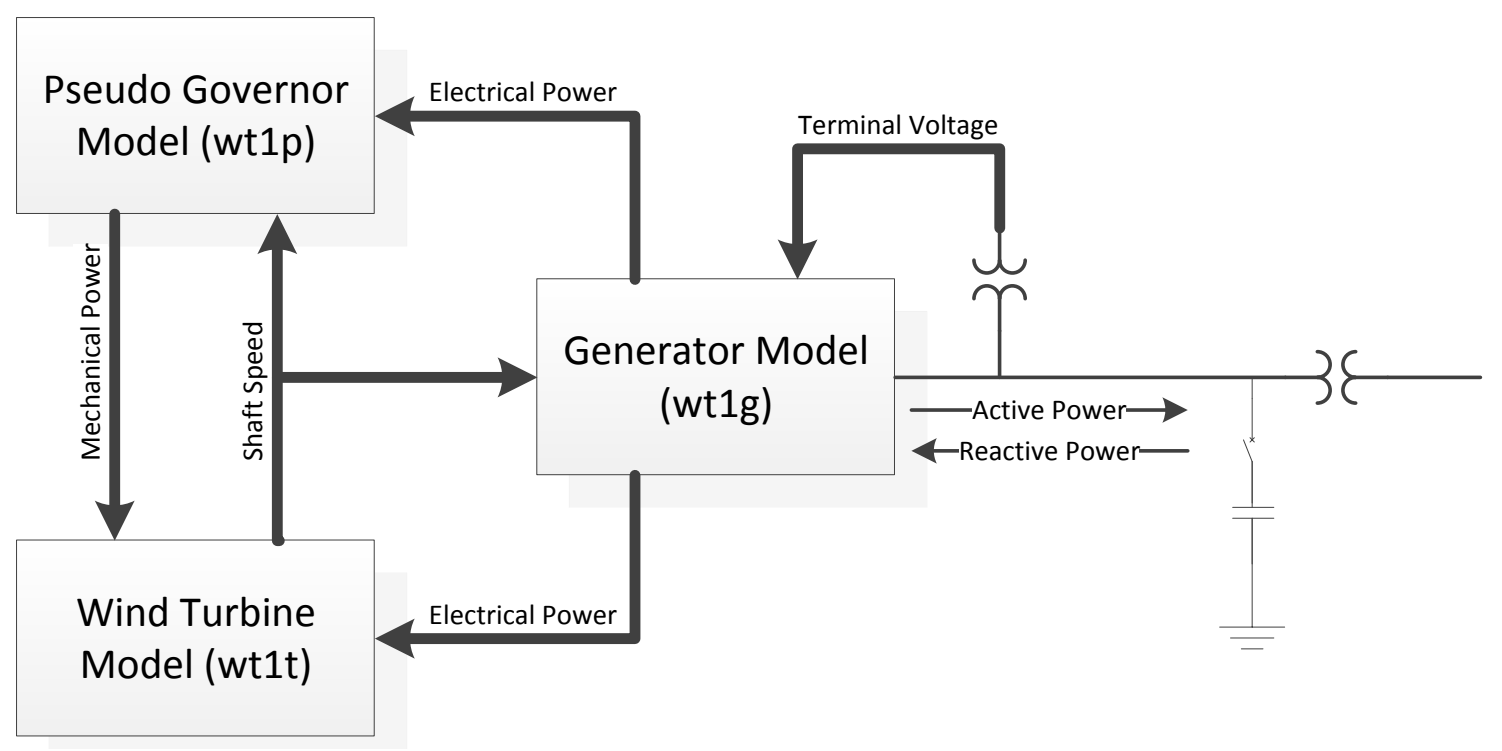

Figure 6-1: Type 1 wind turbine model connectivity diagram in PSLF.

Similar to its counterpart in PSLF the type 1 wind turbine (WT1) is represented by three generic models in PSSE. These models are WT1G, WT12T, and WT12A. The WT1G model includes the induction generator dynamics. The WT12T model includes the double mass shaft compliance model. Finally, the WT12A model contains the pseudo governor model. Figure 6-2 shows the type 1 wind turbine model connectivity diagram in PSSE.

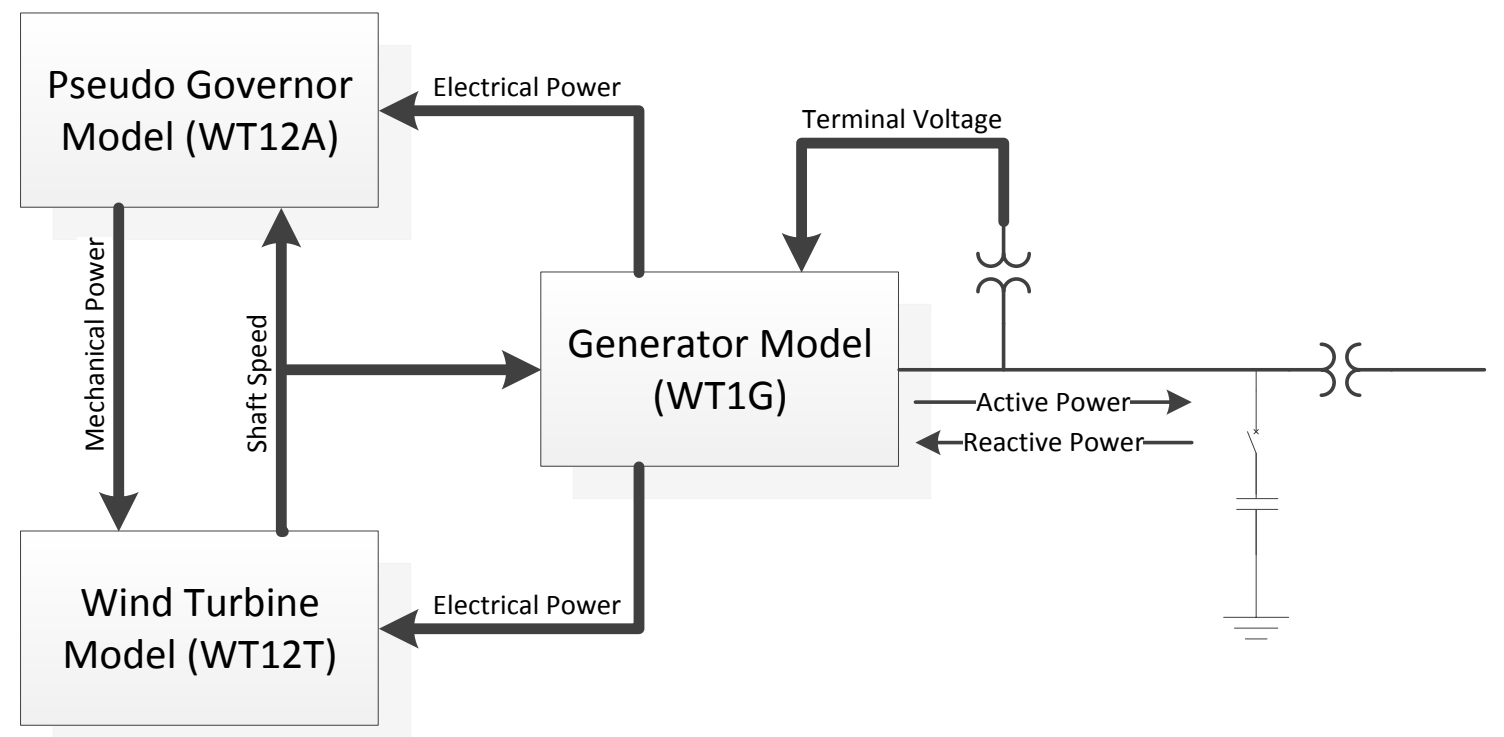

Figure 6-2: Type 1 wind turbine model connectivity diagram in PSSE. 


\subsubsection{Generic Type II Model}

The type 2 wind turbine (wt2) is represented by four generic models in PSLF. These models are wt2g, wt2t, wt2e and wt2p. The wt $2 \mathrm{~g}$ model includes the generator dynamics. The wt2t model includes the wind aerodynamic model and the single or double mass shaft compliance model. The wt $2 p$ includes the pitch controller model. Finally, the wt2e model contains the rotor resistance control model for wound rotor induction wind turbine generator. The generator model represents an induction generator with provisions for adjusting its rotor resistance via the resistance controller. This controller uses rotor speed and generator electrical power as inputs. It computes the resistance to be added to the rotor in the generator model. The wind turbine and pseudo governor models are the same models as those used to represent the generic Type1 WTG. Figure 6-3 shows the type 2 wind turbine model connectivity diagram in PSLF.

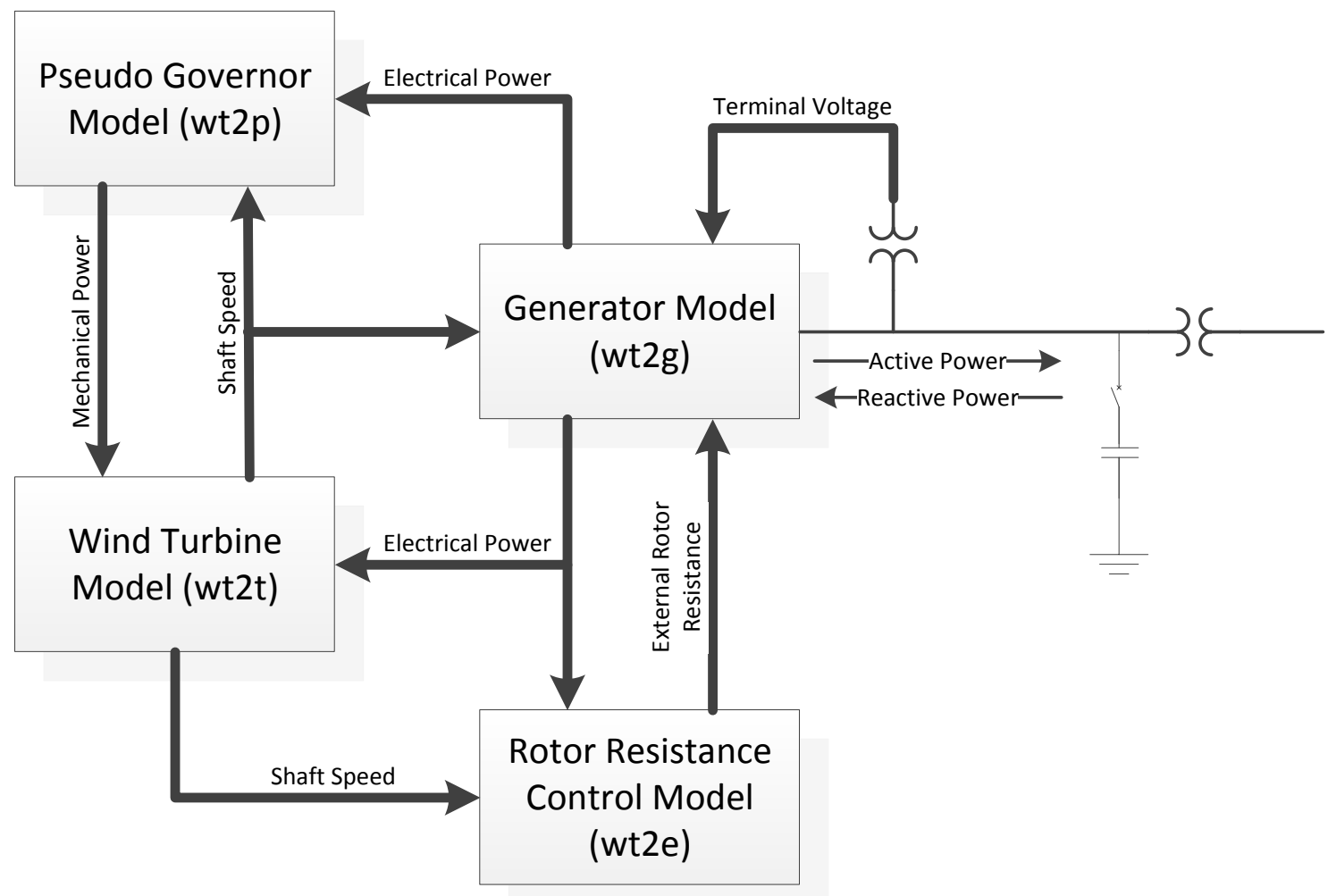

Figure 6-3: Type 2 wind turbine model connectivity diagram in PSLF.

The type 2 wind turbine (WT2) is represented by four generic models in PSSE (similar to PSLF). These models are WT2G, WT2E, WT12T, and WT12A. The WT2G model includes the generator/converters model. The WT2E contains the electrical control model. The WT2T model includes the wind turbine model. Finally, the WT2A model 
contains the pseudo governor model. Figure 6-4 shows the type 2 wind turbine model connectivity diagram in PSSE.

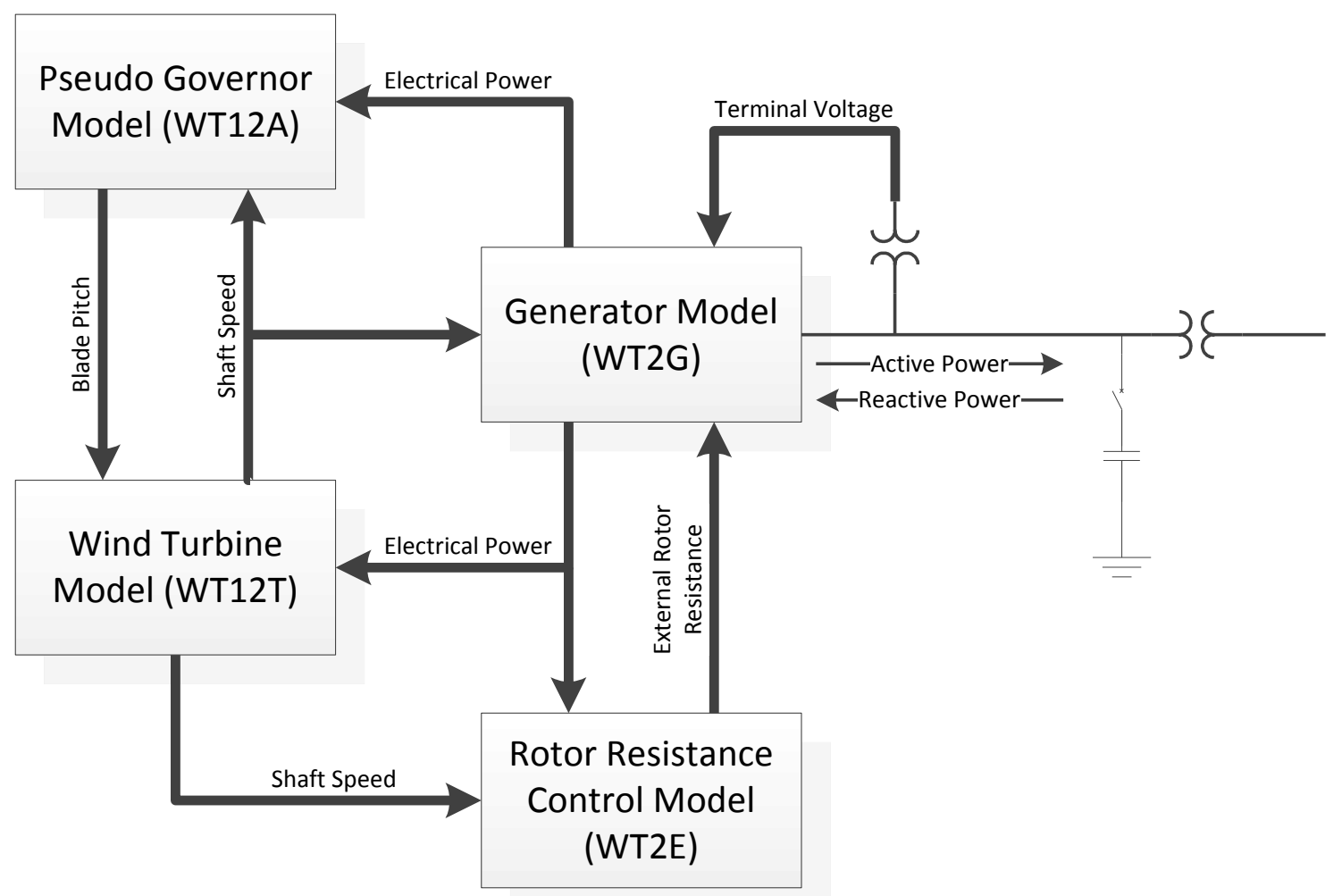

Figure 6-4: Type 2 wind turbine model connectivity diagram in PSSE.

\subsubsection{Generic Type III Model}

The type 3 wind turbine (wt3) is represented by four generic models in PSLF. These models are wt3g, wt3t, and wt3e. The wt3g model includes the generator and converters dynamics. The wt3t model includes the wind aerodynamic model. Finally, the wt3e model contains the real and reactive control models. The generic Type 3 generator/converter model is the equivalent of the generator and field converter, and provides the interface between the WTG and the network. The mechanical state equations are included in the turbine model; furthermore the flux dynamics are eliminated to reflect the rapid response of the converter. The net result is an algebraic, controlled-current source that computes the required injected current into the network in response to commands from the electrical control model. The model also incorporates low voltage power logic and fast-acting converter controls that mitigate over-voltages by reducing reactive current output. The input signals into the generator/converter model are passed through simple low-pass filters. These filters are approximations to the actual fast electronic control system; each filter consists of a first 
order transfer function with a time constant of twenty milliseconds Figure 6-5 shows the type 3 wind turbine model connectivity diagram in PSLF.

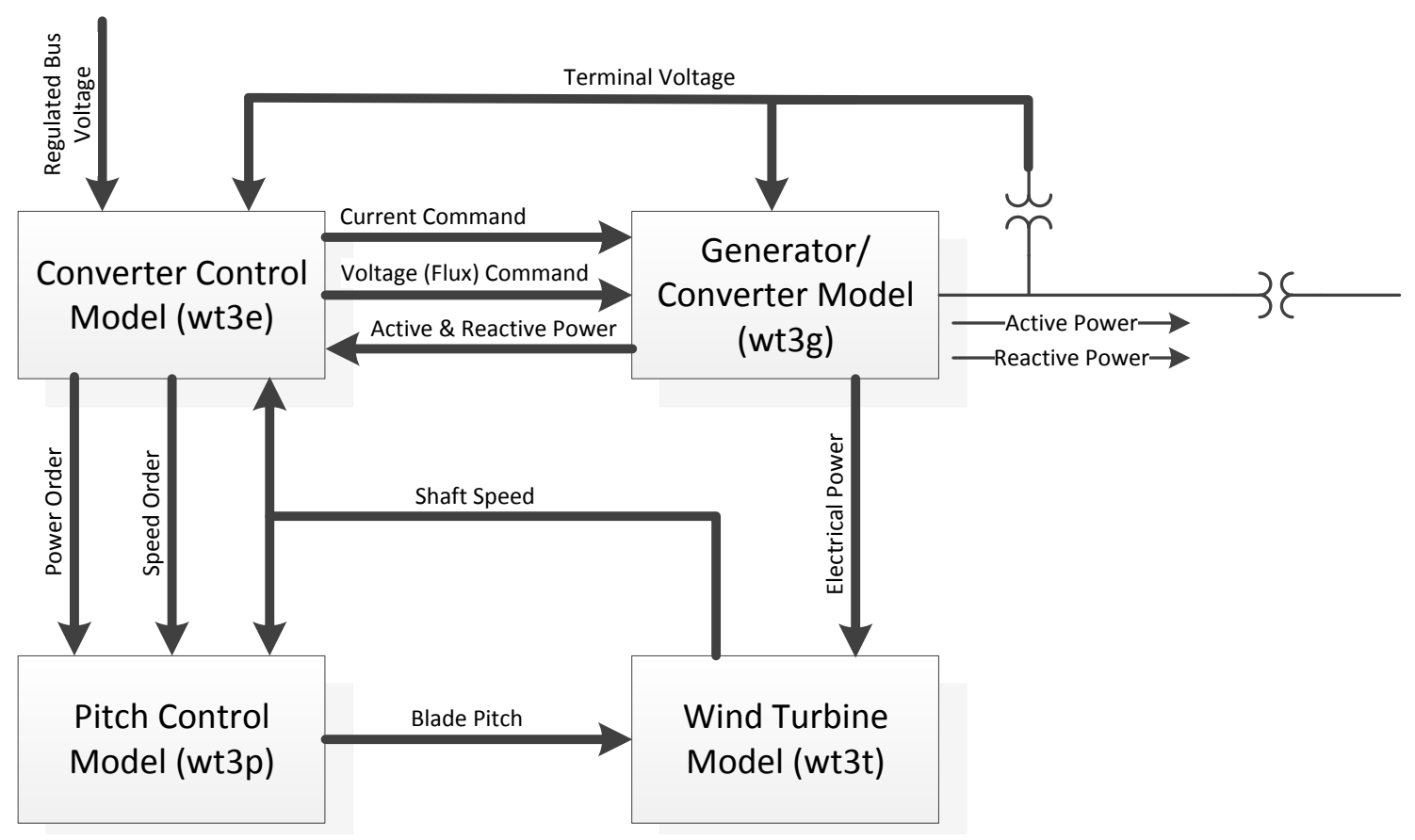

Figure 6-5: Type 3 wind turbine model connectivity diagram in PSLF.

The type 3 wind turbine (WT3) is represented by four generic models in PSSE (similar to PSLF). These models are WT3G1, WT3T1, WT3E1 and WT3P1. The WT3G1 model includes the generator and converters dynamics. The WT3T1 model includes the wind aerodynamic model and the single or double mass shaft compliance model. The WT3P1 includes the pitch controller model. Finally, the WT3E1 model contains the real and reactive control models. Figure 6-6 shows the type 3 wind turbine model connectivity diagram in PSSE. 


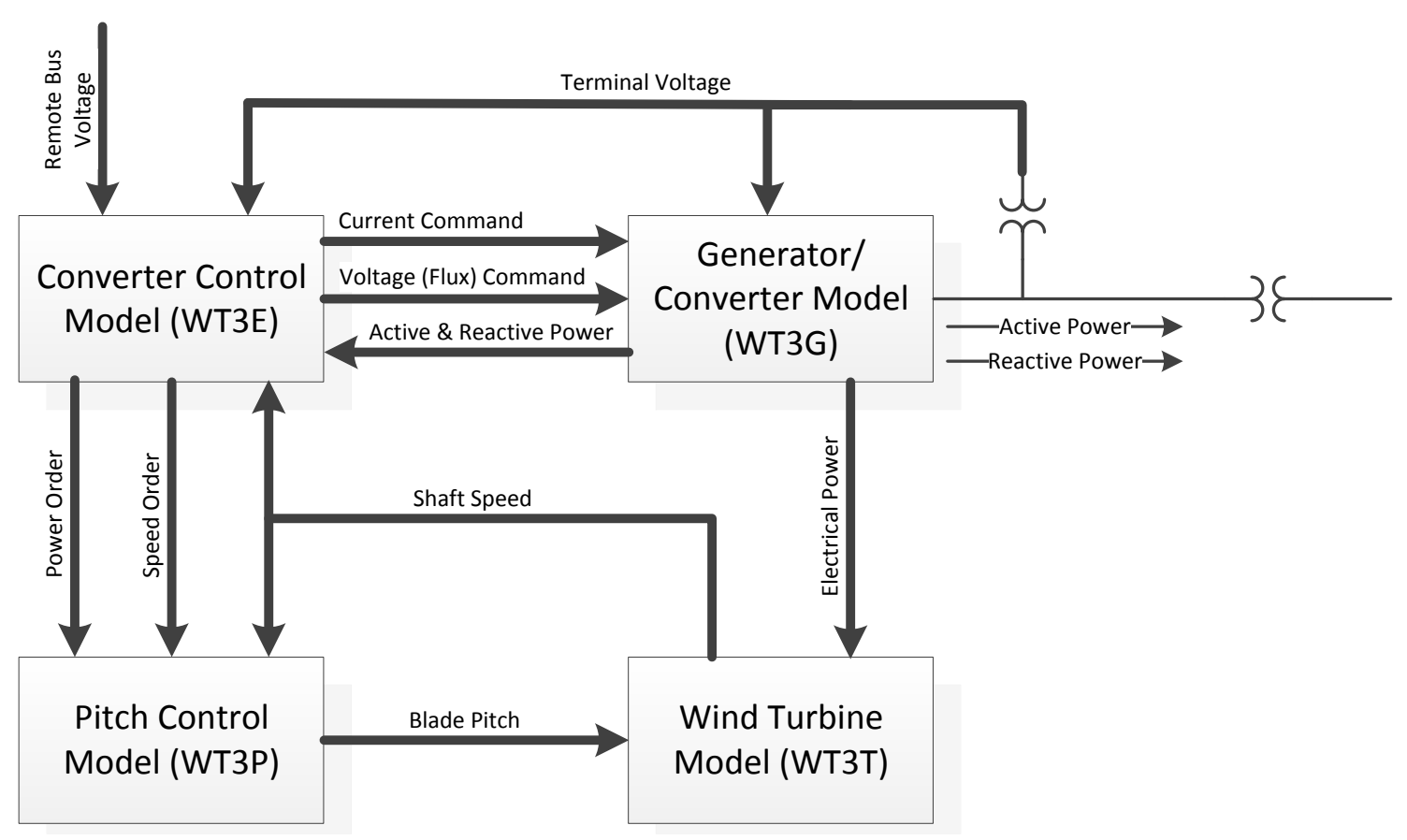

Figure 6-6: Type 3 wind turbine model connectivity diagram in PSSE.

\subsubsection{Generic Type IV Model}

The type 4 wind turbine (wt4) is represented by three generic models in PSLF. These models are wt4g, wt4t, and wt4e. The wt4g model includes the generator and converters dynamics. The wt4t model includes the wind aerodynamic model and the wind turbine model. Finally, the wt4e model contains the real and reactive control models. The converter control model shown computes the active and reactive power to be delivered to the system. The overall structure of the controller is somewhat similar to the Type 3 WTG reactive power control model but it includes logic to determine the current limits. The objective of these limits is to prevent the combination of the real and reactive currents from exceeding converter capability. Depending upon the value of a user-specified P-Q priority flag, either real or reactive power has priority. This flag is dependent upon the equipment features selected, and is normally dictated by the host system grid code. Figure 6-7 shows the type 4 wind turbine model connectivity diagram in PSLF. 


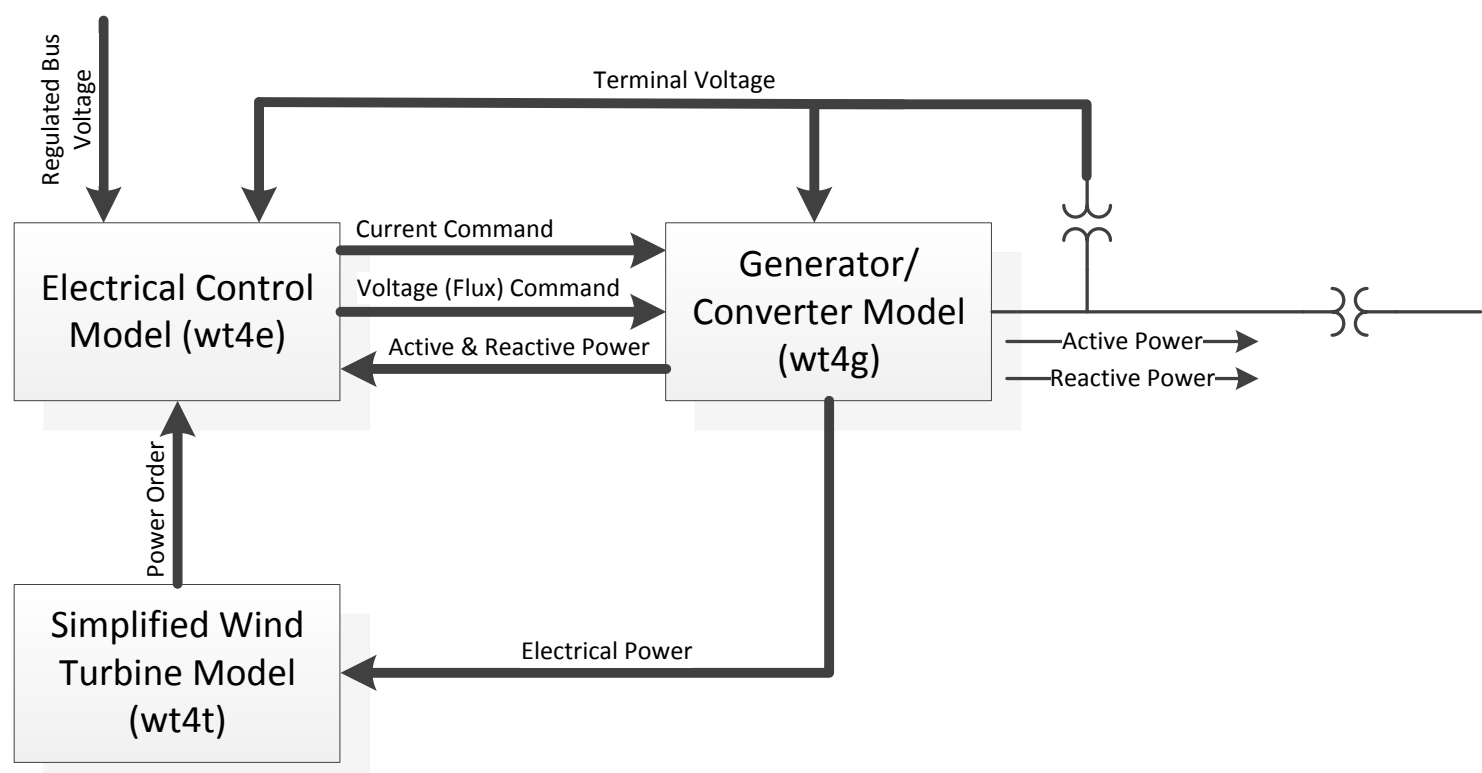

Figure 6-7: Type 4 wind turbine model connectivity diagram in PSLF.

The type 4 wind turbine (WT4) is represented by four generic models in PSSE. These models are WT4G and WT4E. The WT4G model includes the generator and converters dynamics. The WT4E model contains the electrical control model for the type 4 wind generator. Figure 6-8 shows the type 4 wind turbine model connectivity diagram in PSSE.

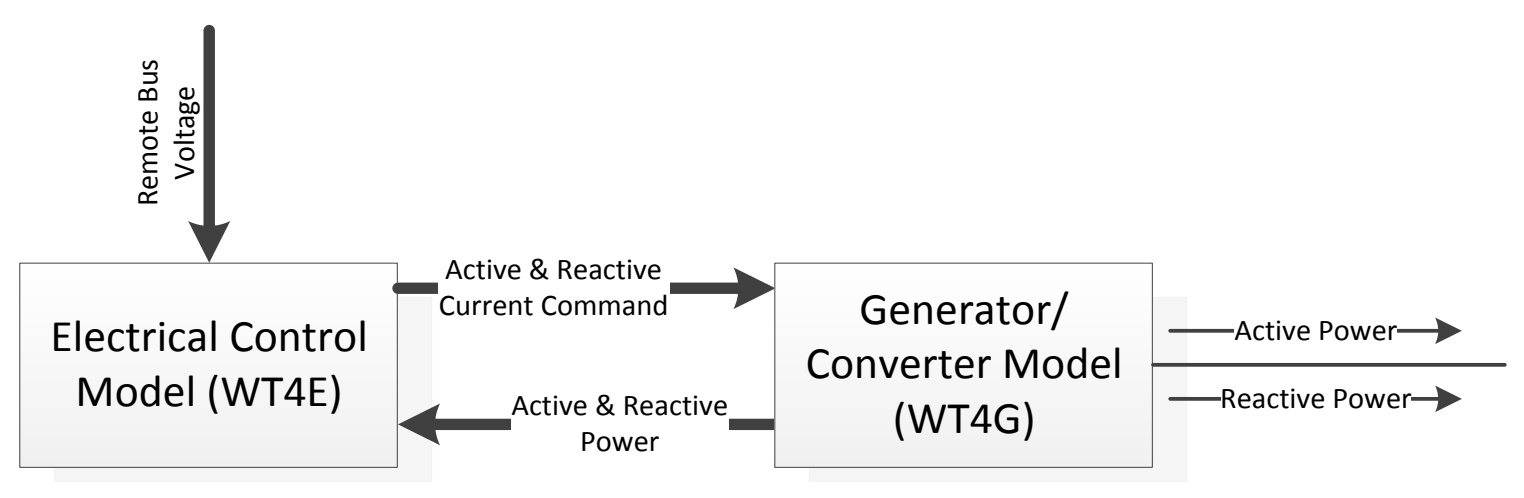

Figure 6-8: Type 4 wind turbine model connectivity diagram in PSSE.

\subsubsection{Summary}

Table 6-1 summarizes the generic wind turbine models in PSLF model library, while Table 6-2 summarizes the generic wind turbine models in PSSE model library. 
Table 6-1: Generic wind turbine models in PSLF

\begin{tabular}{|c|c|c|c|}
\hline Type I & Type II & Type III & Type IV \\
\hline $\begin{array}{l}\qquad \text { wt1g } \\
\text { Generator model } \\
\text { (induction } \\
\text { generator) }\end{array}$ & \begin{tabular}{l}
$\quad$ wt2g \\
\multicolumn{1}{c}{ Generator model } \\
(induction generator \\
with external rotor \\
resistance)
\end{tabular} & $\begin{array}{l}\text { wt3g } \\
\text { Generator/converter } \\
\text { model (doubly-fed) }\end{array}$ & $\begin{array}{l}\text { wt4g } \\
\text { Generator/Converter } \\
\text { model }\end{array}$ \\
\hline-- & $\begin{array}{l}\qquad \text { wt2e } \\
\text { Rotor resistance } \\
\text { Control }\end{array}$ & $\begin{array}{l}\qquad \mathbf{w t 3 e} \\
\text { Excitation (Converter) } \\
\text { Control }\end{array}$ & $\begin{array}{l}\qquad \mathbf{w t 4 e} \\
\text { Excitation (Converter) } \\
\text { Control }\end{array}$ \\
\hline $\begin{array}{l}\text { wt1t } \\
\text { Wind turbine } \\
\text { model }\end{array}$ & $\begin{array}{c}\text { wt2t } \\
\text { Wind turbine model }\end{array}$ & $\begin{array}{c}\text { wt3t } \\
\text { Wind turbine model }\end{array}$ & $\begin{array}{l}\text { wt4t } \\
\text { Simplified wind turbine } \\
\text { model }\end{array}$ \\
\hline $\begin{array}{l}\qquad \text { wt1p } \\
\text { Pseudo governor } \\
\text { model }\end{array}$ & $\begin{array}{l}\qquad \mathbf{w t 2 p} \\
\text { Pseudo governor } \\
\text { model }\end{array}$ & $\begin{array}{c}\text { wt3p } \\
\text { Pitch controller model }\end{array}$ & -- \\
\hline
\end{tabular}

Table 6-2: Generic wind turbine models in PSSE

\begin{tabular}{|c|c|c|c|}
\hline Type I & Type II & Type III & Type IV \\
\hline $\begin{array}{l}\quad \text { WT1G } \\
\text { Generator } \\
\text { model }\end{array}$ & $\begin{array}{l}\text { WT2G } \\
\text { Generator model } \\
\text { (induction generator with } \\
\text { external rotor resistance) }\end{array}$ & $\begin{array}{l}\quad \text { WT3G } \\
\text { Generator/Converter } \\
\text { model (doubly-fed) }\end{array}$ & $\begin{array}{l}\text { WT4G } \\
\text { Generator/Converter } \\
\text { model }\end{array}$ \\
\hline -- & $\begin{array}{l}\qquad \text { WT2E } \\
\qquad \begin{array}{l}\text { Rotor resistance control } \\
\text { model }\end{array}\end{array}$ & $\begin{array}{r}\text { WT3E } \\
\text { Electrical control }\end{array}$ & $\begin{array}{c}\text { WT4E } \\
\text { Electrical control }\end{array}$ \\
\hline \multicolumn{2}{|c|}{$\begin{array}{l}\text { WT12T } \\
\text { Two-Mass turbine model (for Type I and } \\
\text { Type II) }\end{array}$} & $\begin{array}{c}\text { WT3T } \\
\text { Mechanical system model }\end{array}$ & -- \\
\hline-- & -- & $\begin{array}{c}\text { WT3P } \\
\text { Pitch control model }\end{array}$ & -- \\
\hline \multicolumn{2}{|c|}{$\begin{array}{l}\text { WT12A } \\
\text { Pseudo-Governor model (for Type I and } \\
\text { Type II) }\end{array}$} & -- & -- \\
\hline
\end{tabular}




\subsection{Known Issues and Ongoing Developments}

The existing WECC generic models provide a good basis for advancement of standard models for wind turbine generators (WTGs) and wind power plants (WPPs). However, like with any other model, they must be further refined over time to reflect ongoing technological improvements, allow for evaluation of evolving grid codes, and improve the overall accuracy of the model. Based on user experience and consultation with industry, a number of technical specific improvements are being considered. Some of the technical aspects related to the WECC generic model currently under consideration are described in great detail in [8], [14].

\section{- $\quad$ Representation of pitch control for Type 1 and Type 2 WTGs}

The generic Type 1 and Type 2 WTG models use a pseudo-governor model to represent the effect of aerodynamic controls on WTG dynamics. The existing pseudogovernor implementation uses a simple proportional-integral control acting on speed error and power error. This works well for voltage disturbances; however, a sustained frequency change will result in an unintended sustained change in mechanical power reference.

\section{- $\quad$ Generalization of Type 3 and Type 4 WTG Control Structure}

It is known that the control structure of the existing generic Type 3 WTG does not allow for representation of the full range of possible control implementations for doubly-fed asynchronous generators (DFAG). Parameter sensitivity analyses have shown that the existing model does not have sufficient degrees of freedom to adjust the model response. Since the generator is represented simply as excitation voltage behind an equivalent sub-synchronous reactance, similar to a synchronous generator, it is difficult to reproduce active and reactive current modulation that occurs in Type 3 and Type 4 WTGs during a grid disturbance.

\section{- DC link dynamics, unbalanced fault conditions}

The existing WECC Type 3 generic does not keep track DC capacitor voltage or crowbar/dc breaking implementation since detailed representation of dynamics during a fault was considered outside the scope of WECC positive-sequence generic models. Effectively, it is assumed that the rotor-side converter is able to maintain control of the rotor currents even during severe faults. 
Due to inherent limitations, positive sequence programs are not the most suitable platforms to represent dc dynamics and unbalanced fault conditions to the desired extent.

\section{- Active Power Control}

The generic models were designed to be valid for an arbitrary wind speed that is assumed to remain constant for the duration of the simulation. This assumption allowed for simplification of aerodynamic portion of the generic models. Ability to specify active/reactive current priority as well as active current magnitude and rate of recovery are included in the existing generic Type 3 and 4 WTG models. Addition of other active power control functionality is under consideration, including frequency droop and synthetic inertia. 


\section{Wind Turbine Data Base}

\subsection{Introduction}

This Chapter summarizes the vendor specific model (VSM) characterization along with generic model parameterization and validation for the major wind turbines in North America. The purpose of this chapter is to provide a comprehensive overview of the VSM behavior and the ability of the current generic models to mimic this behavior under uniform conditions in both PSLF and PSSE simulation platforms ${ }^{1}$. The results, shown in the following Sections, together with results to be obtained in the future, are intended to be compiled into an online wind turbine data base.

\subsubsection{Test System ${ }^{2}$}

The test system is shown in Figure 7-1. The system includes an equivalent $100 \mathrm{MW}$ wind power plant (WPP) and its associated unit transformer, collector system, and substation transformer. The equivalent of the collector system is represented by the branch between buses 13 and 14, with the main station transformer for the wind farm between buses 12 and 13. The High Voltage Point of Interconnection (POI) is the $230 \mathrm{kV}$ bus 12 . The POI is connected to the load bus 11 by a double circuit line.

Two other generators are also modeled: a 1,000 MVA hydro unit and 100 MVA gas turbine unit. Two loads of 100 MVA and 1,000 MVA are modeled as constant power loads connected to bus 11 .

\footnotetext{
${ }^{1}$ Note, in some cases VSMs were available either in the PSLF or the PSSE platforms. Also in some cases VSMs in neither platform could be obtained or the results are being reviewed by the manufacturing vendor, in these cases the particular wind turbines were characterized using data from public domains (if available).

${ }^{2}$ Test system for characterization and parameterization simulations was designed by Yuriy Kazachkov Siemens Energy.
} 


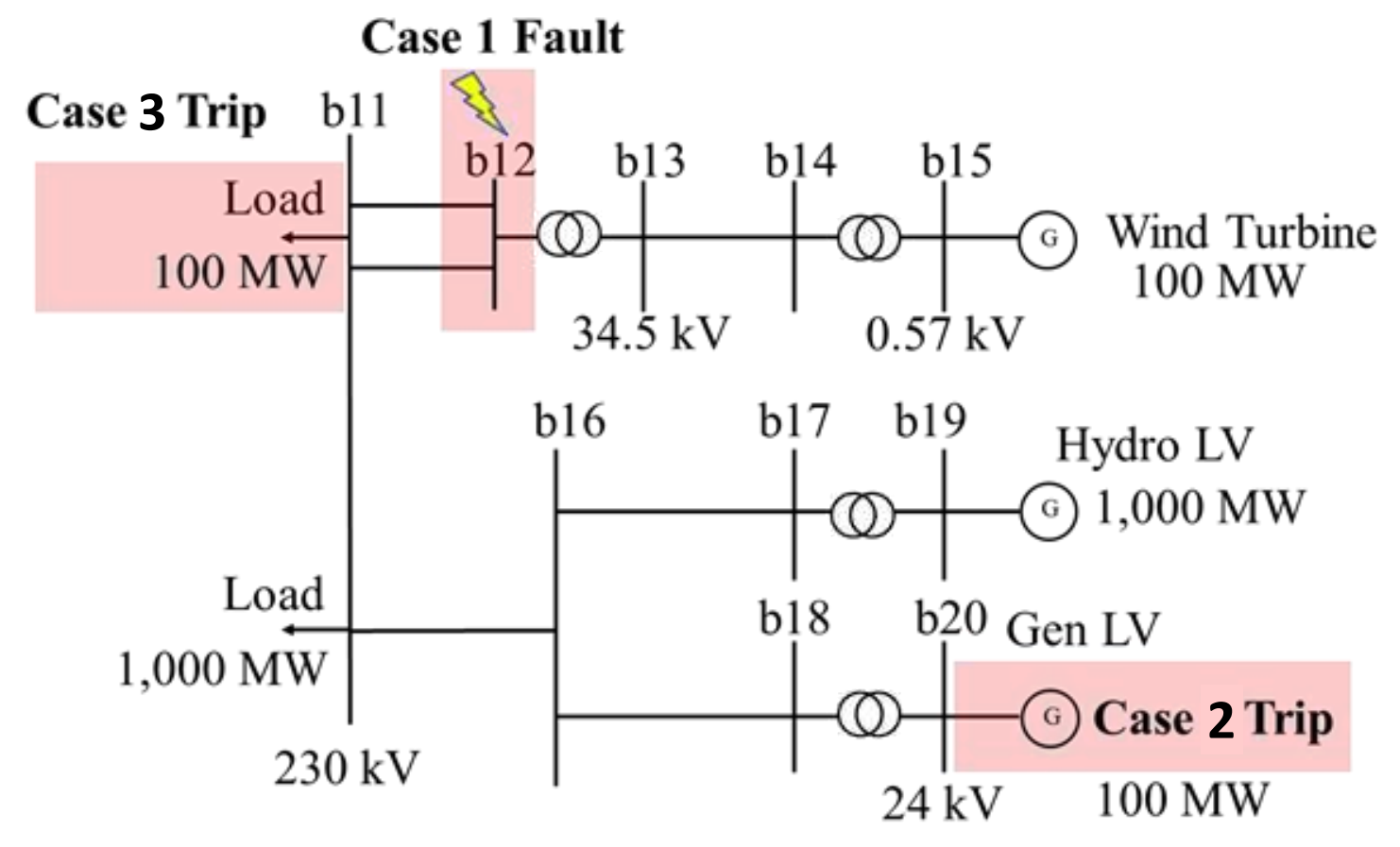

Figure 7-1: One-line diagram of the power system.

\subsubsection{Test Cases}

The following three test cases are documented:

- Case 1: Six-cycle $(0.1 \mathrm{sec}$.$) , three phase fault at bus 12. The fault is cleared$ by tripping one of the two lines between bus 11 and bus 12 .

- Case 2: Trip of a $100 \mathrm{MW}$ generator at bus 20.

- Case 3: Trip of a $100 \mathrm{MW}$ load at bus 11.

For all cases it is assumed that the WPP is generating rated power (100 MW).

The impedance of the double circuit line between buses 11 and 12 was adjusted to ascertain the dynamic performance of the WPP for short circuit ratios (SCRs) of 5 and 10 at the POI.

\subsubsection{Parameterization}

In order for the response of a dynamic model to approximate the response of a given vendor specific model (VSM), the parameters of the generic model typically need to be adjusted. This process is termed parameterization process, i.e., the parameterization process refers to the adjustment of the parameter values of a generic WTG model to best reflect the dynamic characteristics of a VSM. Conceptually, this process is depicted in Figure 7-2. The figure shows a generic WTG and a VSM models 
subject to the same stimulus, e.g., fault, line trip, etc. The parameterization process aims at reducing the difference (error) between the dynamic responses of both models. Reducing the error may entail an algorithmic approach and/or the manual adjustment of parameters. It is recognized that, in general, the responses of the generic and VSM will not be identical. The fundamental issue to be addressed is whether a generic model properly parameterized is a suitable model for application in bulk power system studies of the type often executed in routine planning studies.

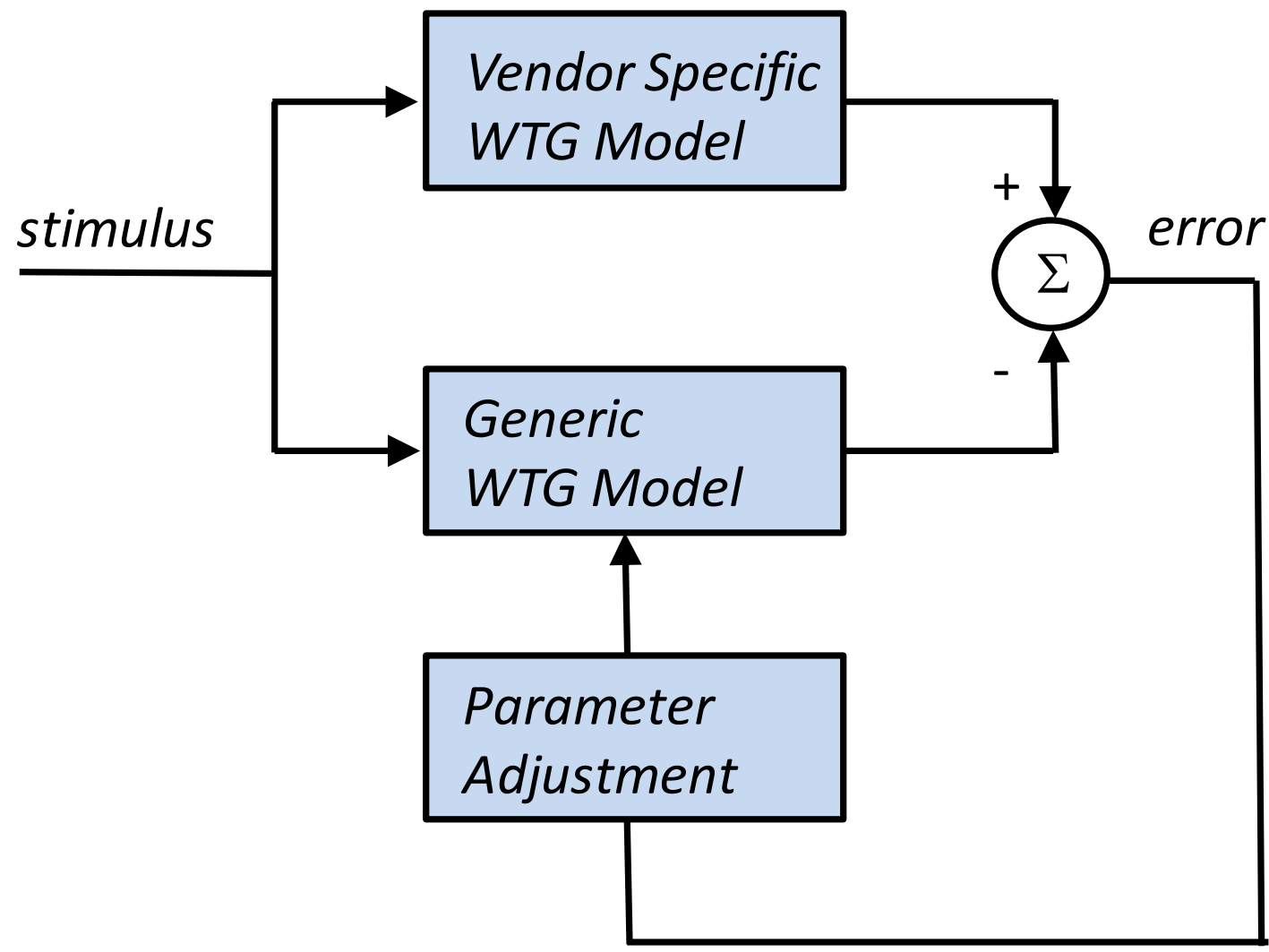

\section{Figure 7-2: Parameterization process.}

The major challenge for the parameterization of generic models comes from the fact that each VSM is designed according to the turbine vendor's specification. This implies that the internal mechanism and control settings can be quite different even for the same type of wind turbine. Furthermore, for confidentiality reasons, critical components and parameters for many VSMs are hard-coded in the models and are not disclosed to users. Often, a VSM is regarded as "blackbox" with essentially unknown characteristics.

The objective of the parameterization part in this report is to facilitate the use of generic models by providing the generic model parameters needed for representing 
VSMs. This part also includes sample time domain simulations that compare the response of VSMs versus the corresponding generic models in PSLF and PSSE platforms. 


\subsection{Clipper - Liberty Series}

\subsubsection{Wind Turbine Characterization}

Clipper's Liberty series is represented by three-blade, upwind, horizontal axis wind turbines with a rated capacity of 2.5-megawatts. Four different models represent the Liberty - 2.5 MW series - C89, C93, C96, and C99, with following features:

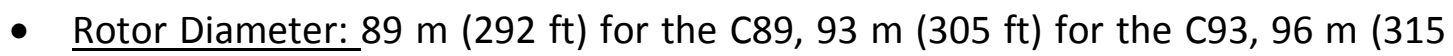

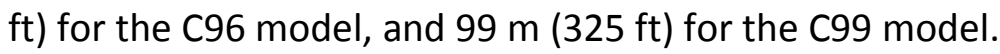

- $\quad$ Hub Heights: 80 m (262 ft)/Other Options Available.

- $\quad$ Cut-in wind speed: $4 \mathrm{~m} / \mathrm{s}(9 \mathrm{mph})$.

- $\quad$ Cut-out wind speed: $25 \mathrm{~m} / \mathrm{s}(55 \mathrm{mph})$.

- Terminal Voltage: 690 VAC +/- 10\% Continuous; 690 VAC + 20\% for 5 Seconds; 690 VAC + 30\% for 0.5 Seconds; 690 VAC - 90\% for 3 Seconds; 690 VAC - 100\% for 150 Milliseconds.

- Power Control: Purpose designed embedded power PC with super capacitor energy storage system as back up for extend through operations.

- Generator: 4 x Synchronous Permanent Magnet (660 kW at 1133 rpm each).

- $\quad$ Power Converter: 4X, Voltage Sourced, IGBT Based 6 Pulse, Inverters.

- Pitch System: 3X DC Electric-Mechanical Gear-motor, Servo Drives and Batteries backup.

- Yaw system: 4 Electro-Mechanical Motors with Planetary Drives with internal gear and ball bearing

- Braking: Dual Disc with Active Hydraulic Brake Calipers

Liberty is a variable speed wind turbine with a four permanent magnet generators distributed powertrain. The rotor on a Liberty turbine is designed to operate in an upwind configuration at 9.6 to 15.5 revolutions per minute (rpm). The Liberty $-2.5 \mathrm{MW}$ series provides the option of a selectable power factor between 0.95 (overexcited/lagging) and 0.95 (underexcited/leading). For example, by configuring the machine to operate at a slightly overexcited (lagging) power factor, the inverters can compensate for reactive power losses within the wind plant collection system, providing 
approximately unity power factor operation at the point of interconnection over a wide range of plant output.

\subsubsection{Vendor Specific Model Characterization}

\subsubsection{Model Performance: Case 1 - Fault Event}

In this case a remote fault is applied to bus 12 for a duration of 6 cycles $(0.1 \mathrm{sec})$.

\section{$\underline{\text { SCR }-5}$}
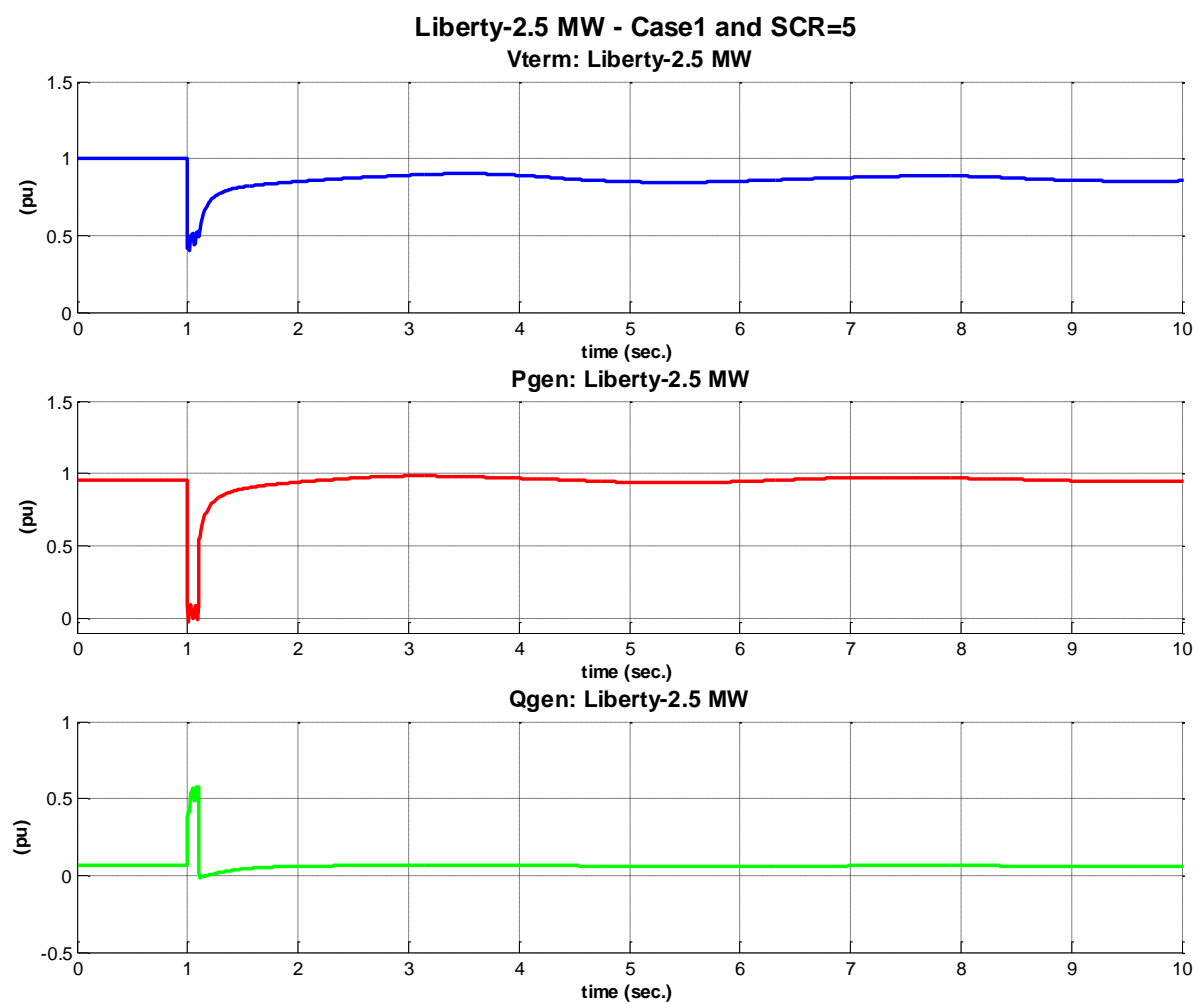

Figure 7-3: Liberty-2.5 MW - Case 1 and $S C R=5\left(V_{\text {Term }}, P, Q\right)$. 

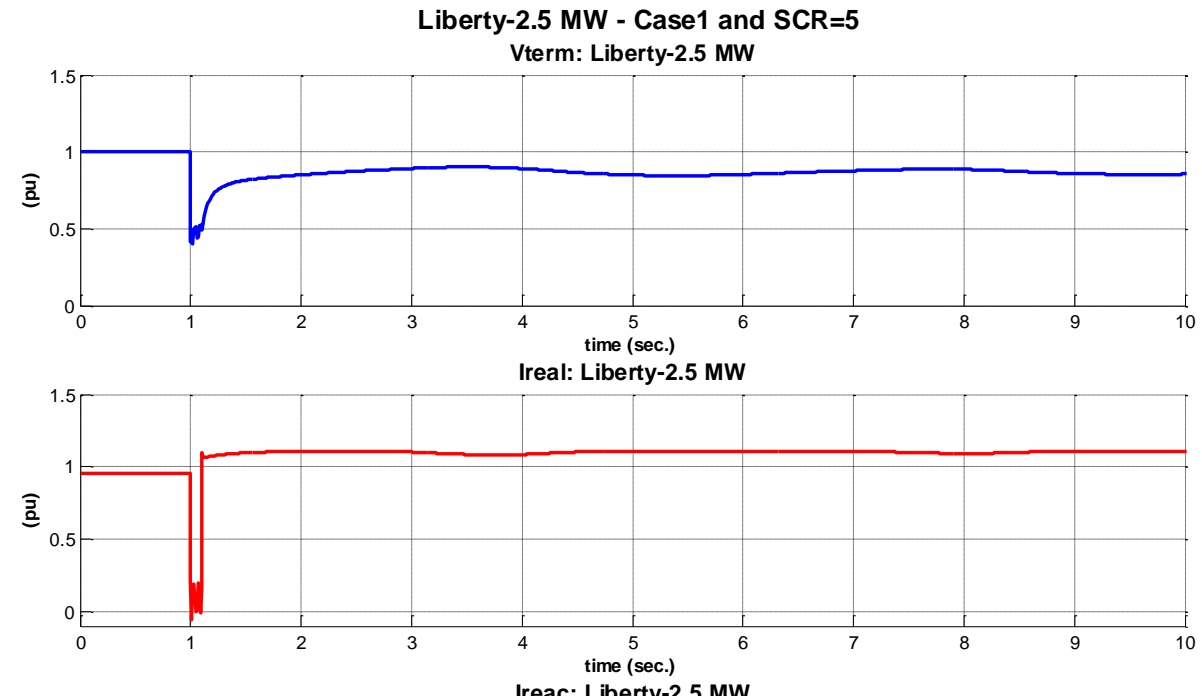

Ireac: Liberty-2.5 MW

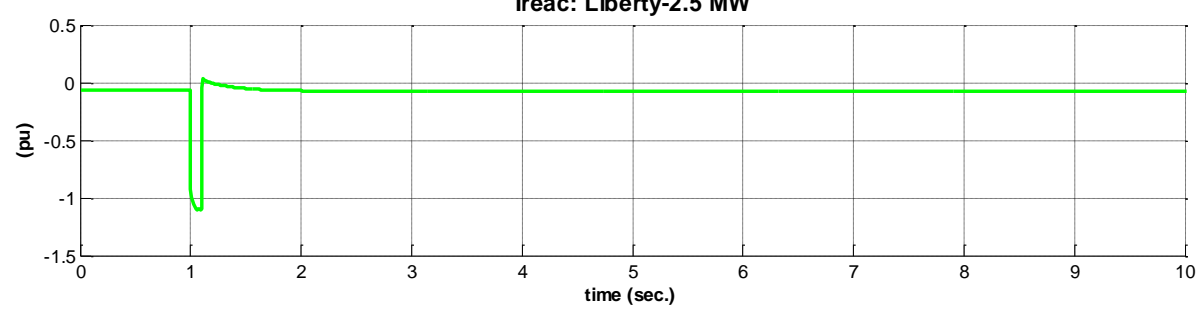

Figure 7-4: Liberty-2.5 MW - Case 1 and SCR=5 ( $\left.V_{\text {Term }}, I_{\text {Real }}, I_{\text {Reac }}\right)$.

SCR - 10
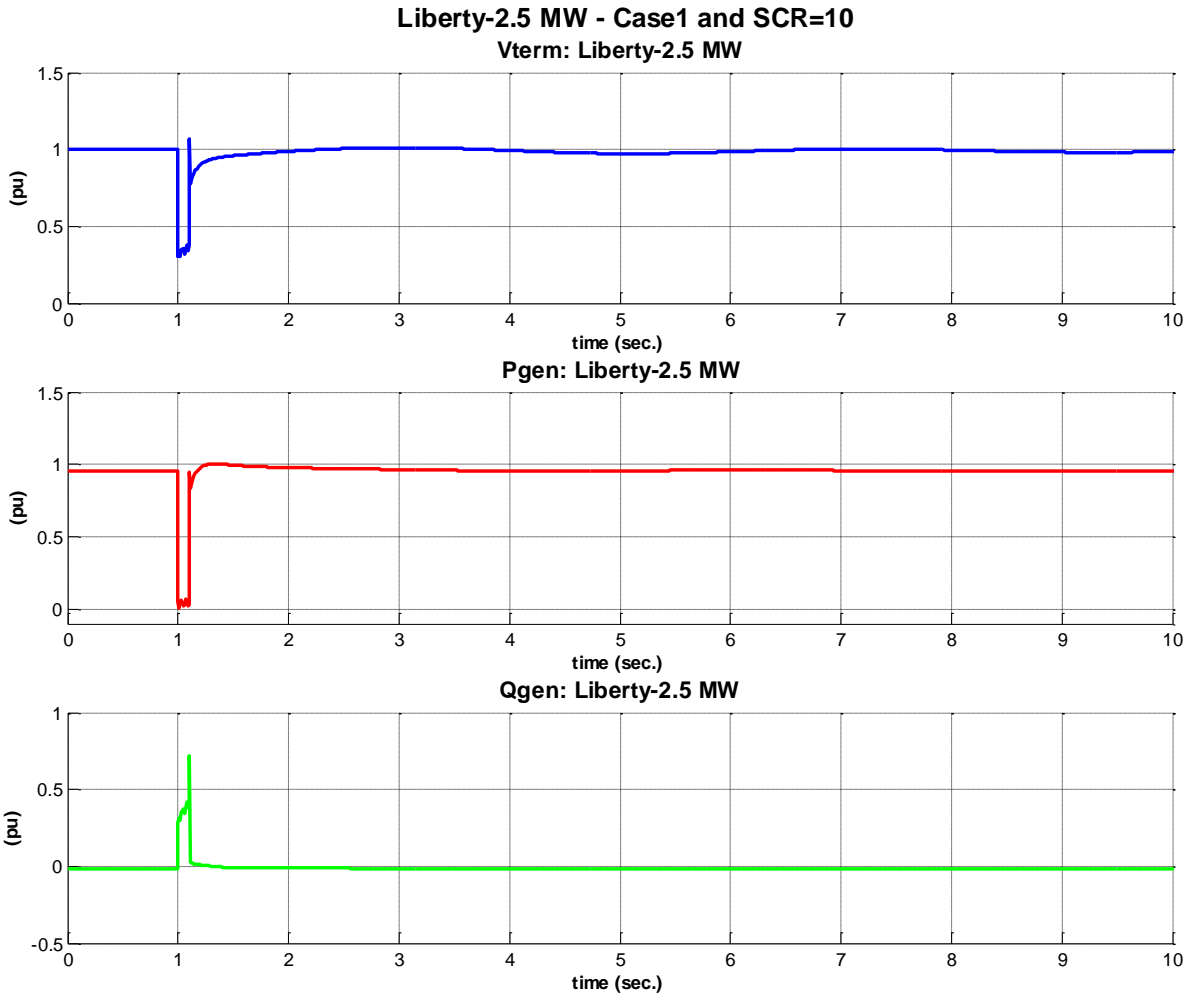

Figure 7-5: Liberty-2.5 MW - Case 1 and $\operatorname{SCR}=10\left(V_{\text {Term }}, P, Q\right)$. 

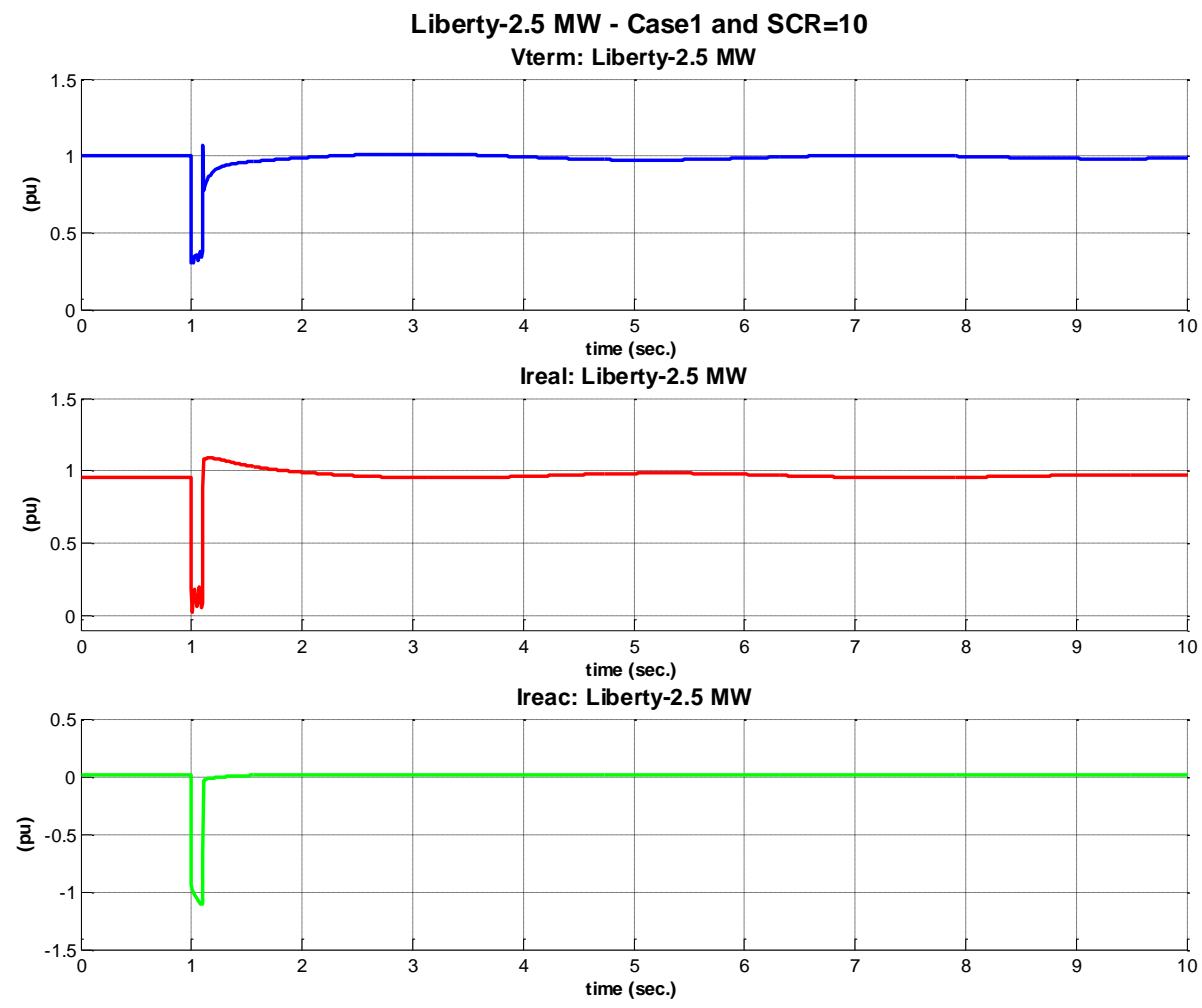

Figure 7-6: Liberty-2.5 MW - Case 1 and $S C R=10\left(V_{\text {Term, }} I_{\text {Real }}, I_{\text {Reac }}\right)$.

For both SCR settings the reactive current output of the WTG increases during the fault. A current limit of 1.11 pu on the 2.5 MVA machine base is implemented to prevent thermal damage to the converter in the event it was subjected to a short circuit near the terminals of the inverter. After the fault is removed the reactive current returns to its steady state condition. 


\subsubsection{Model Performance: Case 2 - Under-Frequency Event}

In this case a under frequency event is created by tripping the 100 MVA generation unit at bus 20 .

\section{SCR -5}
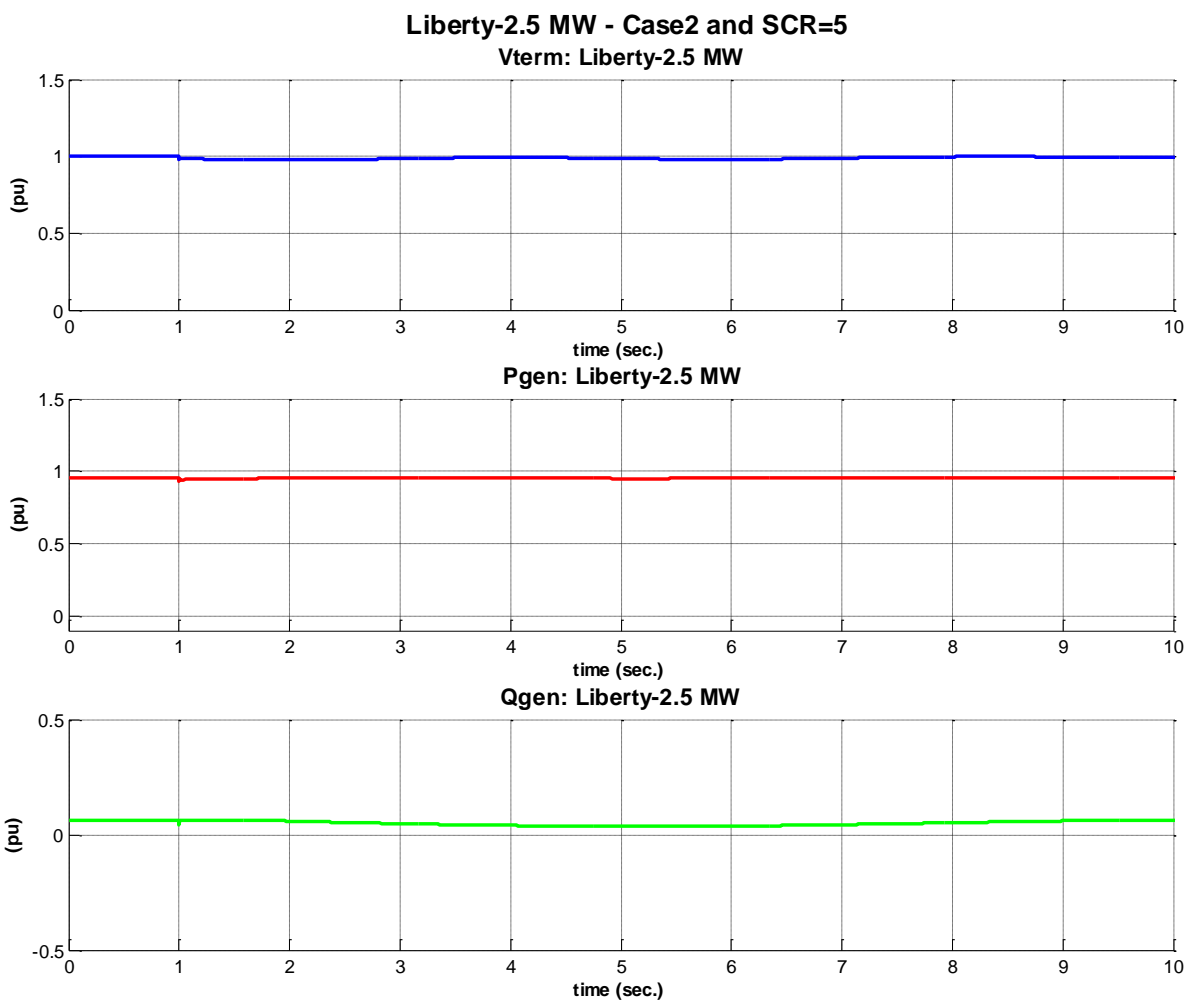

Figure 7-7: Liberty-2.5 MW - Case 2 and SCR=5 (V $\left.\mathrm{V}_{\text {Term }}, \mathrm{P}, \mathrm{Q}\right)$. 

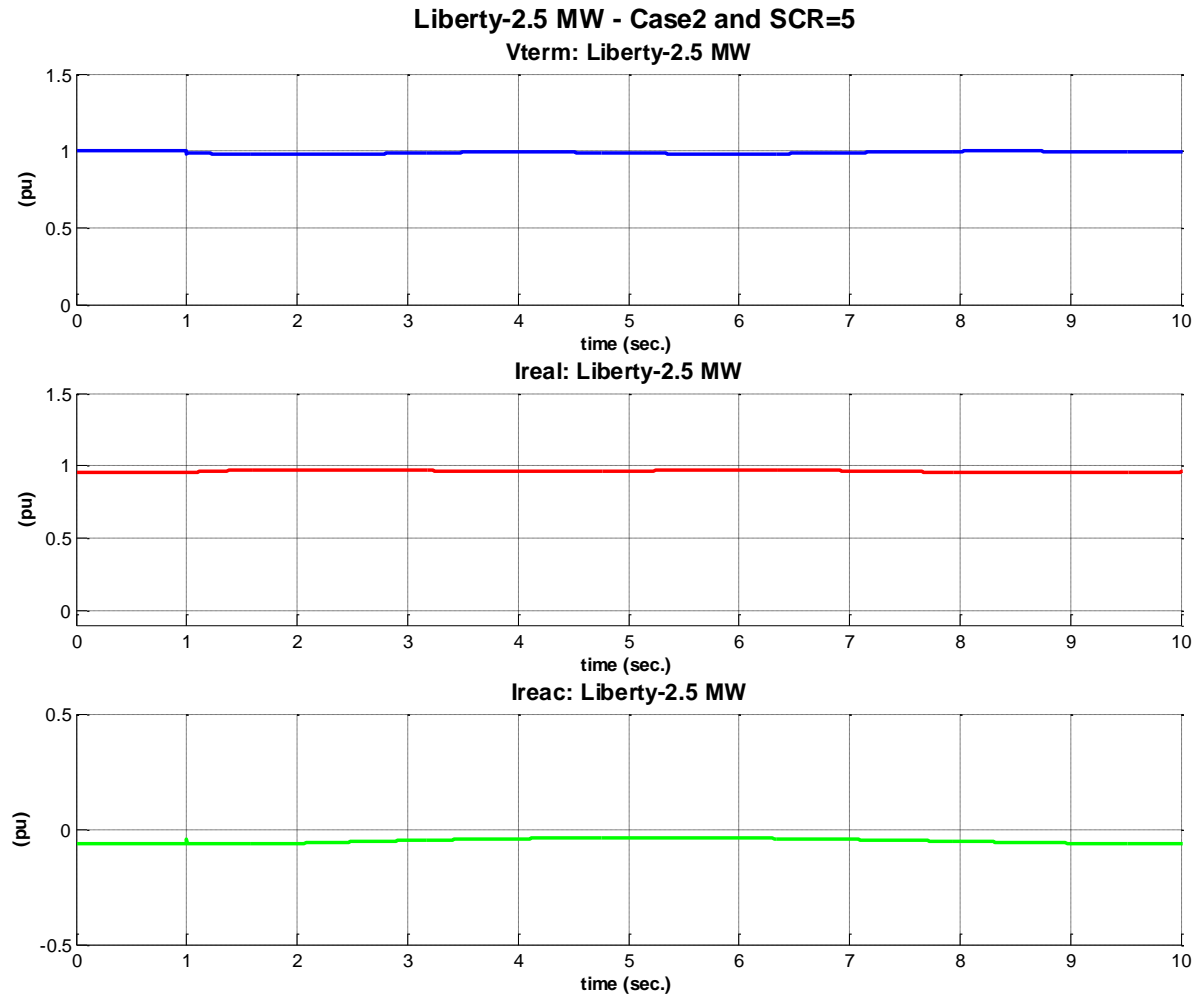

Figure 7-8: Liberty-2.5 MW - Case 2 and SCR=5 ( $\left.\mathrm{V}_{\text {Term }}, \mathrm{I}_{\text {Real }}, \mathrm{I}_{\text {Reac }}\right)$.

$\underline{\text { SCR }-10}$
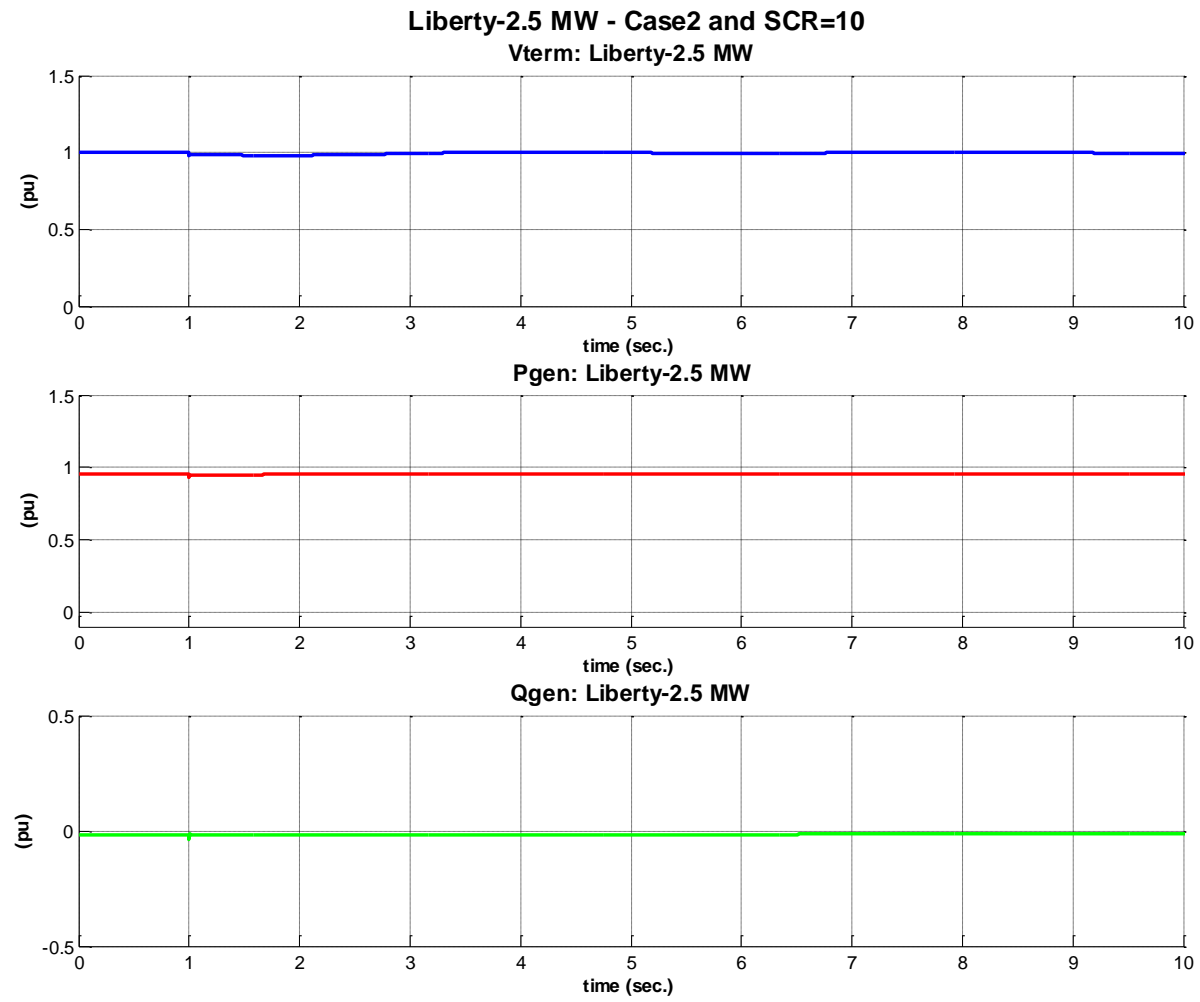

Figure 7-9: Liberty-2.5 MW - Case 2 and $S C R=10\left(V_{\text {Term }}, P, Q\right)$. 

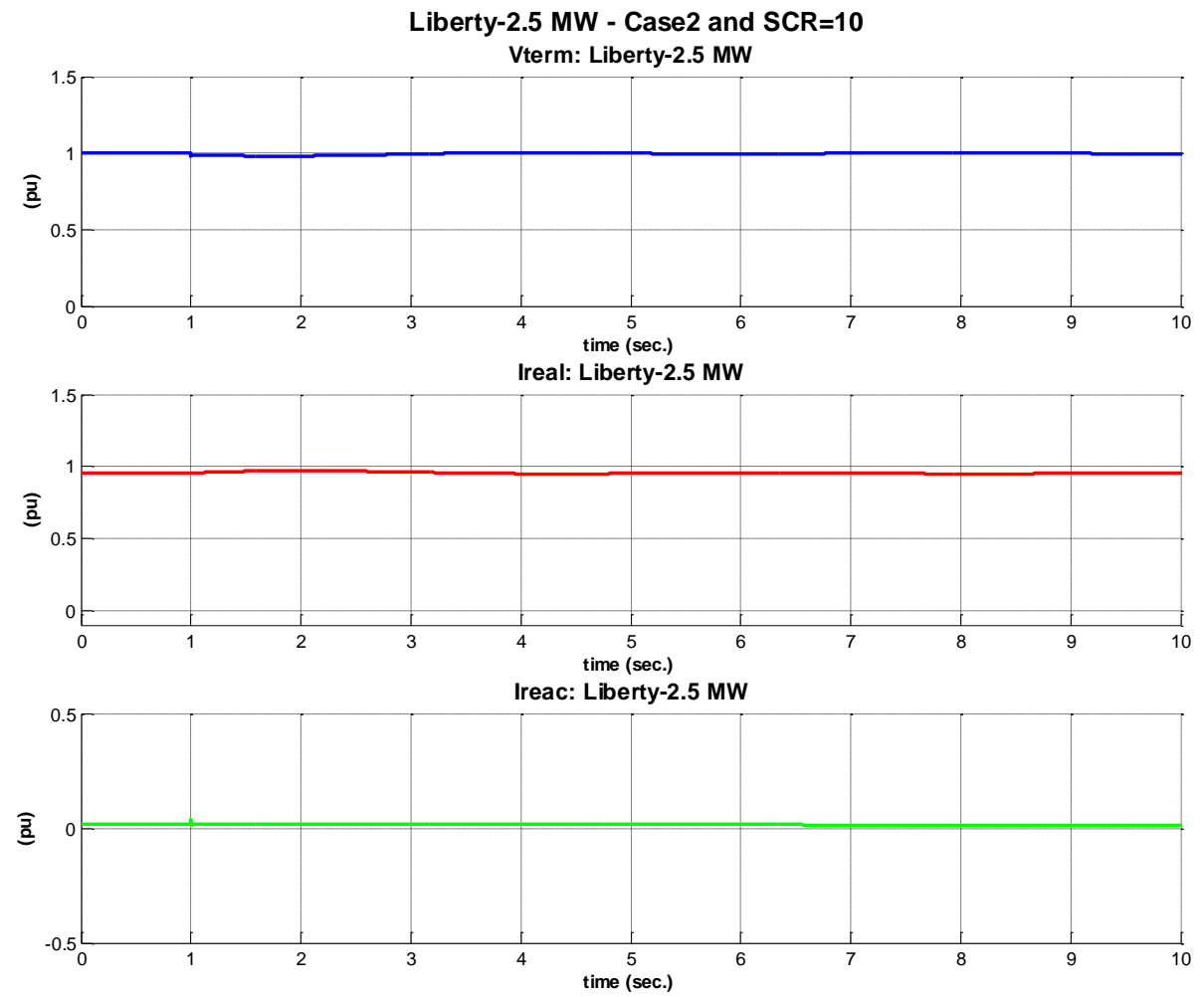

Figure 7-10: Liberty-2.5 MW - Case 2 and $S C R=10\left(V_{\text {Term }}, I_{\text {Real }}, I_{\text {Reac }}\right)$. 


\subsubsection{Model Performance: Case 3-Over-Frequency Event}

In this case a under frequency event is created by tripping the 100 MVA load at bus 11.

$\underline{\mathrm{SCR}-5}$
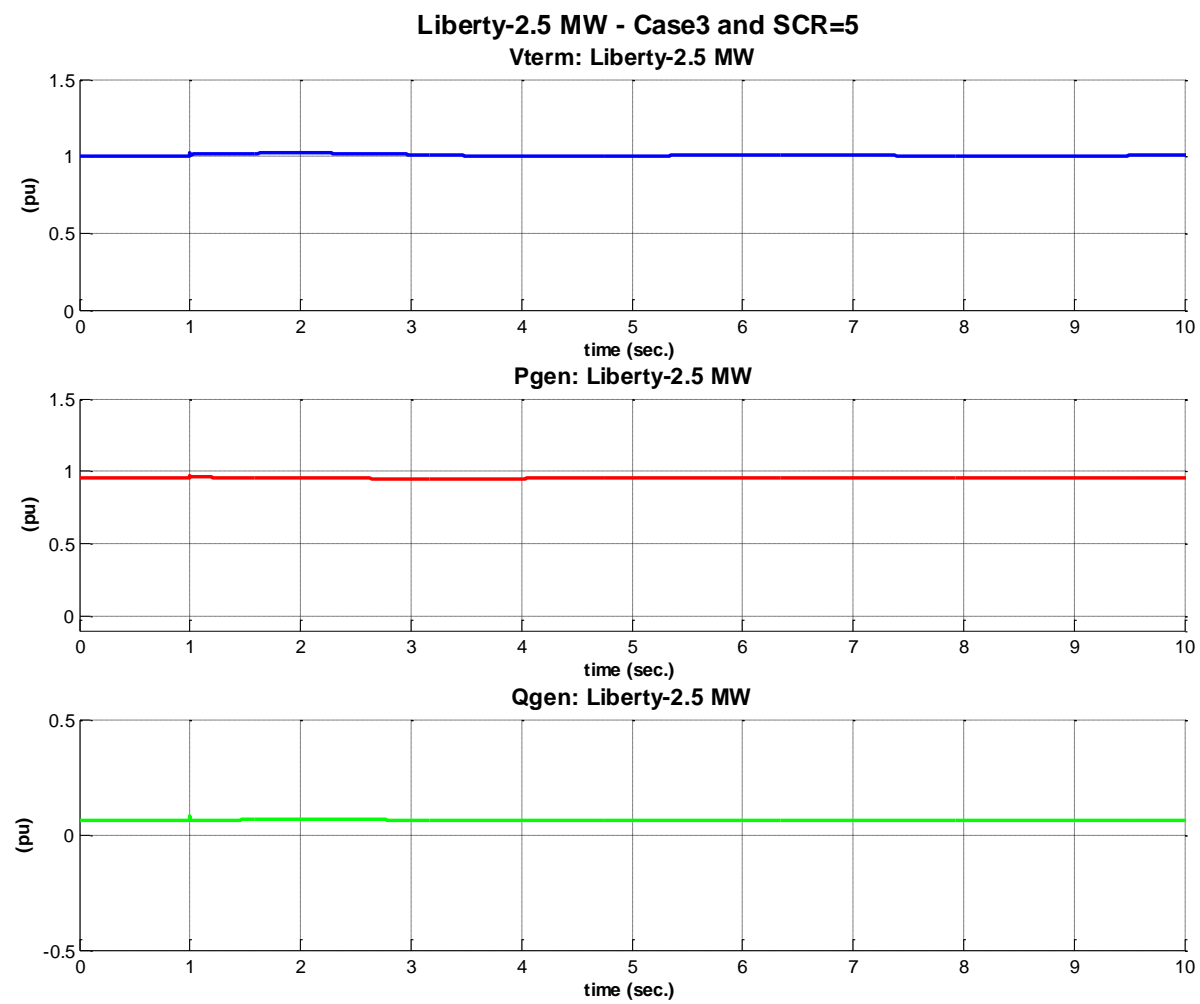

Figure 7-11: Liberty-2.5 MW - Case 3 and $\mathrm{SCR}=5\left(\mathrm{~V}_{\text {Term }}, \mathrm{P}, \mathrm{Q}\right)$. 

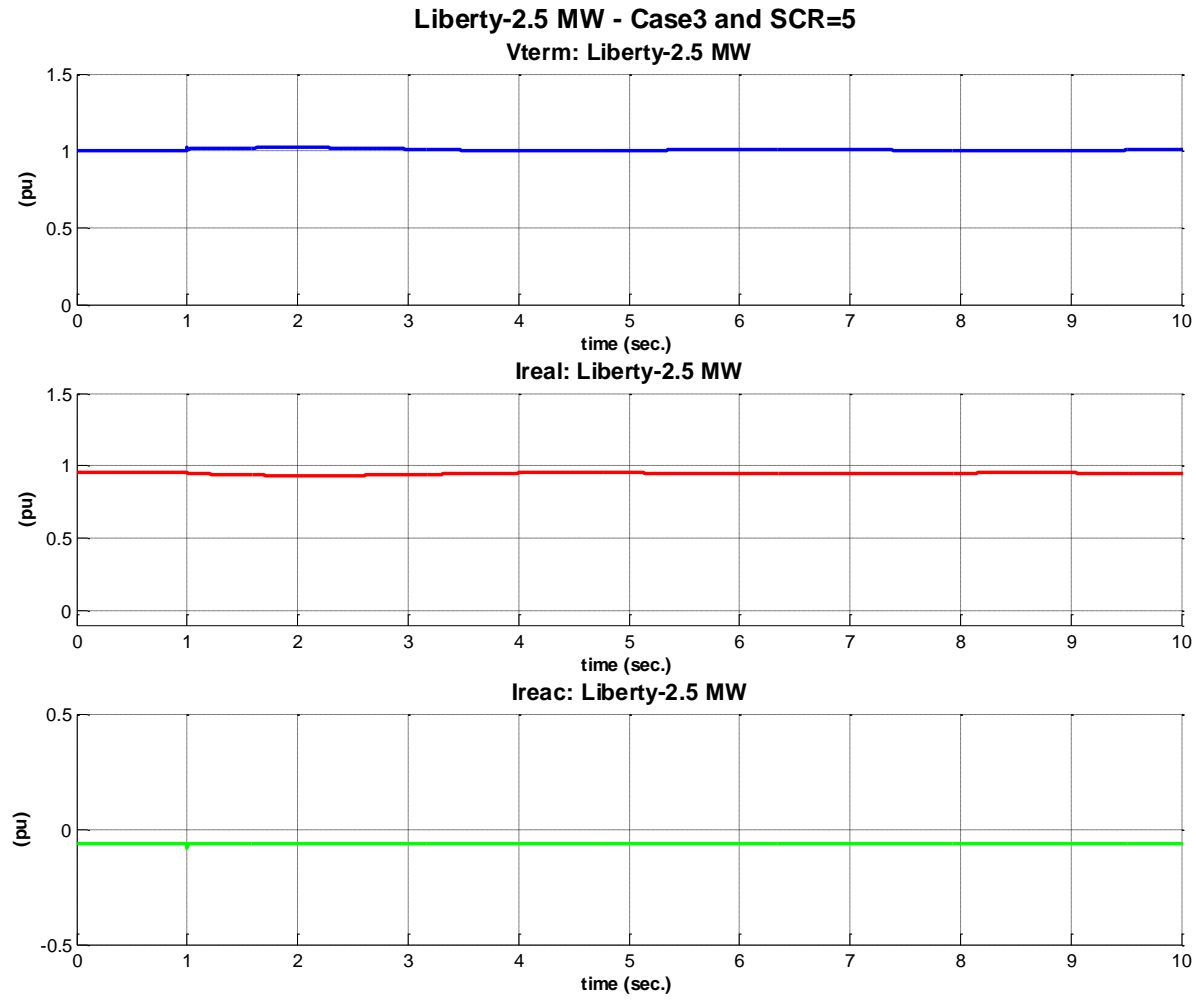

Figure 7-12: Liberty-2.5 MW - Case 3 and $S C R=5\left(V_{\text {Term }}, I_{\text {Real }}, I_{\text {Reac }}\right)$.

$\underline{\text { SCR }-10}$
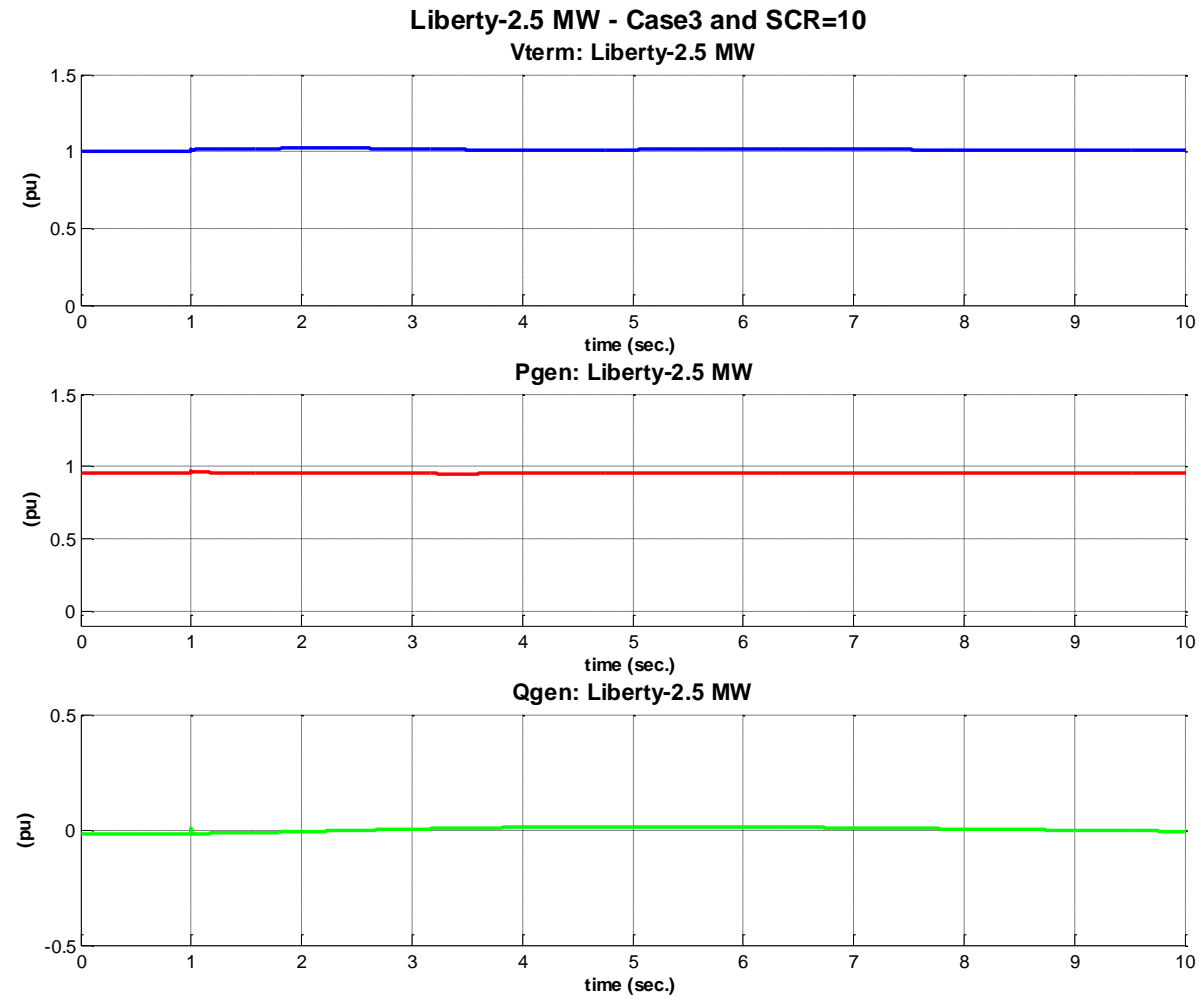

Figure 7-13: Liberty-2.5 MW - Case 3 and $\mathrm{SCR}=10\left(\mathrm{~V}_{\text {Term }}, \mathrm{P}, \mathrm{Q}\right)$. 

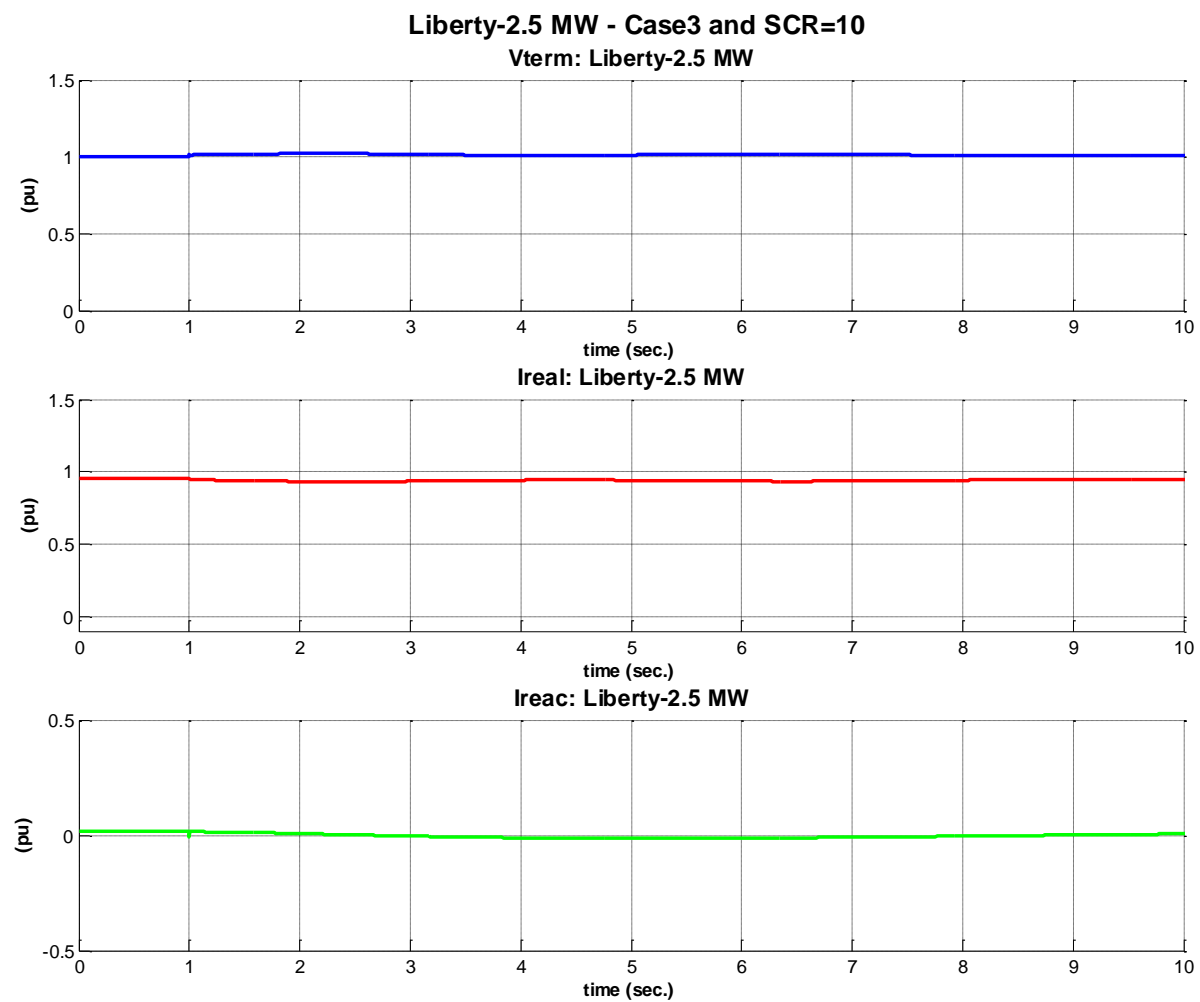

Figure 7-14: Liberty-2.5 MW - Case 3 and SCR=10 ( $\left.V_{\text {Term, }} I_{\text {Real }}, I_{\text {Reac }}\right)$.

\subsubsection{Generic Model Parameterization}

\subsubsection{PSLF Environment}

The PSLF platform generic wind turbine parameters, to mimic the behavior of Clipper's Liberty series, are shown in Table 7-1. The parameter values shown in the table resulted from a compromise between the simulated cases (i.e. SCR 5 and SCR 10) and network conditions (i.e. fault, under- and over frequency). 
Table 7-1: PSLF generic model parameter values.

\begin{tabular}{|c|c|c|c|c|c|}
\hline \multicolumn{2}{|c|}{ wt4g } & \multicolumn{2}{|c|}{ wt4t } & \multicolumn{2}{|c|}{ wt4e } \\
\hline Lvplsw & 1.0 & Tpw & 0.05 & Varflg & 1 \\
\hline Rrpwr & 10.0 & Kpp & 0.08 & Kqi & 0.5 \\
\hline Brkpt & 0.9 & Kip & 0.10 & Kvi & 40 \\
\hline Zerox & 0.4 & Tf & 0.08 & Vmax & 1.1 \\
\hline Lvpll & 1.22 & Kf & 0.00 & Vmin & 0.9 \\
\hline Volim & 1.2 & $\mathrm{dPmx}$ & 0.1 & Qmax & 0.40 \\
\hline Lvpnt1 & 0.8 & $\mathrm{dPmn}$ & -0.1 & Qmin & -0.40 \\
\hline Lvpnt0 & 0.4 & & & $\mathrm{Tr}$ & 0.02 \\
\hline & & & & Tc & 0.15 \\
\hline & & & & Kpv & 18.0 \\
\hline & & & & Kiv & 5.0 \\
\hline & & & & Pfaflg & 0 \\
\hline & & & & $f n$ & 1.0 \\
\hline & & & & TV & 0.05 \\
\hline & & & & Tpwr & 0.05 \\
\hline & & & & Iphl & 1.24 \\
\hline & & & & Iqhl & 1.25 \\
\hline & & & & Pqflag & 0 \\
\hline
\end{tabular}




\subsection{Case 1 - Fault Event}

\section{SCR - 5}
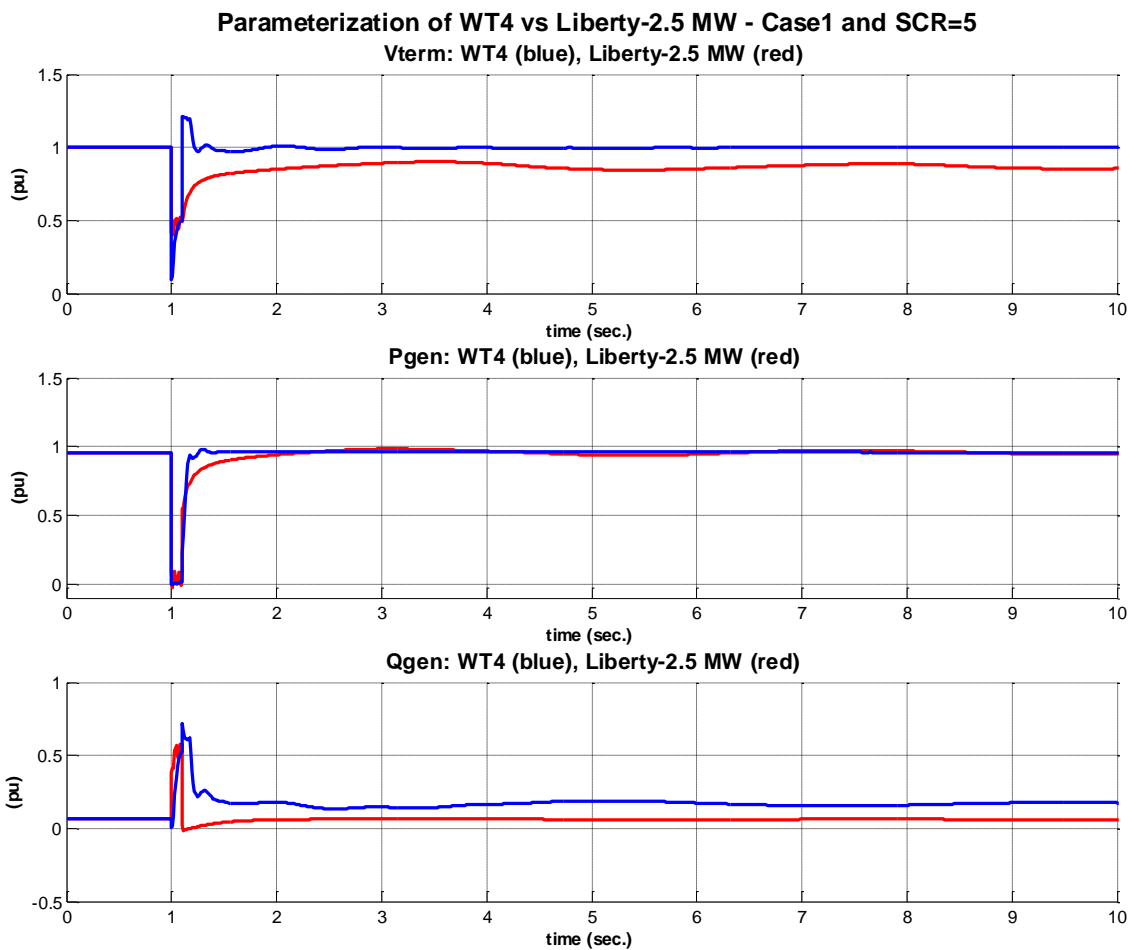

Figure 7-15: Parameterization of WT4 vs Liberty-2.5 MW - Case 1 and SCR=5 ( $\left.\mathrm{V}_{\text {Term }}, \mathrm{P}, \mathrm{Q}\right)$.

Parameterization of WT4 vs Liberty-2.5 MW - Case 1 and SCR= 5 Vterm: WT4 (blue), Liberty-2.5 MW (red)
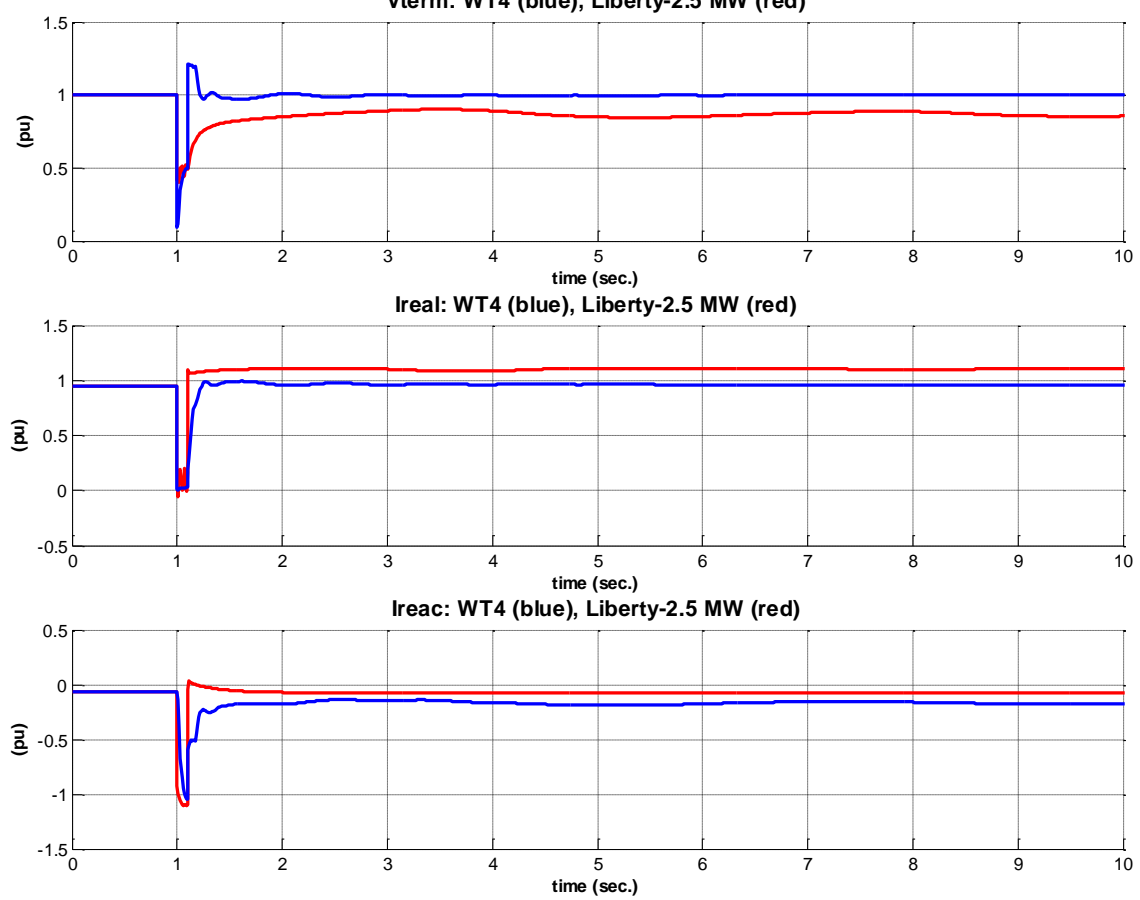

Figure 7-16: Parameterization of WT4 vs Liberty-2.5 MW - Case 1 and SCR=5 $\left(\mathrm{V}_{\text {Term, }}, \mathrm{I}_{\text {Real, }}\right.$, $I_{\text {Reac }}$. 
$\underline{\text { SCR }-10}$
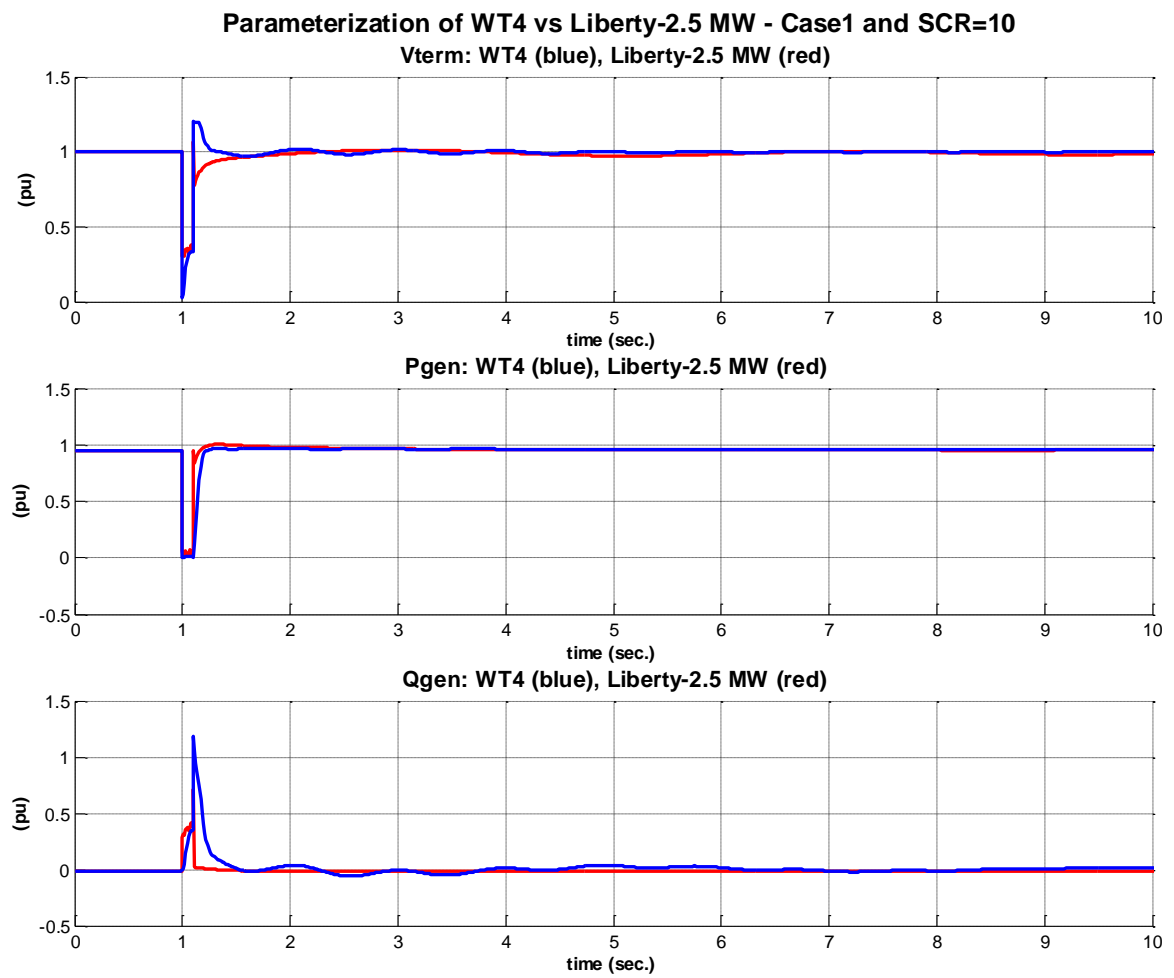

Figure 7-17: Parameterization of WT4 vs Liberty-2.5 MW - Case 1 and SCR=10 ( $\left.V_{\text {Term }}, P, Q\right)$.

Parameterization of WT4 vs Liberty-2.5 MW - Case1 and SCR=10 Vterm: WT4 (blue), Liberty-2.5 MW (red)
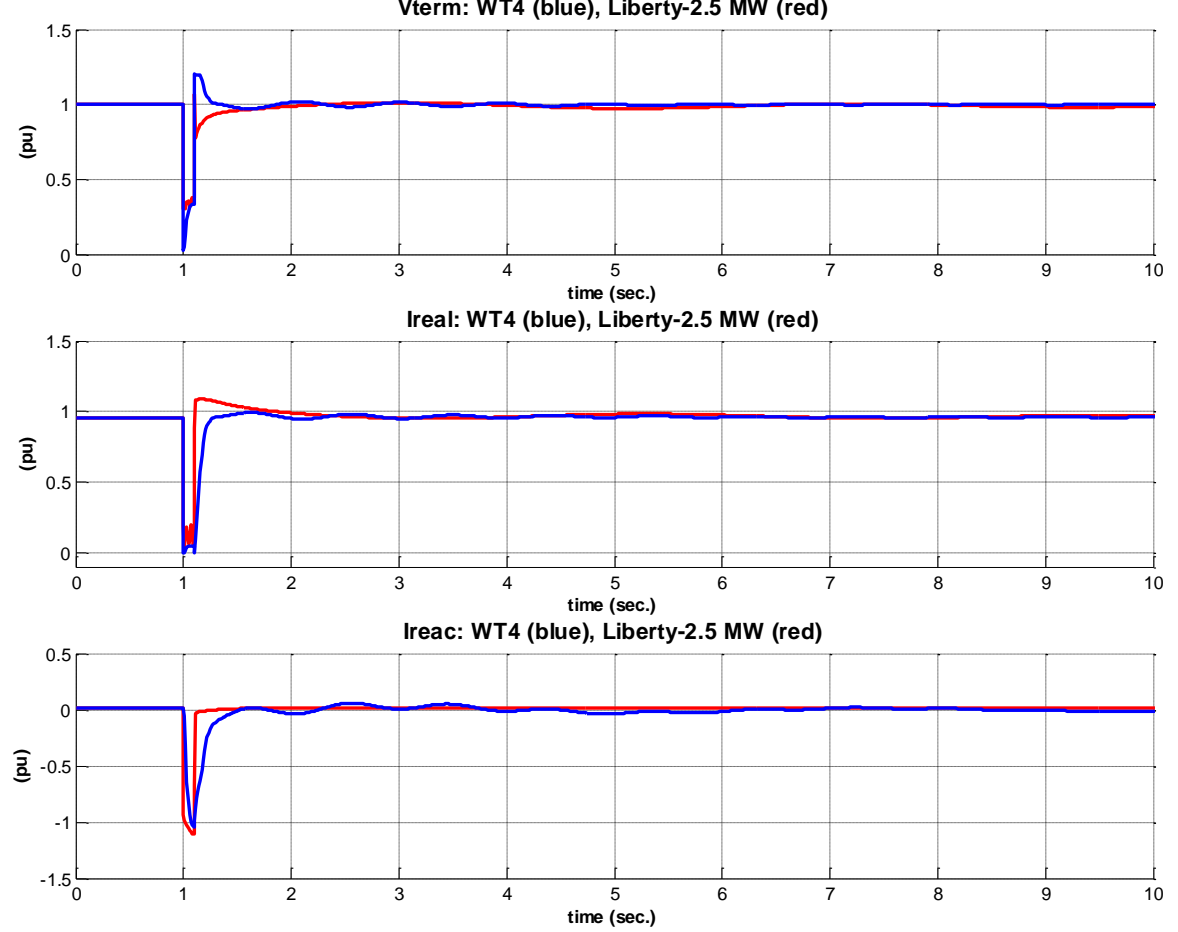

Figure 7-18: Parameterization of WT4 vs Liberty-2.5 MW - Case 1 and $S C R=10\left(V_{\text {Term }}, I_{\text {Real, }}\right.$, $I_{\text {Reac }}$. 


\subsection{Case 2 - Under-Frequency Event}

\section{$\underline{\mathrm{SCR}-5}$}
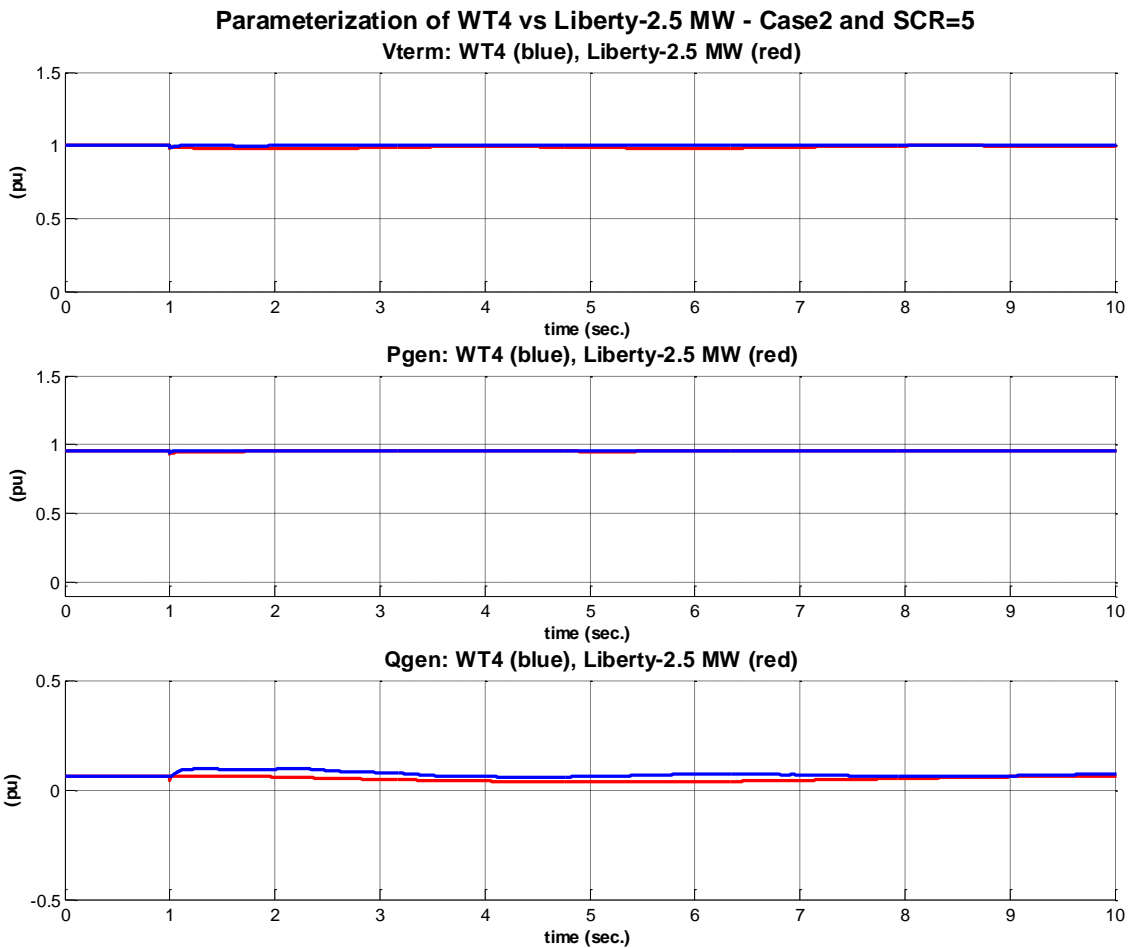

Figure 7-19: Parameterization of WT4 vs Liberty-2.5 MW - Case 2 and SCR=5 $\left(V_{\text {Term }}, P, Q\right)$.

Parameterization of WT4 vs Liberty-2.5 MW - Case2 and SCR=5 Vterm: WT4 (blue), Liberty-2.5 MW (red)
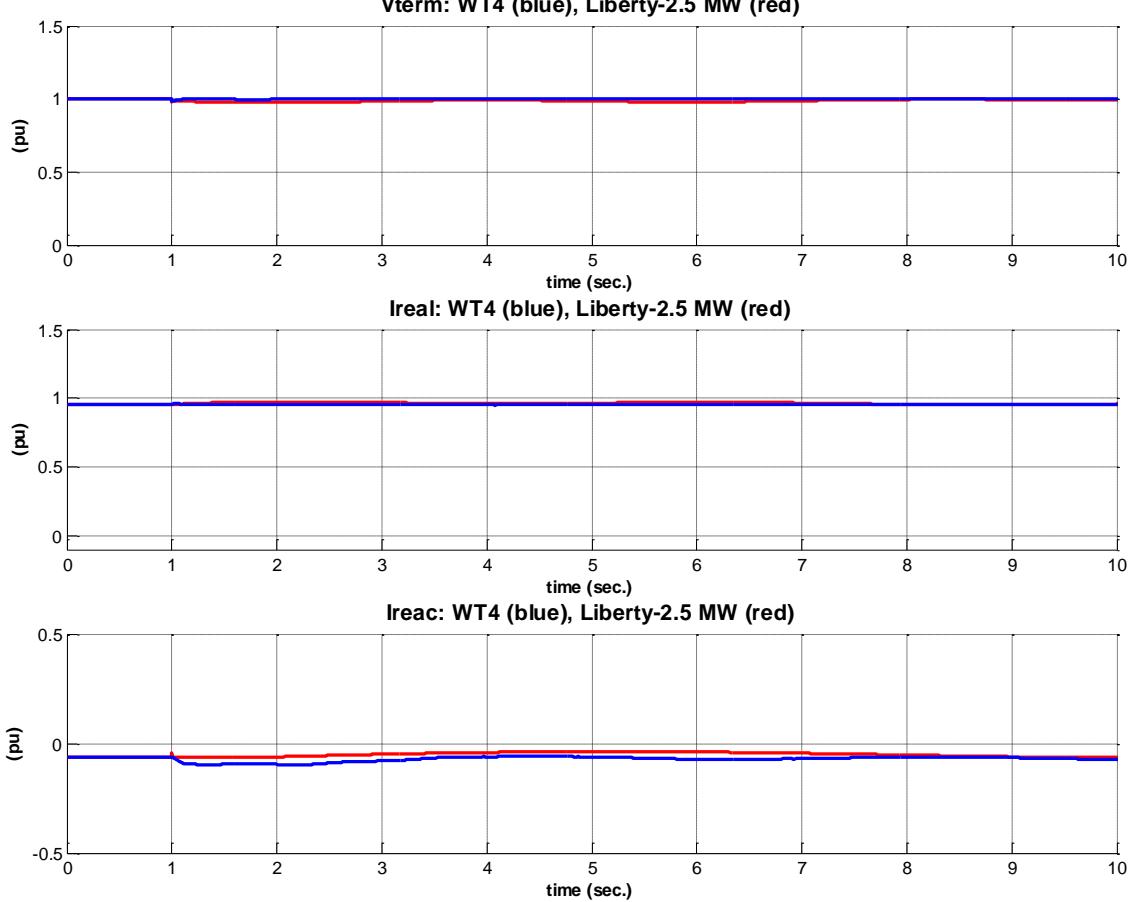

Figure 7-20: Parameterization of WT4 vs Liberty-2.5 MW - Case 2 and SCR=5 $\left(\mathrm{V}_{\text {Term }}, \mathrm{I}_{\text {Real, }}\right.$, I Reac). 
$\underline{\text { SCR }-10}$
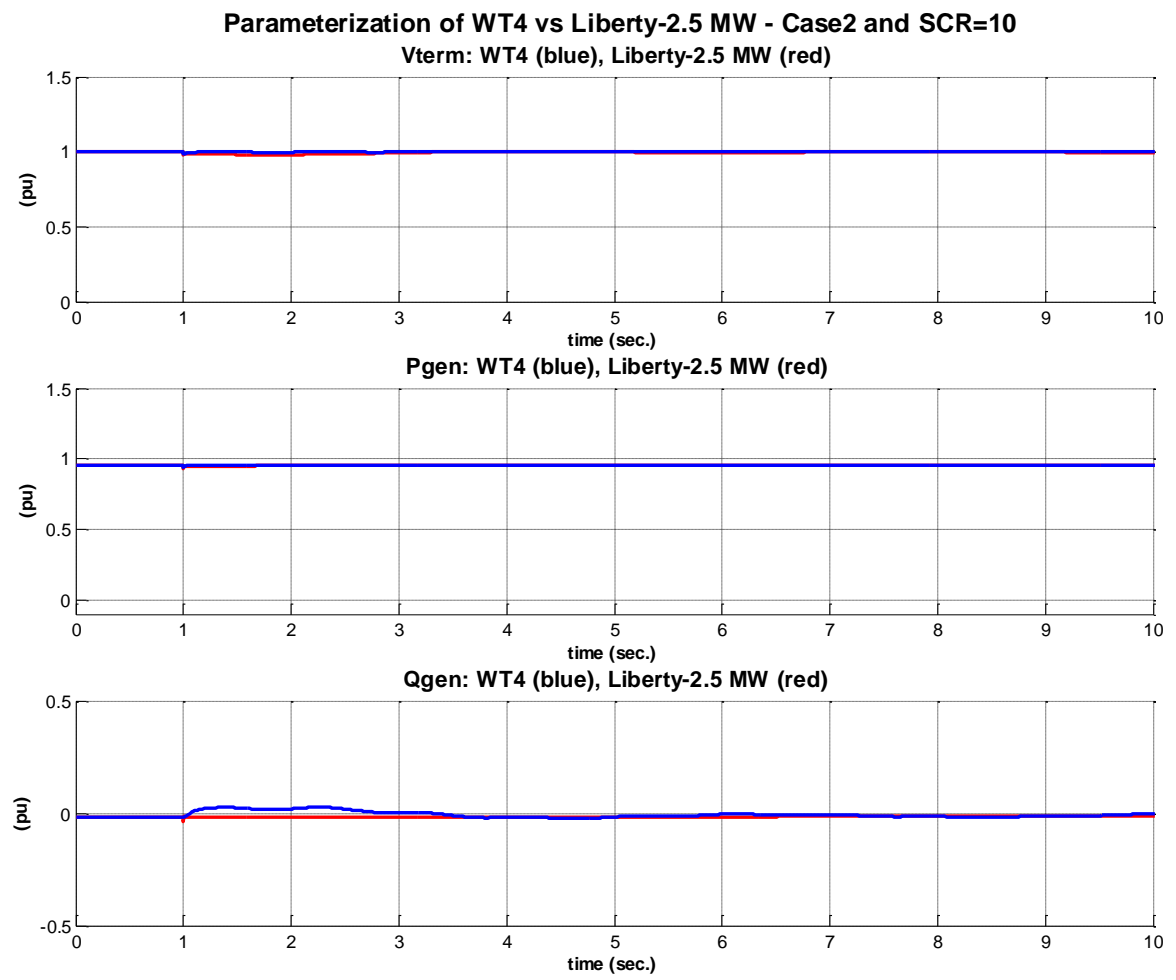

Figure 7-21: Parameterization of WT4 vs Liberty-2.5 MW - Case 2 and SCR=10 ( $\left.V_{\text {Term }}, P, Q\right)$.

Parameterization of WT4 vs Liberty-2.5 MW - Case2 and SCR=10 Vterm: WT4 (blue), Liberty-2.5 MW (red)
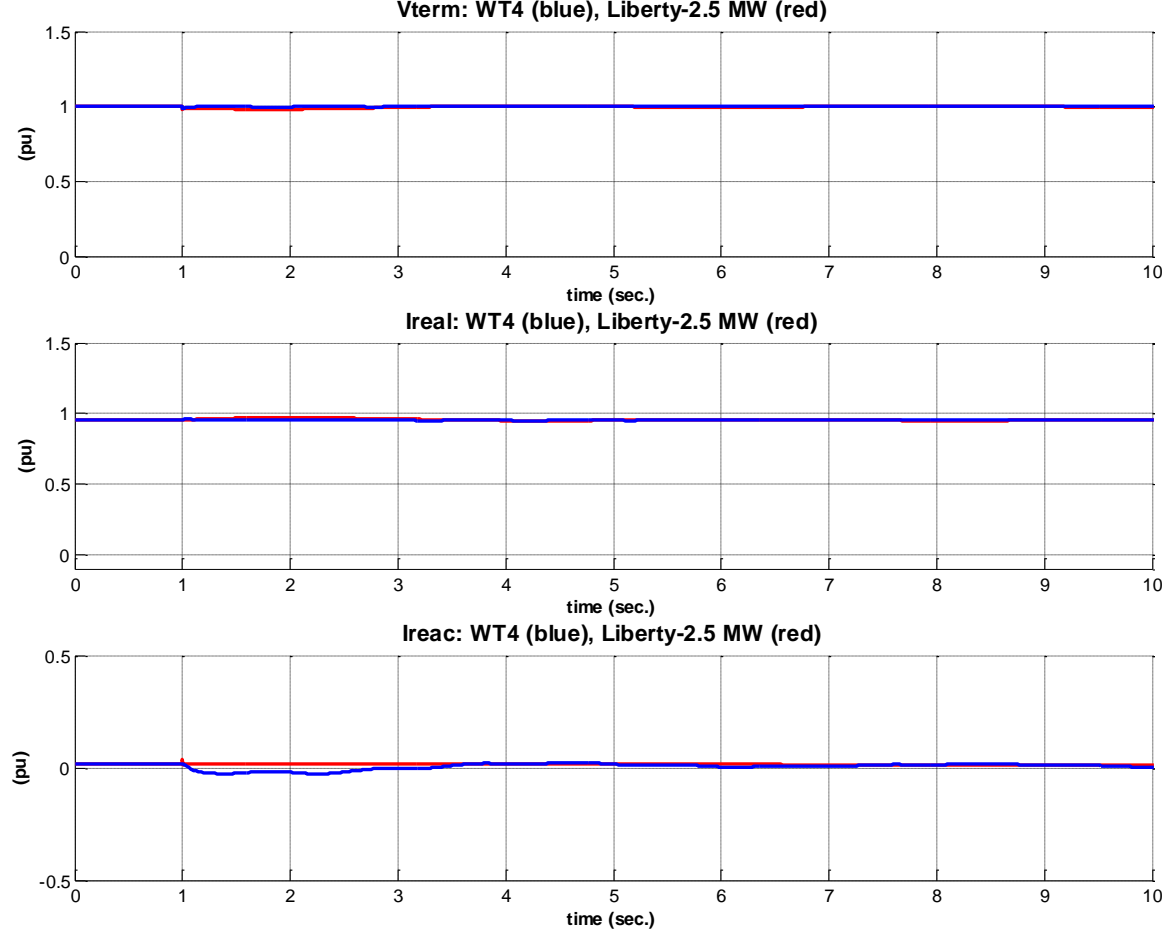

Figure 7-22: Parameterization of WT4 vs Liberty-2.5 MW - Case 2 and $S C R=10\left(V_{\text {Term }}, I_{\text {Real, }}\right.$, $I_{\text {Reac }}$. 


\subsection{Case 3 - Over-Frequency Event}

\section{SCR - 5}
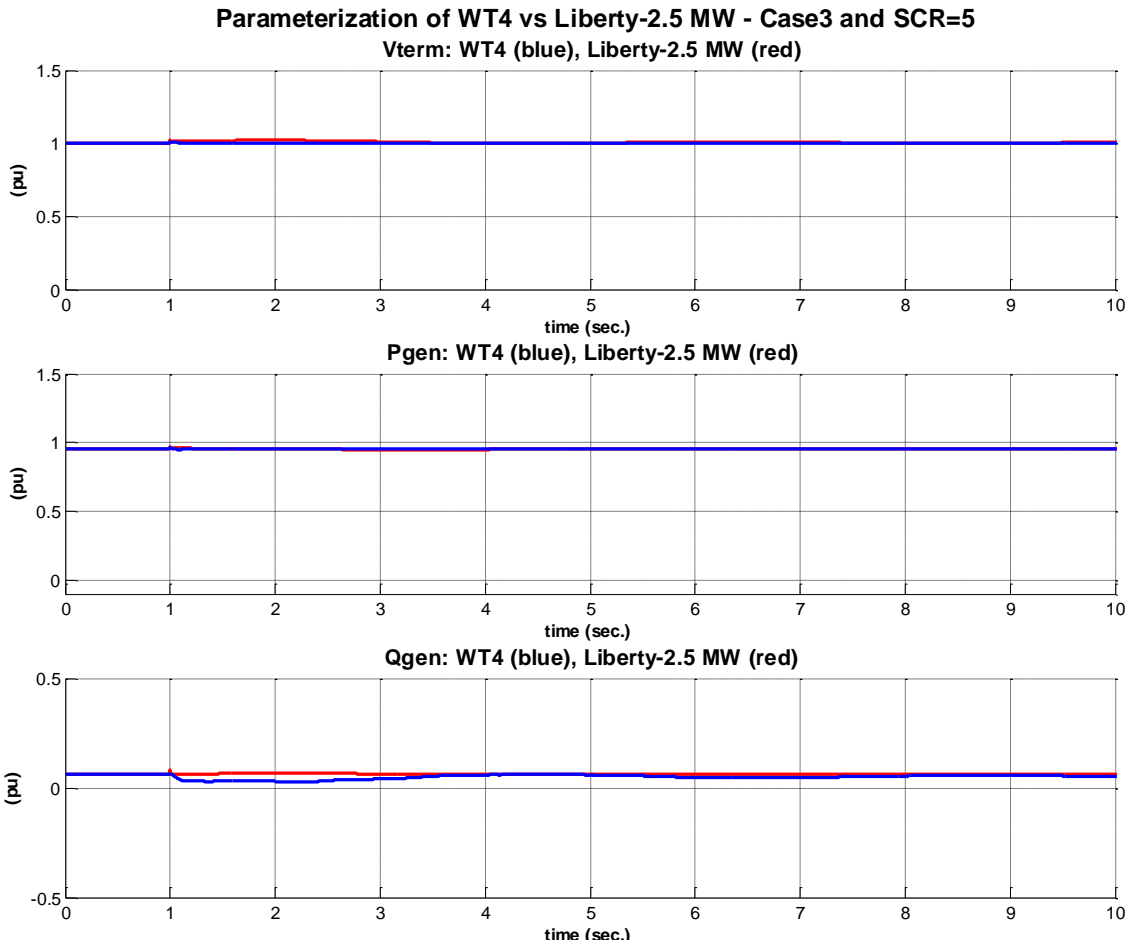

Figure 7-23: Parameterization of WT4 vs Liberty-2.5 MW - Case 3 and $S C R=5\left(V_{\text {Term }}, P, Q\right)$.

Parameterization of WT4 vs Liberty-2.5 MW - Case3 and SCR=5 Vterm: WT4 (blue), Liberty-2.5 MW (red)

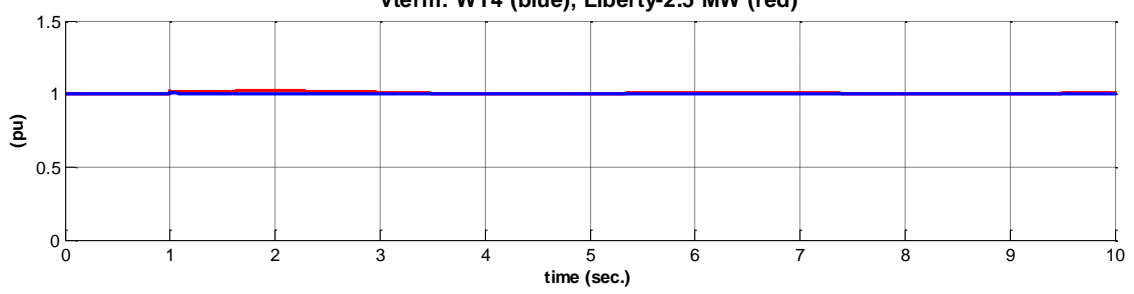

Ireal: WT4 (blue), Liberty-2.5 MW (red)
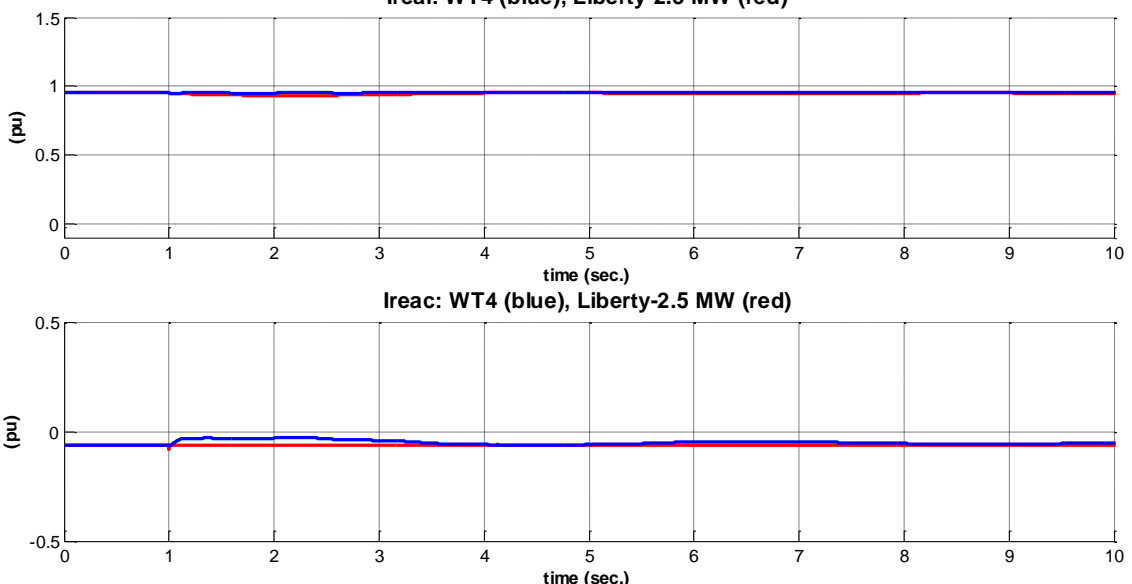

Figure 7-24: Parameterization of WT4 vs Liberty-2.5 MW - Case 3 and SCR=5 $\left(\mathrm{V}_{\text {Term }}, \mathrm{I}_{\text {Real, }}\right.$, $I_{\text {Reac }}$. 
$\underline{\text { SCR }-10}$
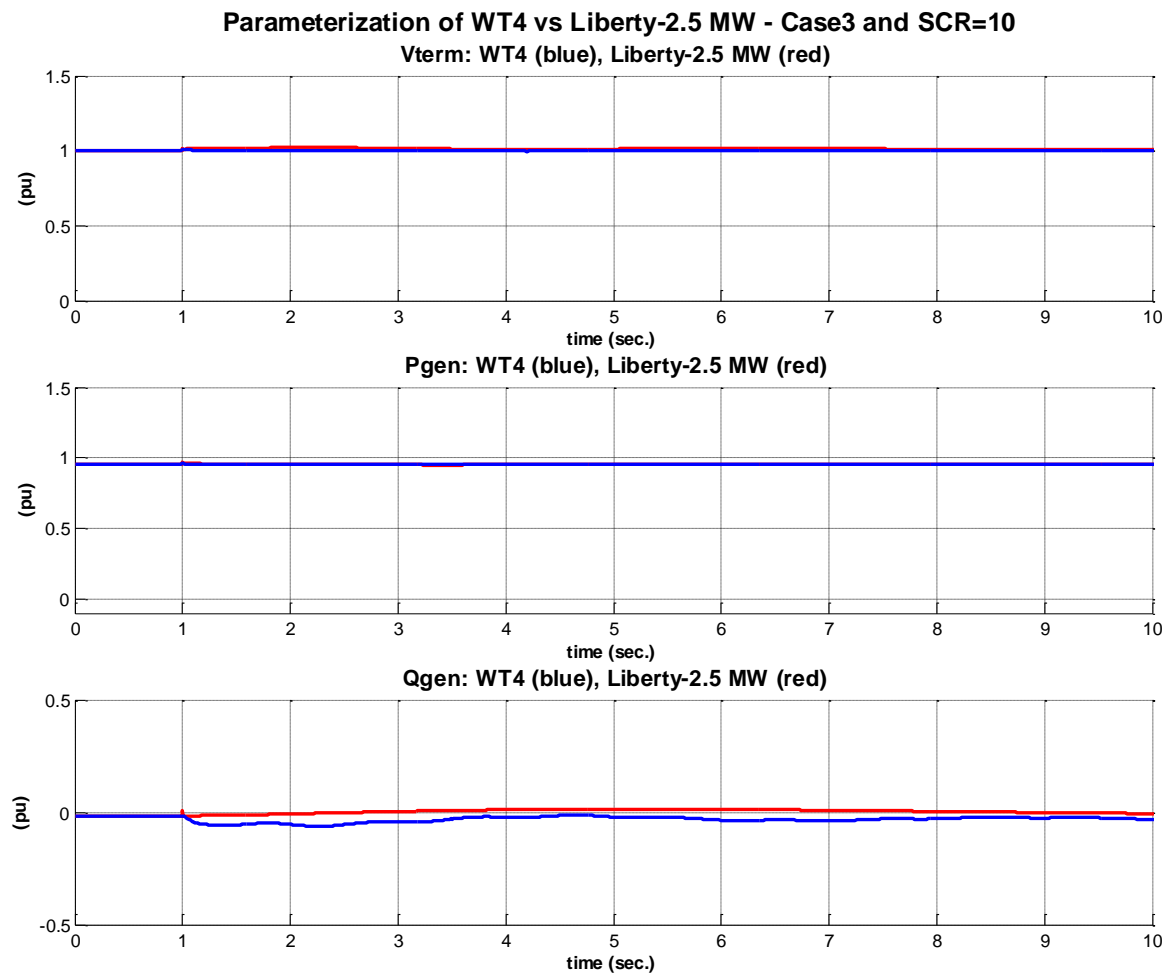

Figure 7-25: Parameterization of WT4 vs Liberty-2.5 MW - Case 3 and SCR=10 ( $\left.V_{\text {Term }}, P, Q\right)$.
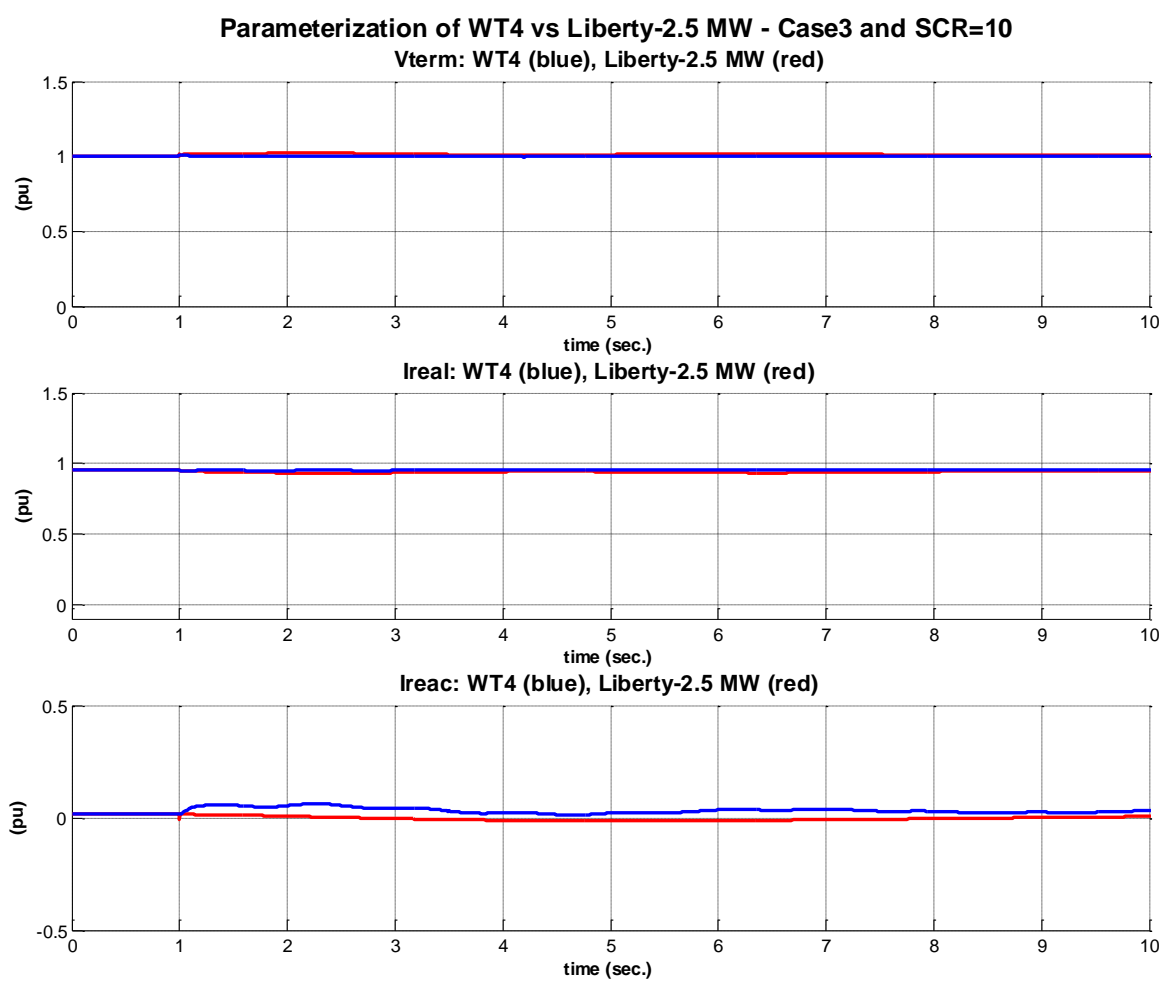

Figure 7-26: Parameterization of WT4 vs Liberty-2.5 MW - Case 3 and SCR=10 ( $V_{\text {Term }}$, I Real, I Reac). 


\subsubsection{Results Validation}

While the parameterization, for the individual cases, can be mostly done with satisfactory results, a universal set of parameters, to cover all cases, is hard to find (if at all).

For cases 1 and $2(S C R=10)$ and case $3(S C R=5)$ the generic model matches the response of the VSM in the frequency range of interest. Thus, for these cases the PSLF model is an appropriate representation of the Liberty - 2.5 MW WTG's behavior for fundamental frequency analysis. However, for all remaining cases and system conditions (i.e. $S C R=5$ and $S C R=10$ ) the generic model does not match the behavior of the VSM.

\subsubsection{Vendor Specific Model Validation}

No data available 


\subsection{Gamesa - G8X Series}

\subsubsection{Wind Turbine Characterization}

Information being reviewed by vendor. Approval for public release is pending.

\subsubsection{Vendor Specific Model Characterization}

\subsubsection{Model Performance: Case 1 - Fault Event}

Information being reviewed by vendor. Approval for public release is pending.

\subsubsection{Model Performance: Case 2 - Under-Frequency Event}

Information being reviewed by vendor. Approval for public release is pending.

\subsubsection{Model Performance: Case 3-Over-Frequency Event}

Information being reviewed by vendor. Approval for public release is pending.

\subsubsection{Generic Model Parameterization}

\subsubsection{PSLF Environment}

\subsection{Case 1 - Fault Event}

Information being reviewed by vendor. Approval for public release is pending.

7.3.3.1.1.2 Case 2-Under-Frequency Event

Information being reviewed by vendor. Approval for public release is pending.

\subsection{Case 3 - Over-Frequency Event}

Information being reviewed by vendor. Approval for public release is pending.

\subsubsection{Results Validation}

Information being reviewed by vendor. Approval for public release is pending.

\subsubsection{Vendor Specific Model Validation}

No data available 


\subsection{General Electric - 1.5 MW Series}

\subsubsection{Wind Turbine Characterization}

GE's 1.5 MW series is represented by three-blade, upwind, horizontal axis wind turbines with a rated capacity of 1.5-megawatts. Three different models represent the 1.5 MW series $-1.5 \mathrm{se}, 1.5 \mathrm{sle}$, and $1.5 x \mathrm{x}$, with following features:

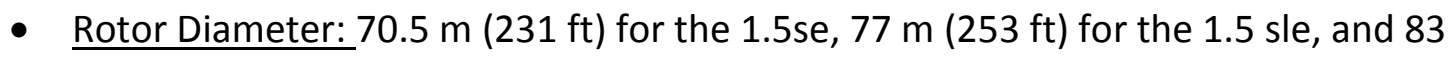
$\mathrm{m}(271 \mathrm{ft})$ for the $1.5 x$ le model.

- $\quad$ Hub Heights: $65 \mathrm{~m}(212 \mathrm{ft})$ to $80 \mathrm{~m}(262 \mathrm{ft})$.

- $\quad$ Cut-in wind speed: 3.5 to $4 \mathrm{~m} / \mathrm{s}$ (8 to $9 \mathrm{mph}$ ).

- Cut-out wind speed: 20 to $25 \mathrm{~m} / \mathrm{s}$ ( 45 to $55 \mathrm{mph}$ ).

- Terminal Voltage: $690 \mathrm{~V}(50 \mathrm{~Hz} / 60 \mathrm{~Hz})$.

- $\quad$ Cut-back-in Wind Speed: 17 to $22 \mathrm{~m} / \mathrm{s}$ (38 to $49 \mathrm{mph}$ )

- $\quad$ Power Control: Active blade pitch control.

- Gearbox: Three-step planetary/helical gear system with a ratio of gear 1:72 (60 $H Z)$.

- Generator: Doubly fed asynchronous generator.

- Power Converter: Pulse-Width-Modulated (PWM) converter in 4-quadrant operation.

- Braking System: Electric drive pitch control with battery backup; Hydraulic parking brake.

The rotor on a GE $1.5 \mathrm{MW}$ turbine is designed to operate in an upwind configuration at 10 to 20 revolutions per minute (rpm). Rotor speed is regulated by a combination of blade pitch angle adjustment and generator/converter torque control. The 1.5 MW series provides the option of a selectable power factor between 0.95 (overexcited) and 0.90 (underexcited) and also a VAR boost, which will override watts production to deliver more VARs during emergency under-voltage conditions. 


\subsubsection{Vendor Specific Model Characterization}

\subsubsection{Model Performance: Case 1 - Fault Event}

In this case a remote fault is applied to bus 12 for a duration of 6 cycles $(0.1 \mathrm{sec})$.

$\underline{\mathrm{SCR}-5}$
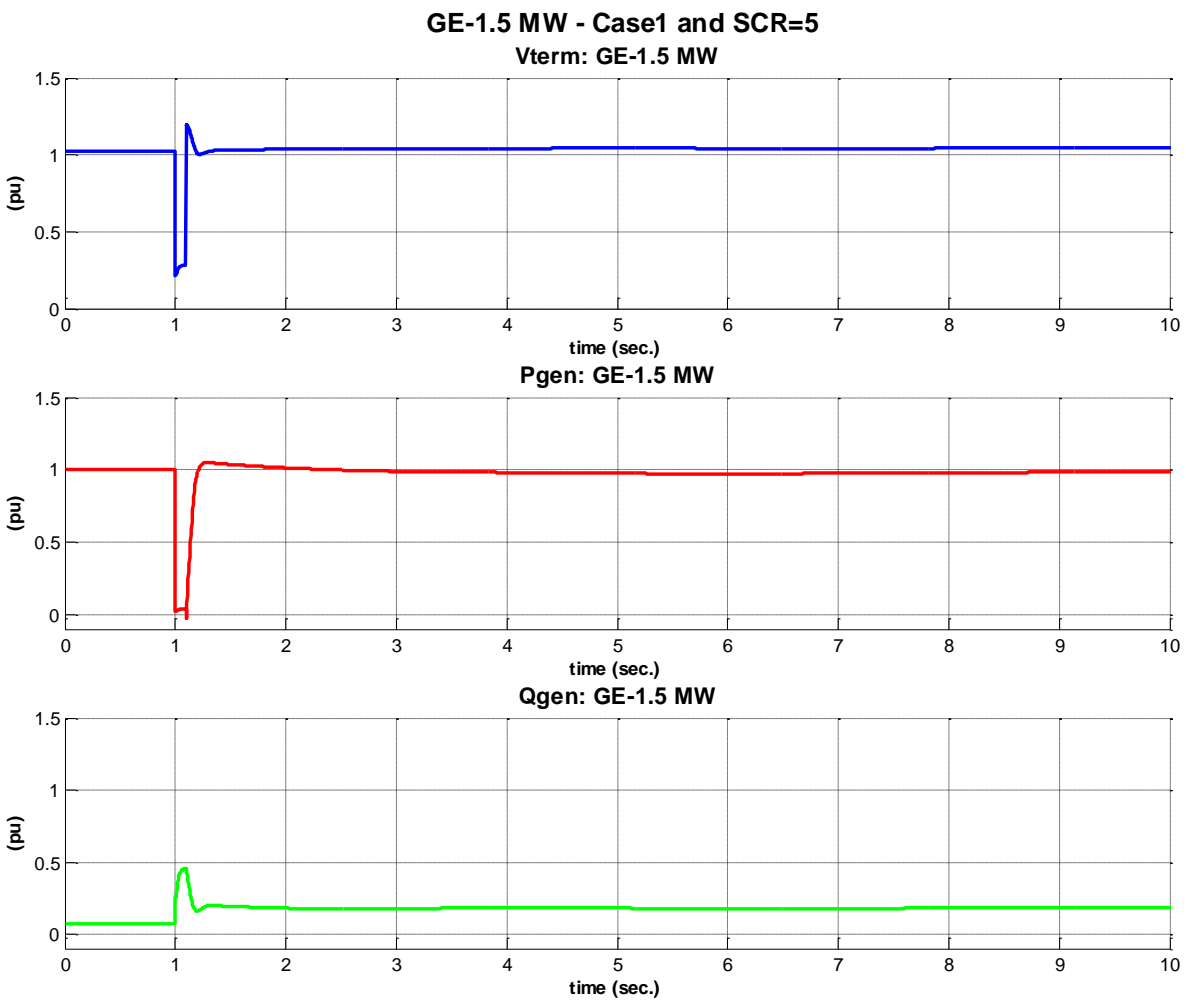

Figure 7-27: GE-1.5 MW - Case 1 and $\mathrm{SCR}=5\left(\mathrm{~V}_{\text {Term }}, \mathrm{P}, \mathrm{Q}\right)$. 

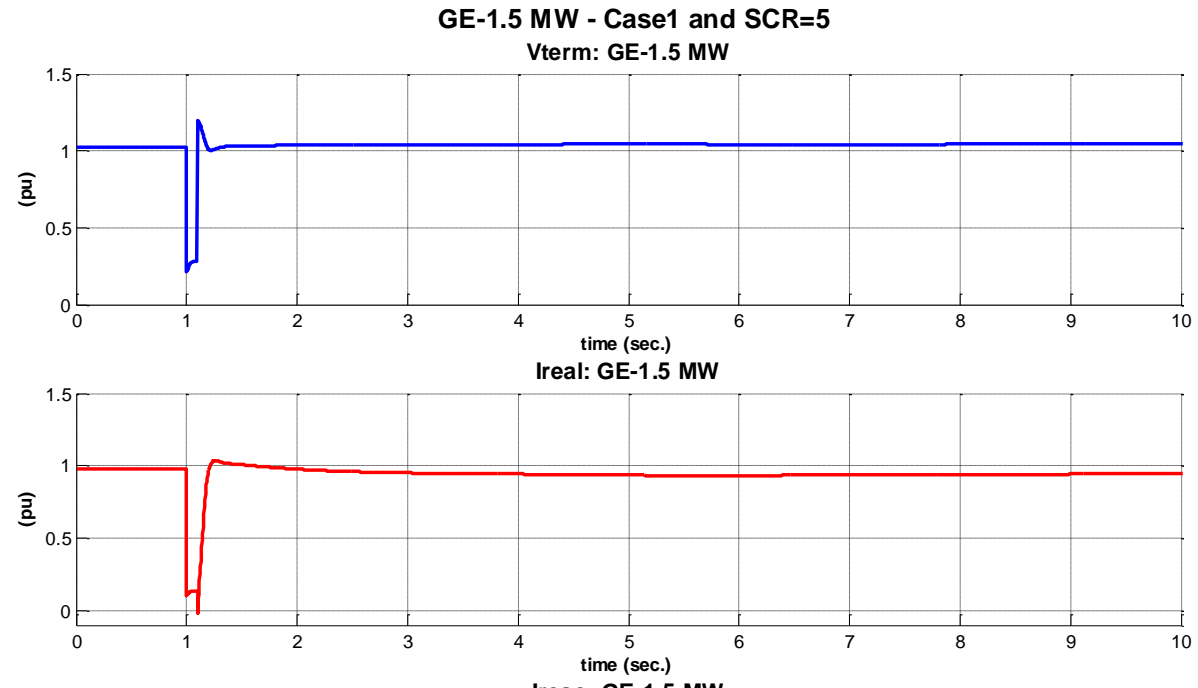

Ireac: GE-1.5 MW

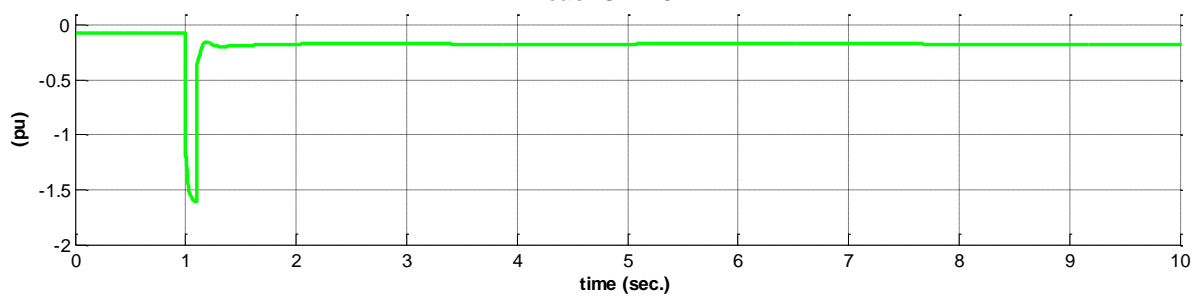

Figure 7-28: GE-1.5 MW - Case 1 and $S C R=5\left(V_{\text {Term, }} I_{\text {Real }}, I_{\text {Reac }}\right)$.

SCR - 10
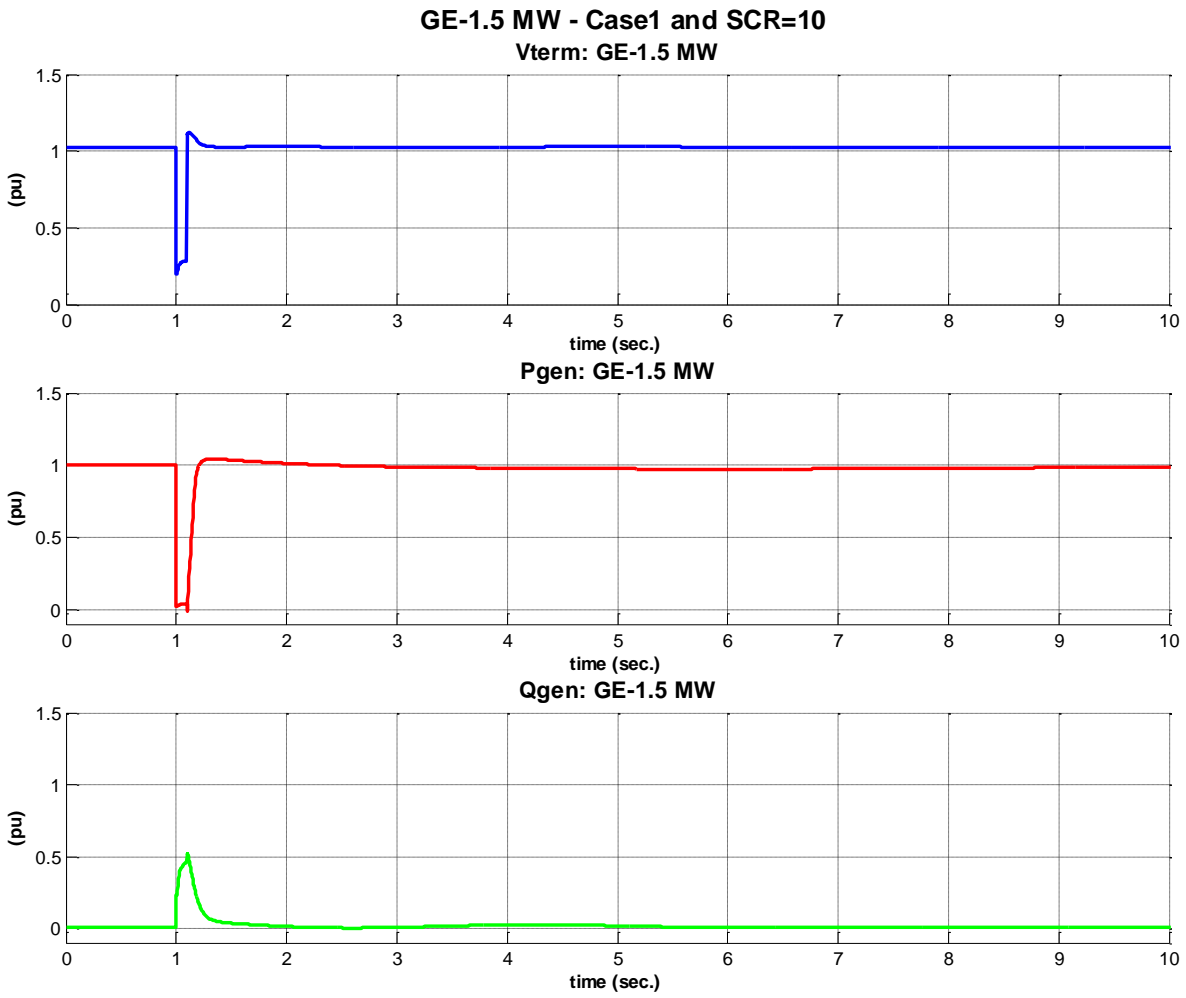

Figure 7-29: GE-1.5 MW - Case 1 and $\mathrm{SCR}=10\left(\mathrm{~V}_{\text {Term }}, \mathrm{P}, \mathrm{Q}\right)$. 

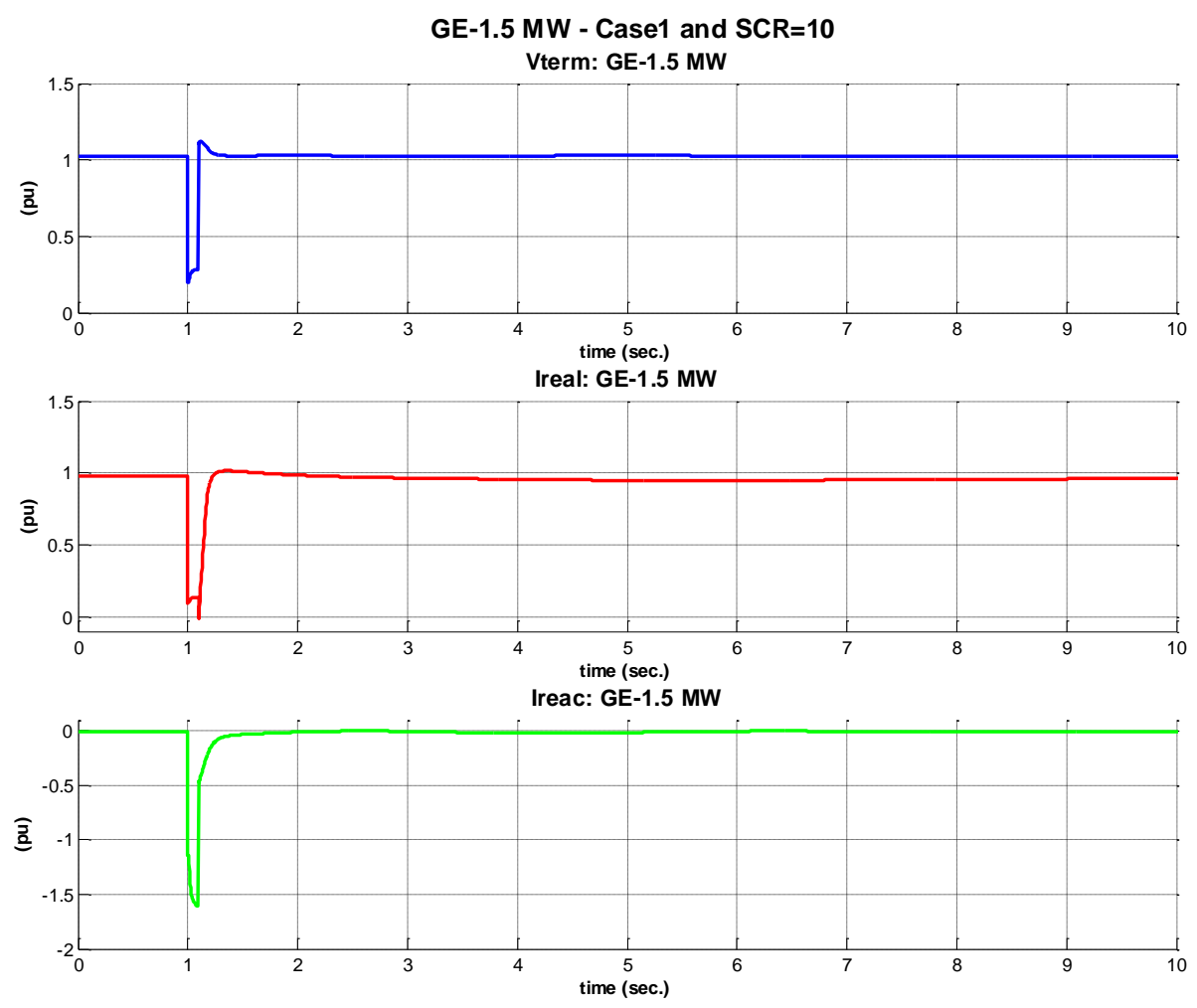

Figure 7-30: GE-1.5 MW - Case 1 and $S C R=10$ ( $\left.\mathrm{V}_{\text {Term }}, I_{\text {Real }}, I_{\text {Reac }}\right)$.

For both SCR settings the reactive power output of the WTG is at or near its maximum during the fault in an effort to regulate voltage. After the fault is removed and the rated voltage level is restored, the reactive power returns to its steady state condition.

\subsubsection{Model Performance: Case 2 - Under-Frequency Event}

In this case a under frequency event is created by tripping the 100 MVA generation unit at bus 20 . 
$\underline{\mathrm{SCR}-5}$

GE-1.5 MW - Case2 and SCR=5

Vterm: GE-1.5 MW
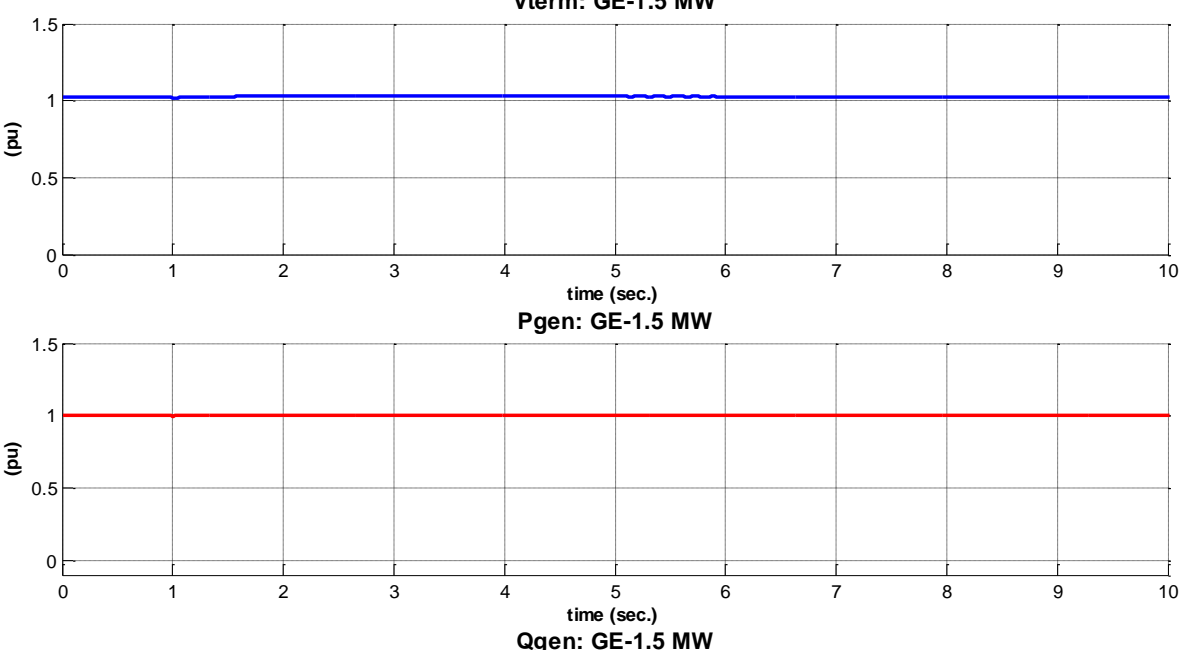

Qgen: GE-1.5 M

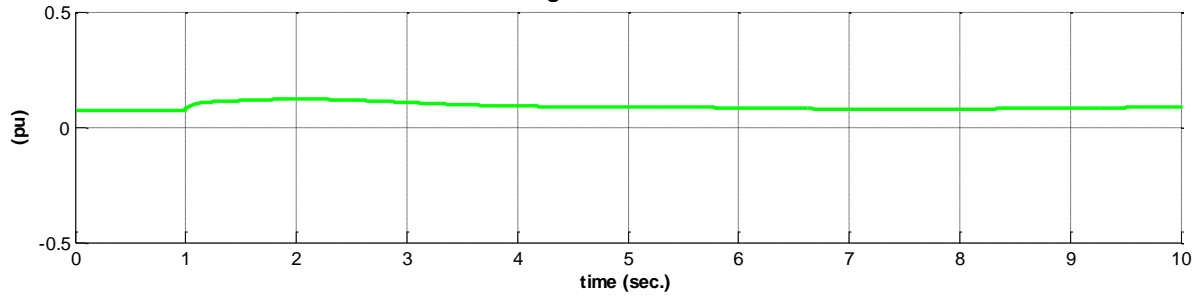

Figure 7-31: GE-1.5 MW - Case 2 and $\mathrm{SCR}=5\left(\mathrm{~V}_{\text {Term }}, \mathrm{P}, \mathrm{Q}\right)$.
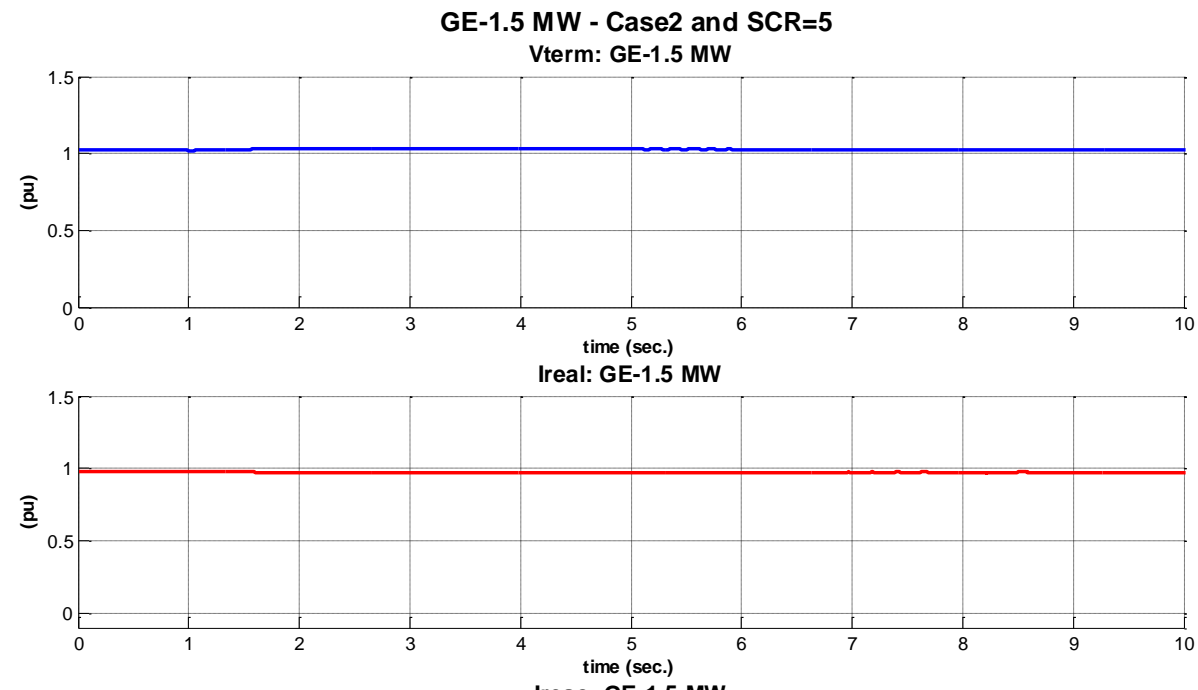

Ireac: GE-1.5 MW

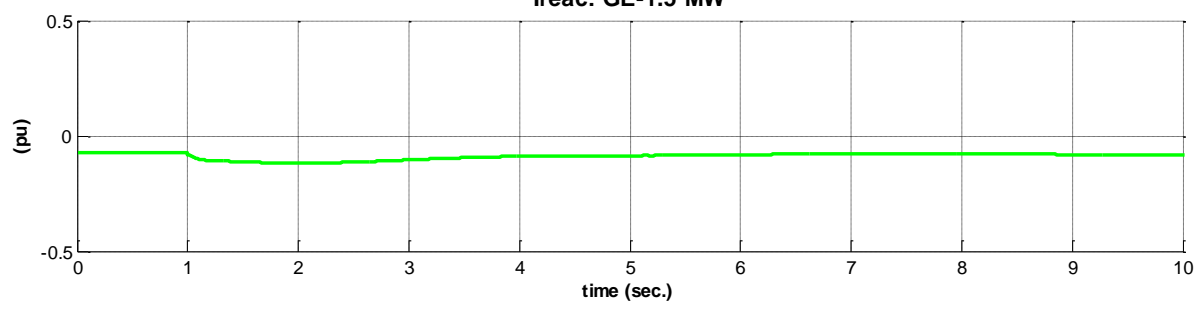

Figure 7-32: GE-1.5 MW - Case 2 and SCR=5 ( $\left.V_{\text {Term }}, I_{\text {Real }}, I_{\text {Reac }}\right)$. 
$\underline{\text { SCR }-10}$
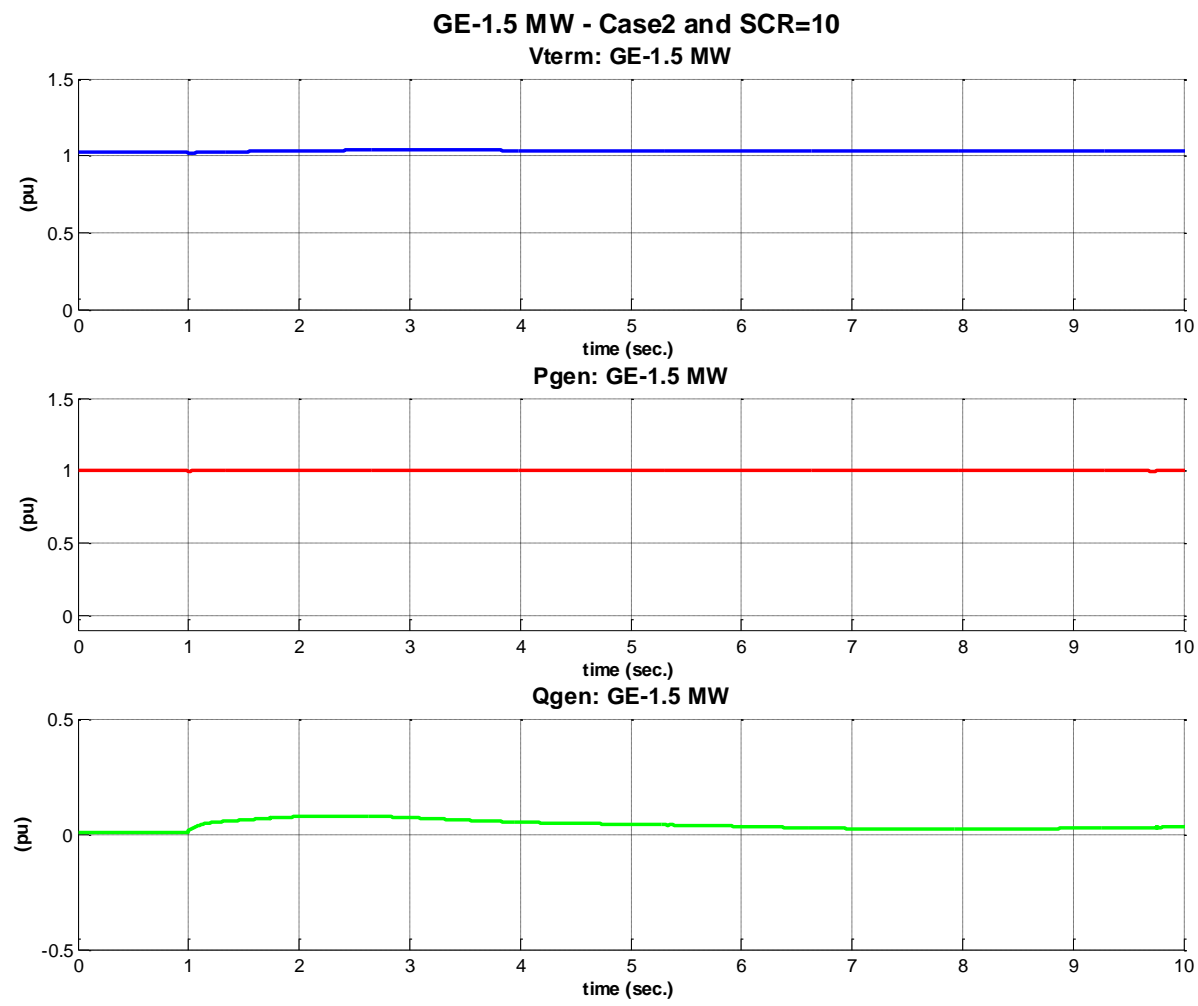

Figure 7-33: GE-1.5 MW - Case 2 and $S C R=10\left(V_{\text {Term }}, P, Q\right)$.
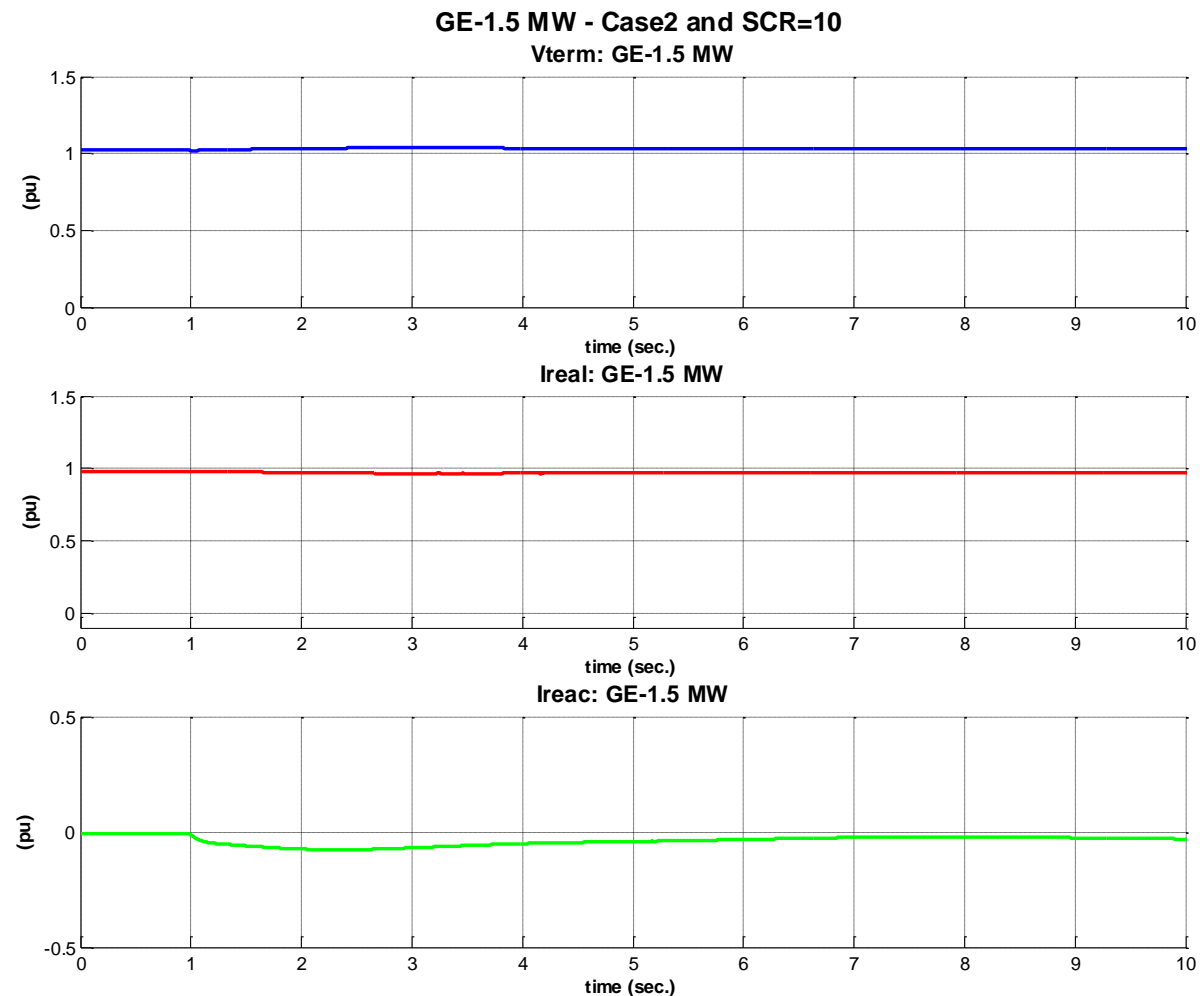

Figure 7-34: GE-1.5 MW - Case 2 and $S C R=10\left(V_{\text {Term }}, I_{\text {Real, }}, I_{\text {Reac }}\right)$. 


\subsubsection{Model Performance: Case 3 - Over-Frequency Event}

In this case a under frequency event is created by tripping the 100 MVA load at bus 11.

$\underline{\mathrm{SCR}-5}$
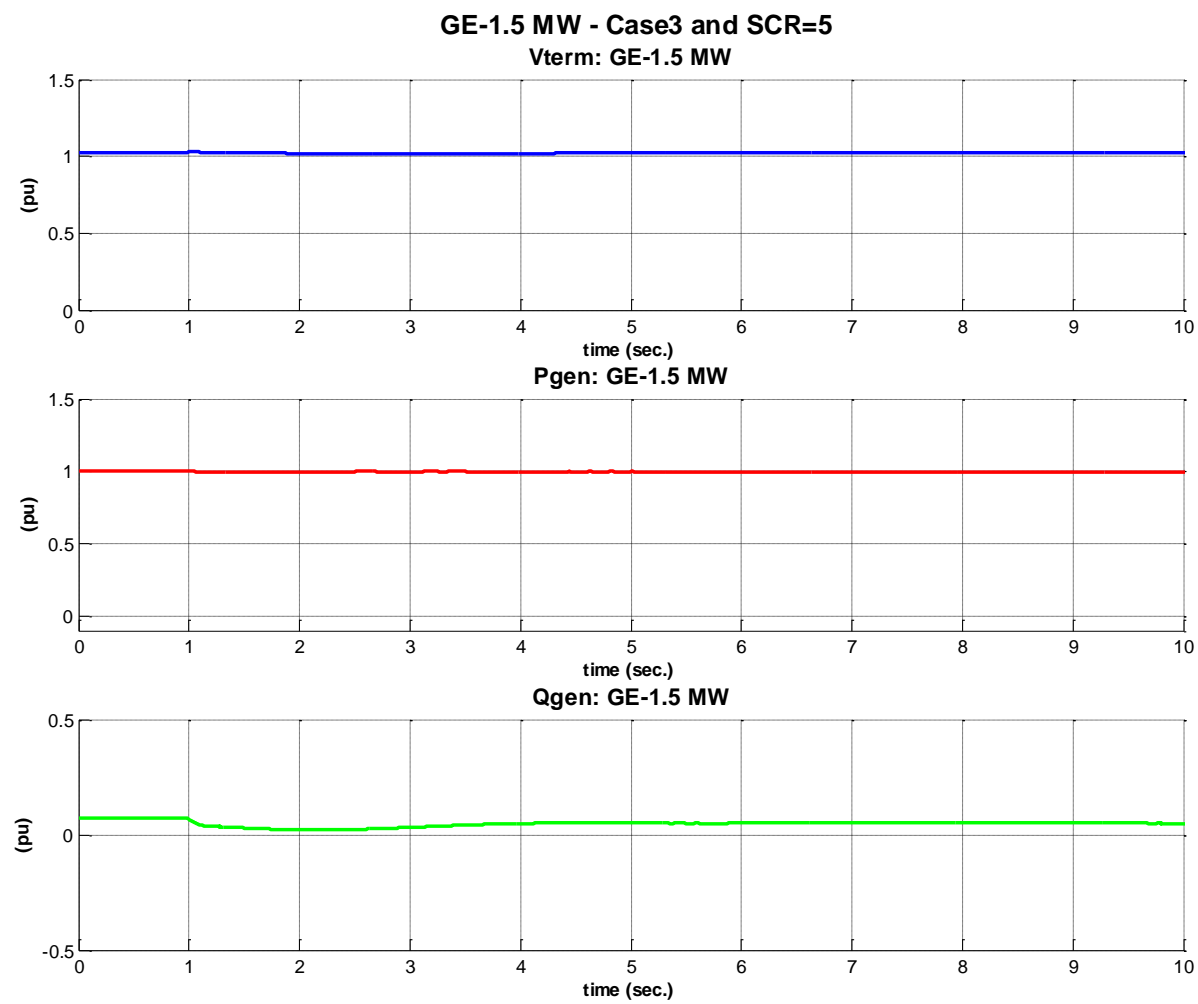

Figure 7-35: GE-1.5 MW - Case 3 and $\mathrm{SCR}=5\left(\mathrm{~V}_{\text {Term }}, \mathrm{P}, \mathrm{Q}\right)$. 

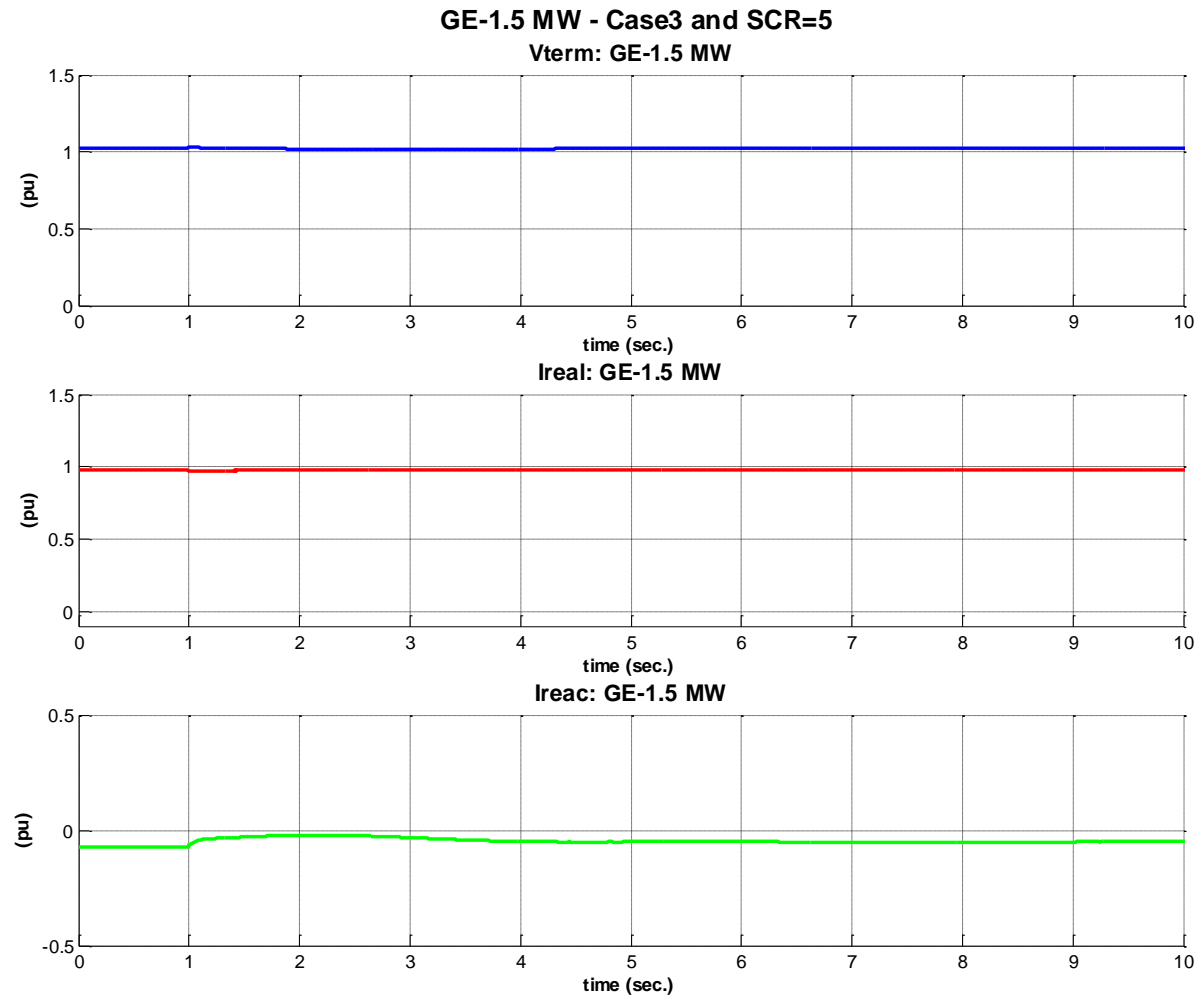

Figure 7-36: GE-1.5 MW - Case 3 and $S C R=5\left(V_{\text {Term, }} I_{\text {Real }}, I_{\text {Reac }}\right)$.

\section{SCR -10}
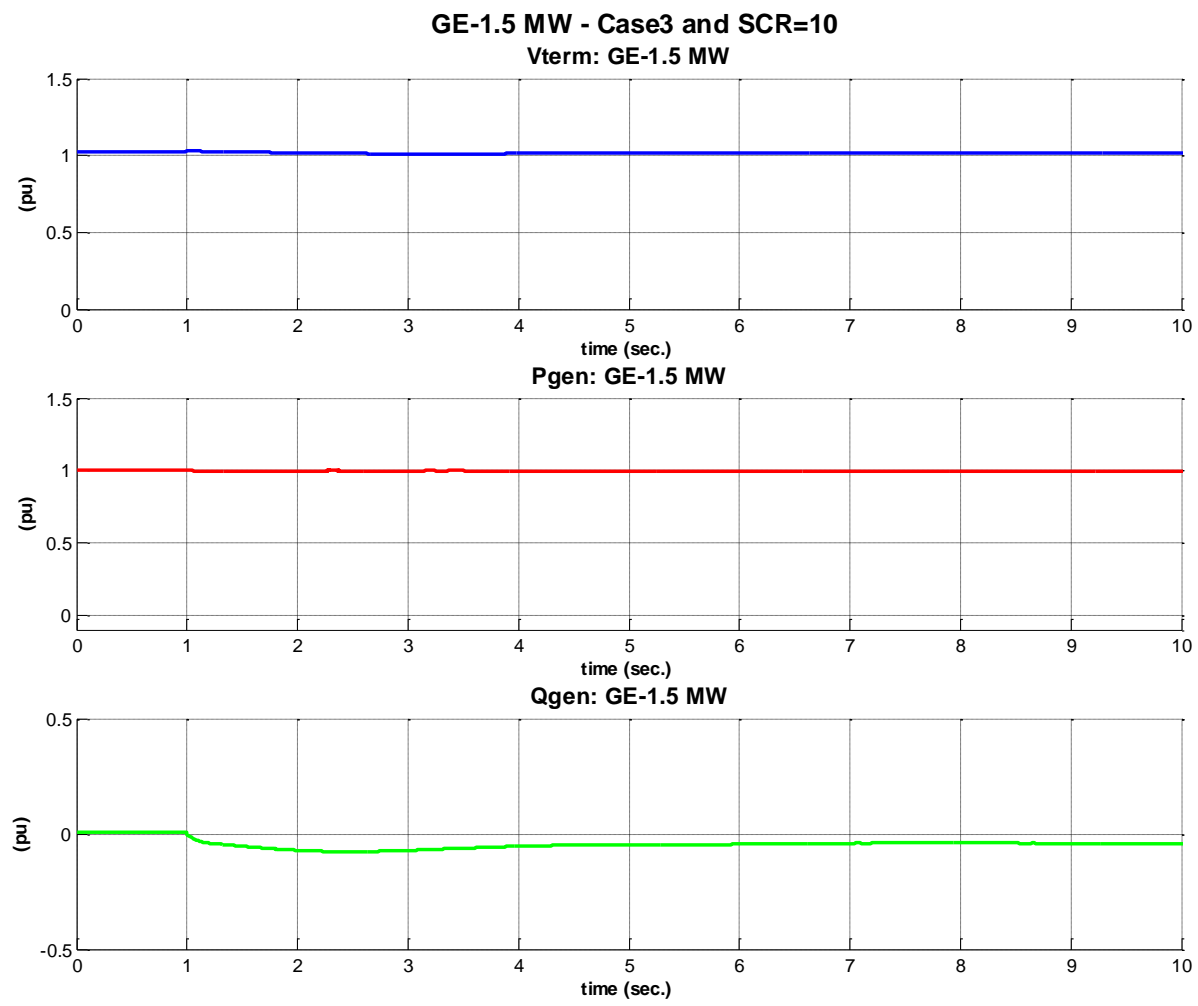

Figure 7-37: GE-1.5 MW - Case 3 and $\mathrm{SCR}=10\left(\mathrm{~V}_{\text {Term }}, \mathrm{P}, \mathrm{Q}\right)$. 

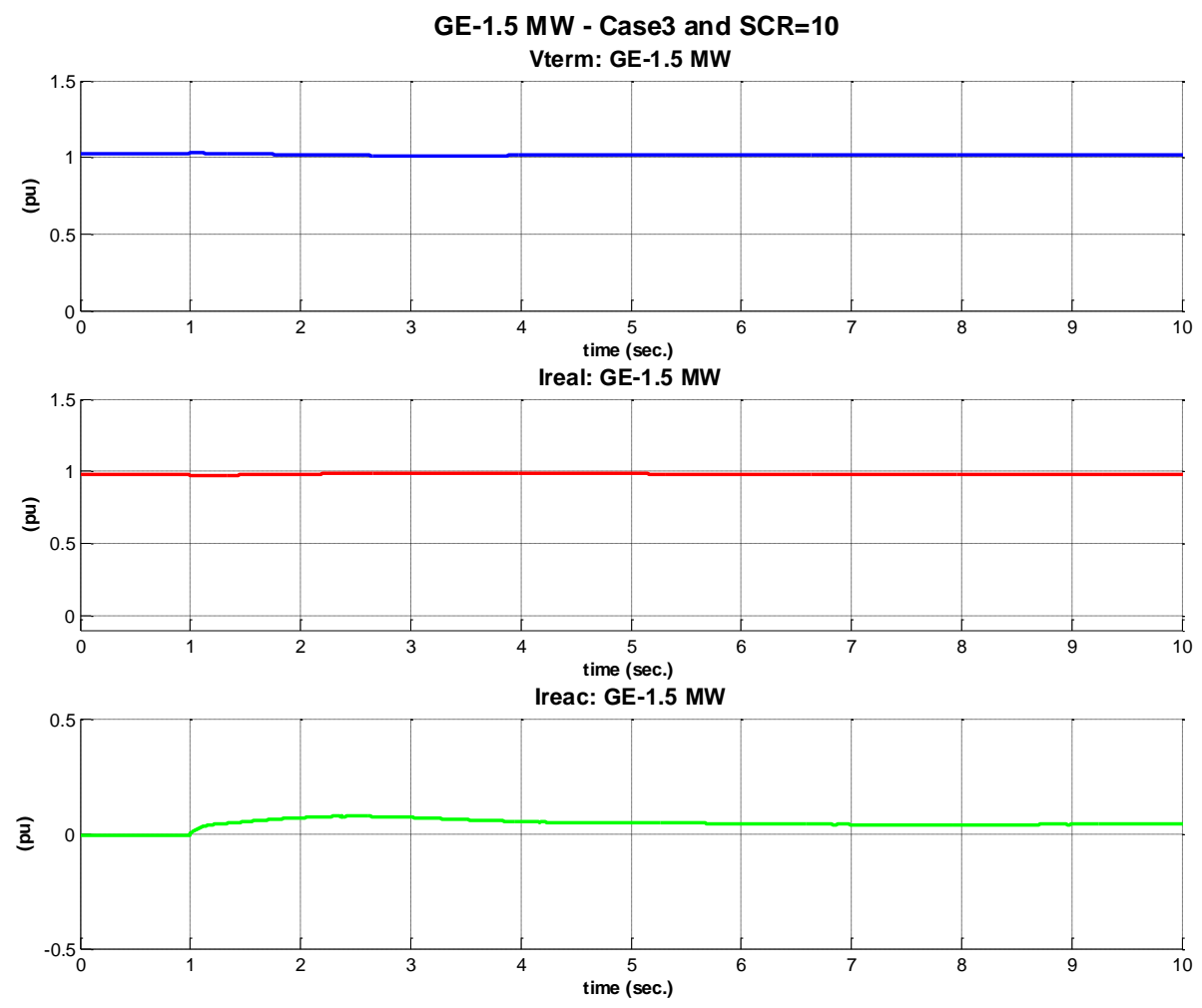

Figure 7-38: GE-1.5 MW - Case 3 and $S C R=10\left(V_{\text {Term }}, I_{\text {Real }}, I_{\text {Reac }}\right)$.

\subsubsection{Generic Model Parameterization}

\subsubsection{PSLF Environment ${ }^{1}$}

The PSLF platform generic wind turbine parameters, to mimic the behavior of GE's 1.5 MW series, are shown in Table 7-2. The parameter values shown in the table resulted from a compromise between the simulated cases (i.e. SCR 5 and SCR 10) and network conditions (i.e. fault, under- and over frequency).

\footnotetext{
${ }^{1}$ Parameterization performed by GE Energy.
} 
Table 7-2: PSLF generic model parameter values

\begin{tabular}{|c|c|c|c|c|c|c|c|}
\hline \multicolumn{2}{|c|}{ wt3g } & \multicolumn{2}{|c|}{ wt3t } & \multicolumn{2}{|c|}{ wt3e } & \multicolumn{2}{|c|}{$w t 3 p$} \\
\hline Lpp & 0.8 & Vw & 1.0 & Varflg & 1 & Kpp & 150.0 \\
\hline Lvplsw & 1.0 & $\mathrm{Ht}$ & 4.94 & Vltflg & 1 & Kip & 25.0 \\
\hline Rrpwr & 10.0 & $\mathrm{D}$ & 0.0 & Tsp & 60.0 & Kpc & 3.0 \\
\hline Brkpt & 0.9 & Kaero & 0.007 & Kptrq & 3.00 & Kic & 30.0 \\
\hline Zerox & 0.5 & Theta2 & 21.98 & Kitrq & 0.6 & Pimax & 27.0 \\
\hline Lvpll & 1.11 & Htfrac & 1.00 & $\mathrm{Tpc}$ & 0.05 & Pimin & 0 \\
\hline Volim & 1.20 & Freq1 & 0.00 & Pmax & 1.12 & Pirat & 10.0 \\
\hline Lvpntl & 0.80 & Dshaft & 0.00 & Pmin & 0.04 & Tpi & 0.3 \\
\hline Lvpnt0 & 0.40 & & & Pwrat & 0.45 & Pset & 1.0 \\
\hline Iolim & -1.30 & & & Ipmax & 1.12 & & \\
\hline Khv & 0.70 & & & Wpmin & 0.69 & & \\
\hline & & & & Wp20 & 0.78 & & \\
\hline & & & & Wp40 & 0.98 & & \\
\hline & & & & Wp60 & 1.12 & & \\
\hline & & & & Pwp100 & 0.74 & & \\
\hline & & & & Wp100 & 1.20 & & \\
\hline & & & & Kqi & 0.10 & & \\
\hline & & & & Kqv & 40.0 & & \\
\hline & & & & Qmax & 0.436 & & \\
\hline & & & & Qmin & -0.436 & & \\
\hline & & & & Vmax & 1.10 & & \\
\hline & & & & $V \min$ & 0.90 & & \\
\hline & & & & Xiqmax & 1.45 & & \\
\hline & & & & Xiqmin & 0.50 & & \\
\hline & & & & $\mathrm{Tp}$ & 0.05 & & \\
\hline & & & & $X_{c}$ & 0.00 & & \\
\hline & & & & $\mathrm{Tr}$ & 0.02 & & \\
\hline & & & & Fn & 1.00 & & \\
\hline & & & & Kiv & 5.00 & & \\
\hline & & & & Kpv & 18.0 & & \\
\hline & & & & Tv & 0.05 & & \\
\hline & & & & Tc & 0.15 & & \\
\hline
\end{tabular}




\subsection{Case 1 - Fault Event}

\section{SCR - 5}

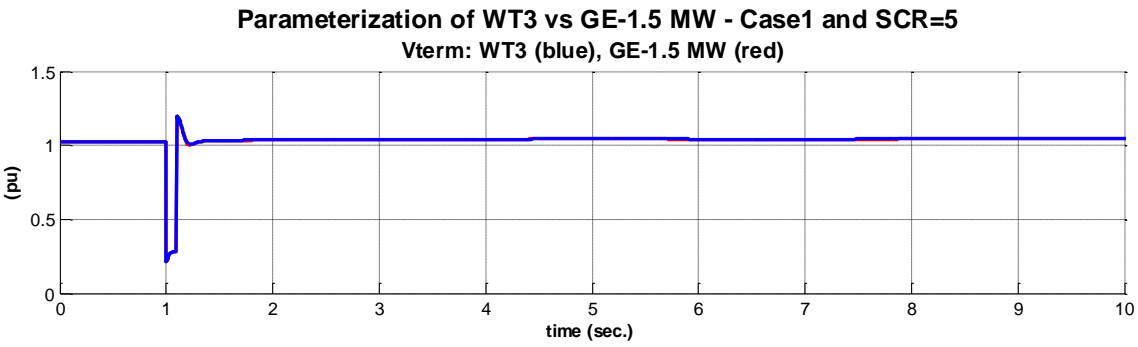

Pgen: WT3 (blue), GE-1.5 MW (red)

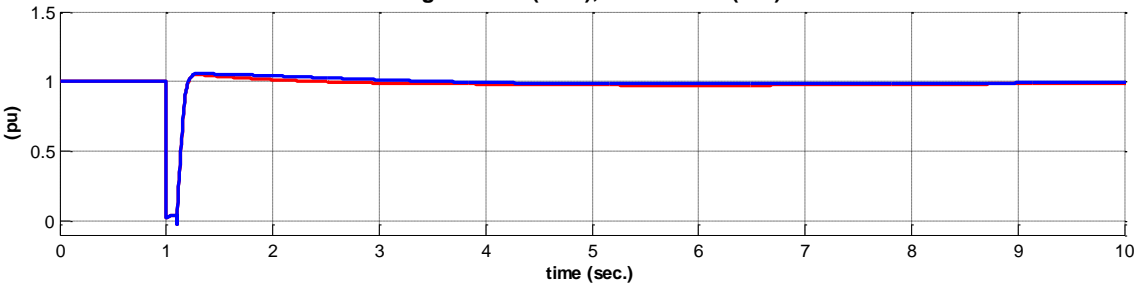

Qgen: WT3 (blue), GE-1.5 MW (red)

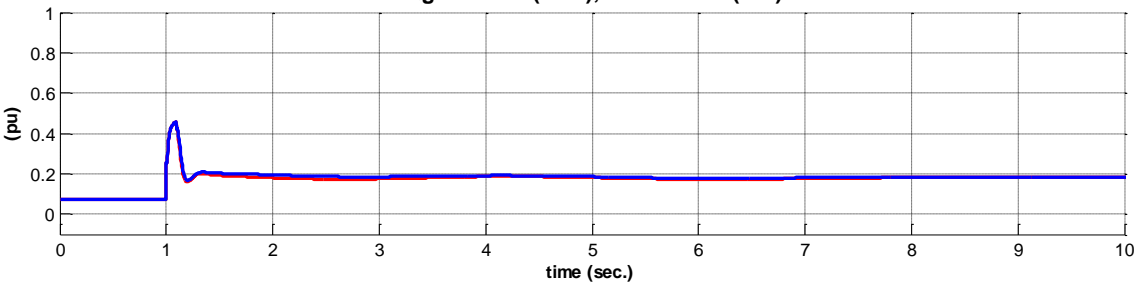

Figure 7-39: Parameterization of WT3 vs GE-1.5 MW - Case 1 and $S C R=5\left(V_{T e r m}, P, Q\right)$.
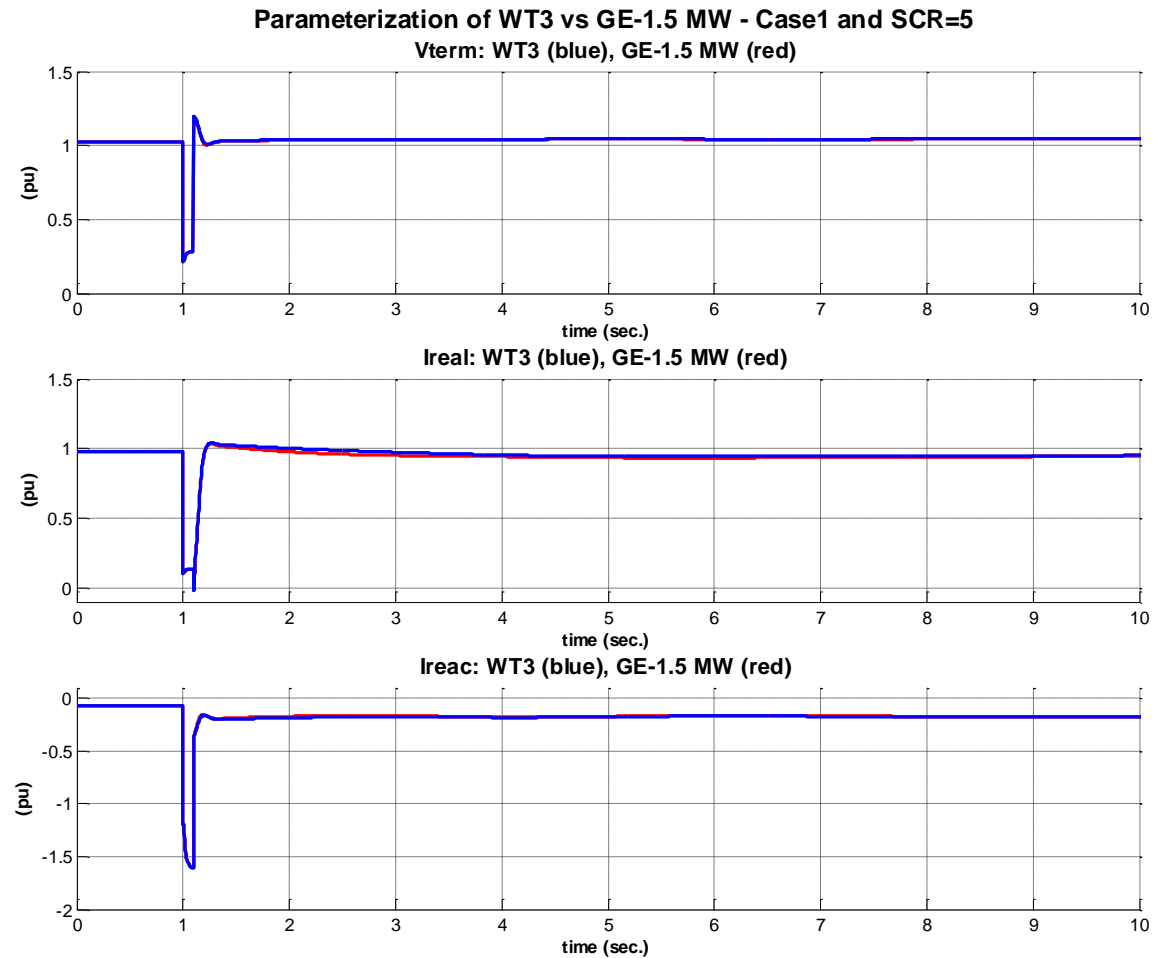

Figure 7-40: Parameterization of WT3 vs GE-1.5 MW - Case 1 and $S C R=5$ (V $\left.V_{\text {Term }}, I_{\text {Real }}, I_{\text {Reac }}\right)$. 
$\underline{\text { SCR }-10}$

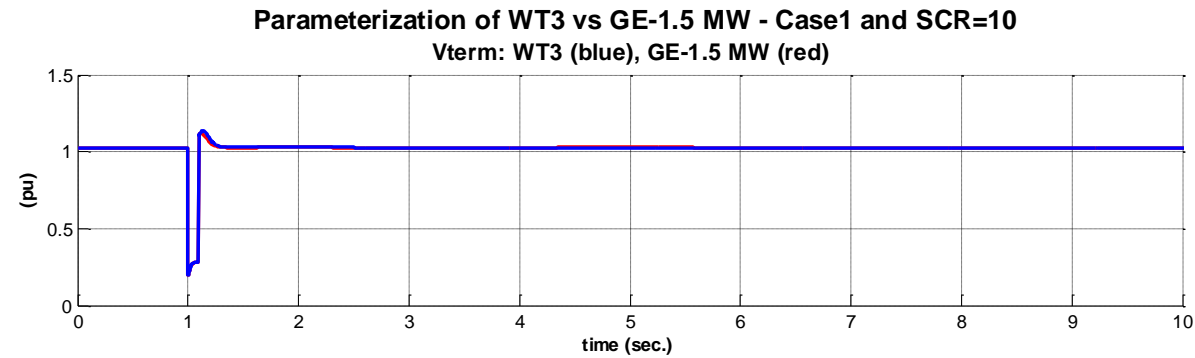

Pgen: WT3 (blue), GE-1.5 MW (red)

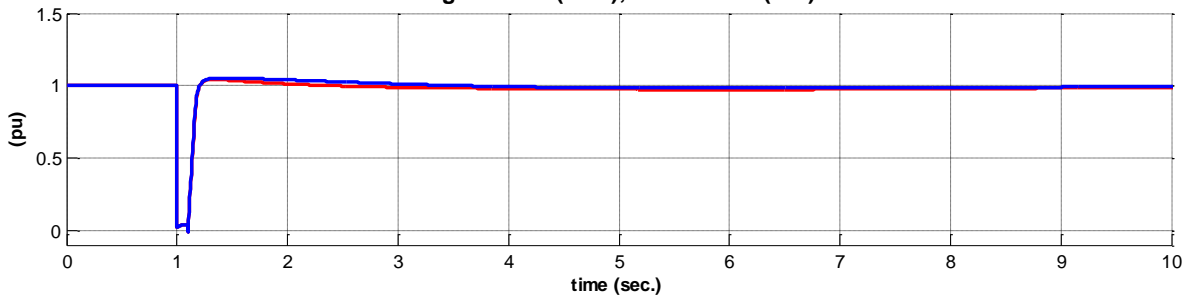

Qgen: WT3 (blue), GE-1.5 MW (red)

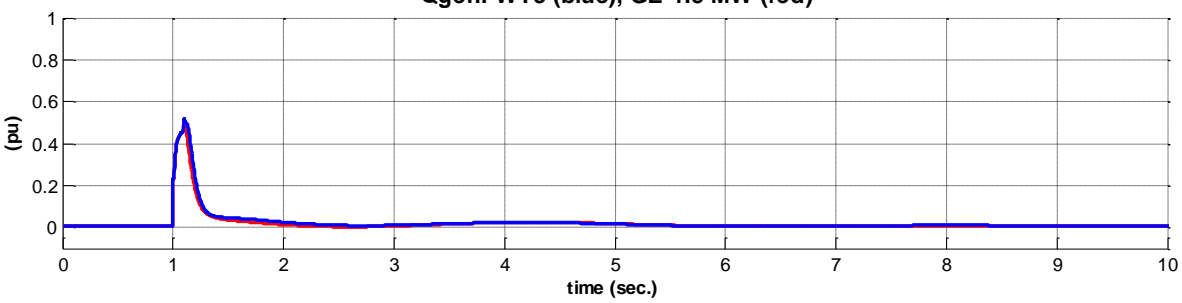

Figure 7-41: Parameterization of WT3 vs GE-1.5 MW - Case 1 and $S C R=10$ ( $\left.V_{\text {Term }}, P, Q\right)$.

Parameterization of WT3 vs GE-1.5 MW - Case1 and SCR=10

Vterm: WT3 (blue), GE-1.5 MW (red)

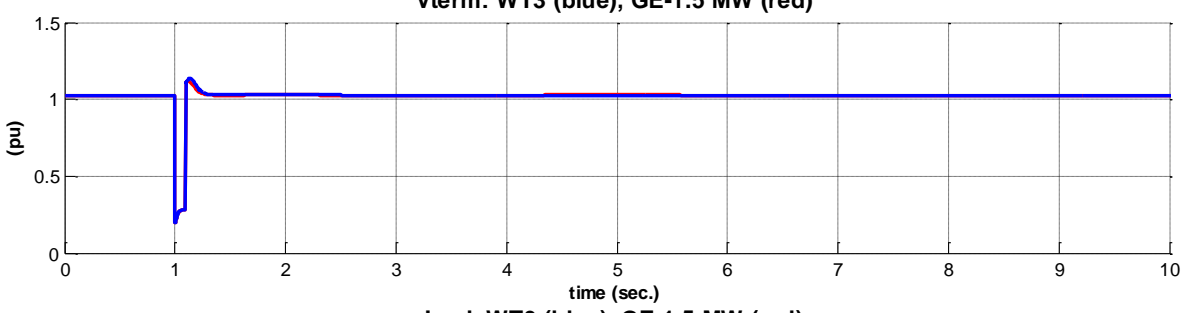

Ireal: WT3 (blue), GE-1.5 MW (red)

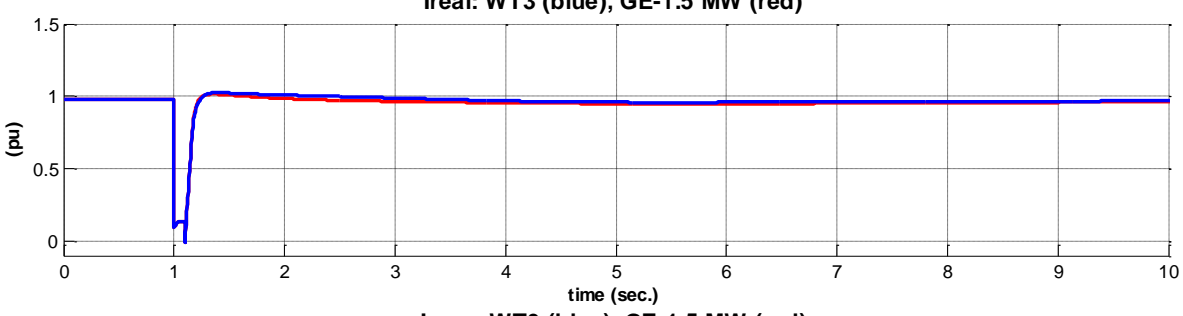

Ireac: WT3 (blue), GE-1.5 MW (red)

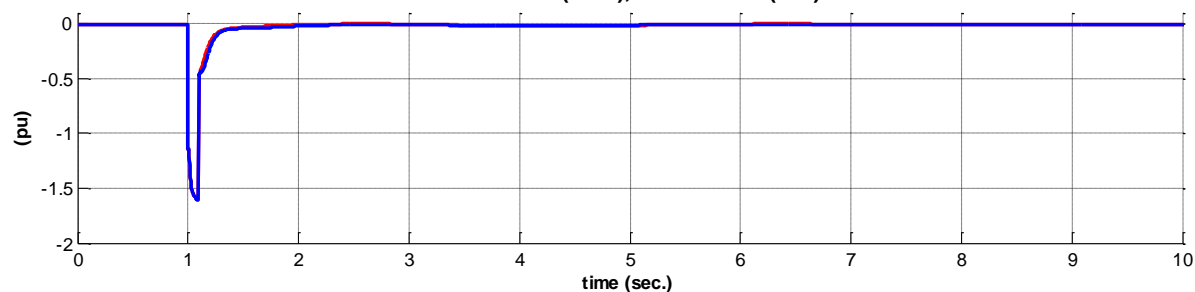

Figure 7-42: Parameterization of WT3 vs GE-1.5 MW - Case 1 and $\mathrm{SCR}=10\left(\mathrm{~V}_{\text {Term }}, \mathrm{I}_{\text {Real }}, \mathrm{I}_{\text {Reac }}\right)$. 


\subsection{Case 2 - Under-Frequency Event}

\section{SCR - 5}

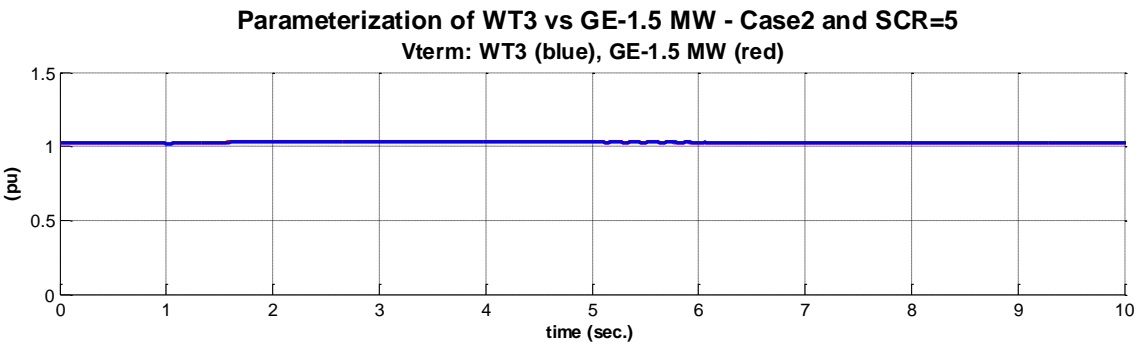

Pgen: WT3 (blue), GE-1.5 MW (red)

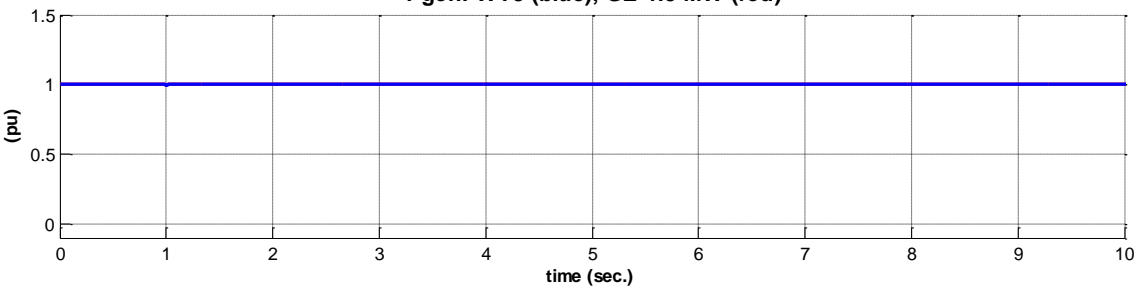

Qgen: WT3 (blue), GE-1.5 MW (red)

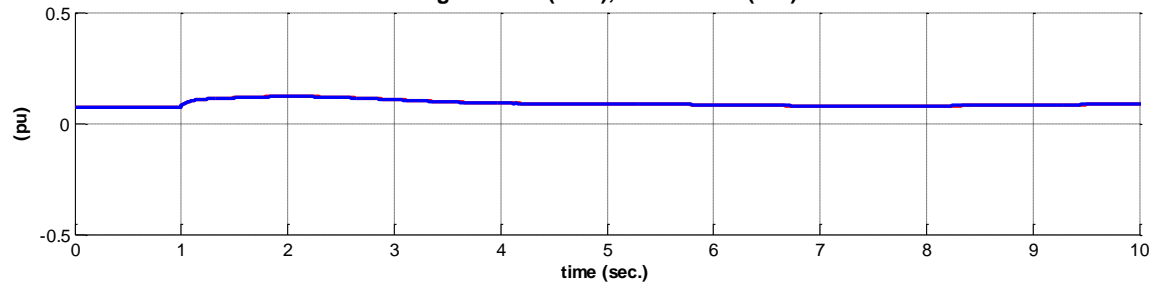

Figure 7-43: Parameterization of WT3 vs GE-1.5 MW - Case 2 and $S C R=5\left(V_{\text {Term }}, P, Q\right)$.
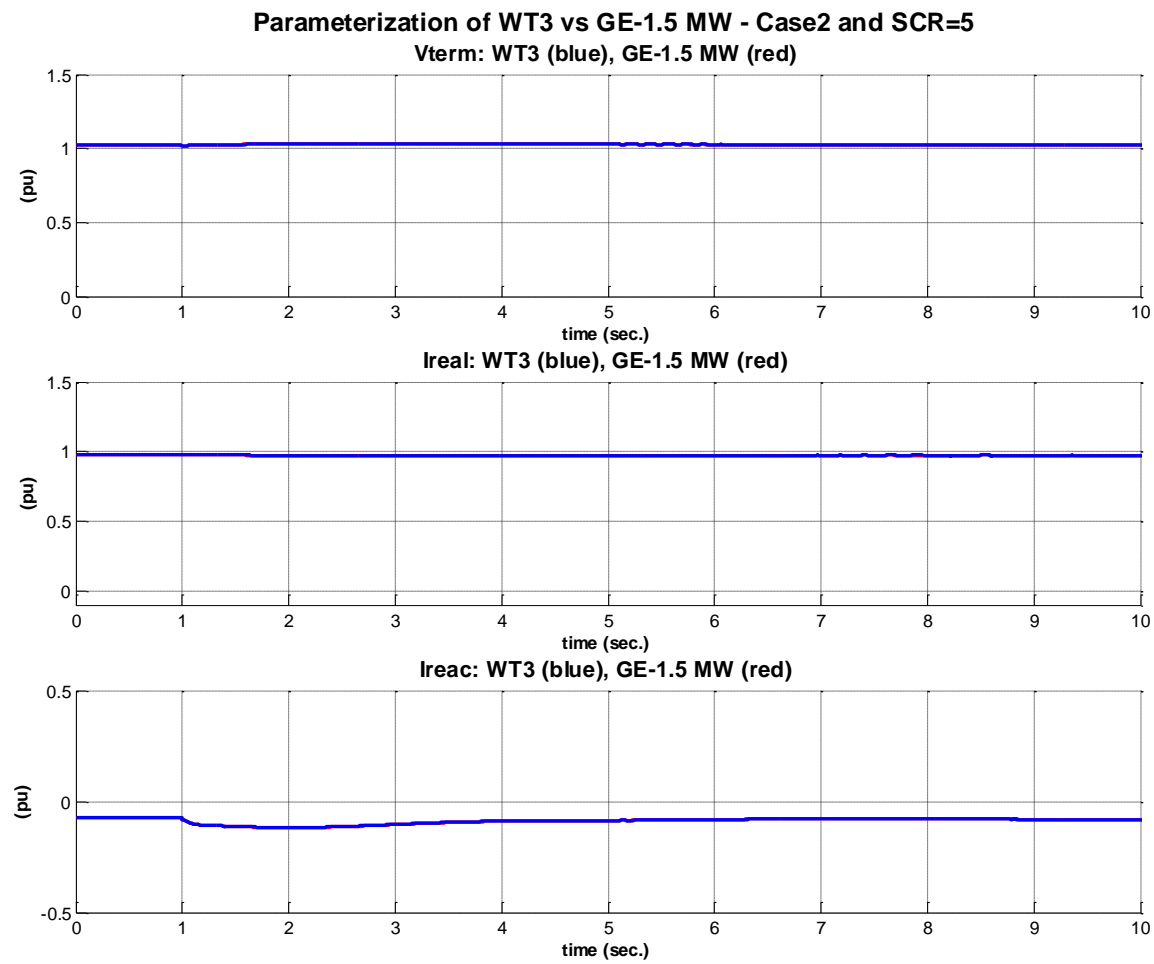

Figure 7-44: Parameterization of WT3 vs GE-1.5 MW - Case 2 and SCR=5 (V $\left.\mathrm{V}_{\text {Term }}, I_{\text {Real }}, I_{\text {Reac }}\right)$. 
$\underline{\text { SCR }-10}$

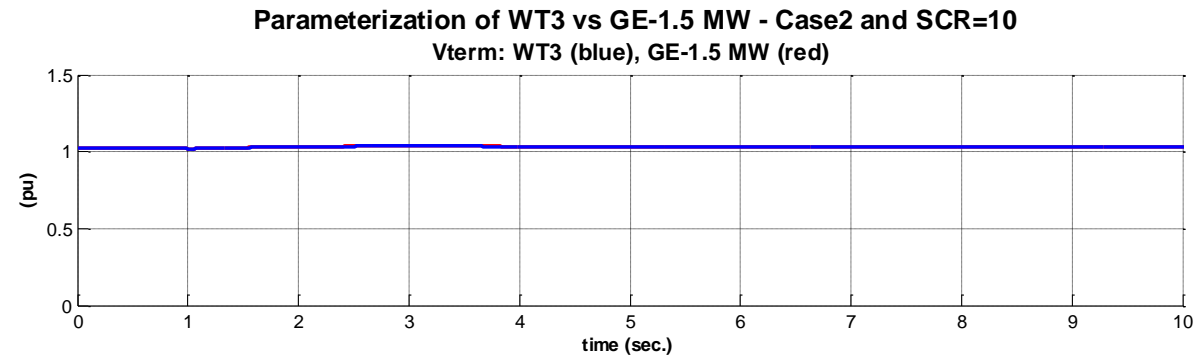

Pgen: WT3 (blue), GE-1.5 MW (red)

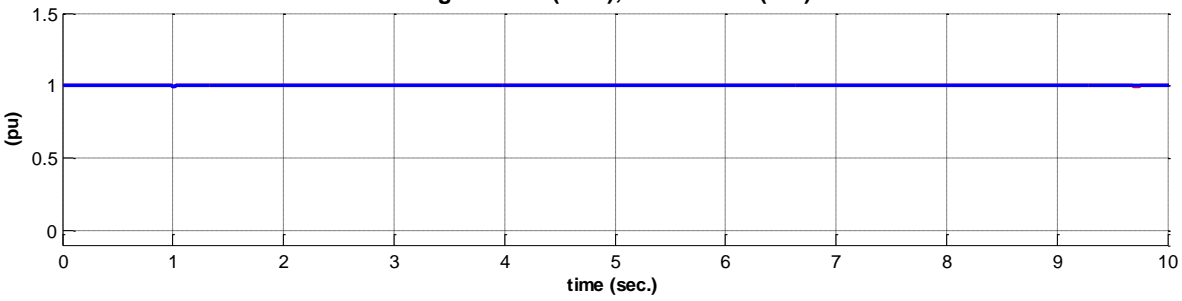

Qgen: WT3 (blue), GE-1.5 MW (red)

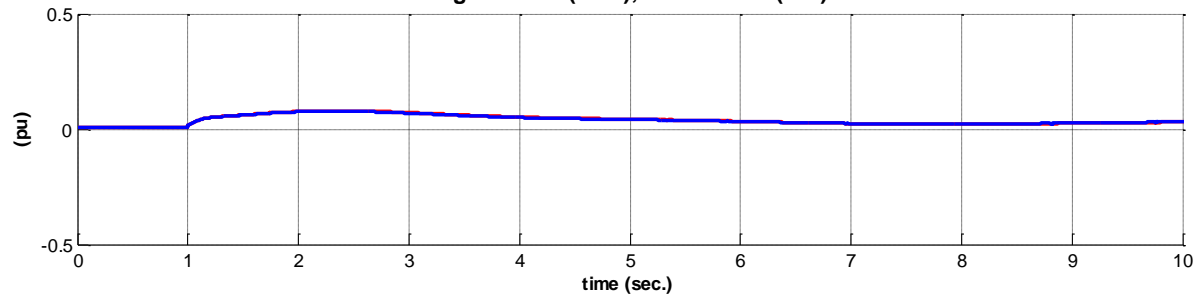

Figure 7-45: Parameterization of WT3 vs GE-1.5 MW - Case 2 and SCR=10 ( $\left.V_{\text {Term }}, P, Q\right)$.

Parameterization of WT3 vs GE-1.5 MW - Case2 and SCR=10

Vterm: WT3 (blue), GE-1.5 MW (red)

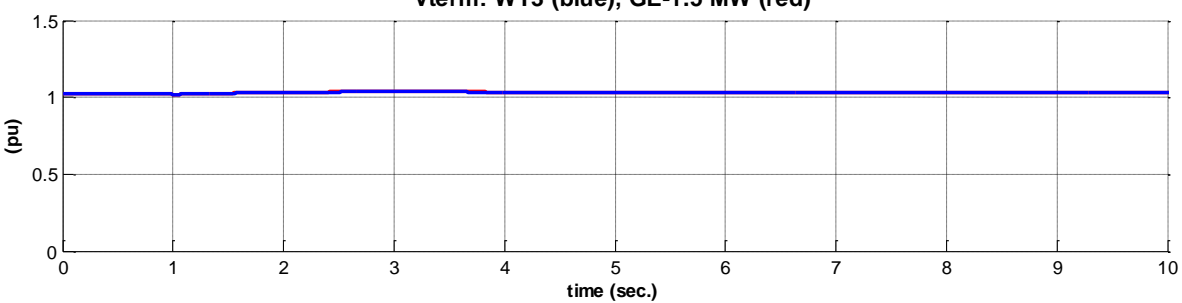

Ireal: WT3 (blue), GE-1.5 MW (red)

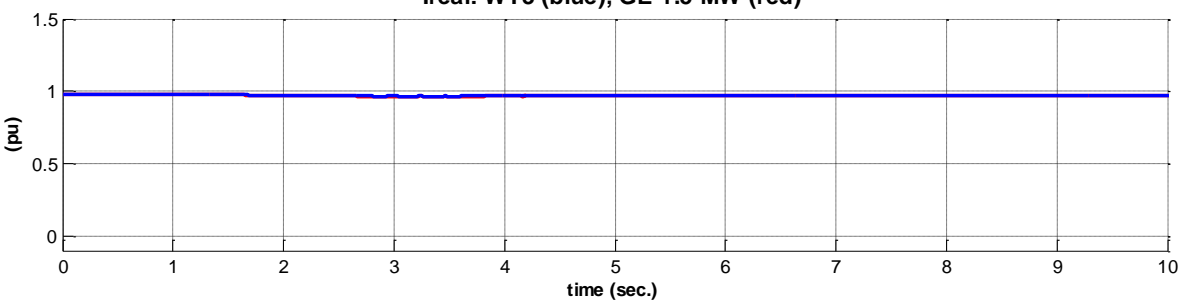

Ireac: WT3 (blue), GE-1.5 MW (red)

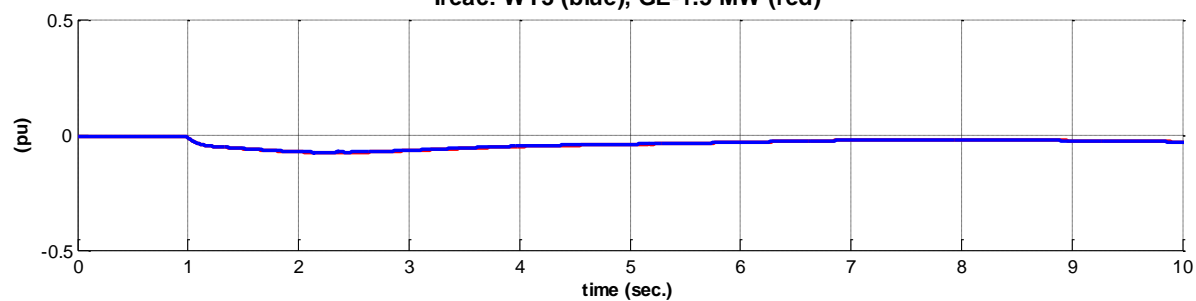

Figure 7-46: Parameterization of WT3 vs GE-1.5 MW - Case 2 and $S C R=10$ ( $\left.V_{\text {Term, }}, I_{\text {Real }}, I_{\text {Reac }}\right)$. 


\subsection{Case 3 - Over-Frequency Event}

$\underline{\text { SCR }-5}$

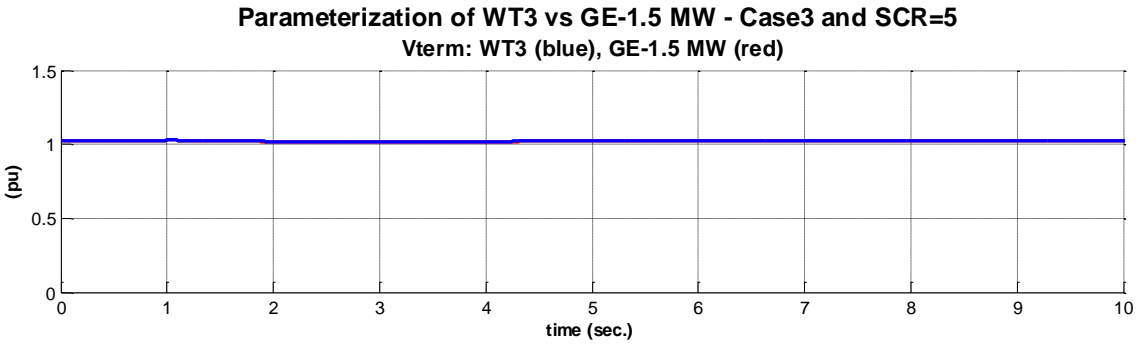

Pgen: WT3 (blue), GE-1.5 MW (red)

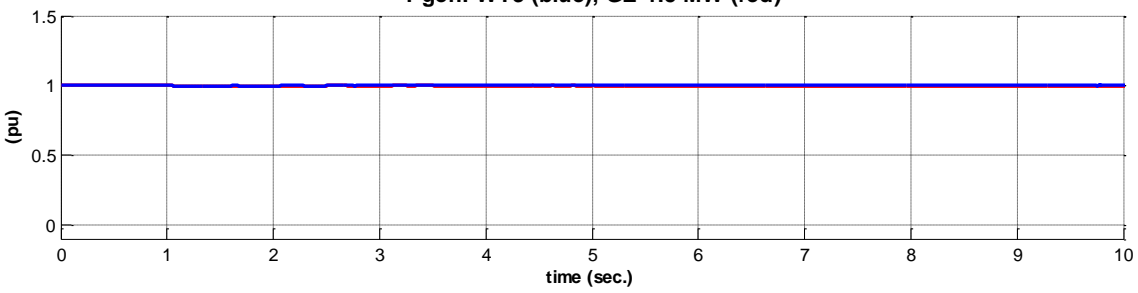

Qgen: WT3 (blue), GE-1.5 MW (red)

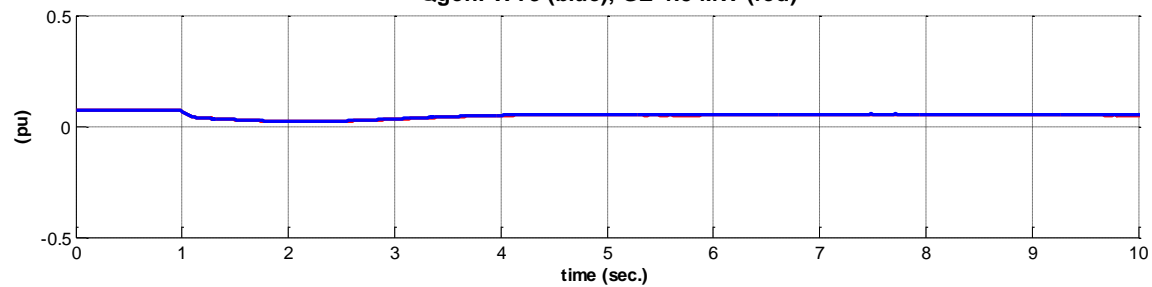

Figure 7-47: Parameterization of WT3 vs GE-1.5 MW - Case 3 and $S C R=5\left(V_{\text {Term }}, P, Q\right)$.
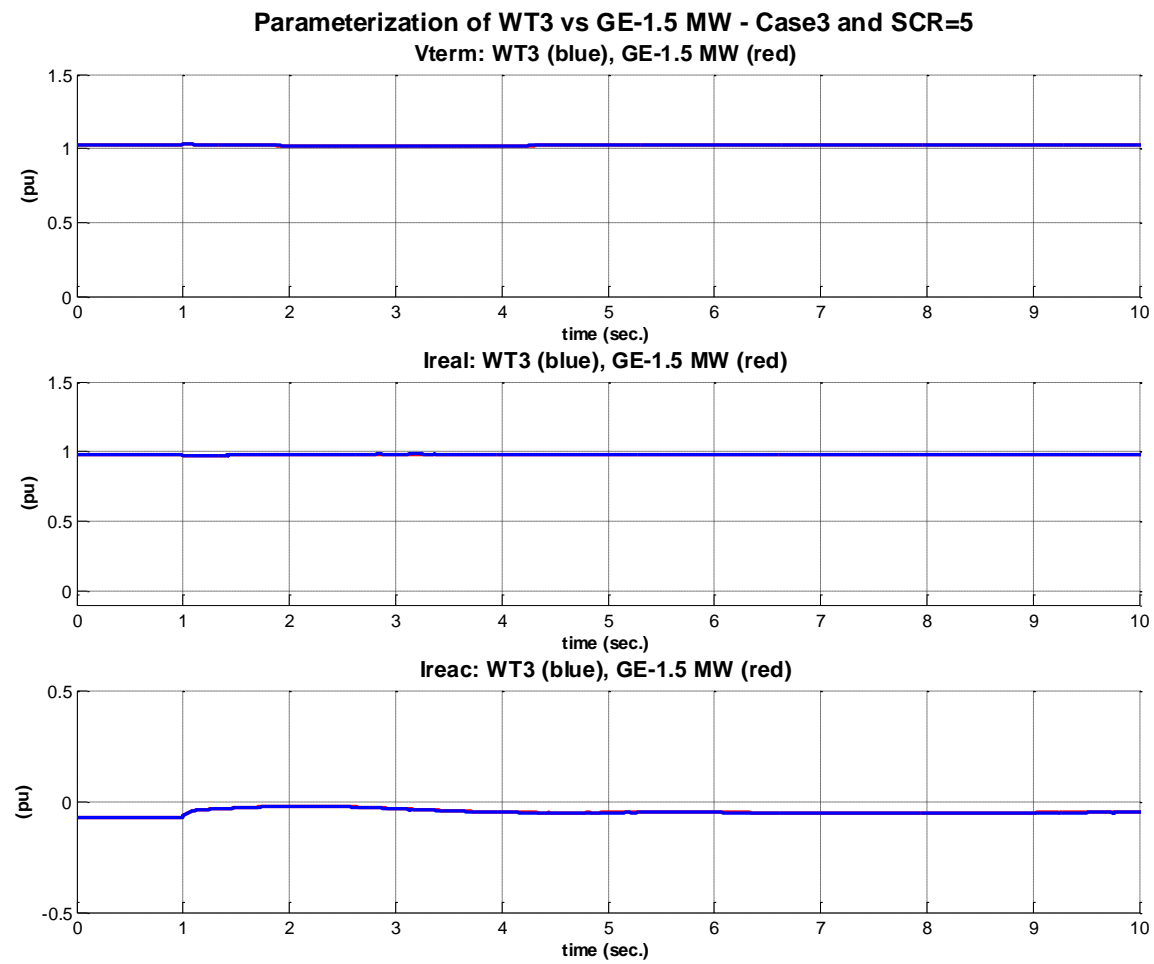

Figure 7-48: Parameterization of WT3 vs GE-1.5 MW - Case 3 and $S C R=5$ (V $\left.V_{\text {Term }}, I_{\text {Real }}, I_{\text {Reac }}\right)$. 
$\underline{\text { SCR }-10}$

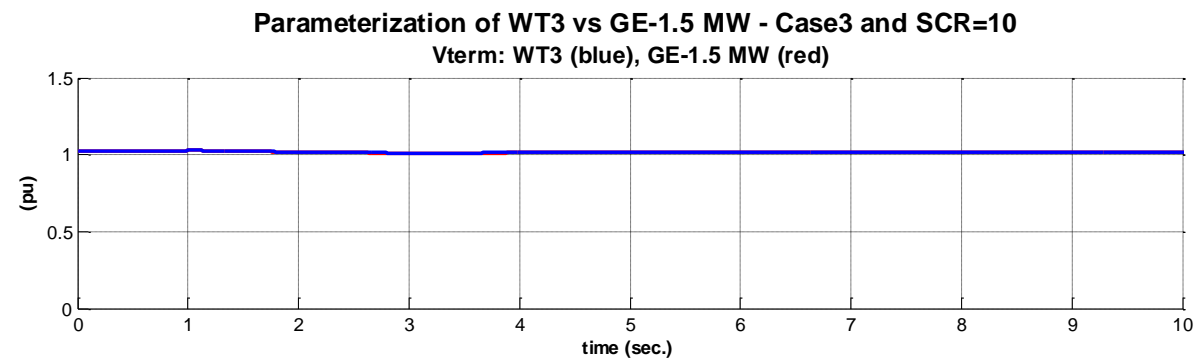

Pgen: WT3 (blue), GE-1.5 MW (red)

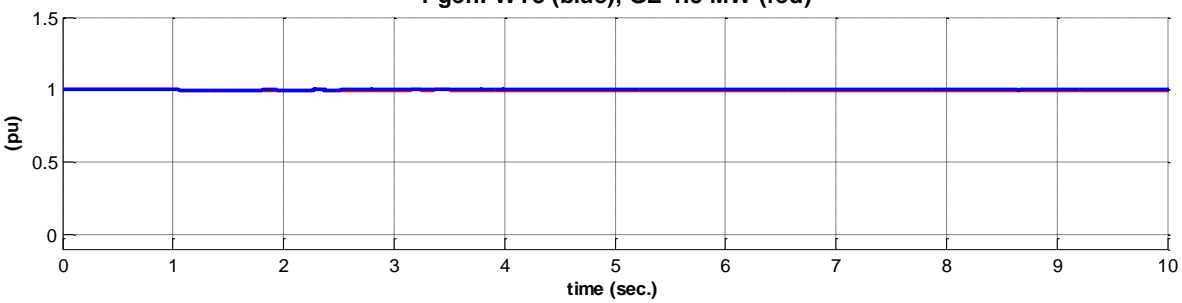

Qgen: WT3 (blue), GE-1.5 MW (red)

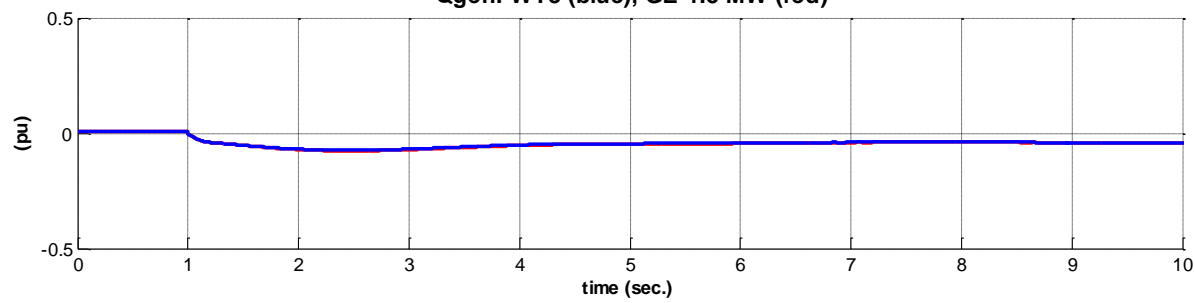

Figure 7-49: Parameterization of WT3 vs GE-1.5 MW - Case 3 and $S C R=10\left(V_{T e r m}, P, Q\right)$.

Parameterization of WT3 vs GE-1.5 MW - Case 3 and SCR $=10$

Vterm: WT3 (blue), GE-1.5 MW (red)

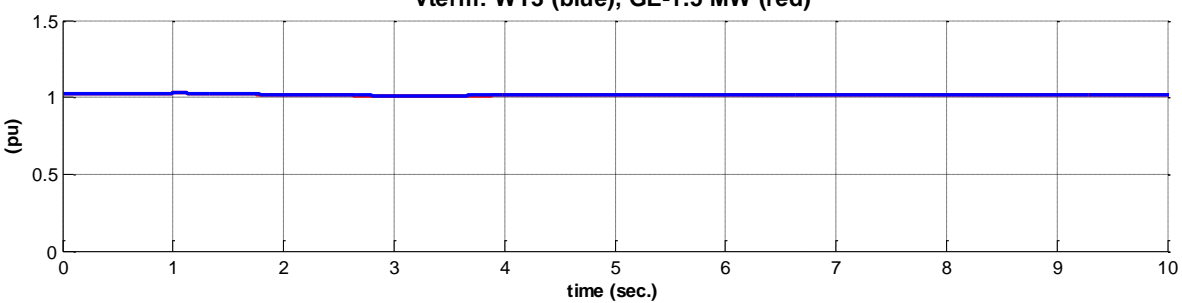

Ireal: WT3 (blue), GE-1.5 MW (red)

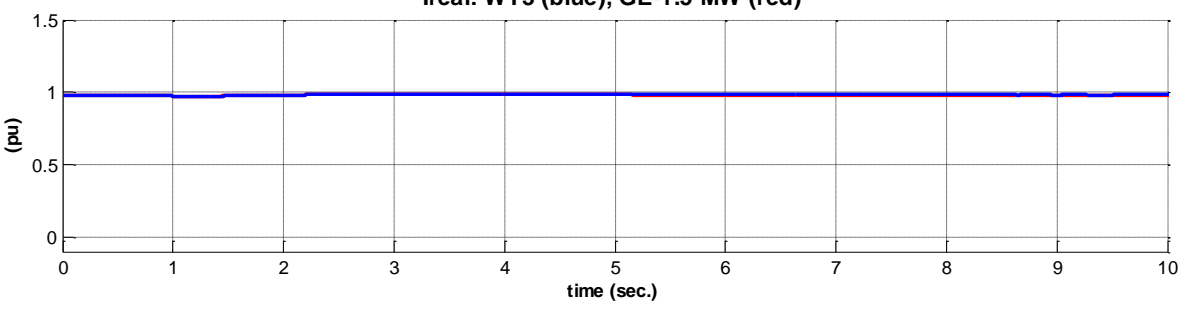

Ireac: WT3 (blue), GE-1.5 MW (red)

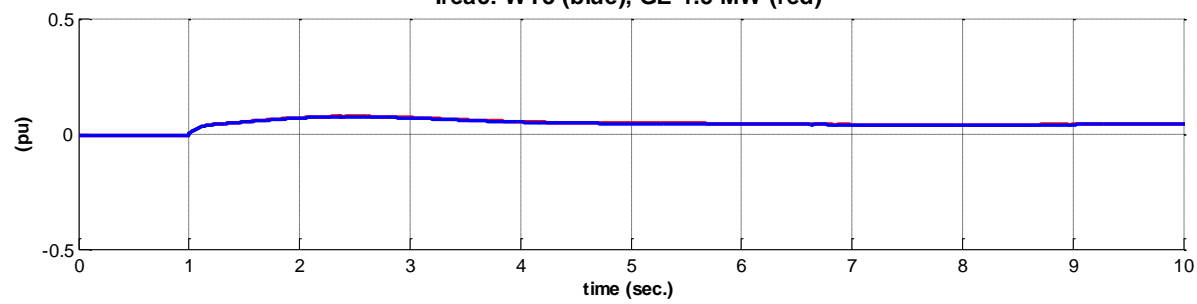

Figure 7-50: Parameterization of WT3 vs GE-1.5 MW - Case 3 and $S C R=10$ ( $\left.V_{\text {Term }}, I_{\text {Real }}, I_{\text {Reac }}\right)$. 


\subsubsection{PSSE Environment ${ }^{1}$}

The PSSE platform generic wind turbine parameters, to mimic the behavior of GE's 1.5 MW series, are shown in Table 7-3. The parameter values shown in the table resulted from a compromise between the simulated cases (i.e. SCR 5 and SCR 10) and network conditions (i.e. fault, under- and over frequency).

Table 7-3: PSSE generic model parameter values.

\begin{tabular}{|c|c|c|c|c|c|c|c|}
\hline \multicolumn{2}{|c|}{ WT3G } & \multicolumn{2}{|c|}{ WT3E } & \multicolumn{2}{|c|}{ WT3T } & \multicolumn{2}{|c|}{ WT3P } \\
\hline Tiqcmd & 0.200 & Tfv & 0.150 & VW & 1.250 & $T p$ & 0.3 \\
\hline Tipcmd & 0.000 & Kpv & 18.00 & $\mathrm{H}$ & 4.940 & Kpp & 150.0 \\
\hline Kpll & 0.000 & Kiv & 5.00 & DAMP & 0.00 & Kip & 25.0 \\
\hline Kipll & 0.000 & Xc & 0.00 & Kaero & 0.01 & Kpc & 3.0 \\
\hline PLLMX & 0.100 & $T f p$ & 0.050 & Theta2 & 21.980 & Kic & 30.0 \\
\hline Prated & 1.500 & Kpp & 3.00 & Htfrac & 0.00 & TetaMin & -4.0 \\
\hline VLVPL1 & 0.500 & Kip & 0.60 & Freq1 & 1.810 & TetaMax & 27.00 \\
\hline VLVPL2 & 0.900 & PMX & 1.120 & DSHAFT & 1.50 & RTetaMax & 10.0 \\
\hline GLVPL & 1.110 & PMN & 0.040 & & & PMX & 1.0 \\
\hline VHVRCR & 1.200 & QMX & 0.4360 & & & & \\
\hline CURHVRCR & 2.000 & QMN & -0.4360 & & & & \\
\hline Rip_LVPL & 10.000 & IPmax & 1.220 & & & & \\
\hline T_LVPL & 0.020 & Trv & 0.020 & & & & \\
\hline & & RPMX & 0.450 & & & & \\
\hline & & RPMN & -0.450 & & & & \\
\hline & & T_Power & 60.00 & & & & \\
\hline & & Kqi & 0.10 & & & & \\
\hline & & VMINCL & 0.90 & & & & \\
\hline & & VMAXCL & 1.10 & & & & \\
\hline & & Kqv & 40.00 & & & & \\
\hline & & $\mathrm{XIQmin}$ & 0.50 & & & & \\
\hline & & XIQmax & 1.450 & & & & \\
\hline & & Tv & 0.050 & & & & \\
\hline & & $T p$ & 0.050 & & & & \\
\hline & & $\mathrm{Fn}$ & 1.00 & & & & \\
\hline & & WPMIN & 0.30 & & & & \\
\hline & & Wp20 & 1.20 & & & & \\
\hline & & Wp40 & 1.20 & & & & \\
\hline & & Wp60 & 1.20 & & & & \\
\hline & & Pwp & 0.740 & & & & \\
\hline & & Wp100 & 1.20 & & & & \\
\hline
\end{tabular}

\footnotetext{
${ }^{1}$ Parameterization performed by Siemens Energy.
} 


\subsection{Case 1 - Fault Event}

\section{SCR - 5}

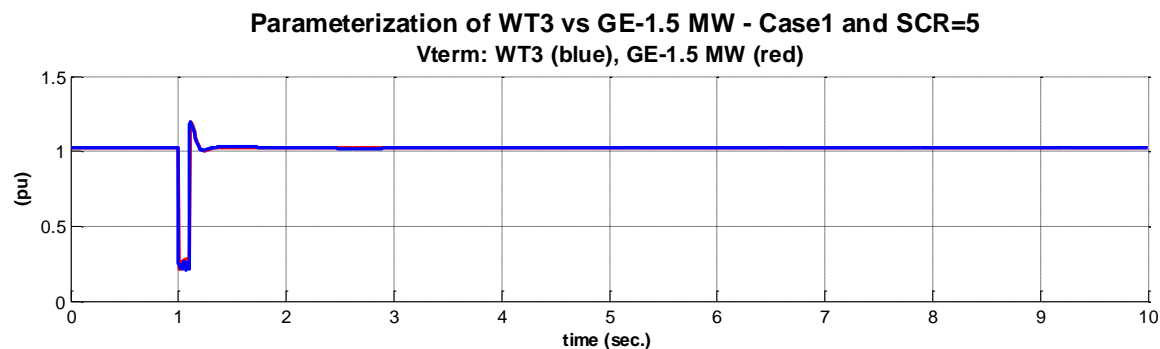

Pgen: WT3 (blue), GE-1.5 MW (red)

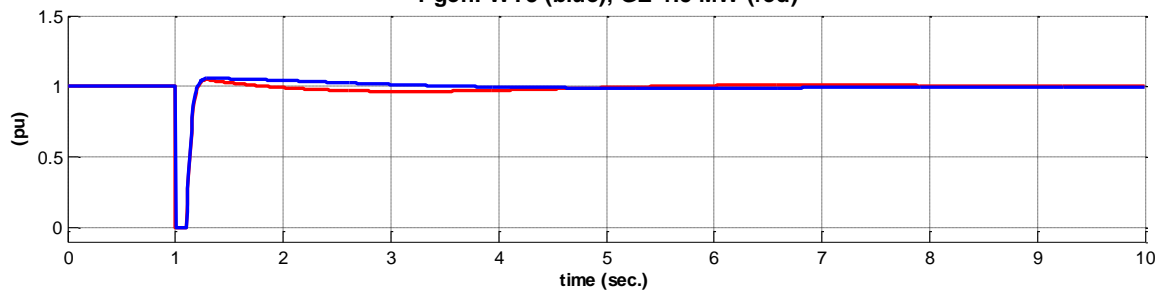

Qgen: WT3 (blue), GE-1.5 MW (red)

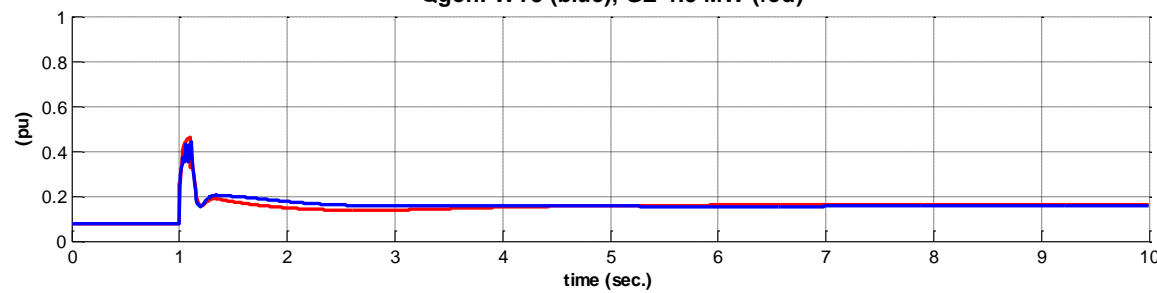

Figure 7-51: Parameterization of WT3 vs GE-1.5 MW - Case 1 and $S C R=5\left(V_{T e r m}, P, Q\right)$.

Parameterization of WT3 vs GE-1.5 MW - Case1 and SCR=5 Vterm: WT3 (blue), GE-1.5 MW (red)

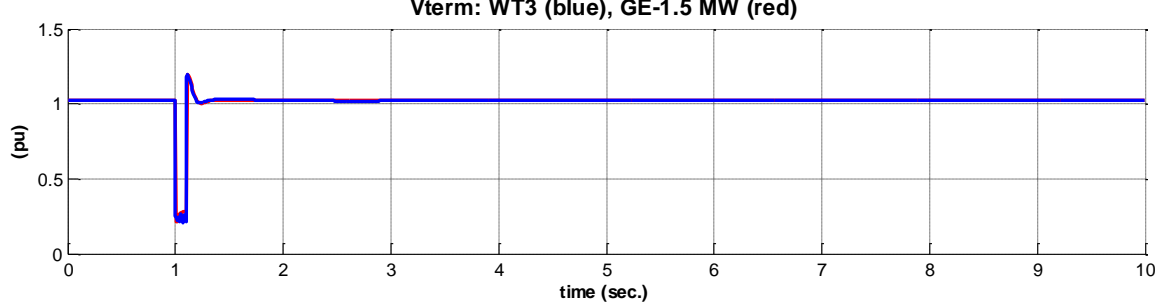

Ireal: WT3 (blue), GE-1.5 MW (red)

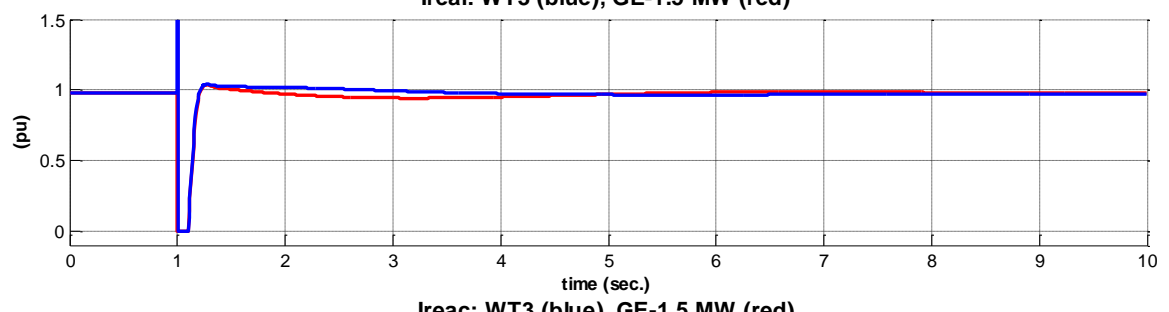

Ireac: WT3 (blue), GE-1.5 MW (red)

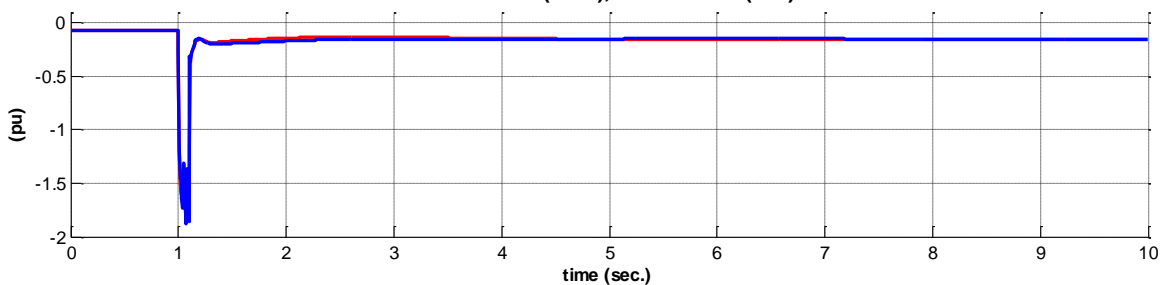

Figure 7-52: Parameterization of WT3 vs GE-1.5 MW - Case 1 and $S C R=5\left(V_{\text {Term }}, I_{\text {Real }}, I_{\text {Reac }}\right)$. 
$\underline{\text { SCR }-10}$
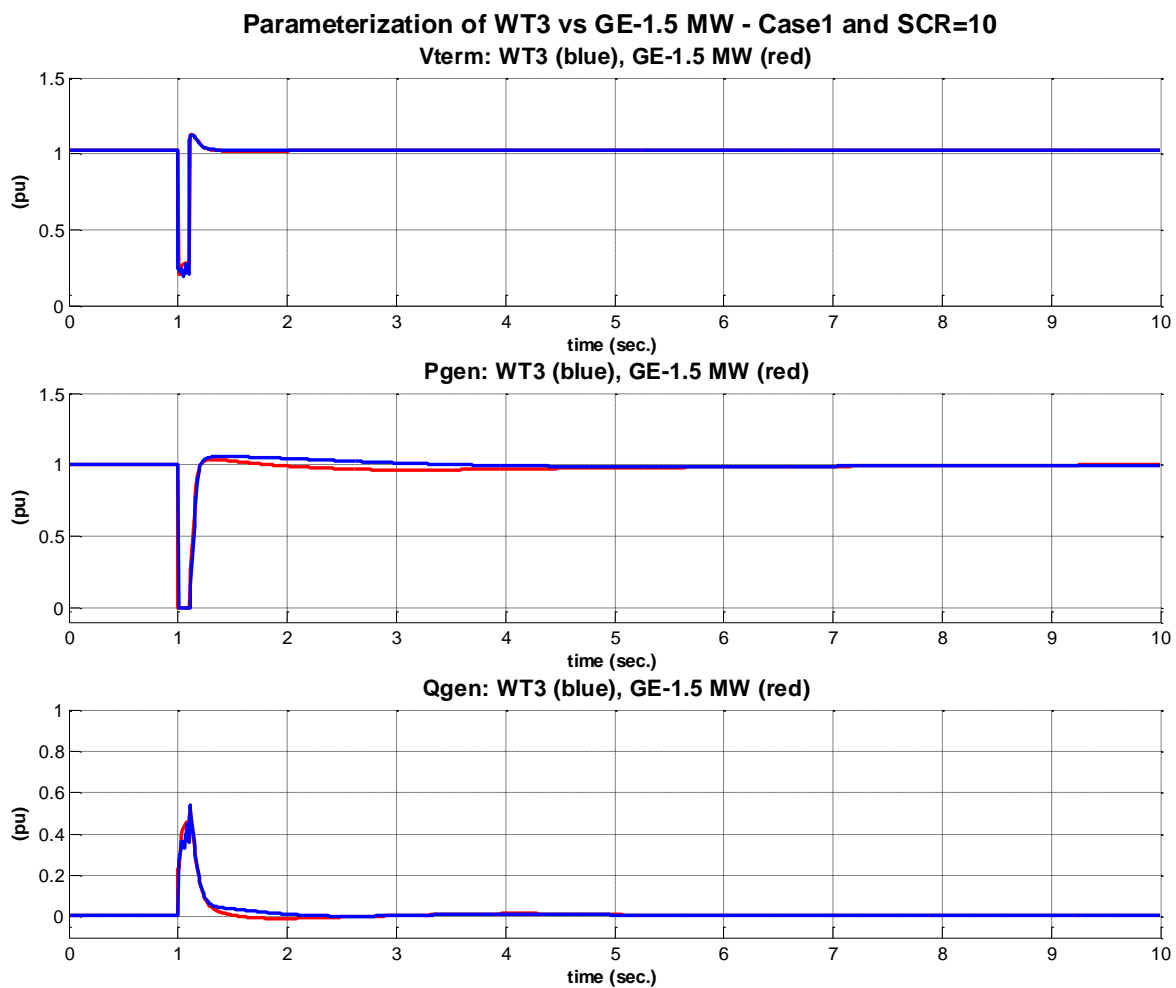

Figure 7-53: Parameterization of WT3 vs GE-1.5 MW - Case 1 and $S C R=10\left(V_{\text {Term }}, P, Q\right)$.
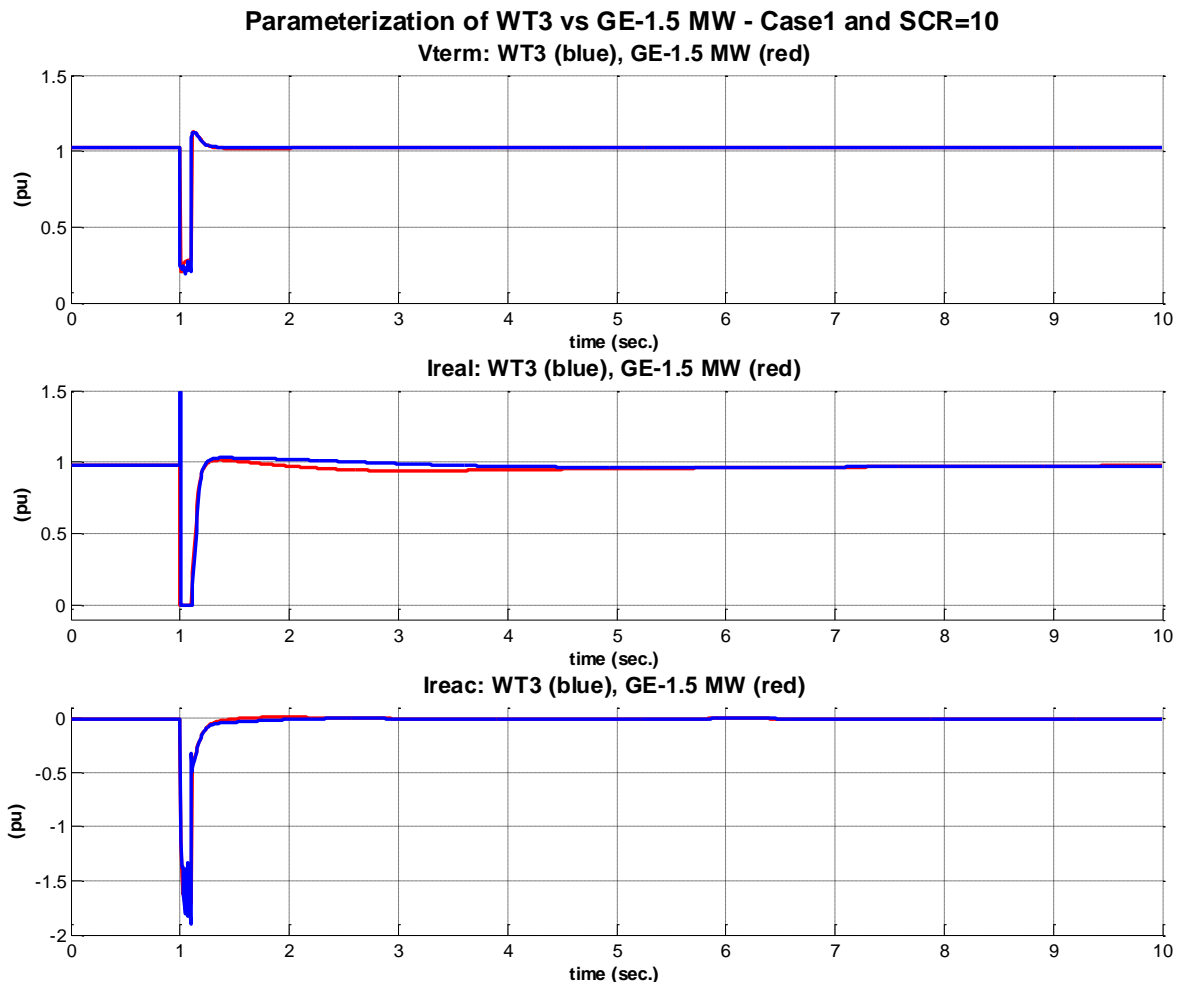

Figure 7-54: Parameterization of WT3 vs GE-1.5 MW - Case 1 and $S C R=10\left(V_{\text {Term, }}, I_{\text {Real }}, I_{\text {Reac }}\right)$. 


\subsection{Case 2 - Under-Frequency Event}

\section{SCR - 5}

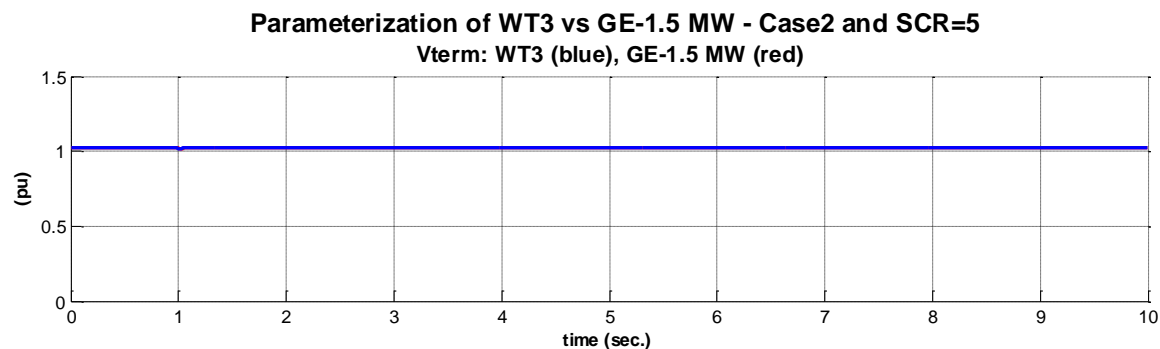

Pgen: WT3 (blue), GE-1.5 MW (red)

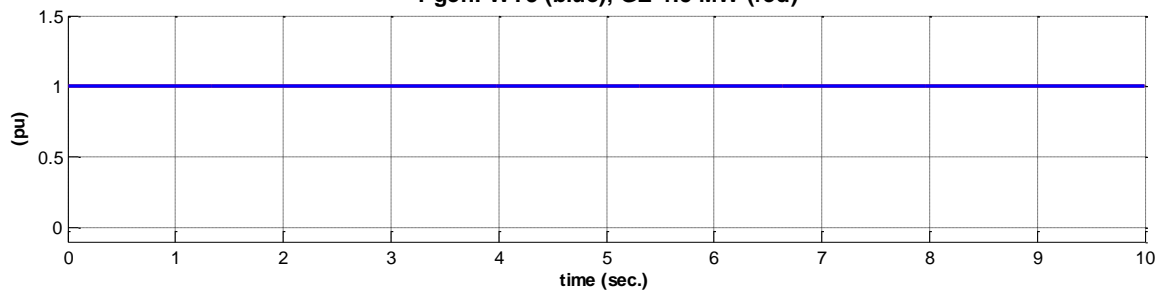

Qgen: WT3 (blue), GE-1.5 MW (red)

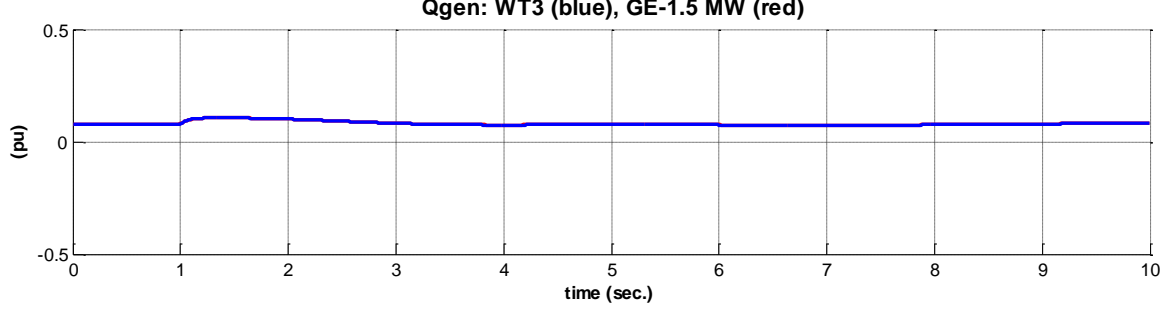

Figure 7-55: Parameterization of WT3 vs GE-1.5 MW - Case 2 and $S C R=5\left(V_{\text {Term }}, P, Q\right)$.
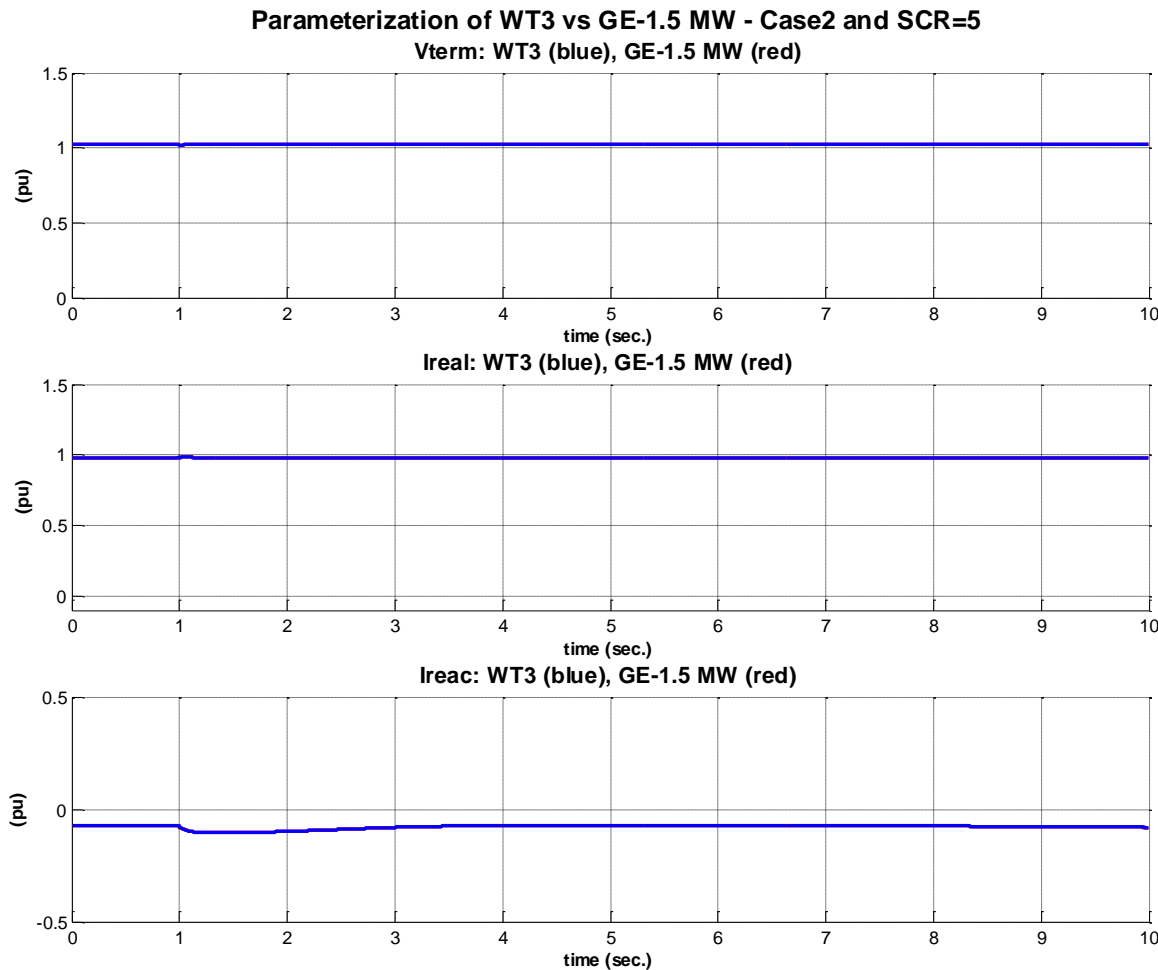

Figure 7-56: Parameterization of WT3 vs GE-1.5 MW - Case 2 and $S C R=5\left(V_{\text {Term }}, I_{\text {Real }}, I_{\text {Reac }}\right)$. 
$\underline{\text { SCR }-10}$

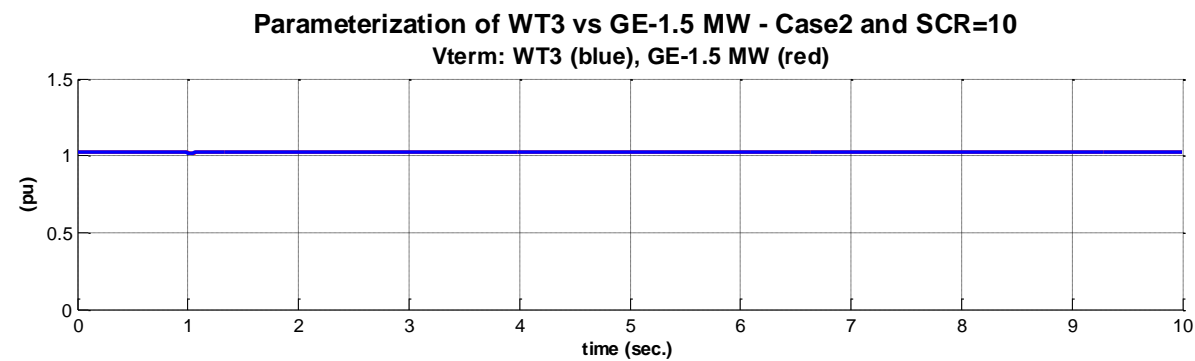

Pgen: WT3 (blue), GE-1.5 MW (red)

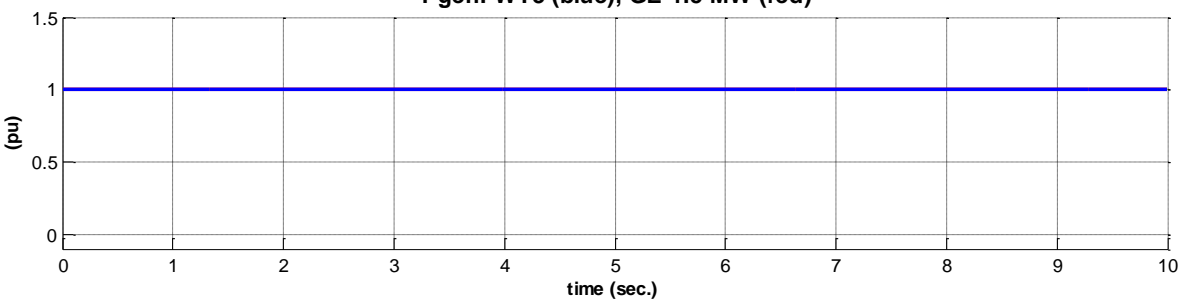

Qgen: WT3 (blue), GE-1.5 MW (red)

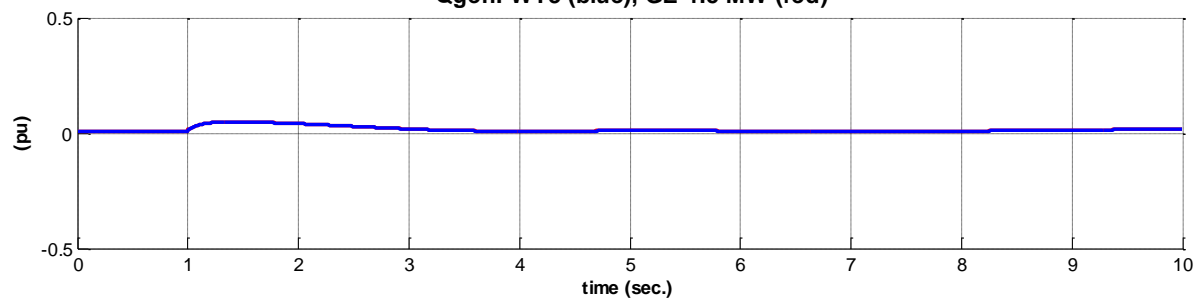

Figure 7-57: Parameterization of WT3 vs GE-1.5 MW - Case 2 and $S C R=10$ ( $\left.V_{\text {Term }}, P, Q\right)$.

Parameterization of WT3 vs GE-1.5 MW - Case2 and SCR=10

Vterm: WT3 (blue), GE-1.5 MW (red)

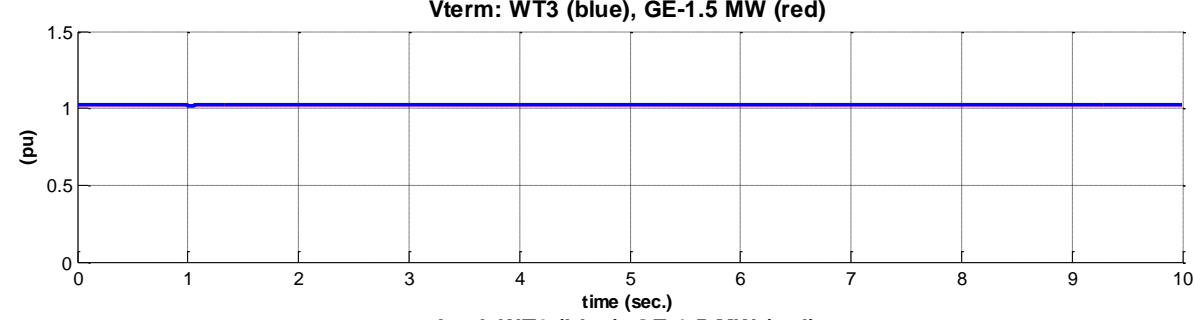

Ireal: WT3 (blue), GE-1.5 MW (red)

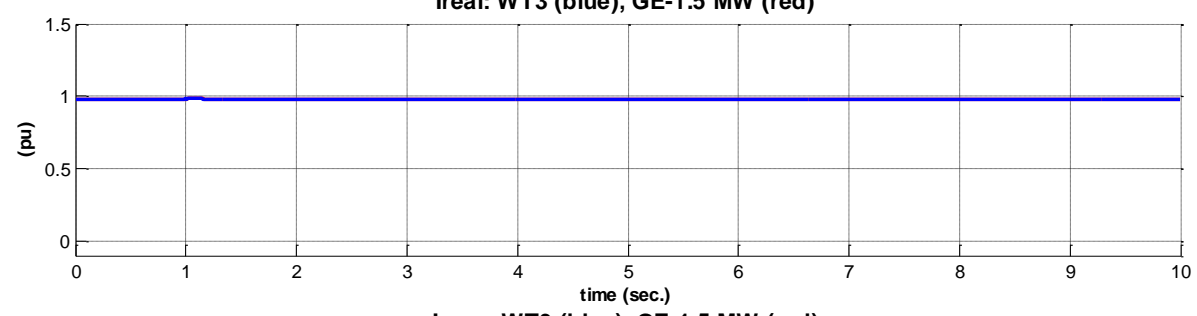

Ireac: WT3 (blue), GE-1.5 MW (red)

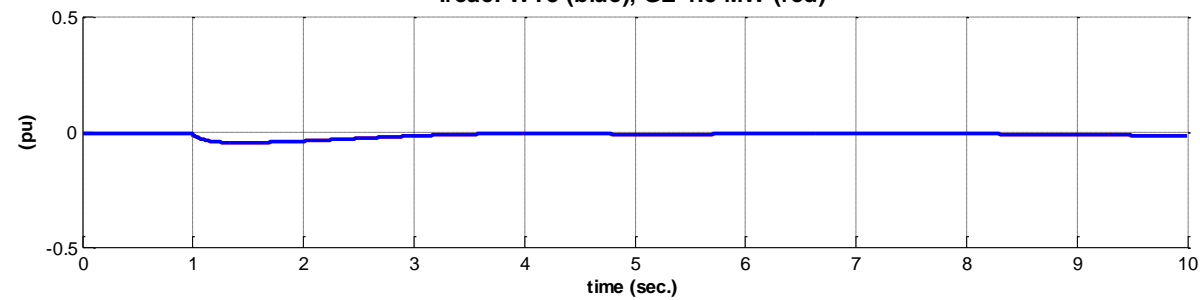

Figure 7-58: Parameterization of WT3 vs GE-1.5 MW - Case 2 and $S C R=10$ ( $\left.V_{\text {Term }}, I_{\text {Real }}, I_{\text {Reac }}\right)$. 


\subsection{Case 3-Over-Frequency Event}

\section{$\underline{\mathrm{SCR}-5}$}

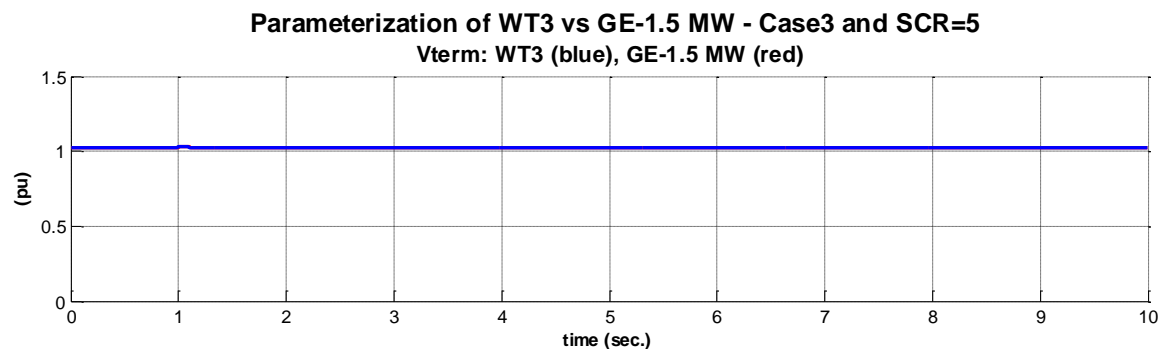

Pgen: WT3 (blue), GE-1.5 MW (red)

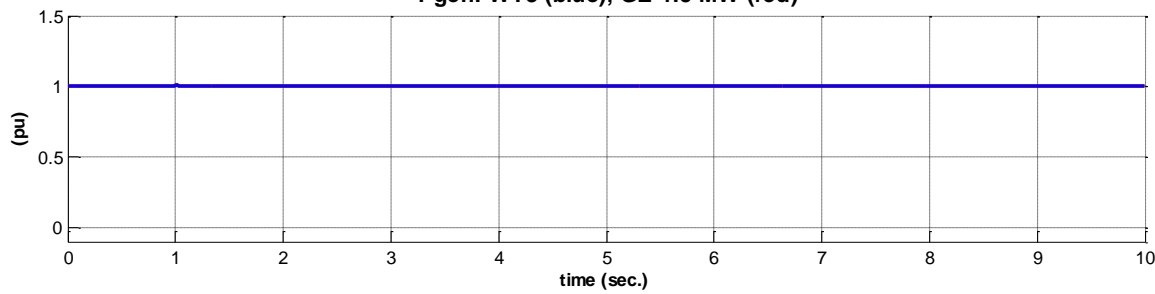

Qgen: WT3 (blue), GE-1.5 MW (red)

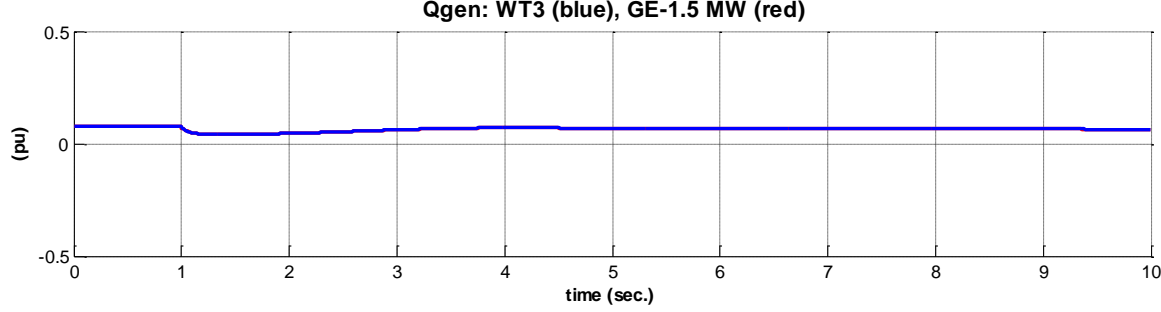

Figure 7-59: Parameterization of WT3 vs GE-1.5 MW - Case 3 and $S C R=5\left(V_{T e r m}, P, Q\right)$.

Parameterization of WT3 vs GE-1.5 MW - Case 3 and SCR=5 Vterm: WT3 (blue), GE-1.5 MW (red)

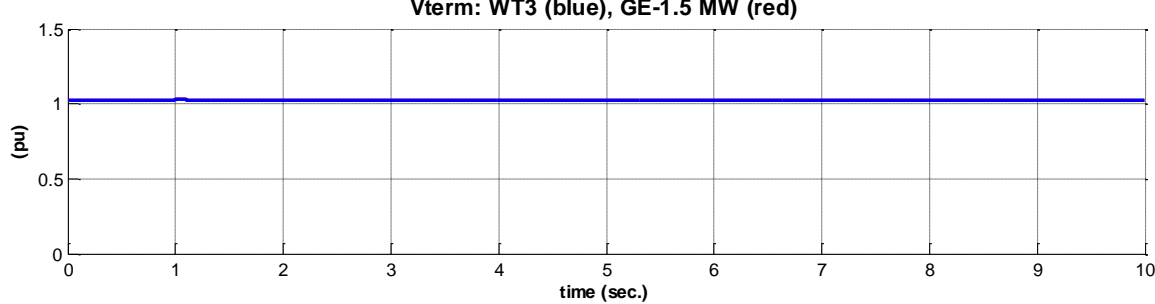

Ireal: WT3 (blue), GE-1.5 MW (red)
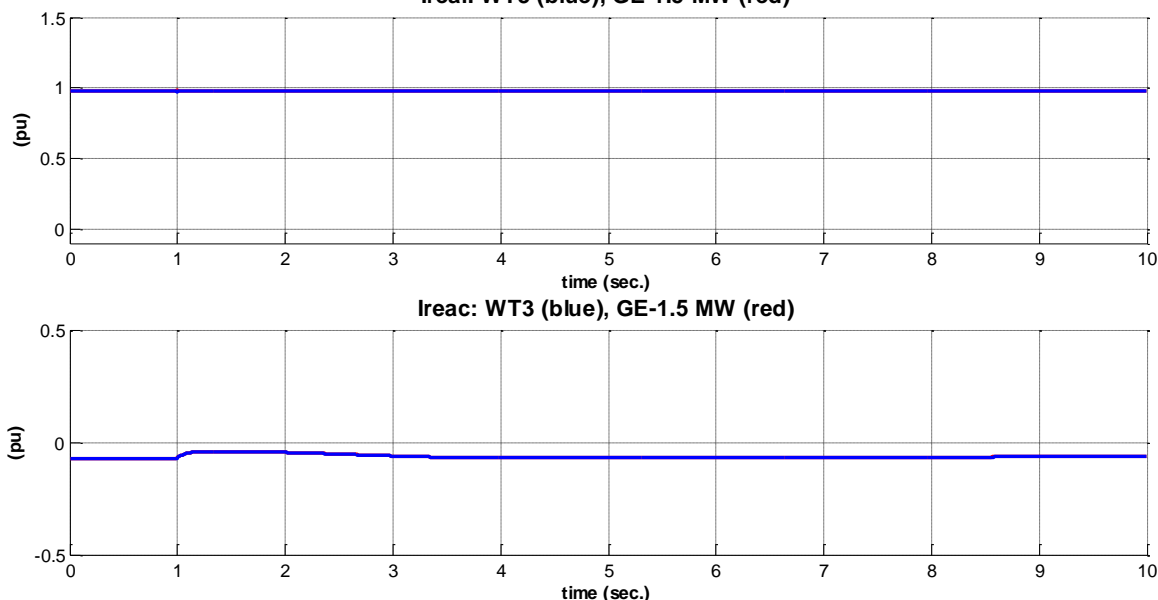

Figure 7-60: Parameterization of WT3 vs GE-1.5 MW - Case 3 and $S C R=5\left(V_{\text {Term }}, I_{\text {Real }}, I_{\text {Reac }}\right)$. 
$\underline{\text { SCR }-10}$

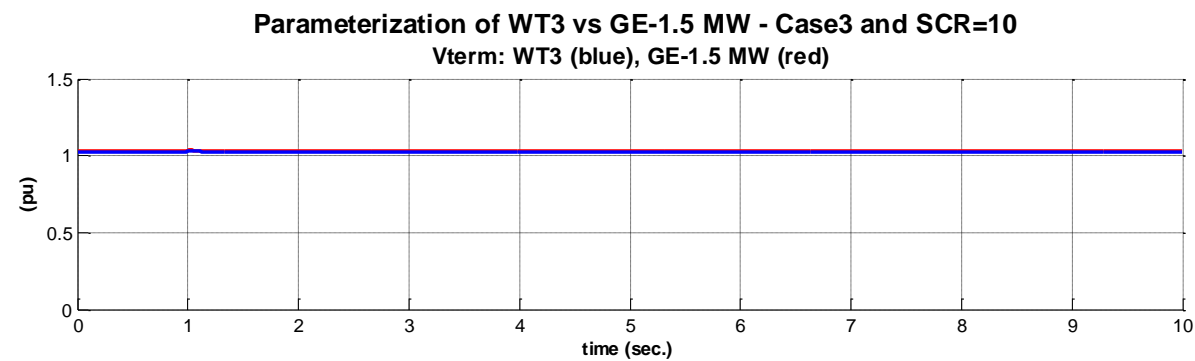

Pgen: WT3 (blue), GE-1.5 MW (red)

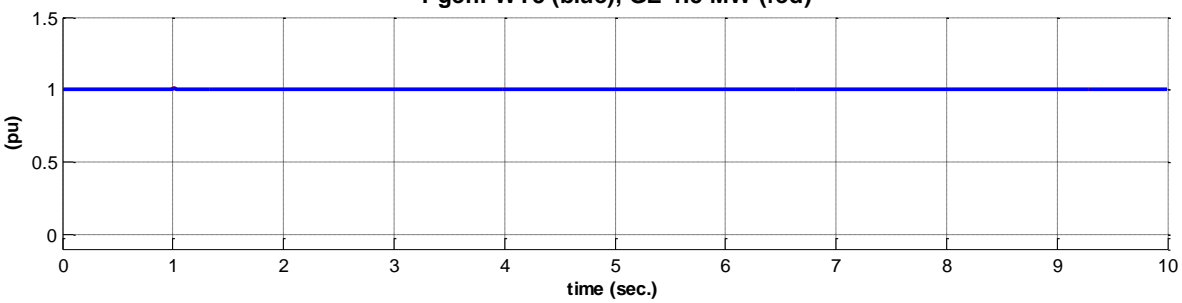

Qgen: WT3 (blue), GE-1.5 MW (red)

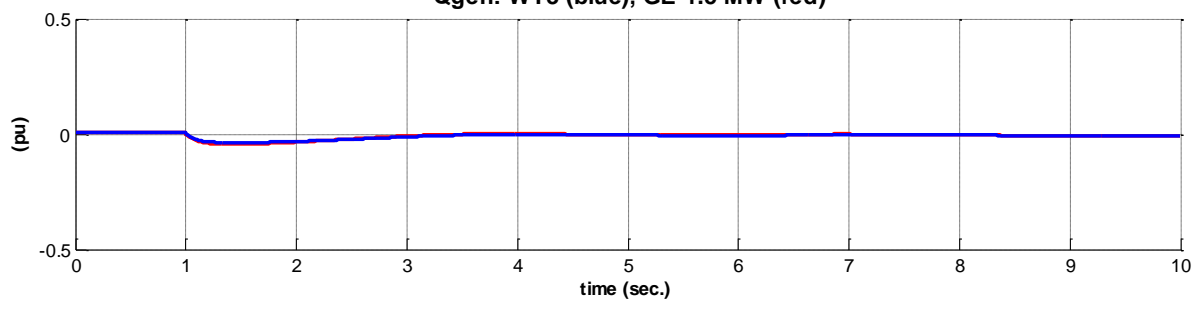

Figure 7-61: Parameterization of WT3 vs GE-1.5 MW - Case 3 and SCR=10 ( $\left.V_{\text {Term }}, P, Q\right)$.

Parameterization of WT3 vs GE-1.5 MW - Case 3 and SCR $=10$

Vterm: WT3 (blue), GE-1.5 MW (red)

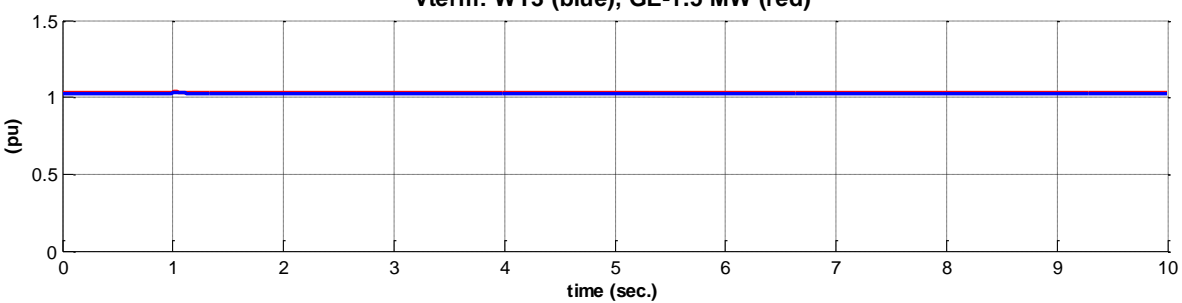

Ireal: WT3 (blue), GE-1.5 MW (red)

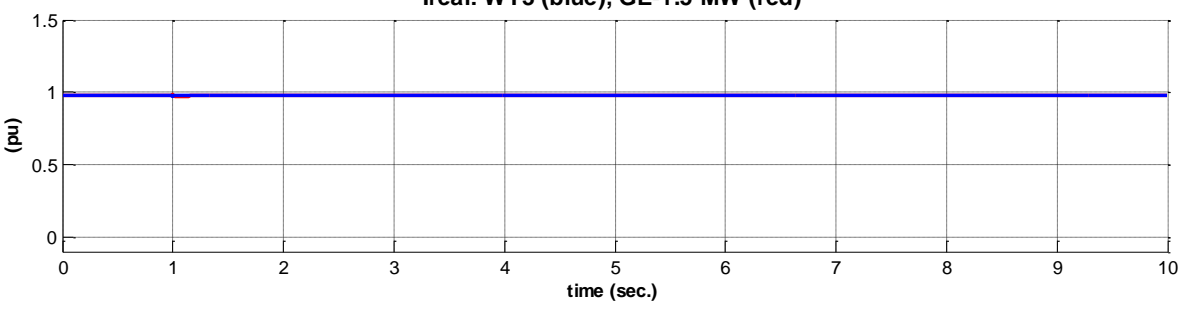

Ireac: WT3 (blue), GE-1.5 MW (red)

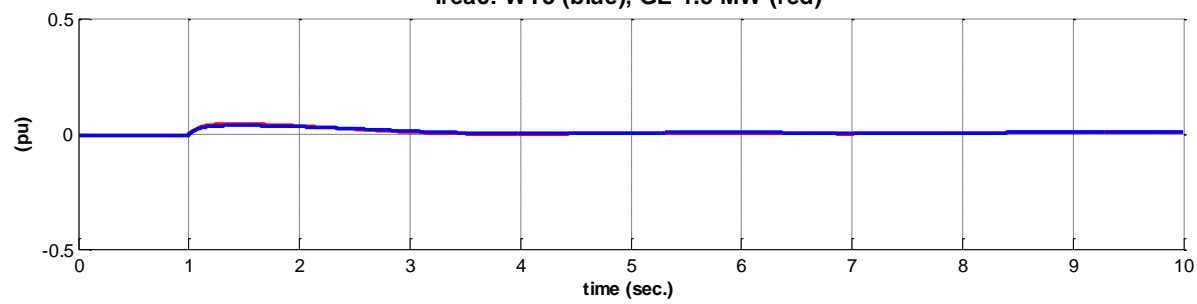

Figure 7-62: Parameterization of WT3 vs GE-1.5 MW - Case 3 and $S C R=10$ ( $\left.V_{\text {Term, }}, I_{\text {Real }}, I_{\text {Reac }}\right)$. 


\subsubsection{Results Validation}

In all figures, the response of the generic model matches the response of the VSM in the frequency range of interest. Thus, both the PSLF and the PSSE models are an appropriate representation of the GE 1.5 MW WTG's behavior for fundamental frequency analysis.

\subsubsection{Vendor Specific Model Validation ${ }^{1}$}

The dynamic performance of the voltage and reactive power at the POI of a WPP for a capacitor switching event is documented in this short section. Specifically, in Figure 7-63 and Figure 7-64, field test measurements are compared against the VSM PSLF GE 1.5 WTG model and a parameterized Generic Type III WTG model. The figures show a close match between the simulated variables and their corresponding measurements and validate the feasibility of using a generic model for the system and event studied.

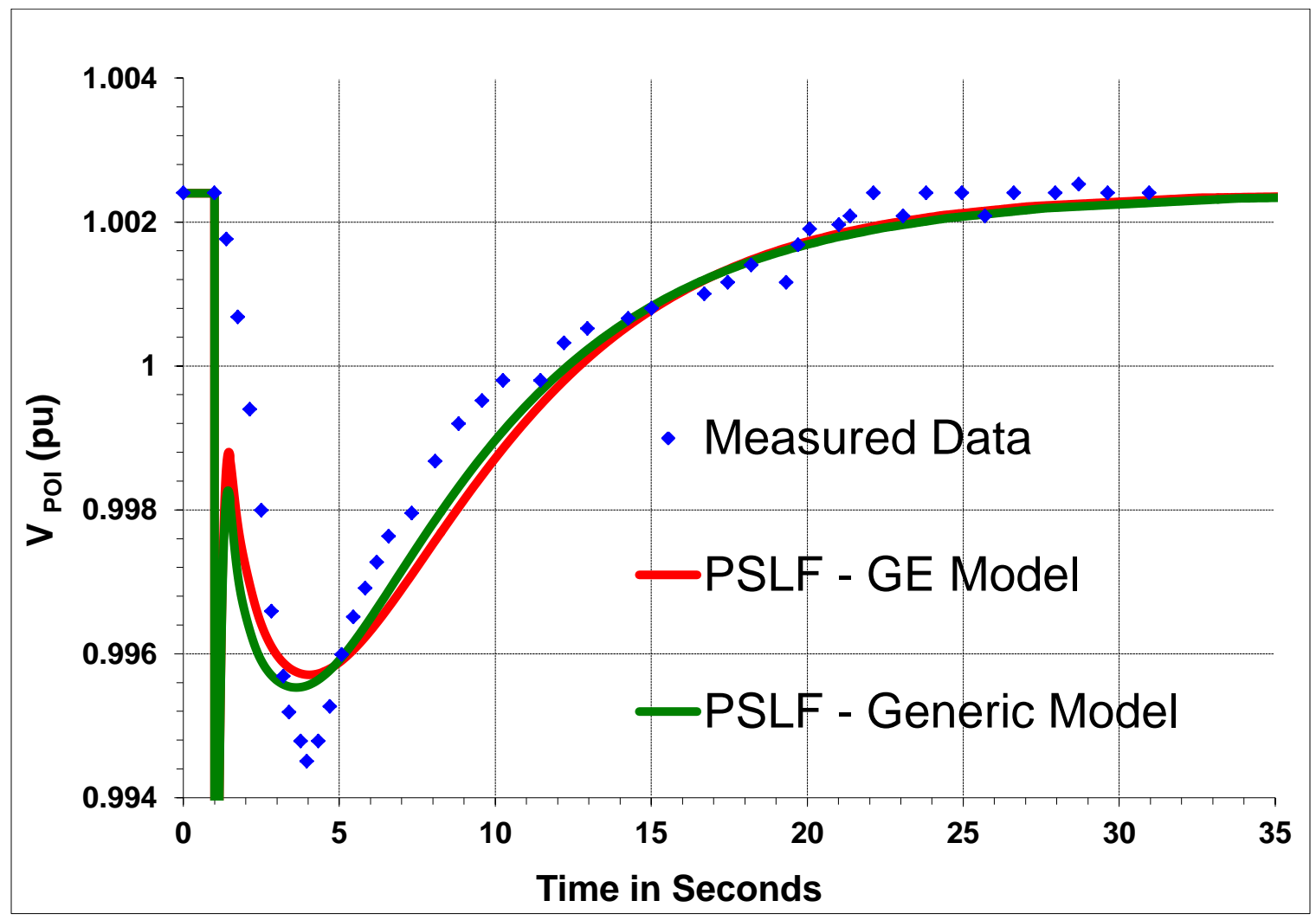

Figure 7-63: POI Voltage - test measurements vs. GE-VSM and Generic Type III simulations.

\footnotetext{
${ }^{1}$ All figures in this Section are courtesy of GE
} 


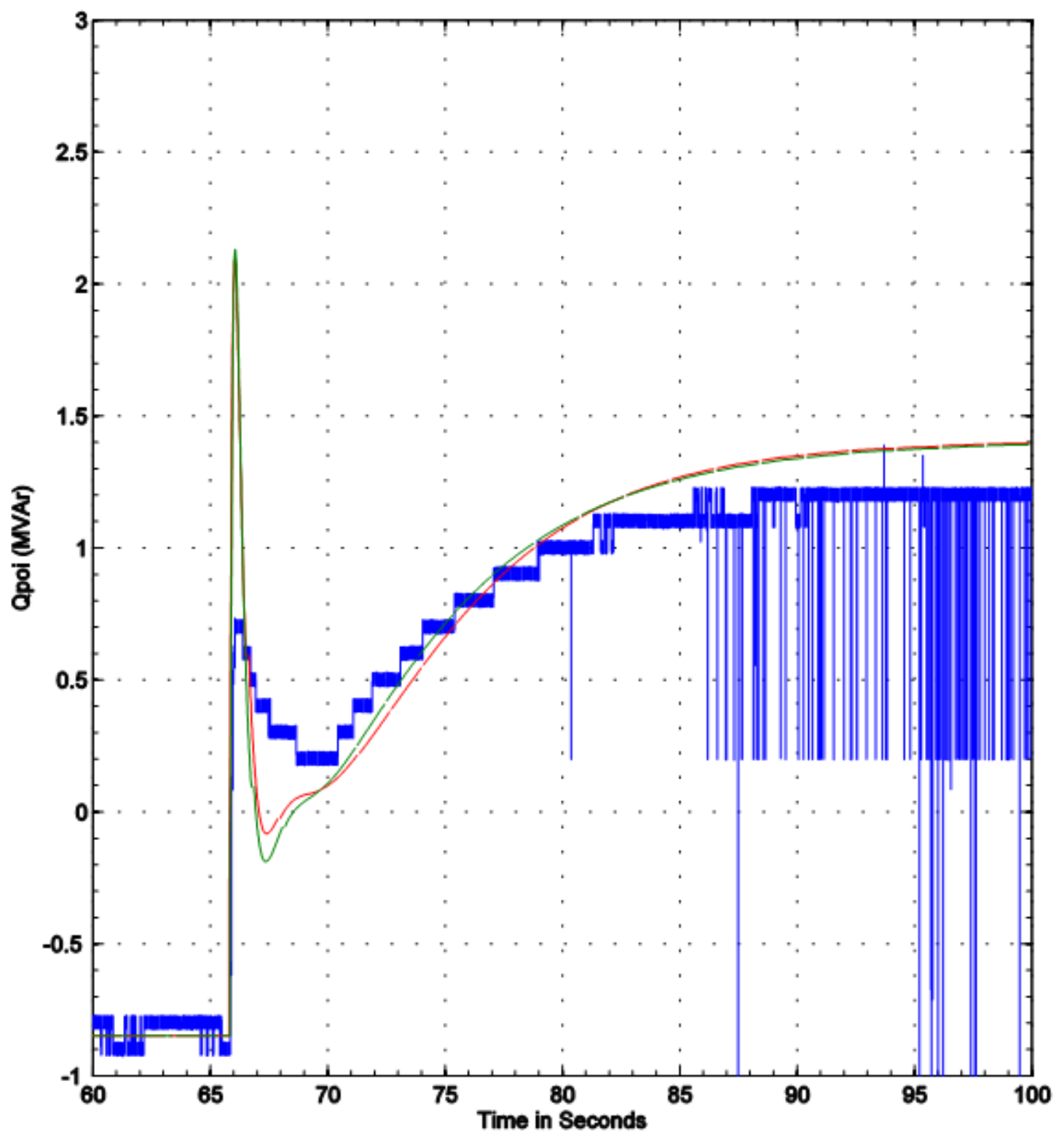

Figure 7-64: POI Reactive Power - test measurements (blue) vs. GE-VSM (red) and Generic Type III (green) simulations. 


\subsection{General Electric - 2.5 MW Series}

\subsubsection{Wind Turbine Characterization}

GE's 2.5 MW series is represented by three-blade, upwind, horizontal axis wind turbines with a rated capacity of 2.5-megawatts, with following features:

- Rotor Diameter: $100 \mathrm{~m}$ and $103 \mathrm{~m}(\mathrm{ft})$.

- Hub Heights: $85 \mathrm{~m}$ (ft).

- $\quad$ Cut-in wind speed: 3.0 (9 mph).

- $\quad$ Cut-out wind speed: $25 \mathrm{~m} / \mathrm{s}(55 \mathrm{mph})$.

- Terminal Voltage: $690 \mathrm{~V}(50 \mathrm{~Hz} / 60 \mathrm{~Hz})$.

- Power Control: Active blade pitch control.

- Generator: Permanent magnet.

- Power Converter: Full converter.

- Braking System: Electric drive pitch control with battery backup; Hydraulic parking brake.

The rotor on a GE $2.5 \mathrm{MW}$ turbine is designed to operate in an upwind configuration at 5 to 14 revolutions per minute (rpm). Rotor speed is regulated by a combination of blade pitch angle adjustment and generator/converter torque control. The 2.5 MW series provides the option of a selectable power factor between 0.90 (overexcited) and 0.90 (underexcited) and also a VAR boost, which will override watts production to deliver more VARs during emergency under-voltage conditions. 


\subsubsection{Vendor Specific Model Characterization}

\subsubsection{Model Performance: Case 1 - Fault Event}

In this case a remote fault is applied to bus 12 for a duration of 6 cycles $(0.1 \mathrm{sec})$.

$\underline{\mathrm{SCR}-5}$
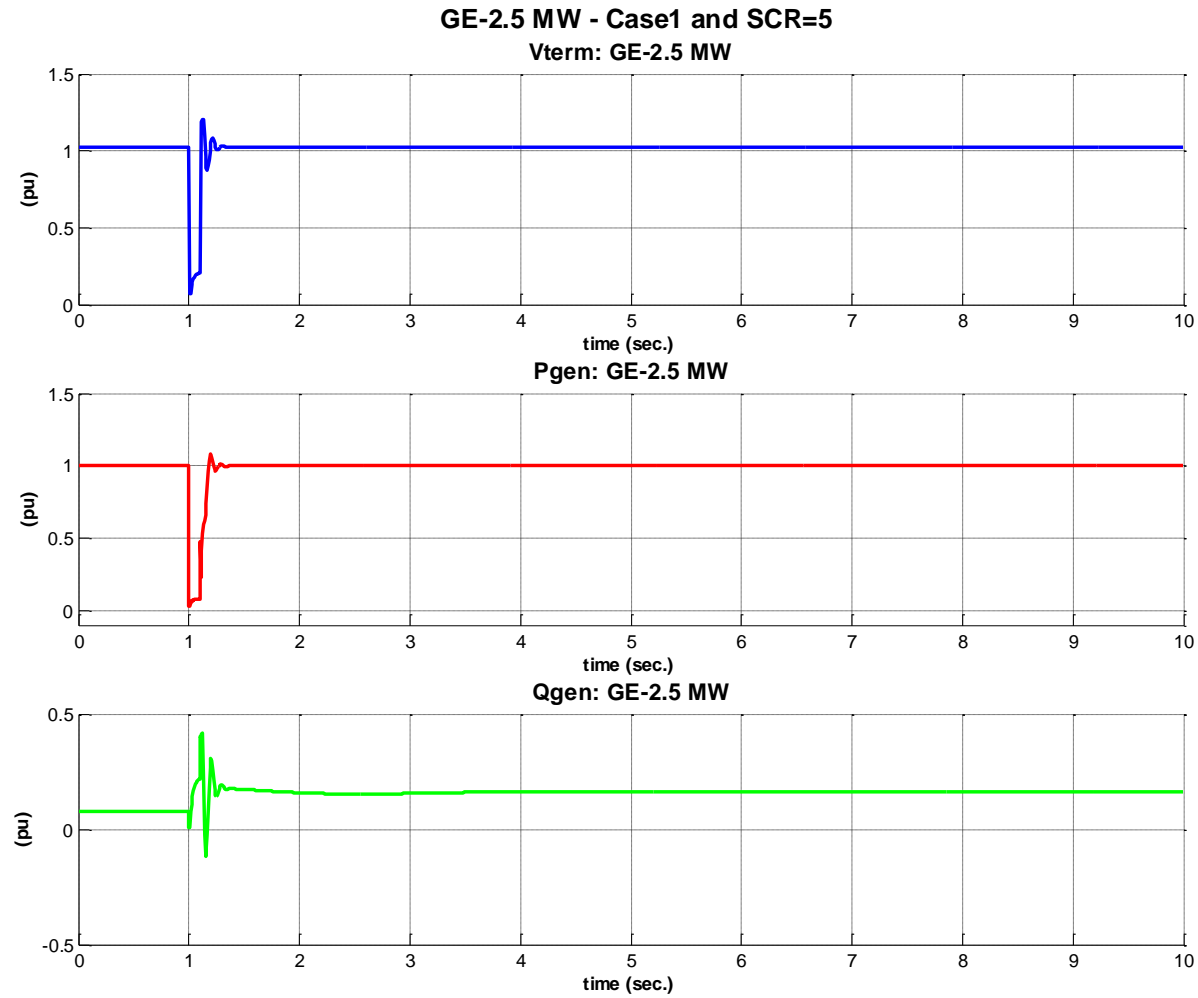

Figure 7-65: GE-2.5 MW - Case 1 and $S C R=5\left(V_{\text {Term }}, P, Q\right)$. 

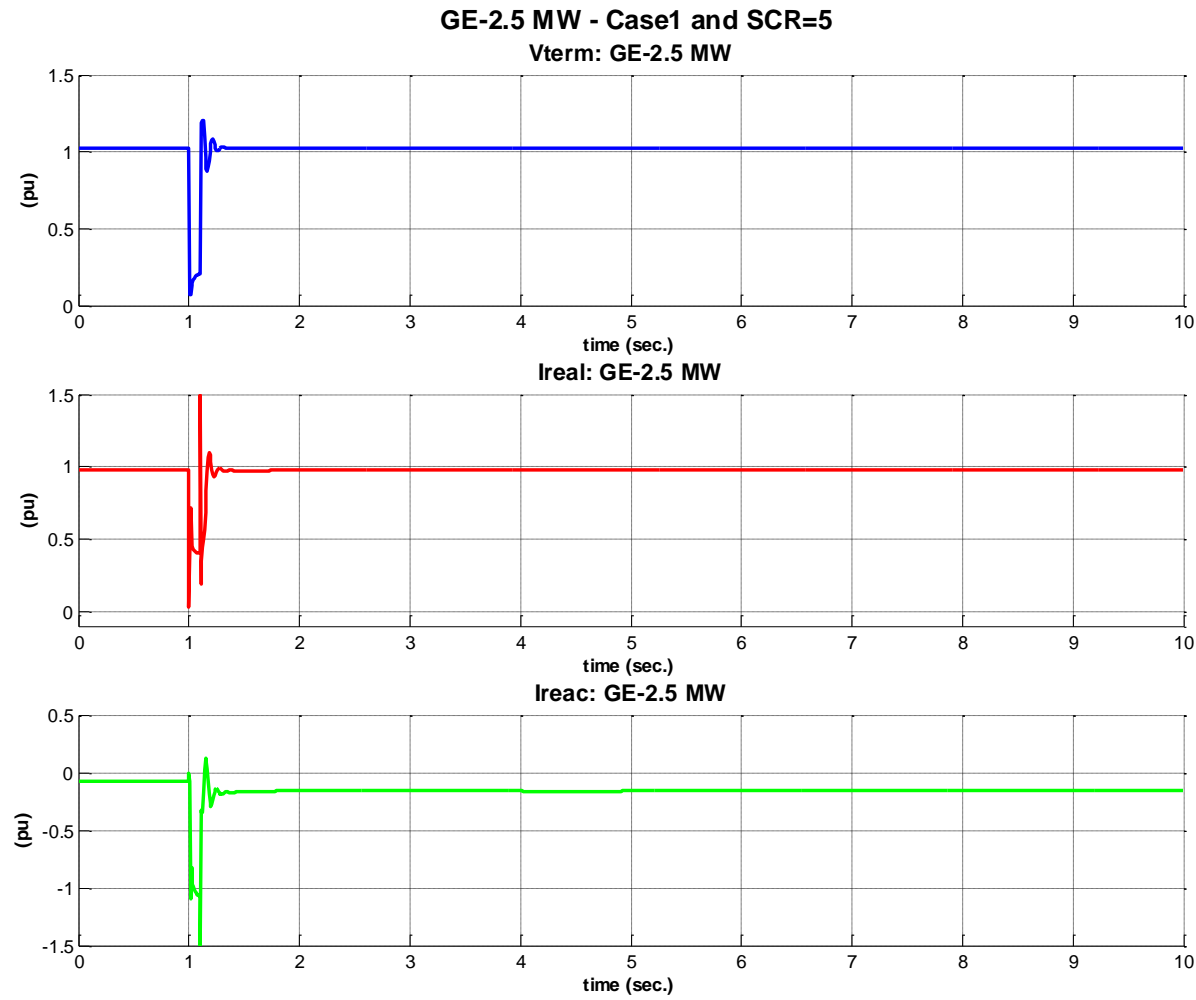

Figure 7-66: GE-2.5 MW - Case 1 and $S C R=5\left(V_{\text {Term, }} I_{\text {Real }}, I_{\text {Reac }}\right)$.

SCR - 10
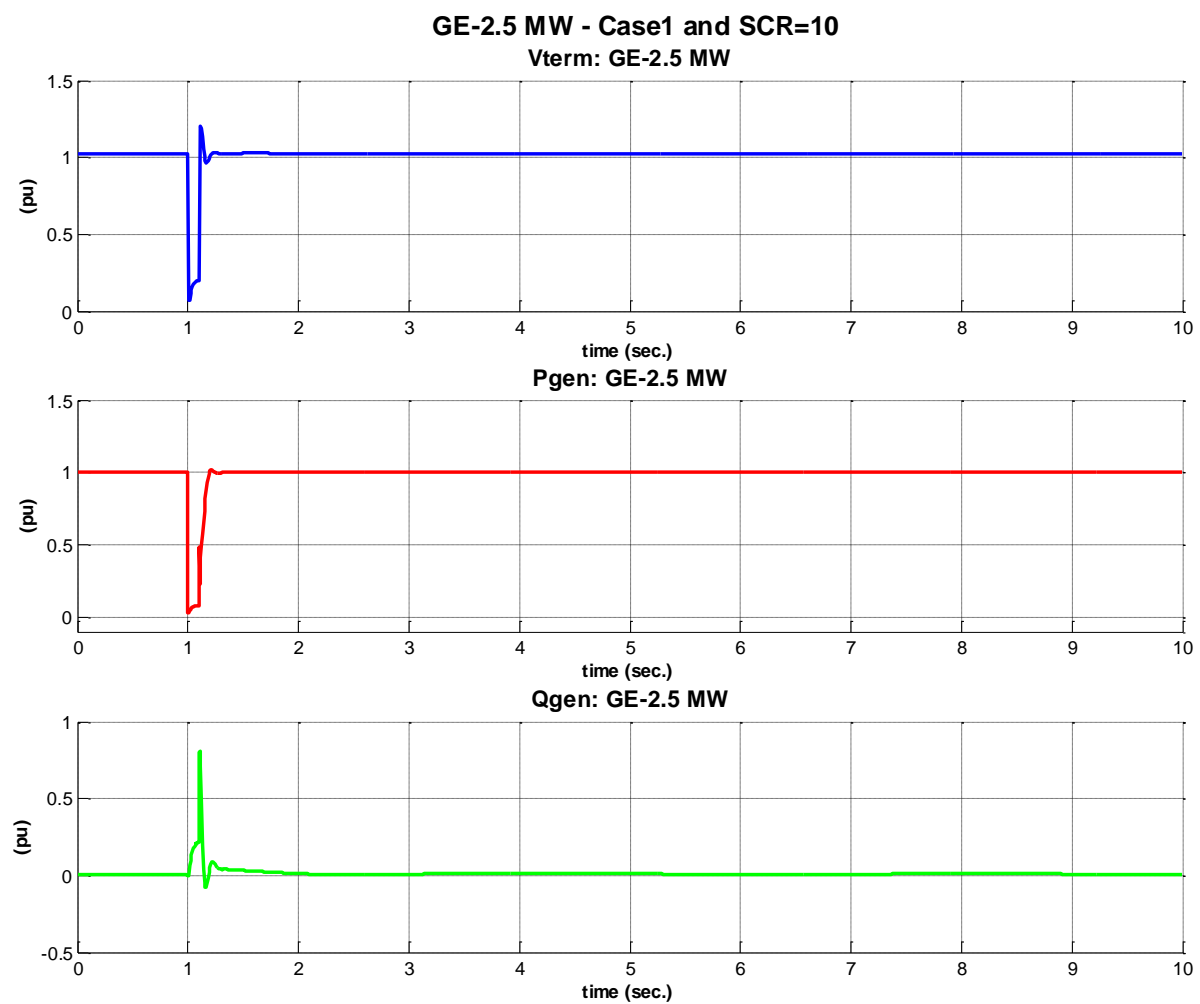

Figure 7-67: GE-2.5 MW - Case 1 and $\mathrm{SCR}=10\left(\mathrm{~V}_{\text {Term }}, \mathrm{P}, \mathrm{Q}\right)$. 

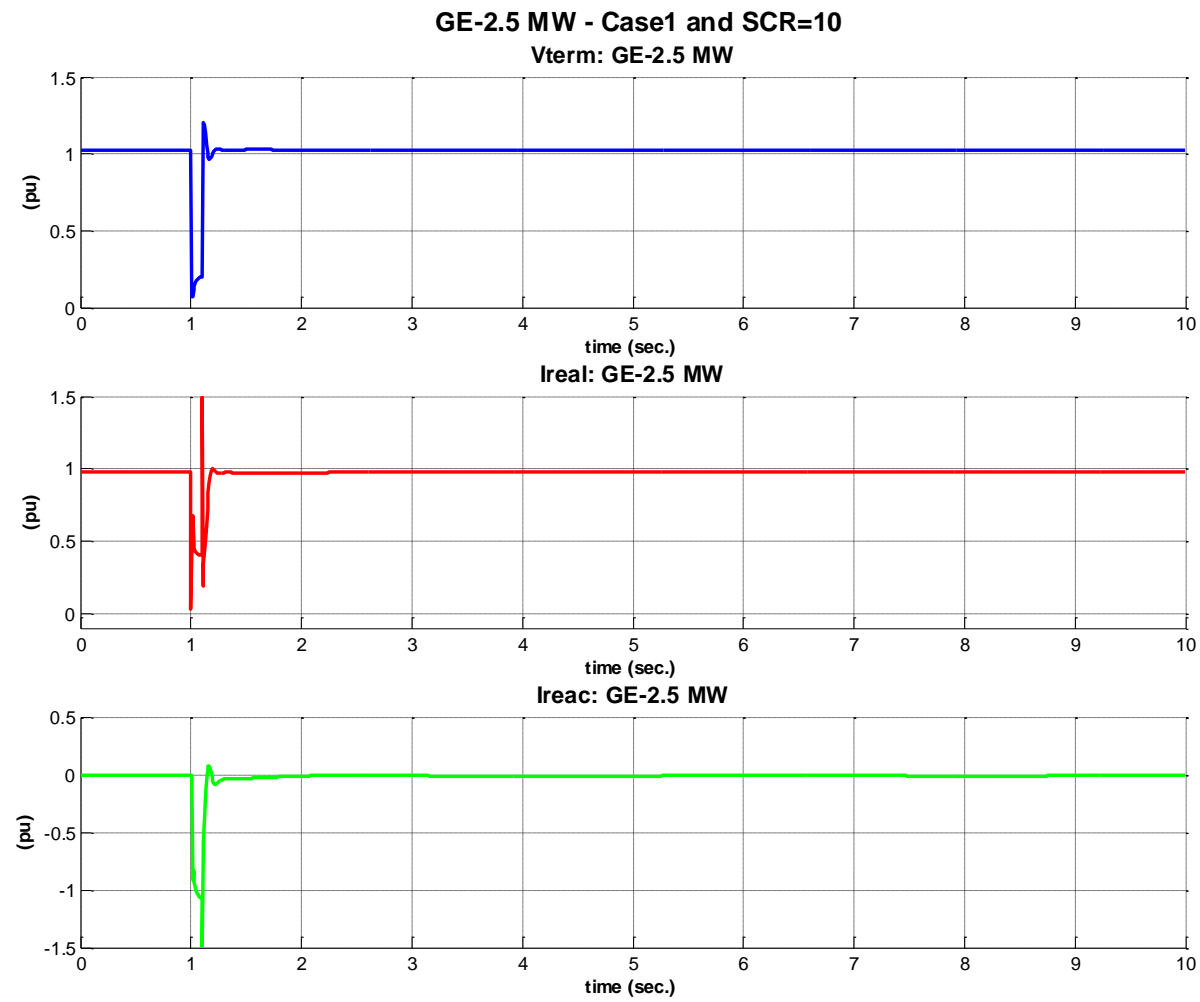

Figure 7-68: GE-2.5 MW - Case 1 and $S C R=10\left(V_{\text {Term }}, I_{\text {Real }}, I_{\text {Reac }}\right)$. 


\subsubsection{Model Performance: Case 2 - Under-Frequency Event}

In this case a under frequency event is created by tripping the 100 MVA generation unit at bus 20 .

\section{SCR -5}
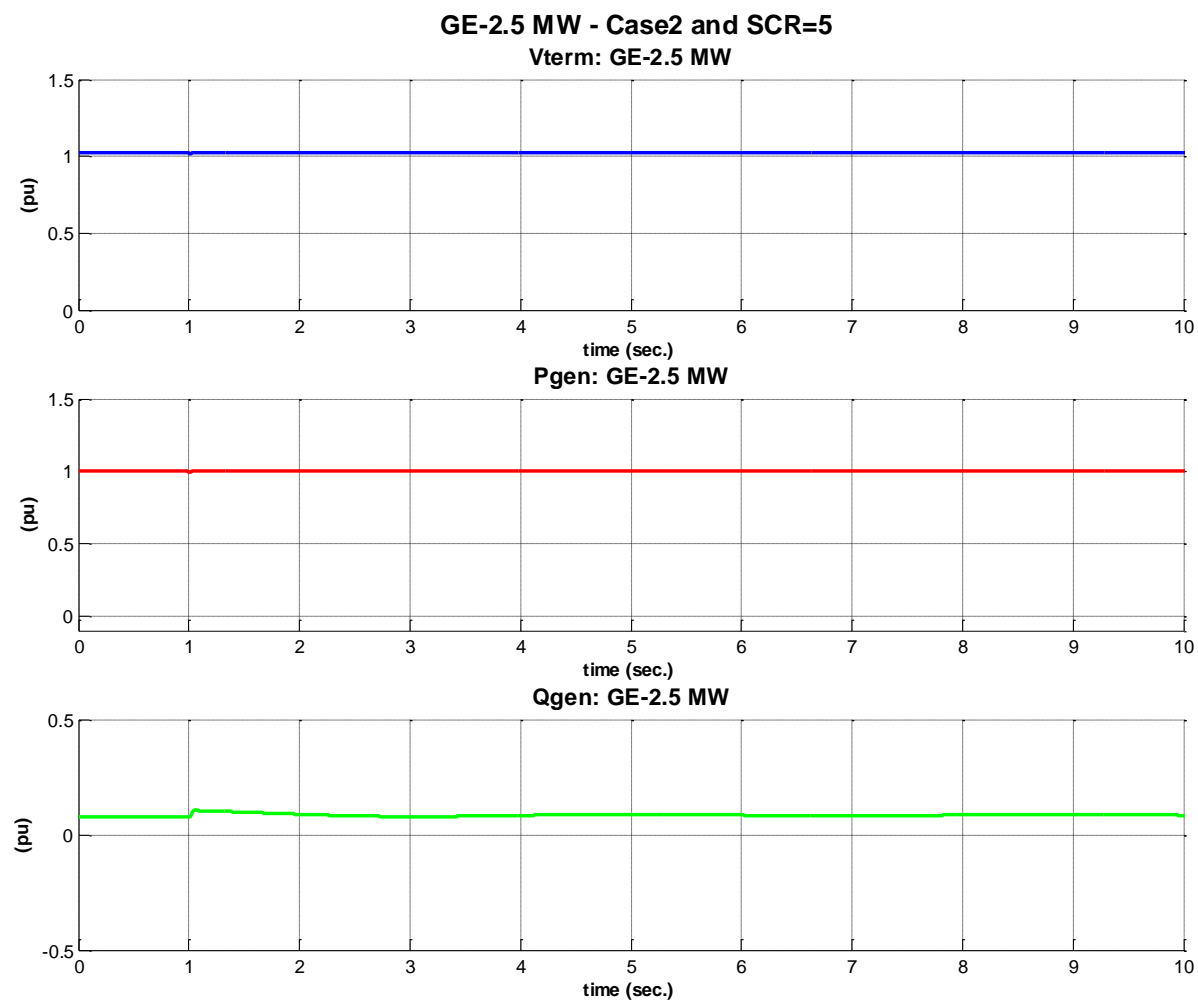

Figure 7-69: GE-2.5 MW - Case 2 and $\mathrm{SCR}=5\left(\mathrm{~V}_{\text {Term }}, \mathrm{P}, \mathrm{Q}\right)$. 

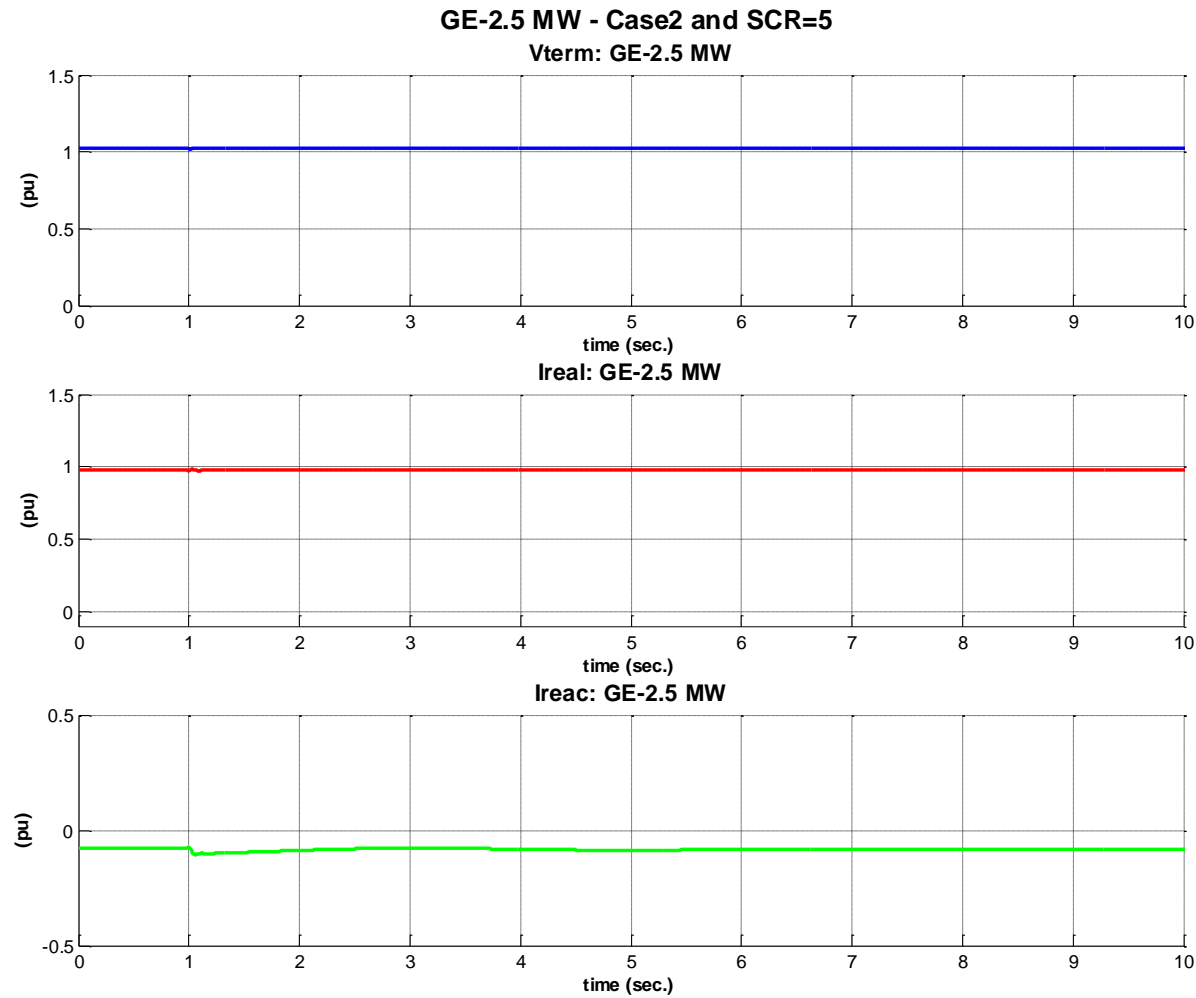

Figure 7-70: GE-2.5 MW - Case 2 and $S C R=5\left(V_{\text {Term, }} I_{\text {Real }}, I_{\text {Reac }}\right)$.

\section{SCR -10}
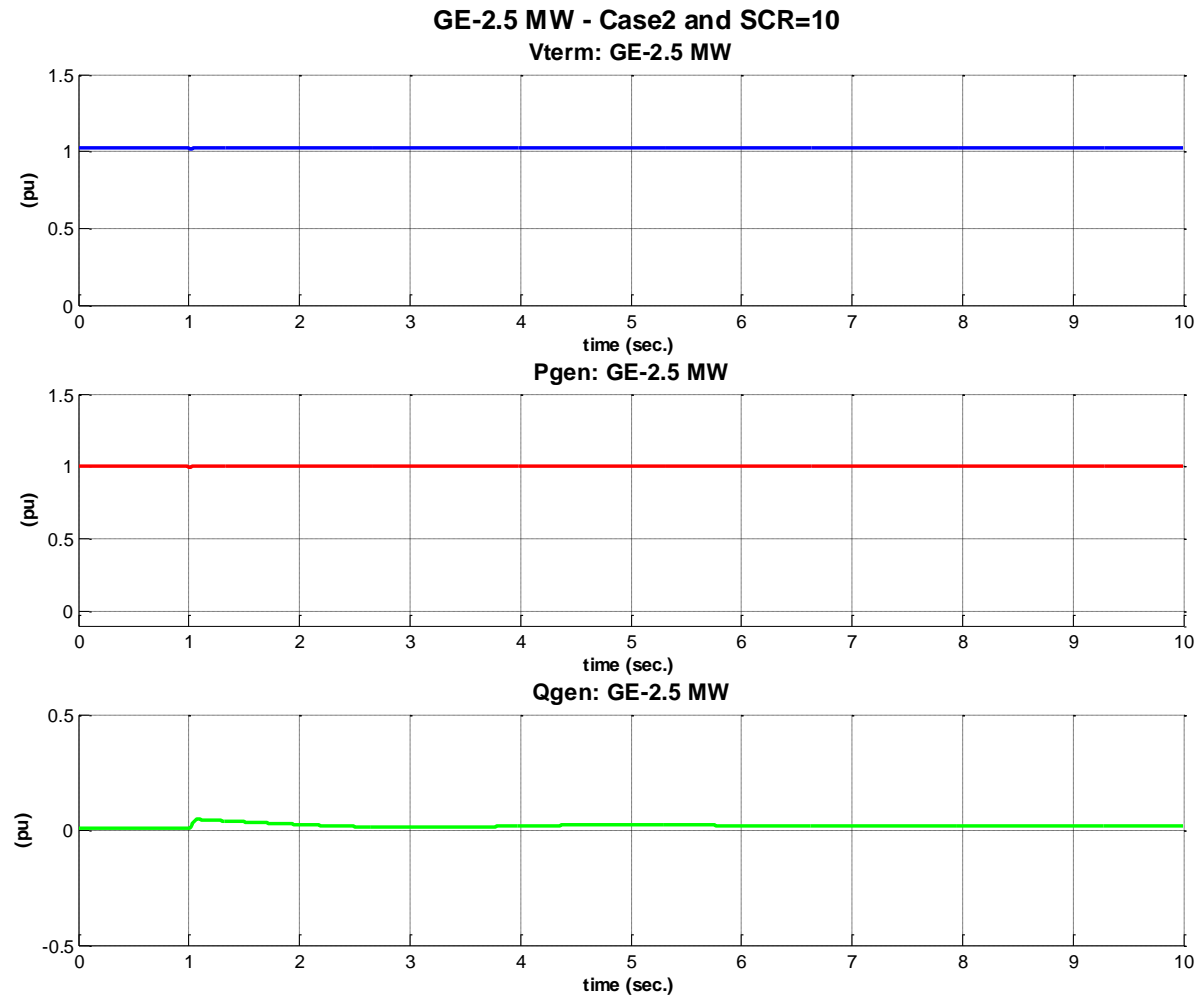

Figure 7-71: GE-2.5 MW - Case 2 and $\mathrm{SCR}=10\left(\mathrm{~V}_{\text {Term }}, \mathrm{P}, \mathrm{Q}\right)$. 

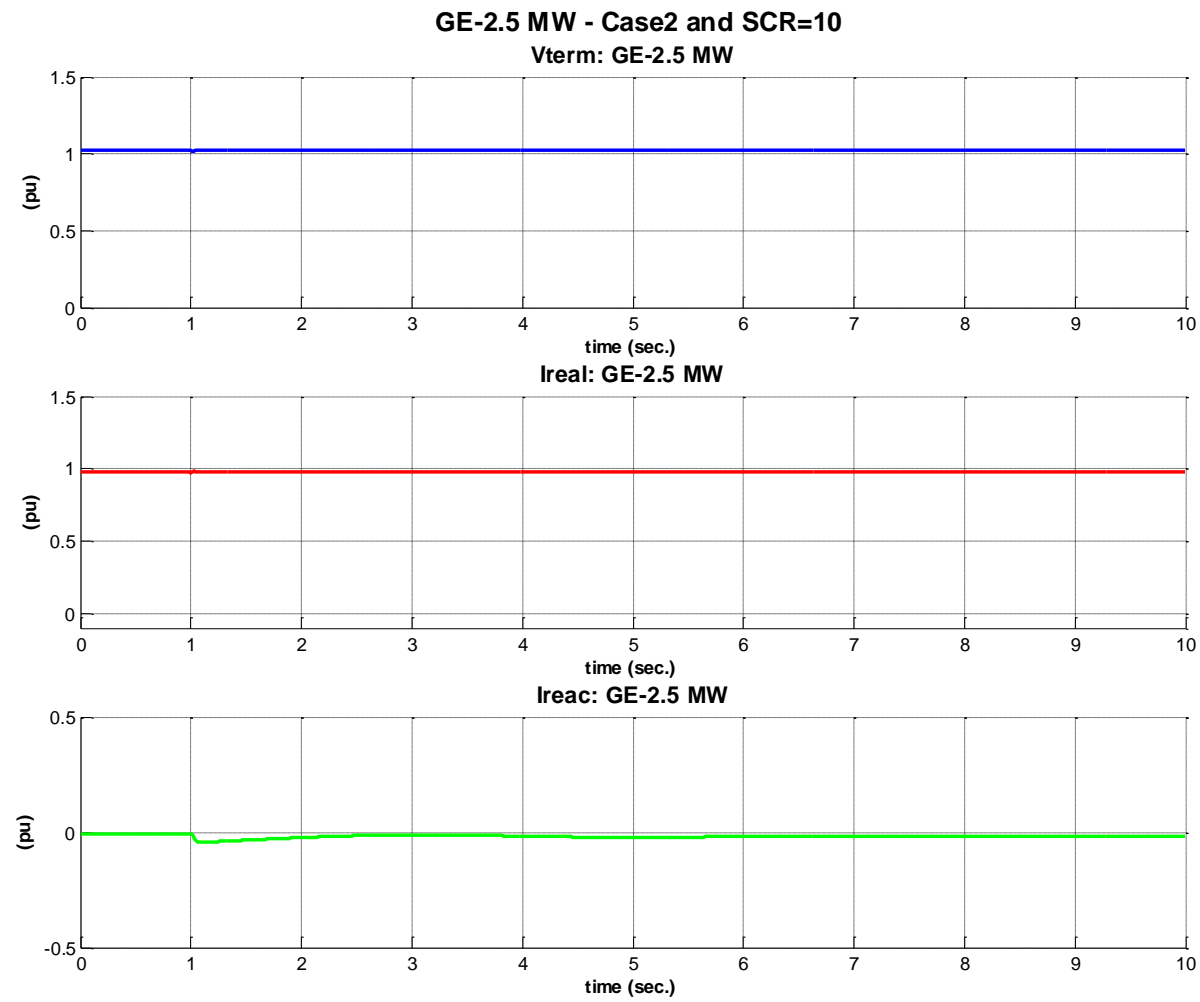

Figure 7-72: GE-2.5 MW - Case 2 and $S C R=10\left(V_{\text {Term }}, I_{\text {Real, }}, I_{\text {Reac }}\right)$. 


\subsubsection{Model Performance: Case 3-Over-Frequency Event}

In this case a under frequency event is created by tripping the 100 MVA load at bus 11.

$\underline{\mathrm{SCR}-5}$
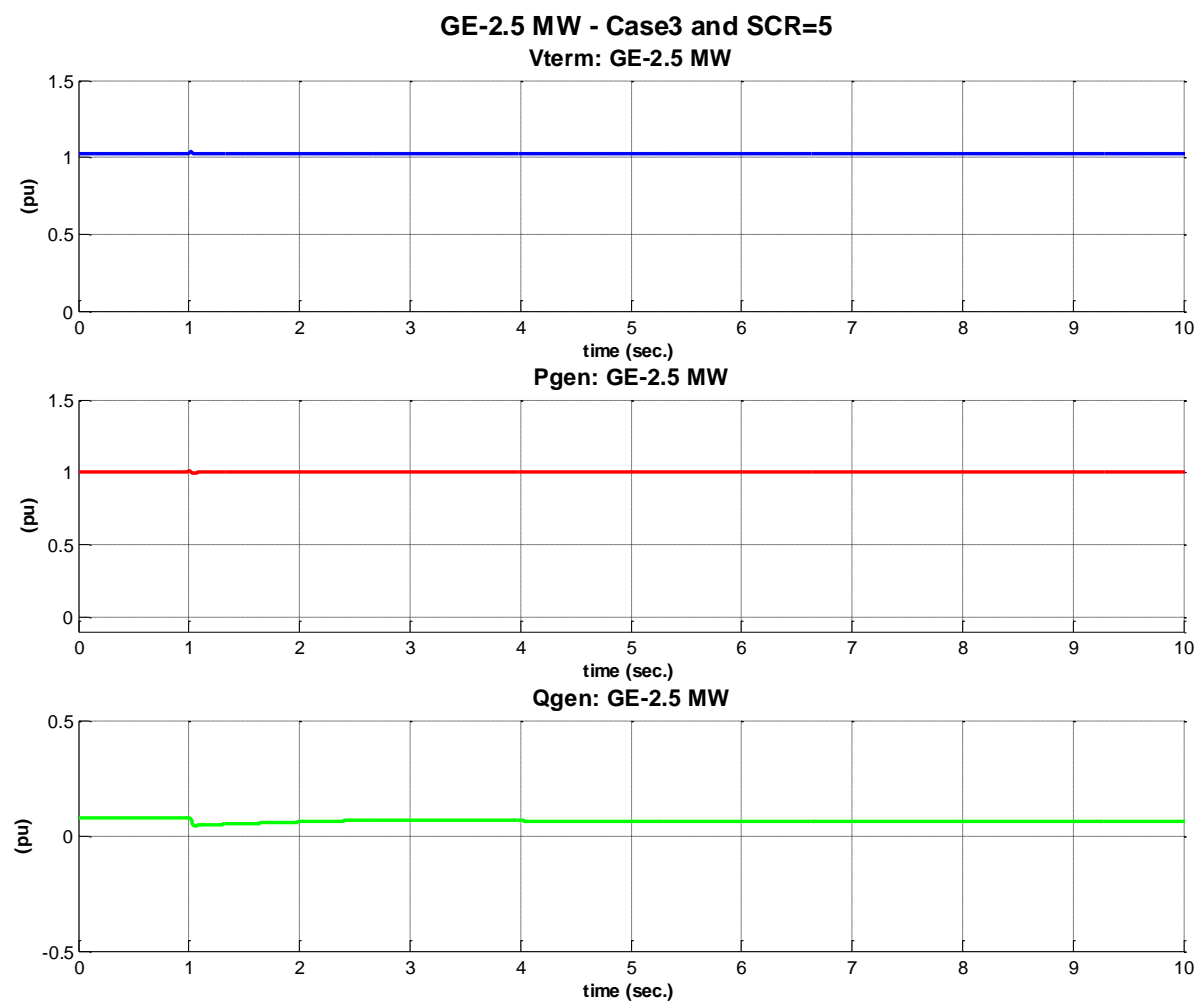

Figure 7-73: GE-2.5 MW - Case 3 and $\mathrm{SCR}=5\left(\mathrm{~V}_{\text {Term }}, \mathrm{P}, \mathrm{Q}\right)$. 

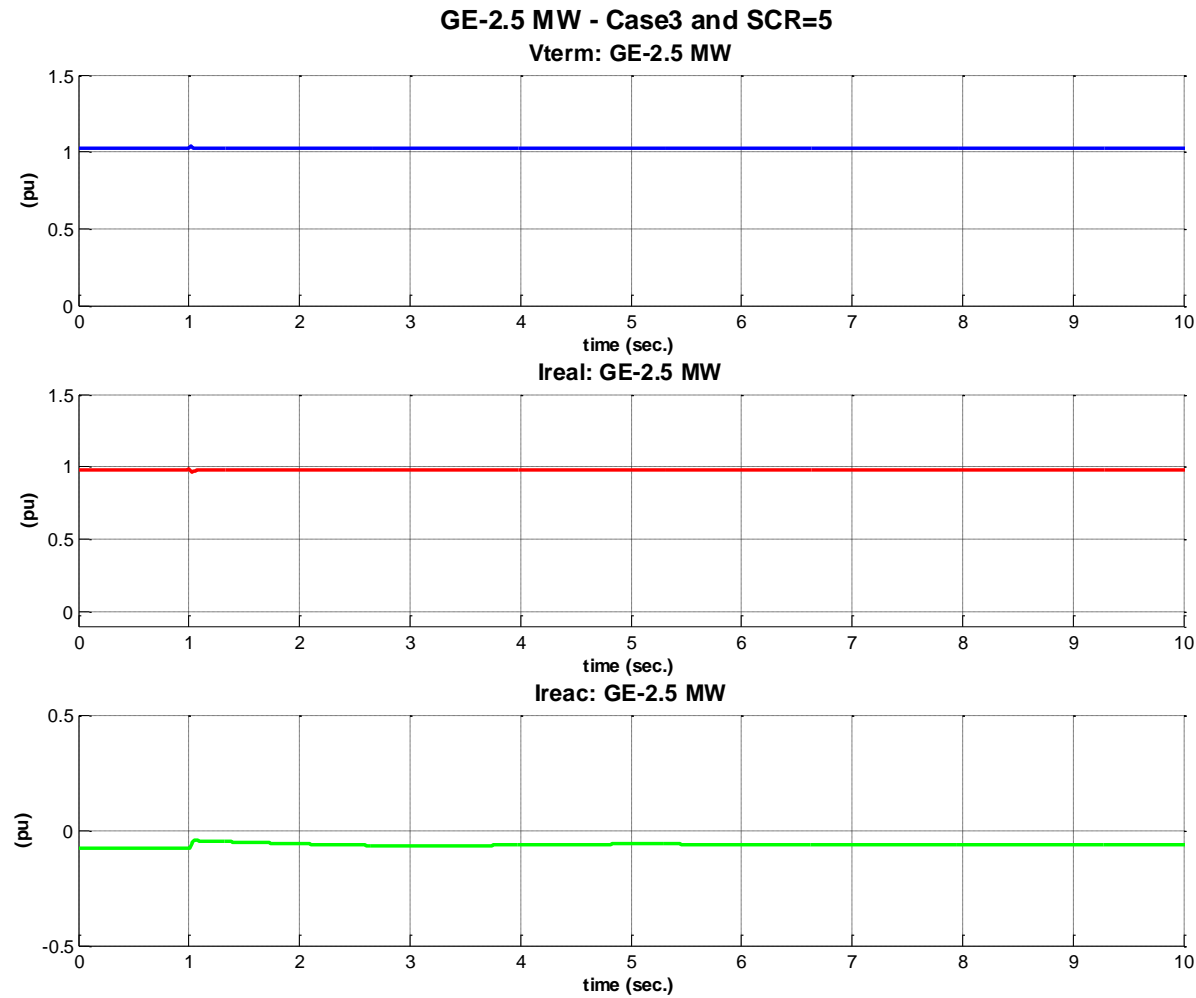

Figure 7-74: GE-2.5 MW - Case 3 and $S C R=5\left(V_{\text {Term, }} I_{\text {Real }}, I_{\text {Reac }}\right)$.

\section{SCR -10}
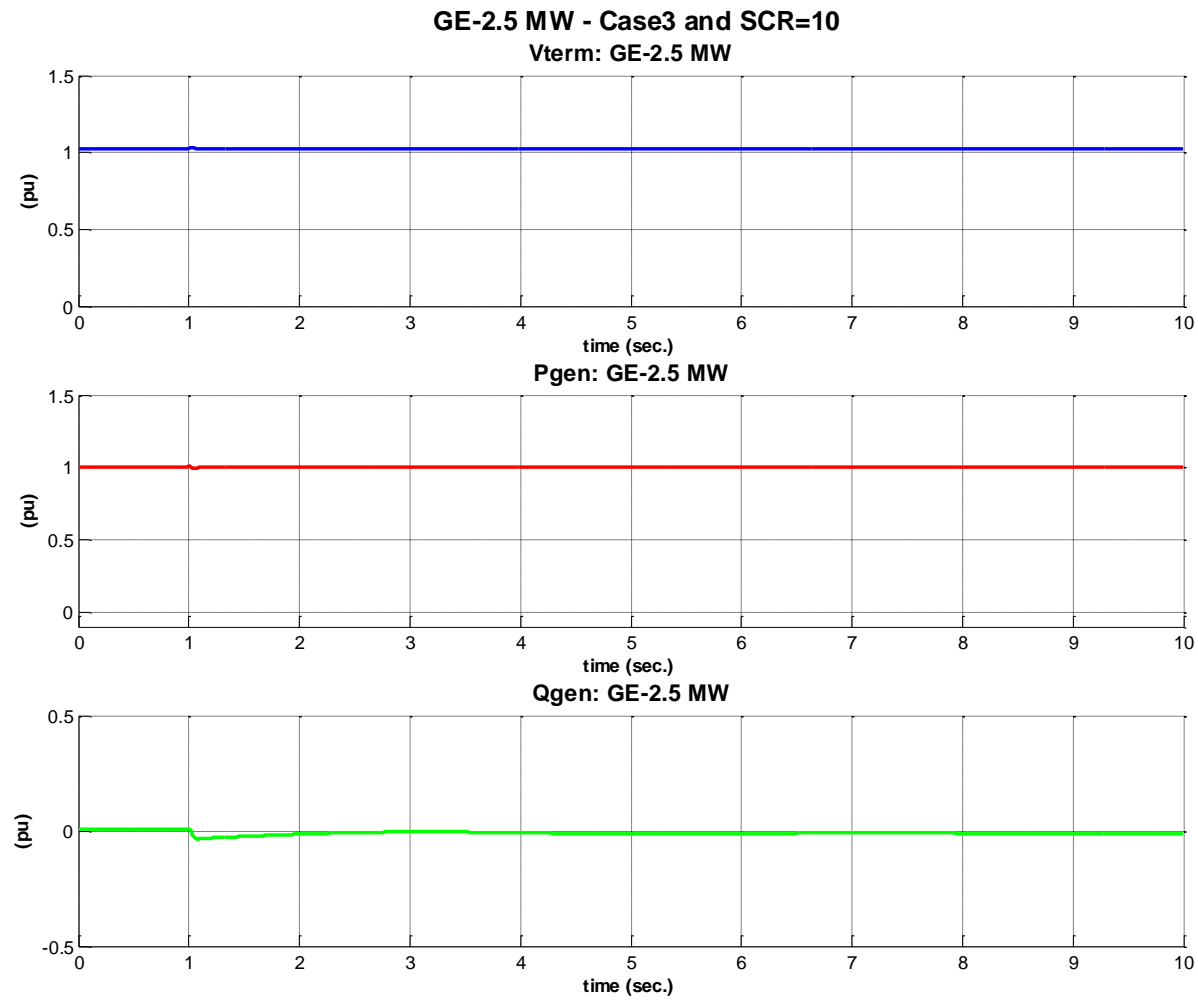

Figure 7-75: GE-2.5 MW - Case 3 and SCR=10 ( $\left.\mathrm{V}_{\text {Term }}, \mathrm{P}, \mathrm{Q}\right)$. 

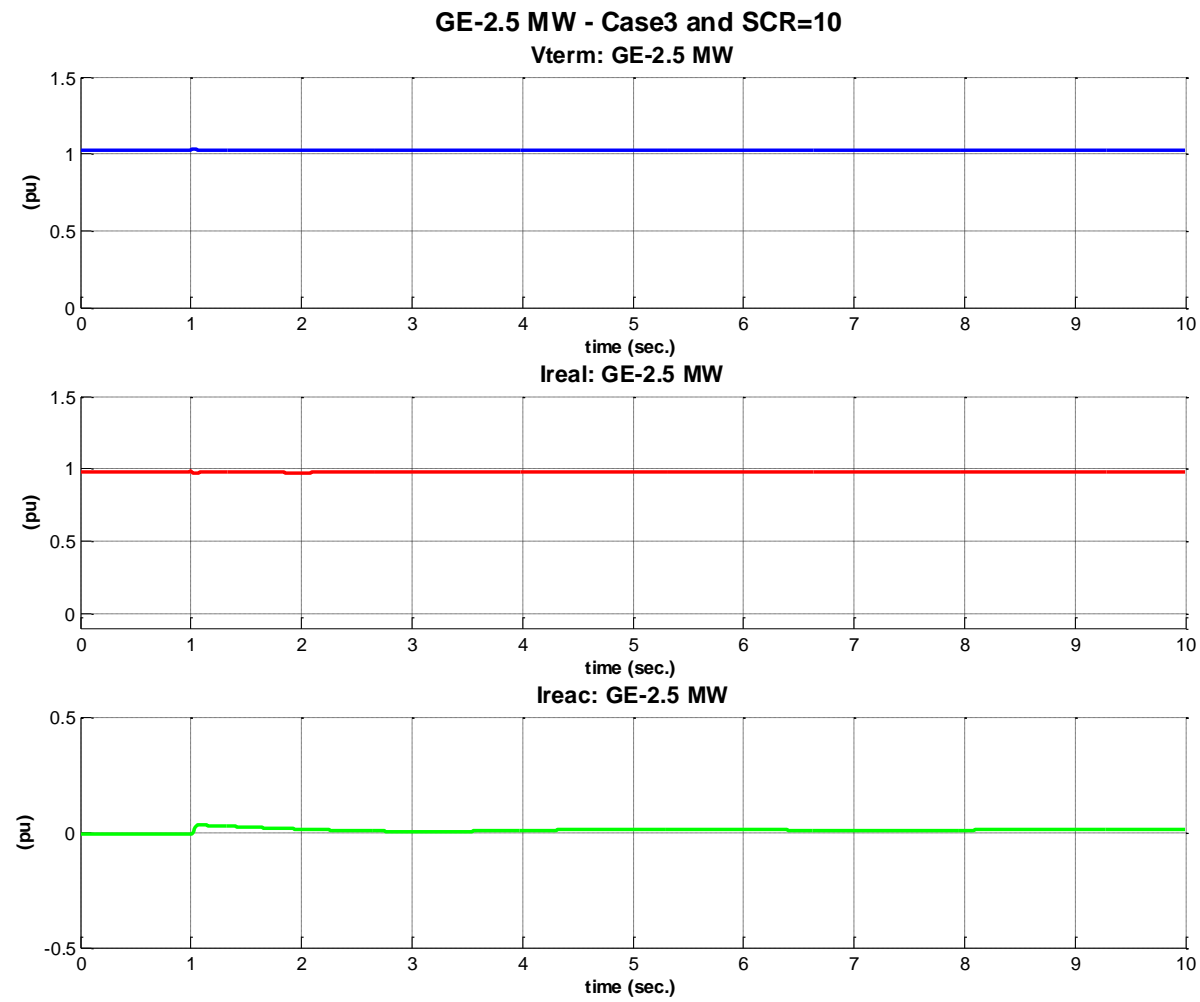

Figure 7-76: GE-2.5 MW - Case 3 and $S C R=10\left(V_{\text {Term }}, I_{\text {Real }}, I_{\text {Reac }}\right)$. 


\subsubsection{Generic Model Parameterization}

\subsubsection{PSLF Environment ${ }^{1}$}

The PSLF platform generic wind turbine parameters, to mimic the behavior of GE's 2.5 MW series, are shown in Table 7-4. The parameter values shown in the table resulted from a compromise between the simulated cases (i.e. SCR 5 and SCR 10) and network conditions (i.e. fault, under- and over frequency).

Table 7-4: PSLF generic model parameter values.

\begin{tabular}{|c|c|c|c|c|c|}
\hline \multicolumn{2}{|c|}{ wt4g } & \multicolumn{2}{|c|}{ wt4t } & \multicolumn{2}{|c|}{ wt4e } \\
\hline Lvplsw & 1.0 & Tpw & 0.05 & Varflg & 1 \\
\hline Rrpwr & 10.0 & Kpp & 0.08 & Kqi & 0.1 \\
\hline Brkpt & 0.9 & Kip & 0.10 & Kvi & 0.001 \\
\hline Zerox & 0.4 & Tf & 0.08 & Vmax & 1.1 \\
\hline Lvpll & 1.22 & $\mathrm{Kf}$ & 0.00 & Vmin & 0.9 \\
\hline Volim & 1.2 & $\mathrm{dPmx}$ & 0.1 & Qmax & 0.40 \\
\hline Lvpnt1 & 0.8 & $\mathrm{dPmn}$ & -0.1 & Qmin & -0.40 \\
\hline \multirow[t]{11}{*}{ Lvpnt0 } & 0.4 & & & $\mathrm{Tr}$ & 0.02 \\
\hline & & & & Tc & 0.15 \\
\hline & & & & Kpv & 18.0 \\
\hline & & & & Kiv & 5.0 \\
\hline & & & & Pfaflg & 0 \\
\hline & & & & $\mathrm{fn}$ & 1.0 \\
\hline & & & & Tv & 0.05 \\
\hline & & & & Tpwr & 0.05 \\
\hline & & & & Iphl & 1.24 \\
\hline & & & & Iqhl & 1.25 \\
\hline & & & & Pqflag & 0 \\
\hline
\end{tabular}

\footnotetext{
${ }^{1}$ Parameterization performed by GE Energy.
} 


\subsection{Case 1 - Fault Event}

\section{SCR - 5}

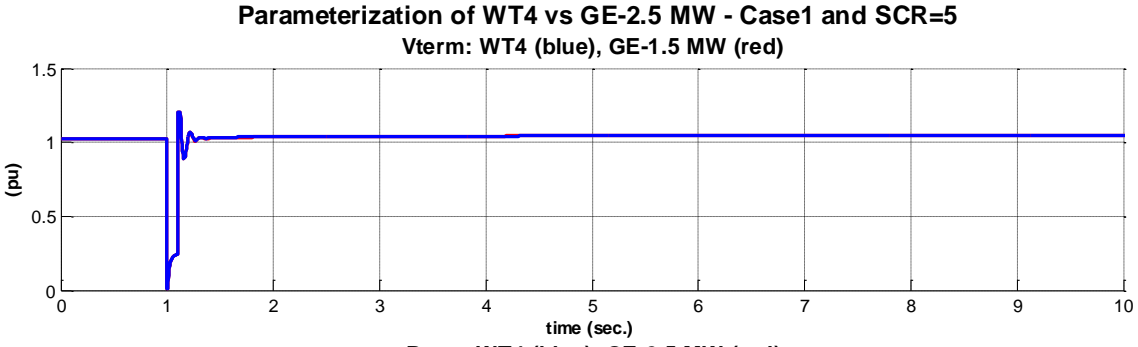

Pgen: WT4 (blue), GE-2.5 MW (red)

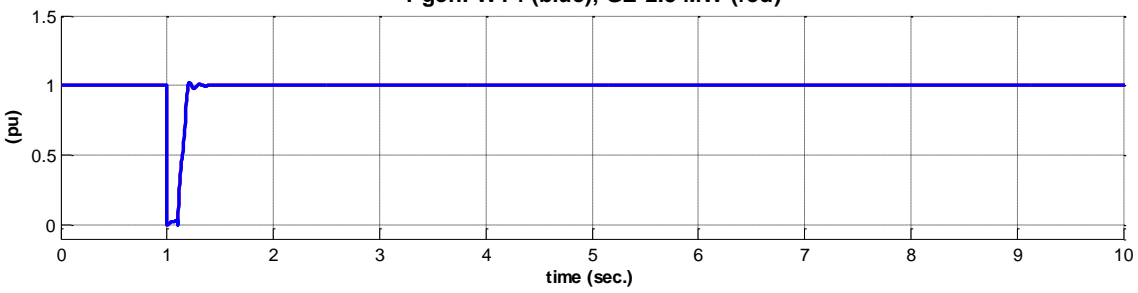

Qgen: WT4 (blue), GE-2.5 MW (red)

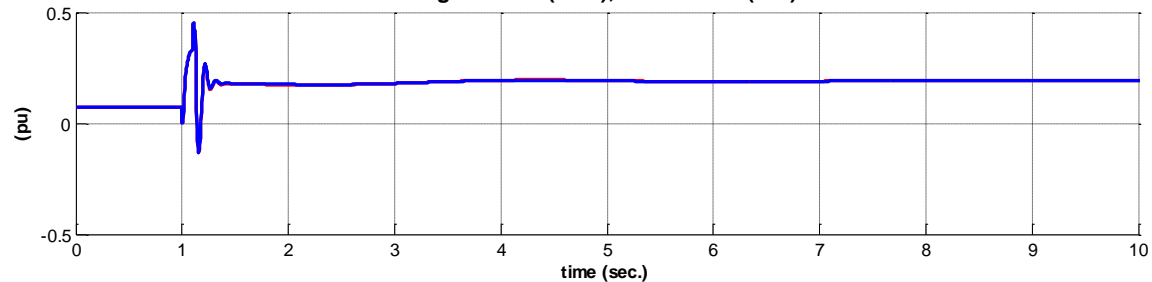

Figure 7-77: Parameterization of WT4 vs GE-2.5 MW - Case 1 and $S C R=5\left(V_{\text {Term }}, P, Q\right)$.
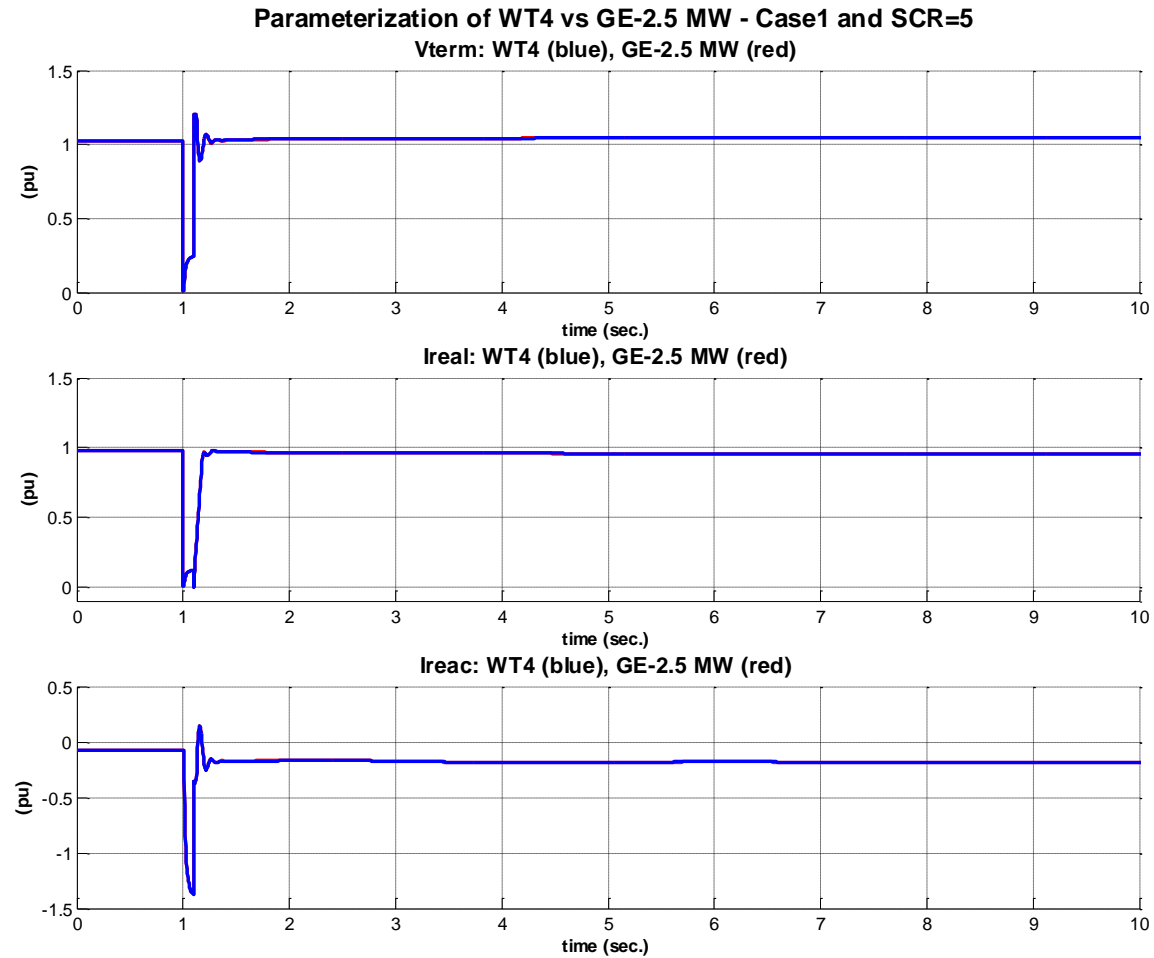

Figure 7-78: Parameterization of WT4 vs GE-2.5 MW - Case 1 and $S C R=5\left(V_{\text {Term }}, I_{\text {Real }}, I_{\text {Reac }}\right)$. 
$\underline{\text { SCR }-10}$

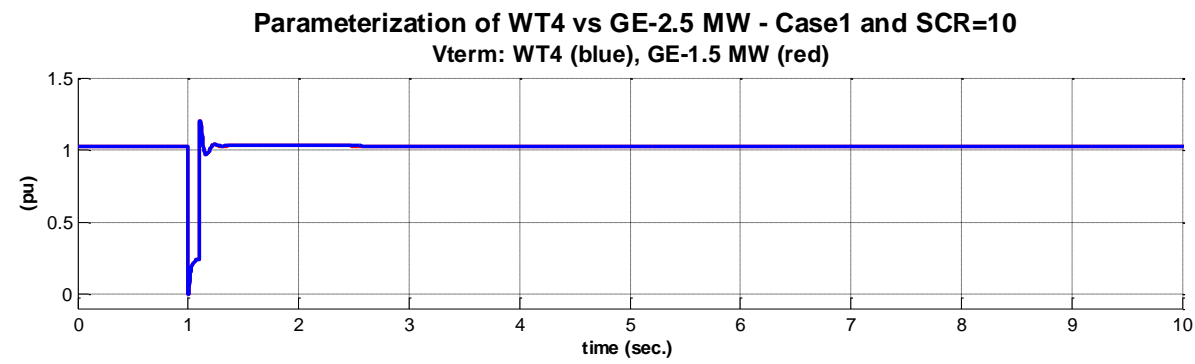

Pgen: WT4 (blue), GE-2.5 MW (red)

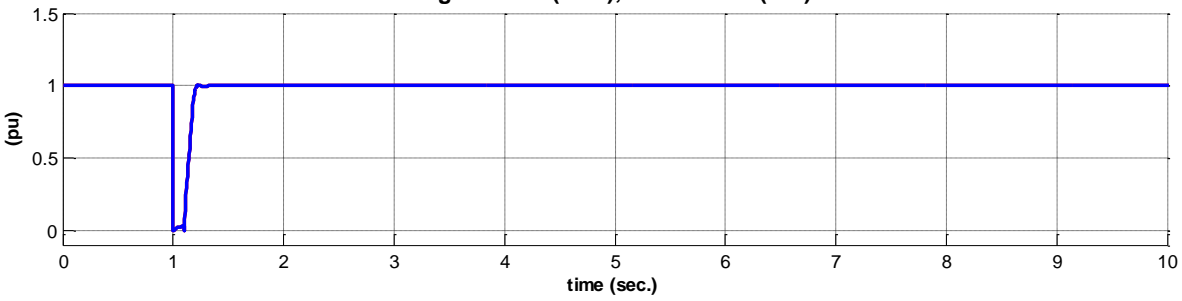

Qgen: WT4 (blue), GE-2.5 MW (red)

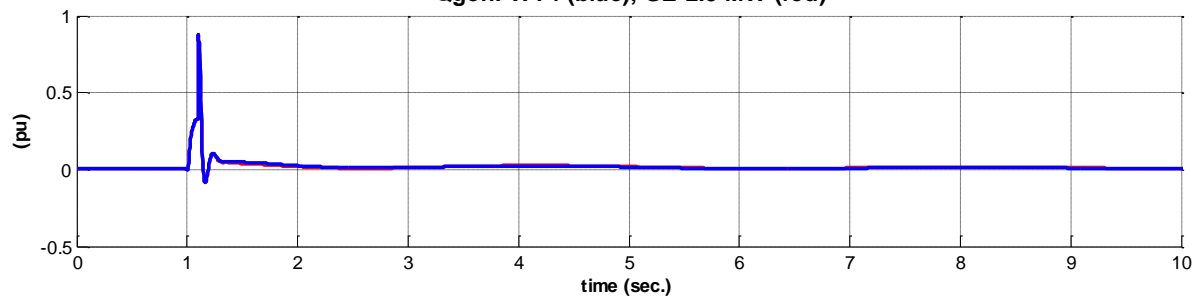

Figure 7-79: Parameterization of WT4 vs GE-2.5 MW - Case 1 and $S C R=10\left(V_{T e r m}, P, Q\right)$.

Parameterization of WT4 vs GE-2.5 MW - Case1 and SCR $=10$

Vterm: WT4 (blue), GE-2.5 MW (red)

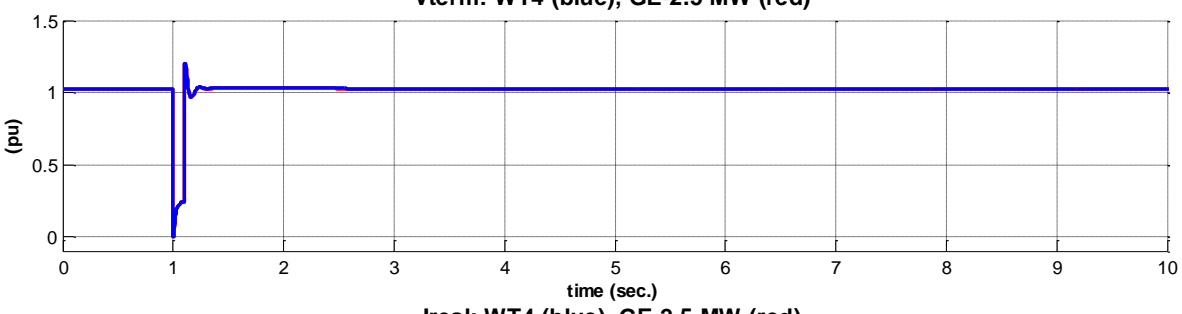

Ireal: WT4 (blue), GE-2.5 MW (red)

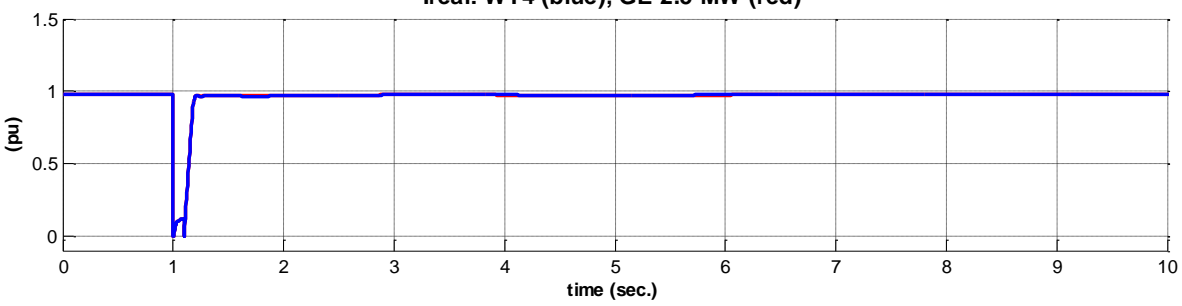

Ireac: WT4 (blue), GE-2.5 MW (red)

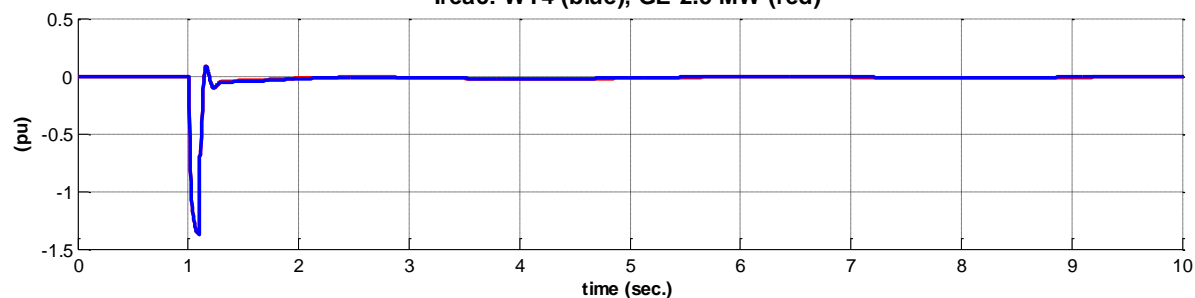

Figure 7-80: Parameterization of WT4 vs GE-2.5 MW - Case 1 and $S C R=10$ ( $\left.V_{\text {Term, }}, I_{\text {Real }}, I_{\text {Reac }}\right)$. 


\subsection{Case 2 - Under-Frequency Event}

\section{$\underline{\text { SCR }-5}$}

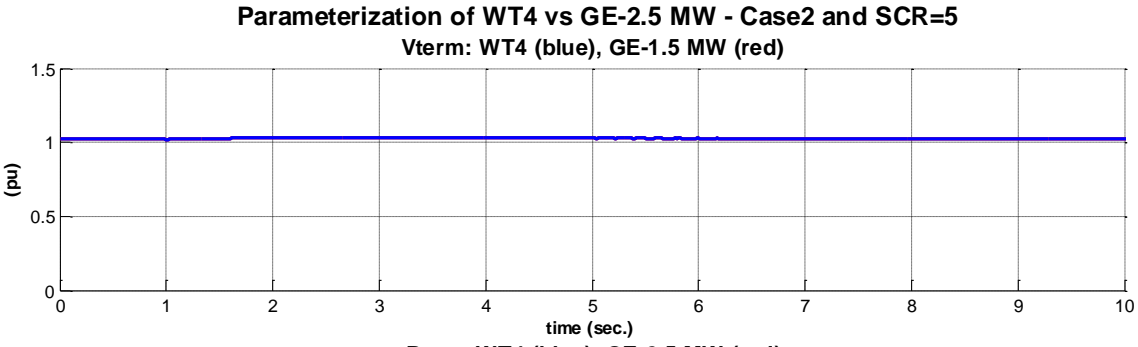

Pgen: WT4 (blue), GE-2.5 MW (red)

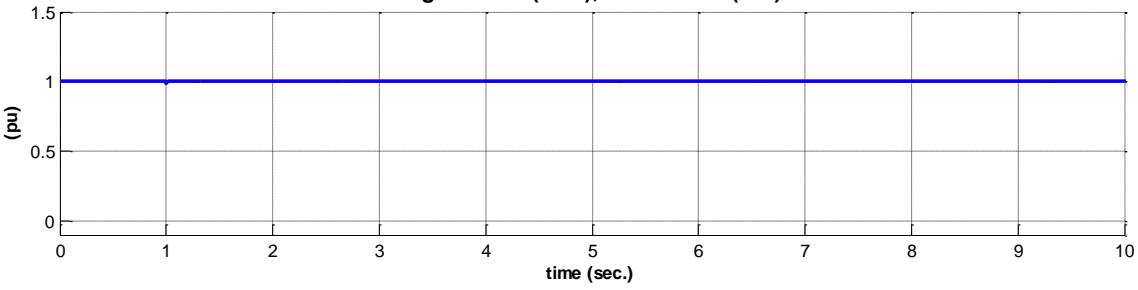

Qgen: WT4 (blue), GE-2.5 MW (red)

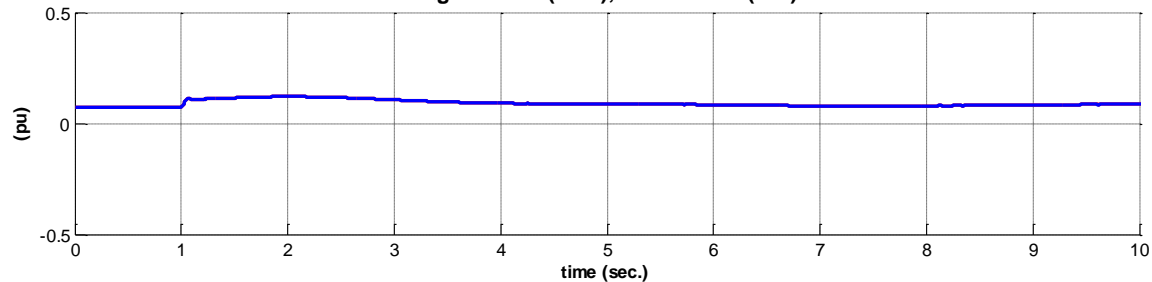

Figure 7-81: Parameterization of WT4 vs GE-2.5 MW - Case 2 and $S C R=5\left(V_{\text {Term }}, P, Q\right)$.
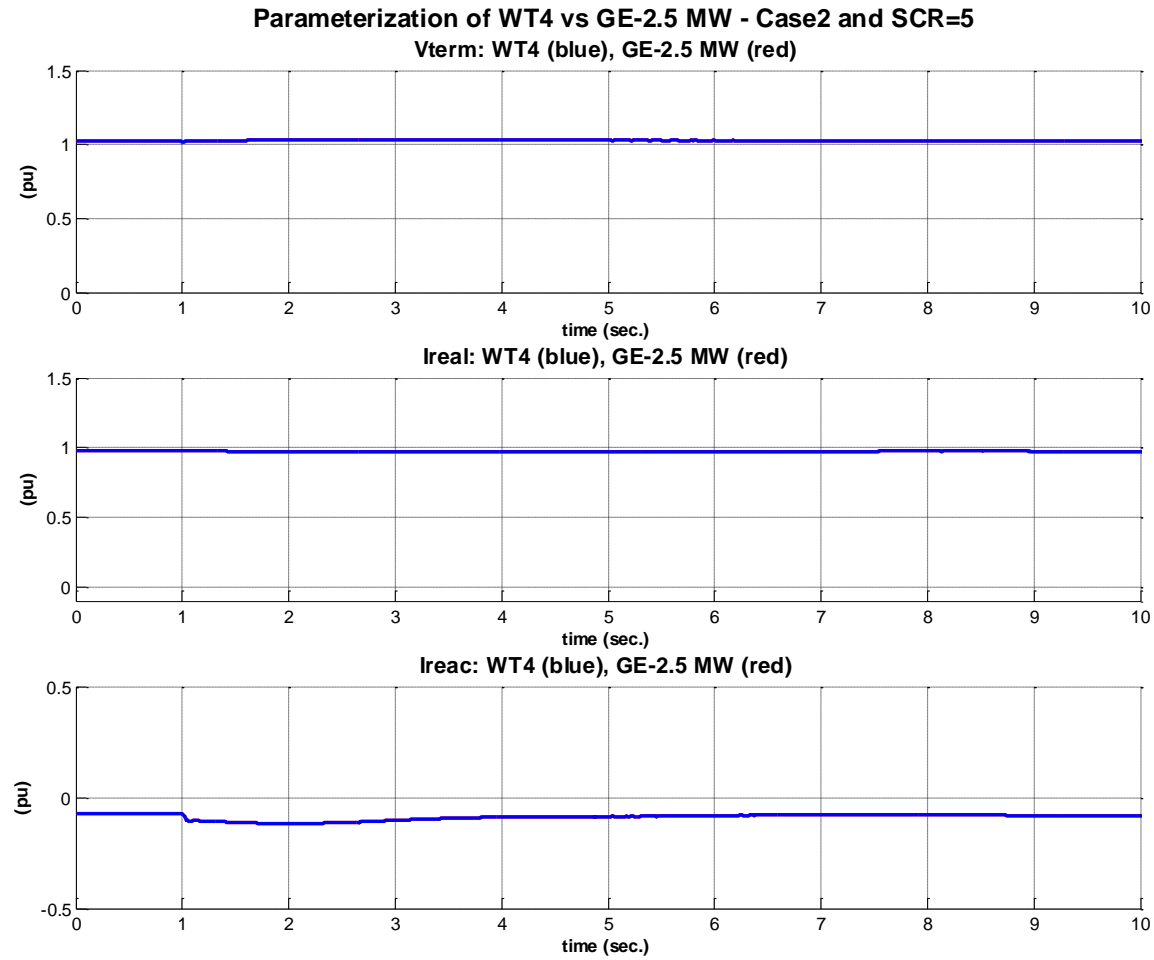

Figure 7-82: Parameterization of WT4 vs GE-2.5 MW - Case 2 and SCR=5 (V $\left.\mathrm{V}_{\text {Term }}, I_{\text {Real }}, I_{\text {Reac }}\right)$. 
$\underline{\text { SCR }-10}$

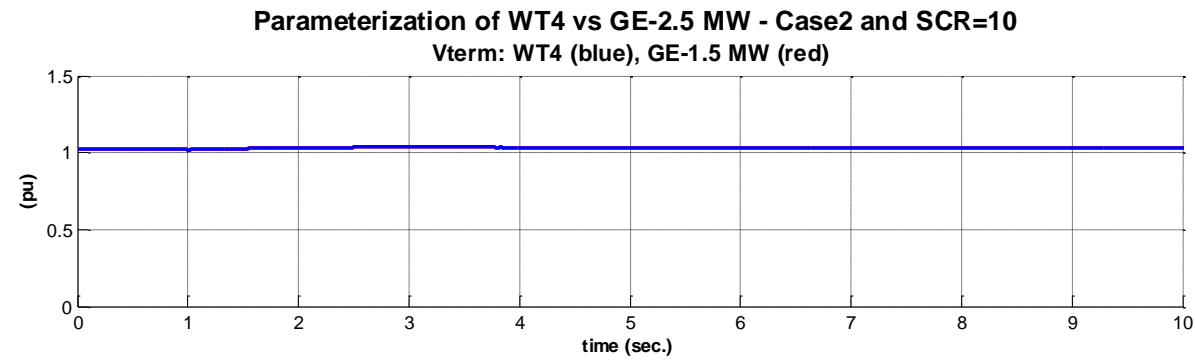

Pgen: WT4 (blue), GE-2.5 MW (red)

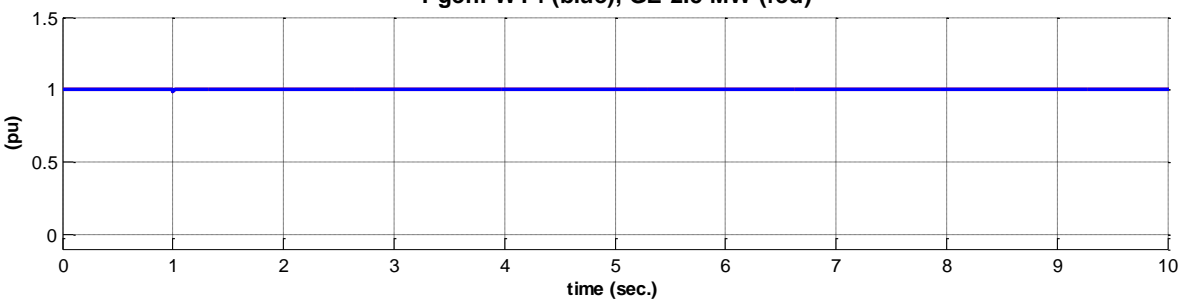

Qgen: WT4 (blue), GE-2.5 MW (red)

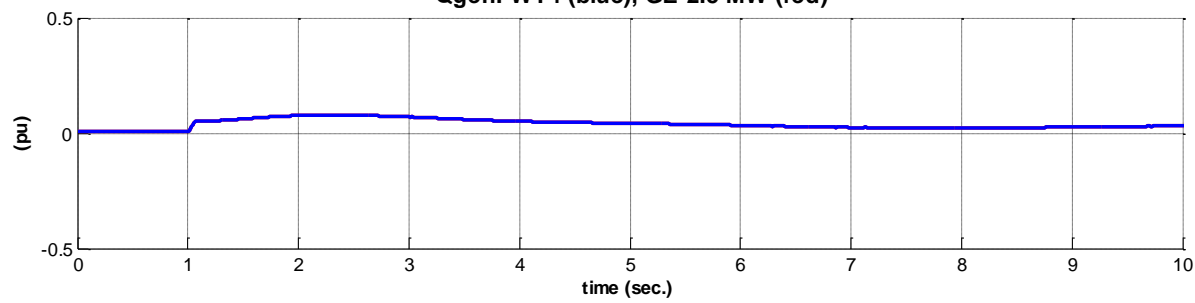

Figure 7-83: Parameterization of WT4 vs GE-2.5 MW - Case 2 and SCR=10 ( $\left.V_{\text {Term }}, P, Q\right)$.

Parameterization of WT4 vs GE-2.5 MW - Case2 and SCR=10

Vterm: WT4 (blue), GE-2.5 MW (red)
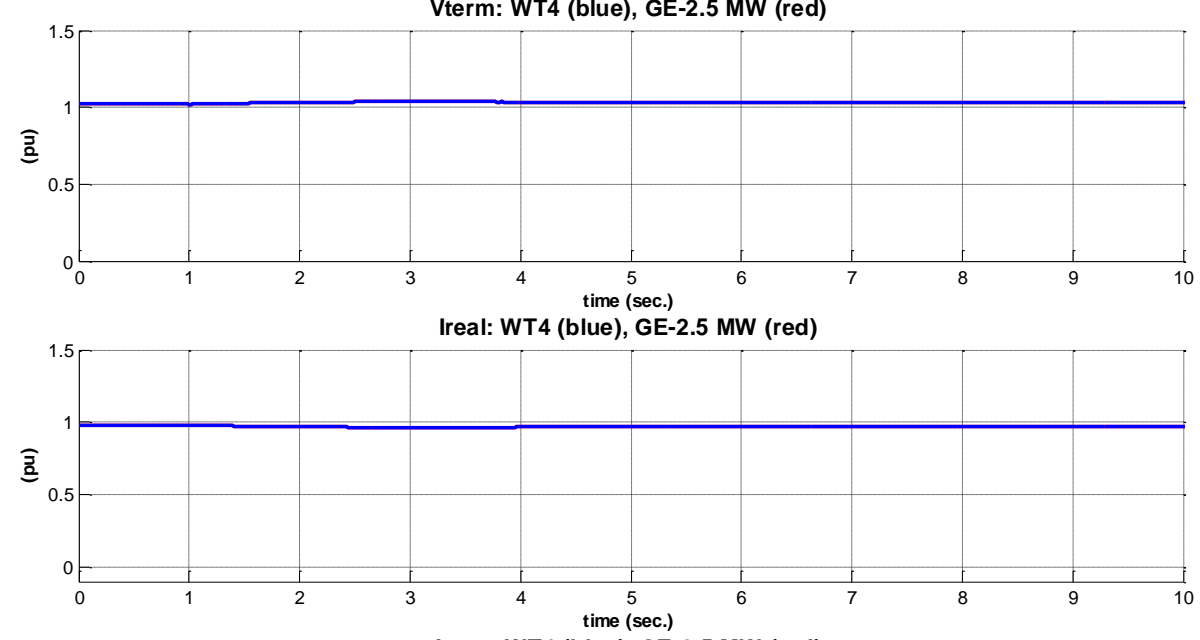

Ireac: WT4 (blue), GE-2.5 MW (red)

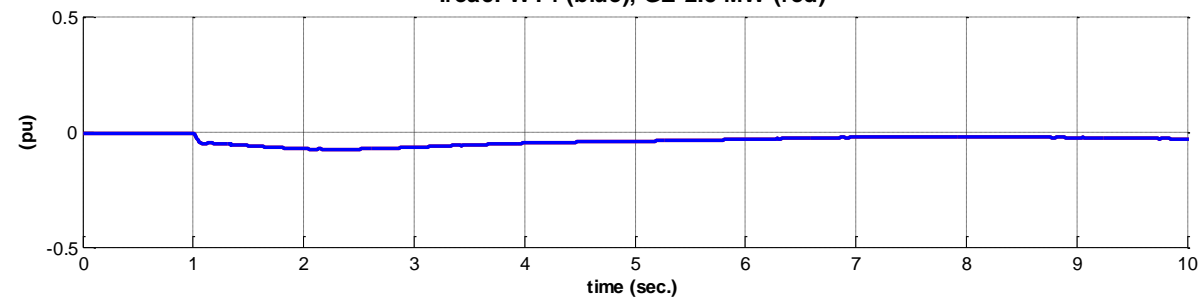

Figure 7-84: Parameterization of WT4 vs GE-2.5 MW - Case 2 and $S C R=10$ ( $\left.\mathrm{V}_{\text {Term }}, I_{\text {Real }}, I_{\text {Reac }}\right)$. 


\subsection{Case 3 - Over-Frequency Event}

$\underline{\text { SCR }-5}$

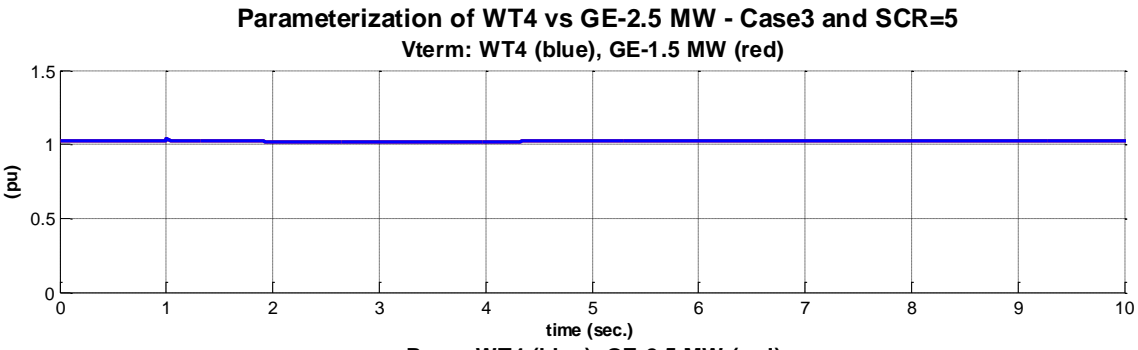

Pgen: WT4 (blue), GE-2.5 MW (red)

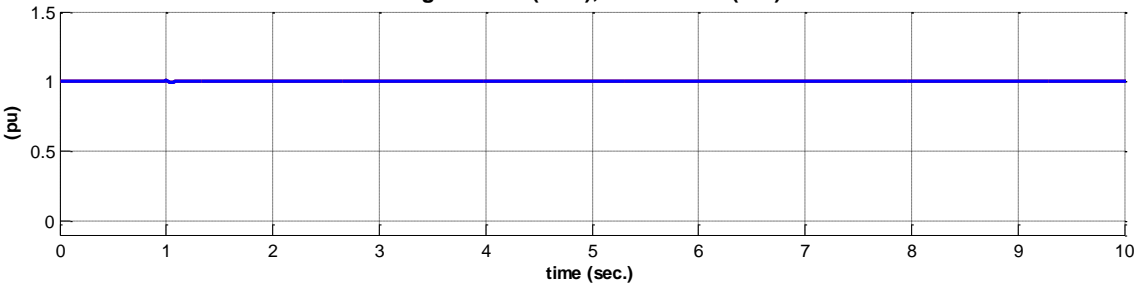

Qgen: WT4 (blue), GE-2.5 MW (red)

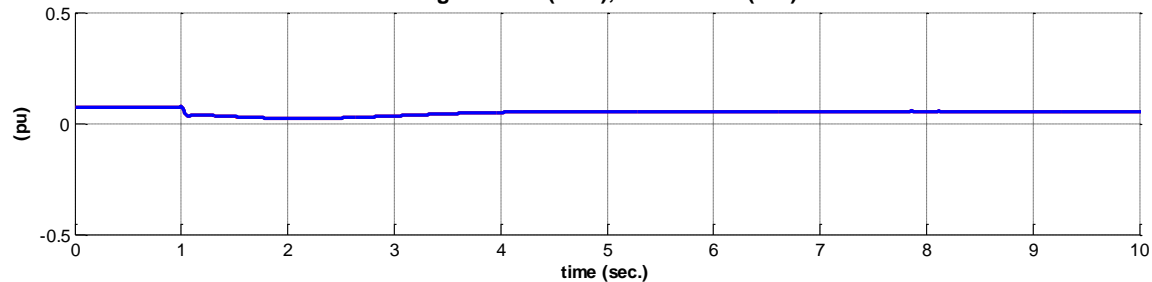

Figure 7-85: Parameterization of WT4 vs GE-2.5 MW - Case 3 and $S C R=5\left(V_{\text {Term }}, P, Q\right)$.
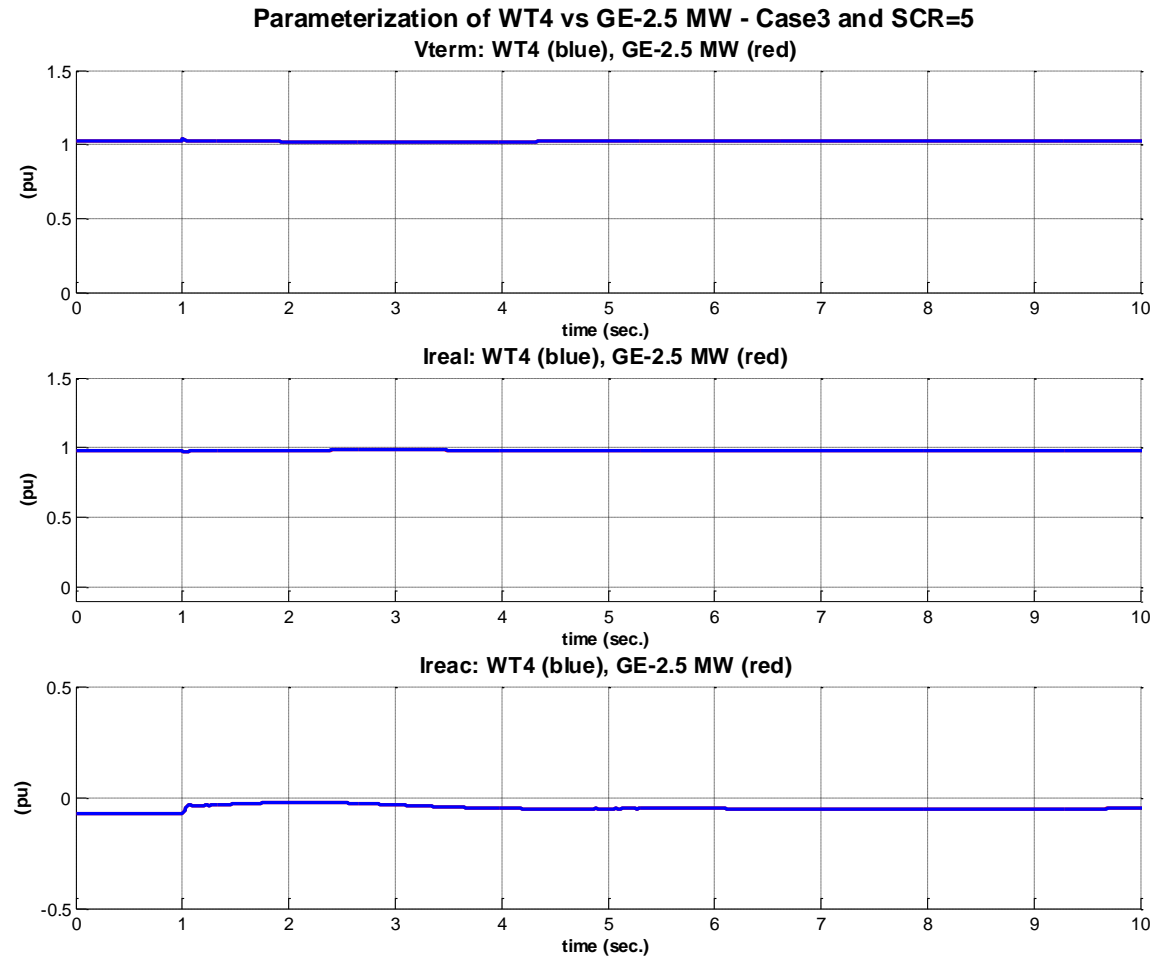

Figure 7-86: Parameterization of WT4 vs GE-2.5 MW - Case 3 and SCR=5 (V $\left.V_{\text {Term }}, I_{\text {Real }}, I_{\text {Reac }}\right)$. 
$\underline{\text { SCR }-10}$

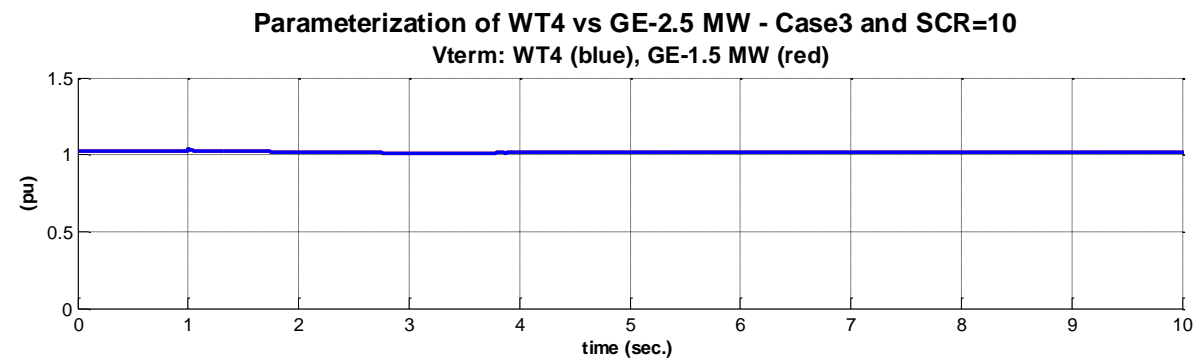

Pgen: WT4 (blue), GE-2.5 MW (red)

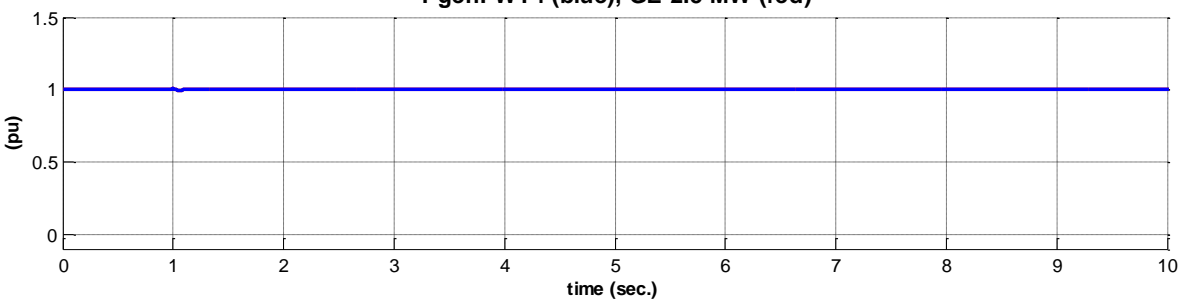

Qgen: WT4 (blue), GE-2.5 MW (red)

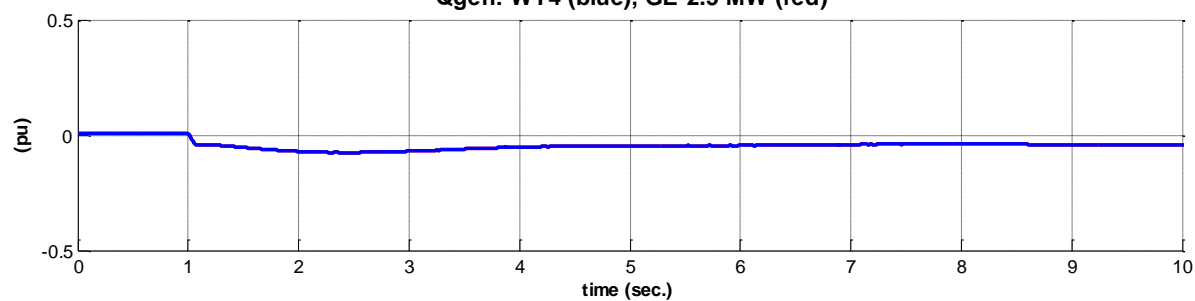

Figure 7-87: Parameterization of WT4 vs GE-2.5 MW - Case 3 and $S C R=10\left(V_{\text {Term }}, P, Q\right)$.

Parameterization of WT4 vs GE-2.5 MW - Case 3 and SCR $=10$

Vterm: WT4 (blue), GE-2.5 MW (red)

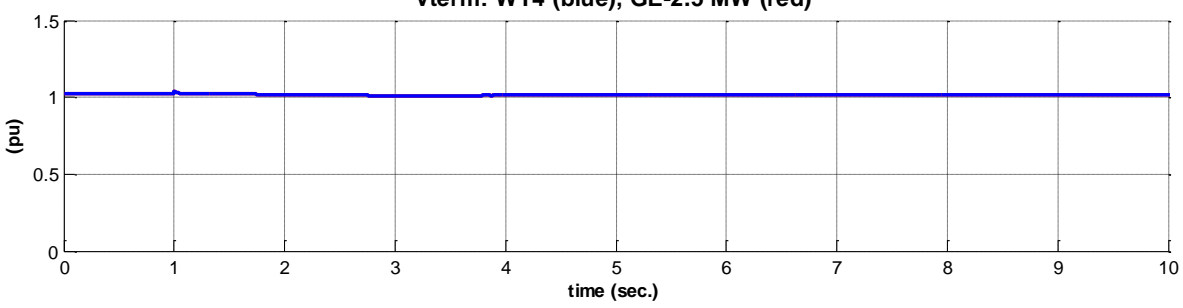

Ireal: WT4 (blue), GE-2.5 MW (red)

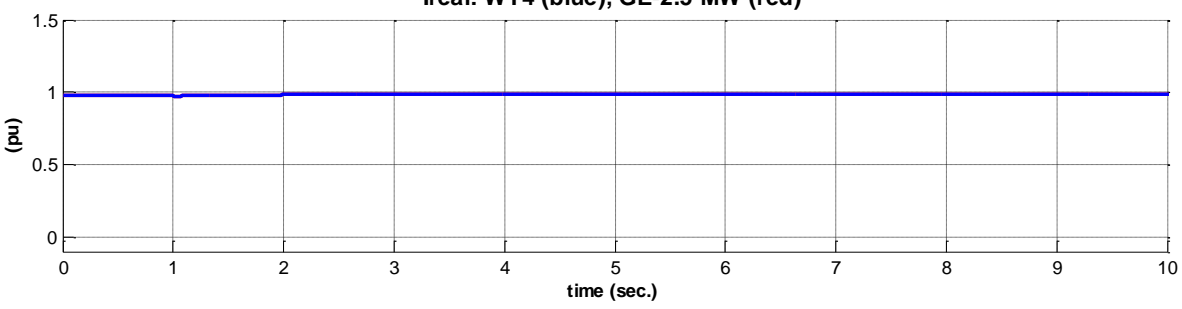

Ireac: WT4 (blue), GE-2.5 MW (red)

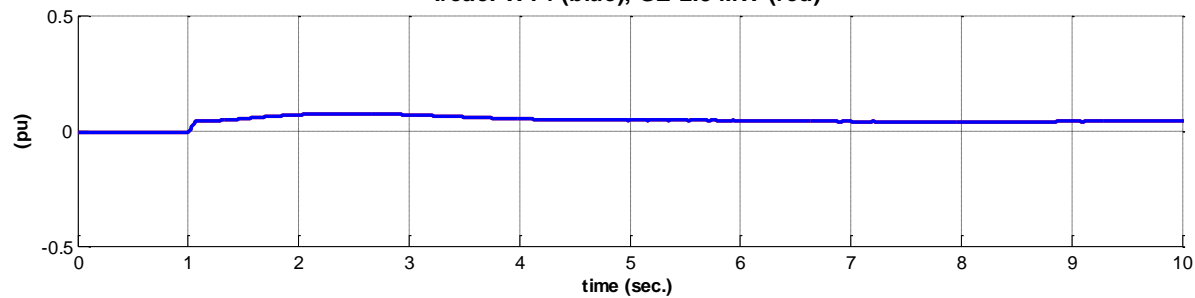

Figure 7-88: Parameterization of WT4 vs GE-2.5 MW - Case 3 and $S C R=10\left(V_{\text {Term }}, I_{\text {Real }}, I_{\text {Reac }}\right)$. 


\subsubsection{PSSE Environment ${ }^{1}$}

The PSSE platform generic wind turbine parameters, to mimic the behavior of GE's 2.5 MW series, are shown in Table 7-5. The parameter values shown in the table resulted from a compromise between the simulated cases (i.e. SCR 5 and SCR 10) and network conditions (i.e. fault, under- and over frequency).

Table 7-5: PSSE generic model parameter values.

\begin{tabular}{|c|c|c|c|}
\hline \multicolumn{2}{|c|}{ WT4G } & \multicolumn{2}{|c|}{ WT4E } \\
\hline Tiqcmd & 0.2000 & Tfv & 0.150 \\
\hline Tipcmd & 0.0200 & Kpv & 18.00 \\
\hline VLVPL1 & 0.087537 & Kiv & 5.00 \\
\hline VLVPL2 & 0.898853 & Kpp & 0.30 \\
\hline GLVPL & 1.111951 & Kip & 0.10 \\
\hline VHVRCR & 1.2000 & $\mathrm{Kf}$ & 0.00 \\
\hline CURHVRCR & 2.0000 & Tf & 0.08 \\
\hline Rip_LVPL & 10.0000 & Qmx & 0.40 \\
\hline T_LVPL & 0.0200 & Qmn & -0.40 \\
\hline & & IPmax & 1.120 \\
\hline & & Trv & 0.020 \\
\hline & & dPMX & 0.50 \\
\hline & & dPMN & -0.50 \\
\hline & & T_Power & 5.00 \\
\hline & & Kqi & 0.10 \\
\hline & & VMINCL & 0.90 \\
\hline & & VMAXCL & 1.10 \\
\hline & & Kvi & 120.0 \\
\hline & & Tv & 0.050 \\
\hline & & $\mathrm{Tp}$ & 0.050 \\
\hline & & ImaxTD & 1.190 \\
\hline & & Iph1 & 1.220 \\
\hline & & lqh1 & 0.830 \\
\hline & & PFAFLG & 0 \\
\hline & & VARFLG & 1 \\
\hline & & PQFLAG & 1 \\
\hline
\end{tabular}

\footnotetext{
${ }^{1}$ Parameterization performed by Siemens Energy.
} 


\subsection{Case 1 - Fault Event}

\section{SCR - 5}

Parameterization of WT4 vs GE-2.5 MW - Case1 and SCR=5

Vterm: WT4 (blue), GE-2.5 MW (red)

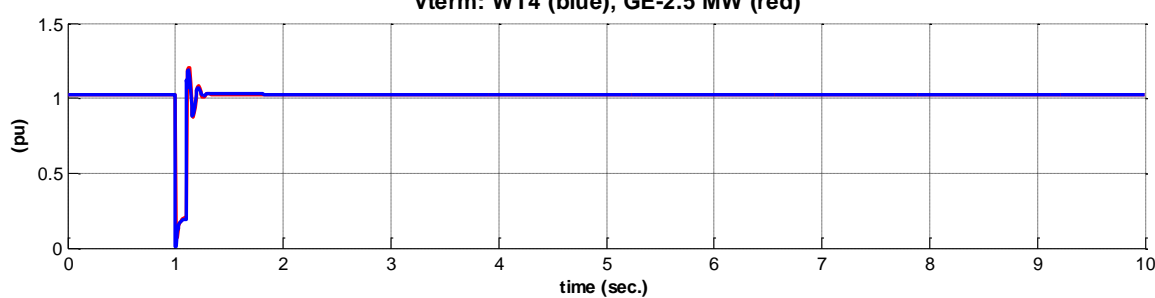

Pgen: WT4 (blue), GE-2.5 MW (red)

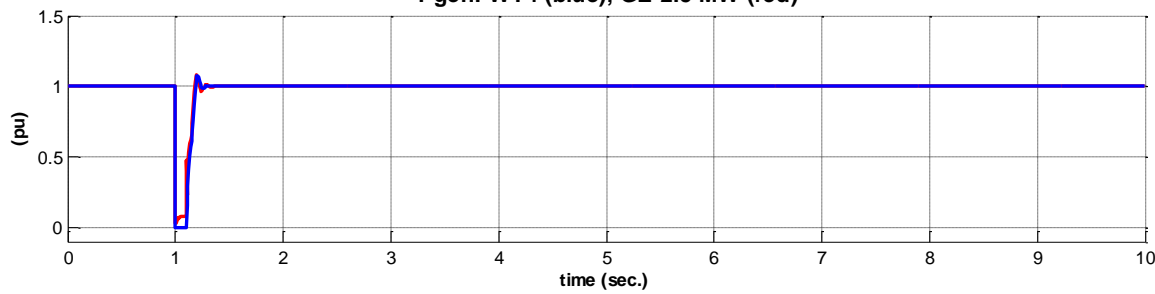

Qgen: WT4 (blue), GE-2.5 MW (red)

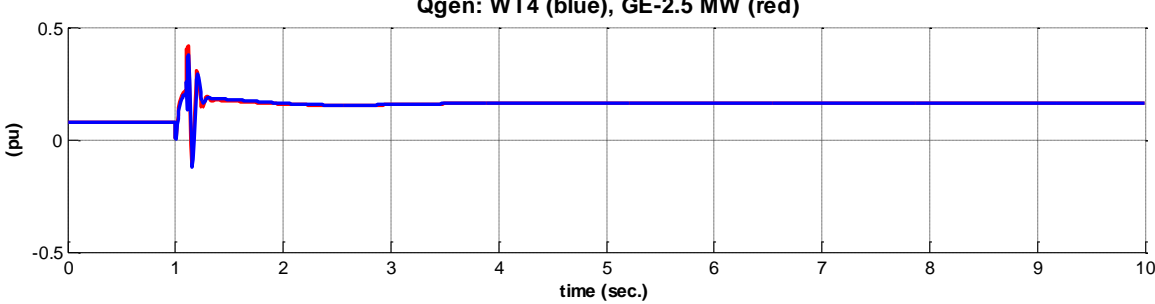

Figure 7-89: Parameterization of WT4 vs GE-2.5 MW - Case 1 and $S C R=5\left(V_{T e r m}, P, Q\right)$.

Parameterization of WT4 vs GE-2.5 MW - Case1 and SCR=5 Vterm: WT4 (blue), GE-2.5 MW (red)

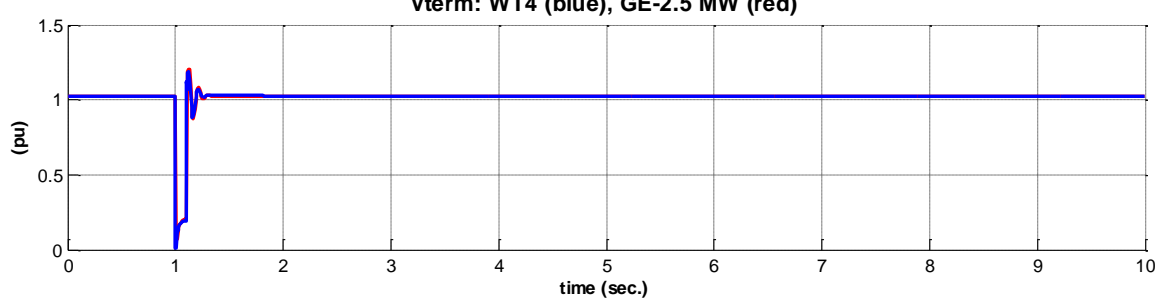

Ireal: WT4 (blue), GE-2.5 MW (red)
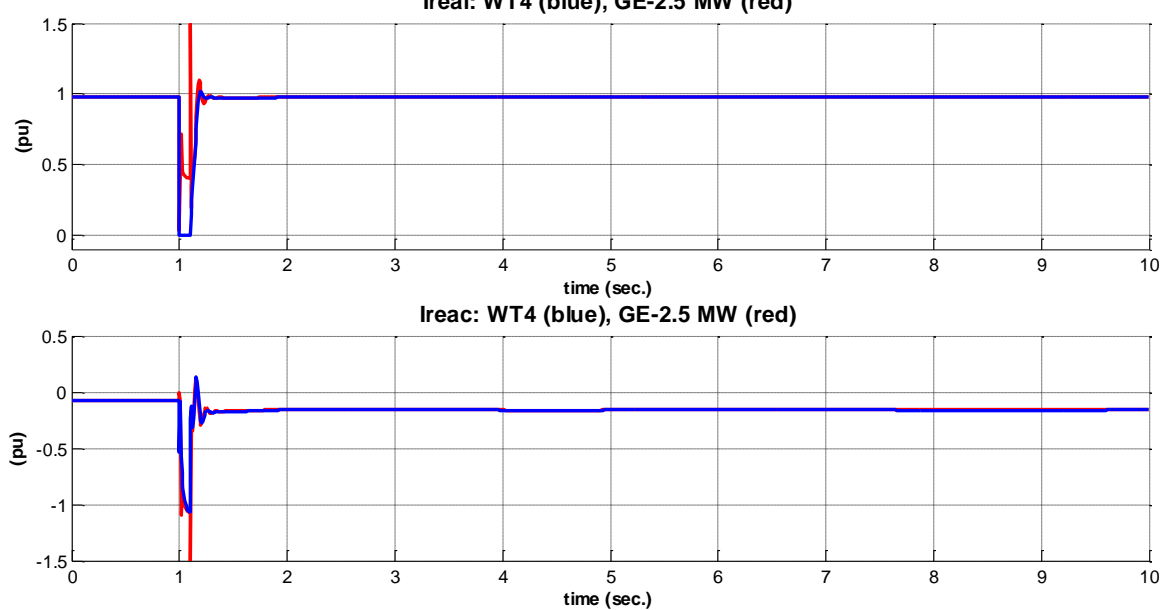

Figure 7-90: Parameterization of WT4 vs GE-2.5 MW - Case 1 and $S C R=5\left(V_{\text {Term }}, I_{\text {Real }}, I_{\text {Reac }}\right)$. 
$\underline{\text { SCR }-10}$

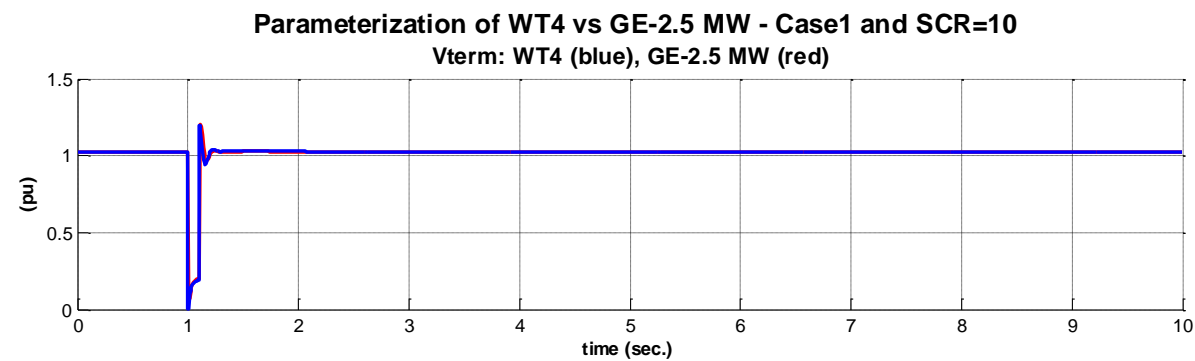

Pgen: WT4 (blue), GE-2.5 MW (red)

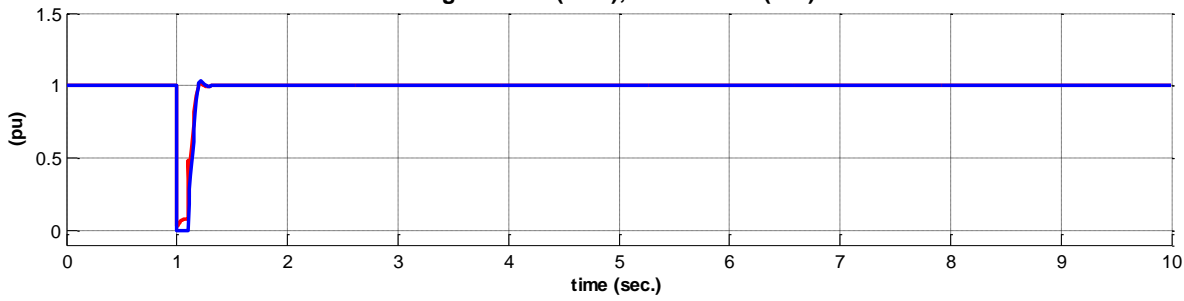

Qgen: WT4 (blue), GE-2.5 MW (red)

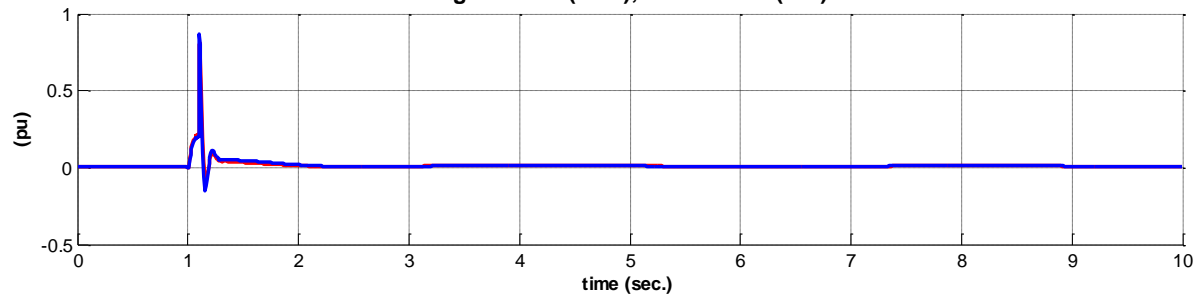

Figure 7-91: Parameterization of WT4 vs GE-2.5 MW - Case 1 and $S C R=10\left(V_{T e r m}, P, Q\right)$.

Parameterization of WT4 vs GE-2.5 MW - Case1 and SCR=10 Vterm: WT4 (blue), GE-2.5 MW (red)

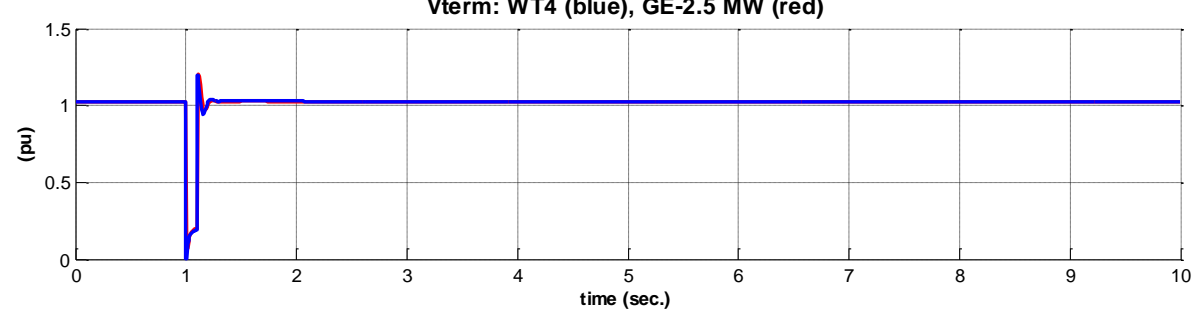

Ireal: WT4 (blue), GE-2.5 MW (red)

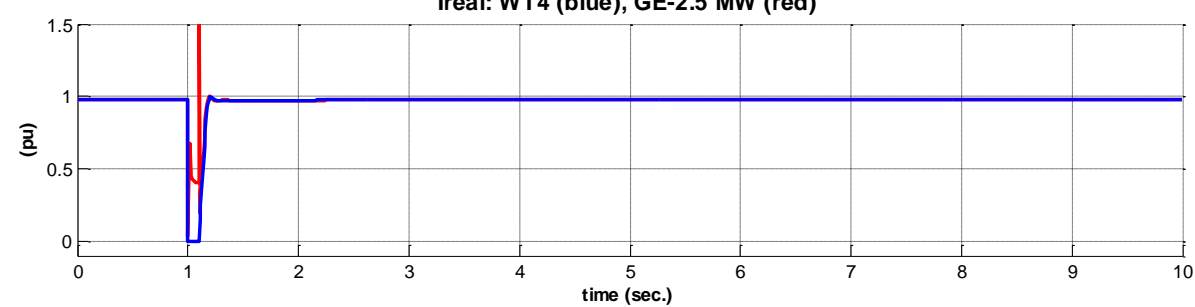

Ireac: WT4 (blue), GE-2.5 MW (red)

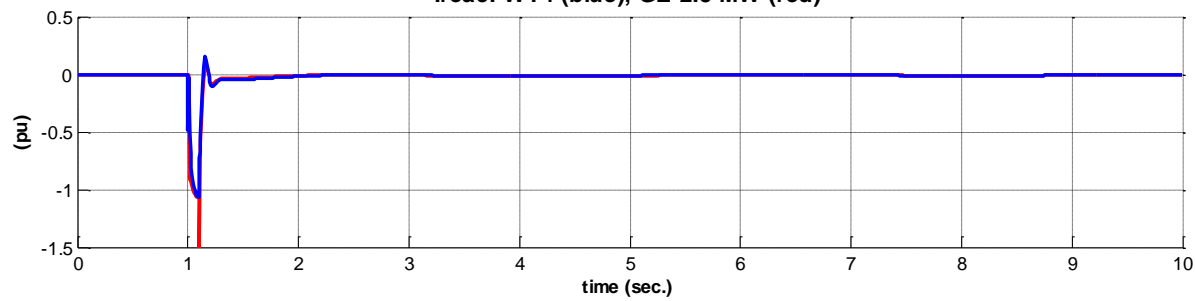

Figure 7-92: Parameterization of WT4 vs GE-2.5 MW - Case 1 and $S C R=10$ ( $\left.V_{\text {Term, }} I_{\text {Real }}, I_{\text {Reac }}\right)$. 


\subsection{Case 2 - Under-Frequency Event}

\section{SCR - 5}

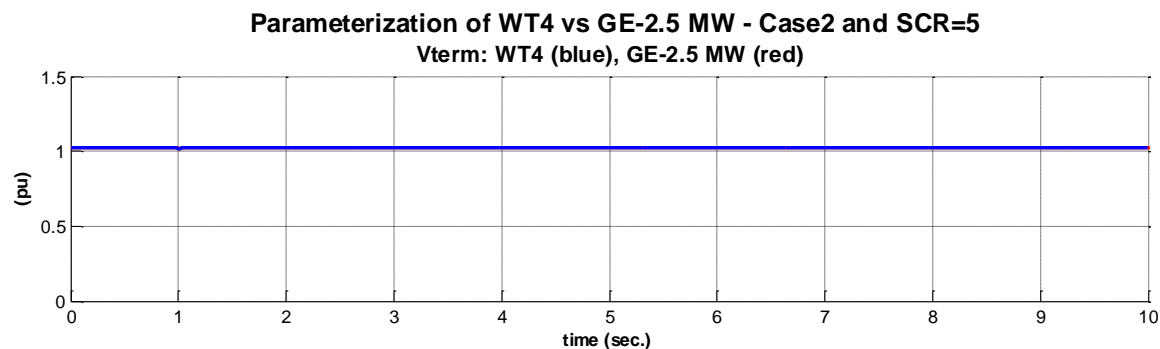

Pgen: WT4 (blue), GE-2.5 MW (red)

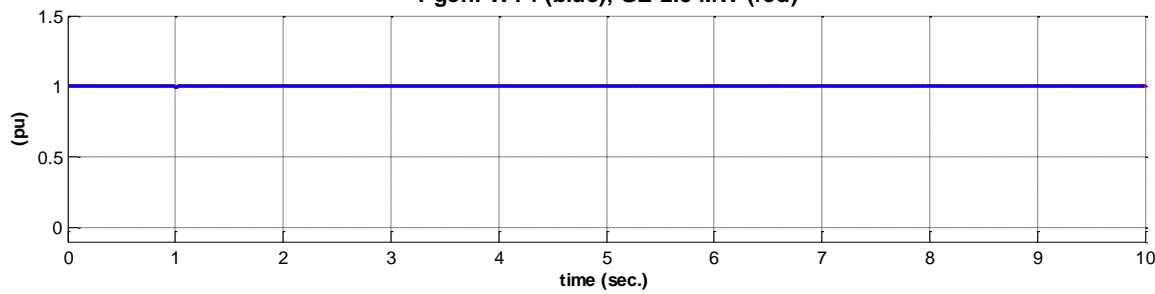

Qgen: WT4 (blue), GE-2.5 MW (red)

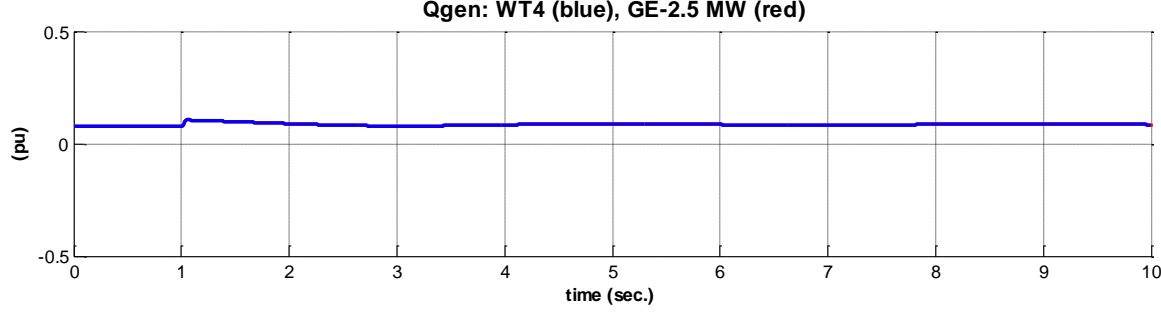

Figure 7-93: Parameterization of WT4 vs GE-2.5 MW - Case 2 and $S C R=5\left(V_{T e r m}, P, Q\right)$.

Parameterization of WT4 vs GE-2.5 MW - Case2 and SCR=5 Vterm: WT4 (blue), GE-2.5 MW (red)

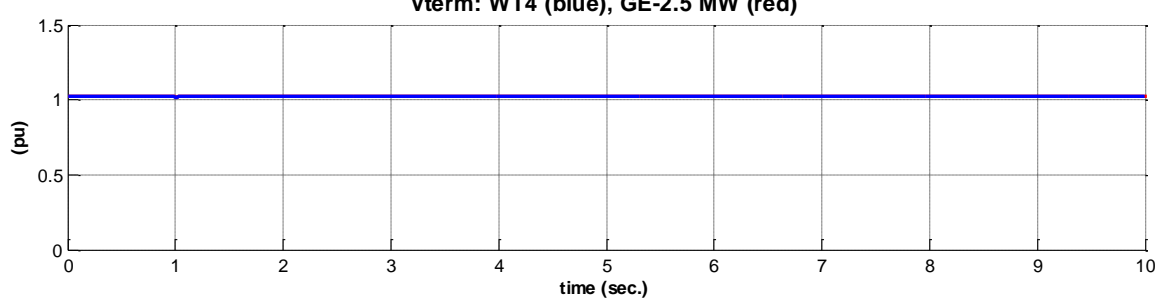

Ireal: WT4 (blue), GE-2.5 MW (red)
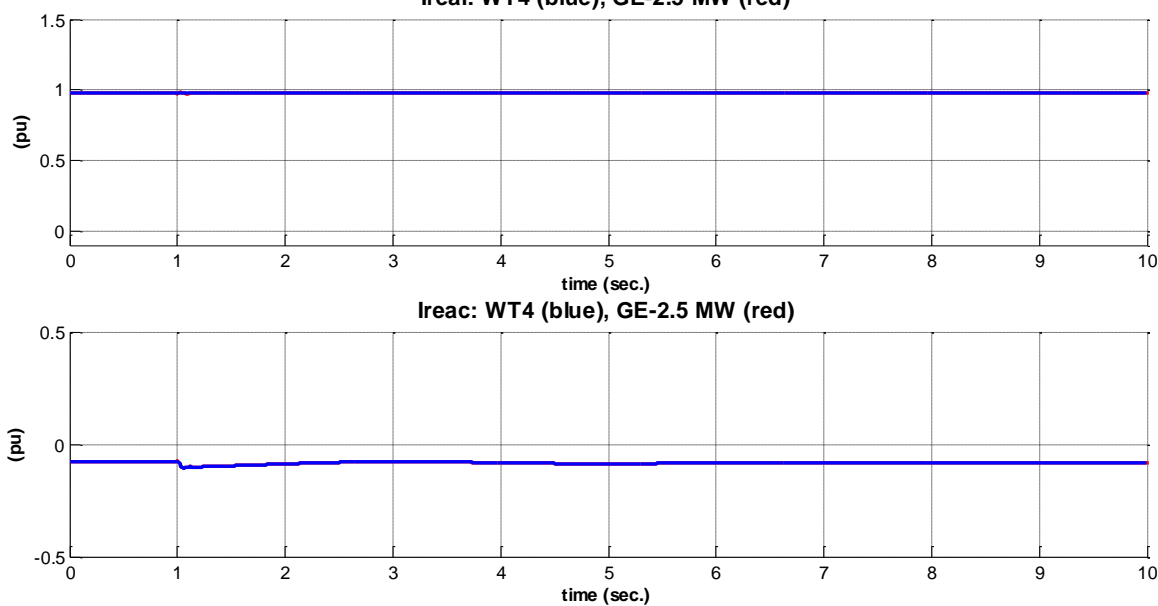

Figure 7-94: Parameterization of WT4 vs GE-2.5 MW - Case 2 and $S C R=5\left(V_{\text {Term }}, I_{\text {Real }}, I_{\text {Reac }}\right)$. 
$\underline{\text { SCR }-10}$
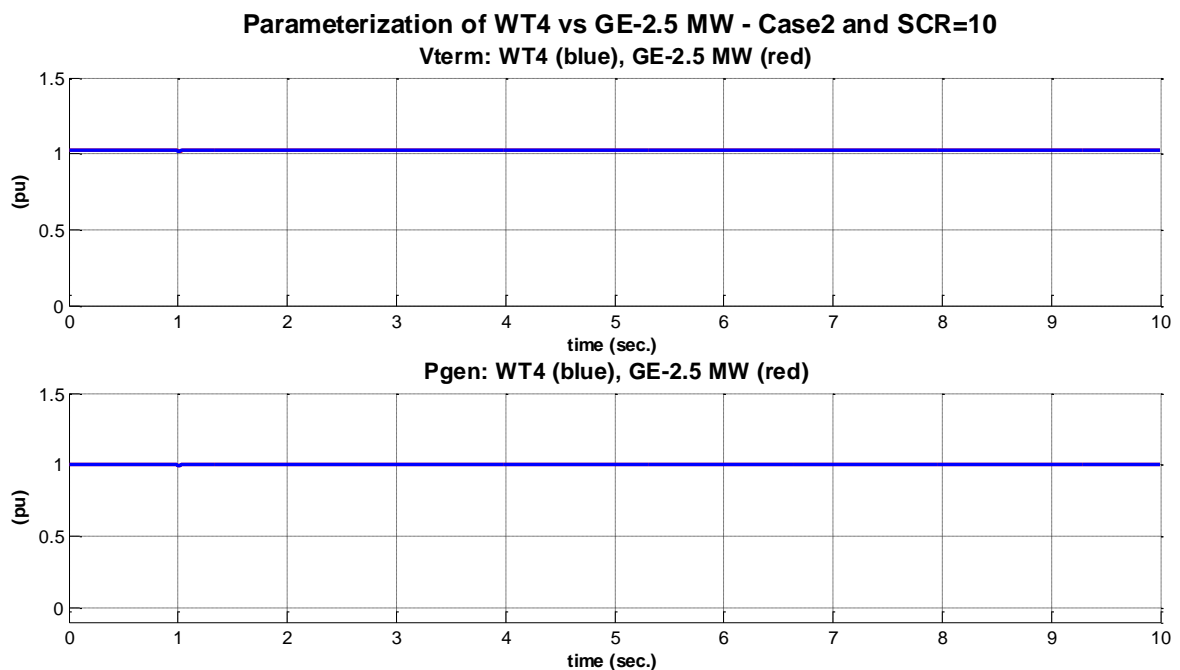

Qgen: WT4 (blue), GE-2.5 MW (red)

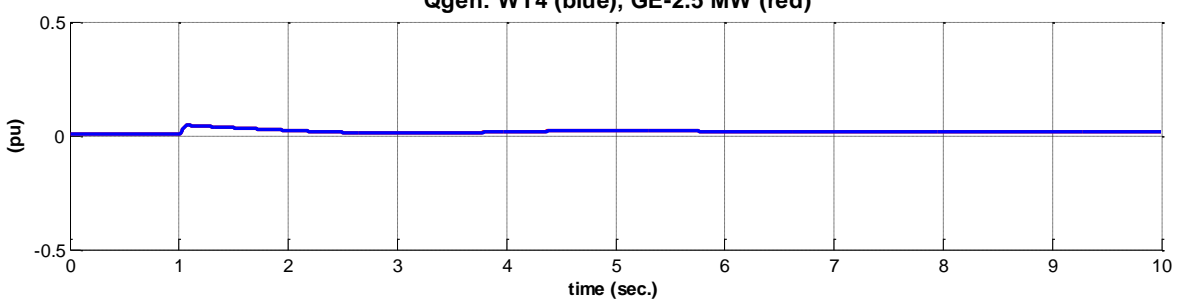

Figure 7-95: Parameterization of WT4 vs GE-2.5 MW - Case 2 and $S C R=10\left(V_{T e r m}, P, Q\right)$.
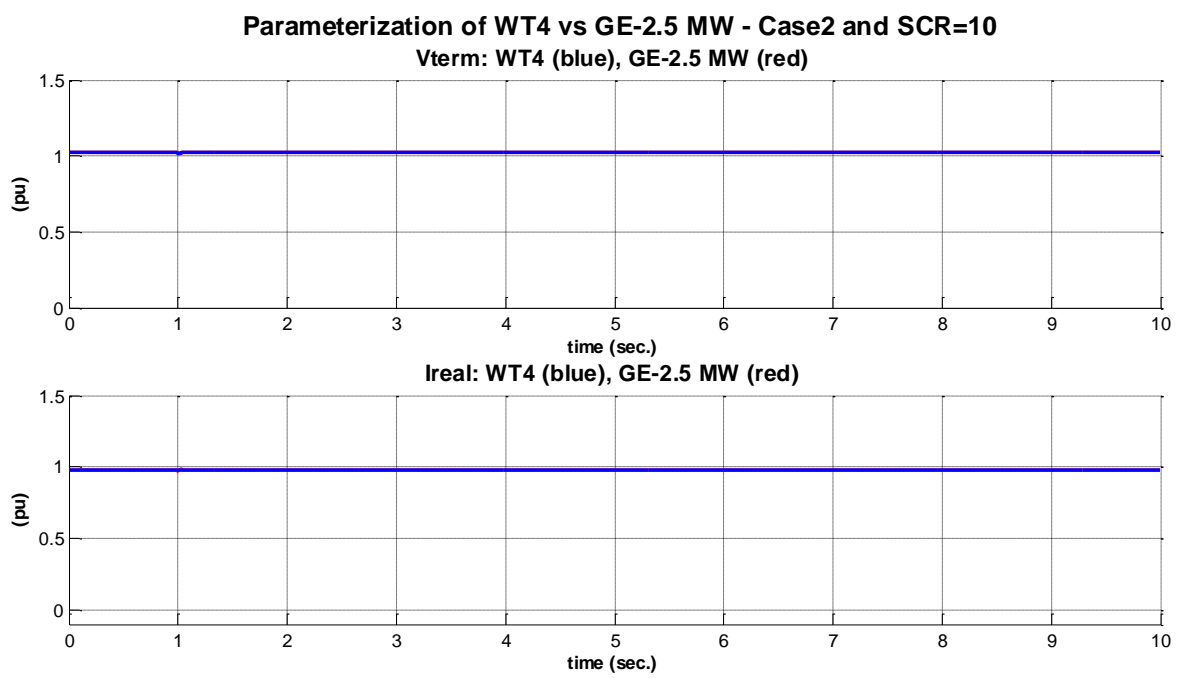

Ireac: WT4 (blue), GE-2.5 MW (red)

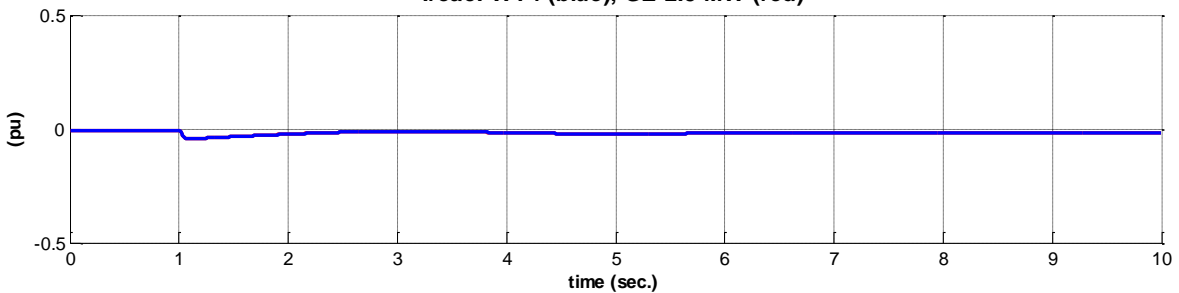

Figure 7-96: Parameterization of WT4 vs GE-2.5 MW - Case 2 and SCR=10 ( $\left.\mathrm{V}_{\text {Term, }} \mathrm{I}_{\text {Real }}, \mathrm{I}_{\text {Reac }}\right)$. 


\subsection{Case 3-Over-Frequency Event}

\section{$\underline{\mathrm{SCR}-5}$}

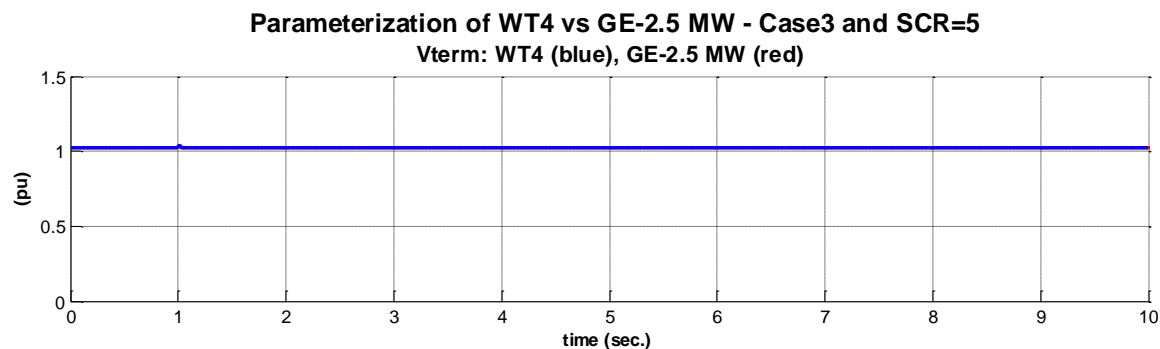

Pgen: WT4 (blue), GE-2.5 MW (red)

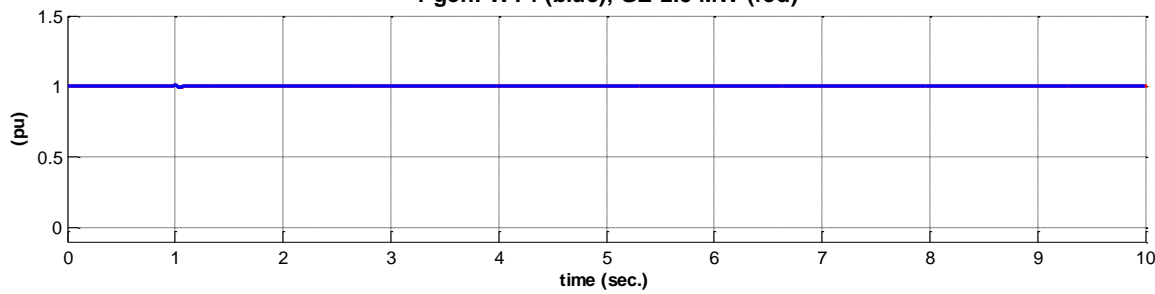

Qgen: WT4 (blue), GE-2.5 MW (red)

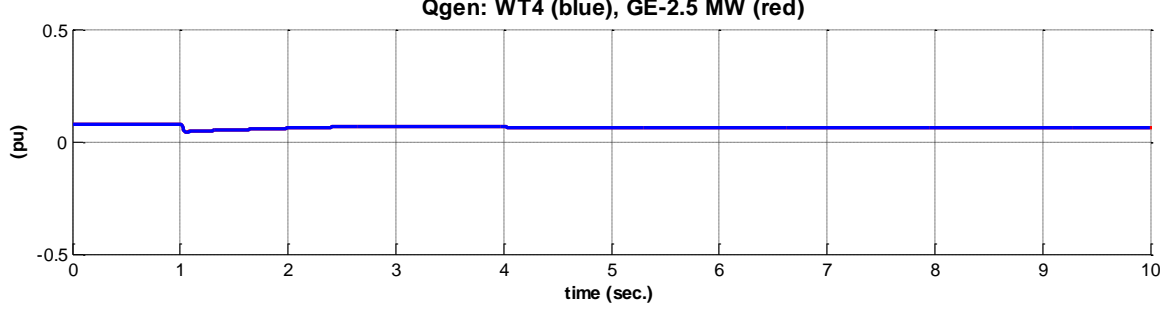

Figure 7-97: Parameterization of WT4 vs GE-2.5 MW - Case 3 and $S C R=5\left(V_{T e r m}, P, Q\right)$.

Parameterization of WT4 vs GE-2.5 MW - Case 3 and SCR=5 Vterm: WT4 (blue), GE-2.5 MW (red)

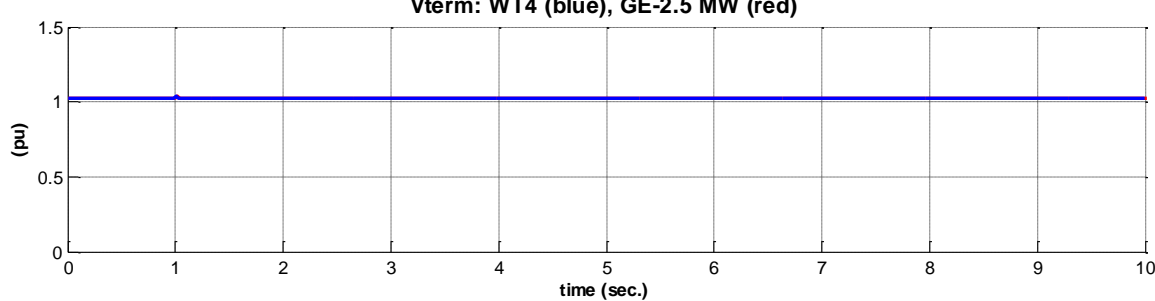

Ireal: WT4 (blue), GE-2.5 MW (red)
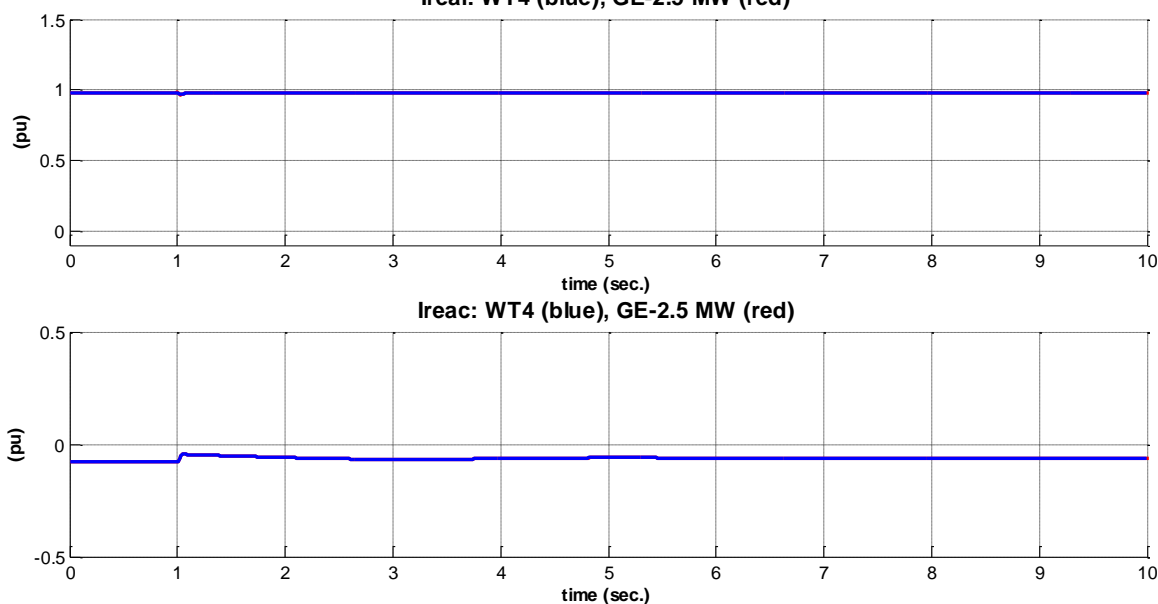

Figure 7-98: Parameterization of WT4 vs GE-2.5 MW - Case 3 and $S C R=5\left(V_{\text {Term }}, I_{\text {Real }}, I_{\text {Reac }}\right)$. 
$\underline{\text { SCR }-10}$
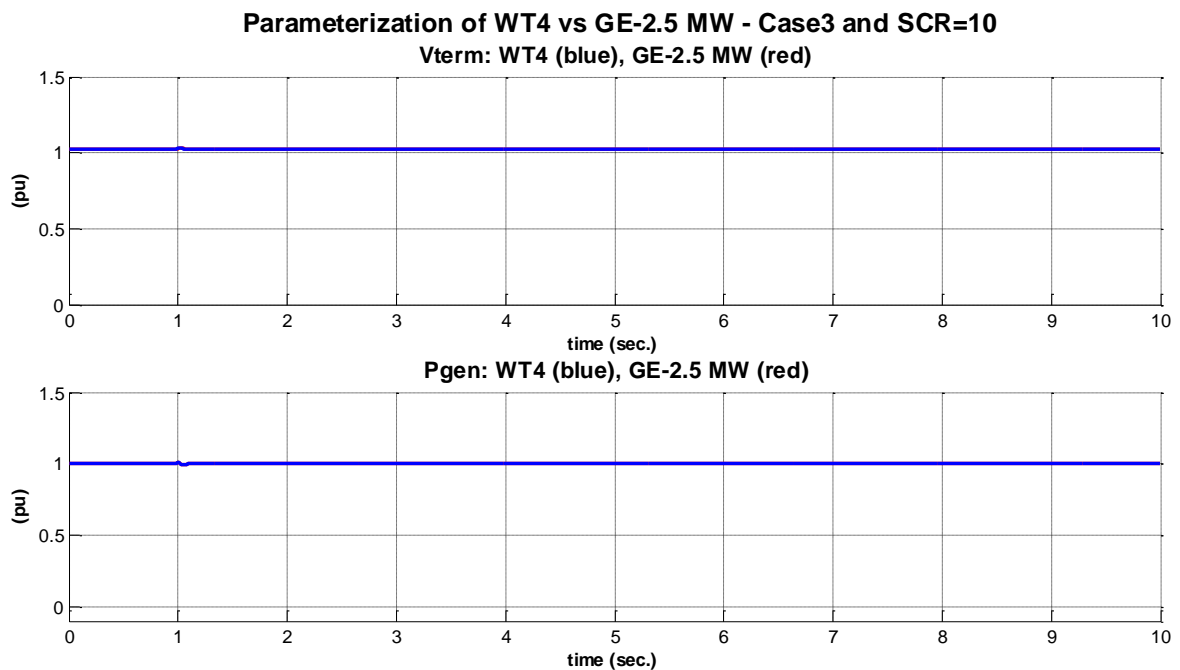

Qgen: WT4 (blue), GE-2.5 MW (red)

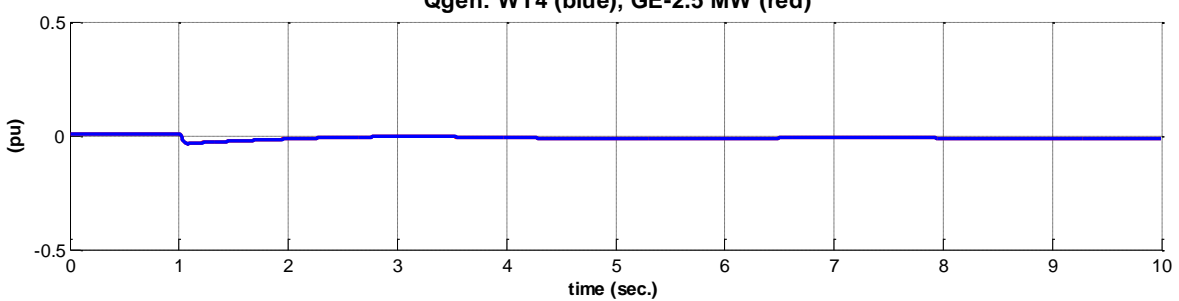

Figure 7-99: Parameterization of WT4 vs GE-2.5 MW - Case 3 and $S C R=10\left(V_{T e r m}, P, Q\right)$.
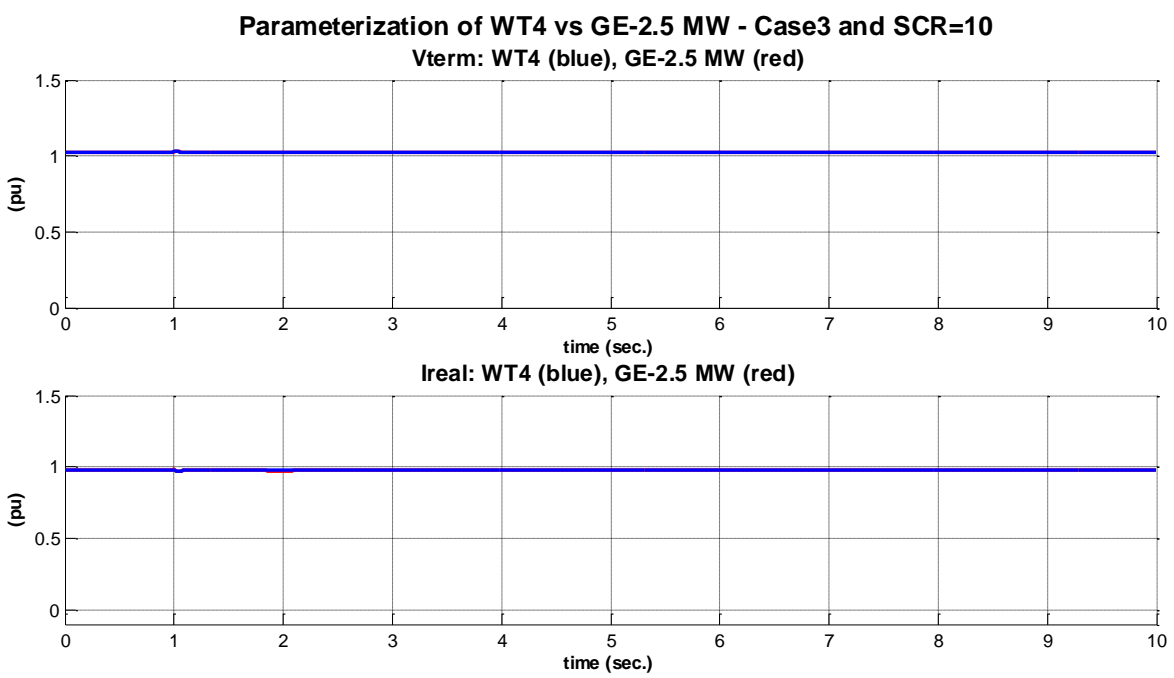

Ireac: WT4 (blue), GE-2.5 MW (red)

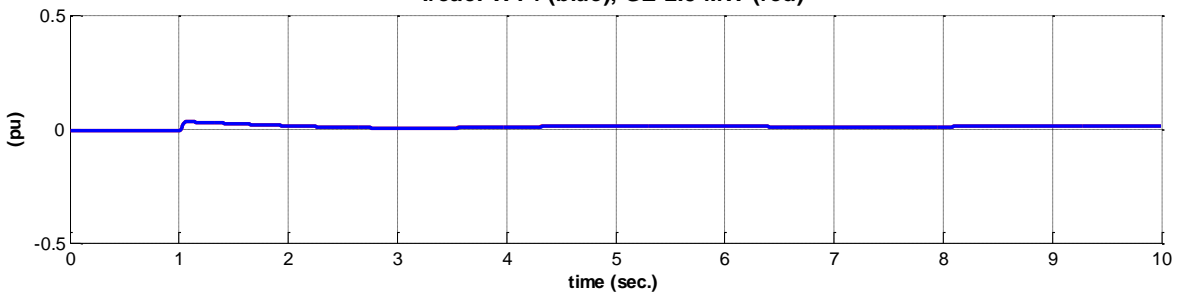

Figure 7-100: Parameterization of WT4 vs GE-2.5 MW - Case 3 and SCR=10 ( $\mathrm{V}_{\text {Term }}, I_{\text {Real }}$, I Reac). 


\subsubsection{Results Validation}

In all figures, the response of the generic model matches the response of the VSM in the frequency range of interest. Thus, both the PSLF and the PSSE models are an appropriate representation of the GE 2.5 MW WTG's behavior for fundamental frequency analysis.

\subsubsection{Vendor Specific Model Validation}

No data available 


\subsection{Mitsubishi - MWT 1000A Series}

\subsubsection{Wind Turbine Characterization}

Mitsubishi's MWT-1000A series is represented by three-blade, upwind, horizontal axis wind turbines with a rated capacity of 1.0-MW, with following features:

- Terminal Voltage: $0.69 \mathrm{kV} / 0.60 \mathrm{kV}(50 \mathrm{~Hz} / 60 \mathrm{~Hz})$.

- $\quad$ Rated power: $1 \mathrm{MW}$.

- Nominal capacity: 1.111 MVA (without shunt capacitors).

- Nominal consumed reactive power: 0.460 MVAr (without shunt capacitors).

- Permanently connected shunt capacitor: 0.34 MVAr.

1) 0.29 MVAr shunt capacitor is connected during the Wind turbine operation.

2) 0.05MVAr shunt capacitor is connected when the generator output is over $1 \mathrm{MW}$ and is disconnected when the output is below $0.5 \mathrm{MW}$.

- $\quad$ Step-up transformer: 1.2 MVA (subject to owner's preferences).

- $\quad$ Step-up transformer reactance: 0.05833 pu (subject to owner's preferences).

- Generator: 4 pole type induction generator.

- $\quad$ Braking system (primary): Aerodynamic brake with blade feathering.

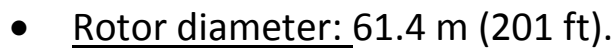

- $\quad$ Cut-in wind speed: $3.0 \mathrm{~m} / \mathrm{s}(6.7 \mathrm{mph})$.

- Cut-out wind speed: $25 \mathrm{~m} / \mathrm{s}(55 \mathrm{mph})$ at $10 \mathrm{~min}$ average and $30 \mathrm{~m} / \mathrm{s}$ (67 mph) at 2 sec average.

- $\quad$ Rated wind speed: $12.5 \mathrm{~m} / \mathrm{s}(28 \mathrm{mph})$

- Wind class: IEC Class IIA

- Power control: Active blade pitch control.

- Hub heights: 55, 60, and $69 \mathrm{~m}(180,197$, and $226 \mathrm{ft})$

- Gearbox: Three stage (1 planetary and 2 parallel) with a ratio of gear 1:76/1:92 $(50 \mathrm{~Hz} / 60 \mathrm{~Hz}$ ) 
The rotor on a MWT-1000A turbine is designed to operate in an upwind configuration at 19.8 revolutions per minute (rpm).

\subsubsection{Vendor Specific Model Characterization}

\subsubsection{Model Performance: Case 1 - Fault Event}

Information being reviewed by vendor. Approval for public release is pending.

\subsubsection{Model Performance: Case 2 - Under-Frequency Event}

Information being reviewed by vendor. Approval for public release is pending.

\subsubsection{Model Performance: Case 3-Over-Frequency Event}

Information being reviewed by vendor. Approval for public release is pending.

\subsubsection{Generic Model Parameterization}

\subsubsection{PSLF Environment}

\subsection{Case 1 - Fault Event}

Information being reviewed by vendor. Approval for public release is pending.

\subsection{Case 2 - Under-Frequency Event}

Information being reviewed by vendor. Approval for public release is pending.

\subsection{Case 3-Over-Frequency Event}

Information being reviewed by vendor. Approval for public release is pending.

\subsubsection{Results Validation}

Information being reviewed by vendor. Approval for public release is pending.

\subsubsection{Vendor Specific Model Validation}

No data available 


\subsection{Mitsubishi - MWT 95 Series}

\subsubsection{Wind Turbine Characterization}

Mitsubishi's MWT-95 series is represented by three-blade, upwind, horizontal axis wind turbines with a rated capacity of 2.4-megawatts, with following features:

- Rotor Diameter: 95 m (312 ft).

- Hub Heights: $80 \mathrm{~m}(262 \mathrm{ft})$.

- $\quad$ Cut-in wind speed: $3 \mathrm{~m} / \mathrm{s}(6.7 \mathrm{mph})$.

- Cut-out wind speed: $25 \mathrm{~m} / \mathrm{s}(55 \mathrm{mph})$ at $10 \mathrm{~min}$ average and $30 \mathrm{~m} / \mathrm{s}(67 \mathrm{mph})$ at 2 sec average.

- Terminal Voltage: $690(50 \mathrm{~Hz} / 60 \mathrm{~Hz})$.

- Generator: Doubly Fed Asynchronous Generator (2,400 kW).

- Power Converter: IGBT Converter.

- Braking System (Primary): Individual Pitch Brake (aerodynamic brake with blade feathering).

MWT-95 is a variable speed wind turbine with a doubly fed asynchronous powertrain. The rotor on a MWT-95 turbine is designed to operate in an upwind configuration at 9.0 to 16.9 revolutions per minute (rpm). These series provides the option of a selectable power factor between 0.9 (inductive) and 0.95 (capacitive). For example, by configuring the machine to operate at a slightly capacitive power factor, the inverters can compensate for reactive power losses within the wind plant collection system, providing approximately unity power factor operation at the point of interconnection over a wide range of plant output.

\subsubsection{Vendor Specific Model Characterization}

\subsubsection{Model Performance: Case 1 - Fault Event}

Information being reviewed by vendor. Approval for public release is pending.

\subsubsection{Model Performance: Case 2 - Under-Frequency Event}

Information being reviewed by vendor. Approval for public release is pending. 


\subsubsection{Model Performance: Case 3 - Over-Frequency Event}

Information being reviewed by vendor. Approval for public release is pending.

\subsubsection{Generic Model Parameterization}

\subsubsection{PSLF Environment}

\subsection{Case 1 - Fault Event}

Information being reviewed by vendor. Approval for public release is pending.

7.7.3.1.1.2 Case 2 - Under-Frequency Event

Information being reviewed by vendor. Approval for public release is pending.

\subsection{Case 3-Over-Frequency Event}

Information being reviewed by vendor. Approval for public release is pending.

\subsubsection{Results Validation}

Information being reviewed by vendor. Approval for public release is pending.

\subsubsection{Vendor Specific Model Validation}

No data available 


\subsection{Nordex - K08 Series}

\subsubsection{Wind Turbine Characterization}

Nordex K08 Gamma series wind turbine is represented by several models of varying size and power. Each type is a three-blade, upwind, horizontal axis wind turbine. These consist of a N90/2.5 MW turbine rated for IEC Class 1a wind sites, a N100/2.5 MW turbine rated for IEC class $2 a$ wind sites version, and a N117/2.4 MW turbine rated for IEC class 3a wind sites. All three of these turbine models are offered with a $50 \mathrm{~Hz}$ and a $60 \mathrm{~Hz}$ variant, as well as an optional cold climate package. These three models include the following features and specifications:

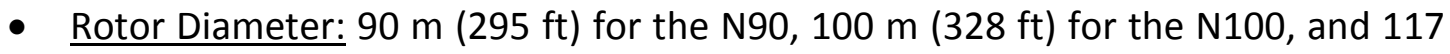
$\mathrm{m}(384 \mathrm{ft})$ for the N117 model.

- $\quad$ Hub Heights: $65 \mathrm{~m}(213 \mathrm{ft}), 75 \mathrm{~m}(246 \mathrm{ft}), 80 \mathrm{~m}(263 \mathrm{ft})$, and $100 \mathrm{~m}$ (328ft) for

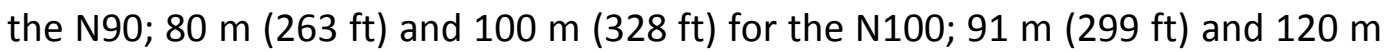
(394 ft) for the N117.

- $\quad$ Cut-in wind speed: $3 \mathrm{~m} / \mathrm{s}(6.7 \mathrm{mph})$.

- Cut-out wind speed: $25 \mathrm{~m} / \mathrm{s}(55.9 \mathrm{mph})$ for the $\mathrm{N} 90$ and N100; $20 \mathrm{~m} / \mathrm{s}$ (44.7 mph) for the N117.

- Terminal Voltage: $660 \mathrm{~V}(50 \mathrm{~Hz} / 60 \mathrm{~Hz})$.

- $\quad$ Cut-back-in Wind Speed: $22 \mathrm{~m} / \mathrm{s}(49.2 \mathrm{mph})$ for the $\mathrm{N} 90$ and N100; $18 \mathrm{~m} / \mathrm{s}(40.3$ $\mathrm{mph}$ ) for the N117

- $\quad$ Power Control: Active blade pitch control of each blade.

- Gearbox: Three-step planetary/helical gear system.

- Generator: Doubly fed asynchronous generator.

- Power Converter: Pulse-Width-Modulated (PWM) converter in 4-quadrant operation.

- Braking System: Electric drive pitch control with battery backup- 3 independent systems, 1 in each blade; Hydraulic parking brake.

Nordex K08 class turbines also offer dynamic power factor range between 0.95 leading and 0.95 lagging, as well as low voltage ride through functions that allow the turbine to continue operating under certain grid fault conditions. All turbines are 
controlled by a software program, and can be accessed world-wide by a remote monitoring network which allows complete control of all turbine parameters through a secure internet gateway.

\subsubsection{Vendor Specific Model Characterization}

\subsubsection{Model Performance: Case 1 - Fault Event}

No data available

\subsubsection{Model Performance: Case 2 - Under-Frequency Event}

No data available

\subsubsection{Model Performance: Case 3-Over-Frequency Event}

No data available

\subsubsection{Generic Model Parameterization}

\subsubsection{PSLF Environment}

No data available

7.8.3.1.1.1 Case 1 - Fault Event

No data available

7.8.3.1.1.2 Case 2-Under-Frequency Event

No data available

\subsection{Case 3-Over-Frequency Event}

No data available

\subsubsection{Results Validation}

No data available

\subsubsection{Vendor Specific Model Validation}

No data available 


\subsection{REPower - MM Series}

\subsubsection{Wind Turbine Characterization}

REpower's MM series is represented by MM 82, MM 92, and MM 100, which are three-blade, upwind, horizontal axis wind turbines with a rated capacity of 2.05megawatts (MM 82 and MM 92) and 1.80-megawatts (MM 100). Following features denote the MM series:

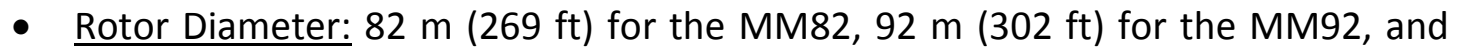
$100 \mathrm{~m}(328 \mathrm{ft})$ for the MM100.

- $\quad$ Rotor Speed: 8.5-17.1 rpm for MM82, 7.8-15 rpm for MM92, 7.8-13.89 rpm for MM100

- $\quad$ Hub Heights: $80 \mathrm{~m}(263 \mathrm{ft})$, and $100 \mathrm{~m}(328 \mathrm{ft})$

- Cut-in wind speed: $3.5 \mathrm{~m} / \mathrm{s}(7.8 \mathrm{mph})$ for the MM82, $3.0 \mathrm{~m} / \mathrm{s}(6.7 \mathrm{mph})$ for the MM92, $3.0 \mathrm{~m} / \mathrm{s}(6.7 \mathrm{mph})$ for the MM100

- Cut-out wind speed: $25 \mathrm{~m} / \mathrm{s}(55.9 \mathrm{mph})$ for the MM82, $24 \mathrm{~m} / \mathrm{s}(53.7 \mathrm{mph})$ for the MM92, $22 \mathrm{~m} / \mathrm{s}$ (49.2 $\mathrm{mph}$ ) for the MM100

- Terminal Voltage: $575 \mathrm{~V}$

- Power Control: Active blade pitch control.

- Gearbox: Combined planetary/spur wheel gearbox.

- Generator: Doubly fed asynchronous generator.

- $\quad$ Power Converter: Pulse width-modulated IGBTs

- Braking System: Electric drive pitch control with battery backup; Hydraulic yaw brake.

- Control Modes: Voltage control, VAR control, and Power Factor control are available.

- $\quad$ Power Factor: 0.95 lead to 0.95 lag is available

\subsubsection{Vendor Specific Model Characterization}

\subsubsection{Model Performance: Case 1 - Fault Event}

No data available 
7.9.2.2 Model Performance: Case 2 - Under-Frequency Event

No data available

7.9.2.3 Model Performance: Case 3-Over-Frequency Event

No data available

\subsubsection{Generic Model Parameterization}

\subsubsection{PSLF Environment}

No data available

7.9.3.1.1.1 Case 1 - Fault Event

No data available

7.9.3.1.1.2 Case 2-Under-Frequency Event

No data available

7.9.3.1.1.3 Case 3-Over-Frequency Event

No data available

\subsubsection{Results Validation}

No data available

\subsubsection{Vendor Specific Model Validation}

No data available 


\subsection{Siemens}

7.10.1 Wind Turbine Characterization

No data available

7.10.2 Vendor Specific Model Characterization

7.10.2.1 Model Performance: Case 1 - Fault Event

No data available

7.10.2.2 Model Performance: Case 2 - Under-Frequency Event

No data available

7.10.2.3 Model Performance: Case 3-Over-Frequency Event

No data available

\subsubsection{Generic Model Parameterization}

\subsubsection{PSLF Environment}

No data available

7.10.3.1.1.1 Case 1 - Fault Event

No data available

7.10.3.1.1.2 Case 2 - Under-Frequency Event

No data available

7.10.3.1.1.3 Case 3 - Over-Frequency Event

No data available

\subsubsection{Results Validation}

No data available

7.10.4 Vendor Specific Model Validation

No data available 


\subsection{Suzlon - S88 Series}

\subsubsection{Wind Turbine Characterization}

Information being reviewed by vendor. Approval for public release is pending.

\subsubsection{Vendor Specific Model Characterization}

\subsubsection{Model Performance: Case 1 - Fault Event}

Information being reviewed by vendor. Approval for public release is pending.

\subsubsection{Model Performance: Case 2 - Under-Frequency Event}

Information being reviewed by vendor. Approval for public release is pending.

\subsubsection{Model Performance: Case 3 - Over-Frequency Event}

Information being reviewed by vendor. Approval for public release is pending.

\subsubsection{Generic Model Parameterization}

\subsubsection{PSLF Environment}

\subsection{Case 1 - Fault Event}

Information being reviewed by vendor. Approval for public release is pending.

\subsection{Case 2 - Under-Frequency Event}

Information being reviewed by vendor. Approval for public release is pending.

\subsection{Case 3 - Over-Frequency Event}

Information being reviewed by vendor. Approval for public release is pending.

\subsubsection{Results Validation}

Information being reviewed by vendor. Approval for public release is pending.

\subsubsection{Vendor Specific Model Validation}

No data available 


\subsection{Suzlon - S9x Series}

\subsubsection{Wind Turbine Characterization}

Information being reviewed by vendor. Approval for public release is pending.

\subsubsection{Vendor Specific Model Characterization}

\subsubsection{Model Performance: Case 1 - Fault Event}

Information being reviewed by vendor. Approval for public release is pending.

\subsubsection{Model Performance: Case 2 - Under-Frequency Event}

Information being reviewed by vendor. Approval for public release is pending.

\subsubsection{Model Performance: Case 3 - Over-Frequency Event}

Information being reviewed by vendor. Approval for public release is pending.

\subsubsection{Generic Model Parameterization}

\subsubsection{PSLF Environment}

\subsection{Case 1 - Fault Event}

Information being reviewed by vendor. Approval for public release is pending.

\subsection{Case 2 - Under-Frequency Event}

Information being reviewed by vendor. Approval for public release is pending.

\subsection{Case 3 - Over-Frequency Event}

Information being reviewed by vendor. Approval for public release is pending.

\subsubsection{Results Validation}

Information being reviewed by vendor. Approval for public release is pending.

\subsubsection{Vendor Specific Model Validation}

No data available 


\subsection{Vestas - Type I Models}

\subsubsection{Example List of Vestas Type I Wind Turbines}

- $\quad \mathrm{V} 82-1.65 \mathrm{MW}$

\subsubsection{Vendor Specific Model Characterization}

\subsubsection{Model Performance: Case 1 - Fault Event}

In this case a remote fault is applied to bus 12 for a duration of 6 cycles $(0.1 \mathrm{sec})$.

In the course of the project when making a parameterization of the WT1 generic model for the system described above, the following observations have been made:

1. For SCR=5, the benchmark Vestas: Type 1 turbine model does not show a stable response to the fault cleared by tripping the line. However it shows a stable response to the fault cleared without a line tripping.

2. So, for the system with $\mathrm{SCR}=5$ it makes sense to parameterize the WT1 model without tripping the line, while for the system with $S C R=10$, the parameterization was carried out for WT1 model with the line tripping.

\section{$\underline{\mathrm{SCR}-5}$}
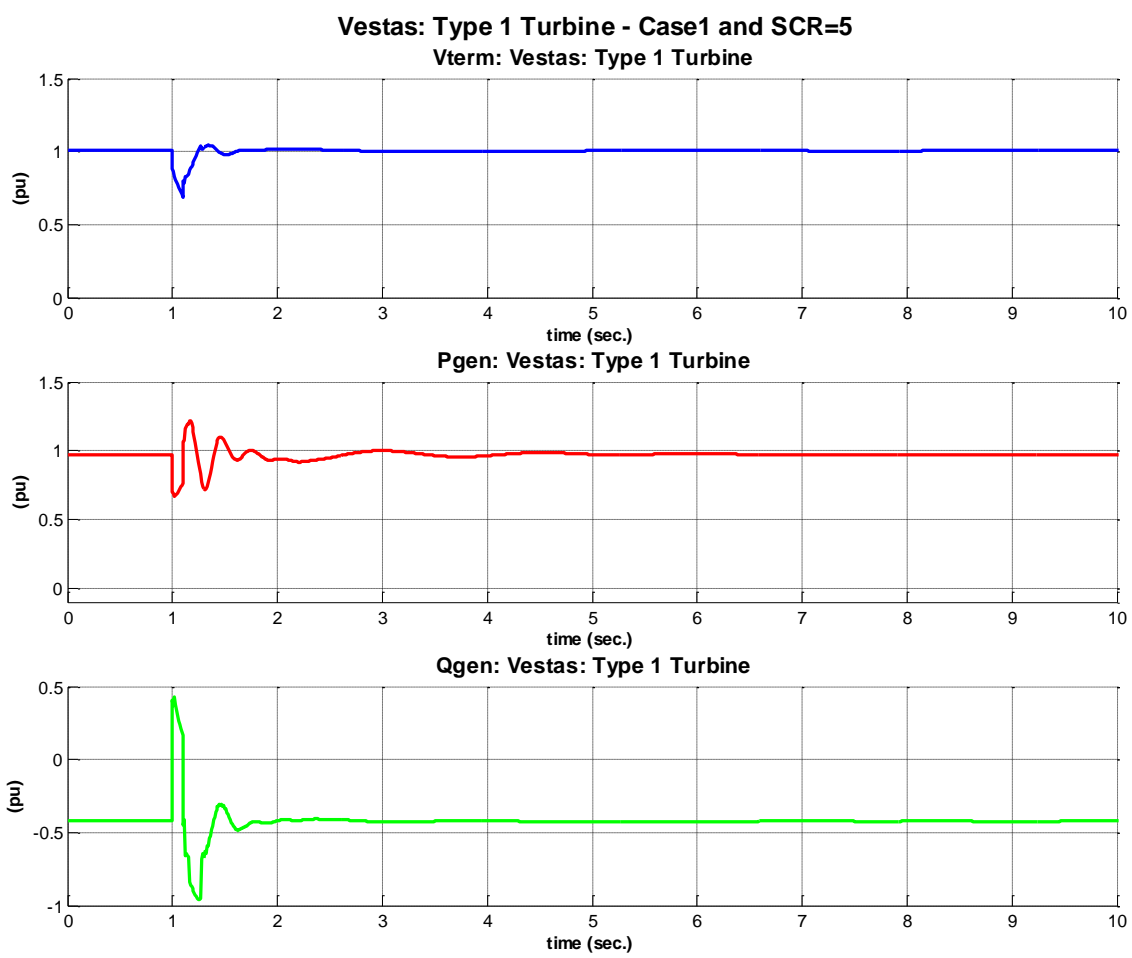

Figure 7-101: Vestas: Type 1 turbine - Case 1 and $S C R=5\left(V_{\text {Term }}, P, Q\right)$. 
Vestas: Type 1 Turbine - Case 1 and SCR $=5$

Vterm: Vestas: Type 1 Turbine

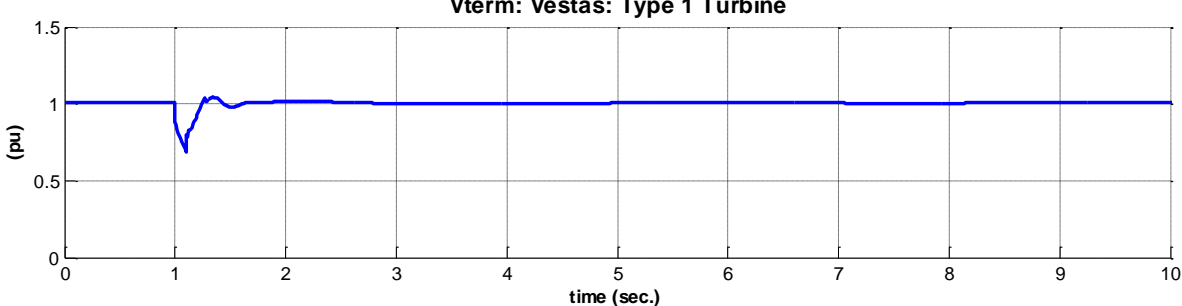

Ireal: Vestas: Type 1 Turbine

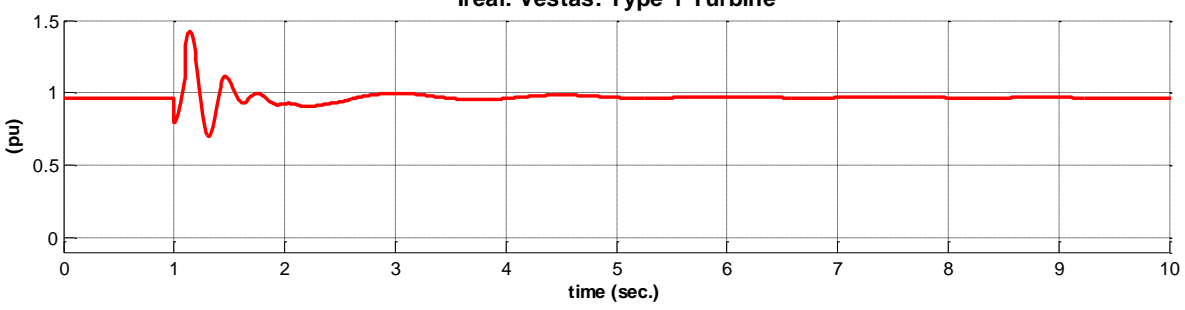

Ireac: Vestas: Type 1 Turbine

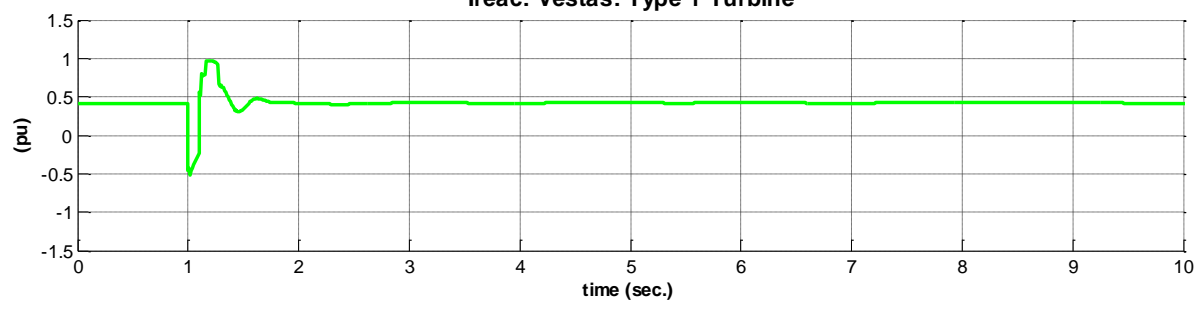

Figure 7-102: Vestas: Type 1 turbine - Case 1 and $S C R=5\left(V_{\text {Term }}, I_{\text {Real }}, I_{\text {Reac }}\right)$.

$\underline{\text { SCR }-10}$

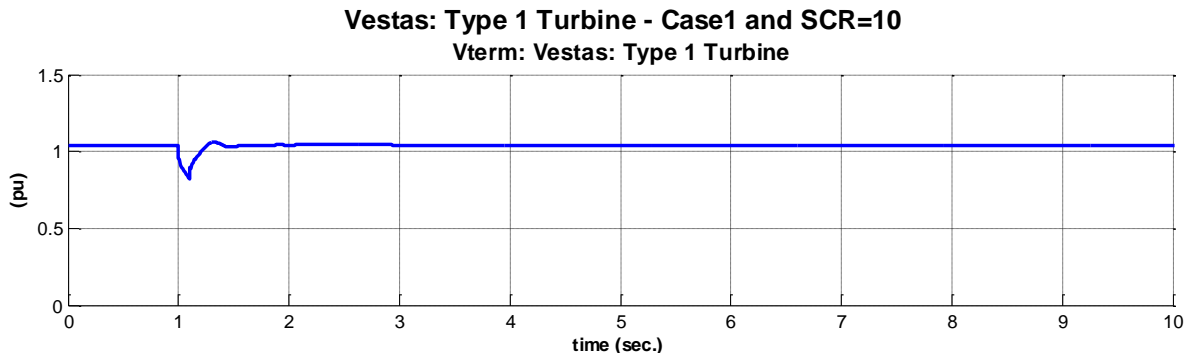

Pgen: Vestas: Type 1 Turbine

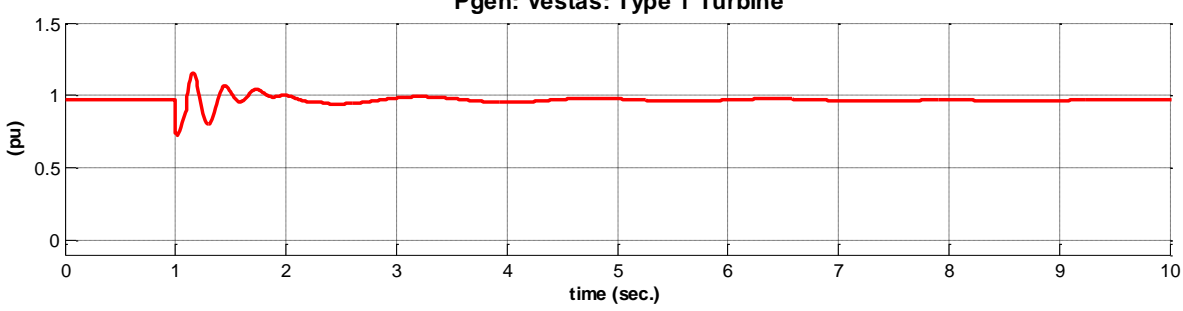

Qgen: Vestas: Type 1 Turbine

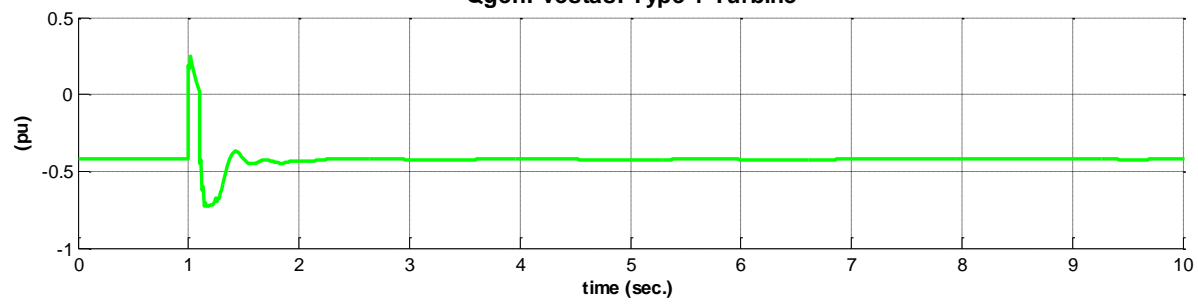

Figure 7-103: Vestas: Type 1 turbine - Case 1 and $\operatorname{SCR}=10\left(V_{\text {Term }}, P, Q\right)$. 

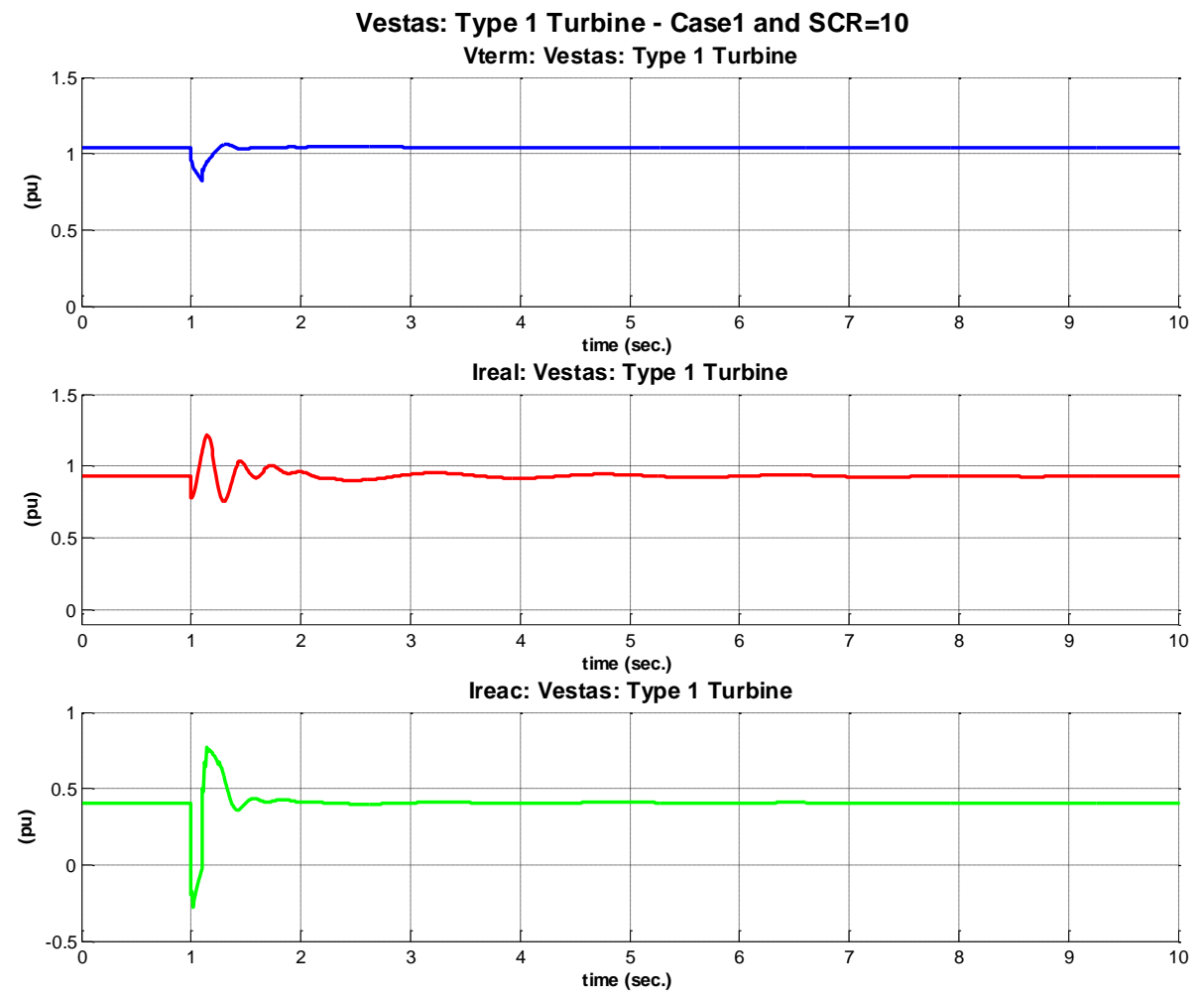

Figure 7-104: Vestas: Type 1 turbine - Case 1 and $S C R=10\left(V_{\text {Term }}, I_{\text {Real }}, I_{\text {Reac }}\right)$. 


\subsubsection{Model Performance: Case 2 - Under-Frequency Event}

In this case a under frequency event is created by tripping the $100 \mathrm{MW}$ generation unit at bus 20 .

\section{SCR -5}
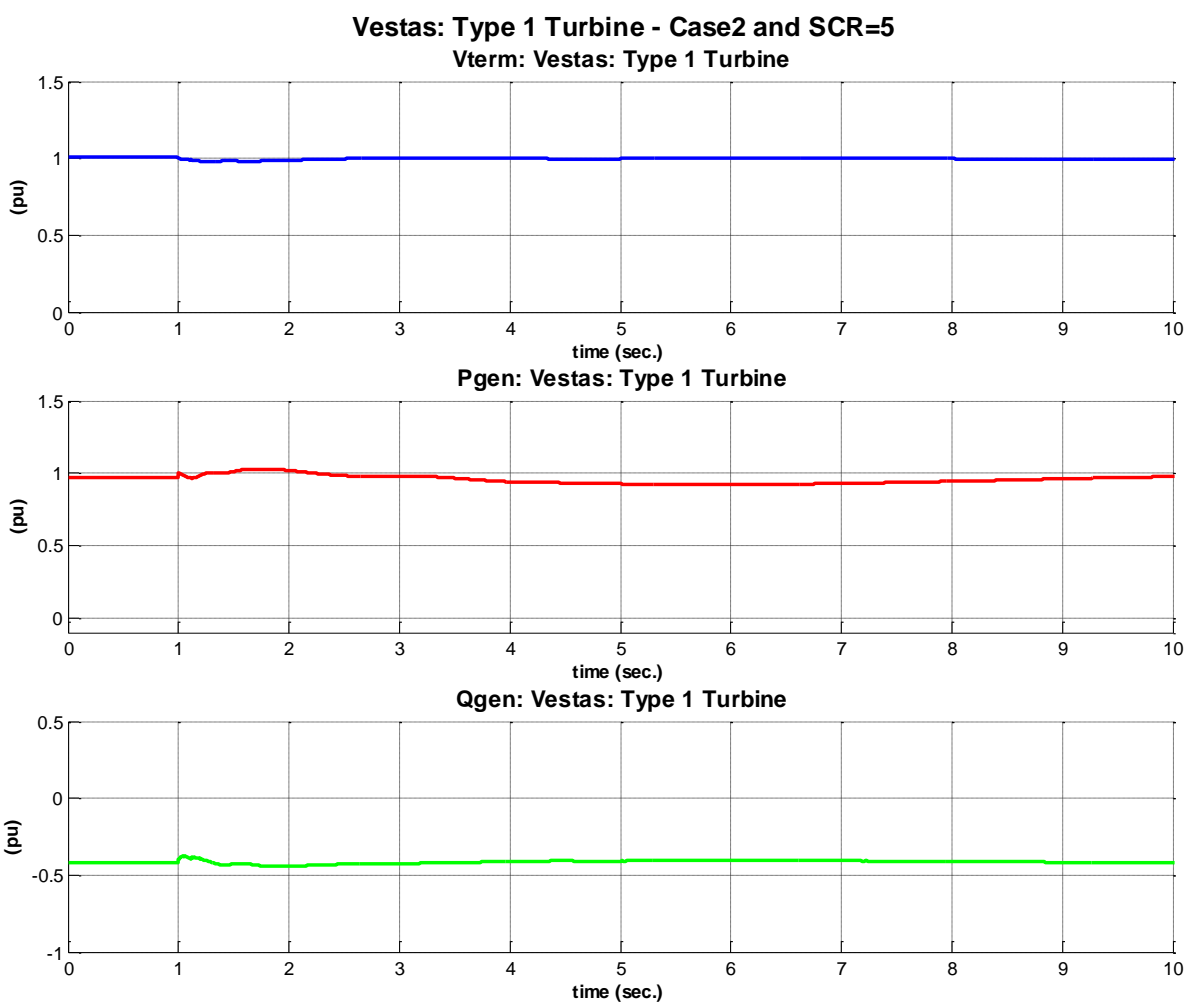

Figure 7-105: Vestas: Type 1 turbine - Case 2 and $S C R=5\left(V_{\text {Term }}, P, Q\right)$. 
Vestas: Type 1 Turbine - Case2 and SCR $=5$

Vterm: Vestas: Type 1 Turbine

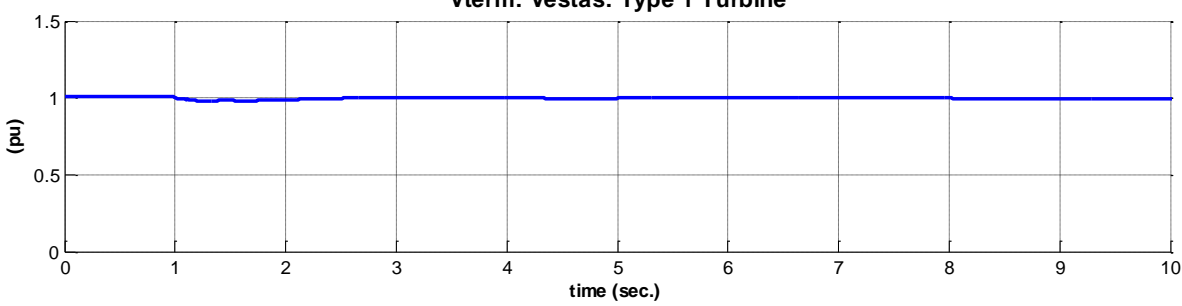

Ireal: Vestas: Type 1 Turbine

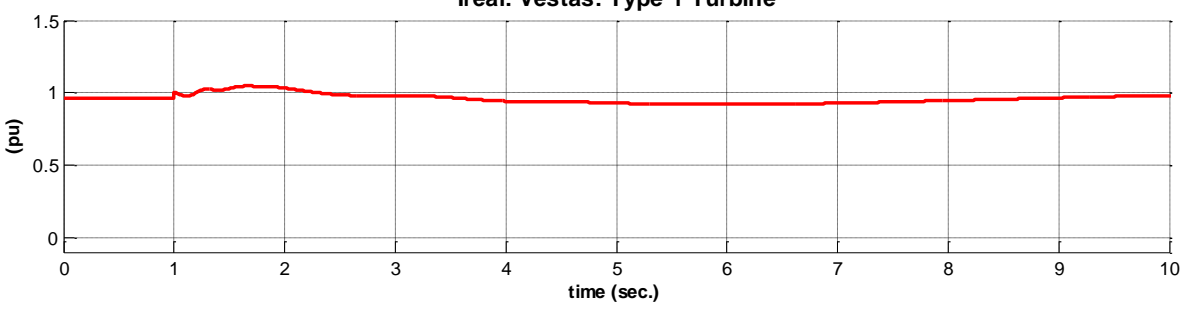

Ireac: Vestas: Type 1 Turbine

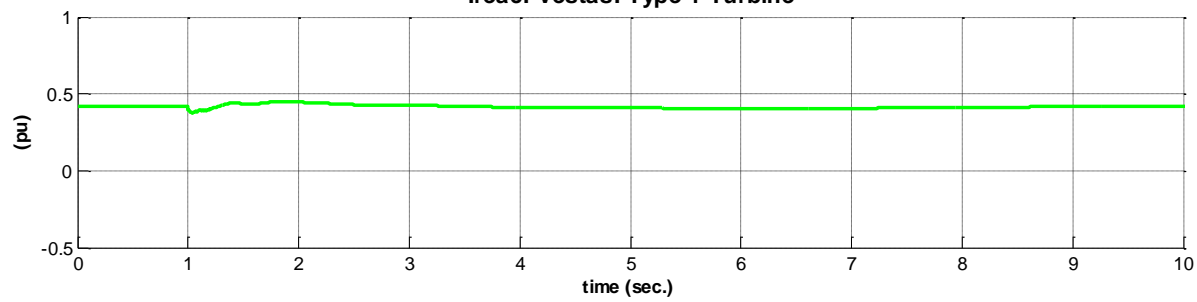

Figure 7-106: Vestas: Type 1 turbine - Case 2 and $S C R=5\left(V_{\text {Term }}, I_{\text {Real }}, I_{\text {Reac }}\right)$.

$\underline{\text { SCR }-10}$

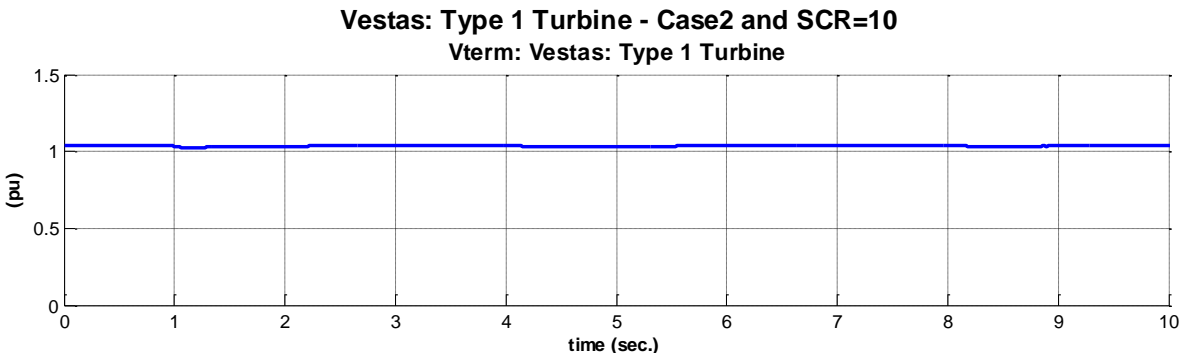

Pgen: Vestas: Type 1 Turbine

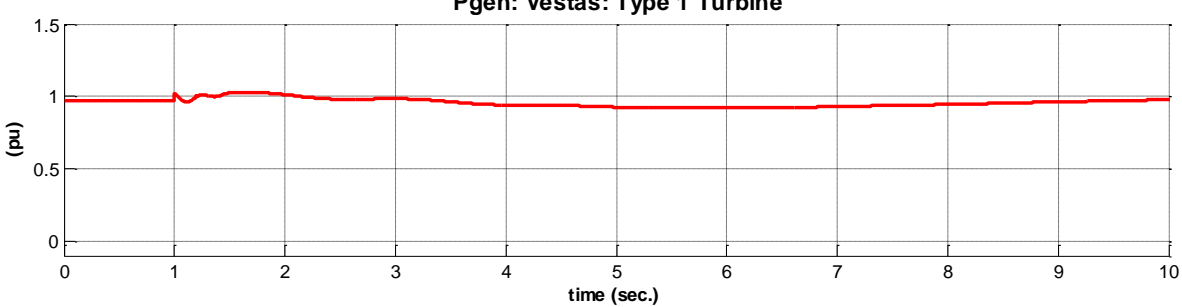

Qgen: Vestas: Type 1 Turbine

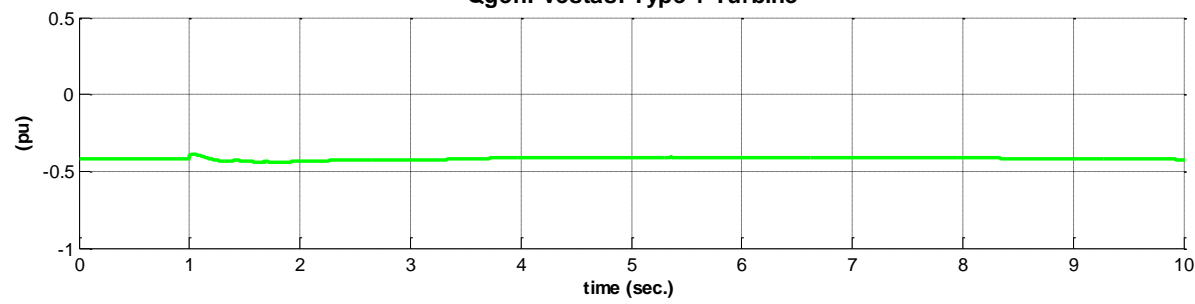

Figure 7-107: Vestas: Type 1 turbine - Case 2 and SCR=10 $\left(V_{\text {Term }}, P, Q\right)$. 

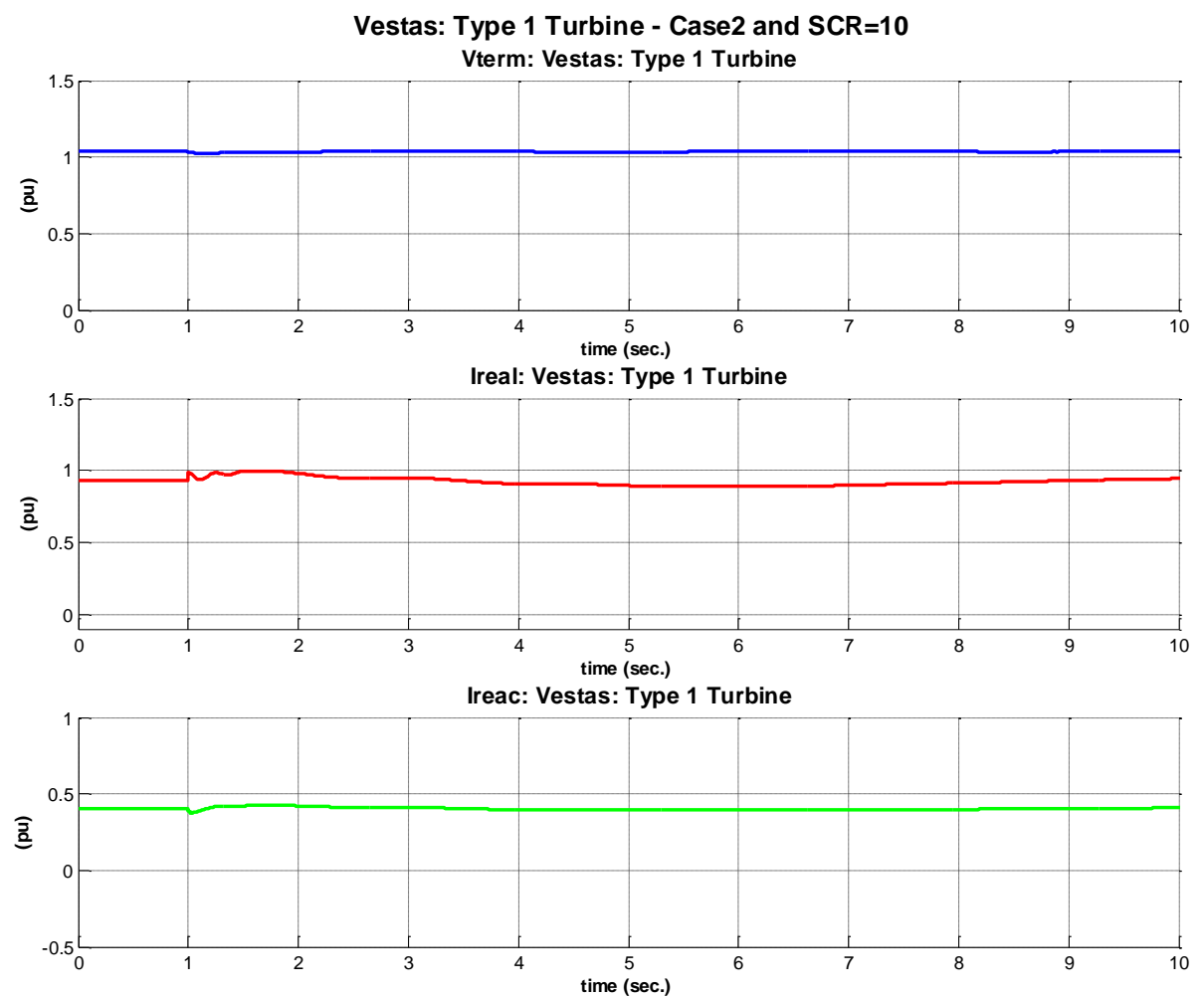

Figure 7-108: Vestas: Type 1 turbine - Case 2 and $S C R=10\left(V_{\text {Term }}, I_{\text {Real }}, I_{\text {Reac }}\right)$. 


\subsubsection{Model Performance: Case 3 - Over-Frequency Event}

In this case a under frequency event is created by tripping the $100 \mathrm{MW}$ load at bus 11.

$\underline{\mathrm{SCR}-5}$
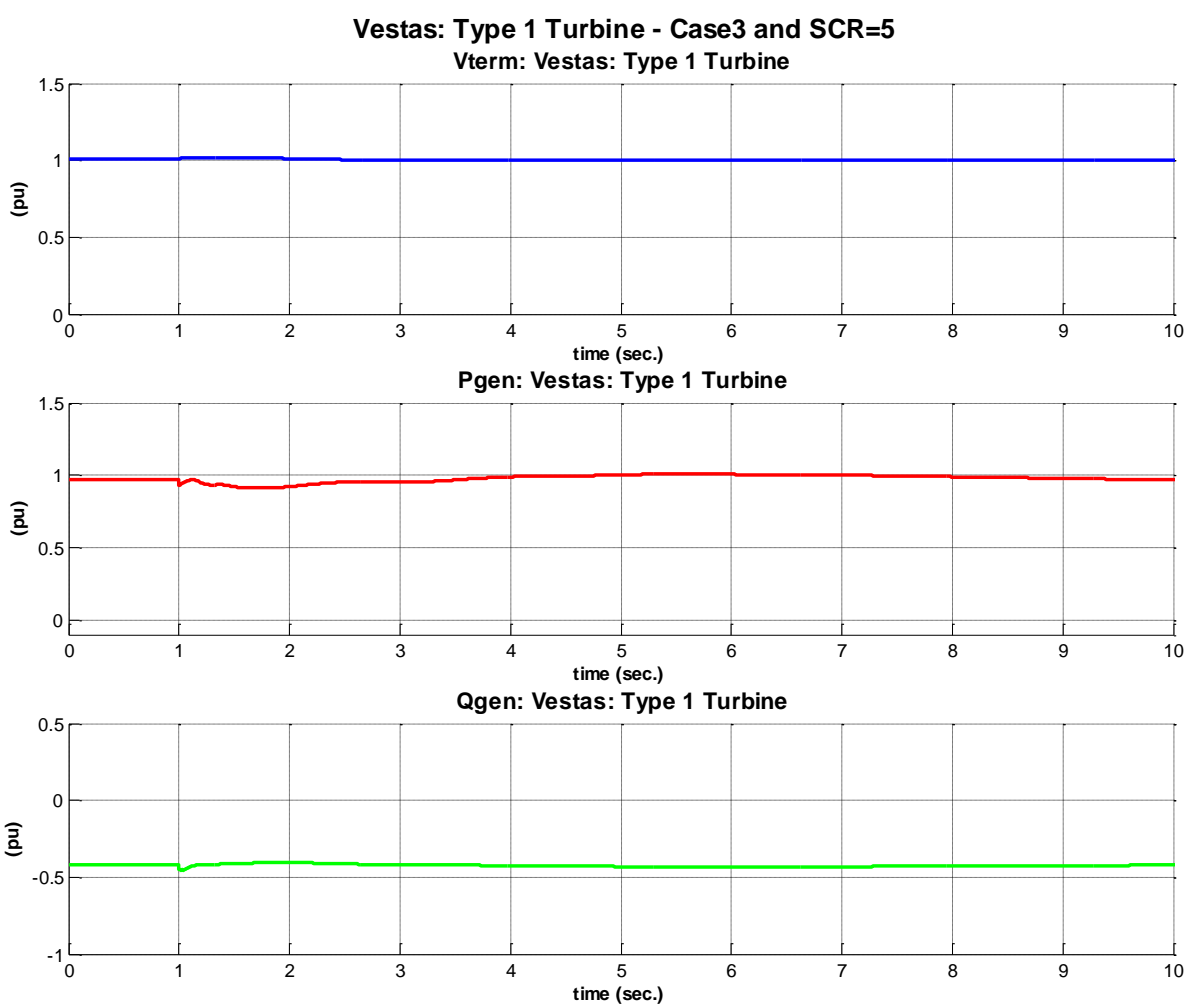

Figure 7-109: Vestas: Type 1 turbine - Case 3 and $S C R=5\left(V_{\text {Term }}, P, Q\right)$. 

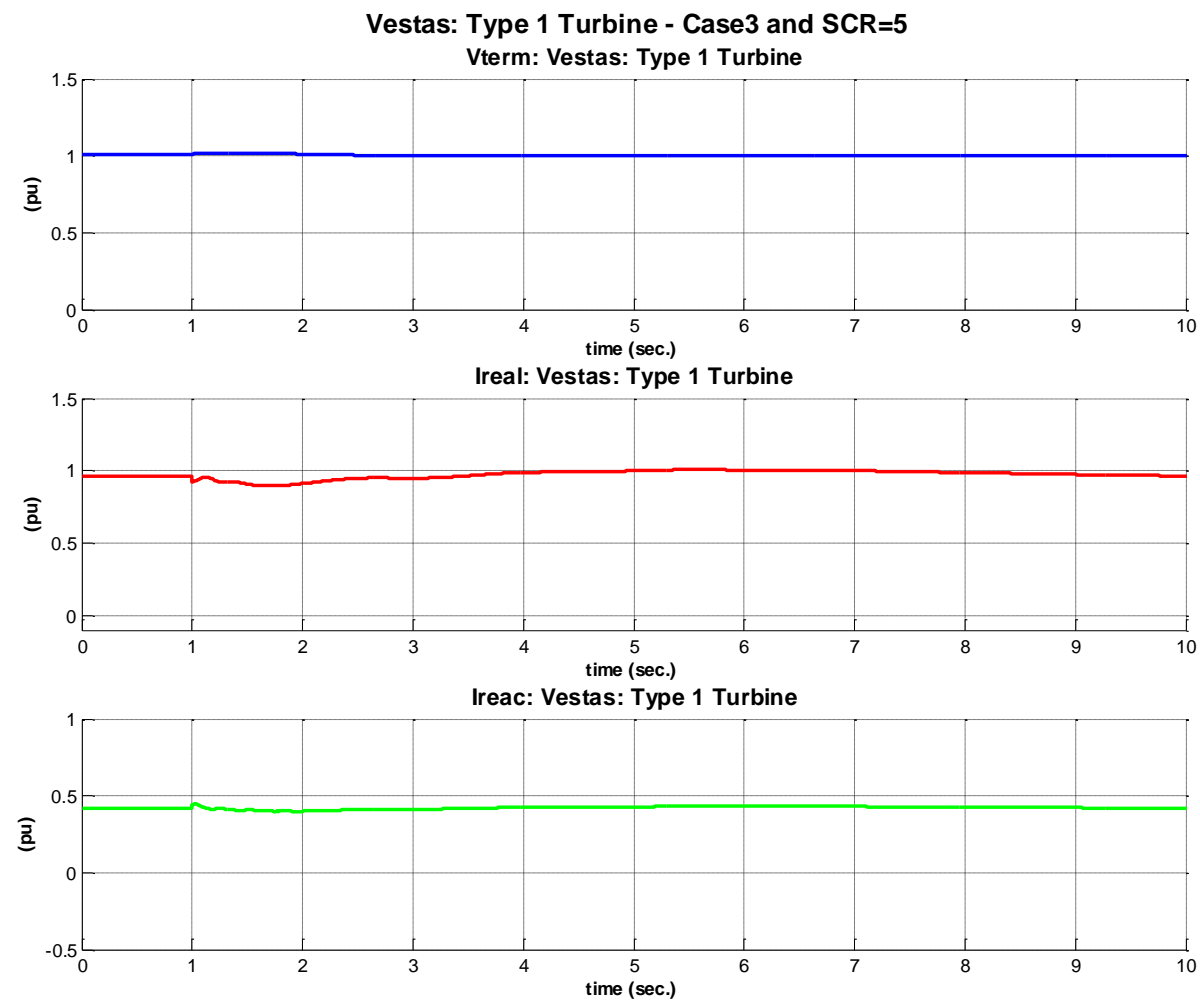

Figure 7-110: Vestas: Type 1 turbine - Case 3 and $S C R=5\left(V_{\text {Term, }} I_{\text {Real }}, I_{\text {Reac }}\right)$.

\section{SCR - 10}

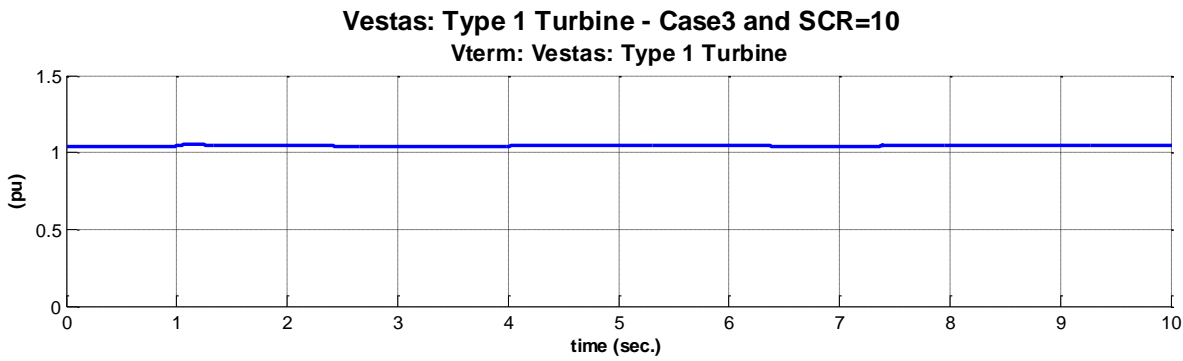

Pgen: Vestas: Type 1 Turbine

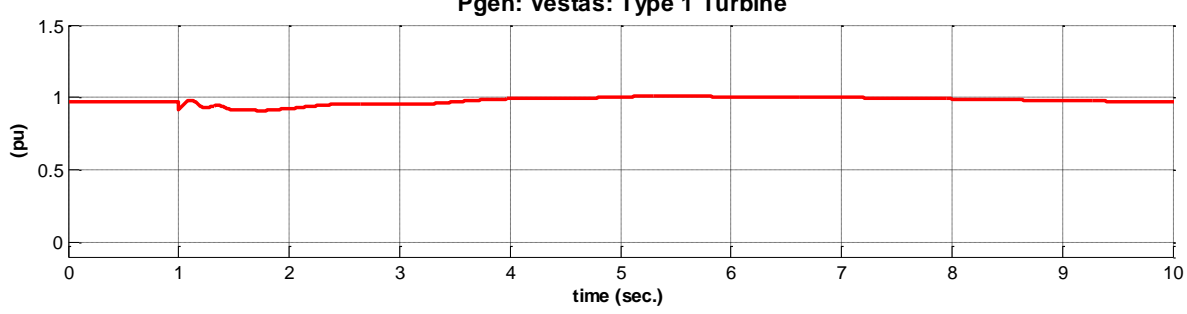

Qgen: Vestas: Type 1 Turbine

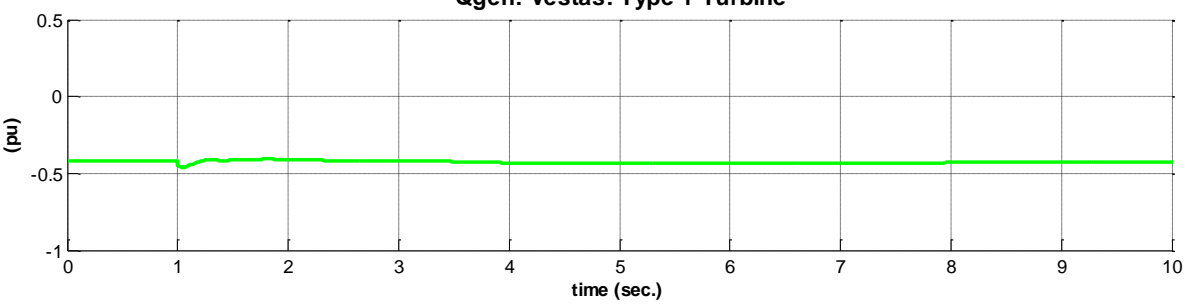

Figure 7-111: Vestas: Type 1 turbine - Case 3 and $\operatorname{SCR}=10\left(V_{\text {Term }}, P, Q\right)$. 

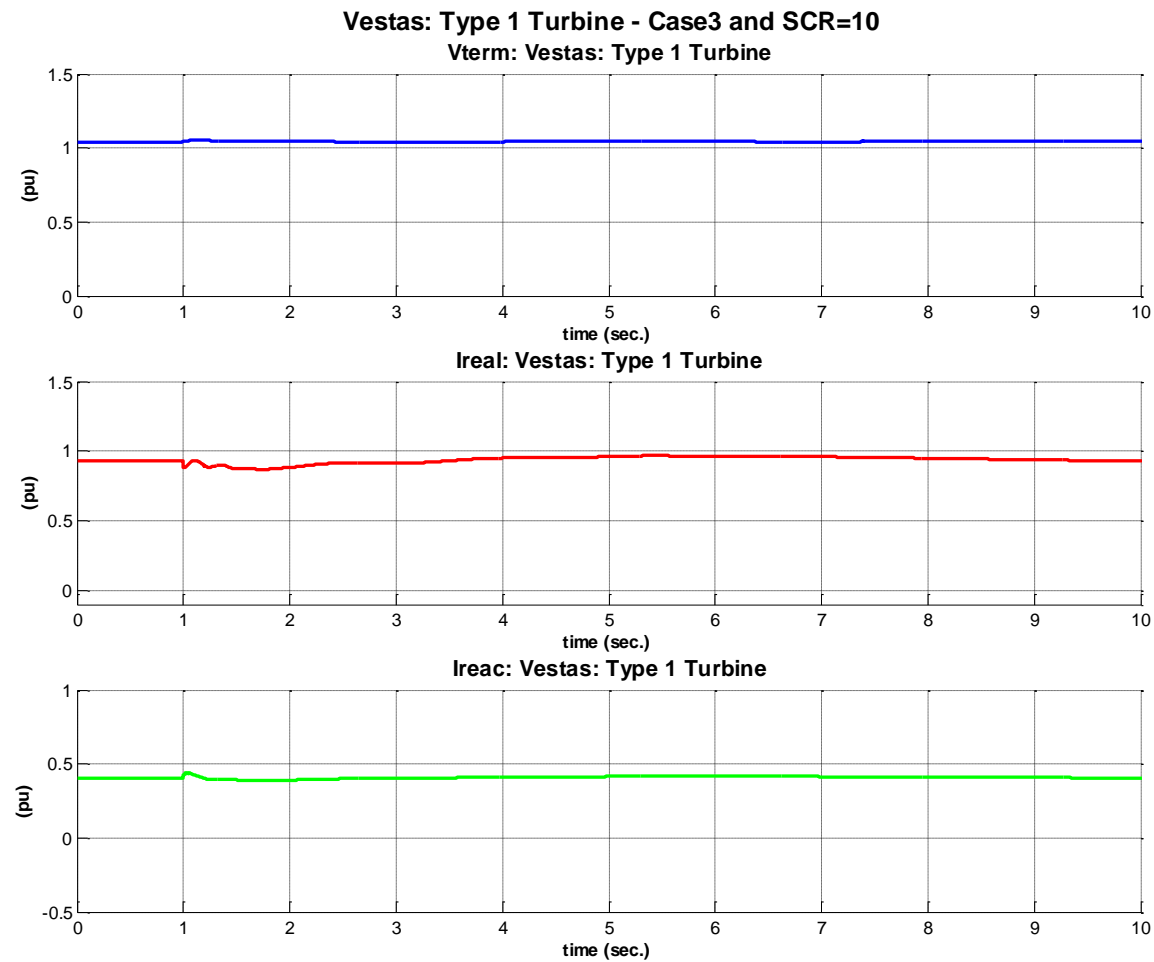

Figure 7-112: Vestas: Type 1 turbine - Case 3 and $S C R=10\left(V_{\text {Term }}, I_{\text {Real, }} I_{\text {Reac }}\right)$.

\subsubsection{Generic Model Parameterization}

\subsubsection{PSSE Environment ${ }^{1}$}

The PSSE platform generic wind turbine parameters, to mimic the behavior of Vestas' type 1 wind turbines, are shown in Table 7-6. The parameter values shown in the table resulted from a compromise between the simulated cases (i.e. SCR 5 and SCR 10) and network conditions (i.e. fault, under- and over frequency).

Table 7-6: PSSE generic model parameter values.

\begin{tabular}{|c|c|c|c|c|c|}
\hline \multicolumn{2}{|c|}{ WT1G } & \multicolumn{2}{|c|}{ WT12A } & \multicolumn{2}{|c|}{ WT12T } \\
\hline $\mathrm{T}^{\prime}$ & 1.262 & DROOP & 8.191367 & $\mathrm{H}$ & 2.94 \\
\hline$T^{\prime \prime}$ & 0.00 & $\mathrm{KP}$ & 0 & DAMP & 0.00 \\
\hline$x$ & 4.01 & $\mathrm{TI}$ & 20.79893 & Htfrac & 0.7279 \\
\hline$X^{\prime}$ & 0.1581 & T1 & 1.161715 & Freq1 & 2.000 \\
\hline$X^{\prime \prime}$ & 0.000 & $\mathrm{~T} 2$ & 0 & DSHAFT & 1.000 \\
\hline$X \mathrm{LL}$ & 0.0873 & TPE & 0 & & \\
\hline E1 & 1.000 & LIMMAX & 0.936986 & & \\
\hline $\mathrm{S}(\mathrm{E} 1)$ & 0.0600 & LIMMIN & 0.25 & & \\
\hline E2 & 1.200 & & & & \\
\hline$S(E 2)$ & 0.15 & & & & \\
\hline
\end{tabular}

\footnotetext{
${ }^{1}$ Parameterization performed by Siemens Energy
} 


\subsection{Case 1 - Fault Event}

\section{SCR - 5}

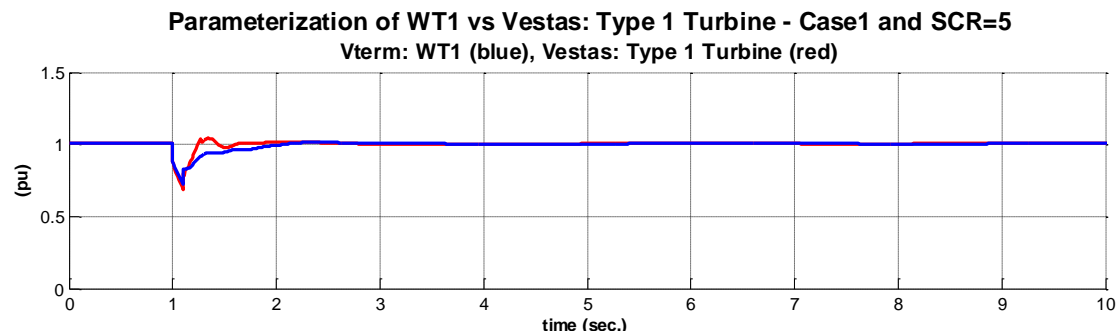

Pgen: WT1 (blue), Vestas: Type 1 Turbine (red)

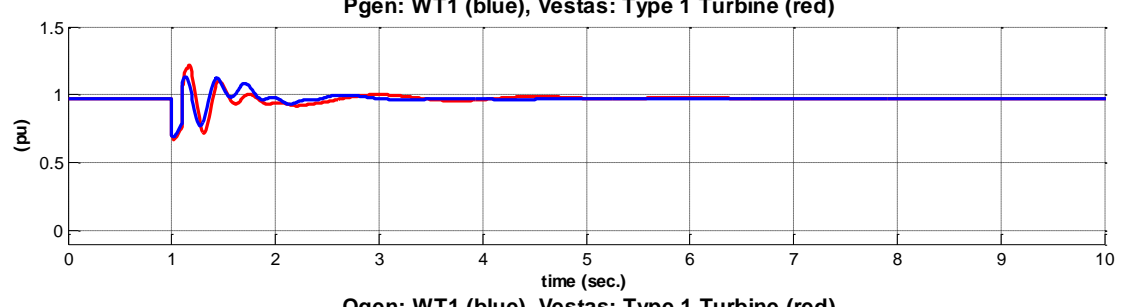

Qgen: WT1 (blue), Vestas: Type 1 Turbine (red)

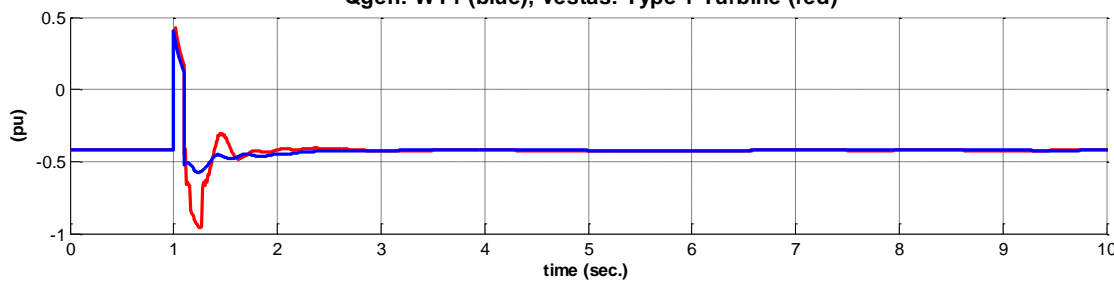

Figure 7-113: Parameterization of WT1 vs Vestas: Type 1 turbine - Case 1 and SCR=5 $\left(\mathrm{V}_{\text {Term }}, \mathrm{P}, \mathrm{Q}\right)$.
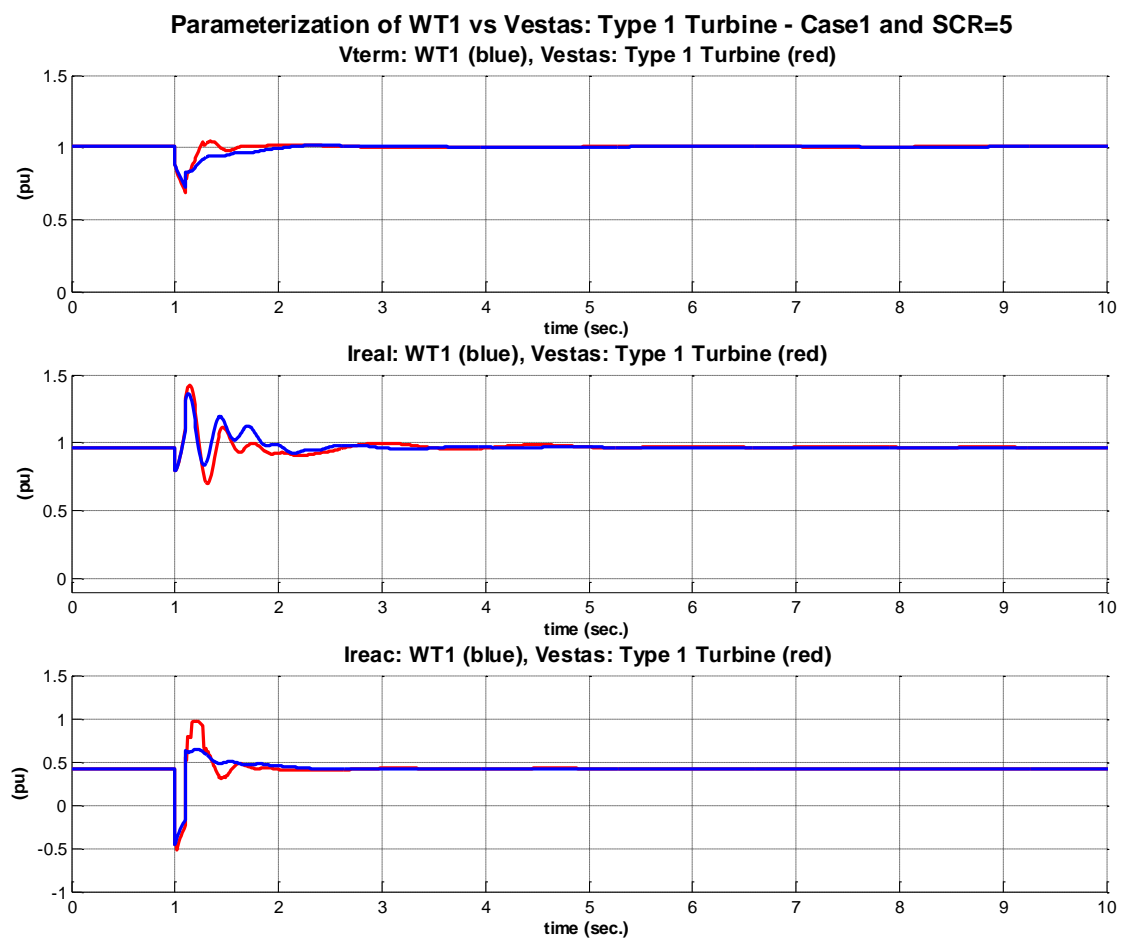

Figure 7-114: Parameterization of WT1 vs Vestas: Type 1 turbine - Case 1 and SCR=5 $\left(V_{\text {Term }}, I_{\text {Real }}, I_{\text {Reac }}\right)$. 
$\underline{\text { SCR }-10}$
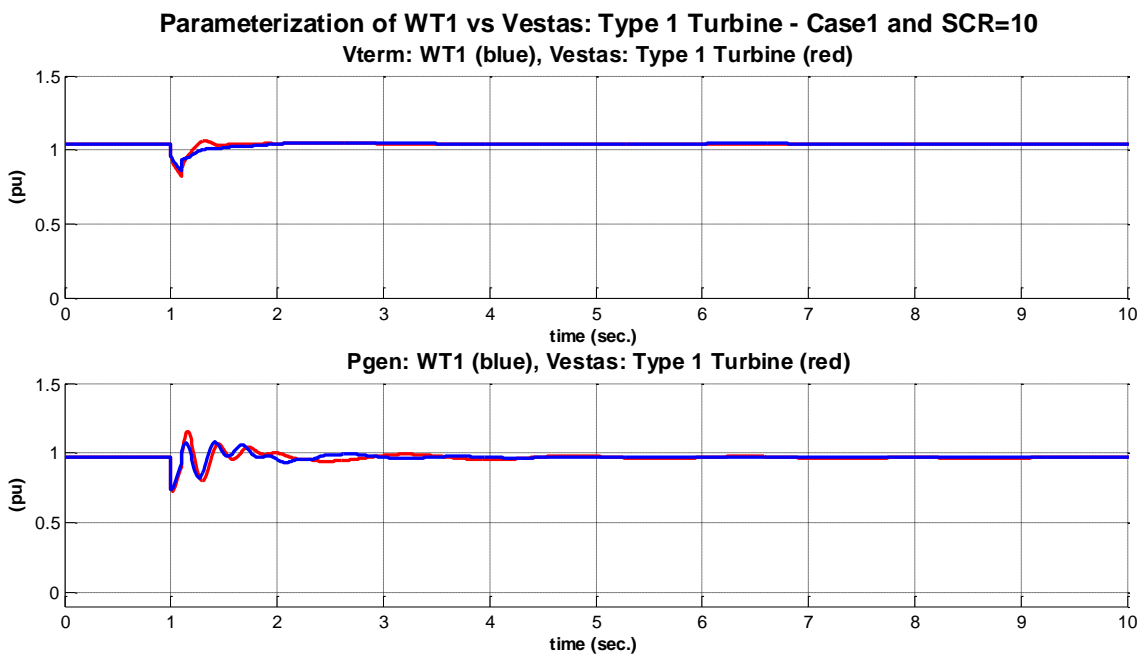

Qgen: WT1 (blue), Vestas: Type 1 Turbine (red)

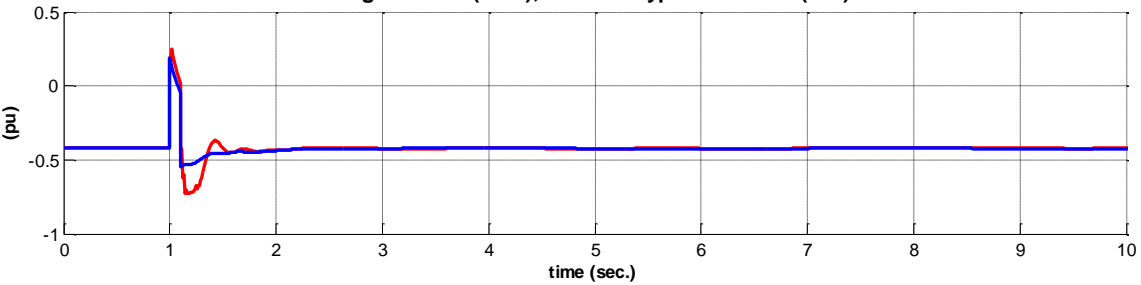

Figure 7-115: Parameterization of WT1 vs Vestas: Type 1 turbine - Case 1 and SCR=10 $\left(\mathrm{V}_{\text {Term }}, \mathrm{P}, \mathrm{Q}\right)$.
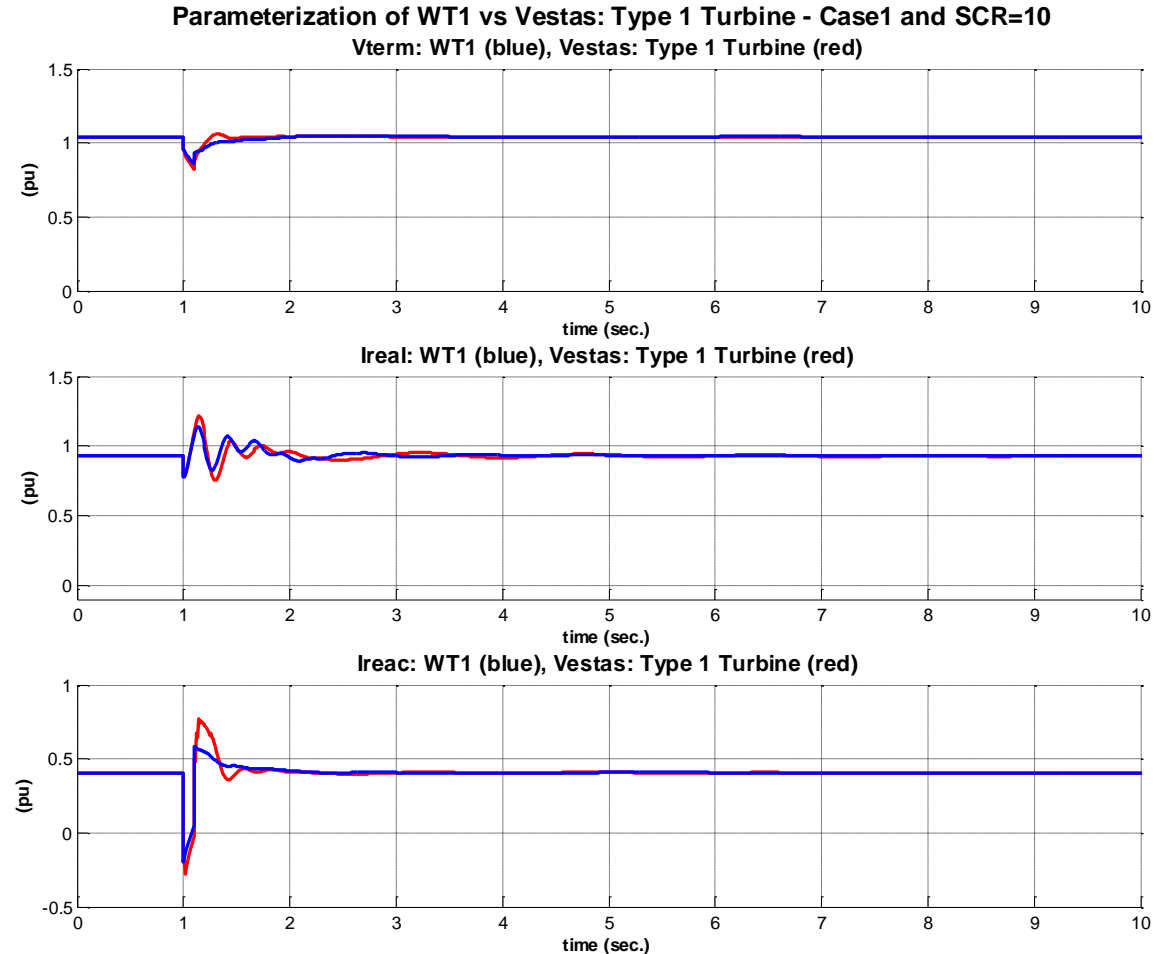

Figure 7-116: Parameterization of WT1 vs Vestas: Type 1 turbine - Case 1 and SCR=10 $\left(\mathrm{V}_{\text {Term, }}, \mathrm{I}_{\text {Real }}, \mathrm{I}_{\text {Reac }}\right)$. 


\subsection{Case 2 - Under-Frequency Event}

\section{$\underline{\mathrm{SCR}-5}$}
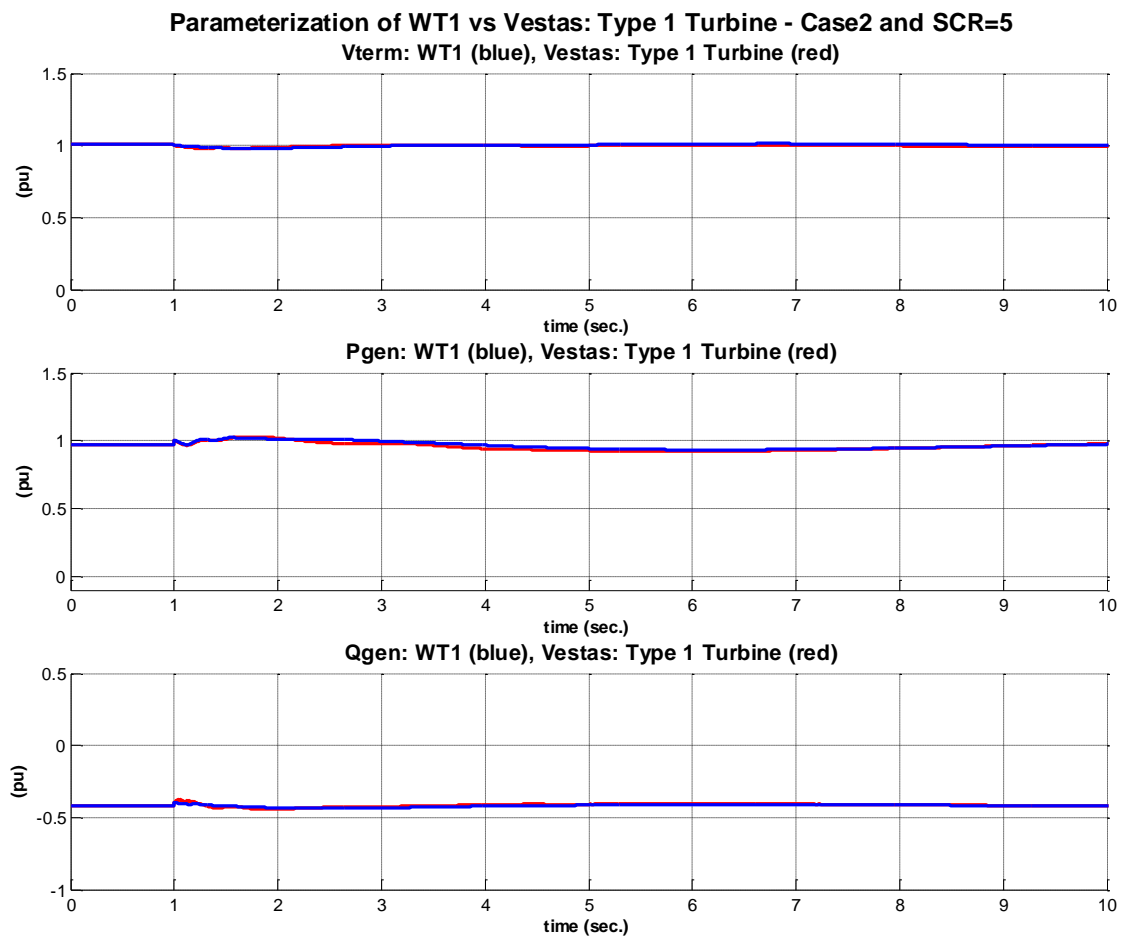

Figure 7-117: Parameterization of WT1 vs Vestas: Type 1 turbine - Case 2 and SCR=5 $\left(\mathrm{V}_{\text {Term }}, \mathrm{P}, \mathrm{Q}\right)$.
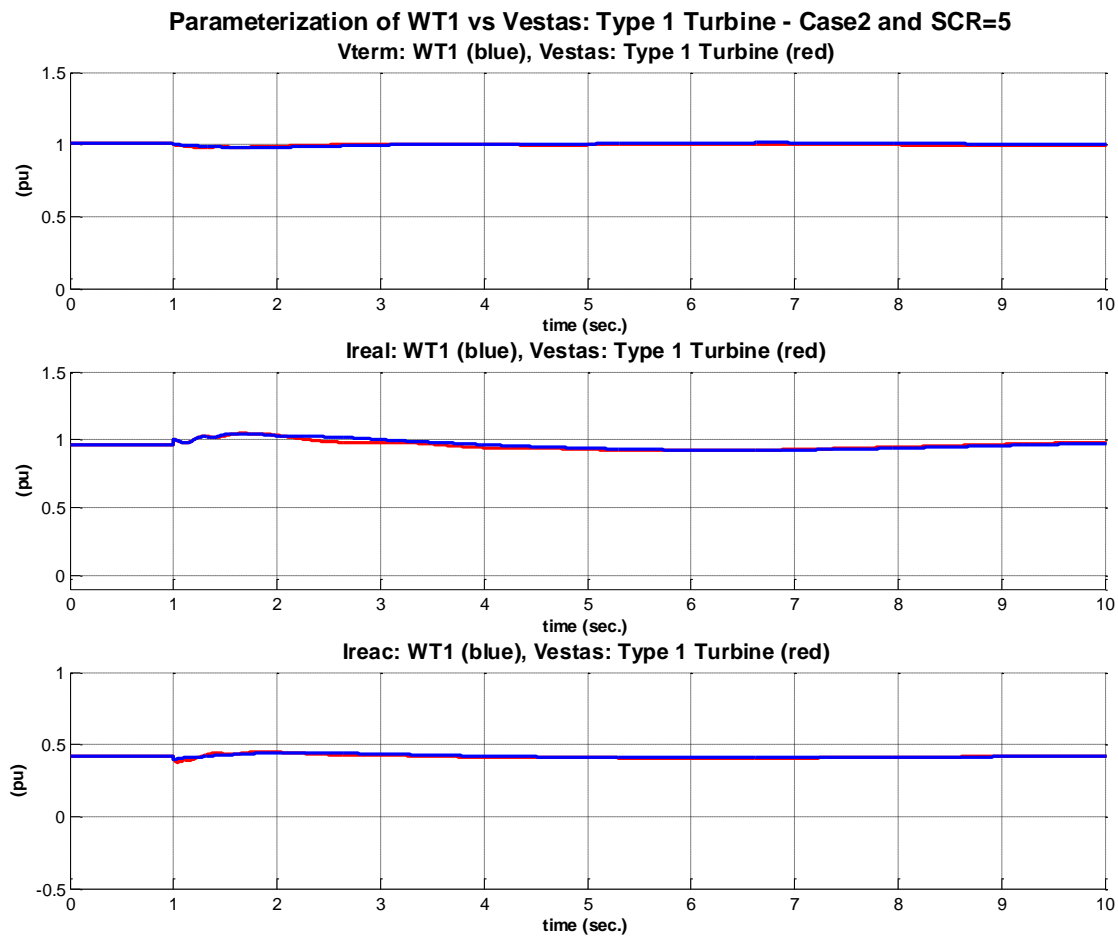

Figure 7-118: Parameterization of WT1 vs Vestas: Type 1 turbine - Case 2 and SCR=5 $\left(V_{\text {Term }}, I_{\text {Real }}, I_{\text {Reac }}\right)$. 
$\underline{\text { SCR }-10}$

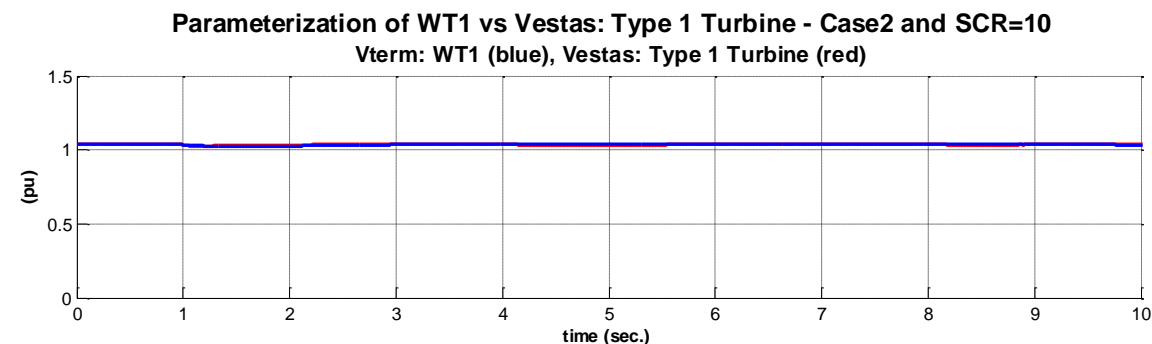

Pgen: WT1 (blue), Vestas: Type 1 Turbine (red)

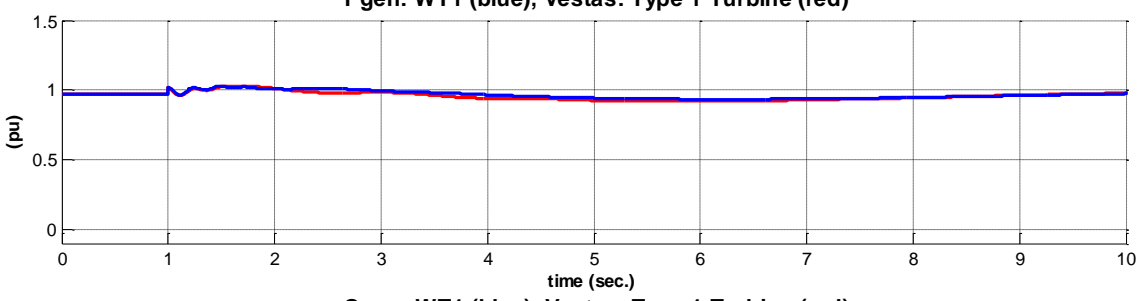

Qgen: WT1 (blue), Vestas: Type 1 Turbine (red)

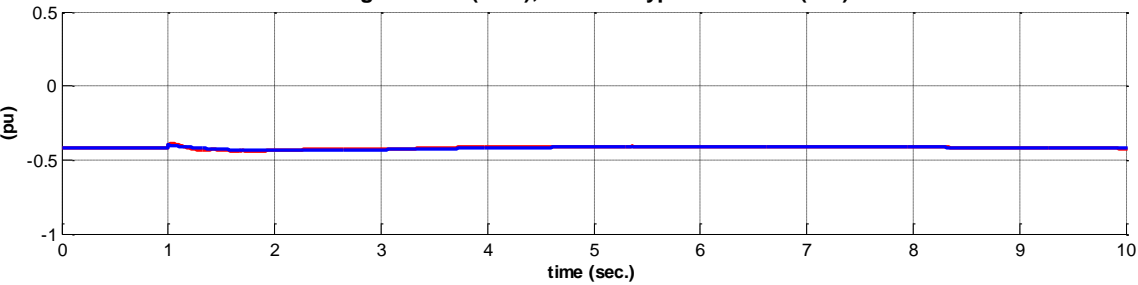

Figure 7-119: Parameterization of WT1 vs Vestas: Type 1 turbine - Case 2 and SCR=10 $\left(\mathrm{V}_{\text {Term }}, \mathrm{P}, \mathrm{Q}\right)$.

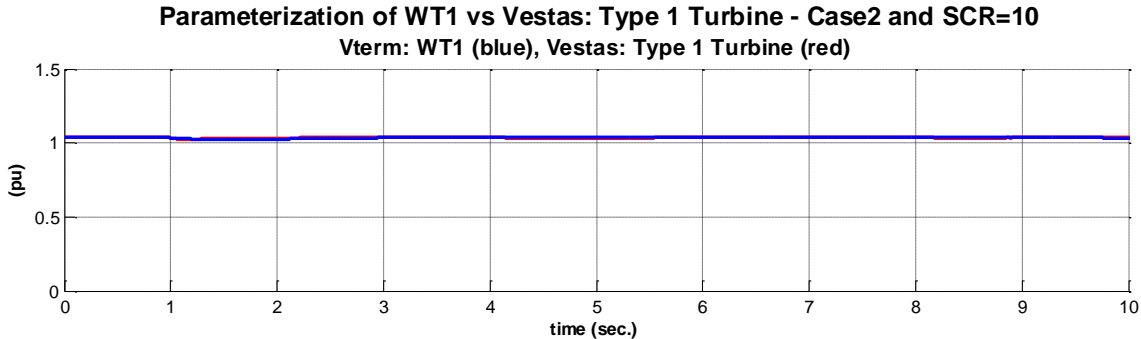

Ireal: WT1 (blue), Vestas: Type 1 Turbine (red)

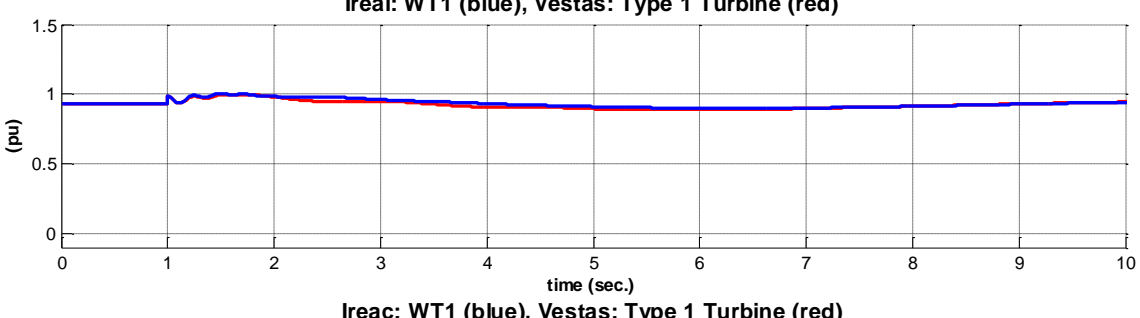

Ireac: WT1 (blue), Vestas: Type 1 Turbine (red)

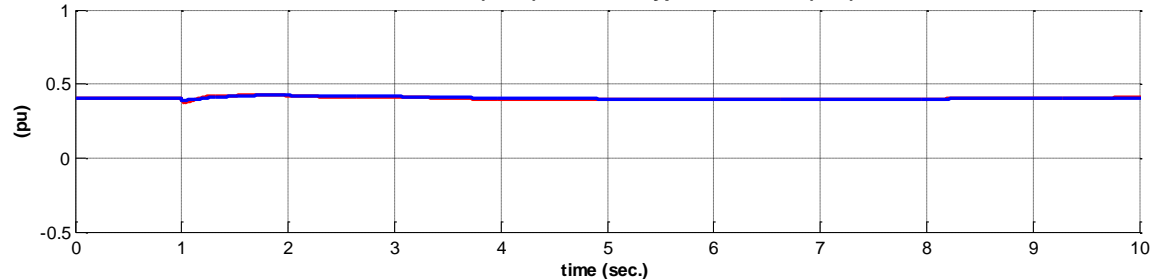

Figure 7-120: Parameterization of WT1 vs Vestas: Type 1 turbine - Case 2 and SCR=10 $\left(\mathrm{V}_{\text {Term, }}, \mathrm{I}_{\text {Real }}, \mathrm{I}_{\text {Reac }}\right)$. 


\subsection{Case 3 - Over-Frequency Event}

\section{SCR - 5}

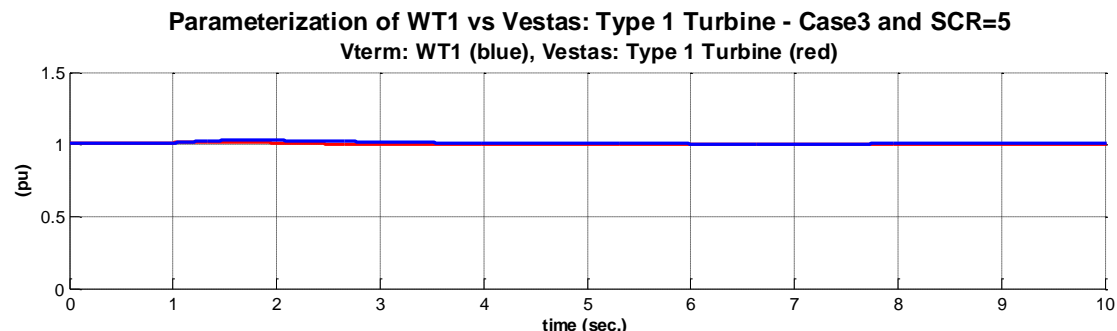

Pgen: WT1 (blue), Vestas: Type 1 Turbine (red)

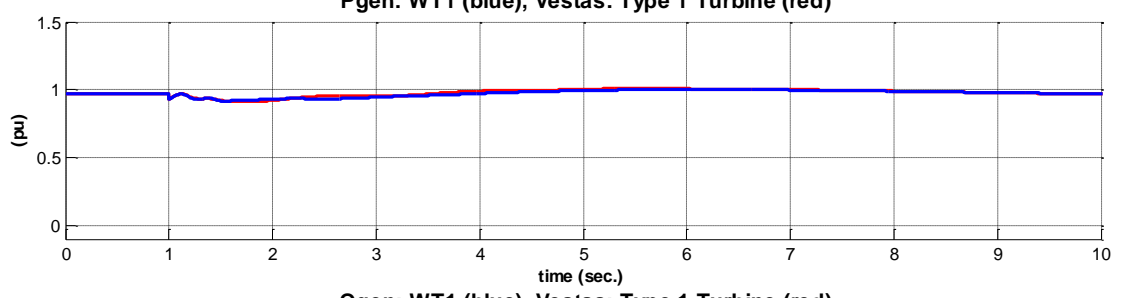

Qgen: WT1 (blue), Vestas: Type 1 Turbine (red)

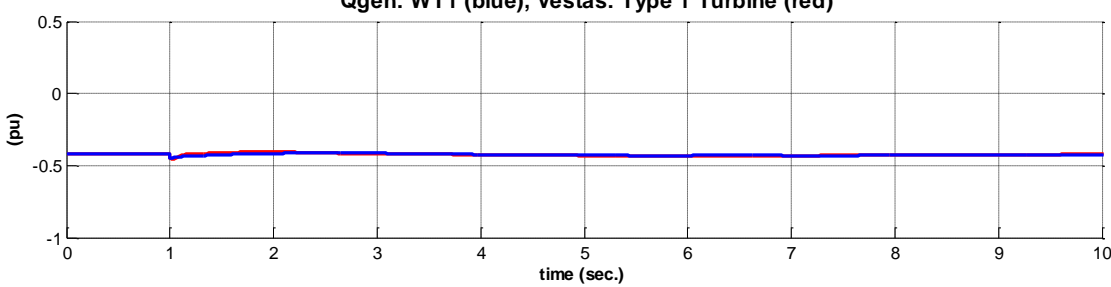

Figure 7-121: Parameterization of WT1 vs Vestas: Type 1 turbine - Case 3 and SCR=5 $\left(\mathrm{V}_{\text {Term }}, \mathrm{P}, \mathrm{Q}\right)$.
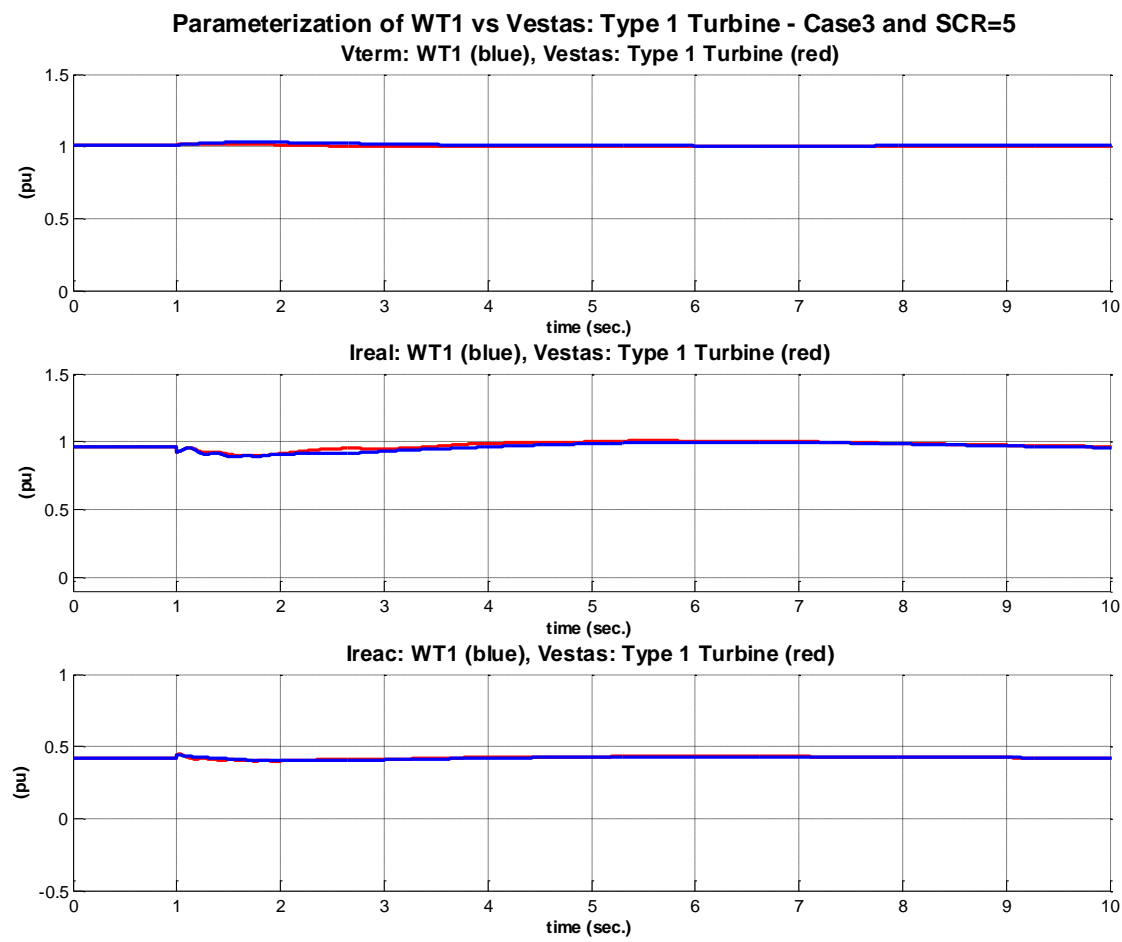

Figure 7-122: Parameterization of WT1 vs Vestas: Type 1 turbine - Case 3 and SCR=5 $\left(\mathrm{V}_{\text {Term }}, \mathrm{I}_{\text {Real }}, \mathrm{I}_{\text {Reac }}\right)$. 
$\underline{\text { SCR }-10}$

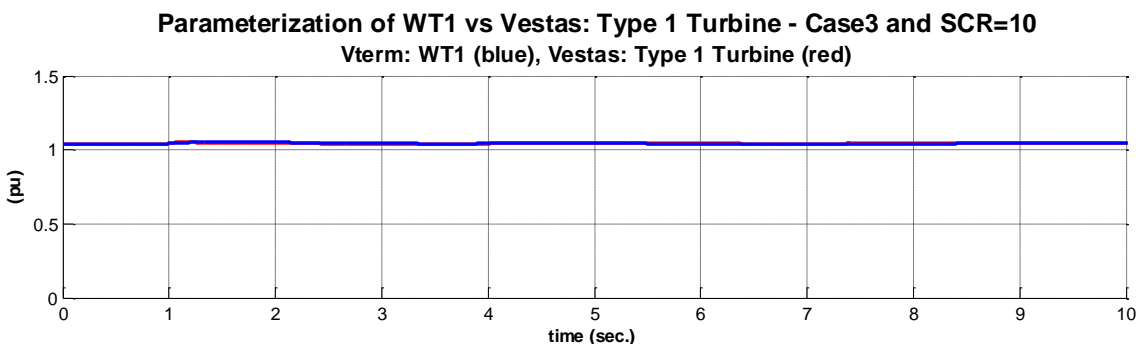

Pgen: WT1 (blue), Vestas: Type 1 Turbine (red)

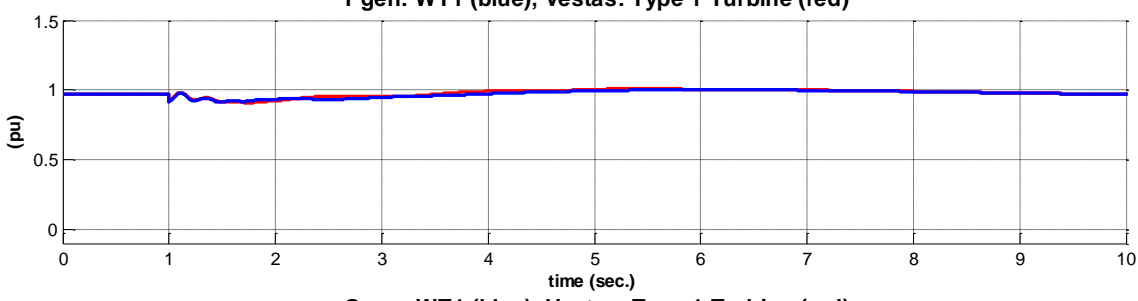

Qgen: WT1 (blue), Vestas: Type 1 Turbine (red)

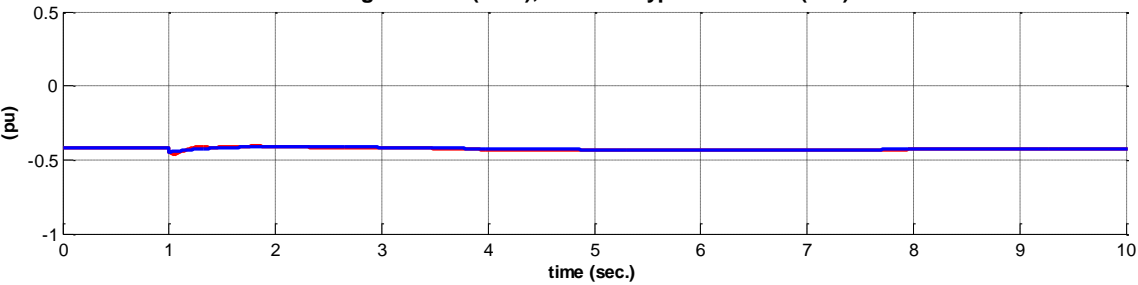

Figure 7-123: Parameterization of WT1 vs Vestas: Type 1 turbine - Case 3 and SCR=10 $\left(\mathrm{V}_{\text {Term }}, \mathrm{P}, \mathrm{Q}\right)$.
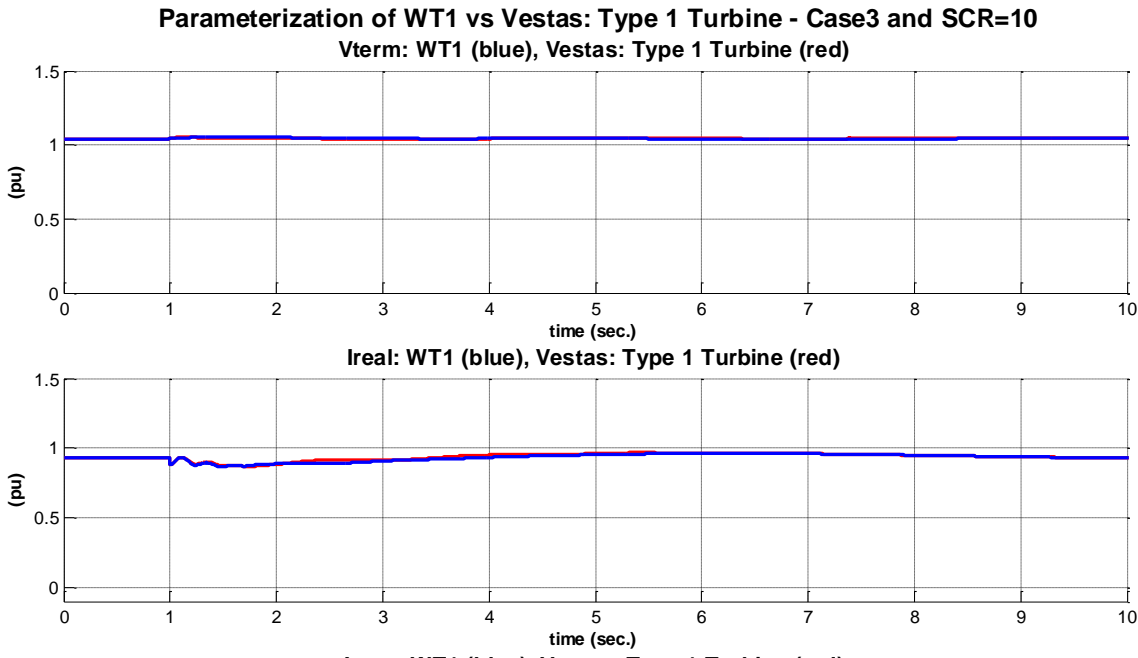

Ireac: WT1 (blue), Vestas: Type 1 Turbine (red)

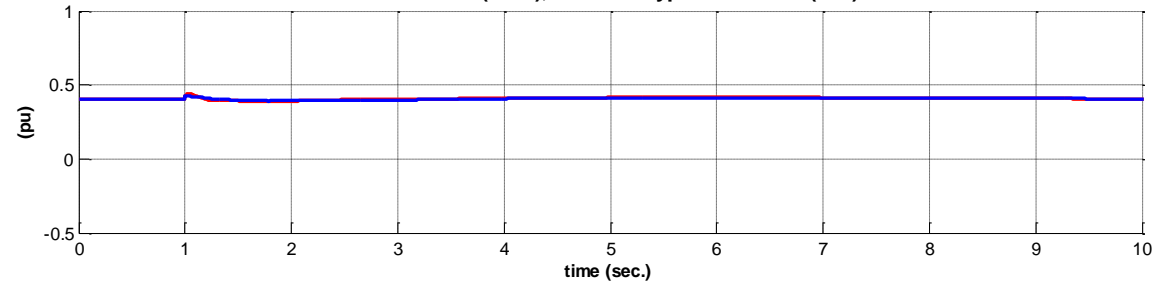

Figure 7-124: Parameterization of WT1 vs Vestas: Type 1 turbine - Case 3 and SCR=10 $\left(\mathrm{V}_{\text {Term, }}, \mathrm{I}_{\text {Real }}, \mathrm{I}_{\text {Reac }}\right)$. 


\subsubsection{Results Validation}

With the fault in case $1 \quad(S C R=5)$ cleared without tripping the line, the parameterization of the WT1 model showed stable and adequate response.

For cases 2 and 3 (over frequency and under frequency events), the response of the generic model matches the response of the VSM in the frequency range of interest. However, for case 1 (fault event), the response of the generic model matches the response of the VSM poorly. In other words, while the generic models are a good representation of the Vestas: Type 1 turbine VSM behavior during frequency events, they are rather a poor alternative for severe fault events.

\subsubsection{Vendor Specific Model Validation}

No data available 


\subsection{Vestas - Type II Models}

\subsubsection{Example List of Vestas Type II Wind Turbines}

- $\mathrm{V} 47-660 \mathrm{~kW}$

- $\mathrm{V} 66-\mathrm{ad}$

- $\mathrm{V} 80-1.8 \mathrm{MW}$

\subsubsection{Vendor Specific Model Characterization}

\subsubsection{Model Performance: Case 1 - Fault Event}

In this case a remote fault is applied to bus 12 for a duration of 6 cycles $(0.1 \mathrm{sec})$.

In the course of the DOE/UWIG/EnerNex project when making a parameterization of the WT2 generic model for the system described above, the following observations have been made:

1. The benchmark Vestas: Type 2 Turbine model does not show the stable or adequate response (if the line trips the system has sustained oscillation for more than 20 seconds) to the fault cleared by tripping the line with SCR=5.

2. For $\mathrm{SCR}=10$, the benchmark Vestas: Type 2 Turbine model shows the stable response to the fault cleared by tripping the line or without tripping the line.

3. So, in terms of the Test 1 (the fault event), it makes sense to parameterize the WT2 model for the system with $S C R=5$ without tripping the line, while for SCR = 10 test, the line was tripped. 
$\underline{\mathrm{SCR}-5}$

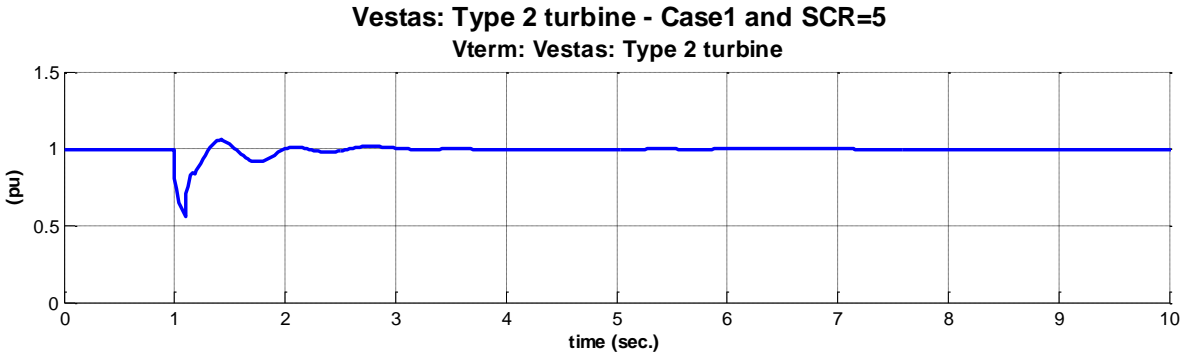

Pgen: Vestas: Type 2 turbine
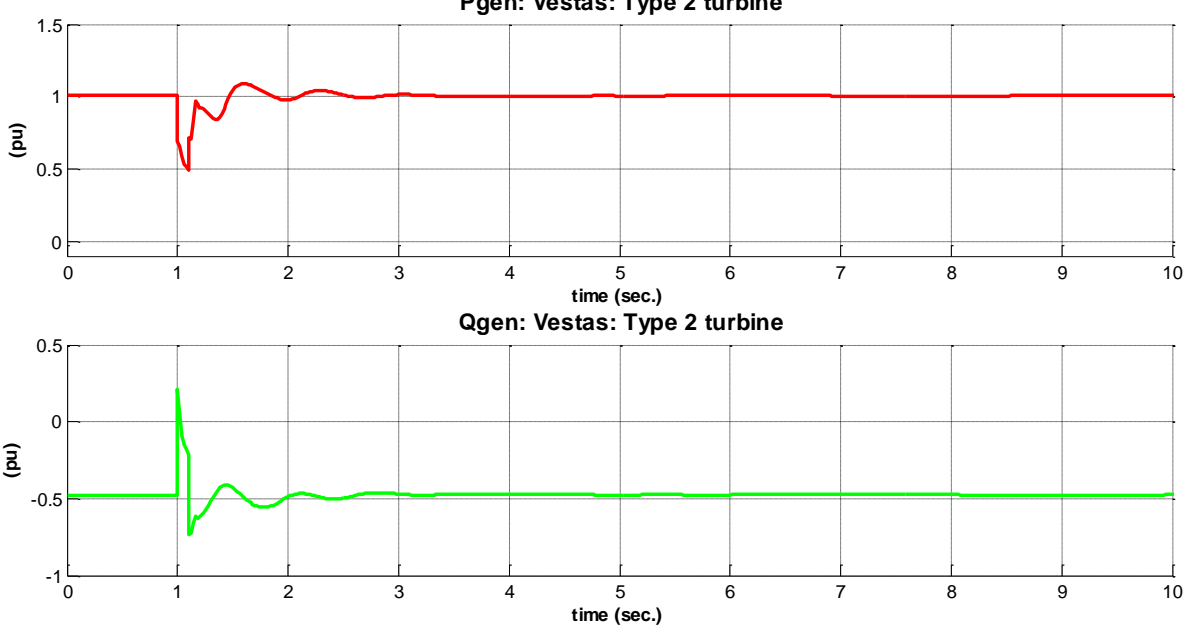

Figure 7-125: Vestas: Type 2 turbine - Case 1 and $S C R=5\left(V_{\text {Term }}, P, Q\right)$.

Vestas: Type 2 turbine - Case1 and SCR=5

Vterm: Vestas: Type 2 turbine

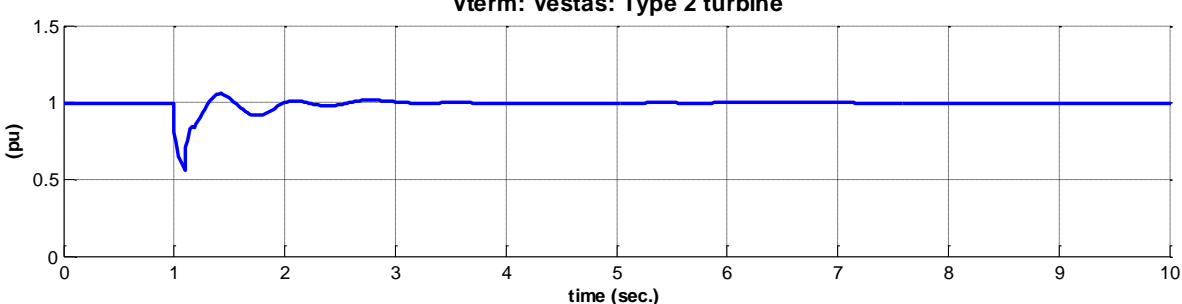

Ireal: Vestas: Type 2 turbine

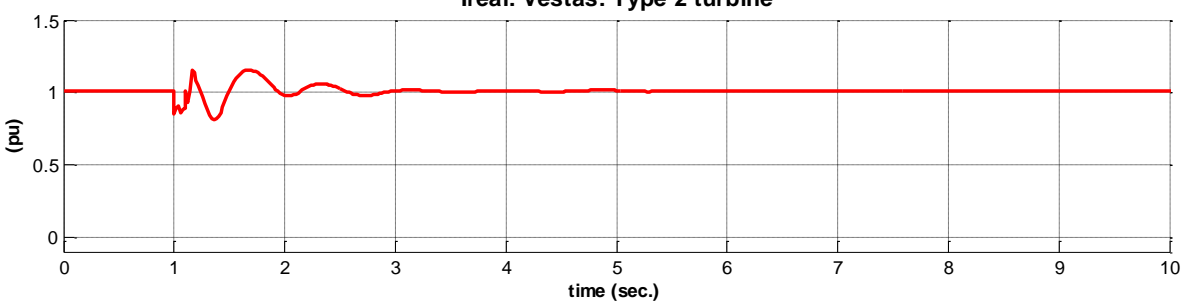

Ireac: Vestas: Type 2 turbine

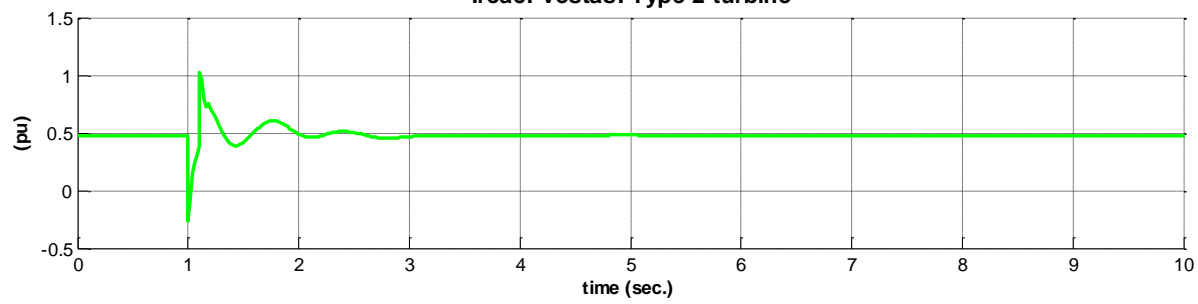

Figure 7-126: Vestas: Type 2 turbine - Case 1 and $S C R=5\left(V_{\text {Term }}, I_{\text {Real }}, I_{\text {Reac }}\right)$. 
$\underline{\text { SCR }-10}$

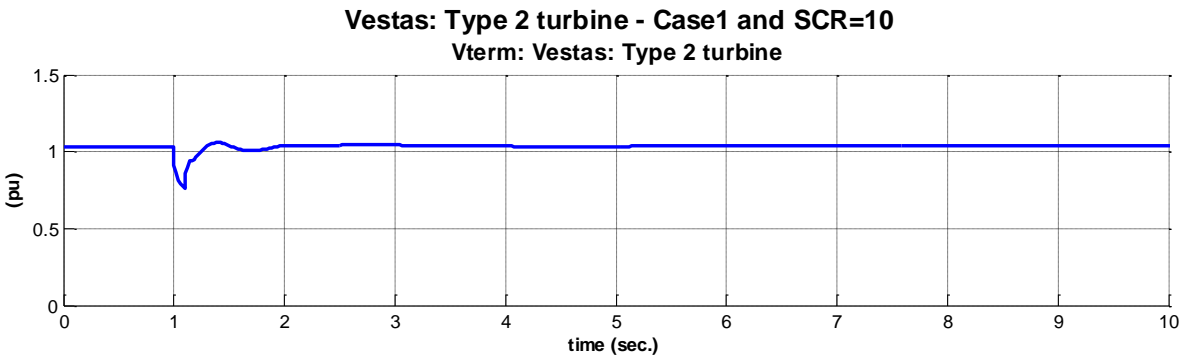

Pgen: Vestas: Type 2 turbine

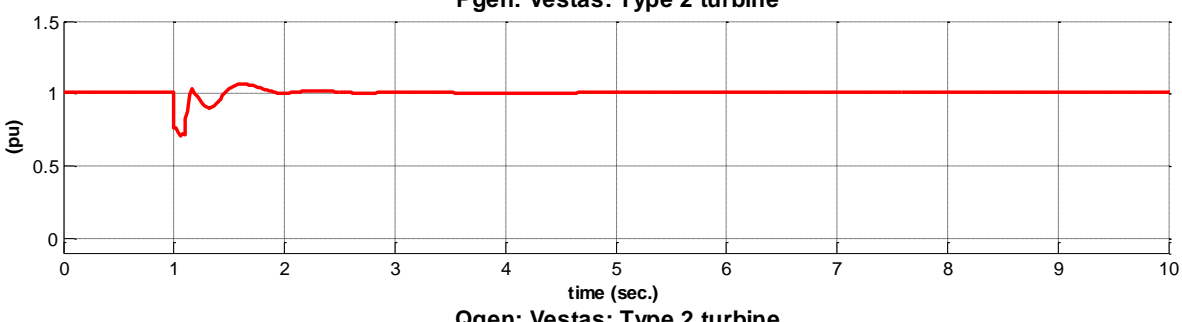

Qgen: Vestas: Type 2 turbine

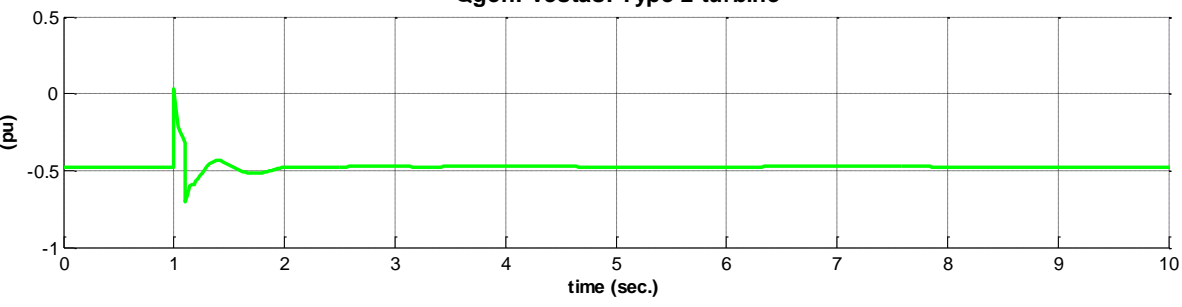

Figure 7-127: Vestas: Type 2 turbine - Case 1 and $\operatorname{SCR}=10\left(V_{\text {Term }}, P, Q\right)$.
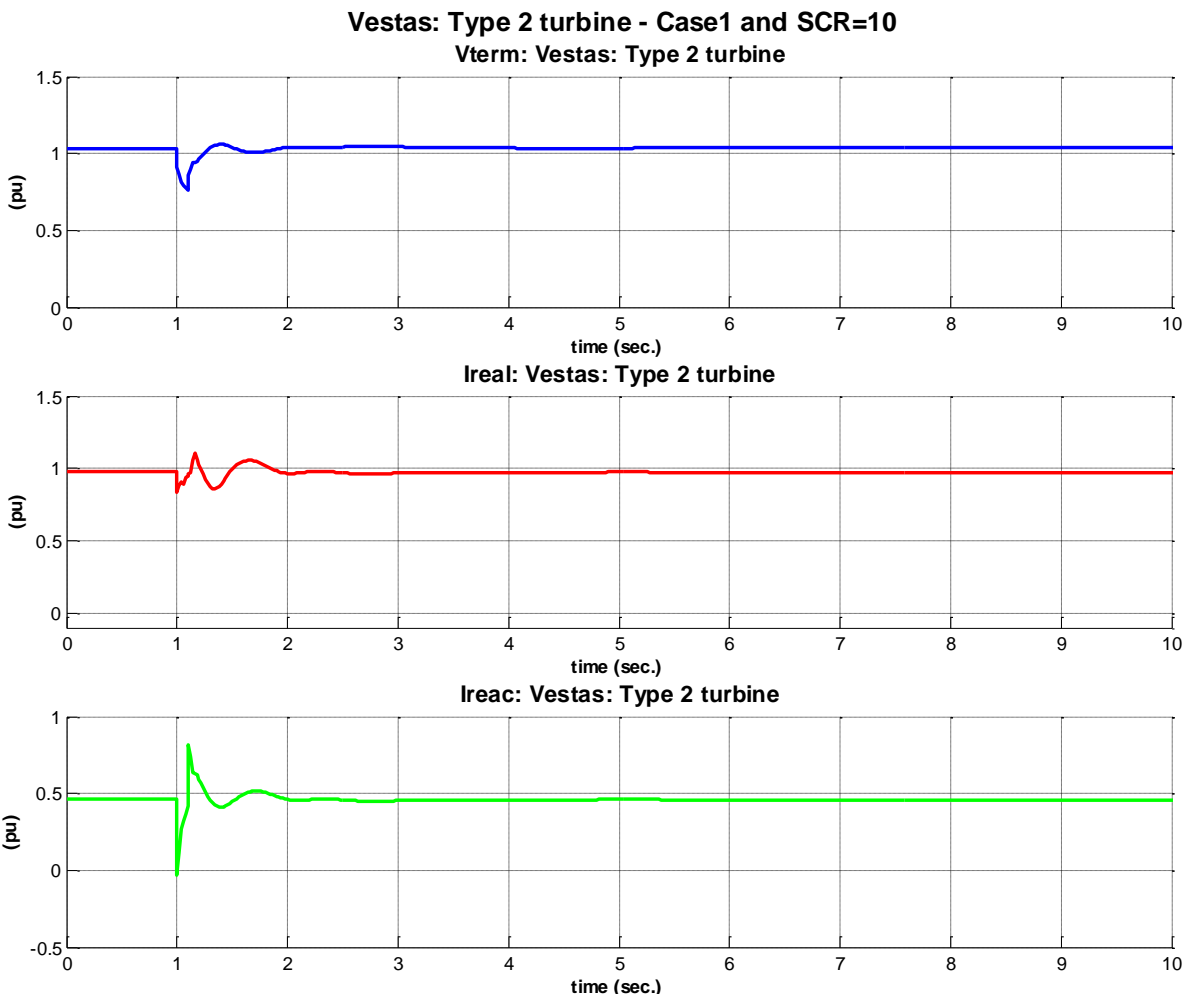

Figure 7-128: Vestas: Type 2 turbine - Case 1 and SCR=10 ( $\left.V_{\text {Term, }} I_{\text {Real }}, I_{\text {Reac }}\right)$. 


\subsubsection{Model Performance: Case 2 - Under-Frequency Event}

In this case a under frequency event is created by tripping the $100 \mathrm{MW}$ generation unit at bus 20 .

Testing the benchmark vendor defined Vestas: Type 2 Turbine model for the test system in Figure 5-1 revealed that, with the recommended parameters, this model shows not stable response to frequency events. Moreover, we could not find any parameters of the benchmark model resulting in stable response. Finally, we found out that the only way to make it stable was to disable the rotor resistance (RCC) control.

We do not know so far what additional features should have been incorporated in the VSM to improve its performance under the frequency events.

\section{$\underline{\mathrm{SCR}-5}$}
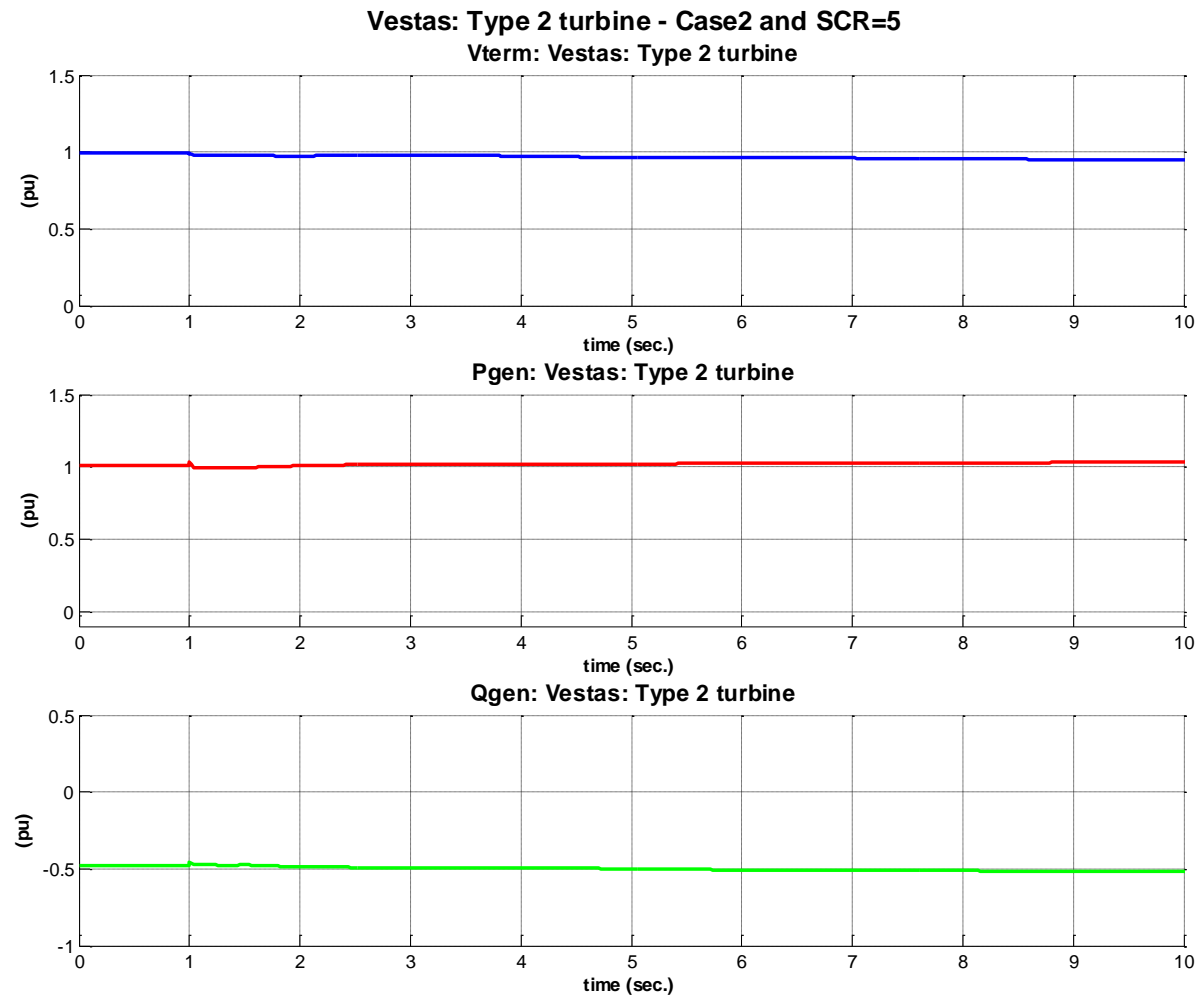

Figure 7-129: Vestas: Type 2 turbine - Case 2 and $S C R=5\left(V_{\text {Term }}, P, Q\right)$. 

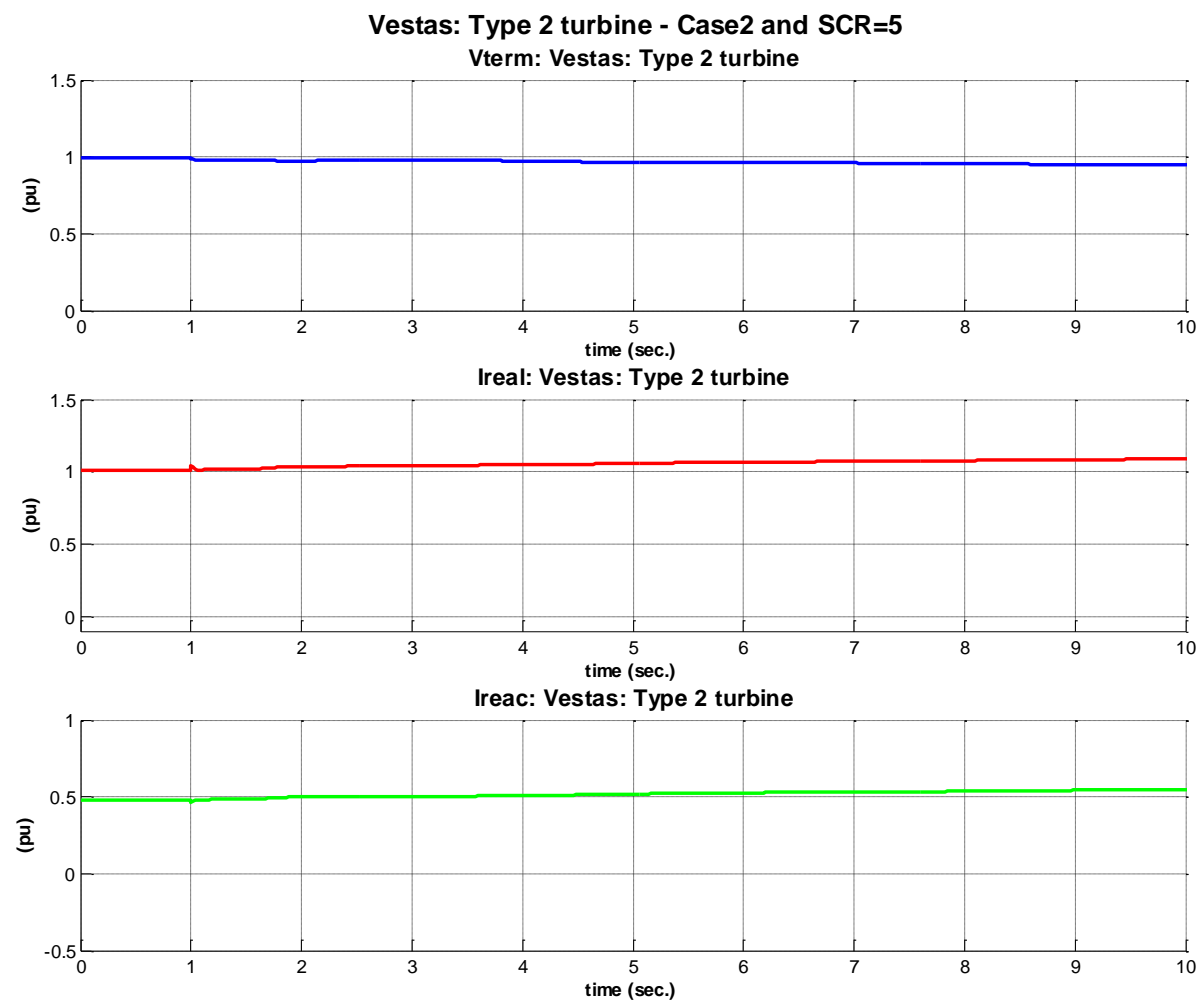

Figure 7-130: Vestas: Type 2 turbine - Case 2 and $S C R=5\left(V_{\text {Term }}, I_{\text {Real }}, I_{\text {Reac }}\right)$.

\section{SCR -10}

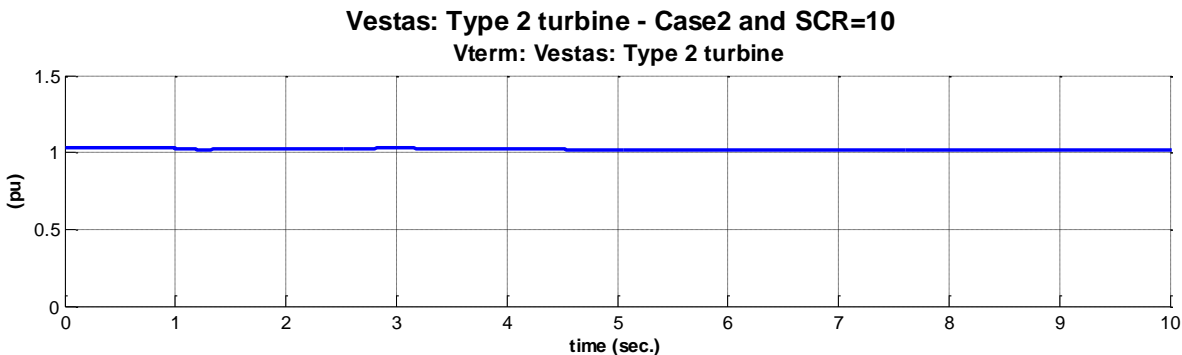

Pgen: Vestas: Type 2 turbine

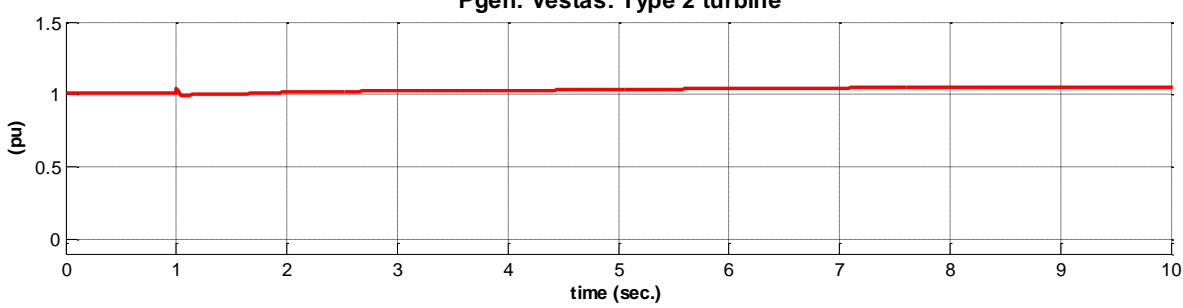

Qgen: Vestas: Type 2 turbine

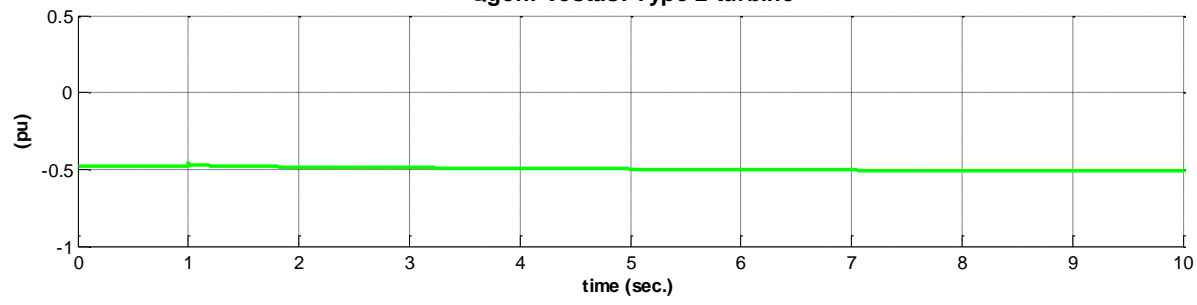

Figure 7-131: Vestas: Type 2 turbine - Case 2 and SCR=10 $\left(V_{\text {Term }}, P, Q\right)$. 

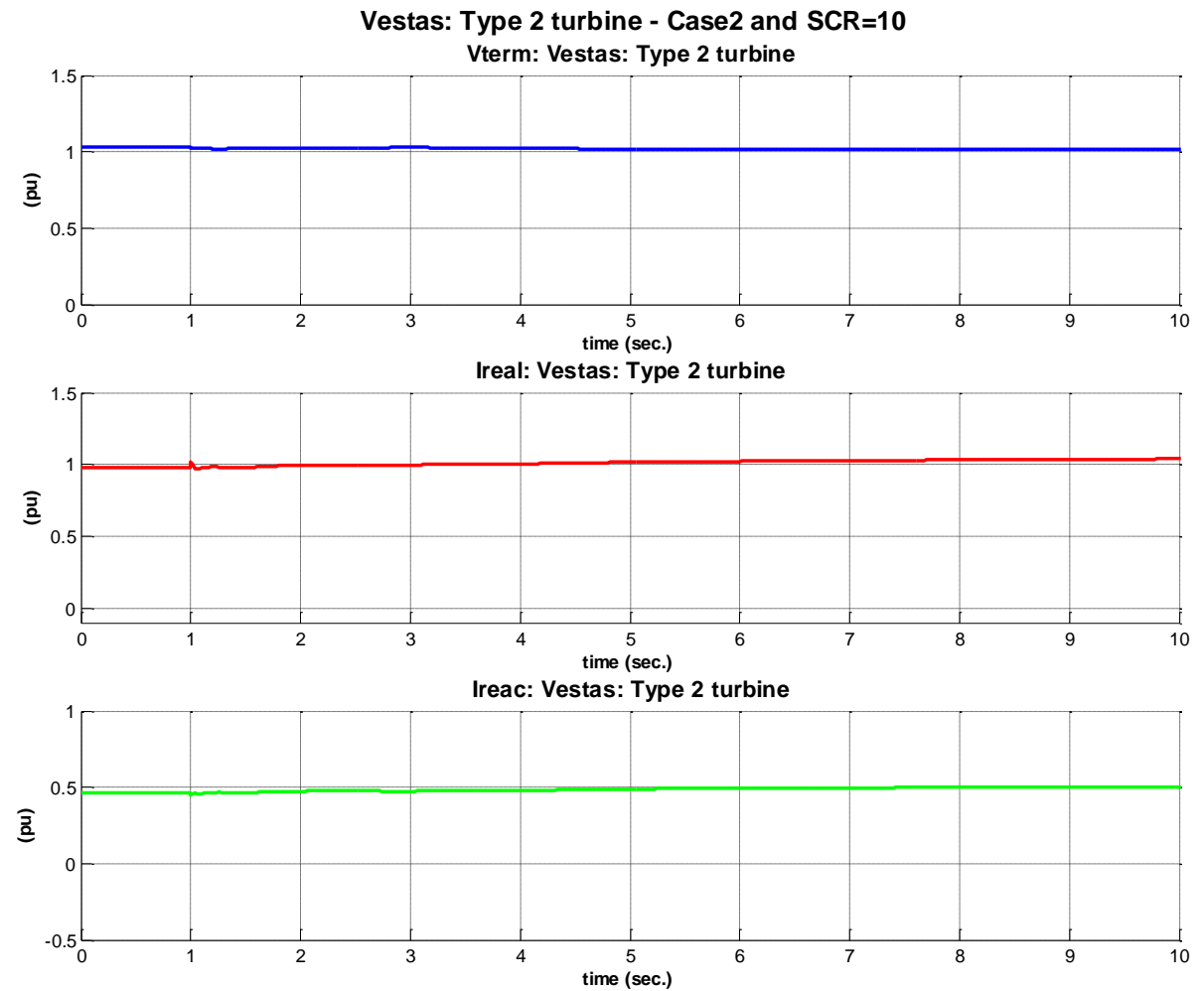

Figure 7-132: Vestas: Type 2 turbine - Case 2 and SCR=10 ( $\left.V_{\text {Term }}, I_{\text {Real }}, I_{\text {Reac }}\right)$. 


\subsubsection{Model Performance: Case 3 - Over-Frequency Event}

In this case a under frequency event is created by tripping the $100 \mathrm{MW}$ load at bus 11.

$\underline{\mathrm{SCR}-5}$
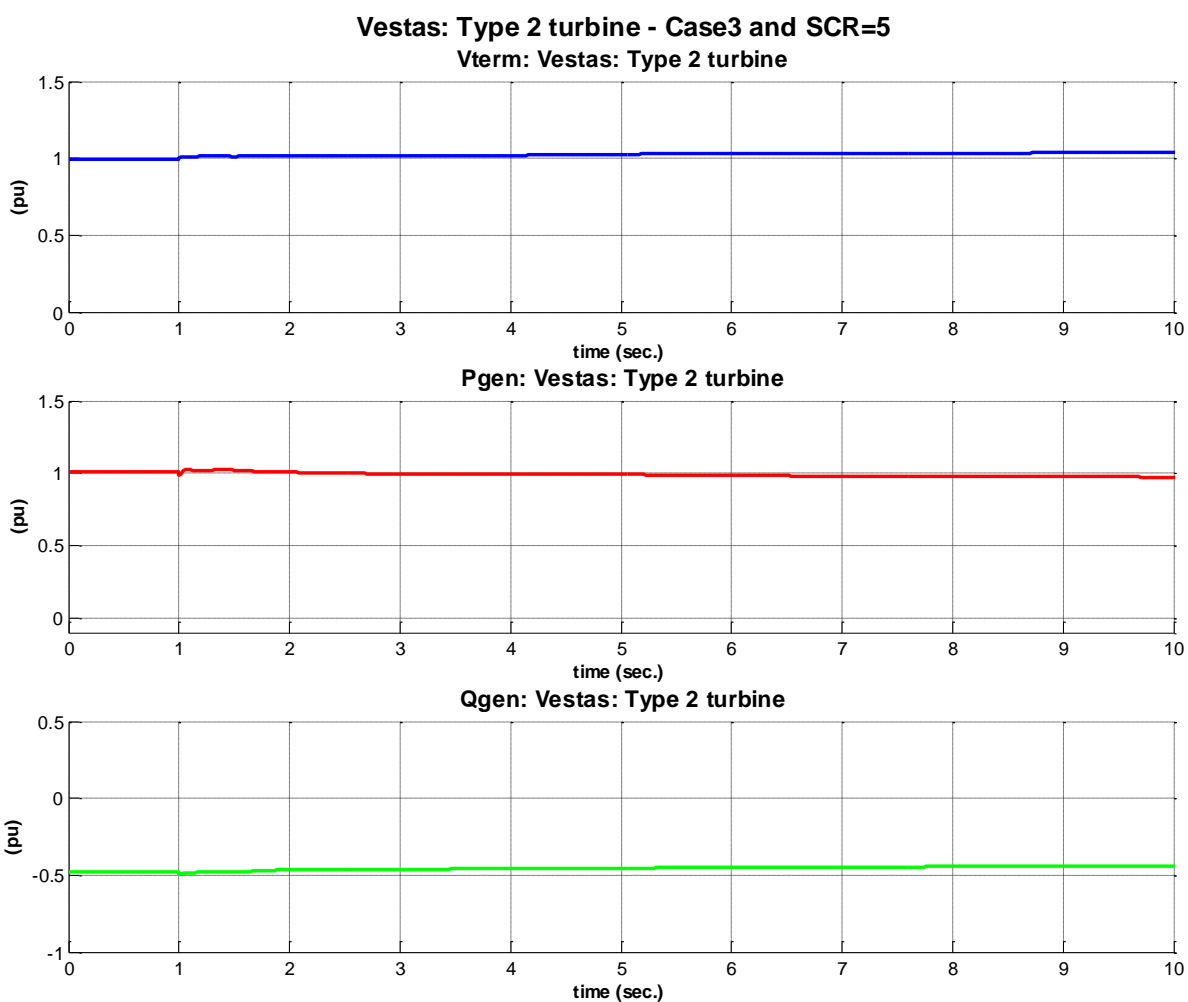

Figure 7-133: Vestas: Type 2 turbine - Case 3 and $\operatorname{SCR}=5\left(V_{\text {Term }}, P, Q\right)$. 

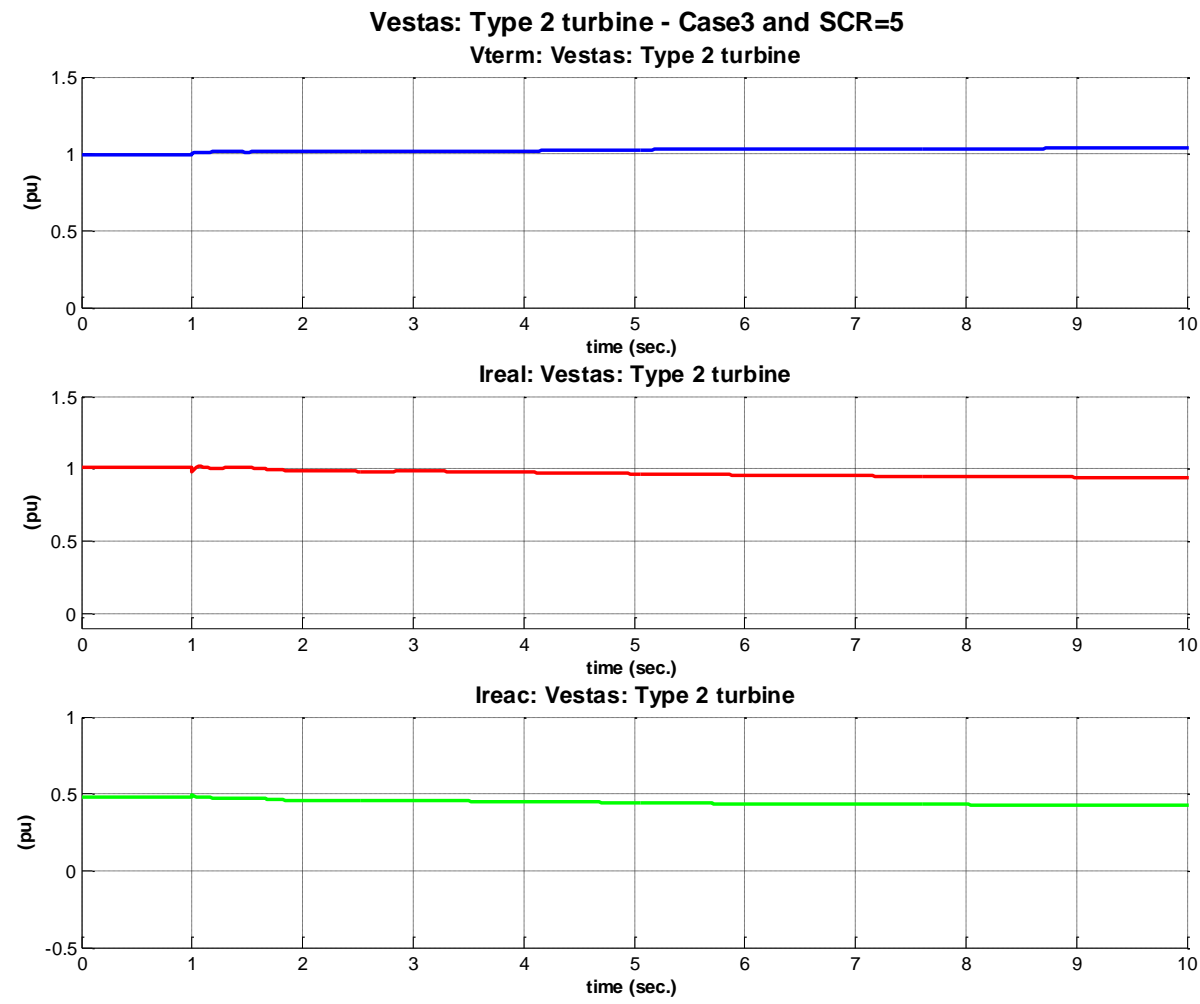

Figure 7-134: Vestas: Type 2 turbine - Case 3 and SCR=5 ( $\left.V_{\text {Term, }} I_{\text {Real }}, I_{\text {Reac }}\right)$.

$\underline{\text { SCR }-10}$

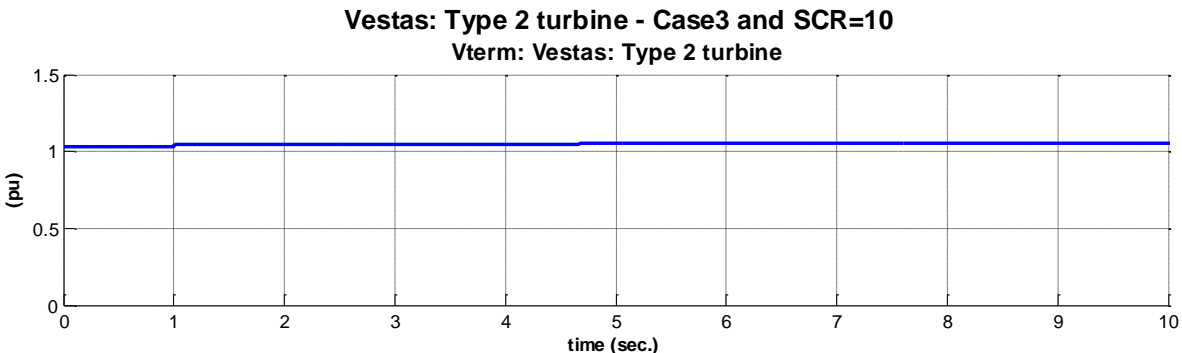

Pgen: Vestas: Type 2 turbine

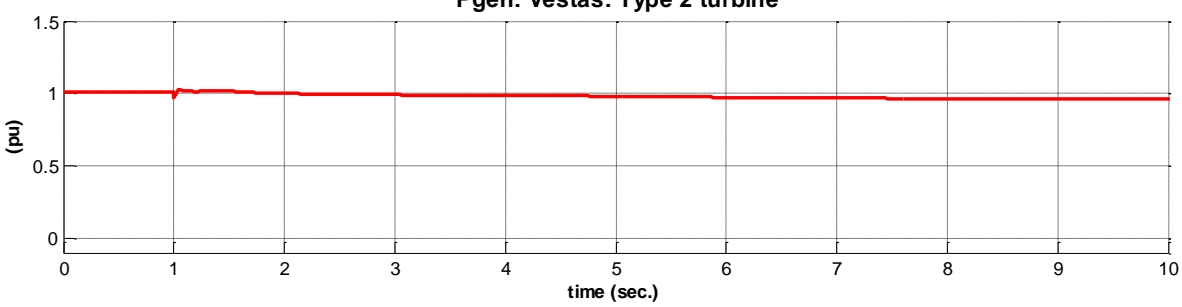

Qgen: Vestas: Type 2 turbine

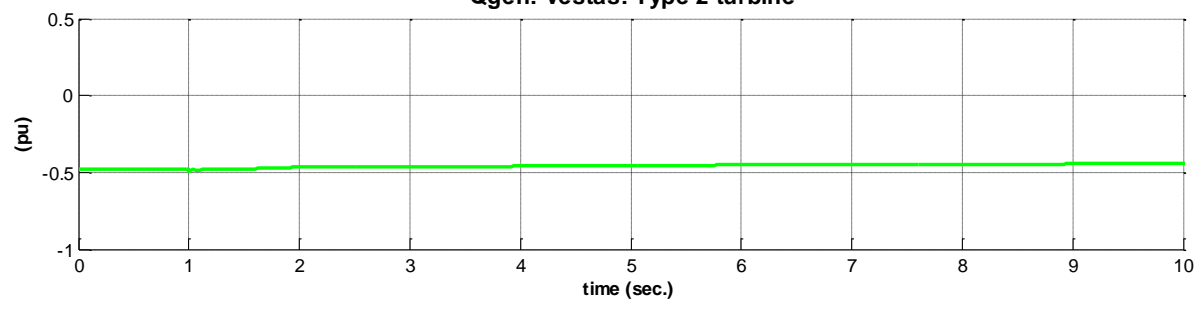

Figure 7-135: Vestas: Type 2 turbine - Case 3 and $\operatorname{SCR}=10\left(V_{\text {Term }}, P, Q\right)$. 

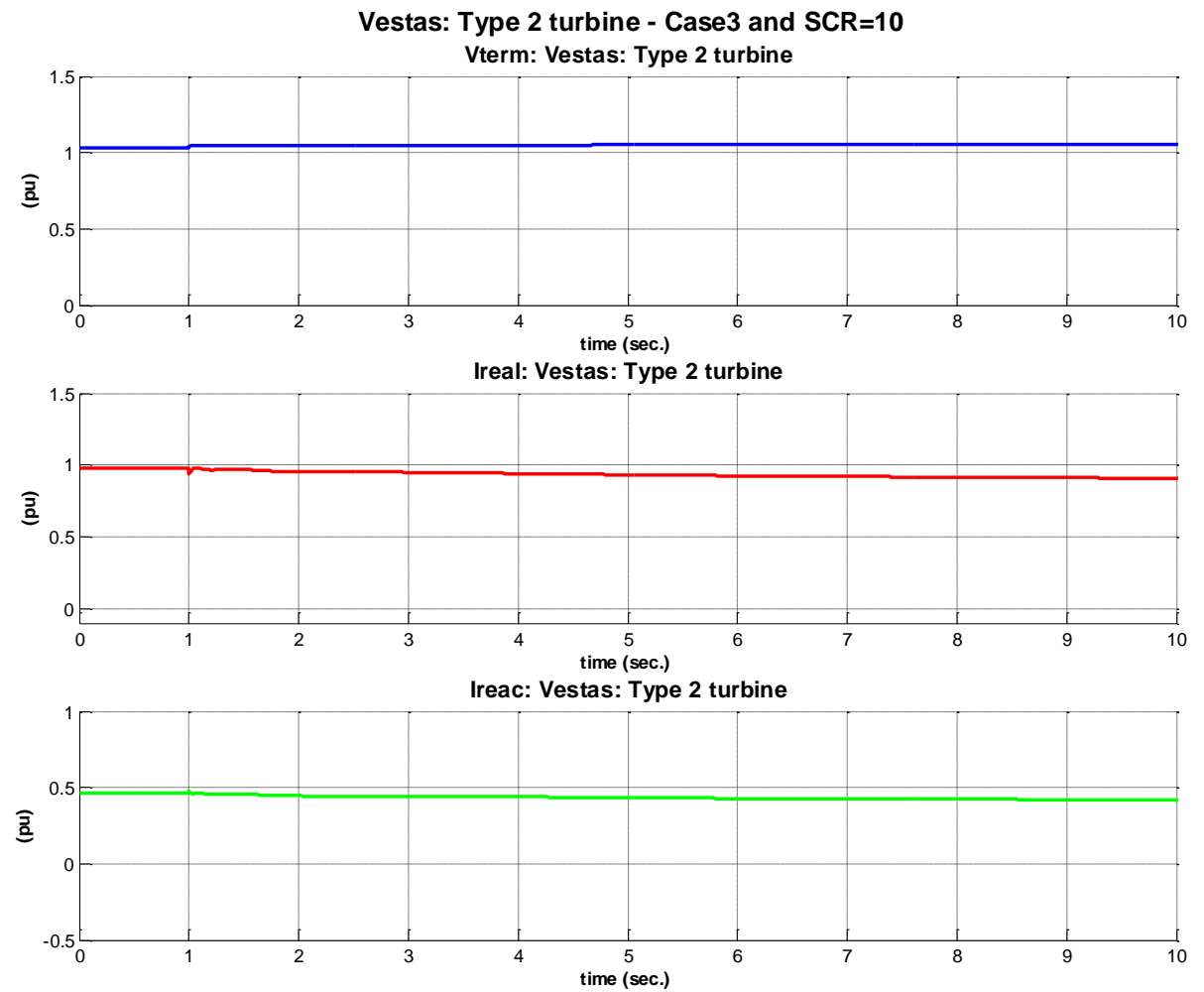

Figure 7-136: Vestas: Type 2 turbine - Case 3 and $S C R=10\left(V_{\text {Term }}, I_{\text {Real }}, I_{\text {Reac }}\right)$. 


\subsubsection{Generic Model Parameterization}

\subsubsection{PSSE Environment ${ }^{1}$}

The PSSE platform generic wind turbine parameters, to mimic the behavior of Vestas' type 2 wind turbines, are shown in Table 7-7. The parameter values shown in the table resulted from a compromise between the simulated cases (i.e. SCR 5 and SCR 10) and network conditions (i.e. fault, under- and over frequency).

Table 7-7: PSSE generic model parameter values.

\begin{tabular}{|c|c|c|c|c|c|c|c|}
\hline \multicolumn{2}{|c|}{ WT2G } & \multicolumn{2}{|c|}{ WT2T } & \multicolumn{2}{|c|}{ WT2E } & \multicolumn{2}{|c|}{ WT2A } \\
\hline XA & 0.126 & $\mathrm{H}$ & 3.46 & Tsp & 0.050 & Droop & 0 \\
\hline XM & 6.840 & Damp & 0.0 & Tpe & 0.050 & $\mathrm{Kp}$ & 18.95796 \\
\hline $\mathrm{X} 1$ & 0.18 & Htfrac & 0.796952 & $\mathrm{Ti}$ & 1.000 & $\mathrm{Ti}$ & 0.056385 \\
\hline R_Rot_Mach & 0.0044 & Freq1 & 1.50 & $\mathrm{Kp}$ & 1.000 & T1 & 0 \\
\hline R_Rot_Mach & 0.1099 & Dshaft & 0.30 & RotRV_Max & 0.990 & $\mathrm{~T} 2$ & 0 \\
\hline $\mathrm{E} 1$ & 1.00 & & & RotRV_Min & 0.050 & Tpe & 0 \\
\hline$S(E 1)$ & 0.00 & & & & & Limmax & 1.000 \\
\hline E2 & 1.20 & & & & & Limmin & 0.250 \\
\hline$S(E 2)$ & 0.00 & & & & & & \\
\hline Power_Ref_1 & 0.00 & & & & & & \\
\hline Power_Ref_2 & 0.217 & & & & & & \\
\hline Power_Ref_3 & 0.8988 & & & & & & \\
\hline Power_Ref_4 & 0.900 & & & & & & \\
\hline Power_Ref_5 & 0.9050 & & & & & & \\
\hline Slip_1 & 0.00 & & & & & & \\
\hline Slip_2 & 0.0054 & & & & & & \\
\hline Slip_3 & 0.020 & & & & & & \\
\hline Slip_4 & 0.040 & & & & & & \\
\hline Slip_5 & 0.100 & & & & & & \\
\hline
\end{tabular}

\footnotetext{
${ }^{1}$ Parameterization performed by Siemens Energy
} 


\subsection{Case 1 - Fault Event}

\section{SCR - 5}

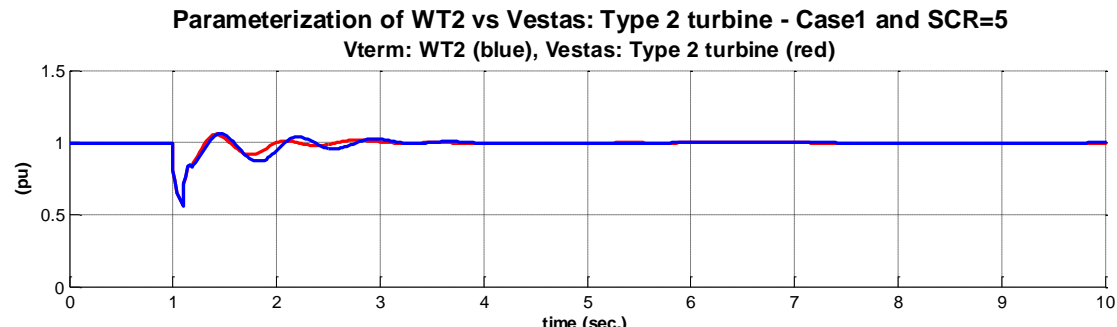

Pgen: WT2 (blue), Vestas: Type 2 turbine (red)

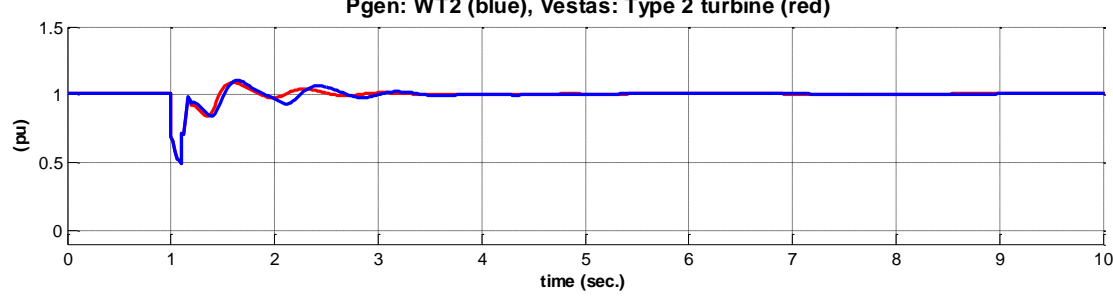

Qgen: WT2 (blue), Vestas: Type 2 turbine (red)

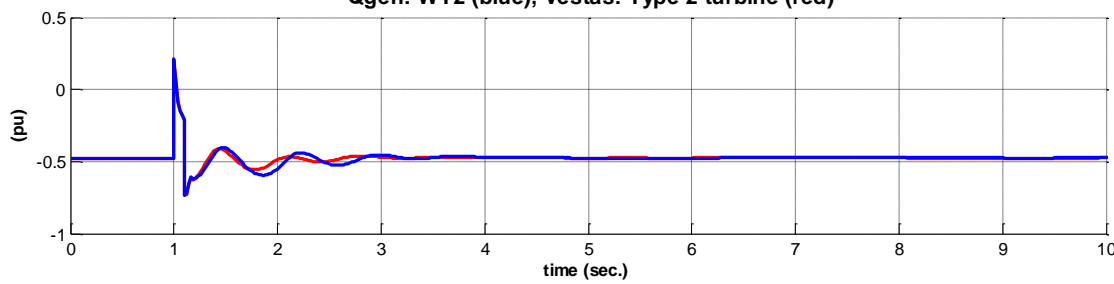

Figure 7-137: Parameterization of WT2 vs Vestas: Type 2 turbine - Case 1 and SCR=5 $\left(\mathrm{V}_{\text {Term }}, \mathrm{P}, \mathrm{Q}\right)$.
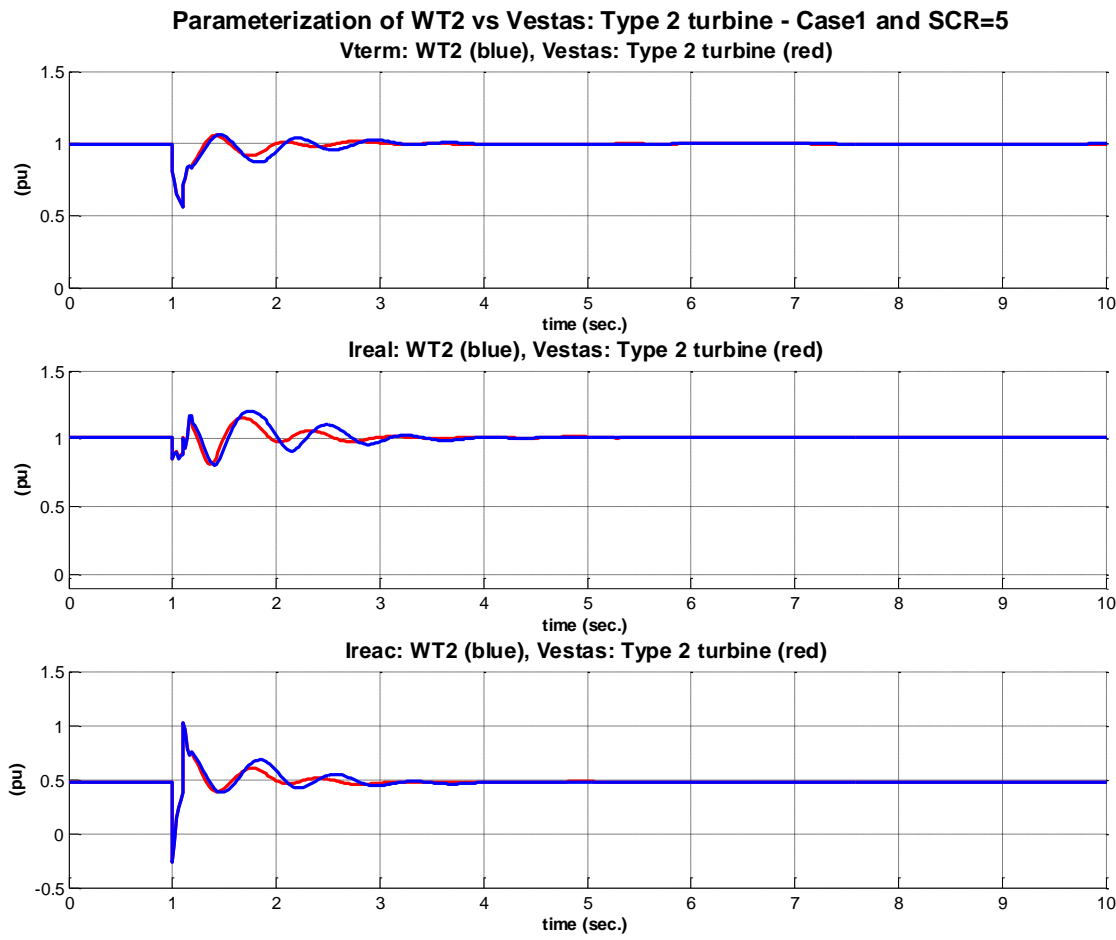

Figure 7-138: Parameterization of WT2 vs Vestas: Type 2 turbine - Case 1 and SCR=5 $\left(V_{\text {Term }}, I_{\text {Real }}, I_{\text {Reac }}\right)$. 
$\underline{\text { SCR }-10}$
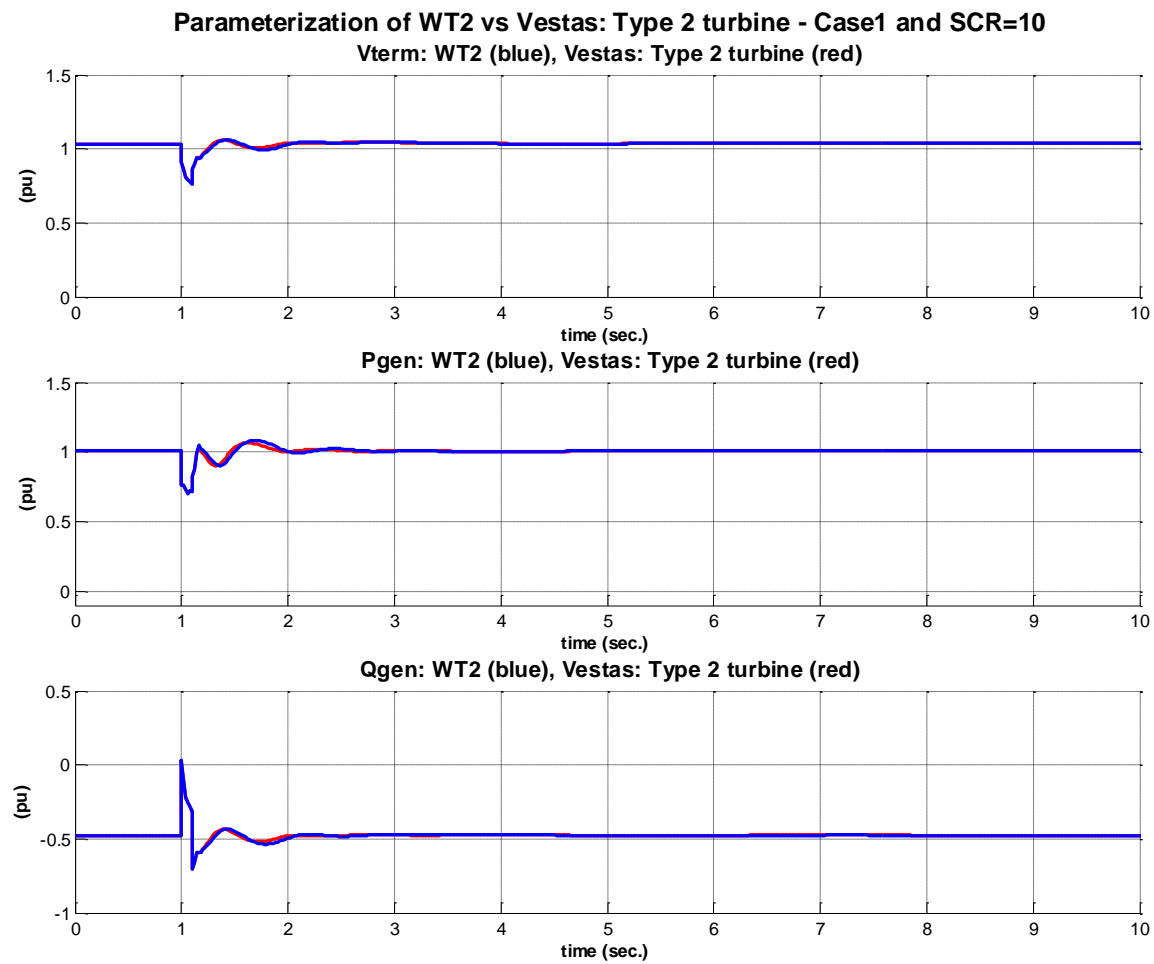

Figure 7-139: Parameterization of WT2 vs Vestas: Type 2 turbine - Case 1 and SCR=10 $\left(\mathbf{V}_{\text {Term }}, \mathbf{P}, \mathbf{Q}\right)$.
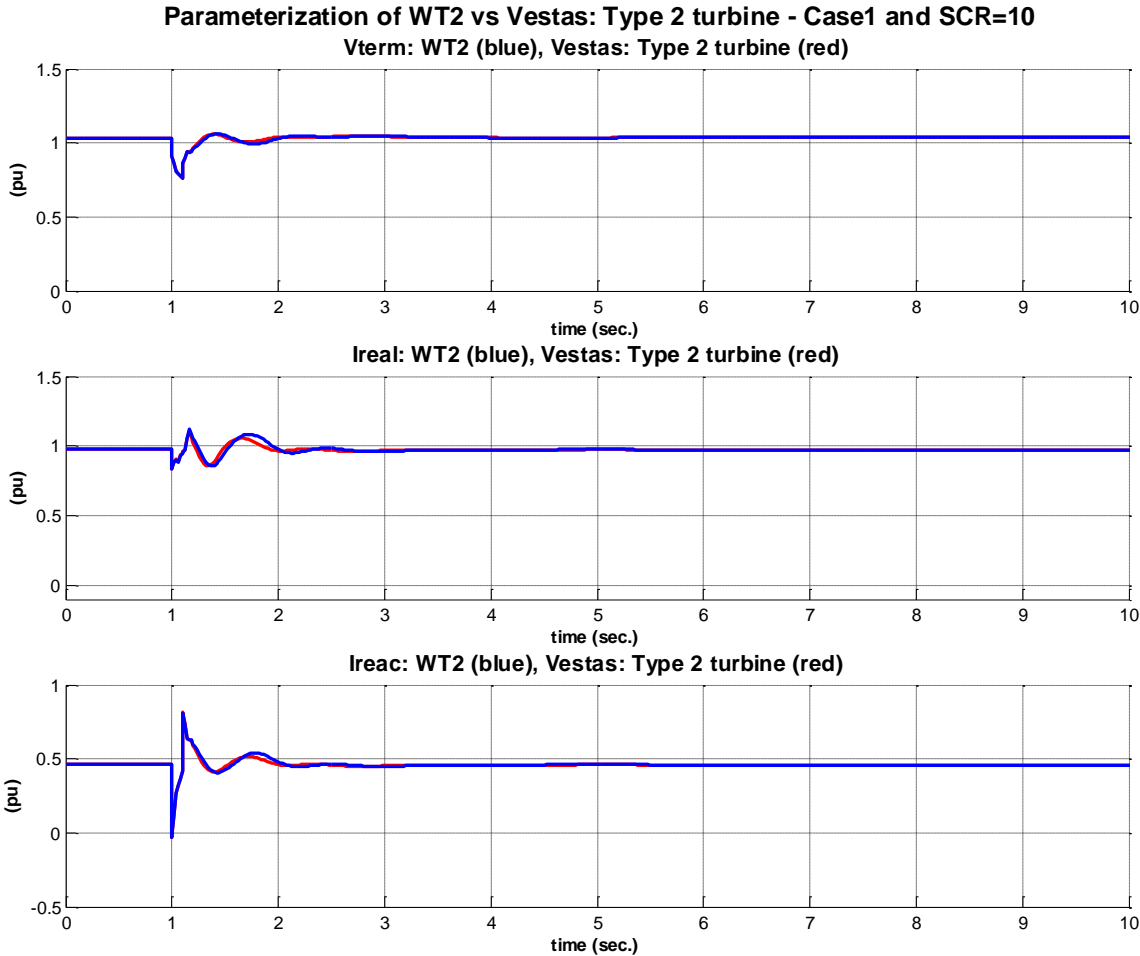

Figure 7-140: Parameterization of WT2 vs Vestas: Type 2 turbine - Case 1 and SCR=10 $\left(\mathrm{V}_{\text {Term, }}, \mathrm{I}_{\text {Real }}, \mathrm{I}_{\text {Reac }}\right)$. 


\subsection{Case 2 - Under-Frequency Event}

\section{$\underline{\mathrm{SCR}-5}$}

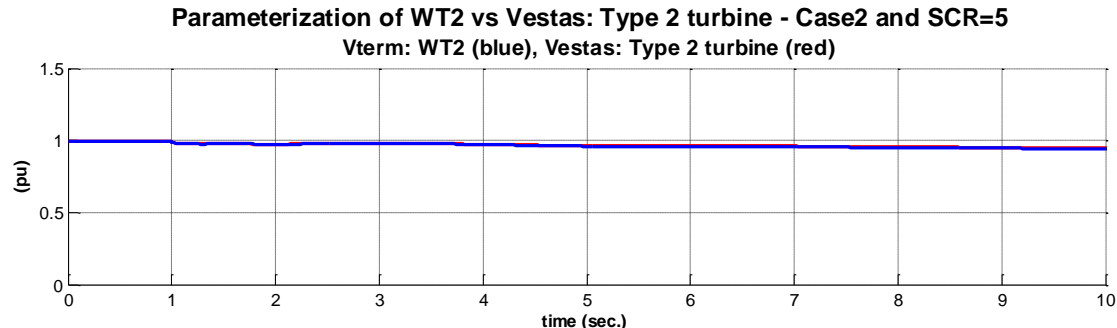

Pgen: WT2 (blue), Vestas: Type 2 turbine (red)

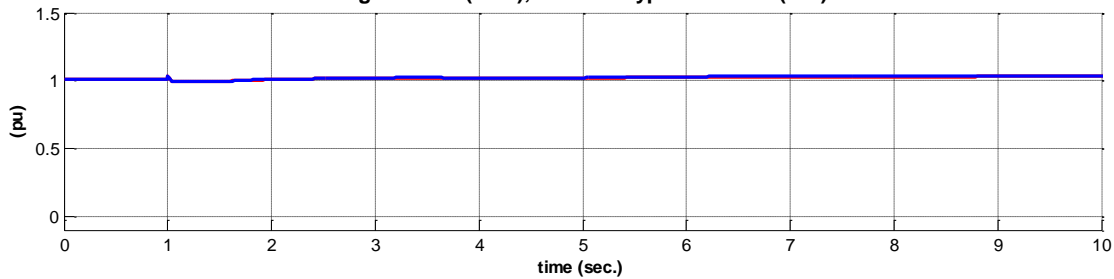

Qgen: WT2 (blue), Vestas: Type 2 turbine (red)

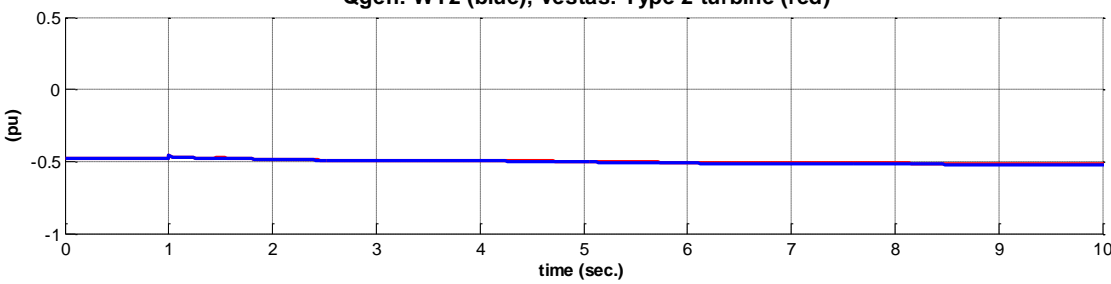

Figure 7-141: Parameterization of WT2 vs Vestas: Type 2 turbine - Case 2 and SCR=5 $\left(\mathrm{V}_{\text {Term }}, \mathrm{P}, \mathrm{Q}\right)$.
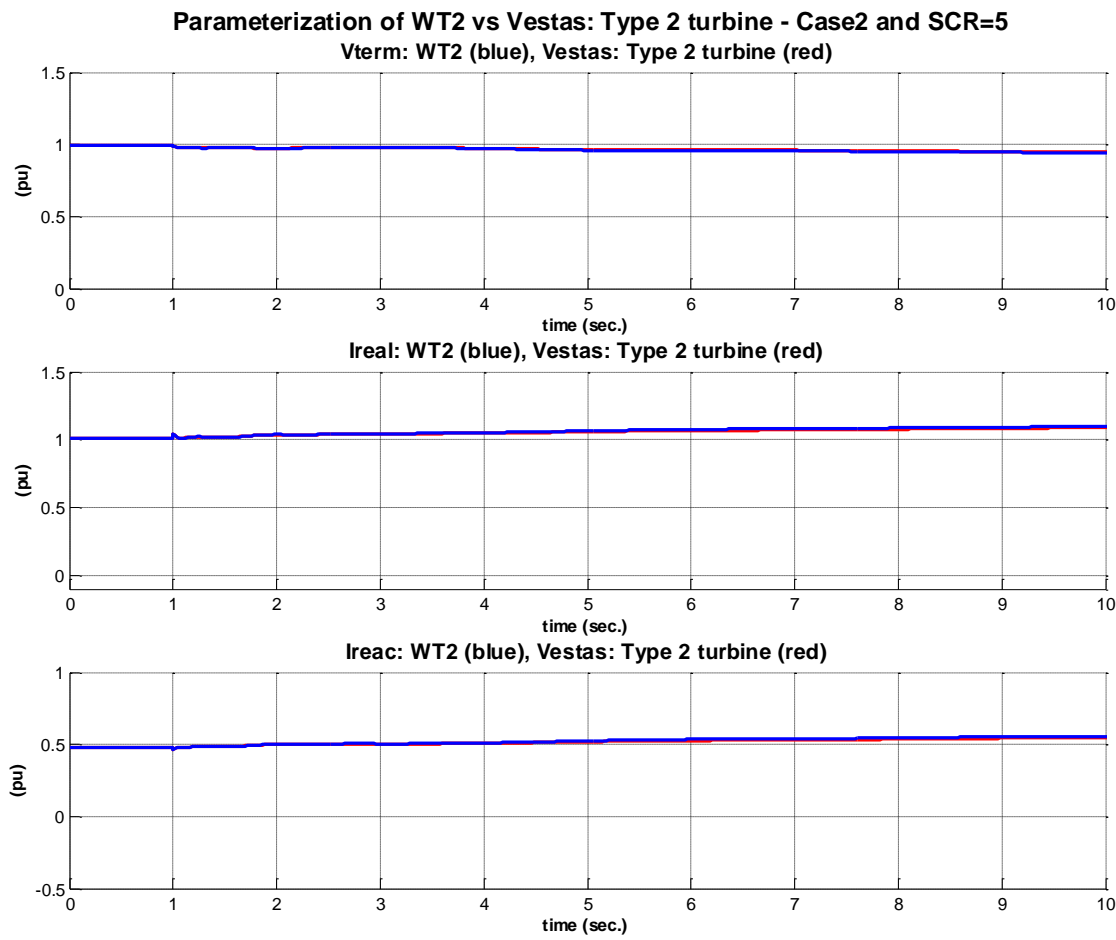

Figure 7-142: Parameterization of WT2 vs Vestas: Type 2 turbine - Case 2 and SCR=5 $\left(V_{\text {Term }}, I_{\text {Real }}, I_{\text {Reac }}\right)$. 
$\underline{\text { SCR }-10}$
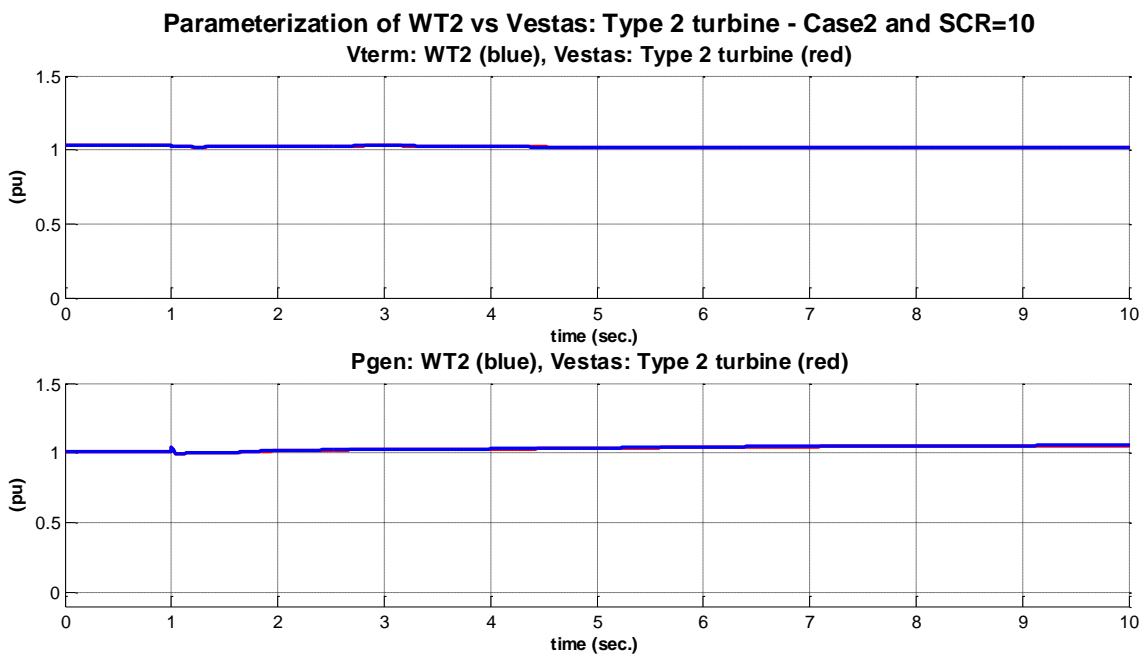

Qgen: WT2 (blue), Vestas: Type 2 turbine (red)

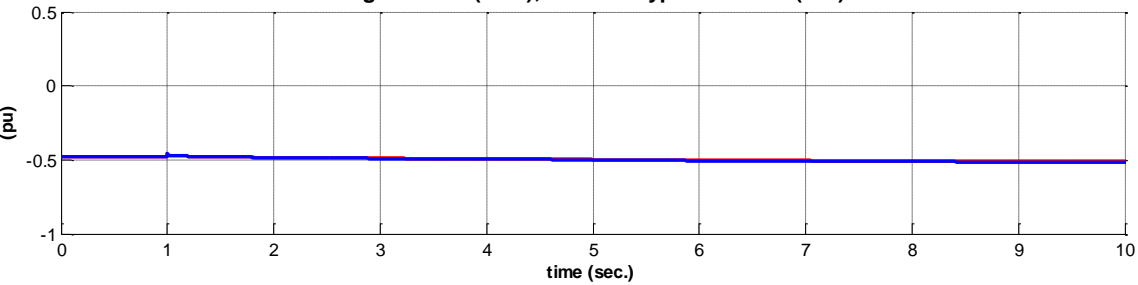

Figure 7-143: Parameterization of WT2 vs Vestas: Type 2 turbine - Case 2 and SCR=10 $\left(\mathrm{V}_{\text {Term }}, \mathrm{P}, \mathrm{Q}\right)$.

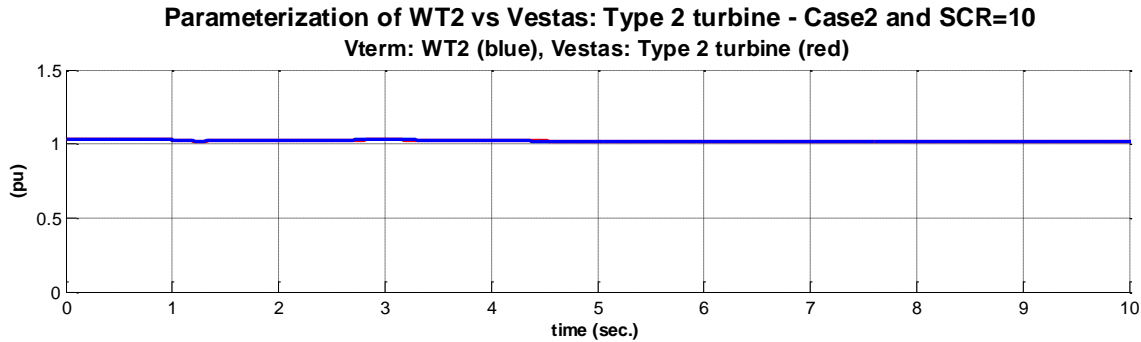

Ireal: WT2 (blue), Vestas: Type 2 turbine (red)

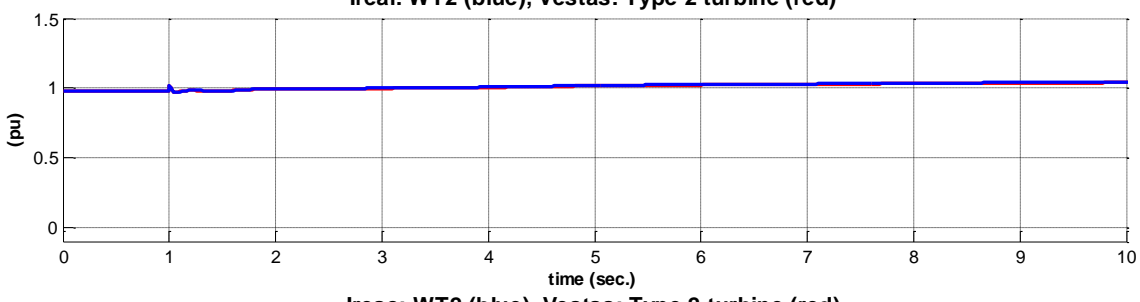

Ireac: WT2 (blue), Vestas: Type 2 turbine (red)

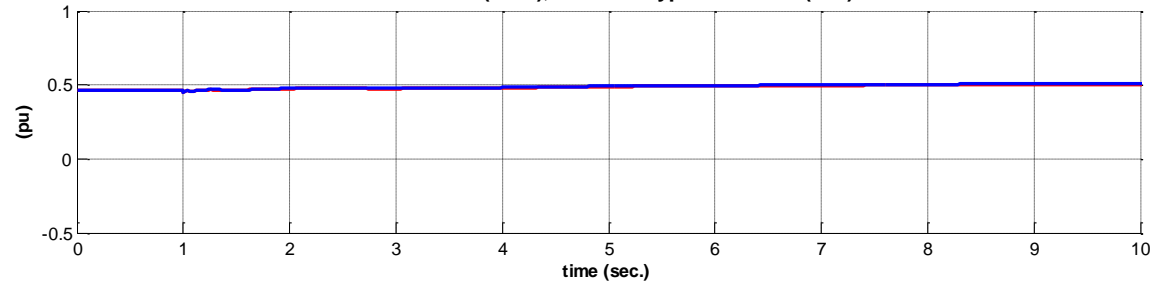

Figure 7-144: Parameterization of WT2 vs Vestas: Type 2 turbine - Case 2 and SCR=10 $\left(\mathrm{V}_{\text {Term }}, \mathrm{I}_{\text {Real }}, \mathrm{I}_{\text {Reac }}\right)$. 


\subsection{Case 3 - Over-Frequency Event}

\section{SCR - 5}

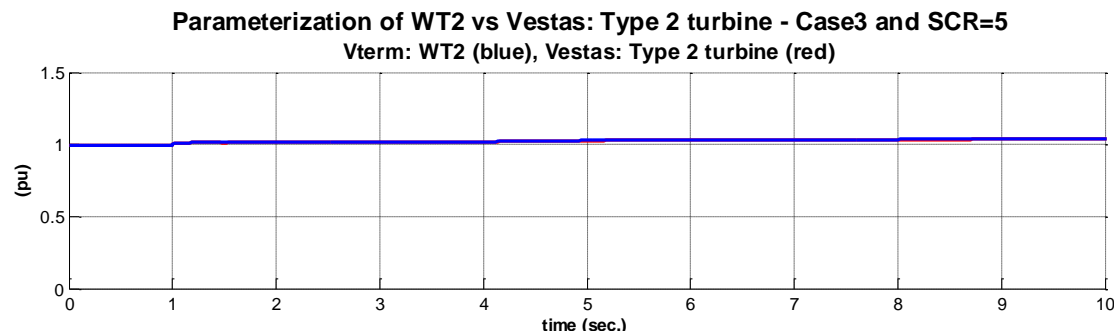

Pgen: WT2 (blue), Vestas: Type 2 turbine (red)

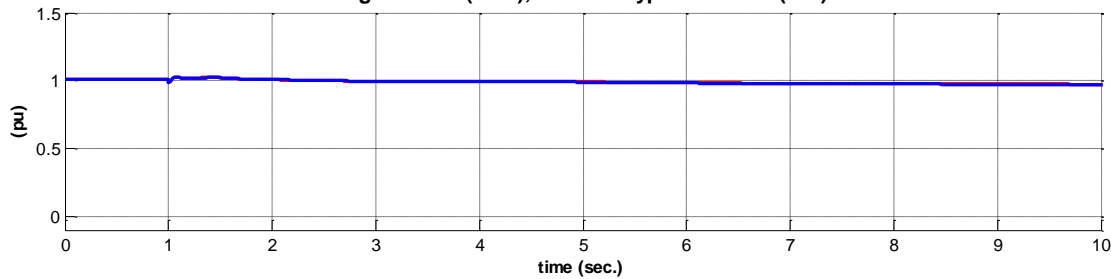

Qgen: WT2 (blue), Vestas: Type 2 turbine (red)

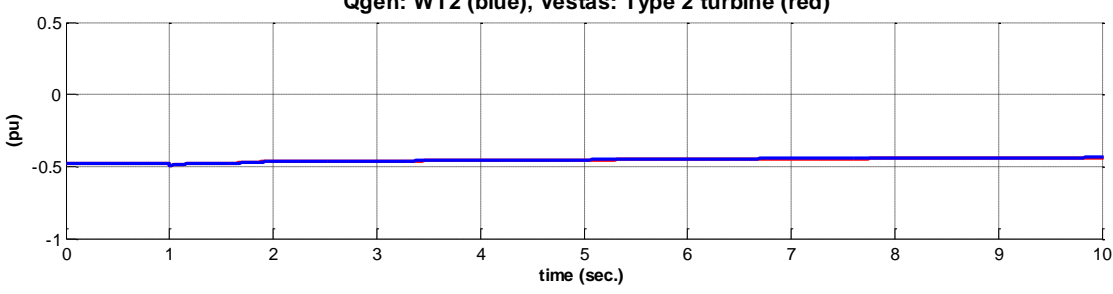

Figure 7-145: Parameterization of WT2 vs Vestas: Type 2 turbine - Case 3 and SCR=5 $\left(\mathrm{V}_{\text {Term }}, \mathrm{P}, \mathrm{Q}\right)$.
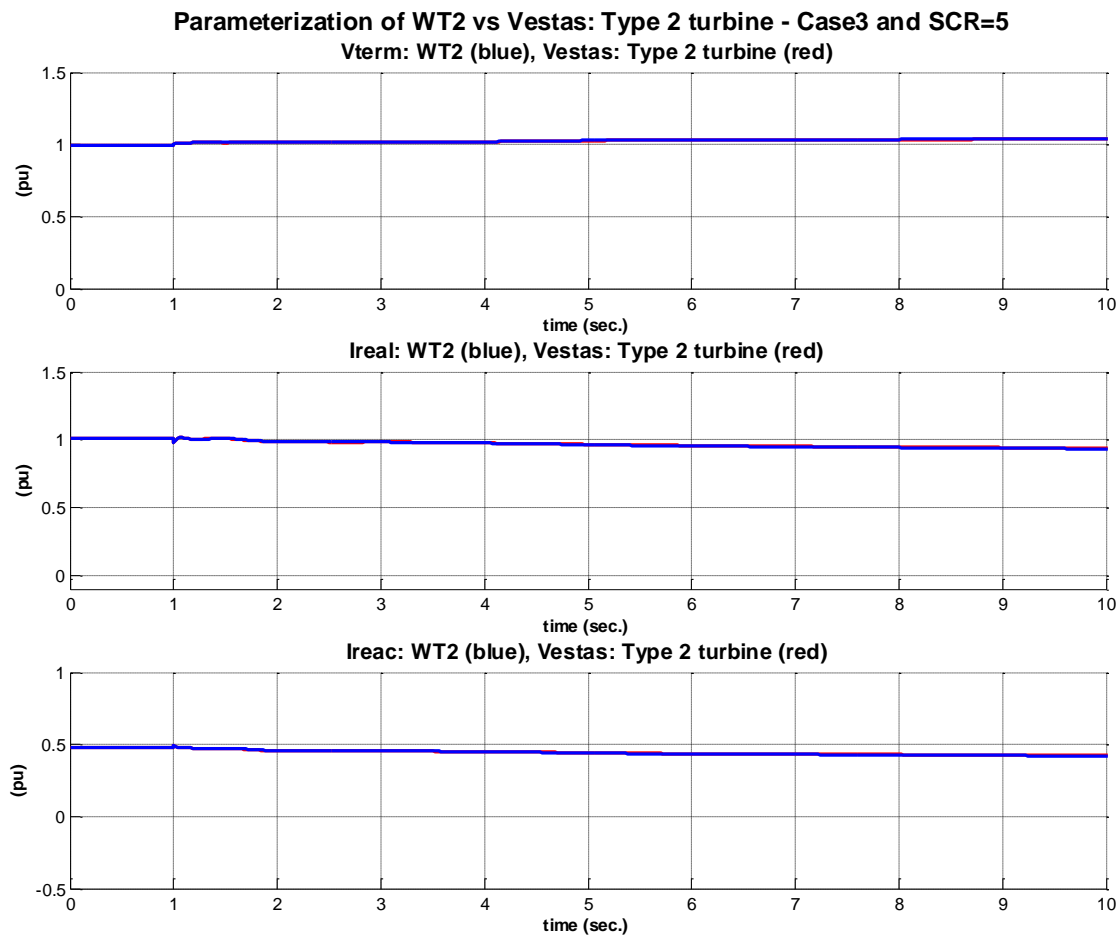

Figure 7-146: Parameterization of WT2 vs Vestas: Type 2 turbine - Case 3 and SCR=5 $\left(\mathrm{V}_{\text {Term }}, \mathrm{I}_{\text {Real }}, \mathrm{I}_{\text {Reac }}\right)$. 
$\underline{\text { SCR }-10}$
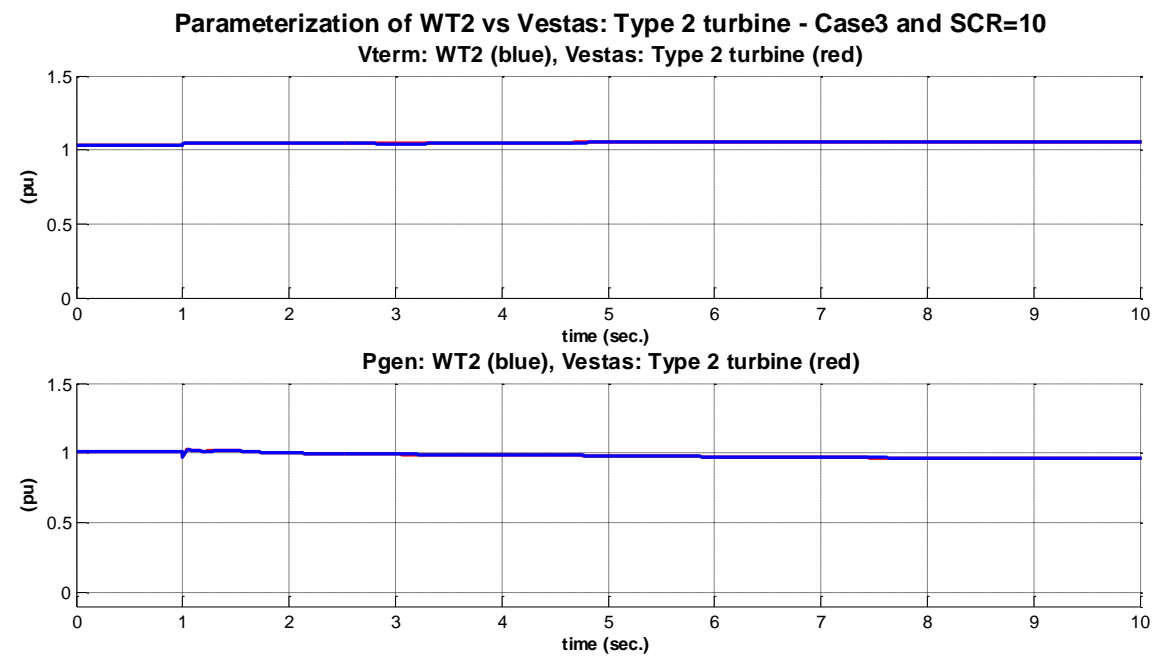

Qgen: WT2 (blue), Vestas: Type 2 turbine (red)

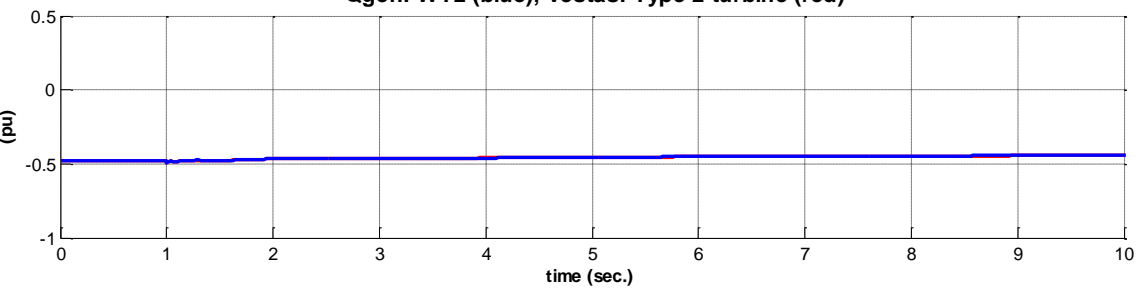

Figure 7-147: Parameterization of WT2 vs Vestas: Type 2 turbine - Case 3 and SCR=10 $\left(\mathbf{V}_{\text {Term }}, \mathbf{P}, \mathbf{Q}\right)$.

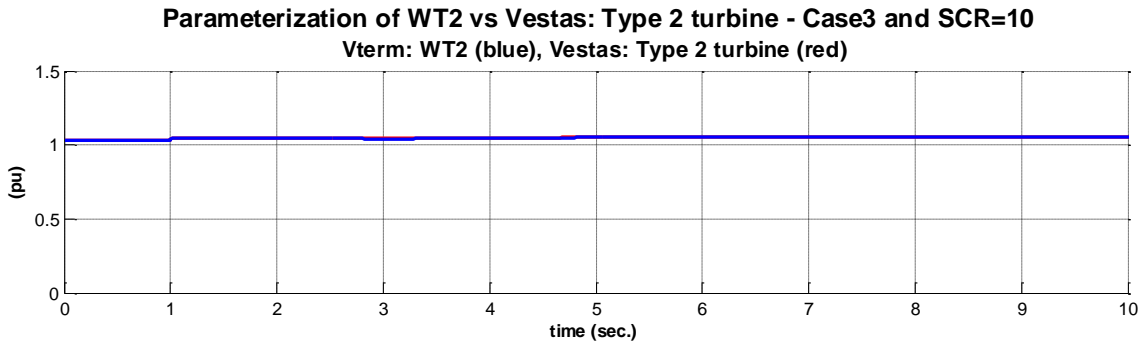

Ireal: WT2 (blue), Vestas: Type 2 turbine (red)

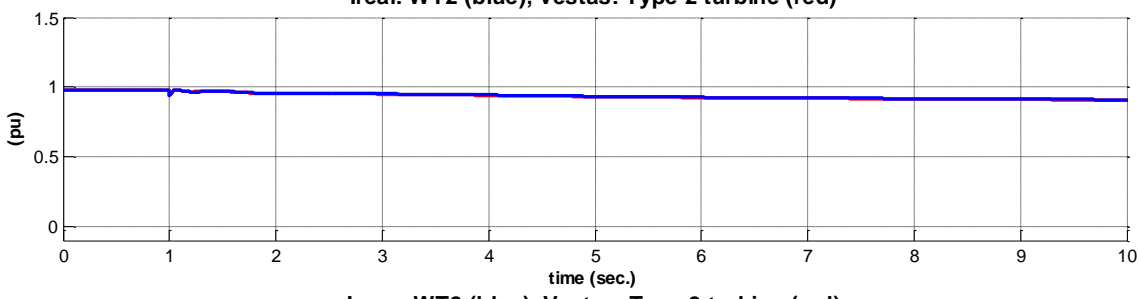

Ireac: WT2 (blue), Vestas: Type 2 turbine (red)

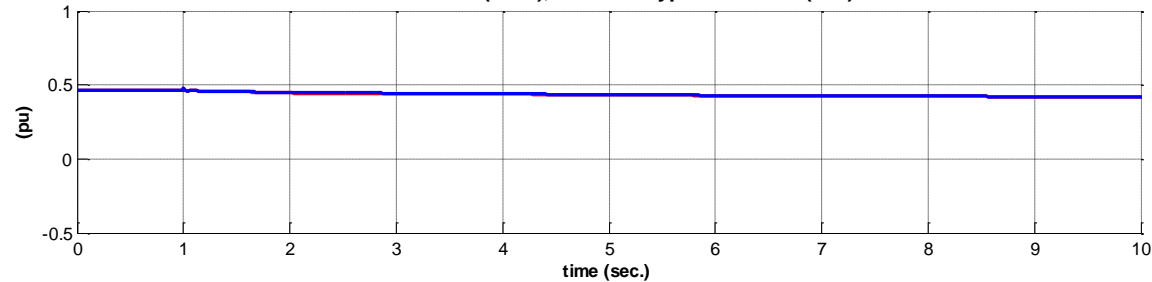

Figure 7-148: Parameterization of WT2 vs Vestas: Type 2 turbine - Case 3 and SCR=10 $\left(\mathrm{V}_{\text {Term, }} \mathrm{I}_{\text {Real }}, \mathrm{I}_{\text {Reac }}\right)$. 


\subsubsection{Results Validation}

With the RCC control disabled for all 3 tests and the fault in case 1 (SCR=5) cleared without tripping the line, the parameterization of the WT2 model showed stable and adequate response. In all figures, the response of the generic model matches the response of the VSM in the frequency range of interest. Thus, both the PSSE model is an appropriate representation of the Vestas: Type 2 WTG's behavior for fundamental frequency analysis.

\subsubsection{Vendor Specific Model Validation}

No data available 


\subsection{Vestas - Type III Models}

\subsubsection{Example List of Vestas Type III Wind Turbines}

- $\quad$ V52-850 kW VCS

- $\quad$ V80 - $2 \mathrm{MW}$ VCS/VCUS

- $\quad \mathrm{V} 90-1.8 / 2 \mathrm{MW}$ VCS

- $\quad$ V90-1.8 MW VCUS

- $\quad \mathrm{V} 90-3 \mathrm{MW}$ VCS/VCRS

- V100 - 1.8 MW VCS/VCUS

\subsubsection{Vendor Specific Model Characterization}

\subsubsection{Model Performance: Case 1 - Fault Event}

In this case a remote fault is applied to bus 12 for a duration of 6 cycles $(0.1 \mathrm{sec})$.

\section{$\underline{\mathrm{SCR}-5}$}
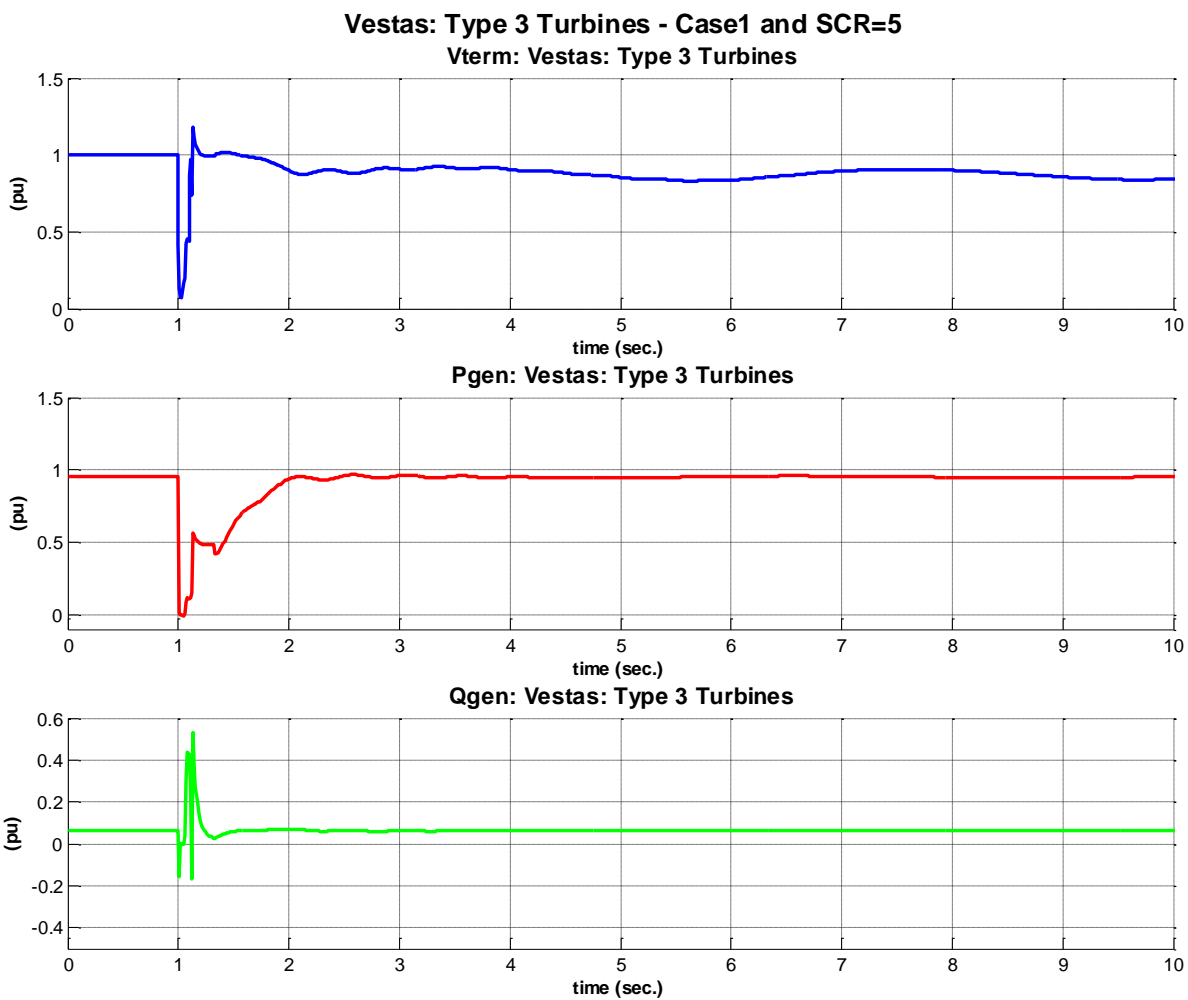

Figure 7-149: Vestas: Type 3 turbine - Case 1 and $S C R=5\left(V_{\text {Term }}, P, Q\right)$. 

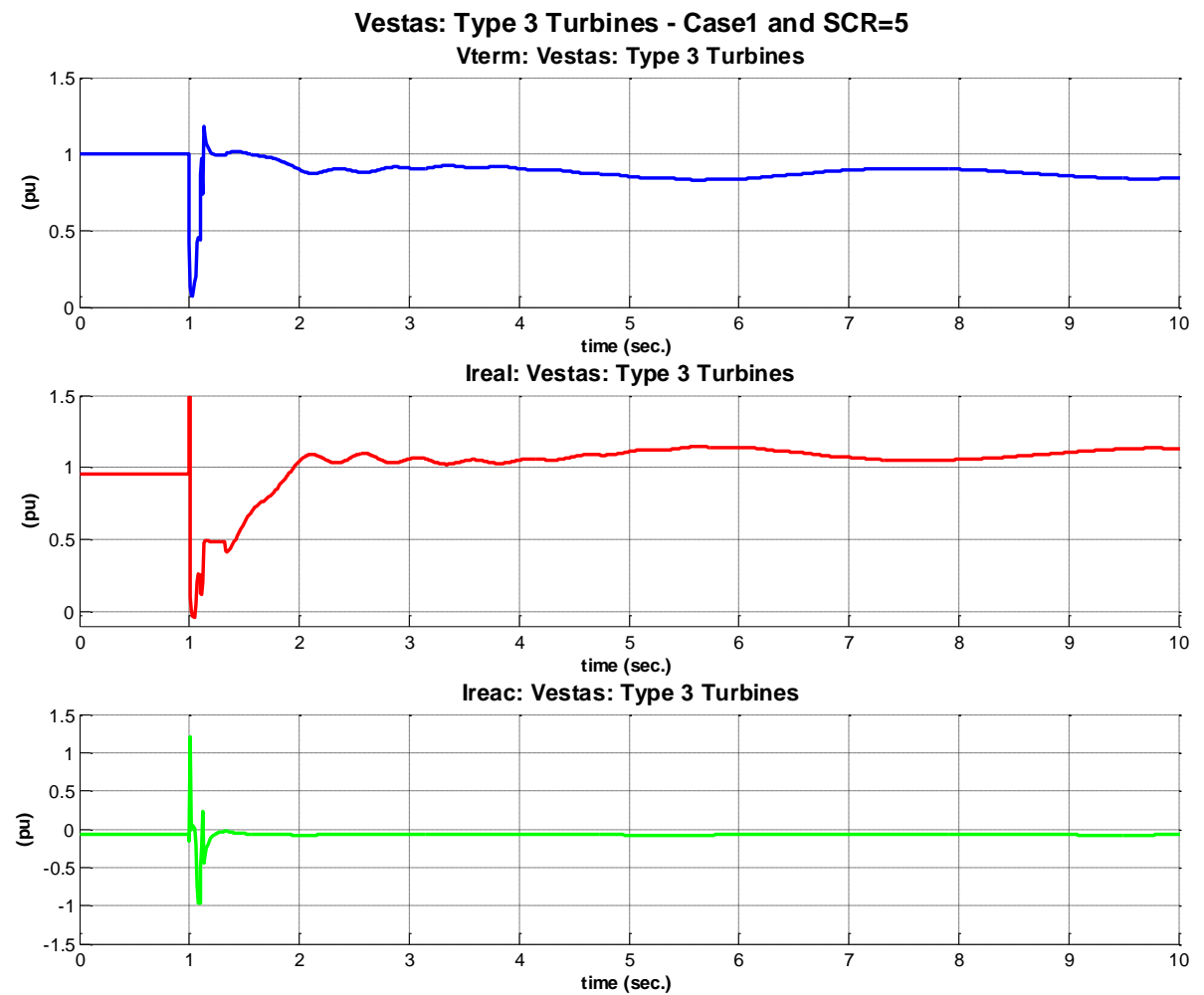

Figure 7-150: Vestas: Type 3 turbine - Case 1 and $S C R=5\left(V_{\text {Term }}, I_{\text {Real }}, I_{\text {Reac }}\right)$.

$\underline{\text { SCR }-10}$
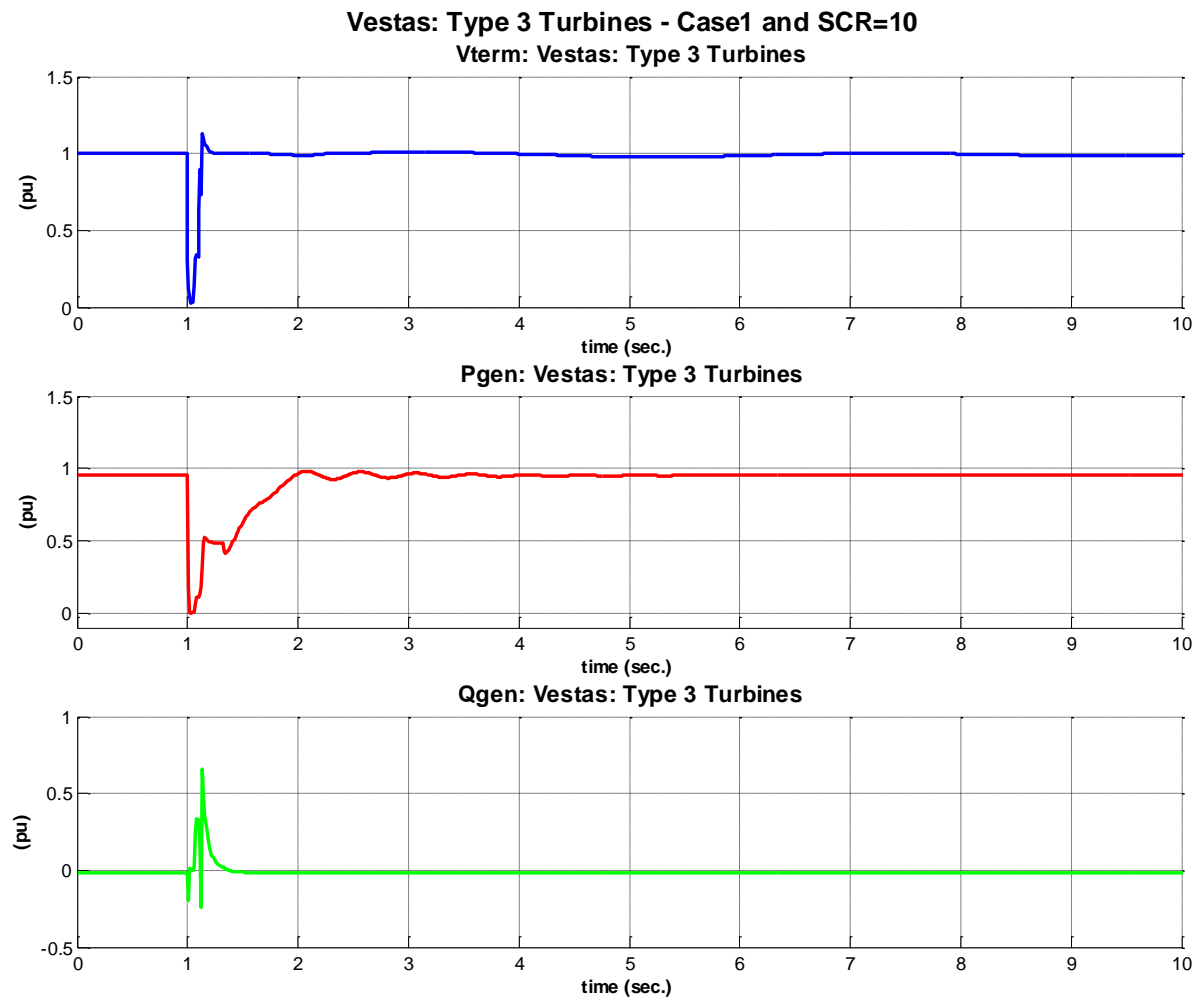

Figure 7-151: Vestas: Type 3 turbine - Case 1 and $\operatorname{SCR}=10\left(V_{\text {Term }}, P, Q\right)$. 

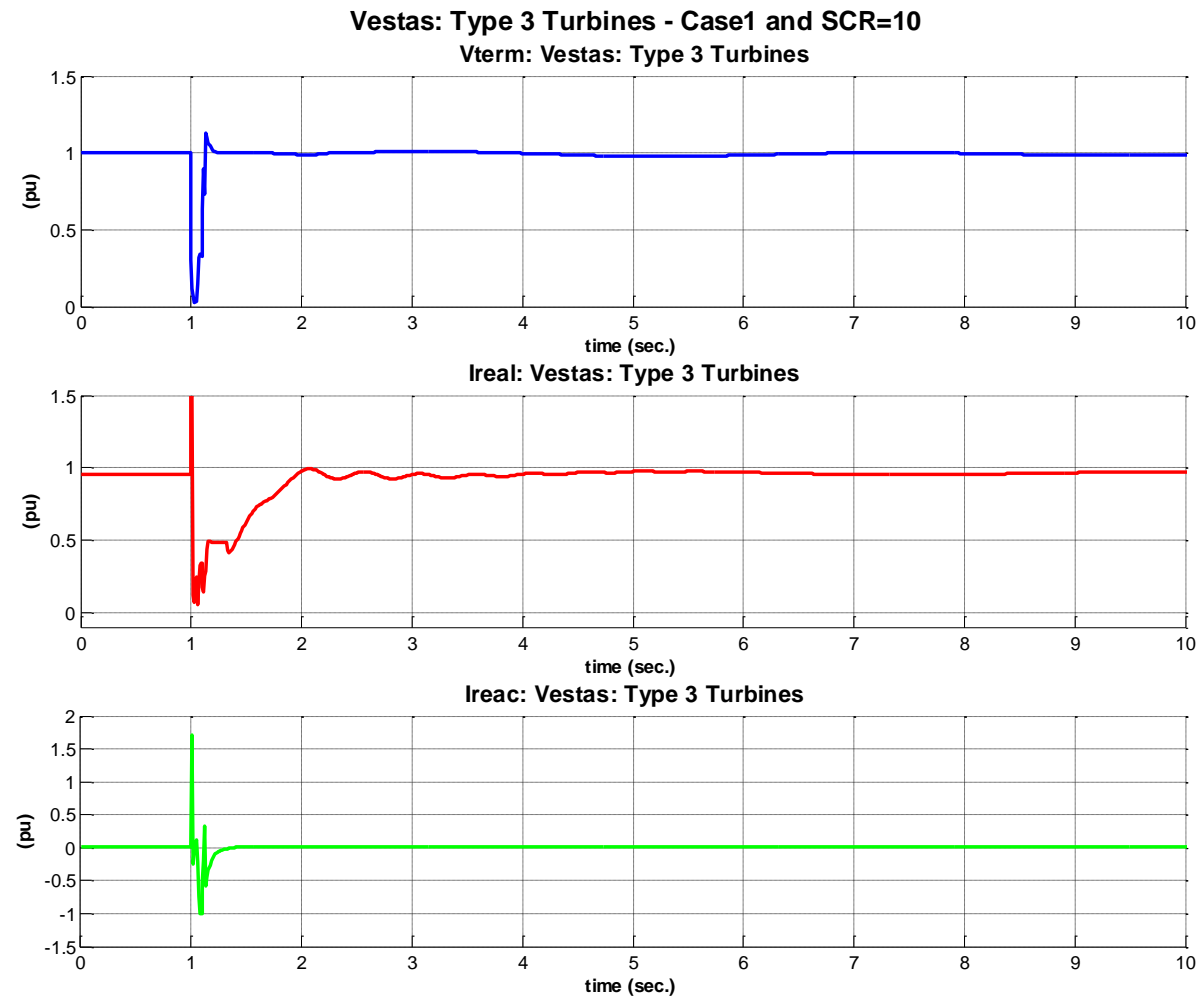

Figure 7-152: Vestas: Type 3 turbine - Case 1 and $S C R=10\left(V_{\text {Term }}, I_{\text {Real }}, I_{\text {Reac }}\right)$.

During low voltage events the turbine switches to current control mode, which gives the priority to the reactive current and injects reactive power to help the Low Voltage Ride Through (LVRT); Once the voltage is recovered above the threshold value of $0.85 \mathrm{pu}$, the turbine remains in the current control mode for additional $0.2 \mathrm{sec}$. After that, the turbine switches back to the power control mode. In this mode the reference power is being ramped up at the slope limit of $1 \mathrm{pu} / \mathrm{sec}$.

\subsubsection{Model Performance: Case 2 - Under-Frequency Event}

In this case a under frequency event is created by tripping the $100 \mathrm{MW}$ generation unit at bus 20 . 
$\underline{\mathrm{SCR}-5}$

Vestas: Type 3 Turbines - Case2 and SCR=5

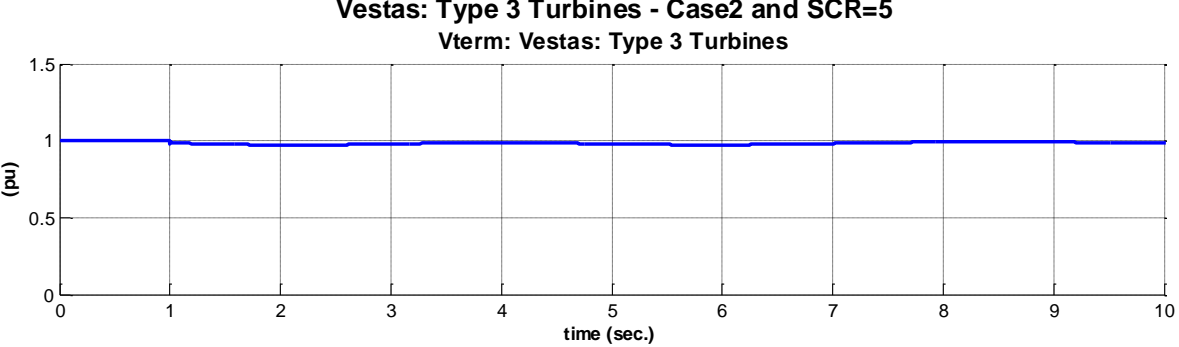

Pgen: Vestas: Type 3 Turbines

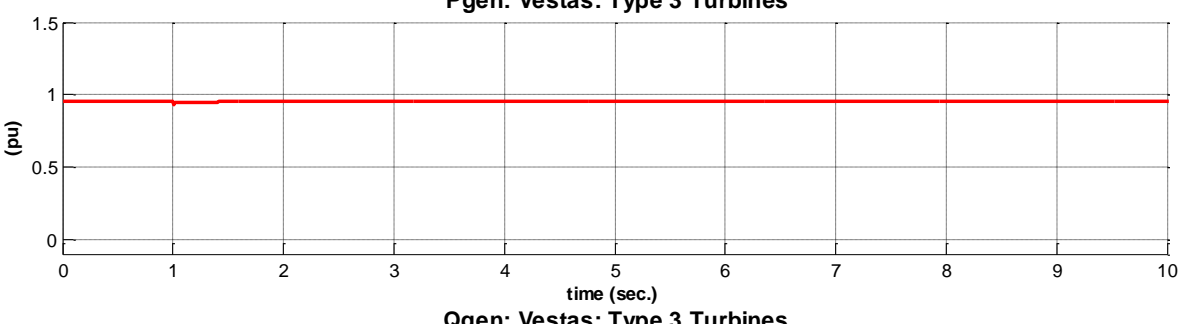

Qgen: Vestas: Type 3 Turbines

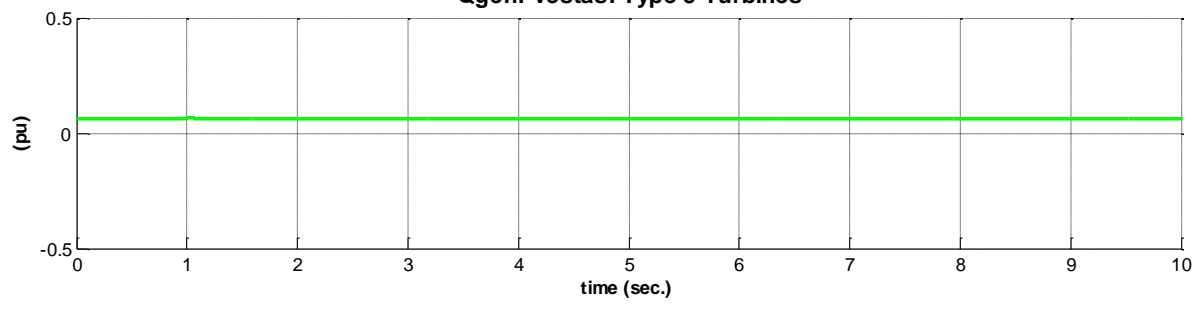

Figure 7-153: Vestas: Type 3 turbine - Case 2 and $S C R=5\left(V_{\text {Term }}, P, Q\right)$.

Vestas: Type 3 Turbines - Case2 and SCR=5 Vterm: Vestas: Type 3 Turbines

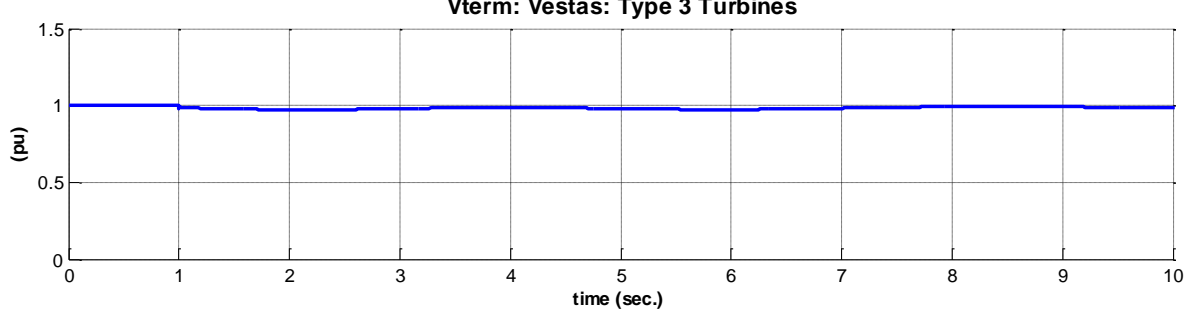

Ireal: Vestas: Type 3 Turbines

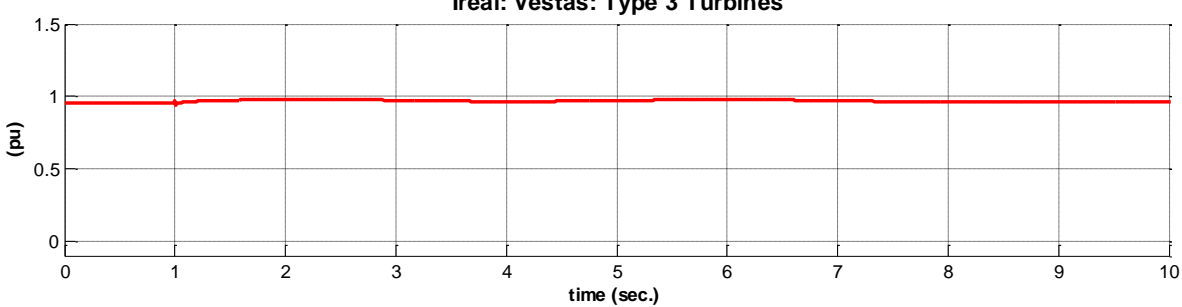

Ireac: Vestas: Type 3 Turbines

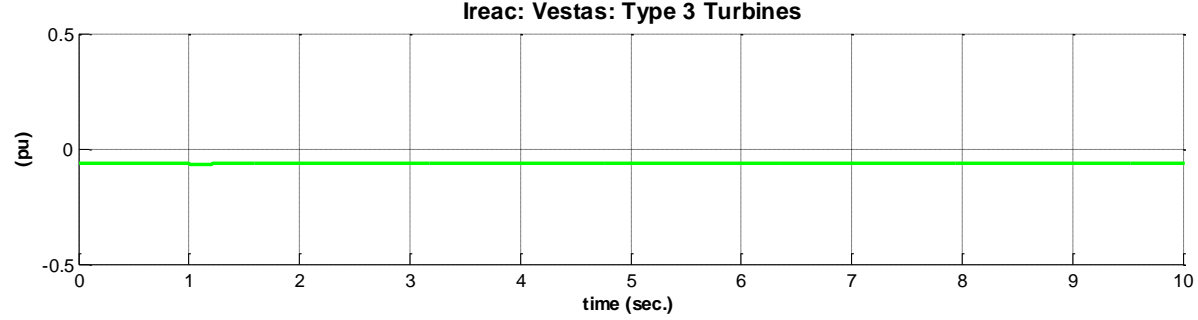

Figure 7-154: Vestas: Type 3 turbine - Case 2 and $S C R=5\left(V_{\text {Term }}, I_{\text {Real }}, I_{\text {Reac }}\right)$. 
$\underline{\text { SCR }-10}$
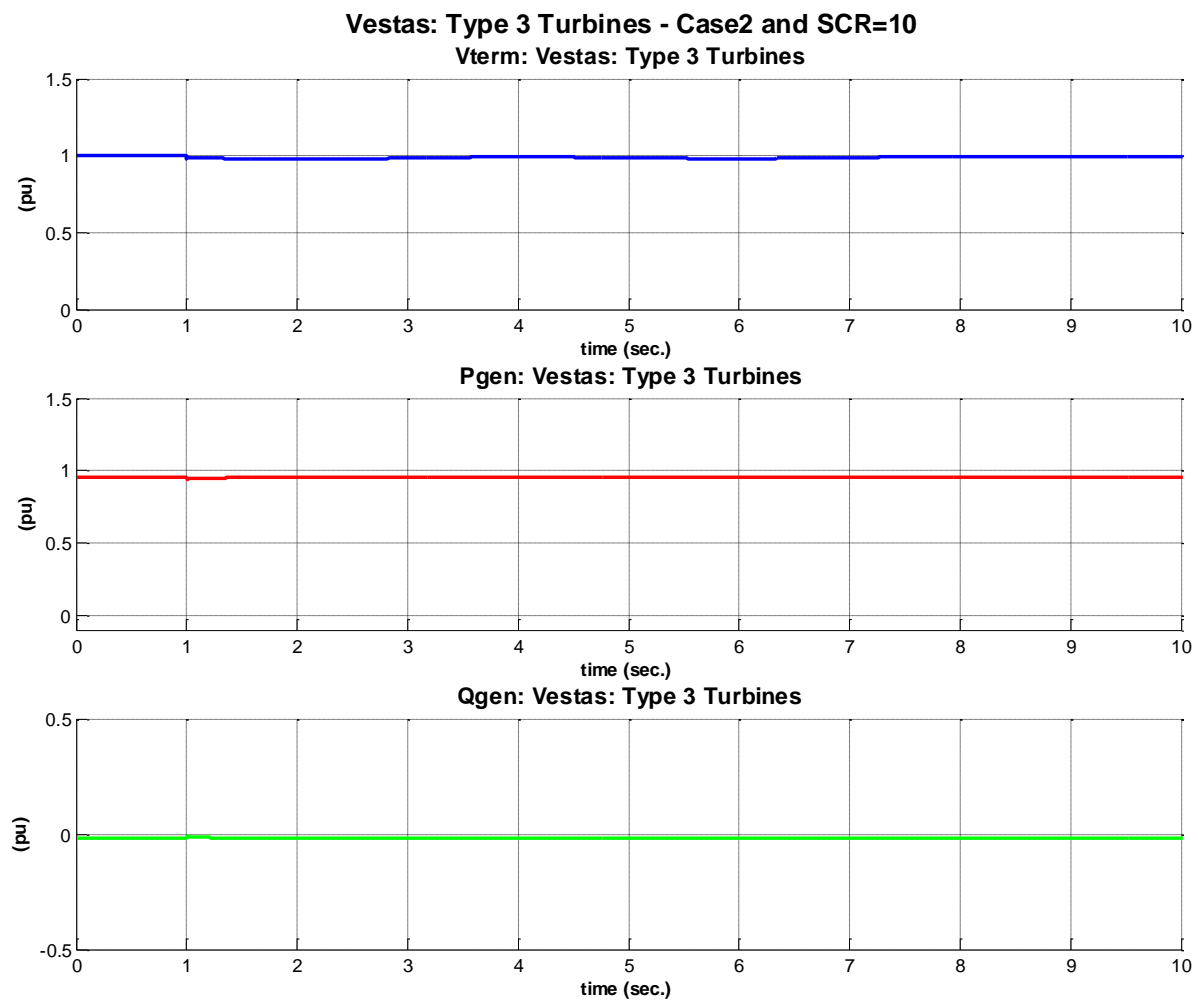

Figure 7-155: Vestas: Type 3 turbine - Case 2 and $S C R=10\left(V_{\text {Term }}, P, Q\right)$.
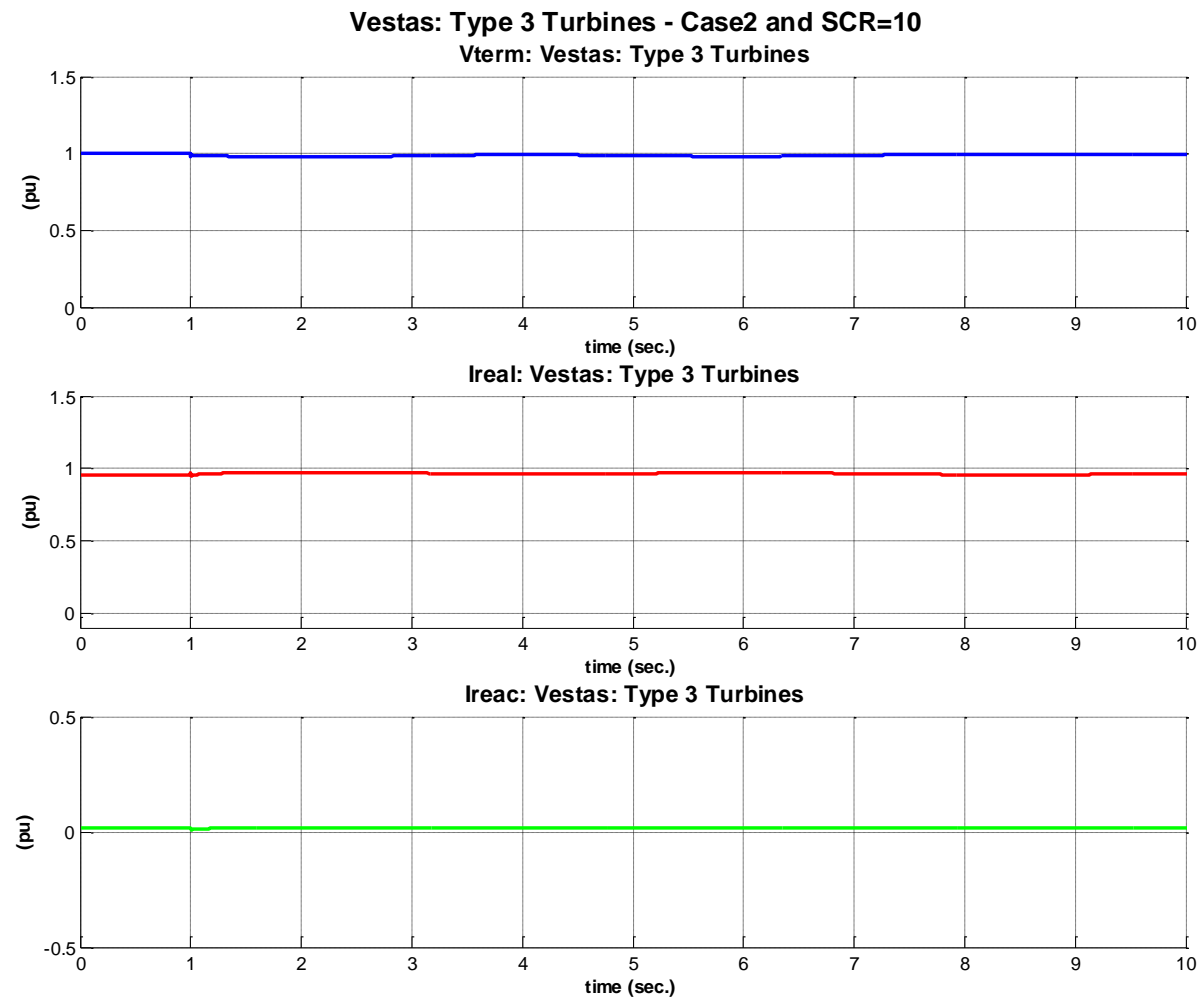

Figure 7-156: Vestas: Type 3 turbine - Case 2 and SCR=10 ( $\left.V_{\text {Term, }} I_{\text {Real }}, I_{\text {Reac }}\right)$. 


\subsubsection{Model Performance: Case 3 - Over-Frequency Event}

In this case a under frequency event is created by tripping the $100 \mathrm{MW}$ load at bus 11.

$\underline{\mathrm{SCR}-5}$
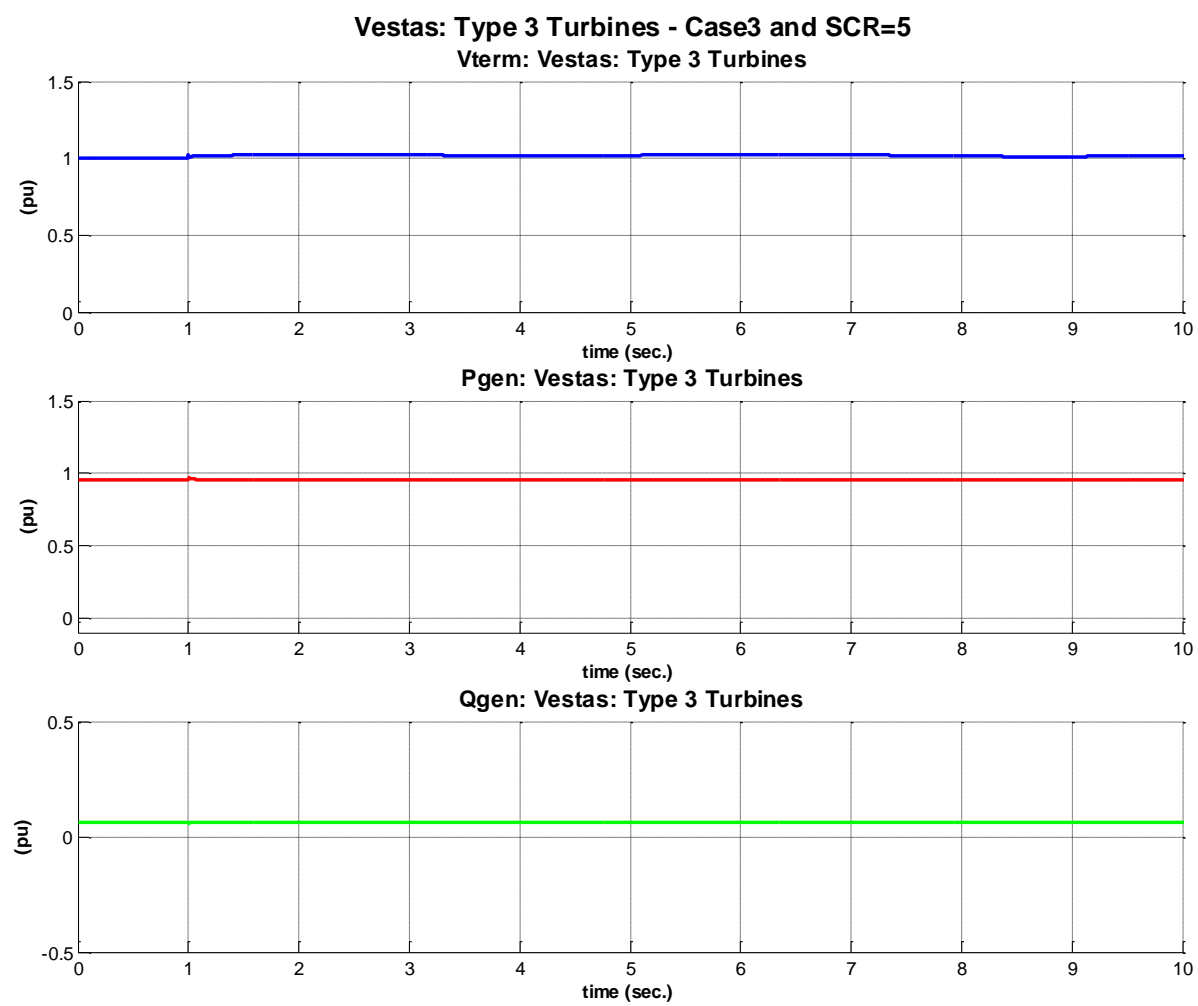

Figure 7-157: Vestas: Type 3 turbine - Case 3 and $S C R=5\left(V_{\text {Term }}, P, Q\right)$. 

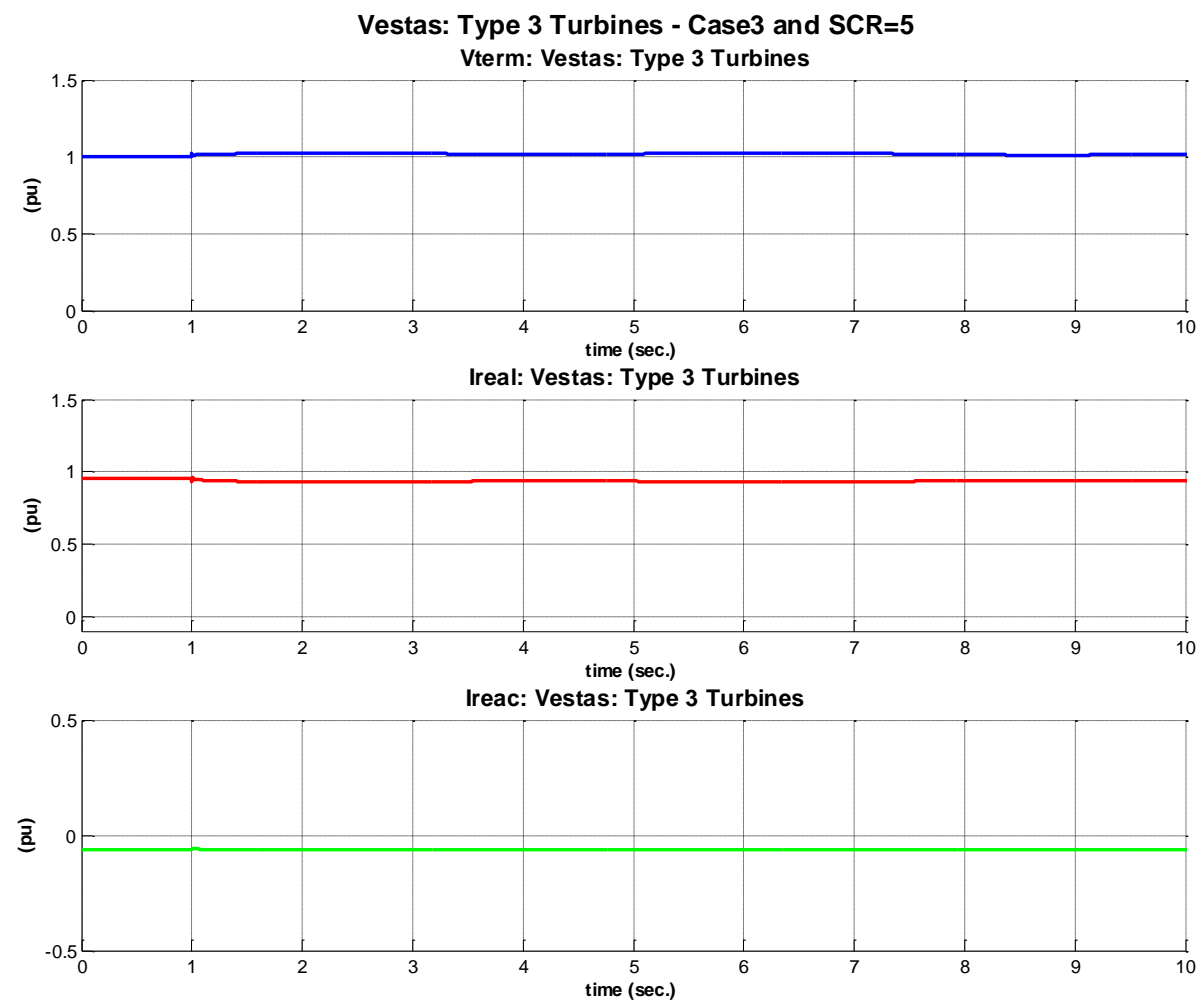

Figure 7-158: Vestas: Type 3 turbine - Case 3 and $S C R=5\left(V_{\text {Term }}, I_{\text {Real }}, I_{\text {Reac }}\right)$.

\section{SCR -10}
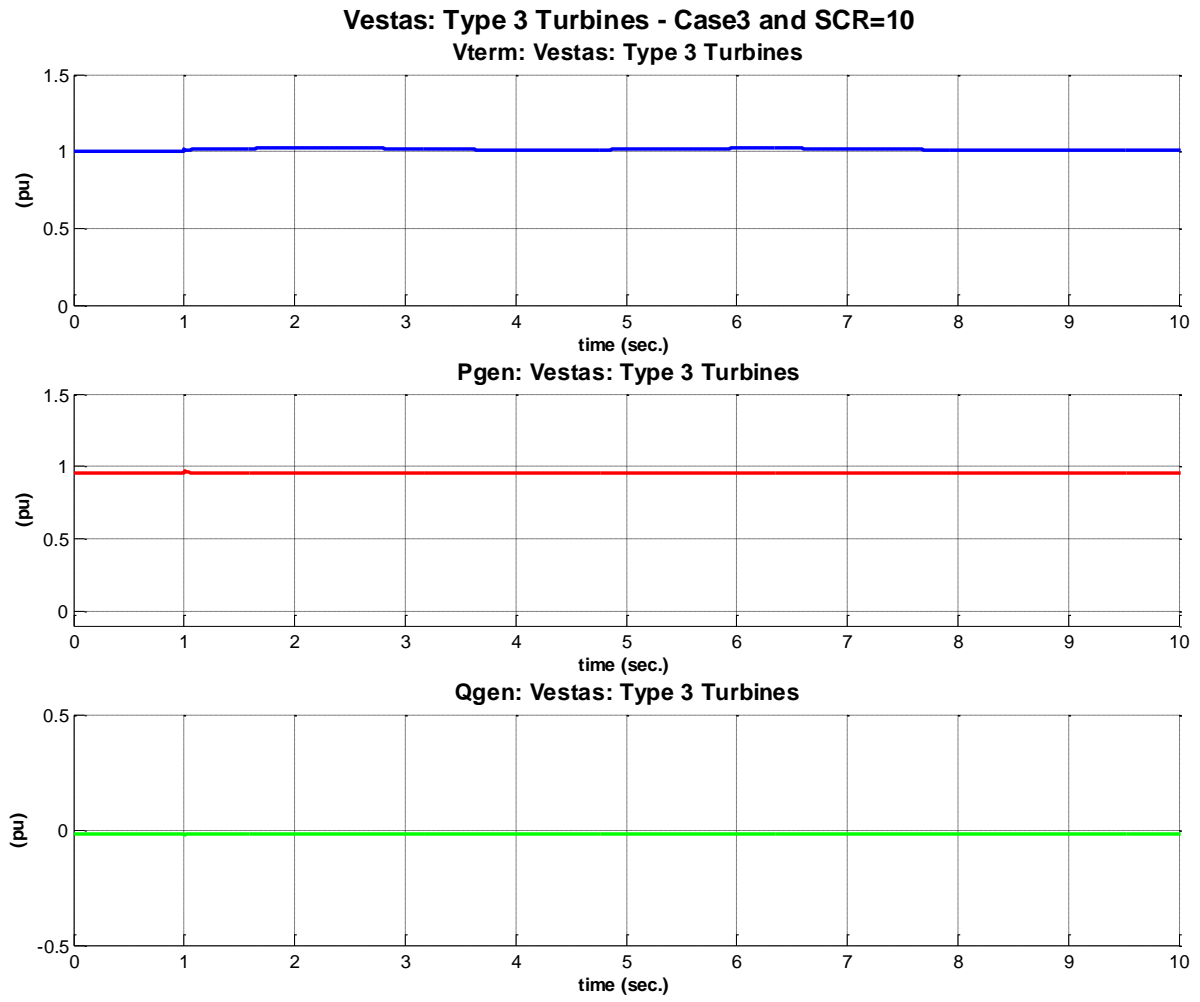

Figure 7-159: Vestas: Type 3 turbine - Case 3 and $S C R=10\left(V_{\text {Term }}, P, Q\right)$. 

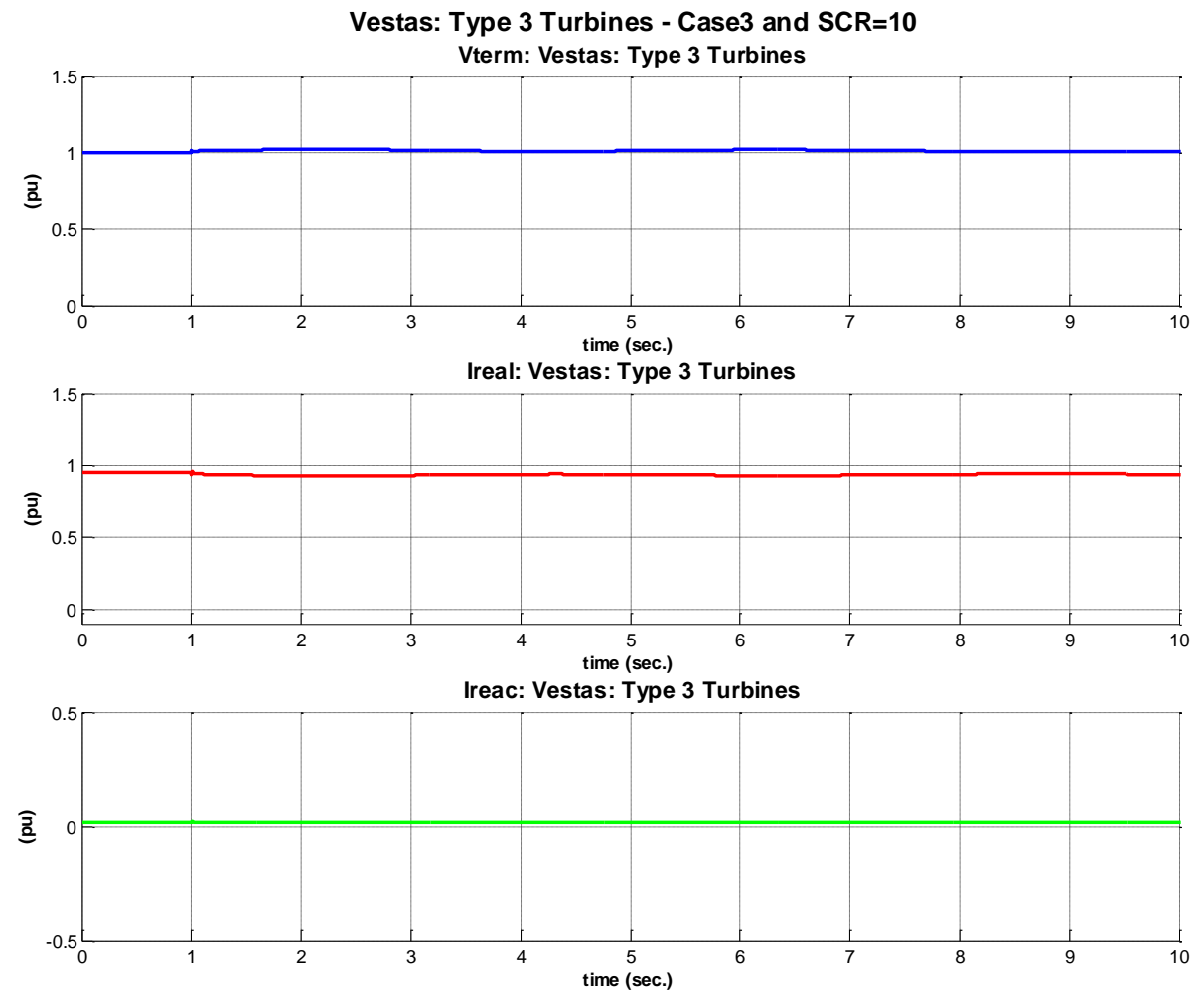

Figure 7-160: Vestas: Type 3 turbine - Case 3 and $S C R=10\left(V_{\text {Term }}, I_{\text {Real }}, I_{\text {Reac }}\right)$. 


\subsubsection{Generic Model Parameterization}

\subsubsection{PSLF Environment}

The PSLF platform generic wind turbine parameters, to mimic the behavior of Vestas' type 3 wind turbines, are shown in Table 7-8. The parameter values shown in the table resulted from a compromise between the simulated cases (i.e. SCR 5 and SCR 10) and network conditions (i.e. fault, under- and over frequency).

Table 7-8: PSLF generic model parameter values.

\begin{tabular}{|c|c|c|c|c|c|c|c|}
\hline \multicolumn{2}{|c|}{ wt3g } & \multicolumn{2}{|c|}{$w t 3 t$} & \multicolumn{2}{|c|}{ wt3e } & \multicolumn{2}{|c|}{ wt3p } \\
\hline Lpp & 0.5 & $V_{w}$ & 1.0 & Varflg & 0 & Kpp & 15.0 \\
\hline Lvplsw & 1.0 & $\mathrm{Ht}$ & 4.94 & Vltflg & 0 & Kip & 2.0 \\
\hline Rrpwr & 10.0 & $\mathrm{D}$ & 0.0 & Tsp & 5.0 & Kpc & 0.30 \\
\hline Brkpt & 0.9 & Kaero & 0.007 & Kptrq & 3.00 & Kic & 0.01 \\
\hline Zerox & 0.5 & Theta2 & 21.98 & Kitrq & 0.6 & Pimax & 27.0 \\
\hline Lvpll & 1.22 & Htfrac & 0.875 & Tpc & 0.05 & Pimin & 0 \\
\hline Volim & 1.20 & Freq1 & 1.80 & Pmax & 1.12 & Pirat & 10.0 \\
\hline Lvpntl & 0.80 & Dshaft & 1.50 & Pmin & 0.04 & Tpi & 0.3 \\
\hline Lvpnt0 & 0.40 & & & Pwrat & 0.45 & Pset & 1.0 \\
\hline & & & & Ipmax & 1.10 & & \\
\hline & & & & Wpmin & 0.69 & & \\
\hline & & & & Wp20 & 0.78 & & \\
\hline & & & & Wp40 & 0.98 & & \\
\hline & & & & Wp60 & 1.12 & & \\
\hline & & & & Pwp100 & 0.74 & & \\
\hline & & & & Wp100 & 1.20 & & \\
\hline & & & & Kqi & 30.0 & & \\
\hline & & & & Kqv & 40.0 & & \\
\hline & & & & Qmax & 0.436 & & \\
\hline & & & & Qmin & -0.436 & & \\
\hline & & & & Vmax & 1.10 & & \\
\hline & & & & Vmin & 0.90 & & \\
\hline & & & & Xiqmax & 1.45 & & \\
\hline & & & & Xiqmin & 0.50 & & \\
\hline & & & & $\mathrm{Tp}$ & 0.05 & & \\
\hline & & & & $X_{c}$ & 0.00 & & \\
\hline & & & & $\mathrm{Tr}$ & 0.02 & & \\
\hline & & & & Fn & 1.00 & & \\
\hline & & & & Kiv & 5.00 & & \\
\hline & & & & Kpv & 18.0 & & \\
\hline & & & & Tv & 0.05 & & \\
\hline & & & & Tc & 0.15 & & \\
\hline
\end{tabular}




\subsection{Case 1 - Fault Event}

\section{SCR - 5}

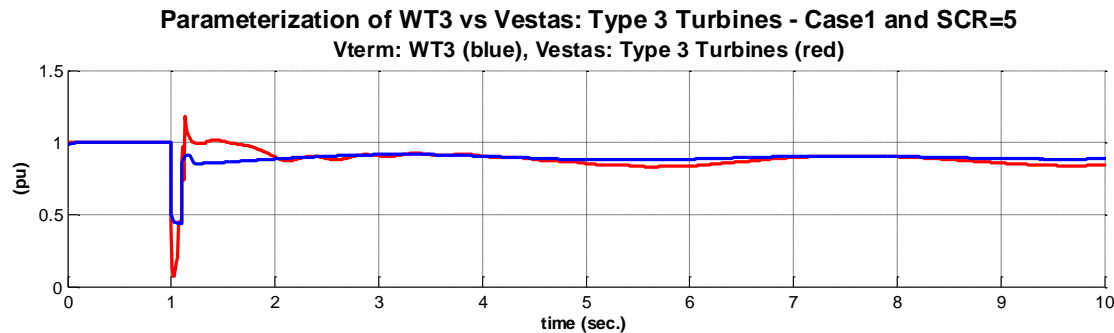

Pgen: WT3 (blue), Vestas: Type 3 Turbines (red)

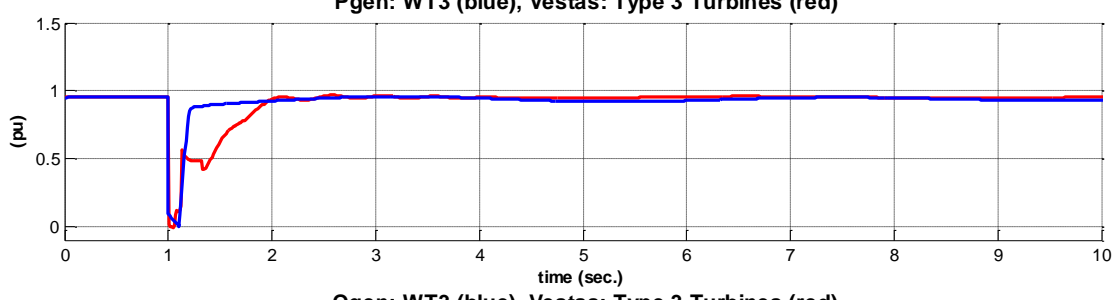

Qgen: WT3 (blue), Vestas: Type 3 Turbines (red)

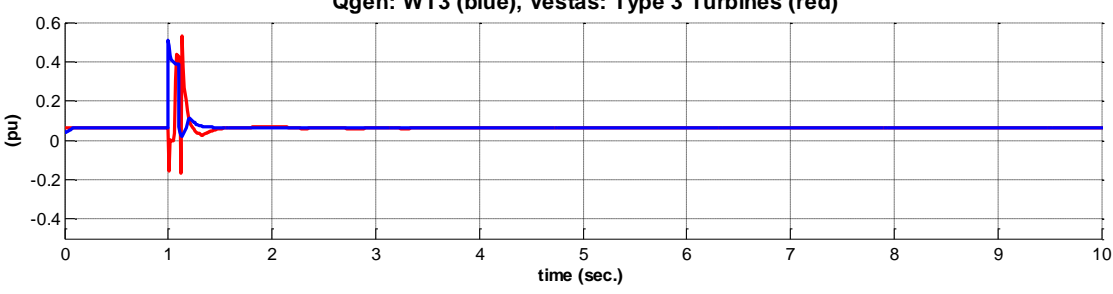

Figure 7-161: Parameterization of WT3 vs Vestas: Type 3 turbine - Case 1 and SCR=5 $\left(\mathrm{V}_{\text {Term }}, \mathrm{P}, \mathrm{Q}\right)$.
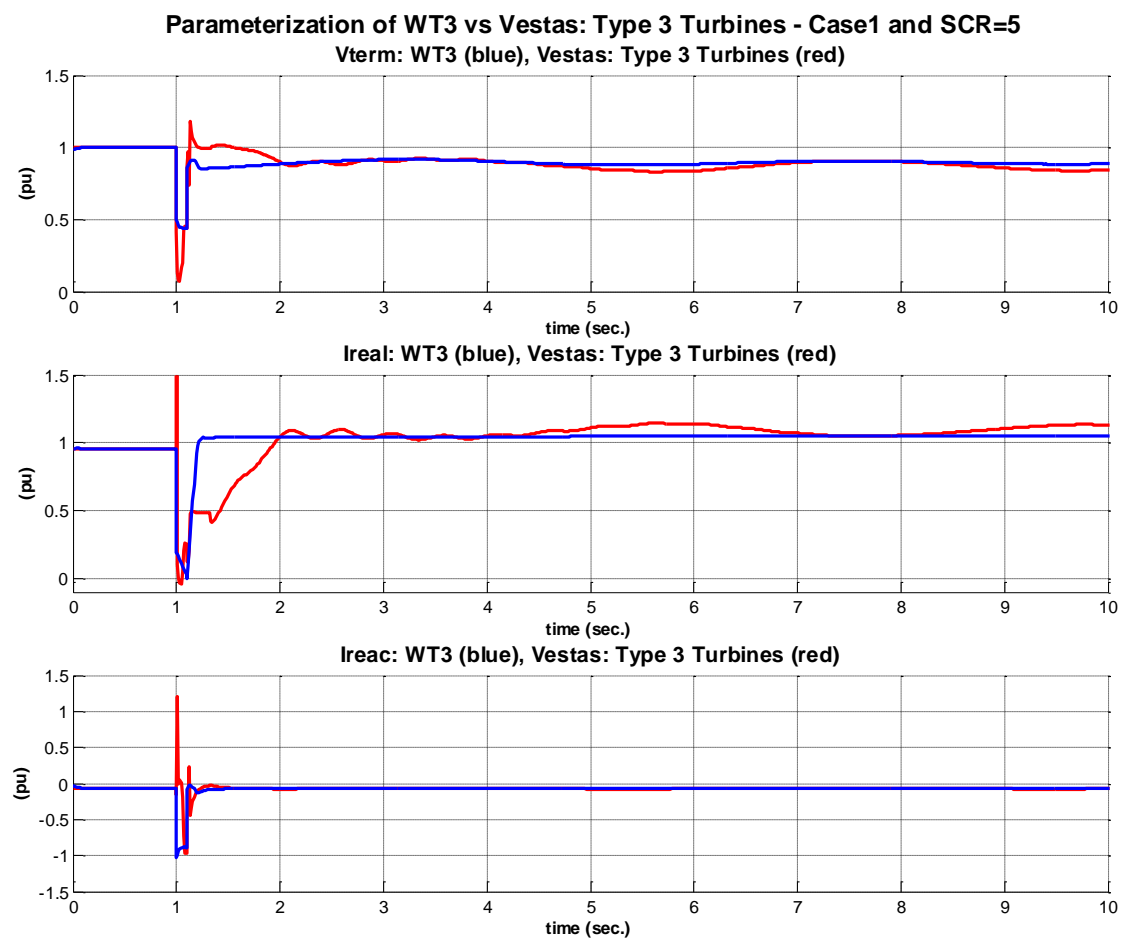

Figure 7-162: Parameterization of WT3 vs Vestas: Type 3 turbine - Case 1 and SCR=5 $\left(\mathrm{V}_{\text {Term }}, \mathrm{I}_{\text {Real }}, \mathrm{I}_{\text {Reac }}\right)$. 
$\underline{\text { SCR }-10}$
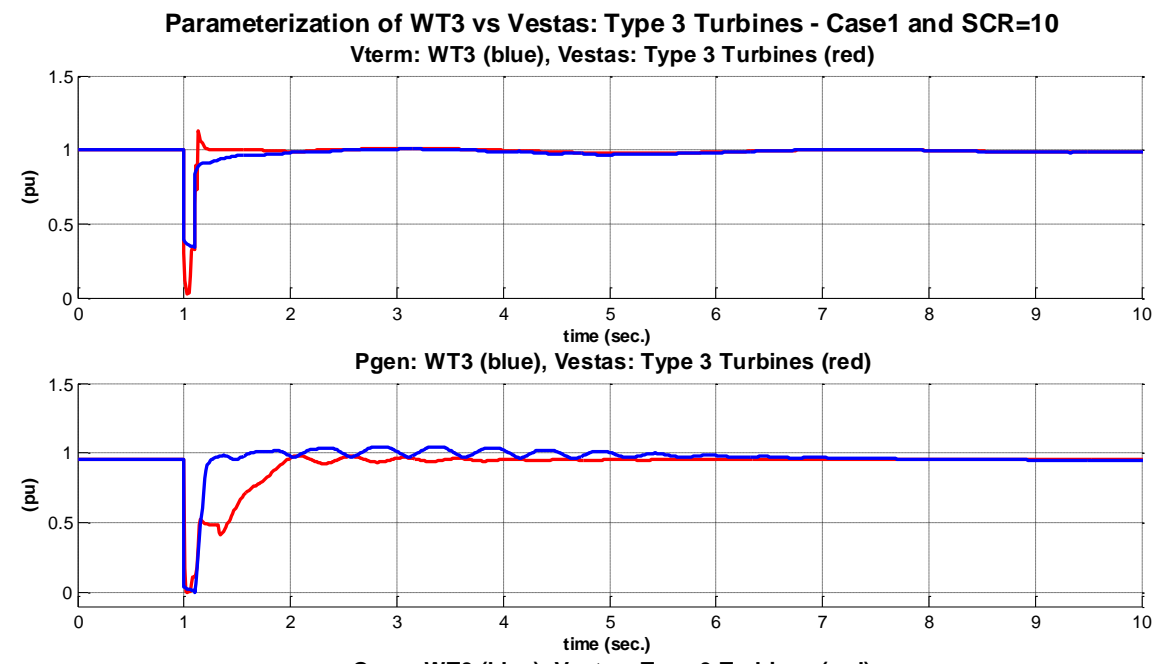

Qgen: WT3 (blue), Vestas: Type 3 Turbines (red)

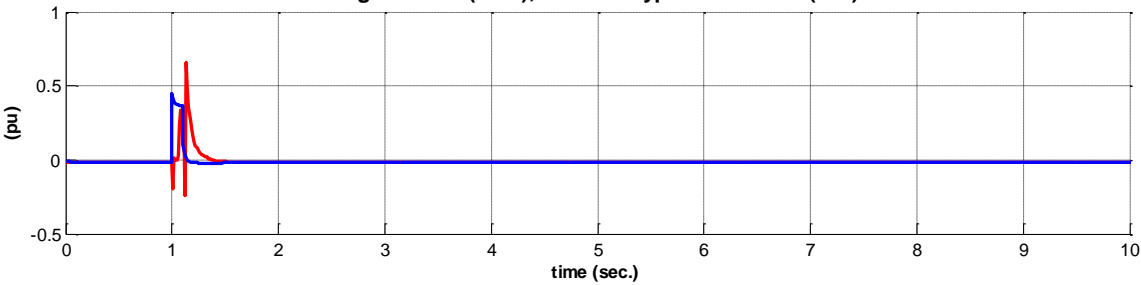

Figure 7-163: Parameterization of WT3 vs Vestas: Type 3 turbine - Case 1 and SCR=10 $\left(\mathrm{V}_{\text {Term }}, \mathrm{P}, \mathrm{Q}\right)$.
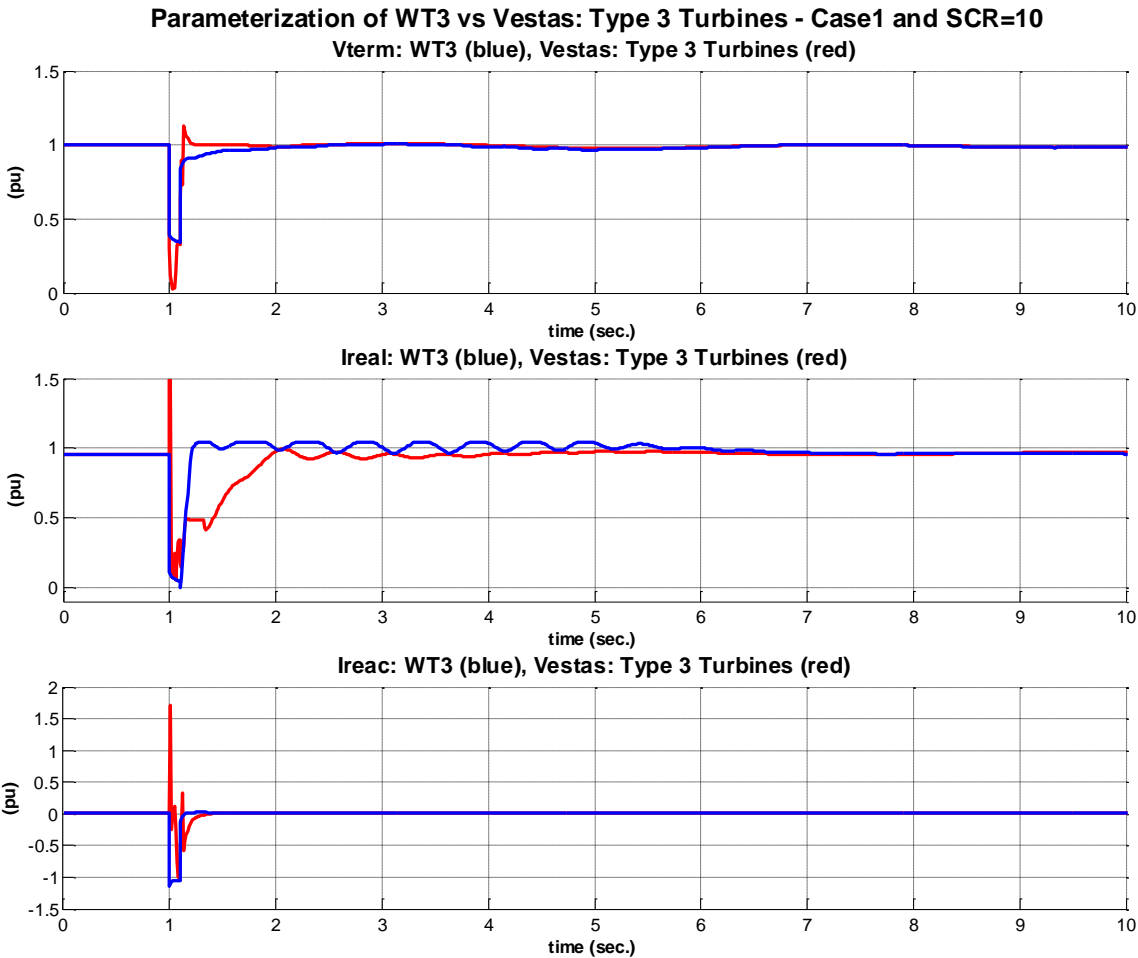

Figure 7-164: Parameterization of WT3 vs Vestas: Type 3 turbine - Case 1 and SCR=10 $\left(V_{\text {Term }}, I_{\text {Real }}, I_{\text {Reac }}\right)$. 


\subsection{Case 2 - Under-Frequency Event}

\section{$\underline{\mathrm{SCR}-5}$}

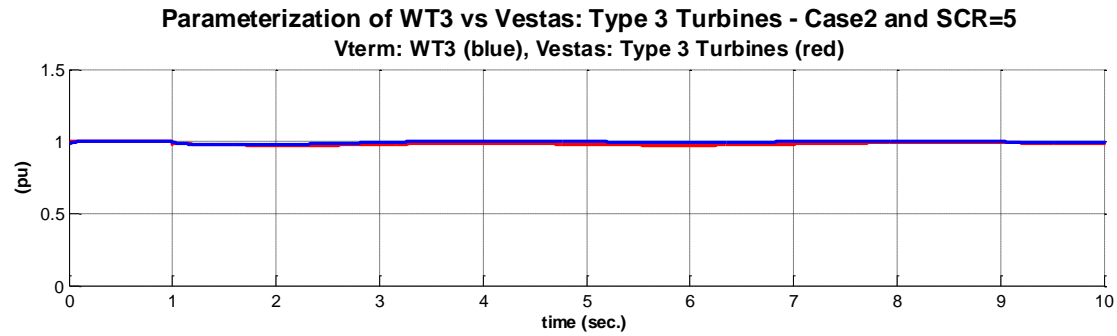

Pgen: WT3 (blue), Vestas: Type 3 Turbines (red)

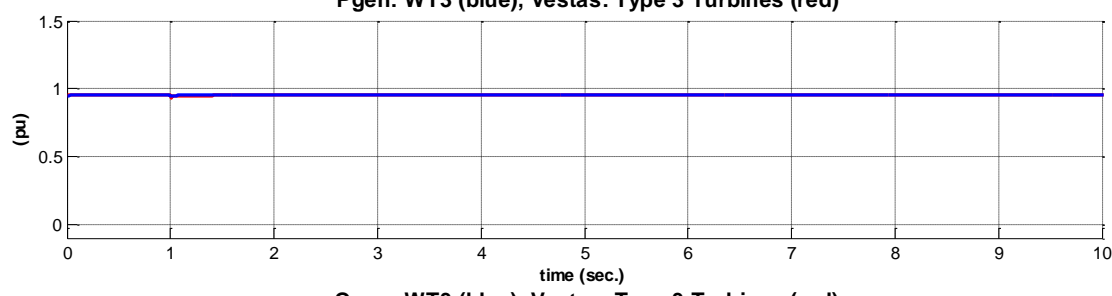

Qgen: WT3 (blue), Vestas: Type 3 Turbines (red)

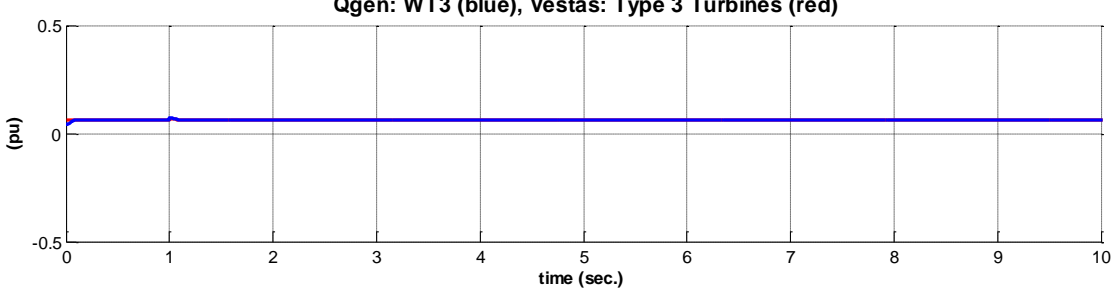

Figure 7-165: Parameterization of WT3 vs Vestas: Type 3 turbine - Case 2 and SCR=5 $\left(\mathrm{V}_{\text {Term }}, \mathrm{P}, \mathrm{Q}\right)$.
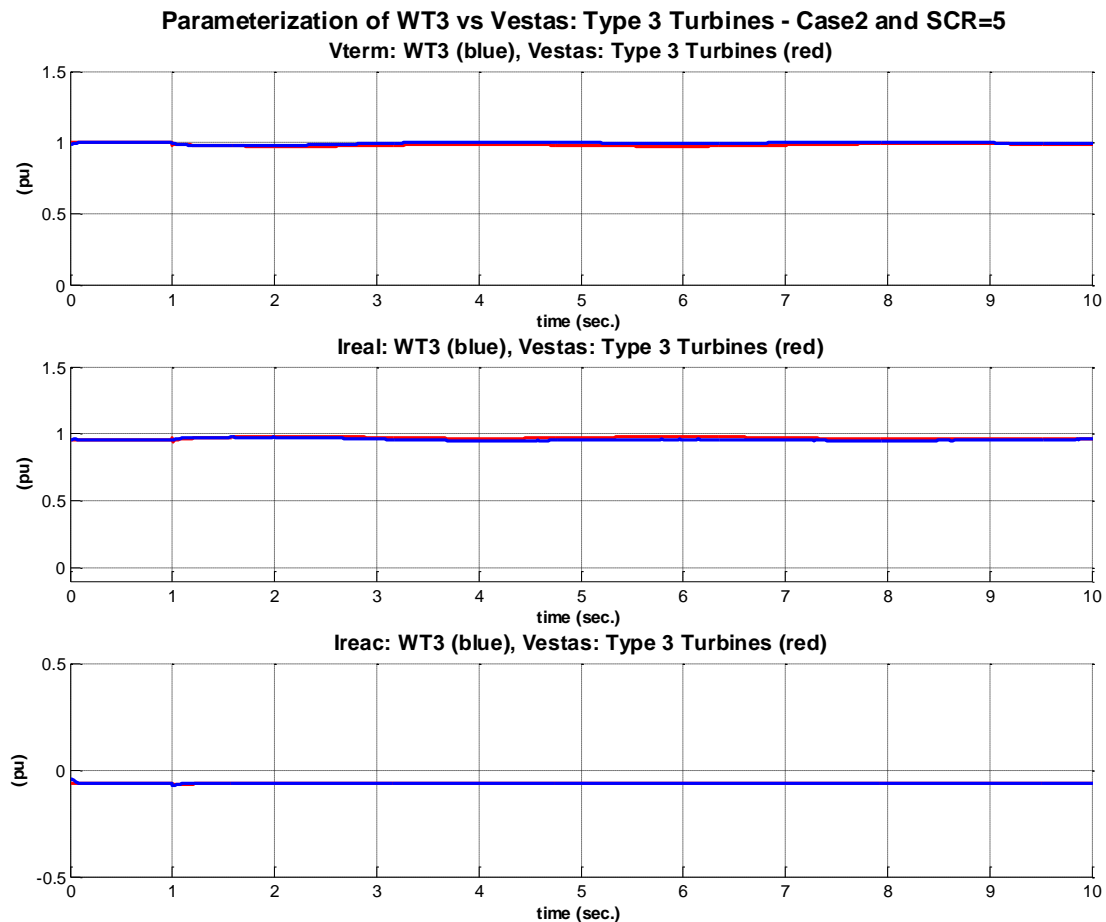

Figure 7-166: Parameterization of WT3 vs Vestas: Type 3 turbine - Case 2 and SCR=5 $\left(\mathrm{V}_{\text {Term }}, \mathrm{I}_{\text {Real }}, \mathrm{I}_{\text {Reac }}\right)$. 
$\underline{\text { SCR }-10}$
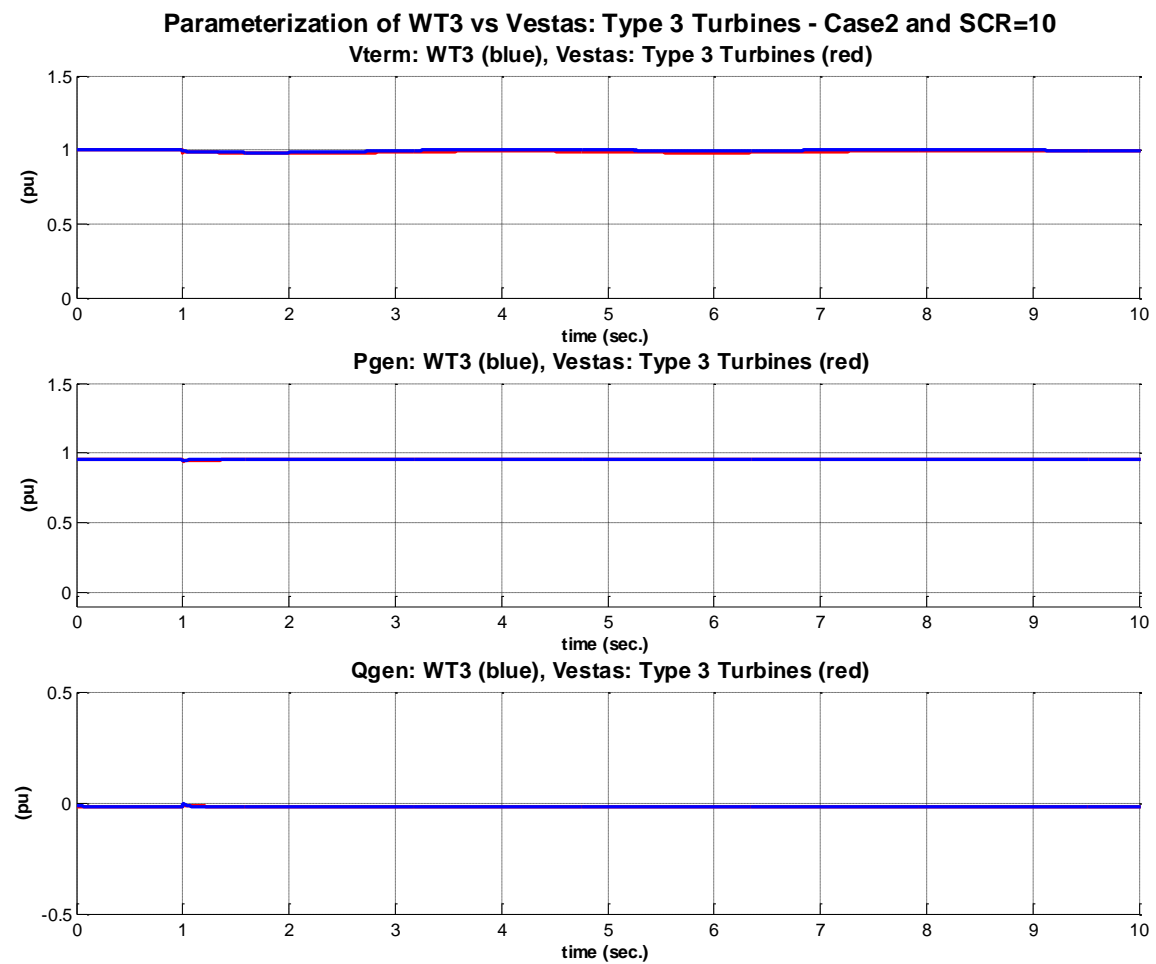

Figure 7-167: Parameterization of WT3 vs Vestas: Type 3 turbine - Case 2 and SCR=10 $\left(\mathrm{V}_{\text {Term }}, \mathrm{P}, \mathrm{Q}\right)$.
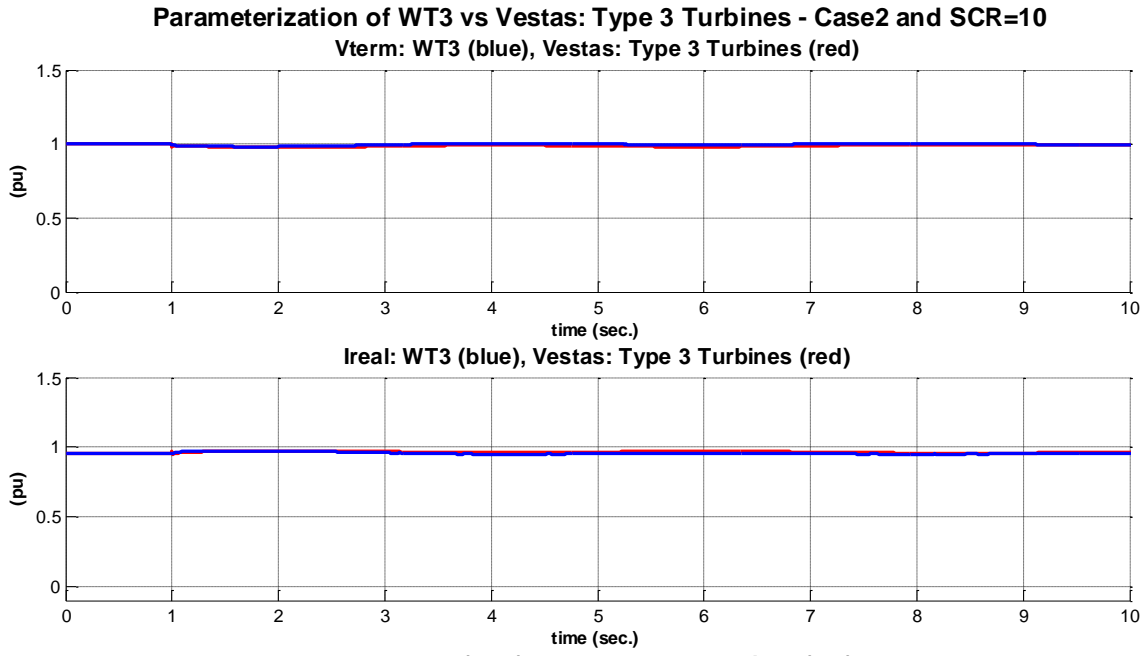

Ireac: WT3 (blue), Vestas: Type 3 Turbines (red)

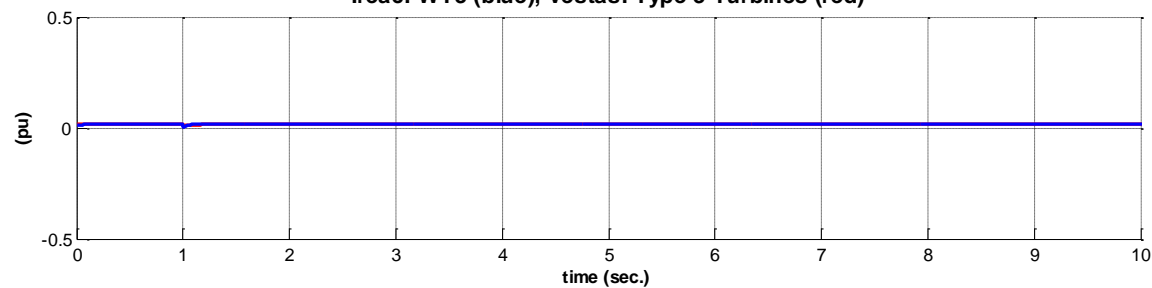

Figure 7-168: Parameterization of WT3 vs Vestas: Type 3 turbine - Case 2 and SCR=10 $\left(V_{\text {Term }}, I_{\text {Real }}, I_{\text {Reac }}\right)$. 


\subsection{Case 3 - Over-Frequency Event}

\section{SCR - 5}
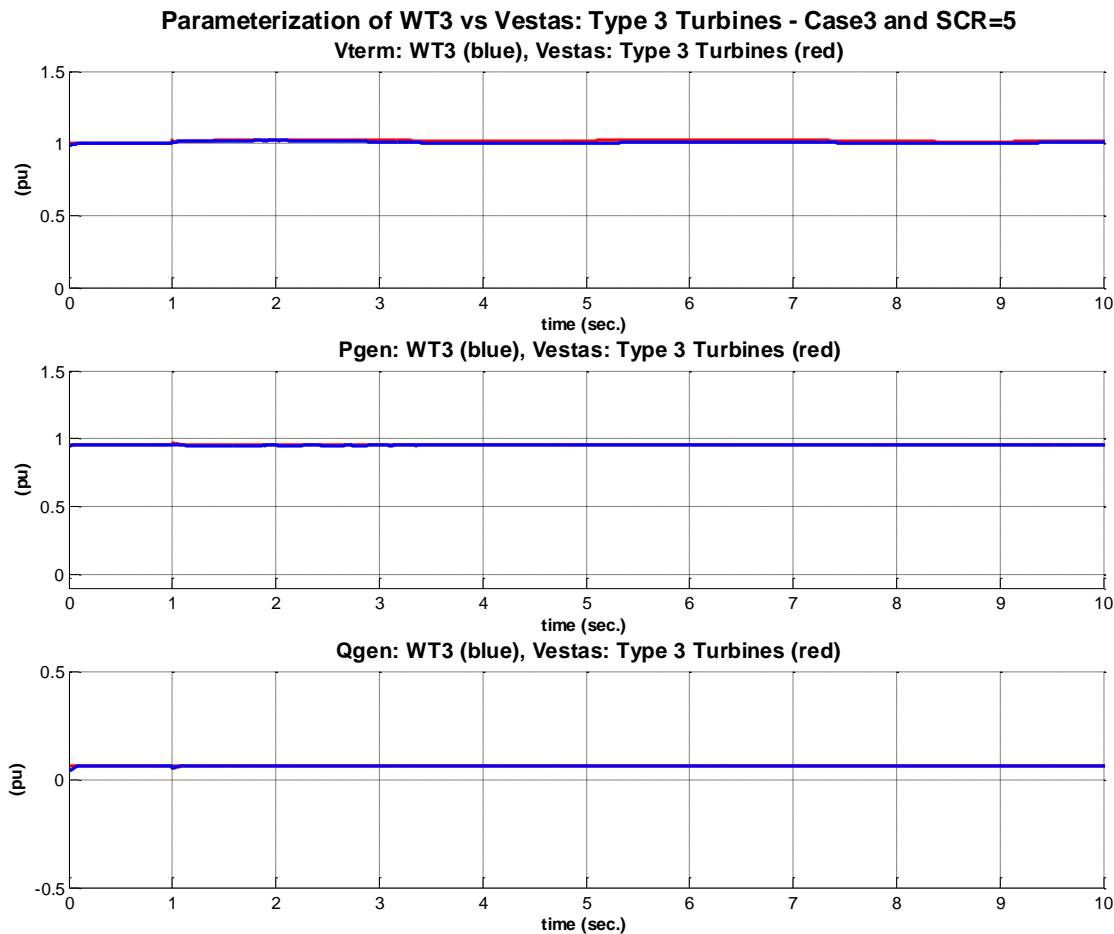

Figure 7-169: Parameterization of WT3 vs Vestas: Type 3 turbine - Case 3 and SCR=5 $\left(\mathrm{V}_{\text {Term }}, \mathrm{P}, \mathrm{Q}\right)$.
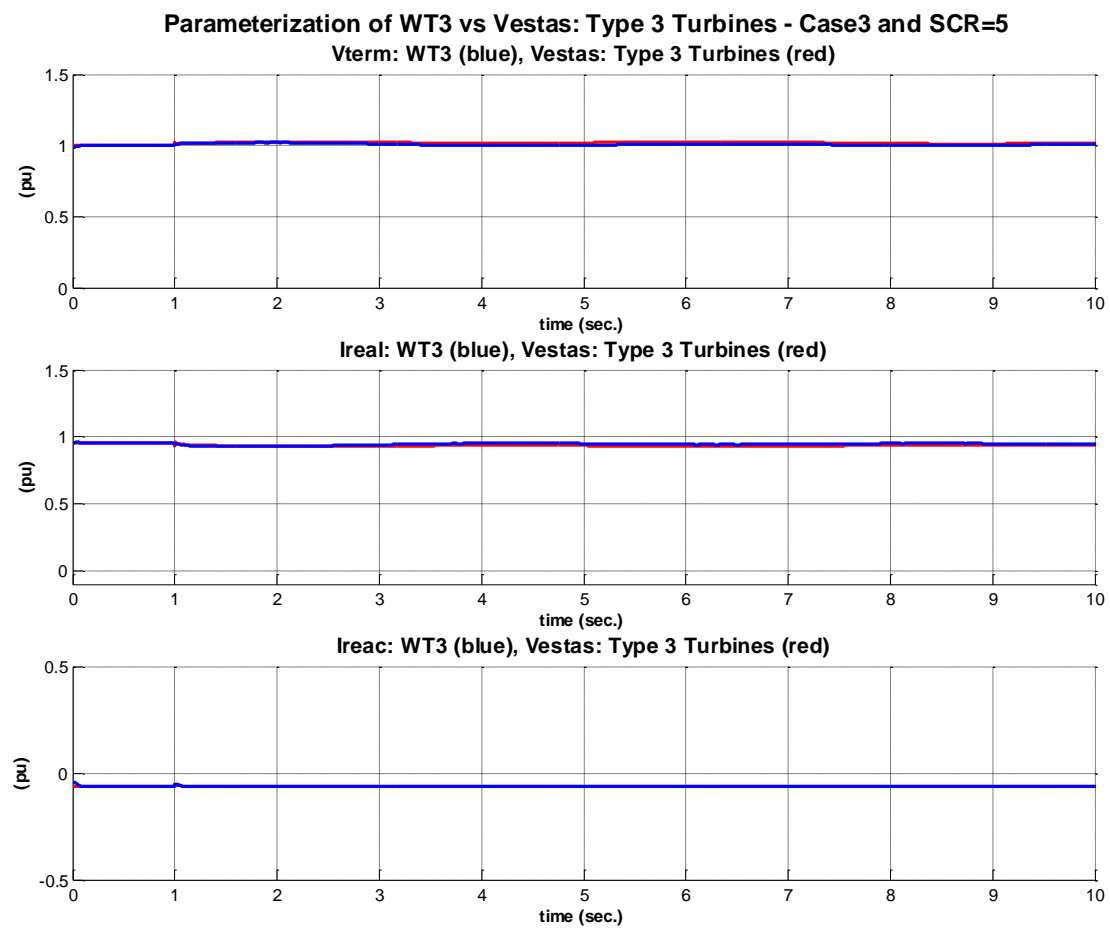

Figure 7-170: Parameterization of WT3 vs Vestas: Type 3 turbine - Case 3 and SCR=5 $\left(\mathrm{V}_{\text {Term }}, \mathrm{I}_{\text {Real }}, \mathrm{I}_{\text {Reac }}\right)$. 
$\underline{\text { SCR }-10}$

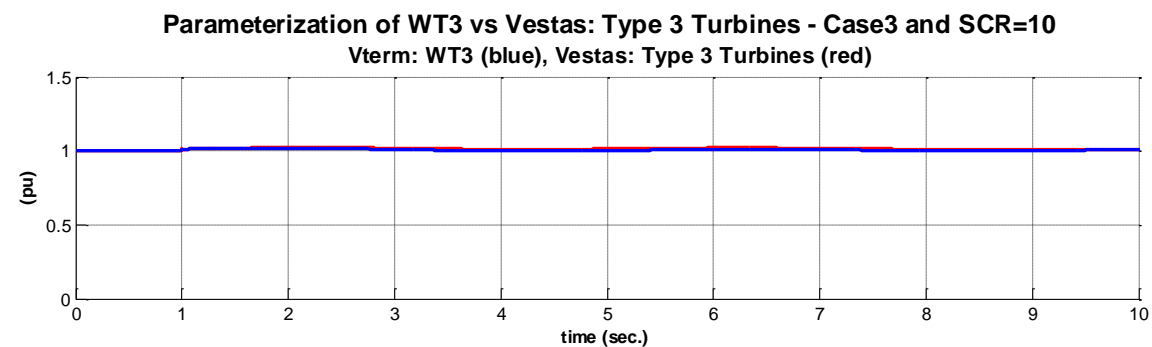

Pgen: WT3 (blue), Vestas: Type 3 Turbines (red)

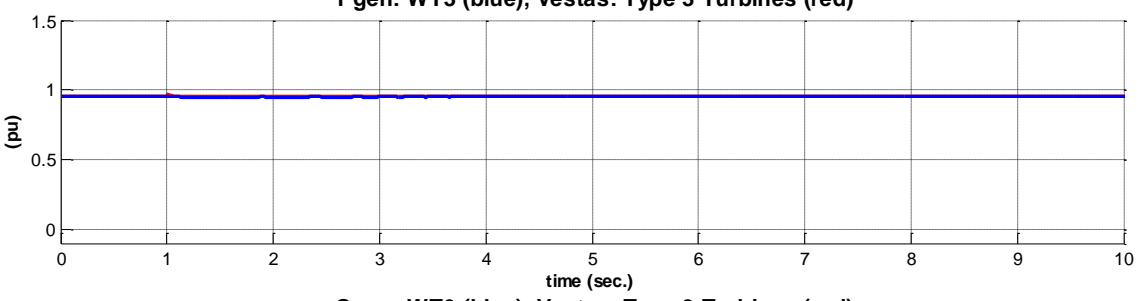

Qgen: WT3 (blue), Vestas: Type 3 Turbines (red)

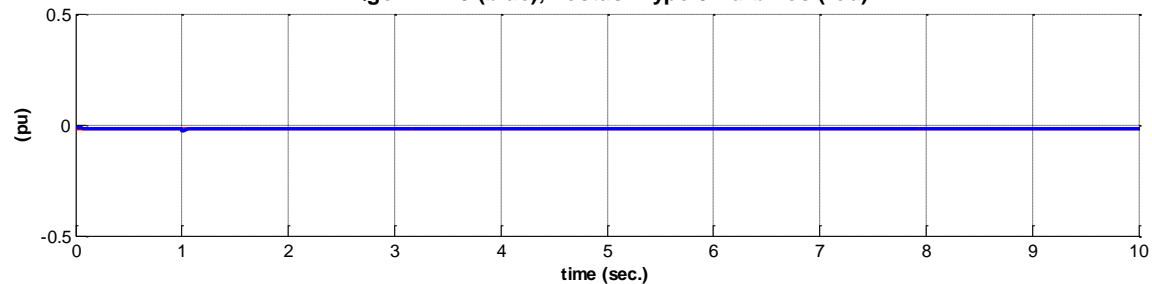

Figure 7-171: Parameterization of WT3 vs Vestas: Type 3 turbine - Case 3 and SCR=10 $\left(\mathrm{V}_{\text {Term }}, \mathrm{P}, \mathrm{Q}\right)$.

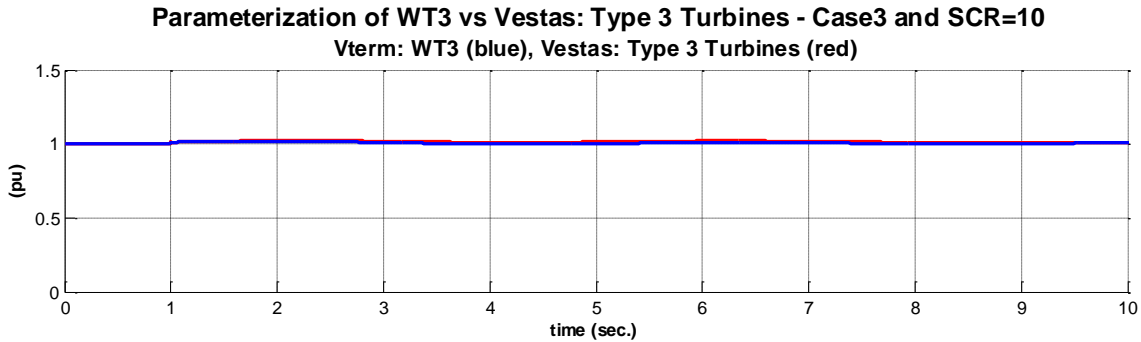

Ireal: WT3 (blue), Vestas: Type 3 Turbines (red)

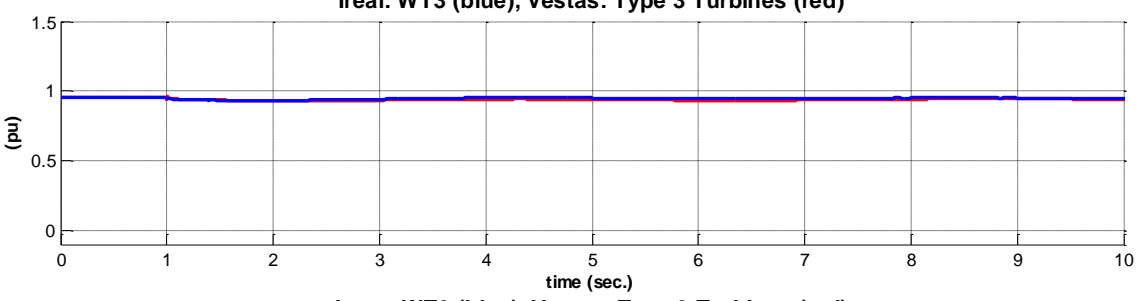

Ireac: WT3 (blue), Vestas: Type 3 Turbines (red)

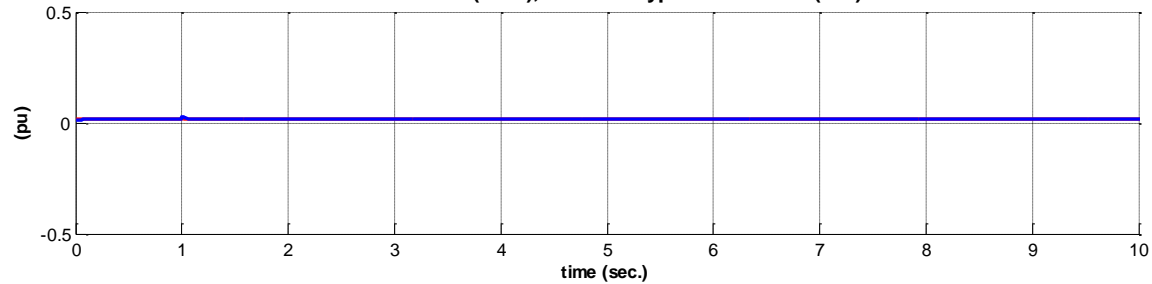

Figure 7-172: Parameterization of WT3 vs Vestas: Type 3 turbine - Case 3 and SCR=10 $\left(\mathrm{V}_{\text {Term }}, \mathrm{I}_{\text {Real }}, \mathrm{I}_{\text {Reac }}\right)$. 


\subsubsection{Results Validation}

For cases 2 and 3 (over frequency and under frequency events), the response of the generic model matches the response of the VSM in the frequency range of interest. However, for case 1 (fault event), the response of the generic model matches the response of the VSM poorly. In other words, while the generic models are a good representation of the Vestas: Type 3 turbine VSM behavior during frequency events, they are rather a poor alternative for severe fault events.

\subsubsection{Vendor Specific Model Validation ${ }^{1}$}

The dynamic Low Voltage Right Through (LVRT) performance during different residual voltage levels and dip durations is documented in this section. Specifically, in Figure 7-173 through Figure 7-178 detailed PSCAD model behavior is compared against the vendor specific PSS/e implementation of type 3 turbines.

\footnotetext{
${ }^{1}$ All figures in this Section are courtesy of Vestas
} 


\section{LVRT Performance During a 0.3 pu Residual Voltage and a Dip Duration of 2 seconds}
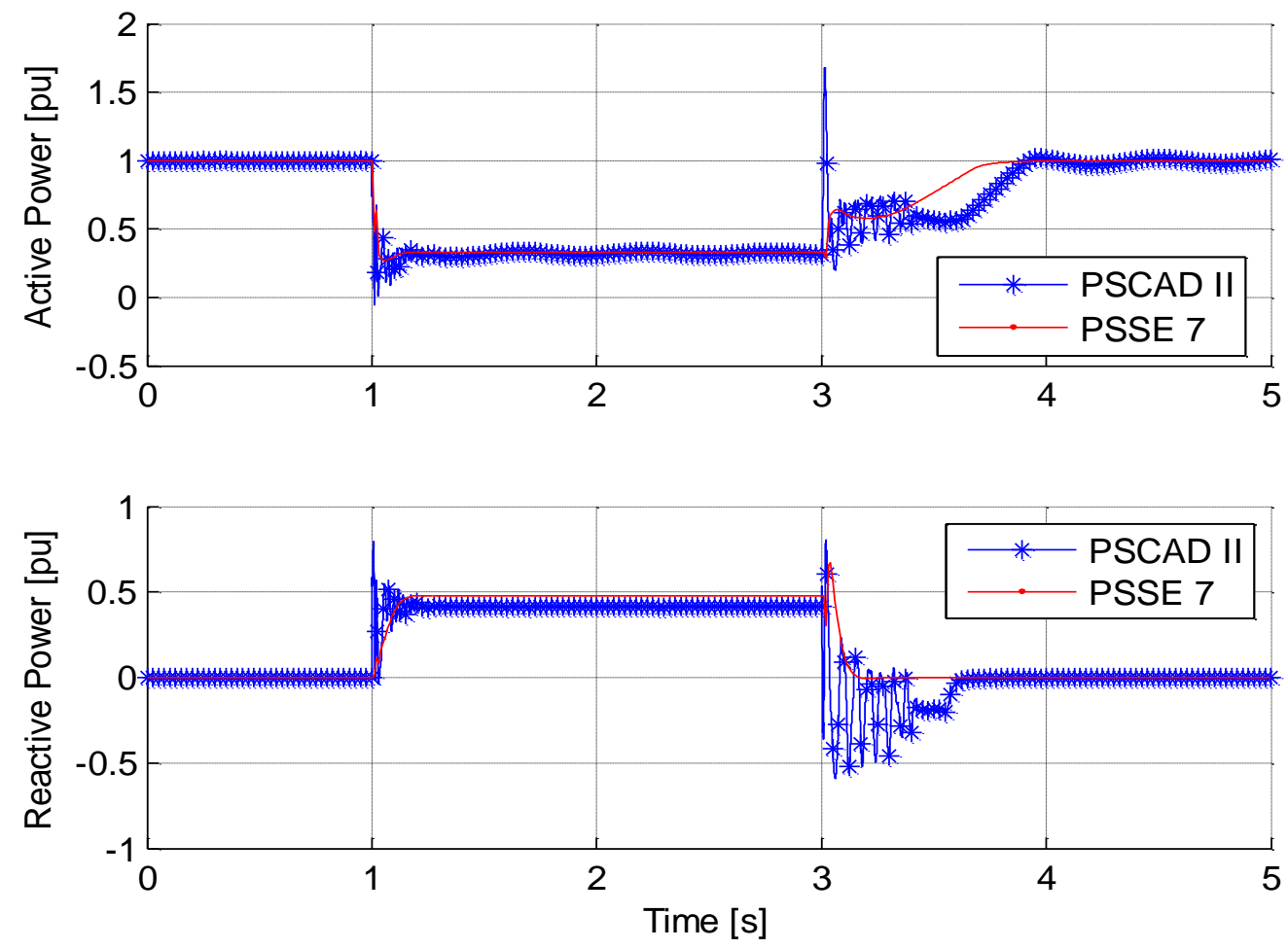

Figure 7-173: Active power and reactive power validation during a 0.3 pu residual voltage and a dip duration of 2 sec.
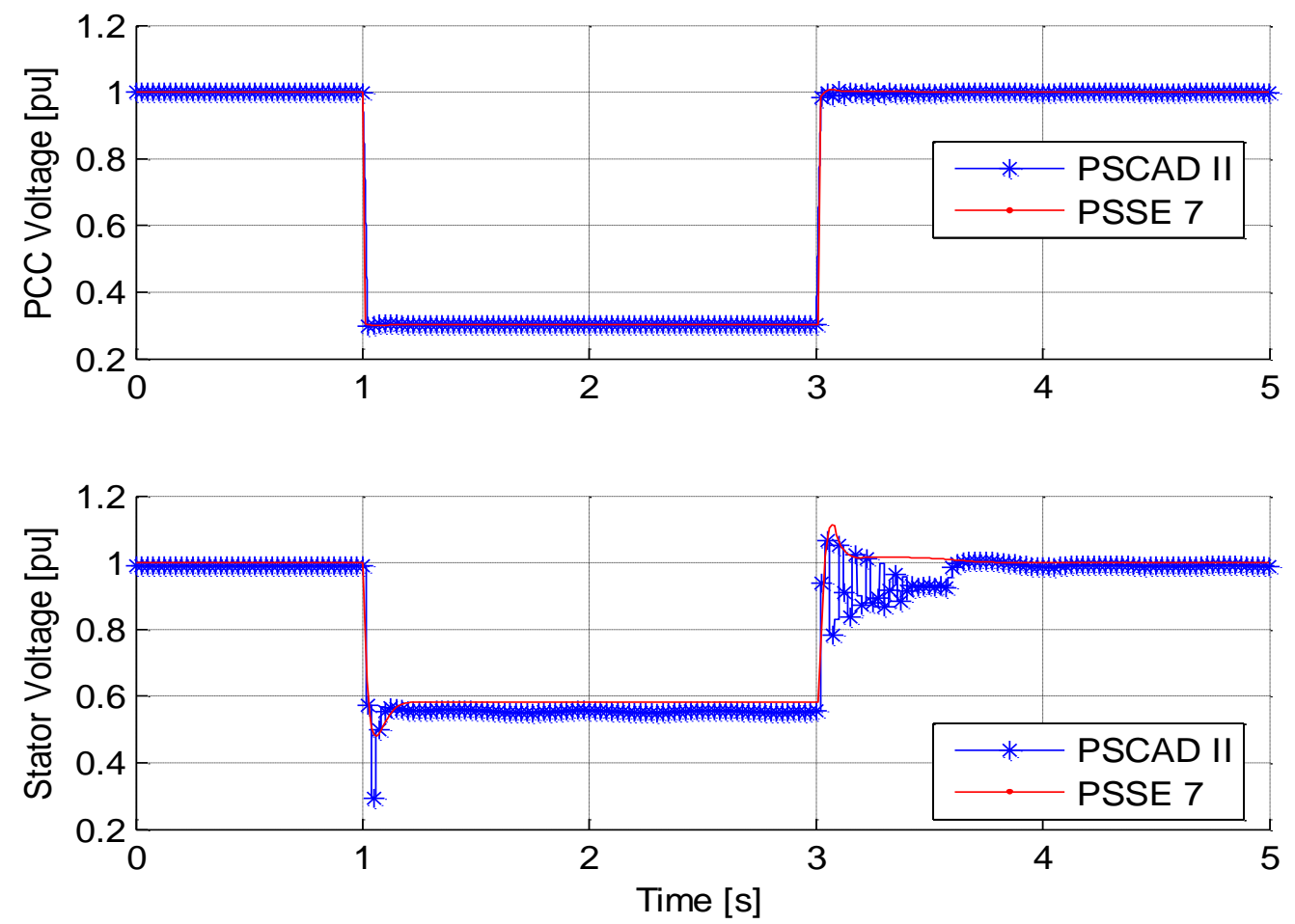

Figure 7-174: Stator voltage and PCC voltage validation during a 0.3 pu residual voltage and a dip duration of 2 sec. 
LVRT Performance During a 0.3 pu Residual Voltage and a Dip Duration of 0.6 seconds
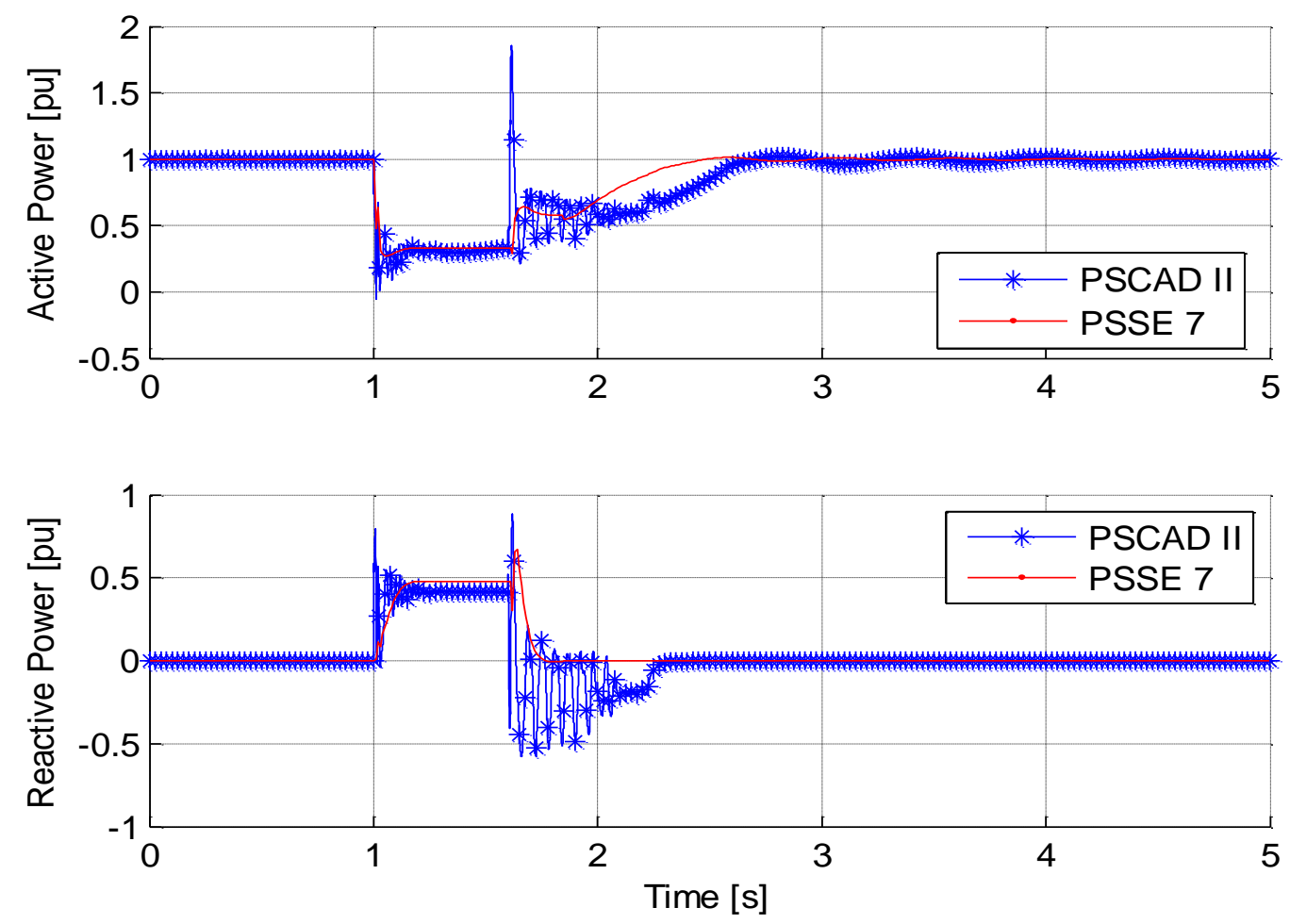

Figure 7-175: Active power and reactive power validation during a 0.3 pu residual voltage and a dip duration of $0.6 \mathrm{sec}$.
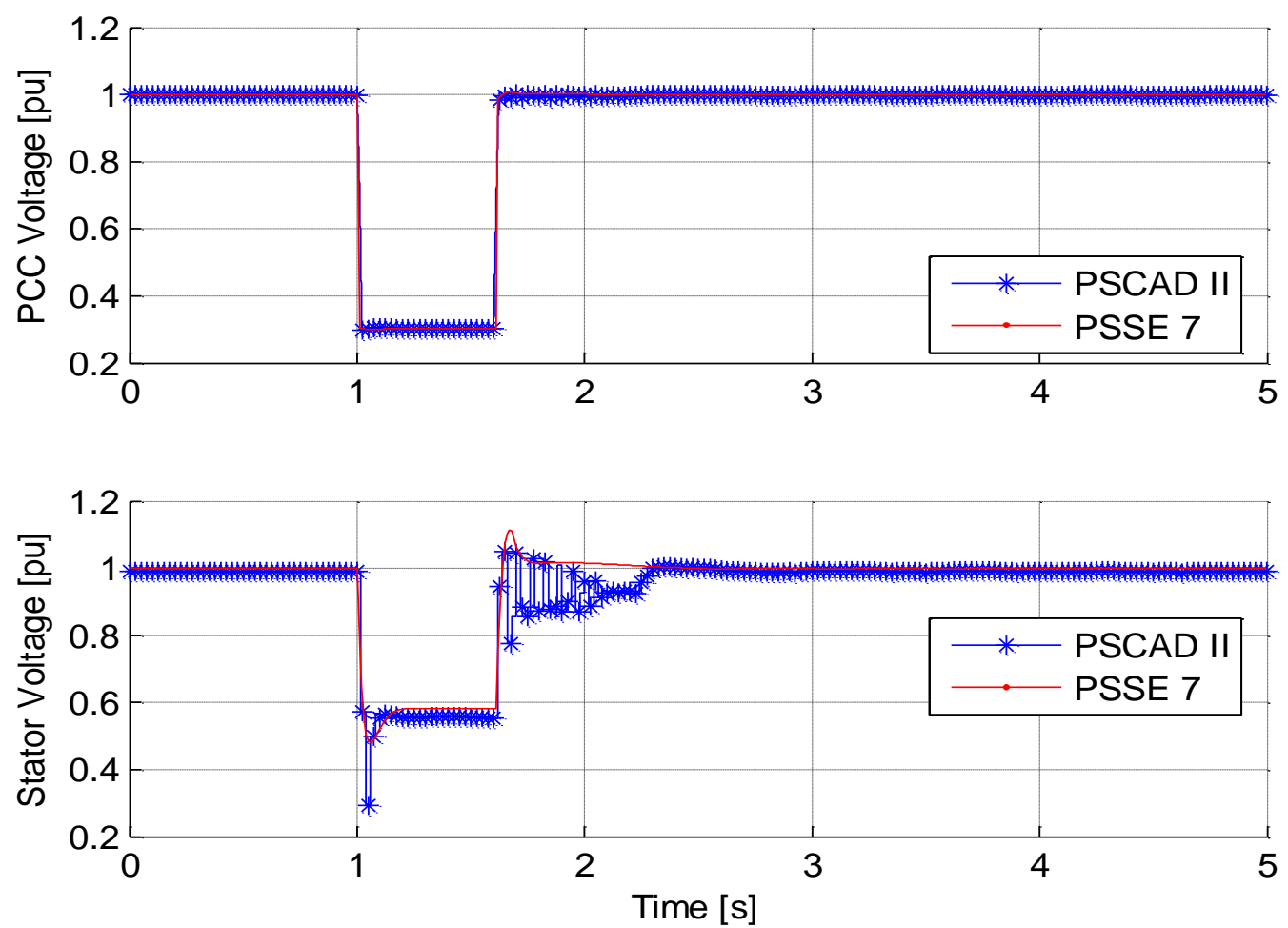

Figure 7-176: PCC voltage and stator voltage validation during a 0.3 pu residual voltage and a dip duration of $0.6 \mathrm{sec}$. 
LVRT Performance During a 0 pu Residual Voltage and a Dip Duration of 0.3 seconds
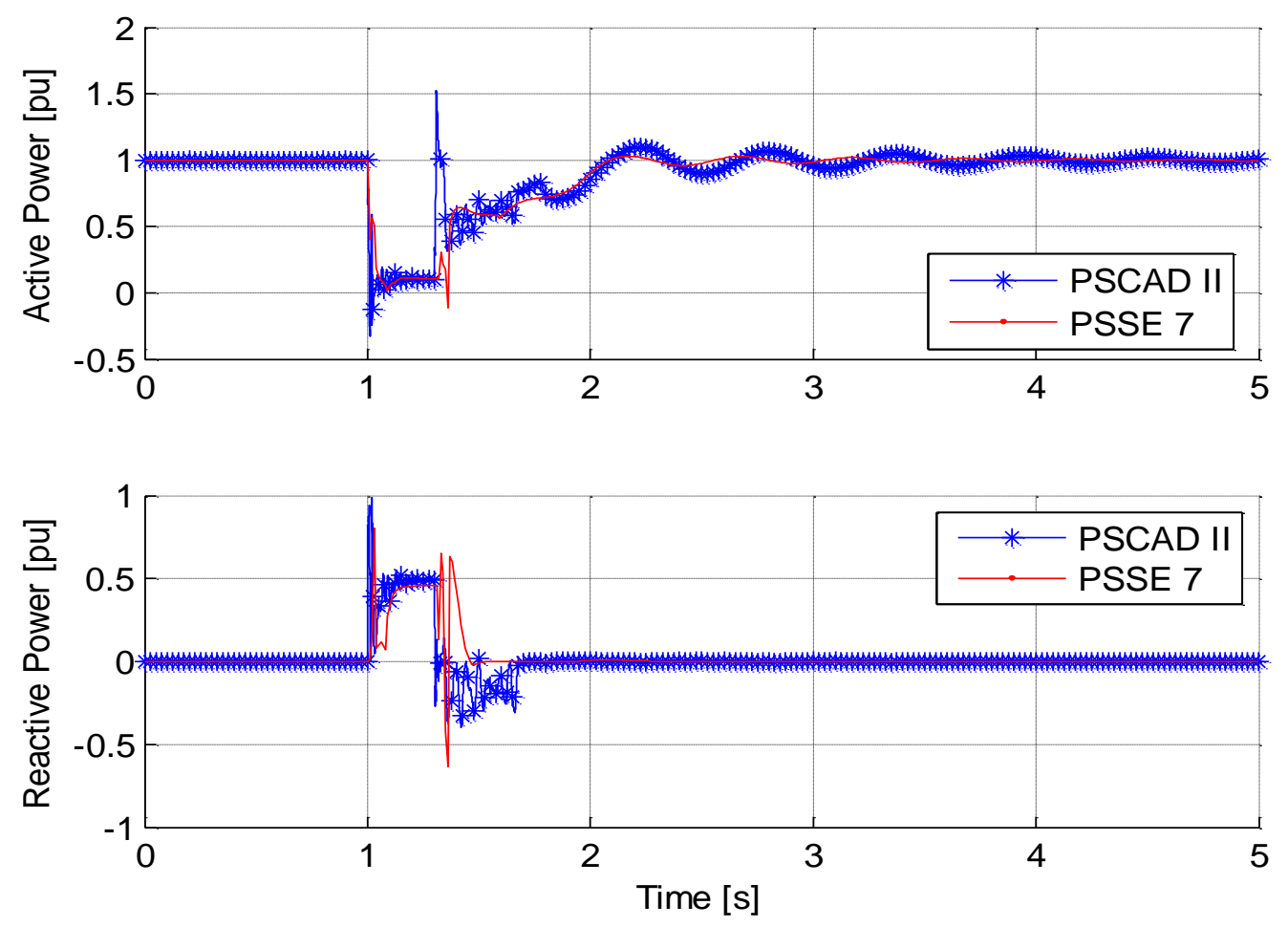

Figure 7-177: Active power and reactive power validation during a 0 pu residual voltage and a dip duration of $0.3 \mathrm{sec}$.
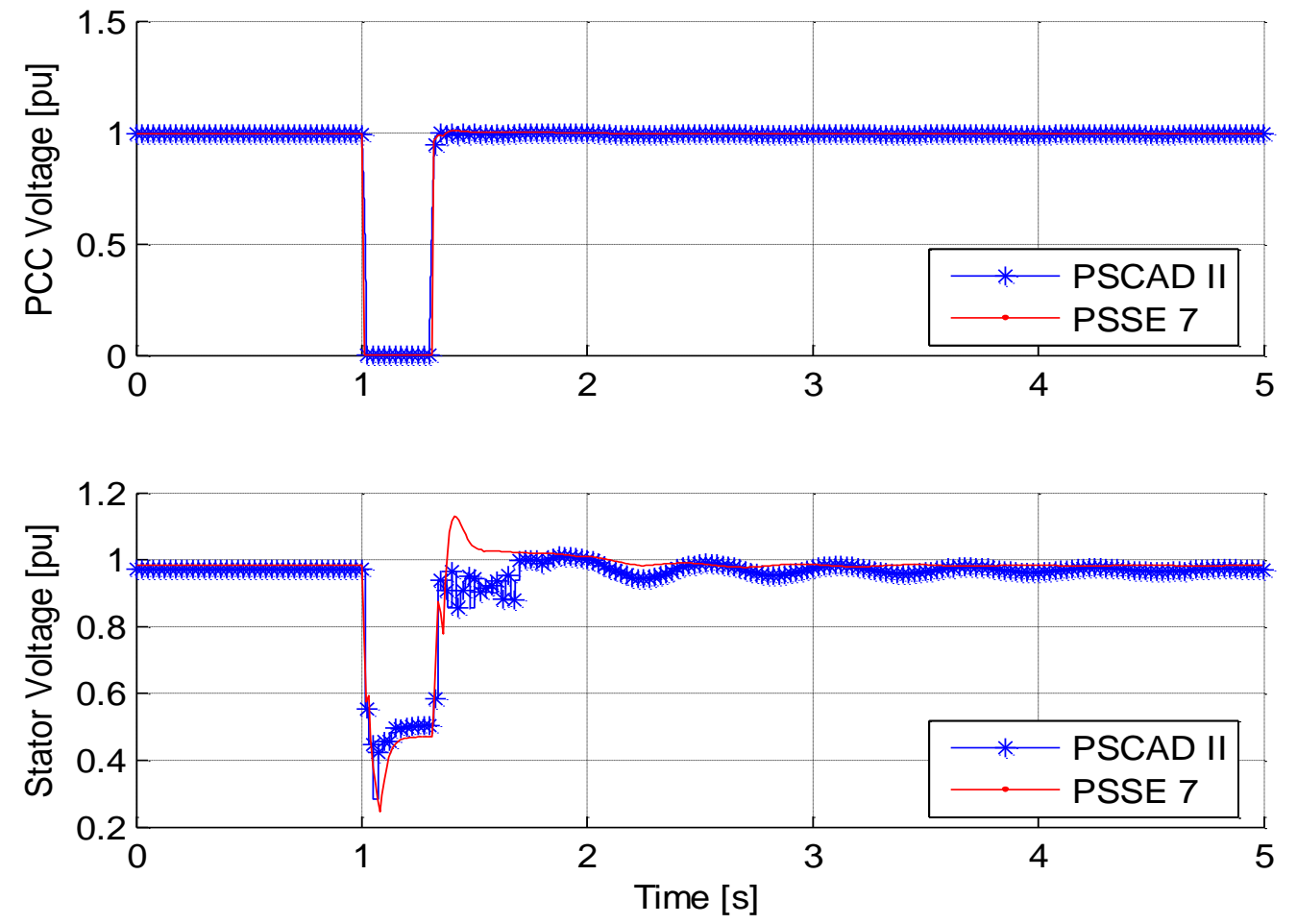

Figure 7-178: PCC voltage and stator voltage validation during a 0 pu residual voltage and a dip duration of $0.3 \mathrm{sec}$. 


\subsection{Vestas - Type IV Models}

7.16.1 Example List of Vestas Type IV Wind Turbines

- $\quad \mathrm{V} 80-2 \mathrm{MW}$ GS

- $\mathrm{V} 90-1.8 / 2 \mathrm{MW}$ GS

- $\quad \mathrm{V} 100-1.8 \mathrm{MW}$ GS

- $\mathrm{V} 112-3 \mathrm{MW}$ GS

\subsubsection{Vendor Specific Model Characterization}

\subsubsection{Model Performance: Case 1 - Fault Event}

In this case a remote fault is applied to bus 12 for a duration of 6 cycles $(0.1 \mathrm{sec})$.

\section{$\underline{\mathrm{SCR}-5}$}
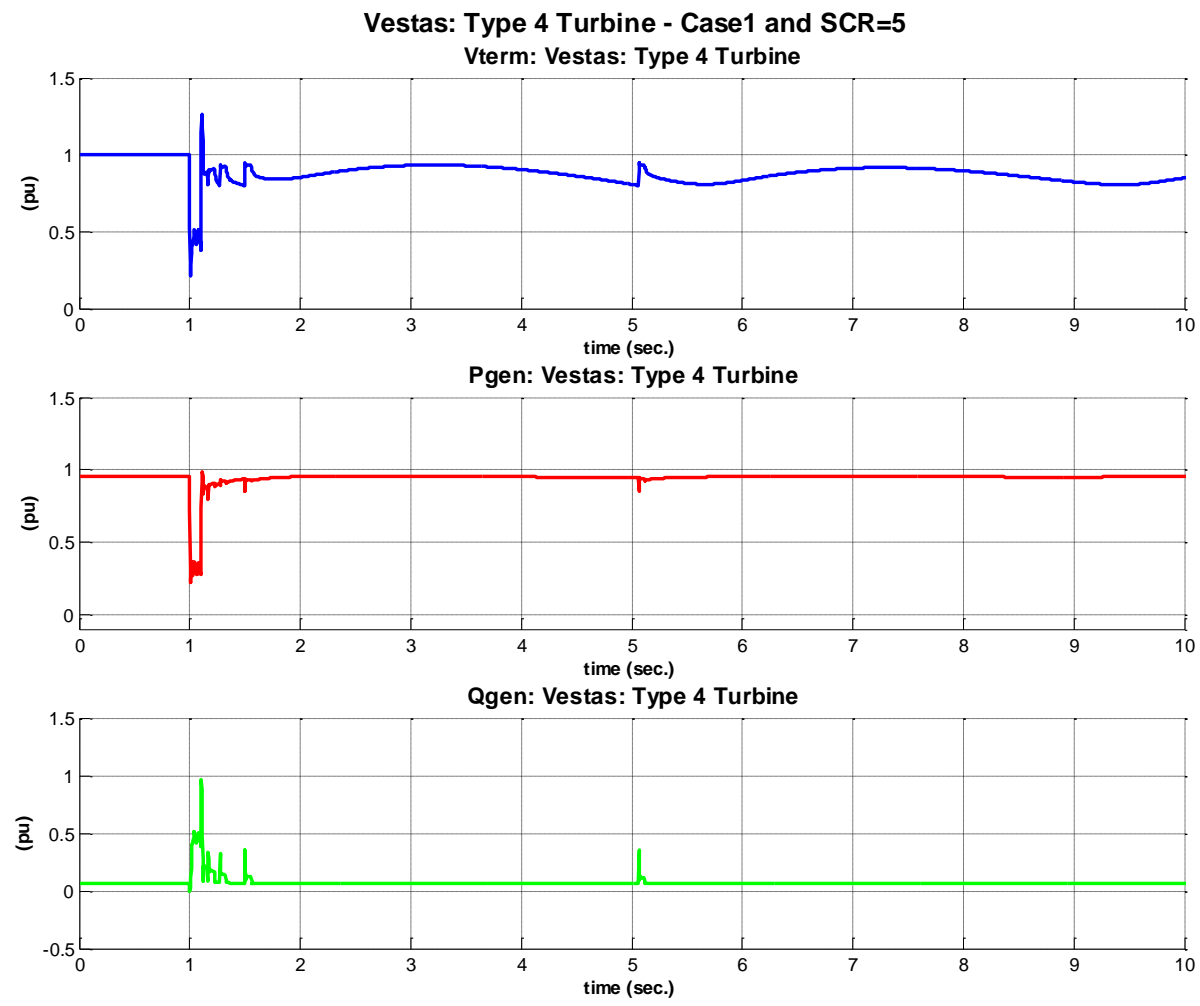

Figure 7-179: Vestas: Type 4 turbine - Case 1 and $\operatorname{SCR}=5\left(V_{\text {Term }}, P, Q\right)$. 

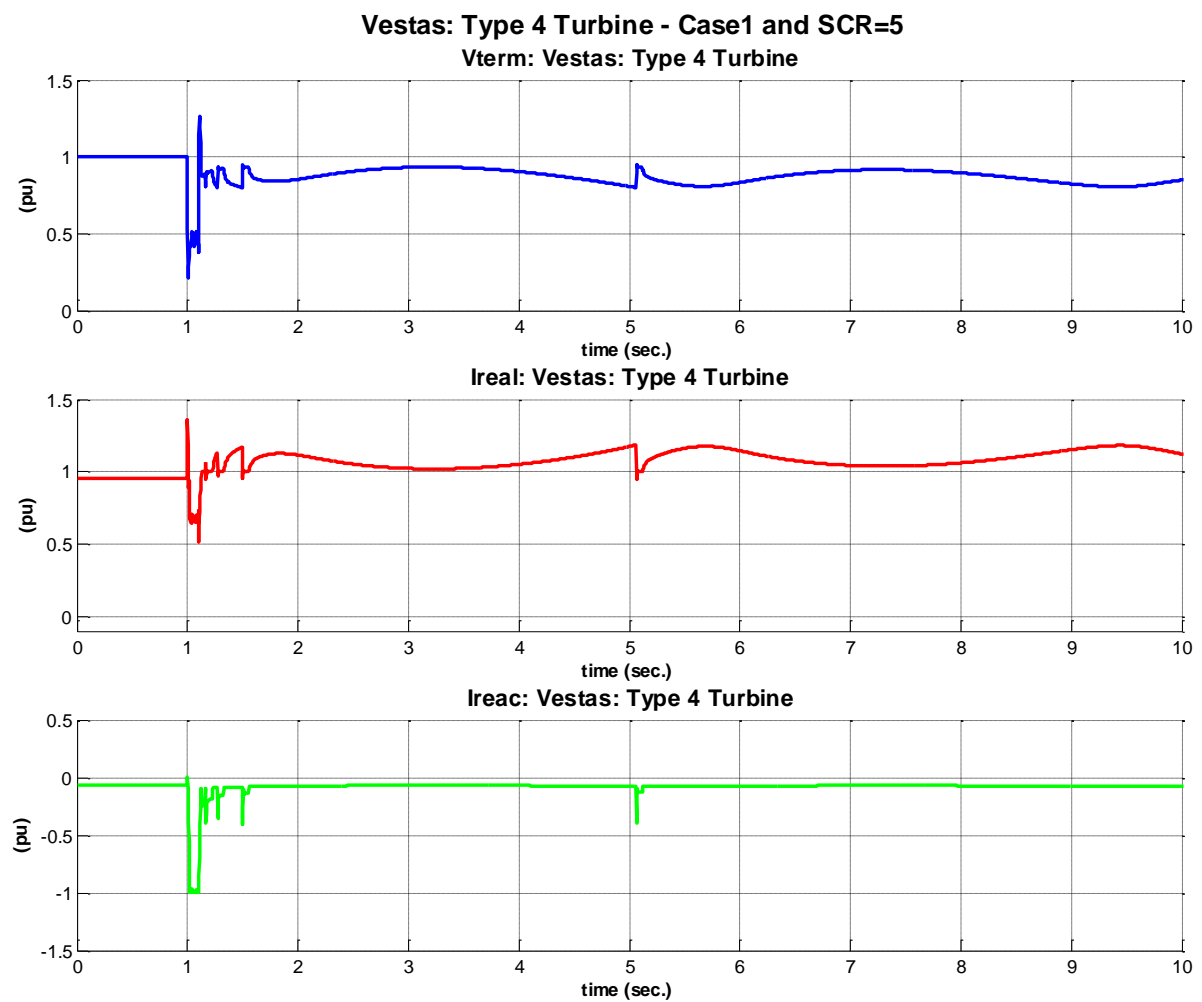

Figure 7-180: Vestas: Type 4 turbine - Case 1 and $S C R=5\left(V_{\text {Term }}, I_{\text {Real }}, I_{\text {Reac }}\right)$.

$\underline{\text { SCR }-10}$
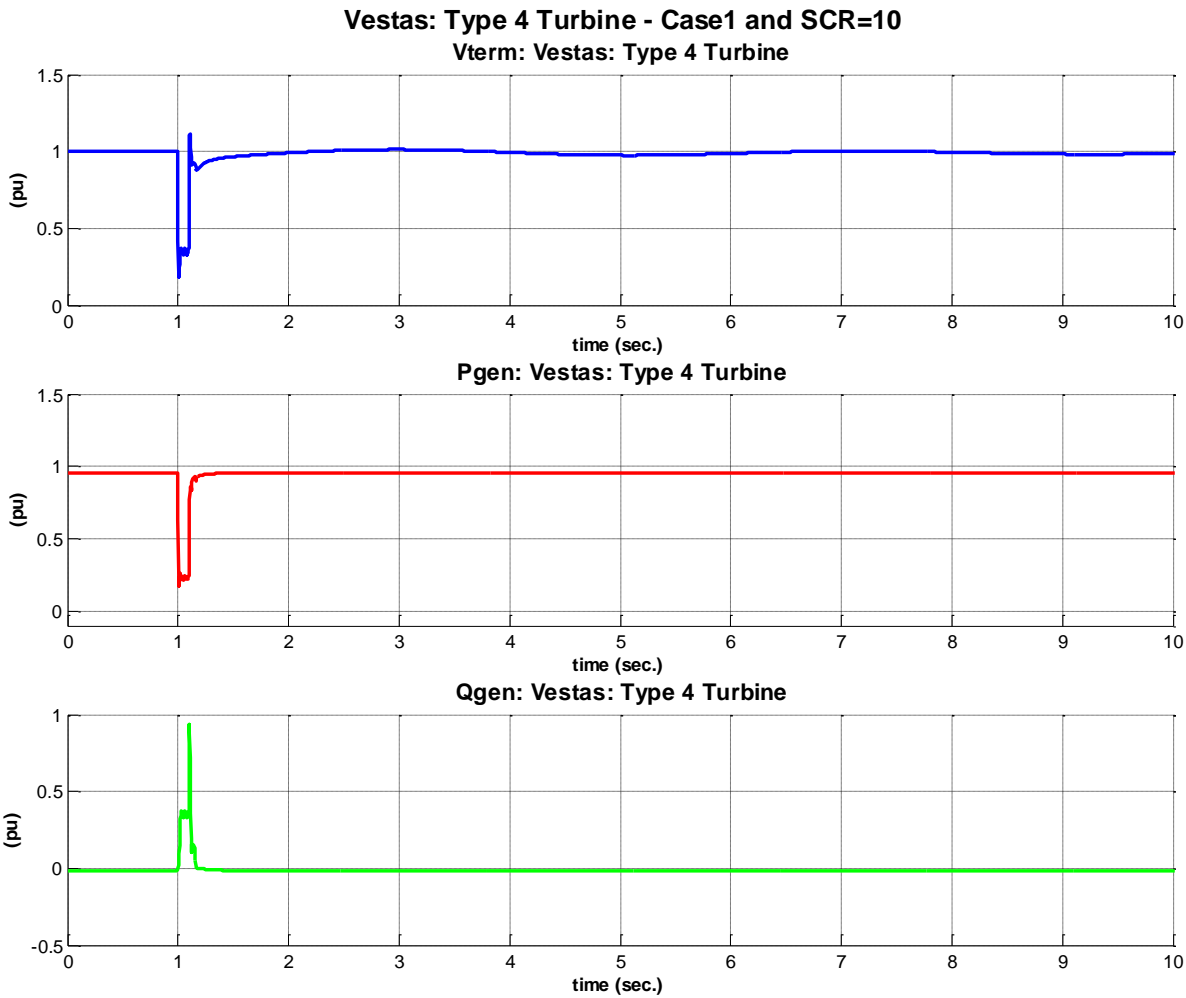

Figure 7-181: Vestas: Type 4 turbine - Case 1 and $S C R=10\left(V_{\text {Term }}, P, Q\right)$. 

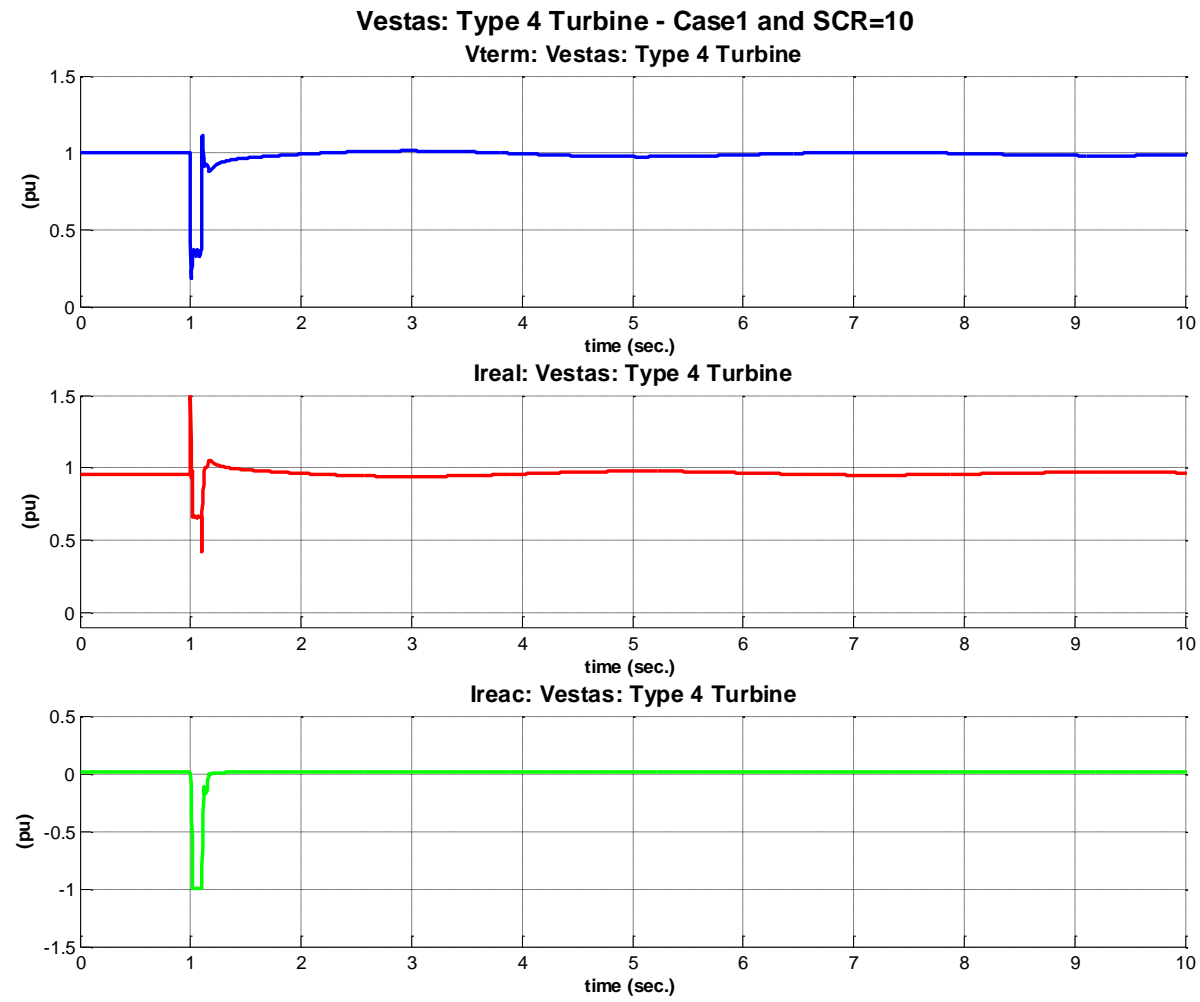

Figure 7-182: Vestas: Type 4 turbine - Case 1 and $S C R=10\left(V_{\text {Term }}, I_{\text {Real }}, I_{\text {Reac }}\right)$.

For the SCR=5 case, after the fault is cleared (tripped line) the reactive power from the turbine and grid is not sufficient to recover the voltage back to the pre-fault value leading to voltage oscillation. At time $=5 \mathrm{sec}$ the voltage drops below a threshold leading to the triggering of the Advanced Grid Option (AGO) - therefore a voltage bump around that time. For the $\mathrm{SCR}=10$ case the grid is stiff enough to provide sufficient reactive power to support the voltage recovery. Thus, a much smother voltage behavior in this case. 


\subsubsection{Model Performance: Case 2 - Under-Frequency Event}

In this case a under frequency event is created by tripping the $100 \mathrm{MW}$ generation unit at bus 20 .

\section{SCR -5}

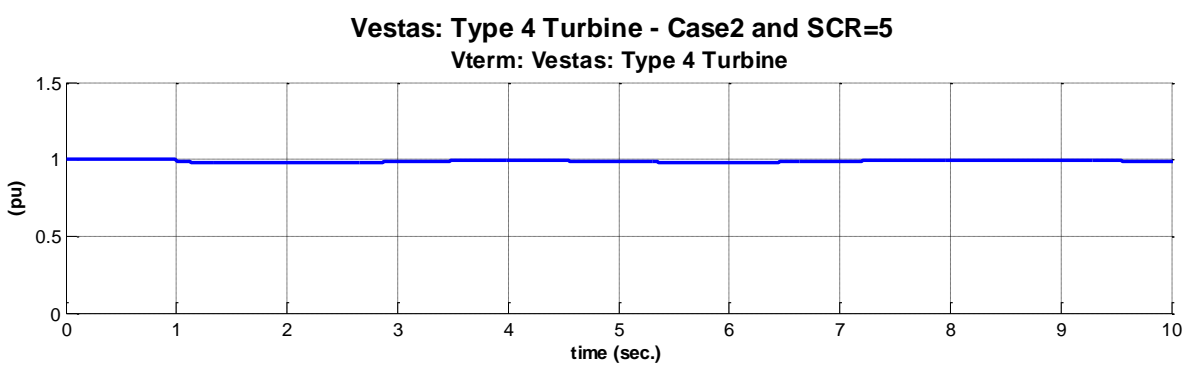

Pgen: Vestas: Type 4 Turbine

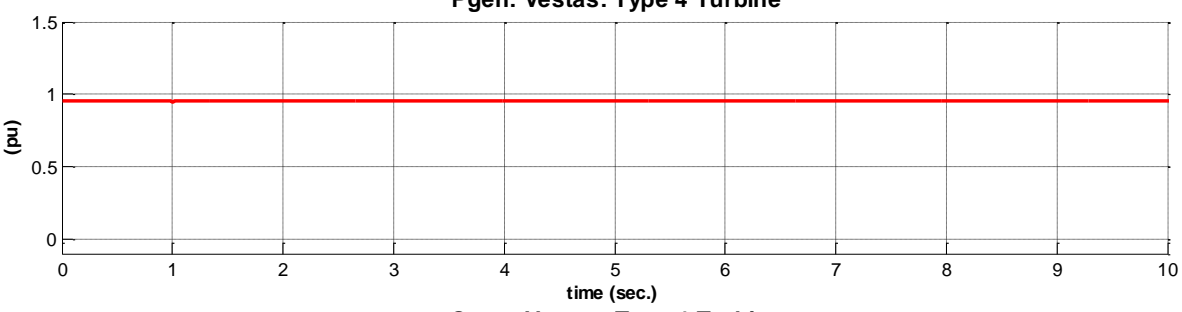

Qgen: Vestas: Type 4 Turbine

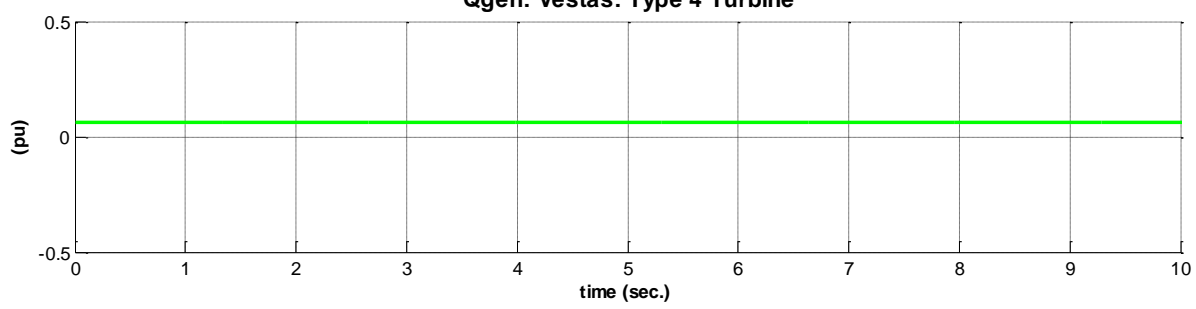

Figure 7-183: Vestas: Type 4 turbine - Case 2 and $S C R=5\left(V_{\text {Term }}, P, Q\right)$. 

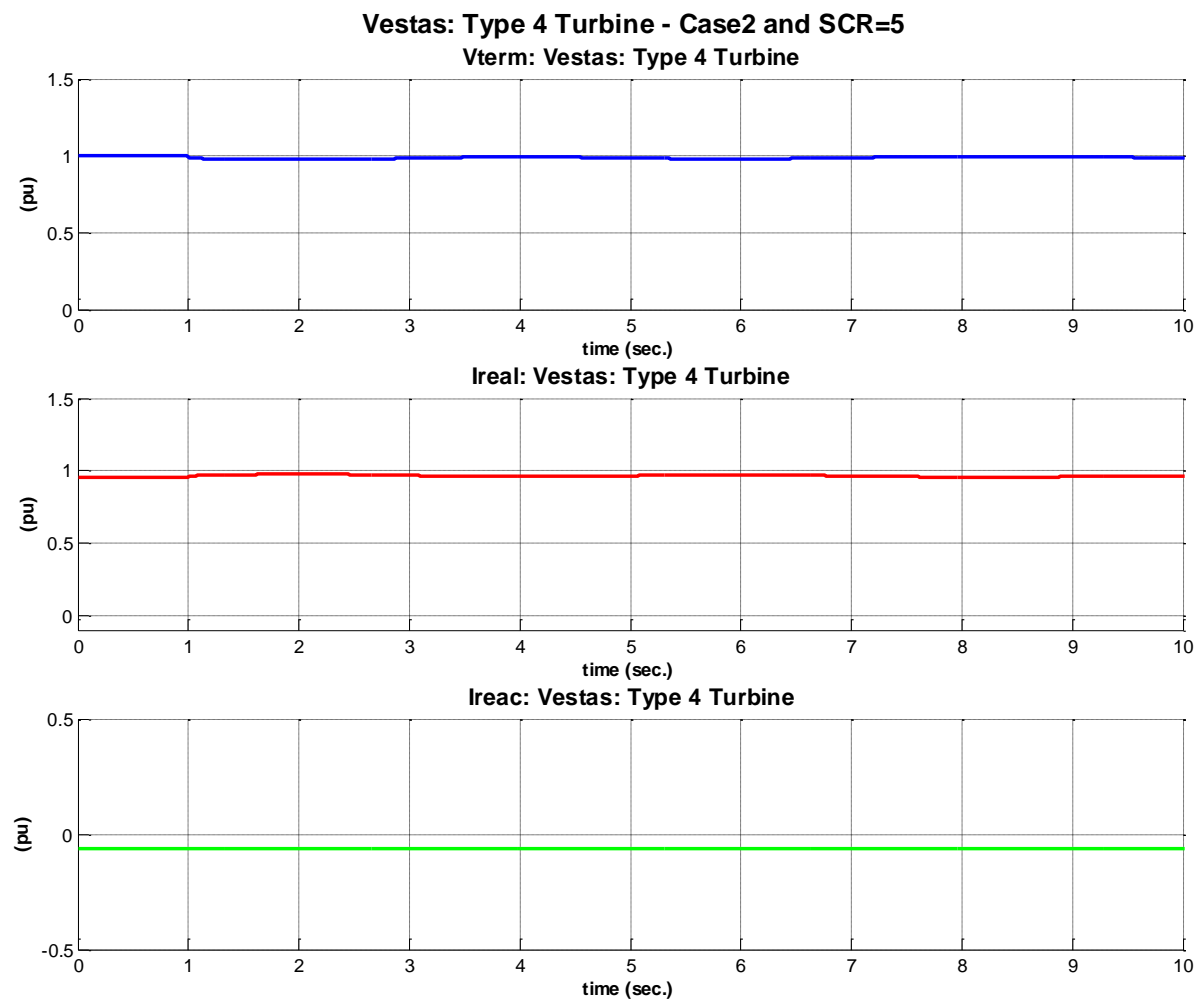

Figure 7-184: Vestas: Type 4 turbine - Case 2 and $S C R=5\left(V_{\text {Term }}, I_{\text {Real }}, I_{\text {Reac }}\right)$.

$\underline{\text { SCR }-10}$
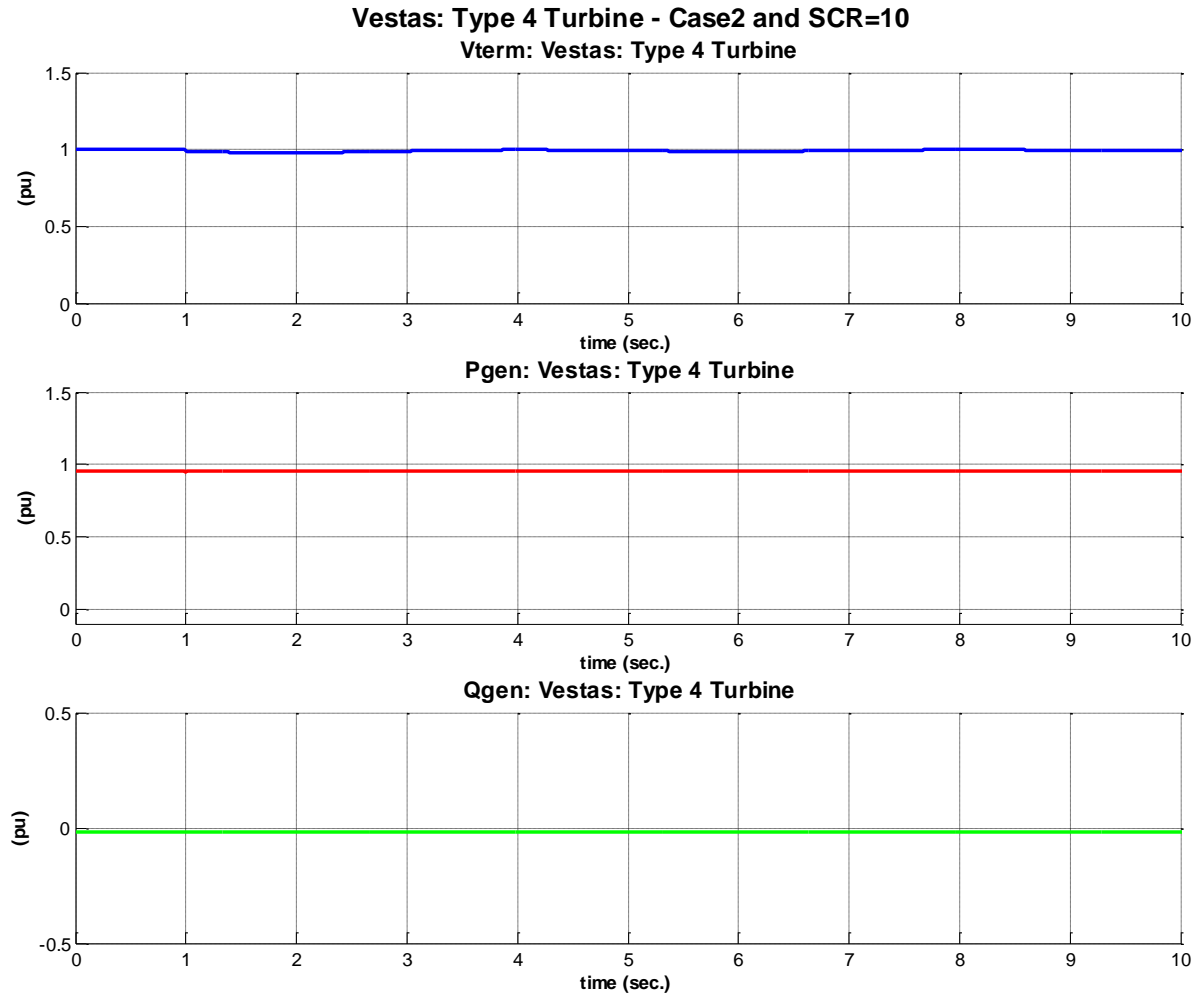

Figure 7-185: Vestas: Type 4 turbine - Case 2 and SCR=10 $\left(V_{\text {Term }}, P, Q\right)$. 

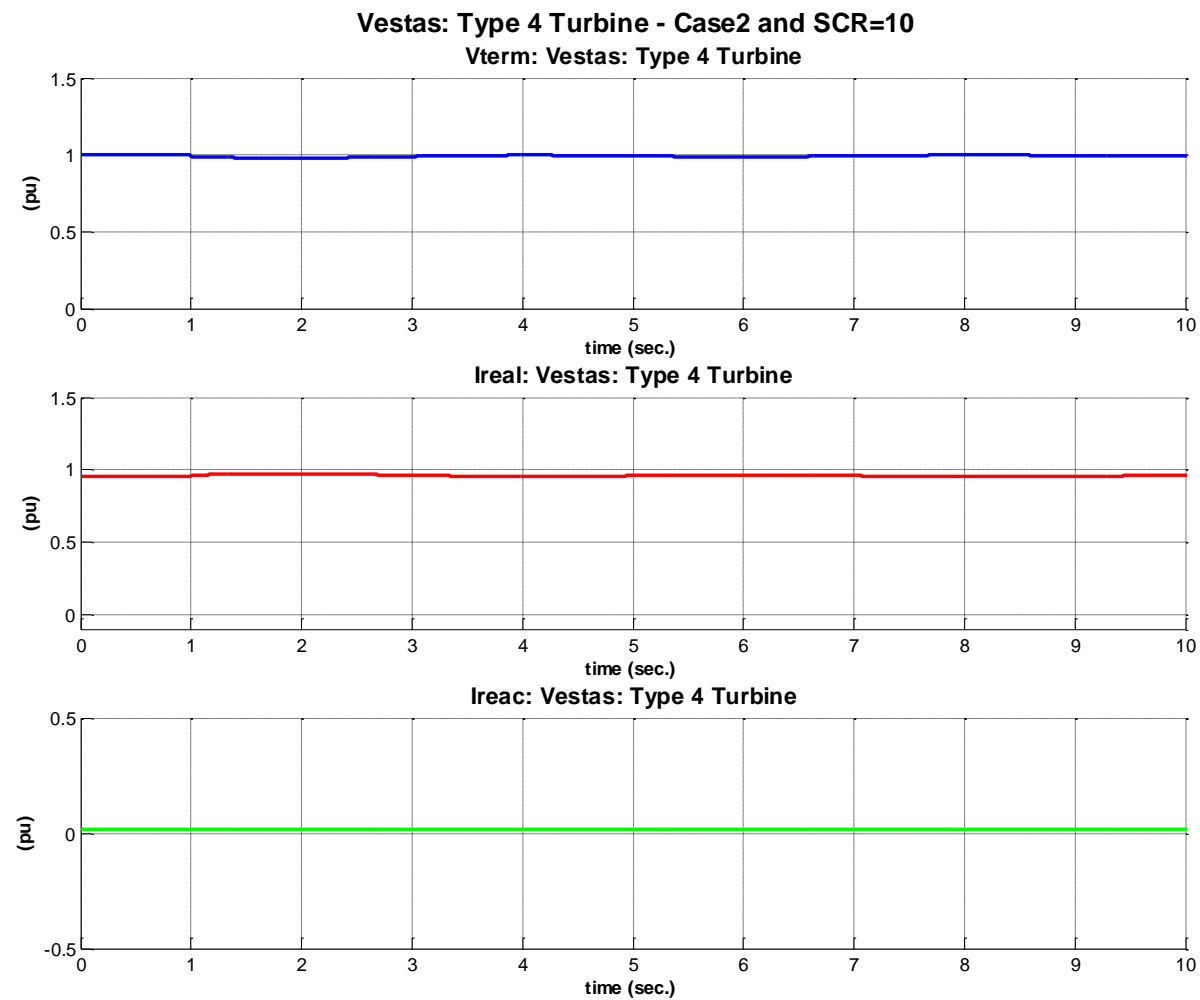

Figure 7-186: Vestas: Type 4 turbine - Case 2 and $S C R=10\left(V_{\text {Term }}, I_{\text {Real }}, I_{\text {Reac }}\right)$. 


\subsubsection{Model Performance: Case 3 - Over-Frequency Event}

In this case a under frequency event is created by tripping the $100 \mathrm{MW}$ load at bus 11.

$\underline{\mathrm{SCR}-5}$
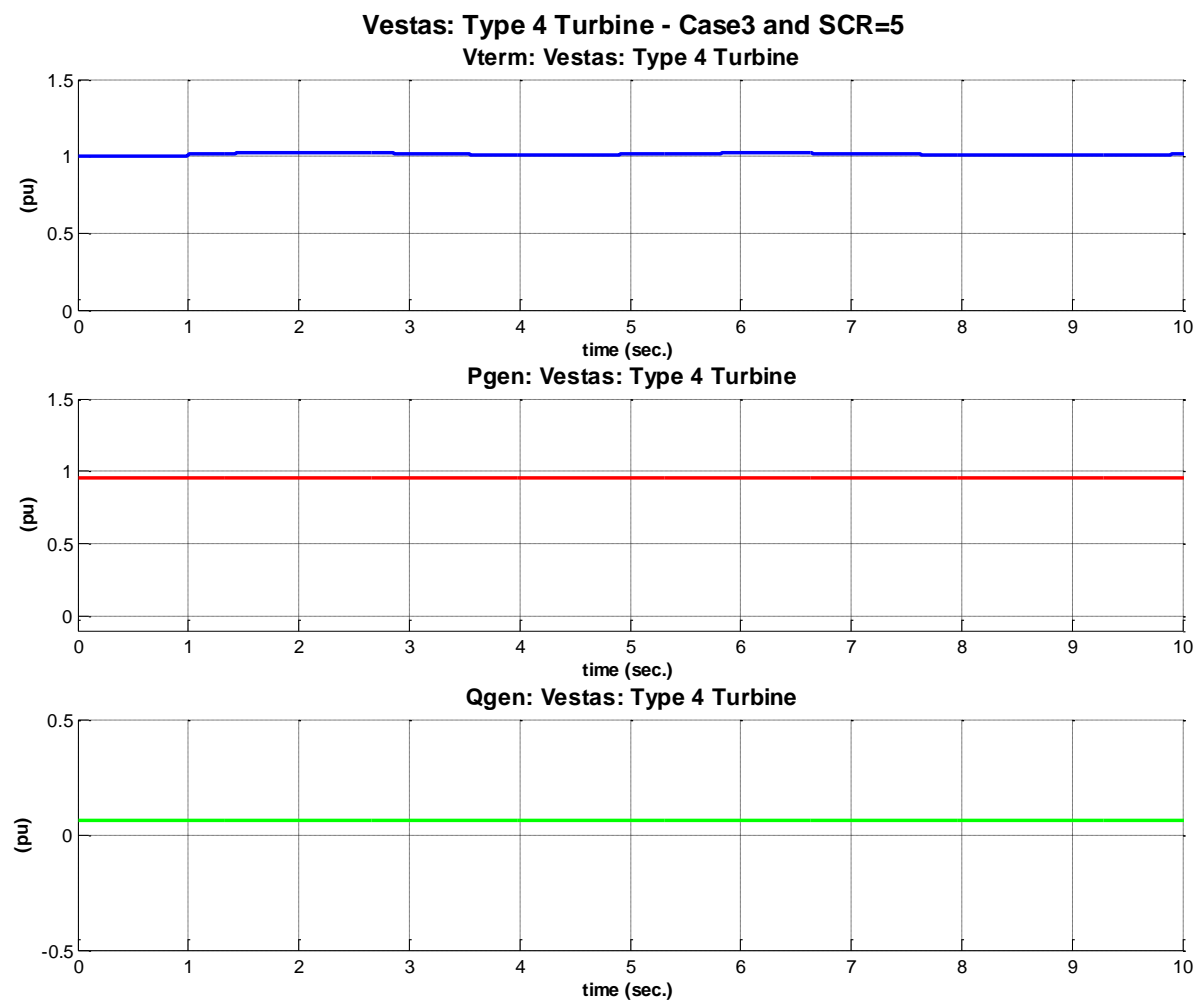

Figure 7-187: Vestas: Type 4 turbine - Case 3 and $S C R=5\left(V_{\text {Term }}, P, Q\right)$. 

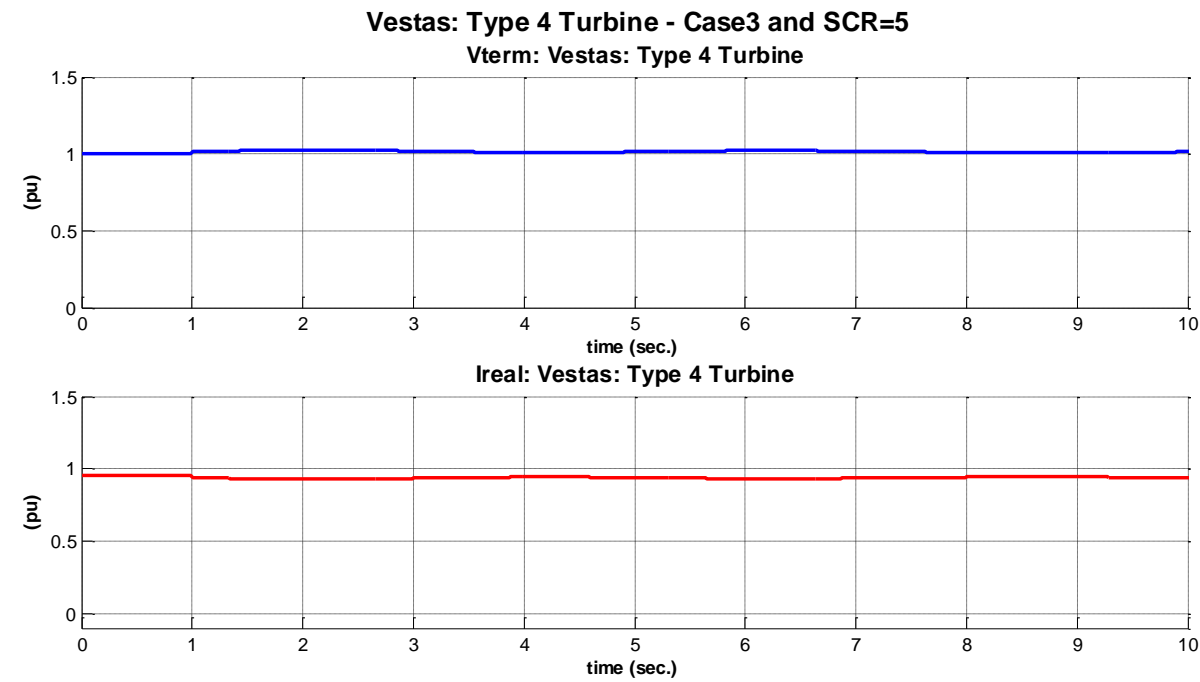

Ireac: Vestas: Type 4 Turbine

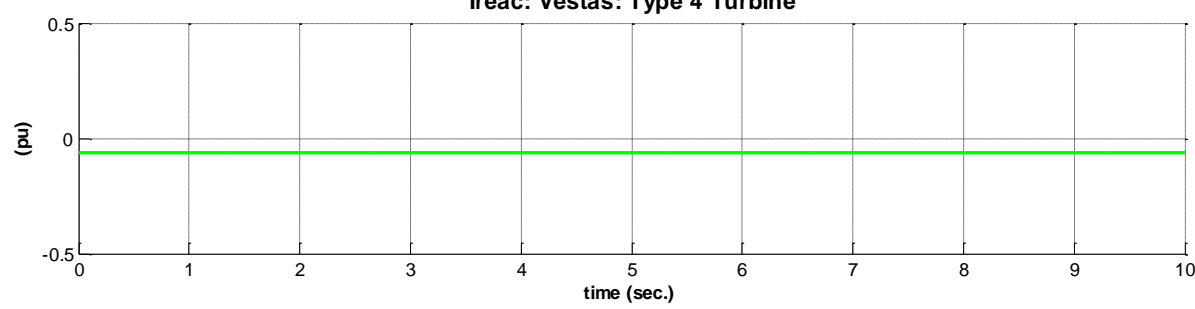

Figure 7-188: Vestas: Type 4 turbine - Case 3 and $S C R=5\left(V_{\text {Term }}, I_{\text {Real }}, I_{\text {Reac }}\right)$.

\section{SCR -10}

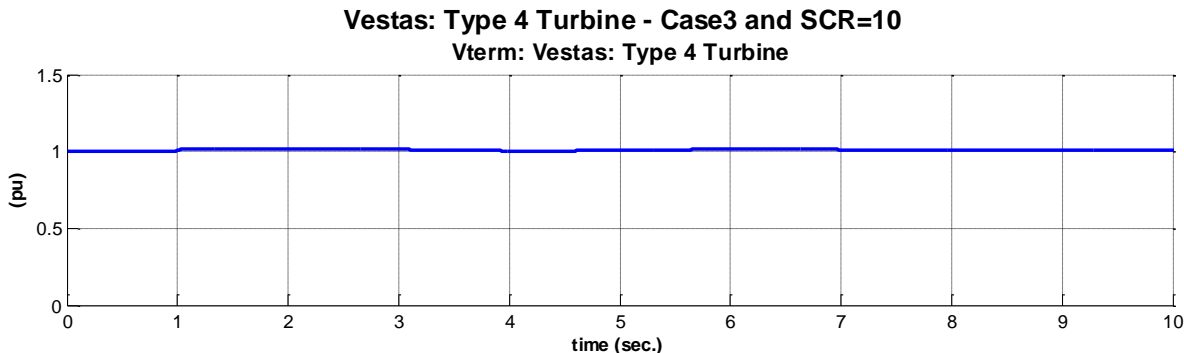

Pgen: Vestas: Type 4 Turbine

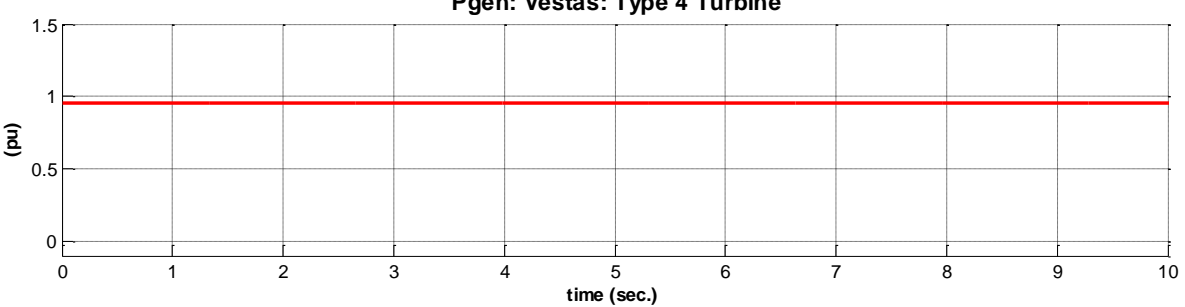

Qgen: Vestas: Type 4 Turbine

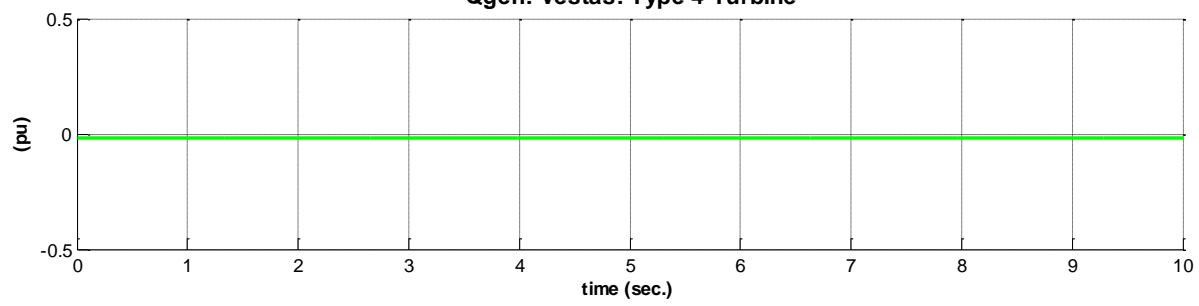

Figure 7-189: Vestas: Type 4 turbine - Case 3 and SCR=10 $\left(V_{\text {Term }}, P, Q\right)$. 

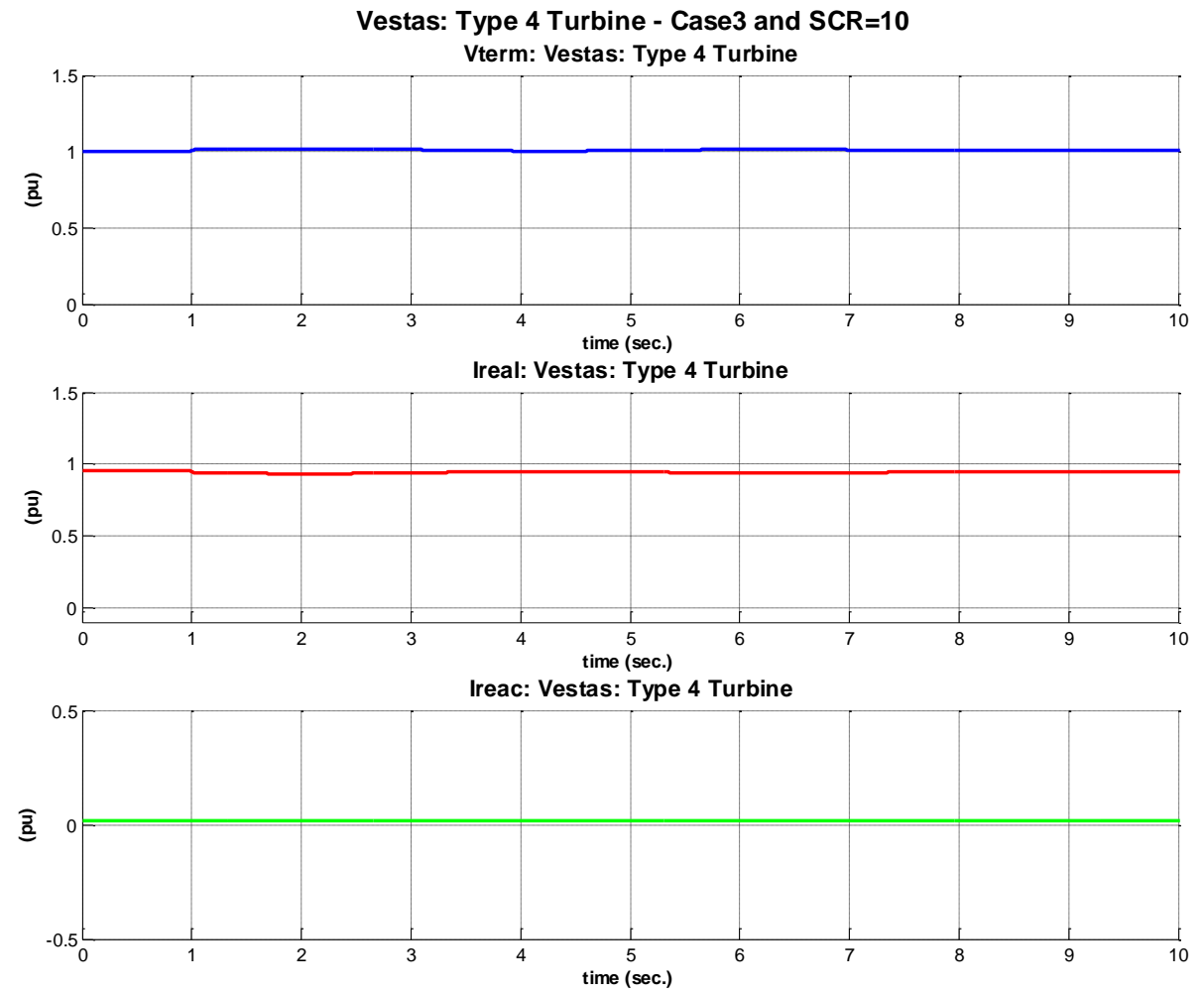

Figure 7-190: Vestas: Type 4 turbine - Case 3 and SCR=10 (VTerm, IReal, IReac). 


\subsubsection{Generic Model Parameterization}

\subsubsection{PSLF Environment}

The PSLF platform generic wind turbine parameters, to mimic the behavior of Vestas' type 4 wind turbines, are shown in Table 7-9. The parameter values shown in the table resulted from a compromise between the simulated cases (i.e. SCR 5 and SCR 10) and network conditions (i.e. fault, under-and over frequency).

Table 7-9: PSLF generic model parameter values.

\begin{tabular}{|c|c|c|c|c|c|}
\hline \multicolumn{2}{|c|}{ wt4g } & \multicolumn{2}{|c|}{ wt4t } & \multicolumn{2}{|c|}{ wt4e } \\
\hline Lvplsw & 1.0 & Tpw & 0.05 & Varflg & 1 \\
\hline Rrpwr & 10.0 & Kpp & 0.08 & Kqi & 0.1 \\
\hline Brkpt & 0.9 & Kip & 0.10 & Kvi & 0.001 \\
\hline Zerox & 0.4 & Tf & 0.08 & Vmax & 1.1 \\
\hline Lvpll & 1.22 & $\mathrm{Kf}$ & 0.00 & Vmin & 0.9 \\
\hline Volim & 1.2 & $\mathrm{dPmx}$ & 0.1 & Qmax & 0.40 \\
\hline Lvpnt1 & 0.8 & $\mathrm{dPmn}$ & -0.1 & Qmin & -0.40 \\
\hline Lvpnt0 & 0.4 & & & $\mathrm{Tr}$ & 0.02 \\
\hline & & & & Tc & 0.15 \\
\hline & & & & Kpv & 18.0 \\
\hline & & & & Kiv & 5.0 \\
\hline & & & & Pfaflg & 0 \\
\hline & & & & $f n$ & 1.0 \\
\hline & & & & $\mathrm{TV}$ & 0.05 \\
\hline & & & & Tpwr & 0.05 \\
\hline & & & & Iphl & 1.24 \\
\hline & & & & Iqhl & 1.25 \\
\hline & & & & Pqflag & 0 \\
\hline
\end{tabular}




\subsection{Case 1 - Fault Event}

\section{SCR - 5}
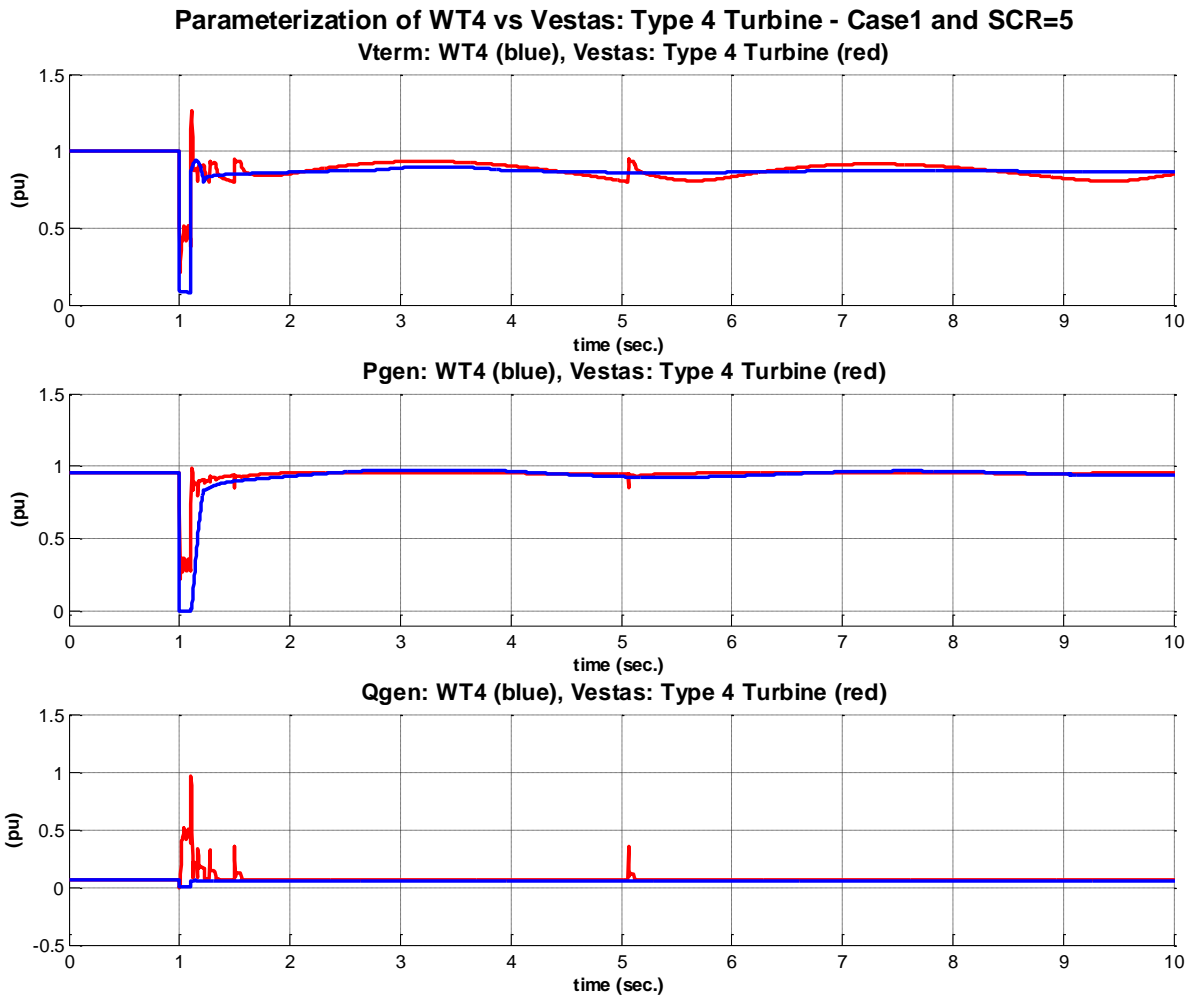

Figure 7-191: Parameterization of WT4 vs Vestas: Type 4 turbine - Case 1 and SCR=5 $\left(\mathrm{V}_{\text {Term }}, \mathrm{P}, \mathrm{Q}\right)$. 

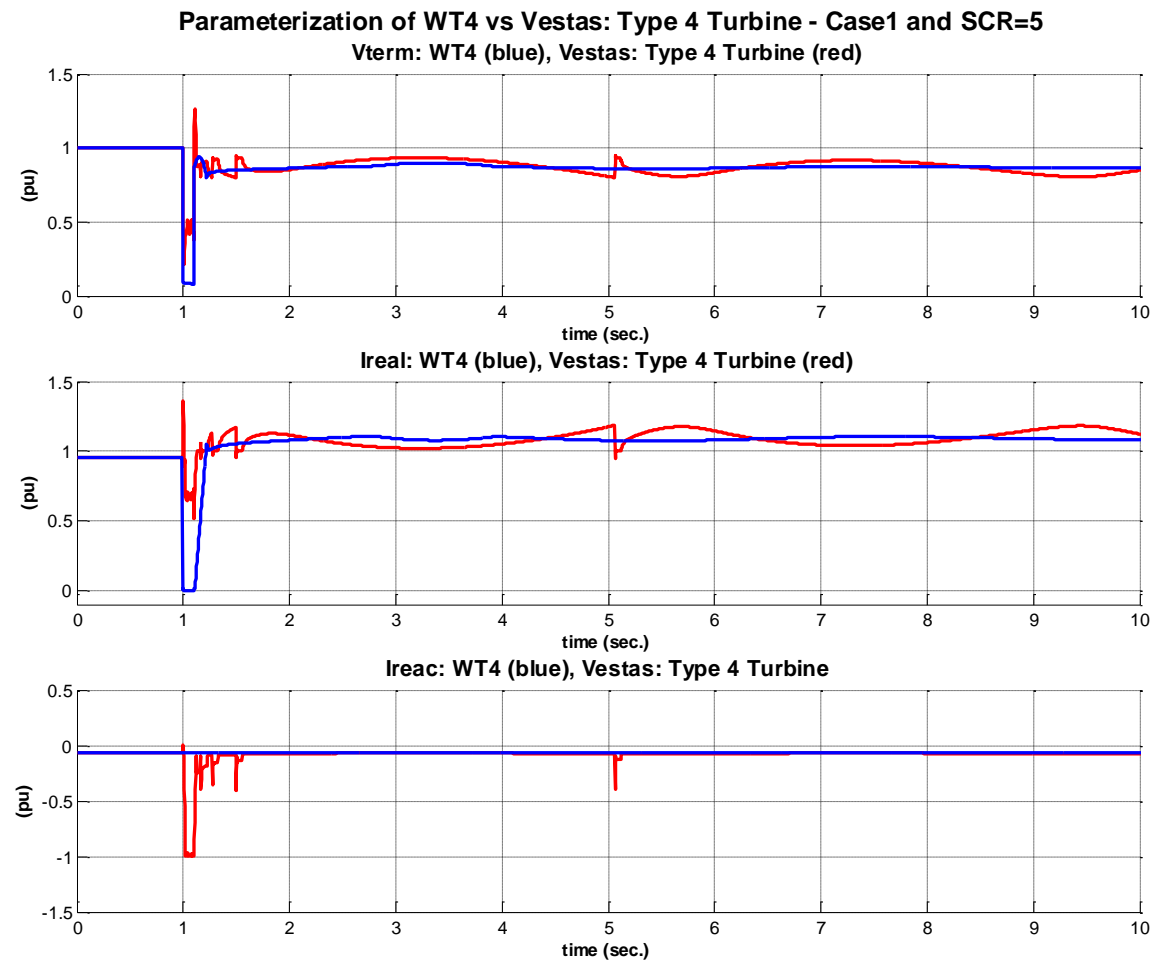

Figure 7-192: Parameterization of WT4 vs Vestas: Type 4 turbine - Case 1 and SCR=5 $\left(V_{\text {Term }}, I_{\text {Real }}, I_{\text {Reac }}\right)$.

\section{SCR -10}
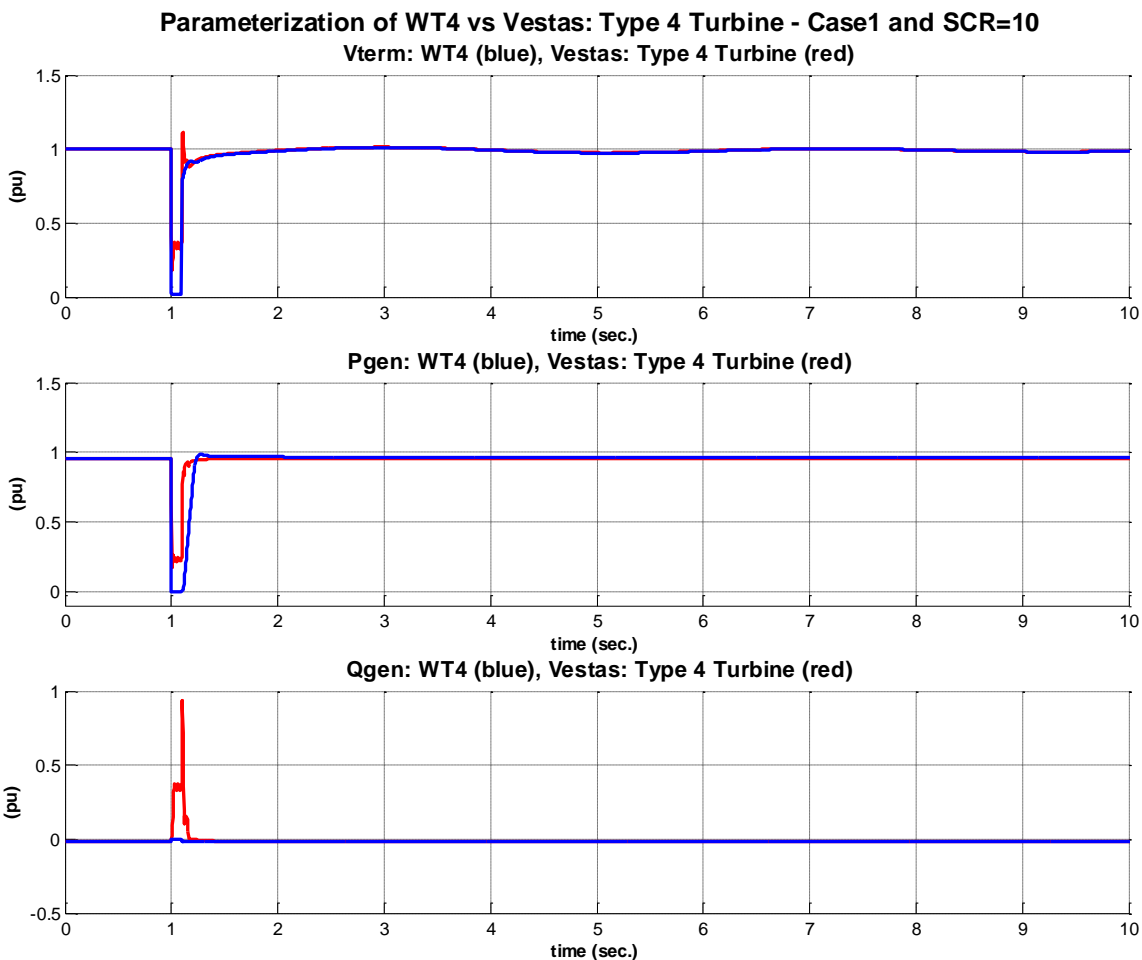

Figure 7-193: Parameterization of WT4 vs Vestas: Type 4 turbine - Case 1 and SCR=10 $\left(\mathrm{V}_{\text {Term }}, \mathbf{P}, \mathbf{Q}\right)$. 

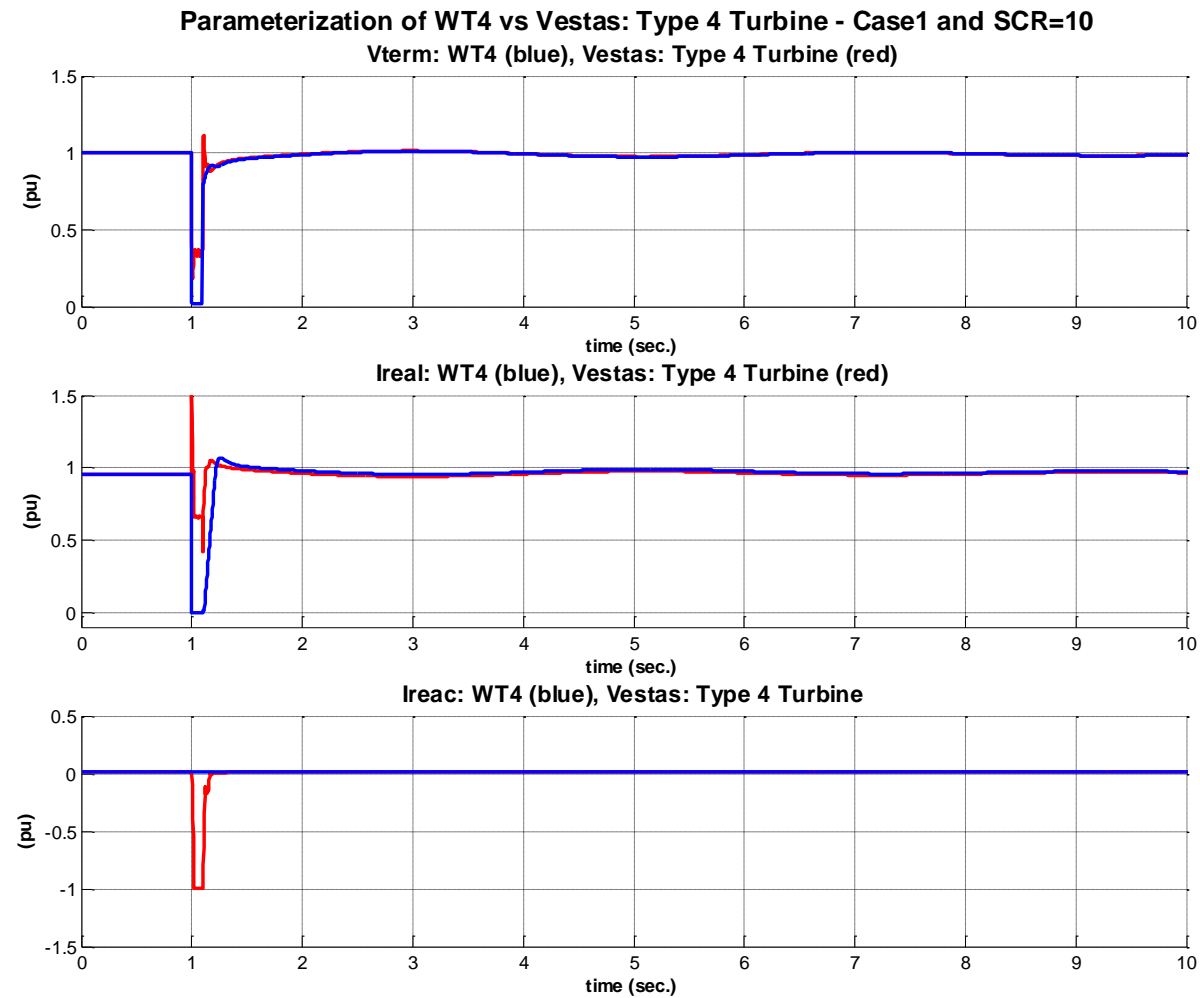

Figure 7-194: Parameterization of WT4 vs Vestas: Type 4 turbine - Case 1 and SCR=10 $\left(\mathrm{V}_{\text {Term }}, \mathrm{I}_{\text {Real }}, \mathrm{I}_{\text {Reac }}\right)$. 


\subsection{Case 2 - Under-Frequency Event}

\section{$\underline{\mathrm{SCR}-5}$}
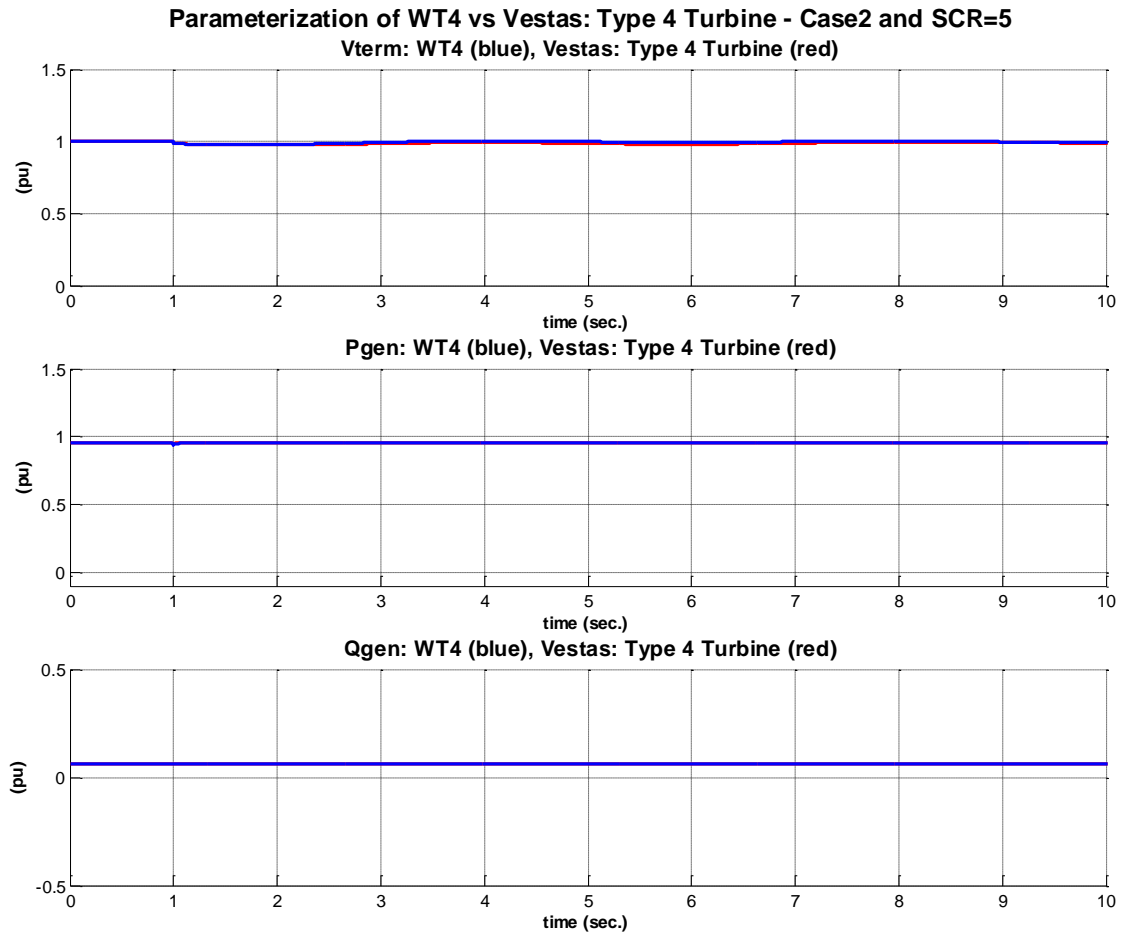

Figure 7-195: Parameterization of WT4 vs Vestas: Type 4 turbine - Case 2 and SCR=5 $\left(\mathrm{V}_{\text {Term }}, \mathrm{P}, \mathrm{Q}\right)$.
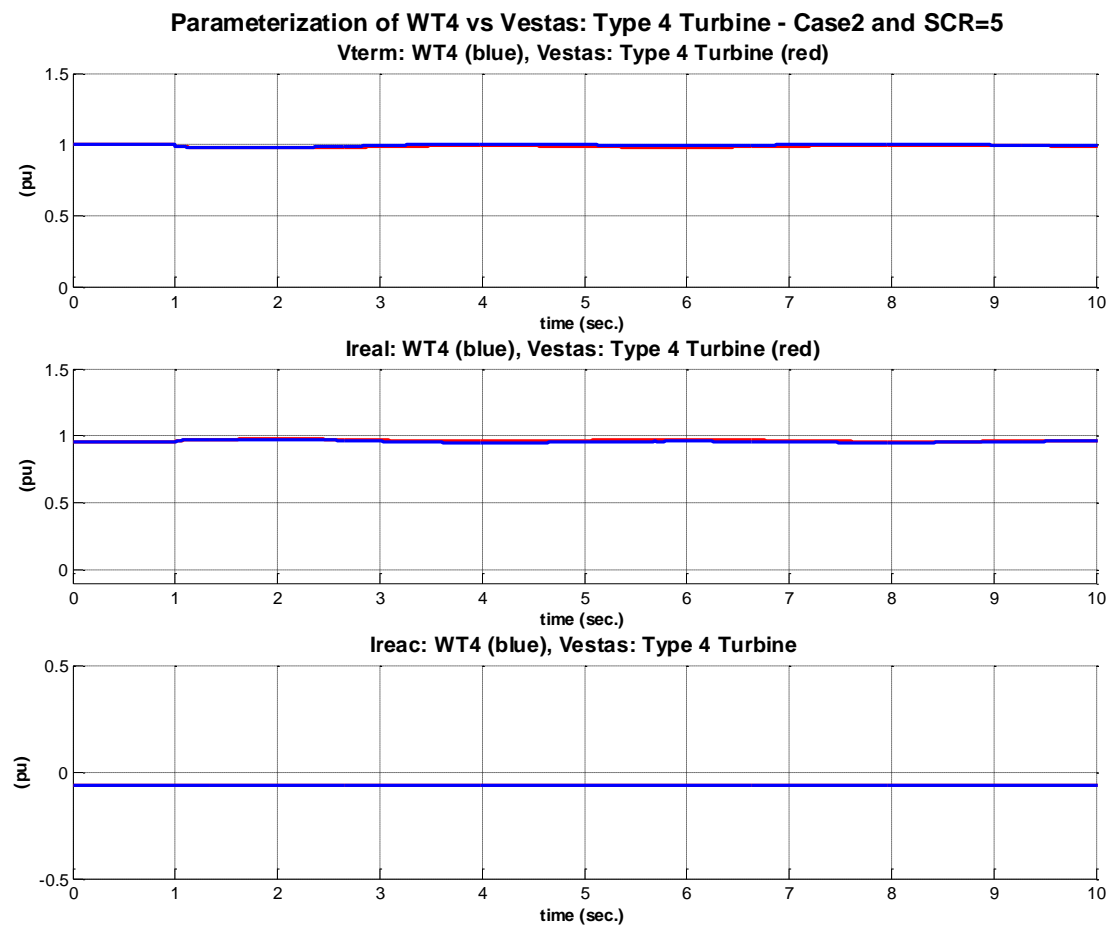

Figure 7-196: Parameterization of WT4 vs Vestas: Type 4 turbine - Case 2 and SCR=5 $\left(V_{\text {Term }}, I_{\text {Real }}, I_{\text {Reac }}\right)$. 
$\underline{\text { SCR }-10}$
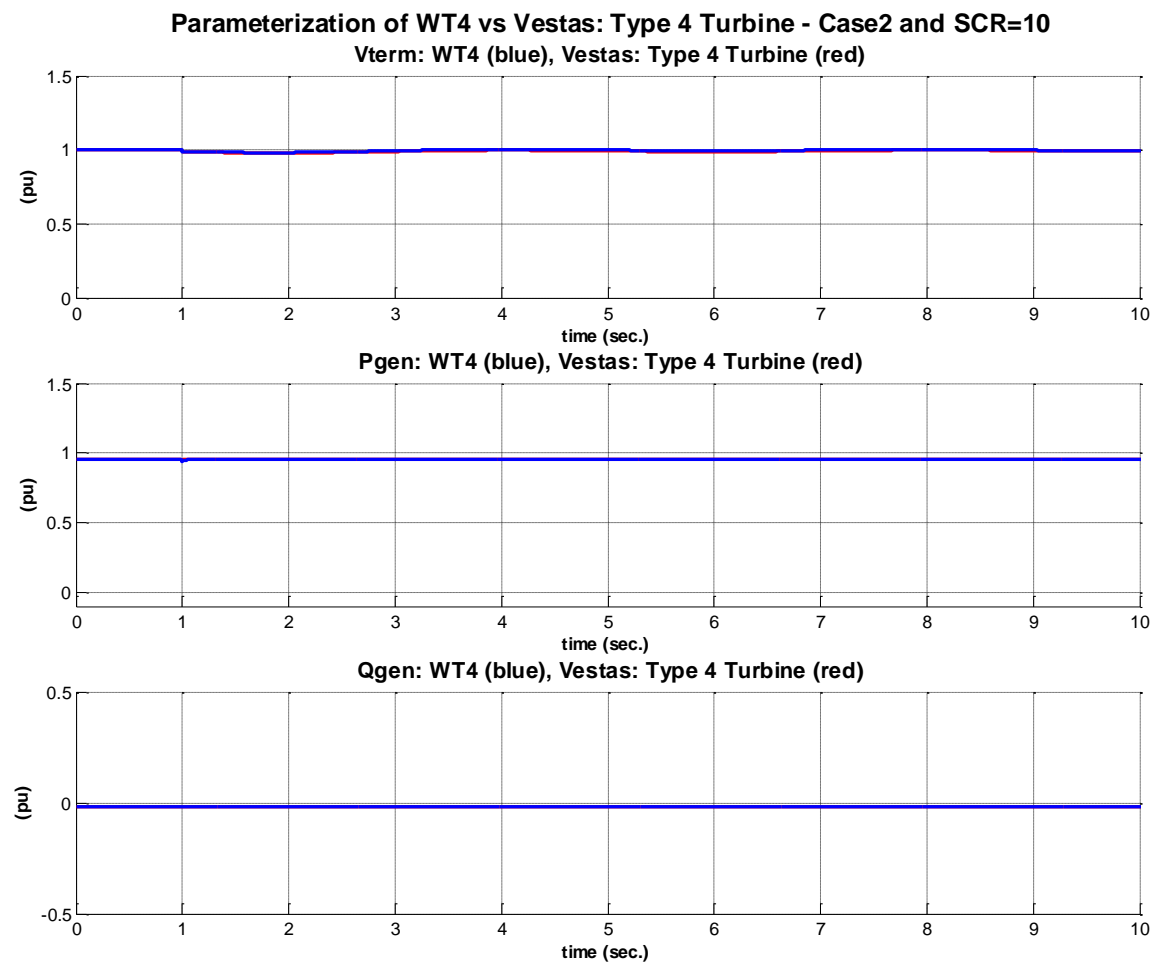

Figure 7-197: Parameterization of WT4 vs Vestas: Type 4 turbine - Case 2 and SCR=10 $\left(\mathbf{V}_{\text {Term }}, \mathbf{P}, \mathbf{Q}\right)$.
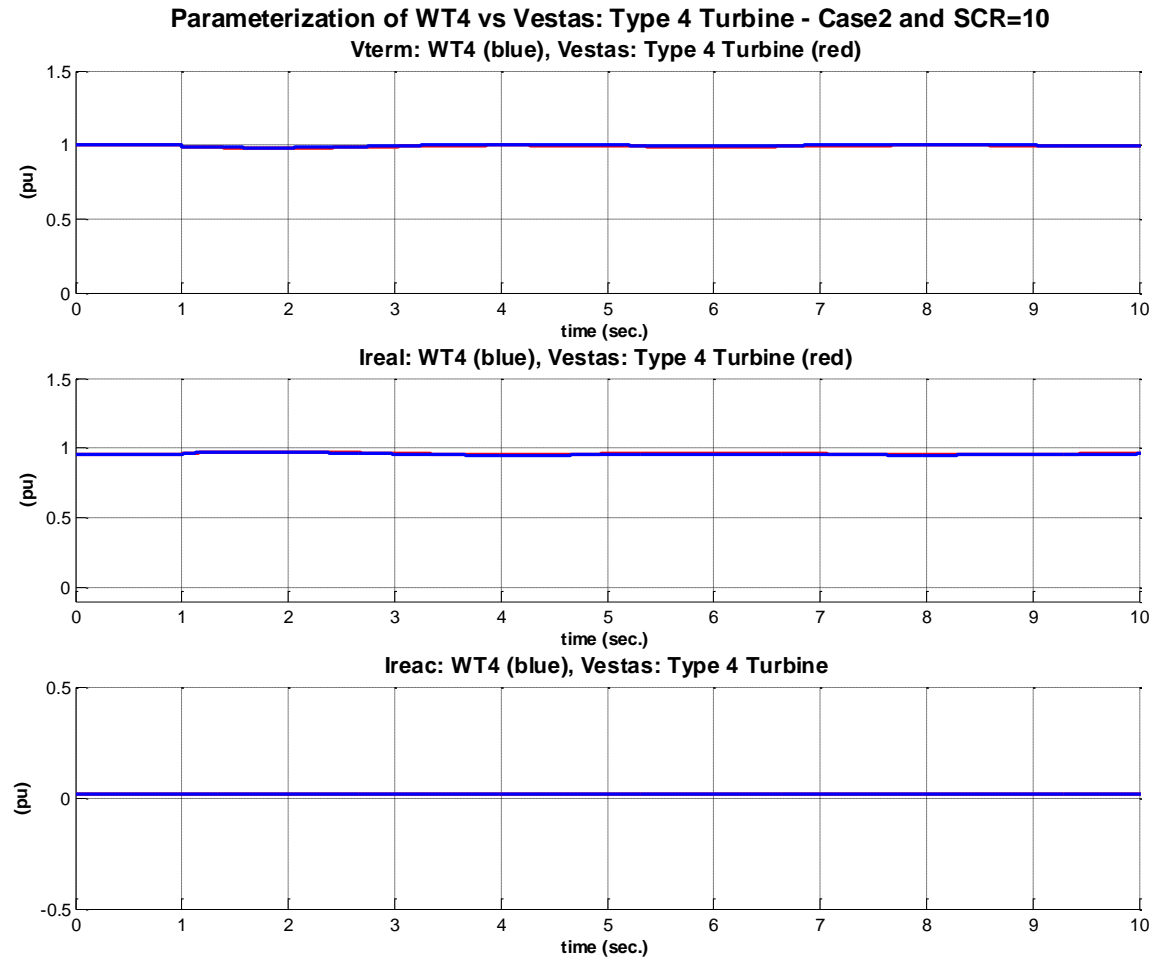

Figure 7-198: Parameterization of WT4 vs Vestas: Type 4 turbine - Case 2 and SCR=10 $\left(V_{\text {Term }}, I_{\text {Real }}, I_{\text {Reac }}\right)$. 


\subsection{Case 3 - Over-Frequency Event}

\section{SCR - 5}
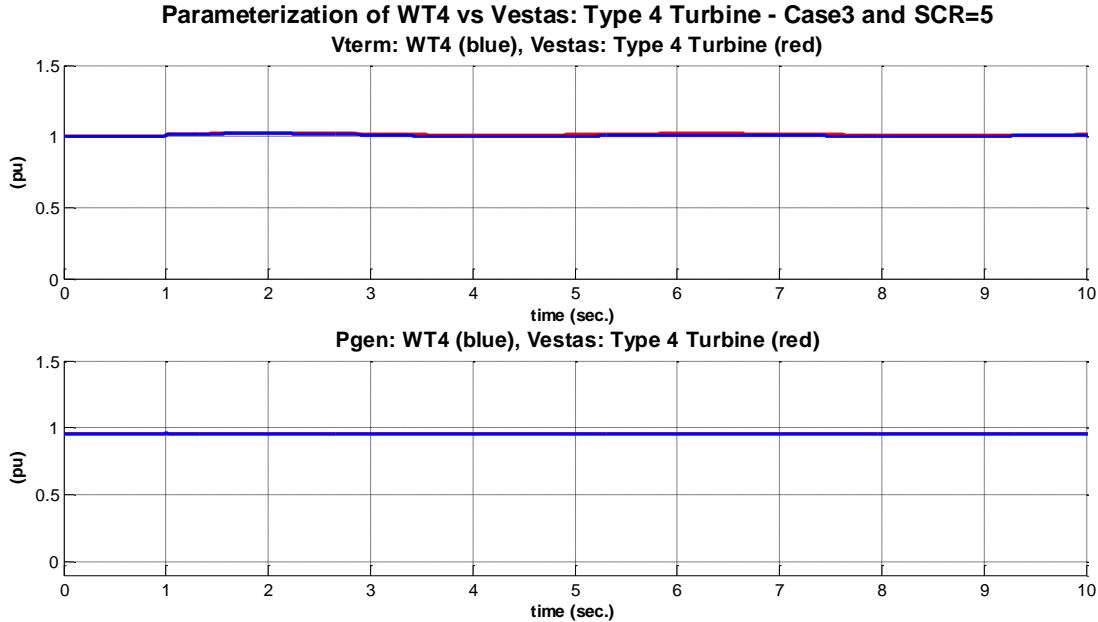

Qgen: WT4 (blue), Vestas: Type 4 Turbine (red)

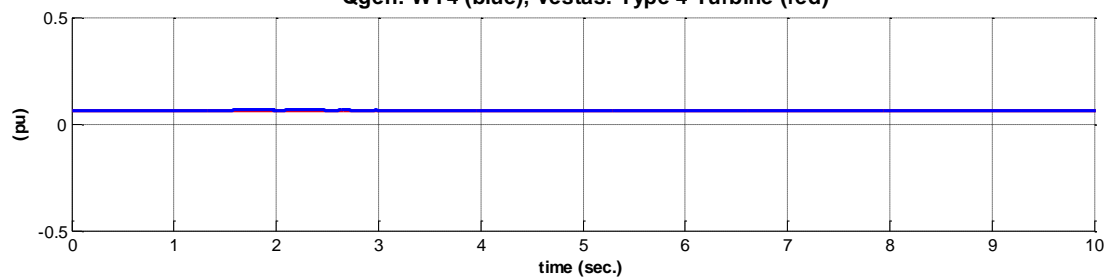

Figure 7-199: Parameterization of WT4 vs Vestas: Type 4 turbine - Case 3 and SCR=5 $\left(\mathrm{V}_{\text {Term }}, \mathrm{P}, \mathrm{Q}\right)$.

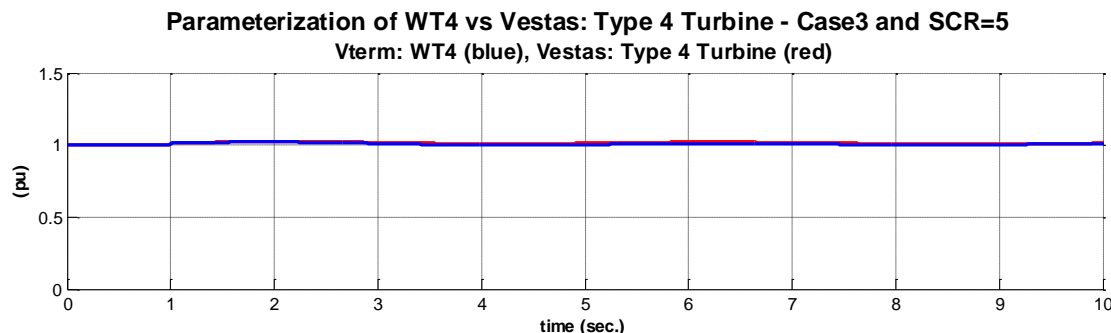

Ireal: WT4 (blue), Vestas: Type 4 Turbine (red)
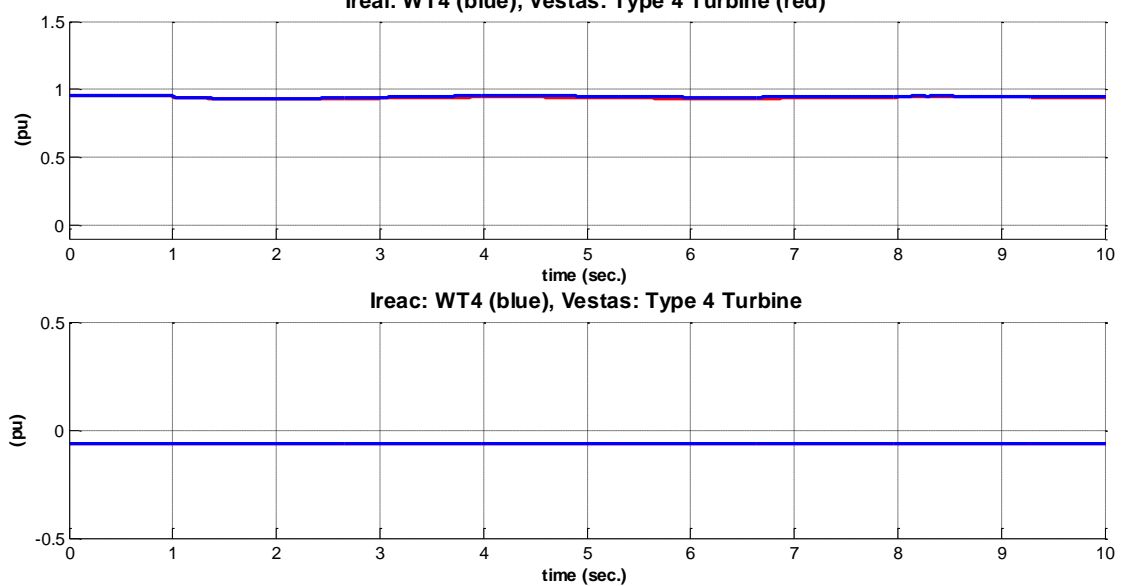

Figure 7-200: Parameterization of WT4 vs Vestas: Type 4 turbine - Case 3 and SCR=5 $\left(V_{\text {Term }}, I_{\text {Real }}, I_{\text {Reac }}\right)$. 
$\underline{\text { SCR }-10}$
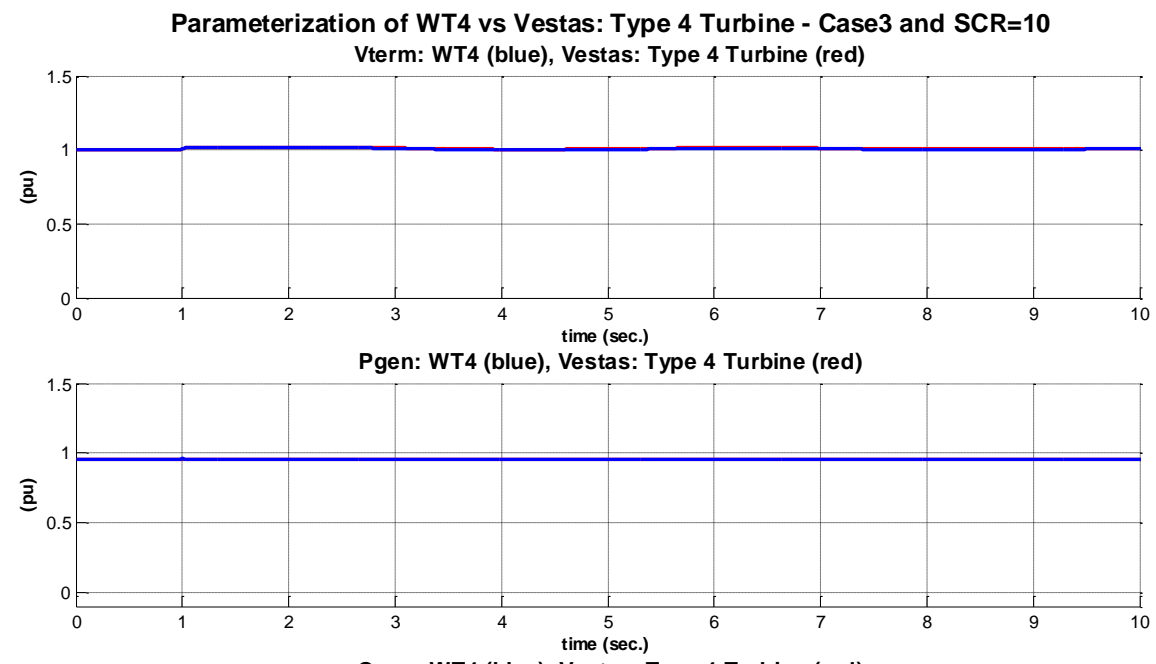

Qgen: WT4 (blue), Vestas: Type 4 Turbine (red)

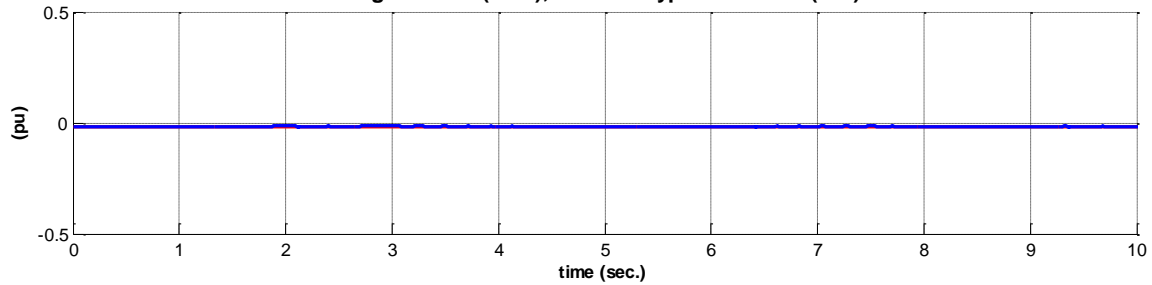

Figure 7-201: Parameterization of WT4 vs Vestas: Type 4 turbine - Case 3 and SCR=10 $\left(\mathrm{V}_{\text {Term }}, \mathrm{P}, \mathrm{Q}\right)$.
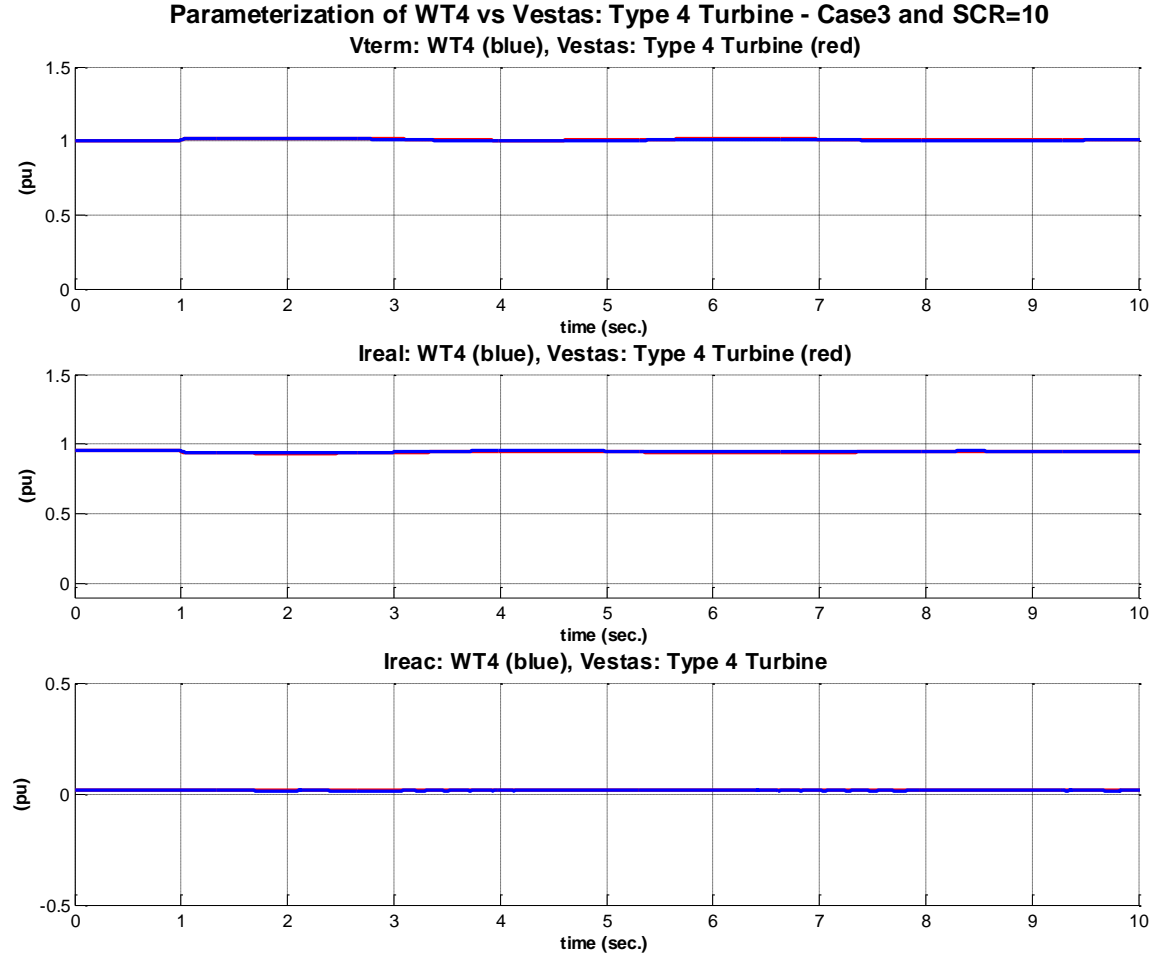

Figure 7-202: Parameterization of WT4 vs Vestas: Type 4 turbine - Case 3 and SCR=10 $\left(V_{\text {Term }}, I_{\text {Real }}, I_{\text {Reac }}\right)$. 


\subsubsection{Results Validation}

For cases 2 and 3 (over frequency and under frequency events), the response of the generic model matches the response of the VSM in the frequency range of interest. However, for case 1 (fault event), the response of the generic model matches the response of the VSM poorly. In other words, while the generic models are a good representation of the Vestas: Type 4 VSM behavior during frequency events, they are rather a poor alternative for severe fault events. 


\subsubsection{Vendor Specific Model Validation ${ }^{1}$}

The dynamic Low Voltage Right Through (LVRT) performance during different residual voltage levels and dip durations is documented in this section. Specifically, in Figure 7-203 through Figure 7-208 detailed PSCAD model behavior is compared against the vendor specific PSS/e implementation of type 4 turbines.

\section{LVRT Performance During a 0.9 pu Residual Voltage and a Dip Duration of 0.5 seconds}
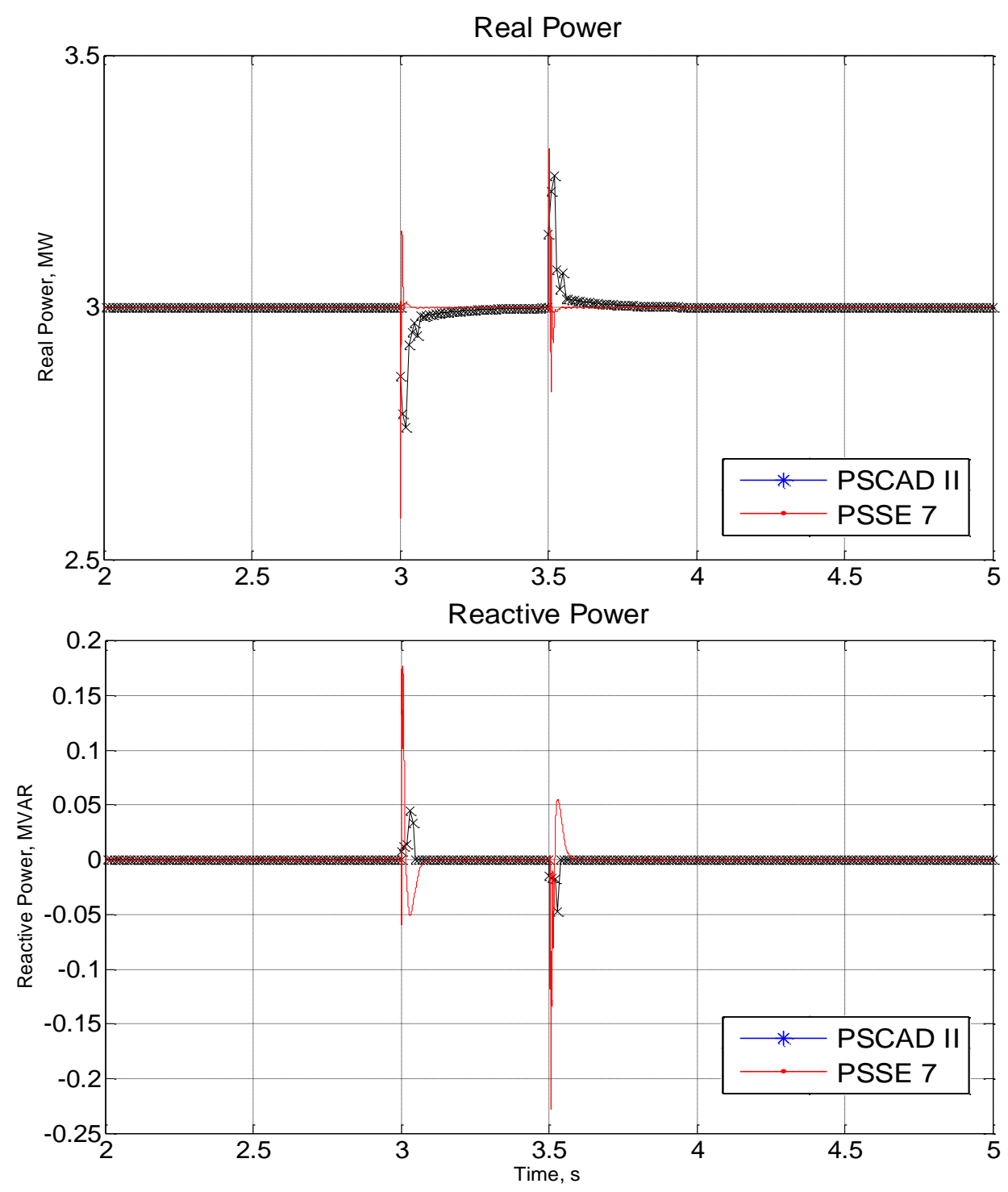

Figure 7-203: Active power and reactive power validation during a 0.9 pu residual voltage and a dip duration of $0.5 \mathrm{sec}$.

\footnotetext{
${ }^{1}$ All figures in this Section are courtesy of Vestas
} 

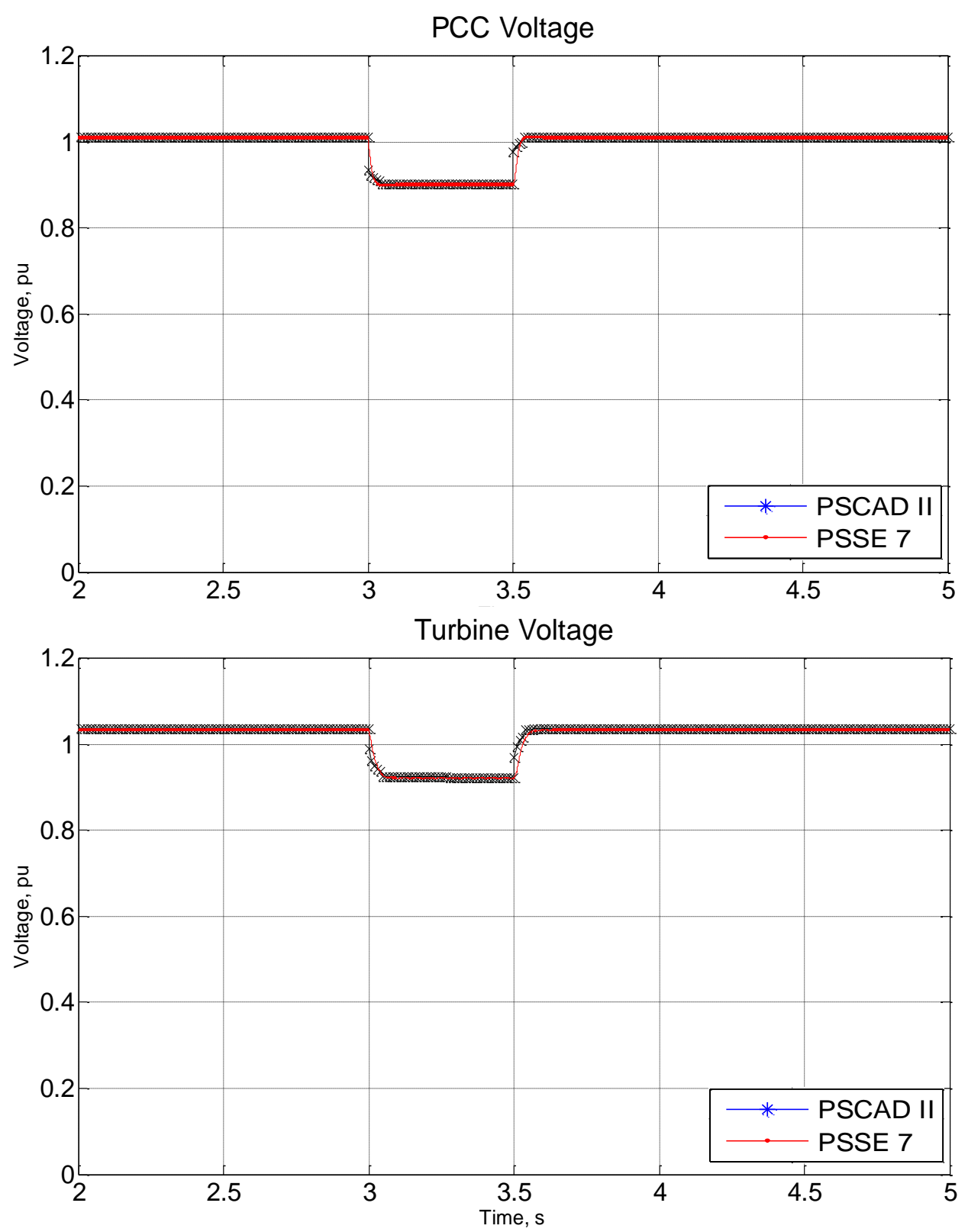

Figure 7-204: PCC voltage and stator voltage validation during a 0.9 pu residual voltage and a dip duration of $0.5 \mathrm{sec}$. 
LVRT Performance During a 0.5 pu Residual Voltage and a Dip Duration of 0.5 seconds
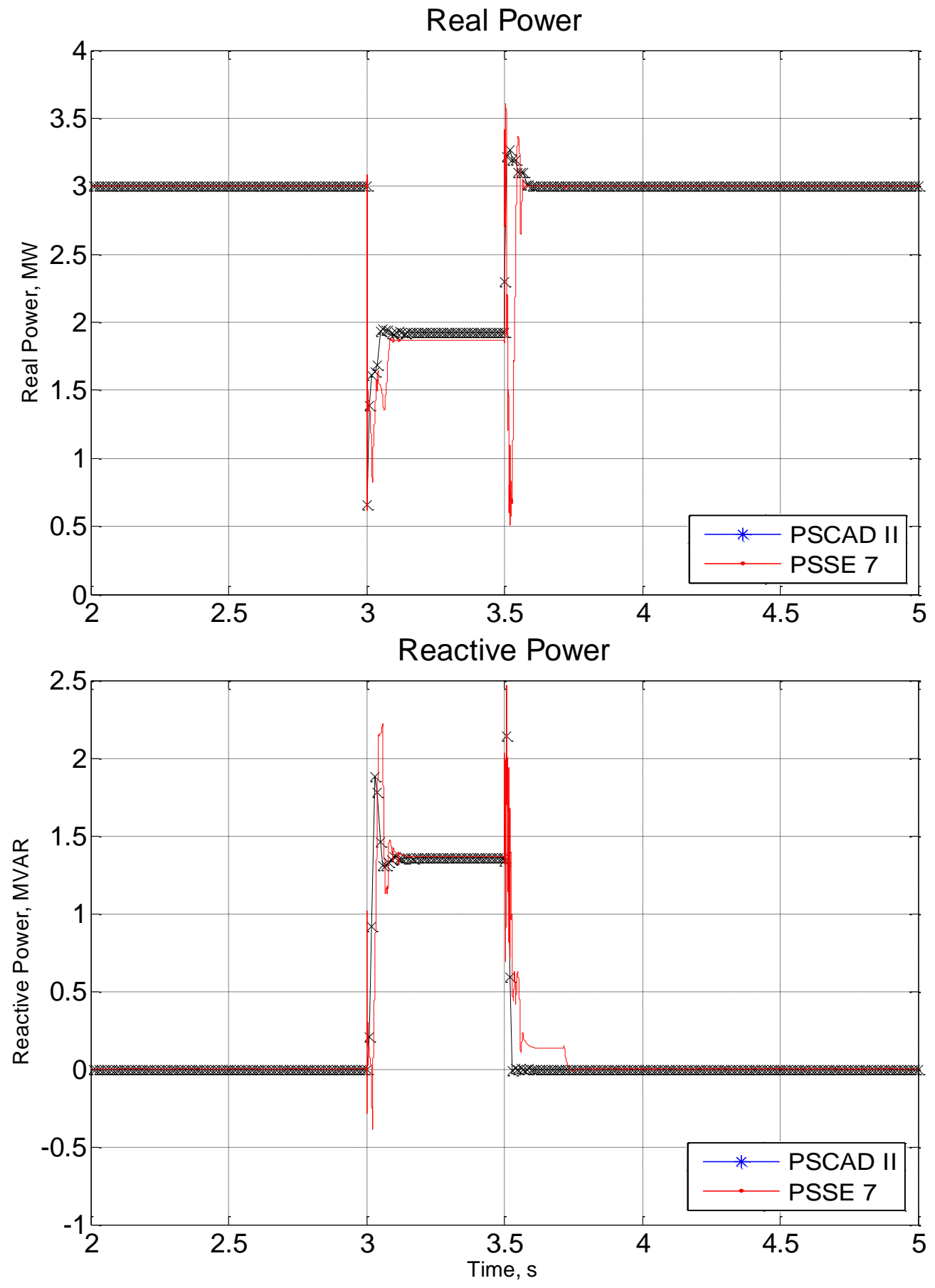

Figure 7-205: Active power and reactive power validation during a 0.5 pu residual voltage and a dip duration of $0.5 \mathrm{sec}$. 

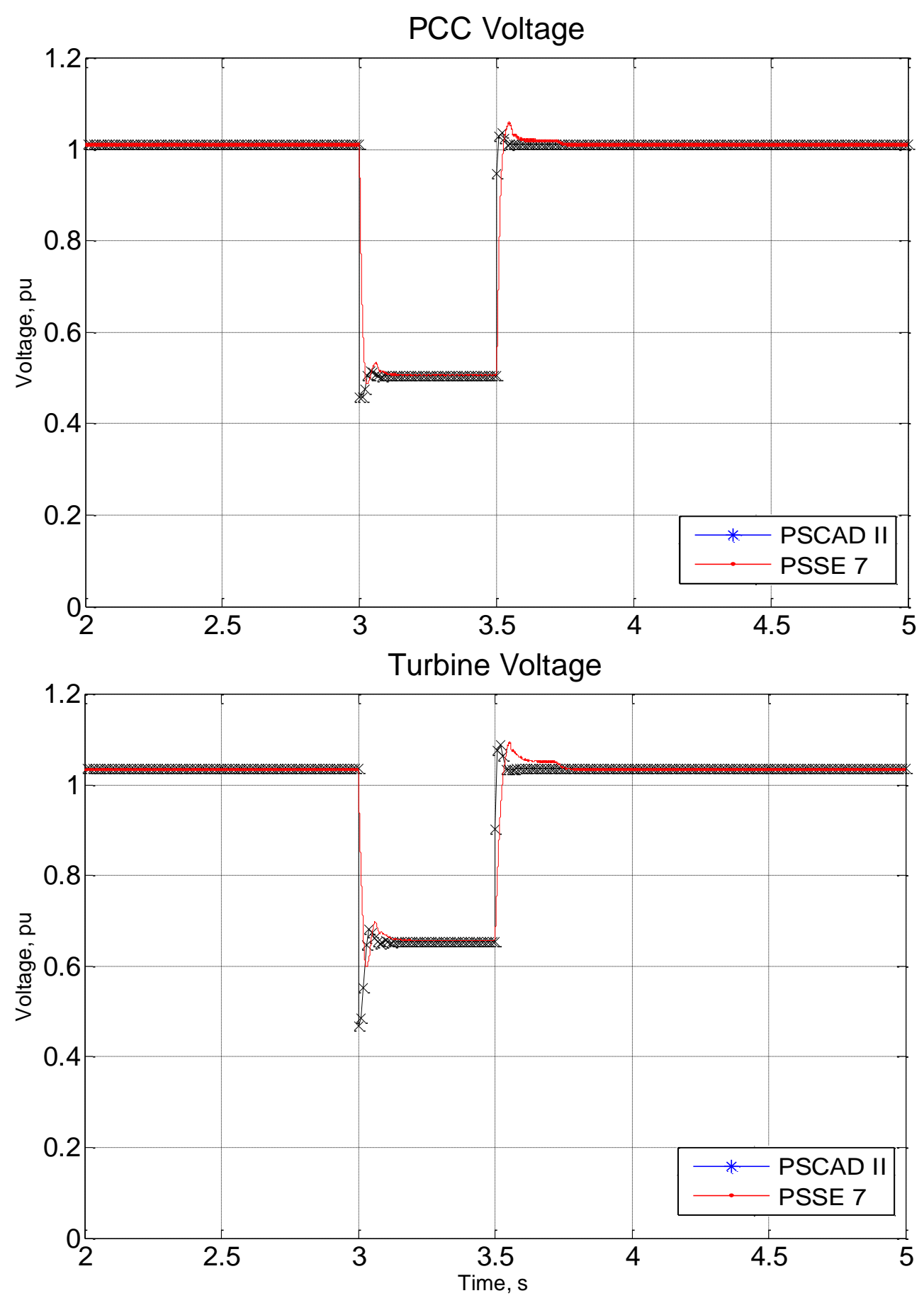

Figure 7-206: PCC voltage and stator voltage validation during a $0.5 \mathrm{pu}$ residual voltage and a dip duration of $0.5 \mathrm{sec}$. 
LVRT Performance During a 0.2 pu Residual Voltage and a Dip Duration of 0.3 seconds
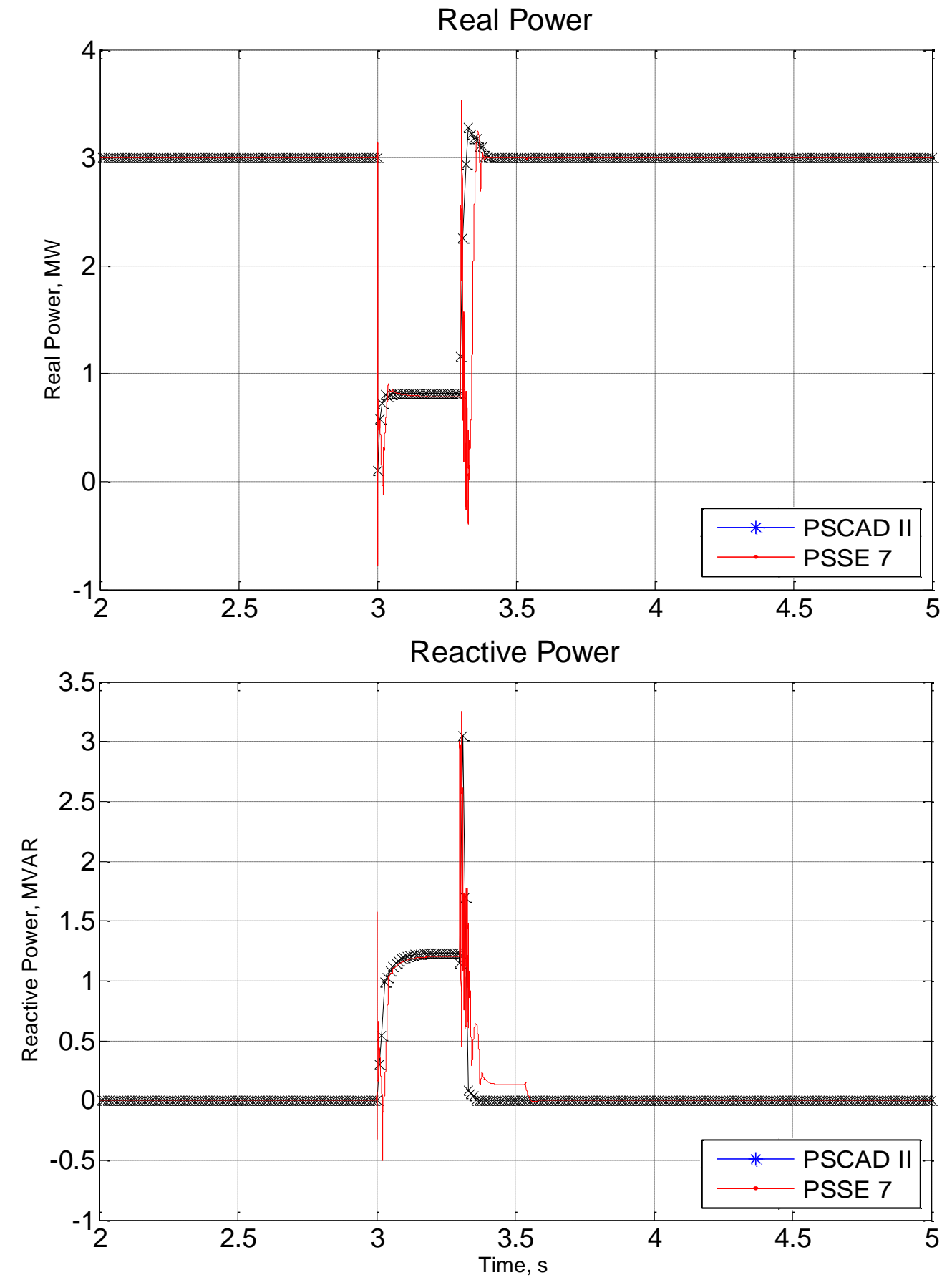

Figure 7-207: Active power and reactive power validation during a 0.2 pu residual voltage and a dip duration of $0.3 \mathrm{sec}$. 

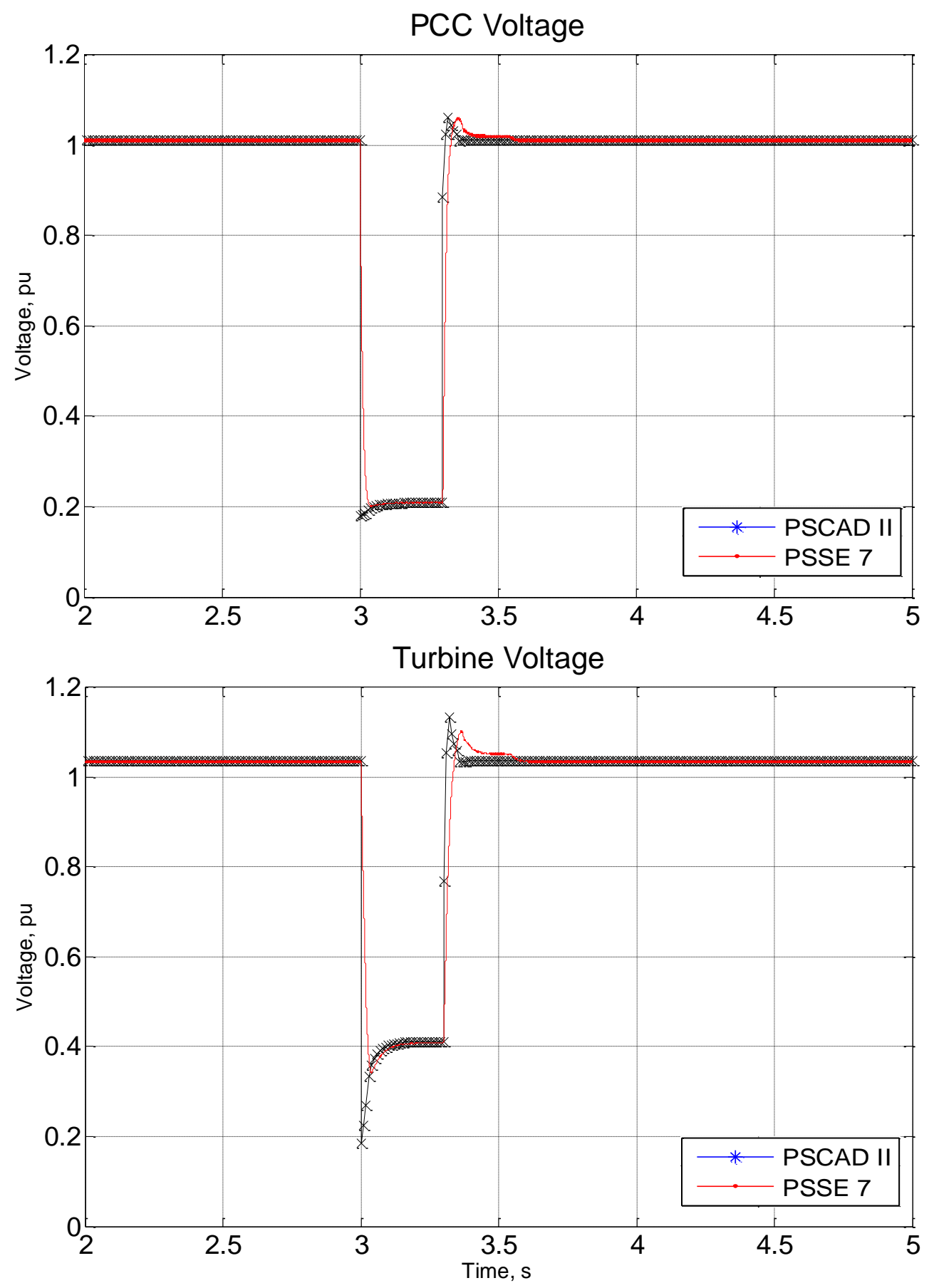

Figure 7-208: PCC voltage and stator voltage validation during a 0.2 pu residual voltage and a dip duration of $0.3 \mathrm{sec}$. 


\section{General Discussion}

\subsection{Summary}

The goal of this project was to accelerate the appropriate use of generic wind turbine models for transmission network analysis. The eventual achievement of the project goal was based on successful execution of the following tasks:

1. Complete characterization and documentation of the four generic models;

2. Comparative testing of the generic models against more detailed vendor specific models;

3. Developing recommended parameters for the generic models to best mimic the performance of specific commercial wind turbines;

4. Acquiring test data from all available sources for the purpose of validating the performance of the appropriately specified vendor specific models in actual case studies;

5. Documenting results of the comparative simulations in an application guide for users;

The project was broken into two distinct phases. The first phase focused on the existing generic models, as well as the substantial number of turbine-specific models that have been developed by vendors over the past several years. The second phase built on the first and took on the difficult task of locating and acquiring test data from the laboratory, field, or much more detailed simulation models to conduct a validation for each generic model application.

The number of turbines that was chosen to be evaluated in the project presented a major challenge. At present, there are approximately nine companies that supply nearly all the turbines to the U.S. market. These include:

- Clipper

- Gamesa

- $\mathrm{GE}$

- Mitsubishi

- Nordex 
- RePower

- Siemens

- Suzlon

- Vestas

At least four of these vendors offer turbines of different topologies (meaning that for purposes of this study, they were considered separately), bringing the total number of turbines of interest to 15.

At the point of submission of this report the evaluation process, that includes characterization, simulation, and analysis, was done for 12 turbines from six companies (i.e. Clipper, Gamesa, GE, Mitsubishi, Suzlon and Vestas). The evaluation of the remaining three models (i.e. manufactured by Nordex, RePower and Siemens) was obstructive due to internal policies implemented by these companies and/or lack of confidence [these companies have] in correct representation of their turbines by the current version of the generic models. As of late August 2012 Gamesa, Mitsubishi and Suzlon were in the process of reviewing and analyzing the provided characterization material. The project team intends to pursue the publication of the not included turbines, once the appropriate information gets released by the manufacturers. Altogether, seven turbines from three manufacturers (i.e. Clipper, GE and Vestas) were included into the current version of the project report.

While developing the recommended parameters for the generic models to best mimic the performance of specific commercial wind turbines following observations have been made (see Chapter 7):

- While the parameterization for the individual cases can be mostly done with satisfactory results, a universal set of parameters (to cover all cases) is hard to find (if at all)

- The behavior of four turbines (i.e. GE's 1.5MW and 2.5MW and Vestas' Type II) could be matched (for all cases) with the responses of the appropriate generic models.

- The behavior of the remaining turbines could only be partially matched with the responses of the appropriate generic models due to the known issues related to the current generation of the generic models (see Section 6.3).

The project team spent a lot of time and effort trying to acquire test data from all available sources for the purpose of validating the performance of the appropriately 
specified vendor specific models in actual case studies. This report contains sets of data validating three turbines (i.e. GE's 1.5MW and Vestas' Type III and Type IV). This task will maintain to be 'work in progress' over an extended period of time with any newly obtained data sets to be published as they become available.

As a part of this project, the project team developed a website - 'Wind Modeling Wiki', which [as of August 2012] contains the entire content from this report. The website can be found under the following URL:

http://www.uwig.org:8080/index.php?title=Main Page

\subsection{Recommendations}

- A greater attention in simulation model development and validation should be given to transmission-connected wind plants containing many dozens or more individual turbines interconnected by an expansive medium voltage network. Wind plant modeling techniques become of more interest to transmission planning engineers, who are specifically interested in the behavior of the plant at the point of interconnections to the transmission network.

- With rising penetration of photovoltaic (pv) generation in distribution and transmission systems similar work has to be done for solar PV systems.

- Significant amounts of work in areas of simulation models development, characterization, and parameterization has been done by national laboratories, federal agencies, electric utilities, research institutes, universities, and other parties. An effort, to identify the available information and to provide a structure for adding this information to appropriate guides, has to be undertaken.

- Going forward a mature encyclopedia-like database, covering a broad spectrum of information (e.g. model development, characterization, simulation, analysis, validation and other related topics), has to be implemented. The 'Wind Modeling Wiki', homepage mentioned above, could become such a database with appropriate coordination, editing, and maintenance efforts. The name would have to be changed to something implying a content that covers wind and pv. 


\section{Works Cited}

[1] UWIG 2006, "Wind Generation in Power Systems: A Short Course on the Integration and Interconnection of Wind Power Plants into Electric Power Systems," in UWIG Fall Workshop, Providence, RI, 2006.

[2] IEEE PES 2008, "Tutorial on Wind Generator Modeling and Controls," in IEEE PES General Meeting, Pittsburgh, PA, 2008.

[3] IEEE PES 2010, "Wind 101: Tutorial on Fundamentals of Wind Energy Conducted by Wind Power Coordinating Committee," in T\&D Conference and Exposition, New Orleans, LA, 2010.

[4] U.S. DOE (Energy Efficiency \& Renewable Energy), "Wind Powering America," 30 June 2011. [Online]. Available:

http://www.windpoweringamerica.gov/wind_installed_capacity.asp. [Accessed 9 January 2012].

[5] AWEA, "U.S. Wind Energy Projects," 30 September 2010. [Online]. Available: http://archive.awea.org/projects/default.aspx. [Accessed 9 January 2012].

[6] The Wind Power, "Wind Turbines and Wind Farms Database," December 2011. [Online]. Available: http://www.thewindpower.net/country-datasheet-zones-4usa.php. [Accessed 9 January 2012].

[7] DOE Energy Efficiency \& Renewable Energy, "2010 Wind Technology Market Report," DOE, 2011.

[8] WG Joint Report - WECC Working Group on Dynamic Performance of Wind Power Generation \& IEEE Working Group on Dynamic Performance of Wind Power Generation, "Description and Technical Specifications for Generic WTG Models - A Status Report," in Power Systems Conference and Exposition (PSCE), Phoenix, AZ, 2011.

[9] GE Energy, PSLF Version 17.0_07 User's Manual, Schenectady, NY, 2010.

[10] Siemens Energy, Inc., PSSE Wind Model Library, Schenectady, NY, 2009.

[11] Y. Kazachkov and S. Stapelton, "Does the Generic Dynamic Simulation Wind Turbine Model Exist?," in WindPower, Denver, CO, 2005.

[12] A. Ellis, E. Muljadi, J. Sanchez-Gasca and Y. Kazachkov, "Generic Models for Simulation of Wind Power Plants in Bulk System Planning Studies," in Proc. Power Engineering Society General Meeting, Detroit, MI, 2011.

[13] A. Ellis, Y. Kazachkov, E. Muljadi, P. Pourbeik and J. Sanchez-Gasca, "Description and Technical Specifications for Generic WTG Models - A Status Report," in IEEE Power Systems Conference and Exposition (PSCE), Phoenix, AZ, 2011.

[14] J. Fortmann, S. Engelhardt, J. Kretschmann, C. Feltes and I. Erlich, "Validation of an RMS DFIG Simulation Model According to New German Model Validation Standard FGW TR4 at Balanced and Unbalanced Grid Faults," in 8th International Workshop 
on Large-Scale Integration of Wind Power into Power Systems as well as on Trasmission Networks for Offshore Wind Farms, Bremen, Germany, 2009.

[15] EPRI, "Generic Models and Model Validation for Wind and Solar PV Generation," EPRI, Palo Alto, CA, 2011.

[16] F. Katiraei and J. R. Aguero, "Solar PV Integration Challenges," IEE Power \& Energy Magazine, pp. 62-71, May/June 2011.

[17] T. McDermott, "Voltage Control and Voltage Fluctuations in Distributed Resource Interconnection Projects," in IEEE PES Transmission and Distribution Conference and Exposition, New Orleans, LA, 19-22 April 2010.

[18] J. Smith, "Analytical Evaluation of Flicker Meter Algorithms," Master's Thesis, Dept. of Electrical and Computer Engineering, Mississippi State University, 1998.

[19] T. A. Short, Distribution Reliability and Power Quality, Boca Raton: CRC Press, 2006.

[20] P. F. Riberio, "Common Misapplications of the IEEE 519 Harmonic Standard: Voltage or Current Limits," 2008.

[21] J. Enslin and P. Heskes, "Harmonic interaction between a large number of distributed power inverters and the distribution network," IEEE Transaction on Power Electronics, Vols. 19, No. 6, November 2004.

[22] T. Short and C. Perry, "Overcurrent Protection Approaches to Improving Distribution Reliability," in IEEE/PES Transmission and Distribution Conference and Exhibition, May 2006.

[23] UWIG, "Application Guide for Distributed Wind Interconnection," Utility Wind Integration Group, 2010.

[24] R. Walling, R. Saint, R. Dugan, J. Burke and L. Kojovic, "Summary of Distributed Resources Impact on Power Delivery Systems," IEEE Transactions on Power Delivery, vol. 23, no. 3, July 2008.

[25] IEEE PES Wind Plant Collector System Design WG, "Wind Power Plant Grounding, Overvoltage Protection, and Insulation Coordination," in PES General Meeting, Calgary, July 2009.

[26] Cooper Power Systems, Electrical Distribution System Protection, 3rd Editition ed., Waukesha, WI: Cooper Power Systems, 1990.

[27] R. Walling, E. Gursoy and B. English, "Current Contributions from Type 3 and Type 4 Wind Turbine Generators During Faults," in PES IEEE General Meeting, Detroit, 2011.

[28] P. Kundur, Power System Stability and Control, McGraw-Hill Inc., 1994.

[29] T. Demiray, Simulation of Power System Dynamics using Dynamic Phasor Models, PhD dissertation for the Swiss Federal Institute of Technology Zurich, 2008.

[30] T. Lund, J. Eek, S. Uski and A. Perdanna, "Dynamic fault simulation of wind turbines using commercial simulation tools," in Fifth International Workshop on Large-Scale Integration of Wind Power and Transmission Networks for Offshore Wind Farms, Glasgow, 2005. 
[31] P. Torcellini, S. Pless, D. M. and C. D., "Zero Energy Buildings: A Critical Look at the Definition," in ACEEE Summary Study, California, 2006.

[32] Ontario Energy Board, "Smart Meters and Time-of-Use (TOU) prices," 20122011. [Online]. Available:

http://www.ontarioenergyboard.ca/OEB/Consumers/Electricity/Smart+Meters. [Accessed 3012 2011].

[33] Ontario Energy Board, "2011 Holiday Schedule for Smart Meter (Time-of-Use) Prices (Regulated Price Plan)," [Online]. Available: http://www.oeb.gov.on.ca/OEB/_Documents/EB-20040205/rpp_2011_tou_holidayschedule.pdf. [Accessed 3012 2011].

[34] Ontario Power Authority, "microFIT Price Schedule - revised August 13, 2010," 138 2010. [Online]. Available: http://microfit.powerauthority.on.ca/pdf/microFITProgram-price-schedule.pdf. [Accessed 3012 2011].

[35] W. Jewell, R. Ramakumar and S. Hill, "A study of dispersed PV Generation on the PSO System," IEEE Transaction on Energy Conversion, vol. 3, no. 3, pp. 473-478, September 1988.

[36] M. Ropp, J. Newmiller, C. Whitaker and B. Norris, "Review of potential problems and utility concerns arising from high penetration levels of photovoltaics in distribution systems," in Photovoltaic Specialists Conference, 2008. PVSC ', 2008.

[37] EPRI, "Photovoltaic Generation Effects on Distribution Feeders, Volume 1: Description of the Gardner, Massachusetts, Twenty-First Century PV Community abd Research Program," EPRI, 1990.

[38] D. Cyganski, J. Orr, A. Chakravorti, A. Emanuel, E. Gulachenski, C. Roots and R. Bellemare, "Current and Voltage Harmonic Measurement at the Gardner Photovoltaic Project," IEEE Transactions on Power Transactions on Power Delivery, pp. 800-809, 1989.

[39] W. Jewell and T. Unruh, "Limits on Cloud-Induced Fluctuation in Photovoltaic Generation," IEEE Transactions on Energy Conversion, pp. 8-14, 1990.

[40] H. Asano, K. Yajima and Y. Kaya, "Influence of Photovoltaic Power Generation on Required Capacity for Load Frequency Control," IEEE Transactions on Energy Conversion , pp. 188-193, 1996.

[41] Povisen, A., "International Energy Agency report IEA PVPS T5-10," IEA, 2002.

[42] General Electric Corporate R\&D, "DG Power Quality, Protection, and Reliability Case Studies Report (NREL report NRELISR-560-34635)," NREL, 2003.

[43] H. Kobayashi and M. Takasaki, "Demonstration Study of Autonomous Demand Area Power System," in Power Engineering Society Transmission and Distribution Conference, 2006.

[44] Dispower, "Distributed Generation with High Penetration of Renewable Energy Sources, Final Public Report," Dispower, 2006.

[45] S. Chalmers, M. Hitt, J. Underhill, P. Anderson, P. Vogt and R. Ingersoll, "The effect of photovoltaic power generation on utility operation," IEEE Transactions on Power 
Apparatus and Systems, vol. 104, no. 3, pp. 524-530., March 1985.

[46] N. Patapoff and D. Mattijetz, "Utility Interconnection Experience with an Operating Central Station MW-Sized Photovoltaic Plant," IEEE Transactions on Power Systems and Apparatus, vol. 104, no. 8, pp. 2020-2024, August 1985.

[47] D. Garrett and S. Jeter, "A Photovoltaic Voltage Regulation Impact Investigation Technique: Part I-Model Development," IEEE Transactions on Energy Conversion, vol. 4, no. 1, pp. 47-53, 1989.

[48] IEEE Std. 1547-2003, IEEE Standard for Interconnecting Distributed Resources with Electric Power Systems, 2003.

[49] IEEE Std. 1547.2-2008, IEEE Application Guide for IEEE Std. 1547, 2008.

[50] C. Klumpner, "A Power Electronic Controlled Dump Load with Negligible Harmonics for Accurate Loading Used in Testing Small Wind Turbines," in IEEE International Symposium on Industrial Electonics (ISIE), 2010.

[51] J. D. Glover, M. S. Sarma and T. Overbye, Power Systems Analysis and Design, Thompson, 2008. 